\title{
HTR-Proteus Pebble Bed Experimental Program Cores 9 \& 10: Columnar Hexagonal Point-on- Point Packing with a 1:1 Moderator-to-Fuel Pebble Ratio
}

John D. Bess

James W. Sterbentz

J. Blair Briggs

Hans D. Gougar

Luka Snoj

Igor Lengar

Oliver Köberl

March 2014

The INL is a U.S. Department of Energy National Laboratory operated by Battelle Energy Alliance

Idaho National 
INL/EXT-12-26334

Rev. 1

\title{
HTR-Proteus Pebble Bed Experimental Program Cores 9 \& 10: Columnar Hexagonal Point-on-Point Packing with a 1:1 Moderator-to-Fuel Pebble Ratio
}

\author{
John D. Bess \\ James W. Sterbentz \\ J. Blair Briggs \\ Hans D. Gougar \\ Luka Snoj $^{1}$ \\ Igor Lengar ${ }^{1}$ \\ Oliver Köberl ${ }^{2}$ \\ 1Jožef Stefan Institute \\ ${ }^{2}$ Paul Scherrer Institut
}

March 2014

Idaho National Laboratory

Idaho Falls, Idaho 83415

http://www.inl.gov

Prepared for the

U.S. Department of Energy

Office of Nuclear Energy

Under DOE Idaho Operations Office

Contract DE-AC07-05ID14517 


\title{
HTR-PROTEUS PEBBLE BED EXPERIMENTAL PROGRAM CORES 9 \& 10: COLUMNAR HEXAGONAL POINT-ON- POINT PACKING WITH A 1:1 MODERATOR-TO-FUEL PEBBLE RATIO
}

\author{
Evaluator \\ John D. Bess \\ Idaho National Laboratory \\ Internal Reviewers \\ James W. Sterbentz (CRIT) \\ J. Blair Briggs (REAC) \\ Hans D. Gougar (REAC) \\ Idaho National Laboratory
}

\author{
Independent Reviewers \\ Luka Snoj \\ Igor Lengar \\ Jožef Stefan Institute \\ Oliver Köberl \\ Paul Scherrer Institut
}


Gas Cooled (Thermal) Reactor - GCR

PROTEUS-GCR-EXP-004

CRIT-REAC

\section{ACKNOWLEDGMENTS}

The author would like to express gratitude for the initial efforts performed by Barbara H. Dolphin, William K. Terry, and Charles A. Wemple at the Idaho National Laboratory as well as Luka Snoj and Igor Lengar at the Jožef Stefan Institute.

This project was supported by the U.S. Department of Energy, Assistant Secretary for Nuclear Energy, under DOE Idaho Operations Office Contract DE-AC07-05ID14517.

The author would particularly like to acknowledge the indispensable work of Christine E. White in the preparation and deciphering of most of the drawings and graphics. IRPhEP working group provided much appreciated assistance, useful comments, and observations in the final preparation of this evaluation. 
NEA/NSC/DOC(2006)1

Gas Cooled (Thermal) Reactor - GCR

PROTEUS-GCR-EXP-004

CRIT-REAC

Status of Compilation / Evaluation / Peer Review

\begin{tabular}{|c|c|c|c|c|c|}
\hline \multicolumn{2}{|r|}{ Section 1} & \multirow[t]{2}{*}{ Compiled } & \multirow[t]{2}{*}{$\begin{array}{c}\text { Independent } \\
\text { Review }\end{array}$} & \multirow[t]{2}{*}{$\begin{array}{c}\text { Working Group } \\
\text { Review }\end{array}$} & \multirow[t]{2}{*}{ Approved } \\
\hline 1.0 & DETAILED DESCRIPTION & & & & \\
\hline 1.1 & $\begin{array}{l}\text { Description of the Critical and / or } \\
\text { Subcritical Configuration }\end{array}$ & YES & YES & YES & YES \\
\hline 1.2 & $\begin{array}{l}\text { Description of Buckling and } \\
\text { Extrapolation Length Measurements }\end{array}$ & NA & NA & NA & NA \\
\hline 1.3 & $\begin{array}{l}\text { Description of Spectral Characteristics } \\
\text { Measurements }\end{array}$ & NA & NA & NA & NA \\
\hline 1.4 & $\begin{array}{l}\text { Description of Reactivity Effects } \\
\text { Measurements }\end{array}$ & YES & YES & YES & YES \\
\hline 1.5 & $\begin{array}{l}\text { Description of Reactivity Coefficient } \\
\text { Measurements }\end{array}$ & NA & NA & NA & NA \\
\hline 1.6 & Description of Kinetics Measurements & NA & NA & NA & NA \\
\hline 1.7 & $\begin{array}{l}\text { Description of Reaction-Rate } \\
\text { Distribution Measurements }\end{array}$ & NA & NA & NA & NA \\
\hline 1.8 & $\begin{array}{l}\text { Description of Power Distribution } \\
\text { Measurements }\end{array}$ & NA & NA & NA & NA \\
\hline 1.9 & Description of Isotopic Measurements & NA & NA & NA & NA \\
\hline 1.10 & $\begin{array}{l}\text { Description of Other Miscellaneous } \\
\text { Types of Measurements }\end{array}$ & NA & NA & NA & NA \\
\hline & Section 2 & Evaluated & $\begin{array}{c}\text { Independent } \\
\text { Review }\end{array}$ & $\begin{array}{c}\text { Working Group } \\
\text { Review }\end{array}$ & Approved \\
\hline 2.0 & $\begin{array}{l}\text { EVALUATION OF EXPERIMENTAL } \\
\text { DATA }\end{array}$ & & & & \\
\hline 2.1 & $\begin{array}{l}\text { Evaluation of Critical and / or Subcritical } \\
\text { Configuration Data }\end{array}$ & YES & YES & YES & YES \\
\hline 2.2 & $\begin{array}{l}\text { Evaluation of Buckling and Extrapolation } \\
\text { Length Data }\end{array}$ & NA & NA & NA & NA \\
\hline 2.3 & $\begin{array}{l}\text { Evaluation of Spectral Characteristics } \\
\text { Data }\end{array}$ & NA & NA & NA & NA \\
\hline 2.4 & Evaluation of Reactivity Effects Data & YES & YES & YES & YES \\
\hline 2.5 & Evaluation of Reactivity Coefficient Data & NA & NA & NA & NA \\
\hline 2.6 & $\begin{array}{l}\text { Evaluation of Kinetics Measurements } \\
\text { Data }\end{array}$ & NA & NA & NA & NA \\
\hline 2.7 & $\begin{array}{l}\text { Evaluation of Reaction Rate } \\
\text { Distributions }\end{array}$ & NA & NA & NA & NA \\
\hline 2.8 & Evaluation of Power Distribution Data & NA & NA & NA & NA \\
\hline 2.9 & Evaluation of Isotopic Measurements & NA & NA & NA & NA \\
\hline 2.10 & $\begin{array}{l}\text { Evaluation of Other Miscellaneous Types } \\
\text { of Measurements }\end{array}$ & NA & NA & NA & NA \\
\hline
\end{tabular}


NEA/NSC/DOC(2006)1

\section{Gas Cooled (Thermal) Reactor - GCR \\ PROTEUS-GCR-EXP-004 \\ CRIT-REAC}

\begin{tabular}{|c|c|c|c|c|c|}
\hline \multicolumn{2}{|r|}{ Section 3} & \multirow[t]{2}{*}{ Compiled } & \multirow[t]{2}{*}{$\begin{array}{c}\text { Independent } \\
\text { Review }\end{array}$} & \multirow[t]{2}{*}{$\begin{array}{c}\text { Working } \\
\text { Group Review }\end{array}$} & \multirow[t]{2}{*}{ Approved } \\
\hline 3.0 & BENCHMARK SPECIFICATIONS & & & & \\
\hline 3.1 & $\begin{array}{l}\text { Benchmark-Model Specifications for } \\
\text { Critical and / or Subcritical } \\
\text { Measurements }\end{array}$ & YES & YES & YES & YES \\
\hline 3.2 & $\begin{array}{l}\text { Benchmark-Model Specifications for } \\
\text { Buckling and Extrapolation Length } \\
\text { Measurements }\end{array}$ & NA & NA & NA & NA \\
\hline 3.3 & $\begin{array}{l}\text { Benchmark-Model Specifications for } \\
\text { Spectral Characteristics Measurements }\end{array}$ & NA & NA & NA & NA \\
\hline 3.4 & $\begin{array}{l}\text { Benchmark-Model Specifications for } \\
\text { Reactivity Effects Measurements }\end{array}$ & YES & YES & YES & YES \\
\hline 3.5 & $\begin{array}{l}\text { Benchmark-Model Specifications for } \\
\text { Reactivity Coefficient Measurements }\end{array}$ & NA & NA & NA & NA \\
\hline 3.6 & $\begin{array}{l}\text { Benchmark-Model Specifications for } \\
\text { Kinetics Measurements }\end{array}$ & NA & NA & NA & NA \\
\hline 3.7 & $\begin{array}{l}\text { Benchmark-Model Specifications for } \\
\text { Reaction-Rate Distribution } \\
\text { Measurements }\end{array}$ & NA & NA & NA & NA \\
\hline 3.8 & $\begin{array}{l}\text { Benchmark-Model Specifications for } \\
\text { Power Distribution Measurements }\end{array}$ & NA & NA & NA & NA \\
\hline 3.9 & $\begin{array}{l}\text { Benchmark-Model Specifications for } \\
\text { Isotopic Measurements }\end{array}$ & NA & NA & NA & NA \\
\hline 3.10 & $\begin{array}{l}\text { Benchmark-Model Specifications of } \\
\text { Other Miscellaneous Types of } \\
\text { Measurements }\end{array}$ & NA & NA & NA & NA \\
\hline & Section 4 & Compiled & $\begin{array}{c}\text { Independent } \\
\text { Review }\end{array}$ & $\begin{array}{c}\text { Working } \\
\text { Group Review }\end{array}$ & Approved \\
\hline 4.0 & $\begin{array}{l}\text { RESULTS OF SAMPLE } \\
\text { CALCULATIONS }\end{array}$ & & & & \\
\hline 4.1 & $\begin{array}{l}\text { Results of Calculations of the Critical or } \\
\text { Subcritical Configurations }\end{array}$ & YES & YES & YES & YES \\
\hline 4.2 & $\begin{array}{l}\text { Results of Buckling and Extrapolation } \\
\text { Length Calculations }\end{array}$ & NA & NA & NA & NA \\
\hline 4.3 & $\begin{array}{l}\text { Results of Spectral Characteristics } \\
\text { Calculations }\end{array}$ & NA & NA & NA & NA \\
\hline 4.4 & Results of Reactivity Effect Calculations & YES & YES & YES & YES \\
\hline 4.5 & $\begin{array}{l}\text { Results of Reactivity Coefficient } \\
\text { Calculations }\end{array}$ & NA & NA & NA & NA \\
\hline 4.6 & $\begin{array}{l}\text { Results of Kinetics Parameter } \\
\text { Calculations }\end{array}$ & NA & NA & NA & NA \\
\hline 4.7 & $\begin{array}{l}\text { Results of Reaction-Rate Distribution } \\
\text { Calculations }\end{array}$ & NA & NA & NA & NA \\
\hline 4.8 & $\begin{array}{l}\text { Results of Power Distribution } \\
\text { Calculations }\end{array}$ & NA & NA & NA & NA \\
\hline 4.9 & Results of Isotopic Calculations & NA & NA & NA & NA \\
\hline 4.10 & $\begin{array}{l}\text { Results of Calculations of Other } \\
\text { Miscellaneous Types of Measurements }\end{array}$ & NA & NA & NA & NA \\
\hline Sectio & on 5 & Compiled & $\begin{array}{c}\text { Independent } \\
\text { Review }\end{array}$ & $\begin{array}{c}\text { Working Group } \\
\text { Review }\end{array}$ & Approved \\
\hline 5.0 & REFERENCES & YES & YES & YES & YES \\
\hline $\begin{array}{l}\text { Apper } \\
\text { Typic }\end{array}$ & $\begin{array}{l}\text { endix A: Computer Codes, Cross Sections, and } \\
\text { cal Input Listings }\end{array}$ & YES & YES & YES & YES \\
\hline
\end{tabular}


NEA/NSC/DOC(2006)1

Gas Cooled (Thermal) Reactor - GCR

PROTEUS-GCR-EXP-004

CRIT-REAC

\title{
HTR-PROTEUS PEBBLE BED EXPERIMENTAL PROGRAM CORES 9 \& 10: COLUMNAR HEXAGONAL POINT-ON-POINT PACKING WITH A 1:1 MODERATOR-TO-FUEL PEBBLE RATIO
}

\author{
IDENTIFICATION NUMBER: PROTEUS-GCR-EXP-004 \\ CRIT-REAC
}

KEY WORDS: control rod worths, critical facility, graphite-moderated, graphite-reflected, intermediate enriched uranium dioxide, Paul Scherrer Institut, pebble bed arrangement, PROTEUS, TRISO, zero-power experiment

\section{SUMMARY}

\subsection{DETAILED DESCRIPTION}

PROTEUS is a zero-power research reactor based on a cylindrical graphite annulus with a central cylindrical cavity; it is a part of the Paul Scherrer Institute (formerly EIR, Eidgenossisches Institut fur Reaktorforschung) and is situated near Würenlingen in the canton of Aargau in northern Switzerland. The graphite annulus remains basically the same for all experimental programs, but the contents of the central cavity are changed according to the type of reactor being investigated. Through most of its service history, PROTEUS has represented light-water reactors, but from 1992 to 1996 PROTEUS was configured as a pebble-bed reactor (PBR) critical facility and designated as HTR-PROTEUS. The nomenclature was used to indicate that this series consisted of High Temperature Reactor experiments performed in the PROTEUS assembly. During this period, seventeen critical configurations were assembled and various reactor physics experiments were conducted. These experiments included measurements of criticality, differential and integral control rod and safety rod worths, kinetics, reaction rates, water ingress effects, and small sample reactivity effects (Ref. 3 ).

HTR-PROTEUS was constructed, and the experimental program was conducted, for the purpose of providing experimental benchmark data for assessment of reactor physics computer codes. Considerable effort was devoted to benchmark calculations as a part of the HTR-PROTEUS program. References 1 and 2 provide detailed data for use in constructing models for codes to be assessed. Reference 3 is a comprehensive summary of the HTR-PROTEUS experiments and the associated benchmark program. This document draws freely from these references. 
NEA/NSC/DOC(2006)1

\section{Gas Cooled (Thermal) Reactor - GCR \\ PROTEUS-GCR-EXP-004 \\ CRIT-REAC}

Four benchmark reports were prepared to document evaluation of the experimental configurations according to core packing and the moderator-to-fuel pebble ratios:

- PROTEUS-GCR-EXP-001

- $\quad$ Cores 1, 1A, 2, and 3

- Hexagonal Close Packing

- 1:2 Moderator-to-Fuel Pebble Ratio

- PROTEUS-GCR-EXP-002

- Core 4

- Random Packing

- 1:1 Moderator-to-Fuel Pebble Ratio

- PROTEUS-GCR-EXP-003

- $\quad$ Cores 5, 6, 7, and 8

- Columnar Hexagonal Point-On-Point Packing

- 1:2 Moderator-to-Fuel Pebble Ratio

- PROTEUS-GCR-EXP-004

- Cores 9 and 10

- Columnar Hexagonal Point-On-Point Packing

- 1:1 Moderator-to-Fuel Pebble Ratio

In its deployment as a pebble bed reactor critical facility from 1992 to 1996, the reactor was designated as HTR-PROTEUS. This experimental program was performed as part of an International Atomic Energy Agency (IAEA) Coordinated Research Project (CRP) on the Validation of Safety Related Physics Calculations for Low Enriched HTGRs (High Temperature Gas-cooled Reactors). Additional historical data regarding this IAEA CRP and the PROTEUS facility are provided in Appendix D (Ref. 3). Figure 1.0-1 shows a generic HTR-PROTEUS configuration.

Within this project, critical experiments were conducted for graphite moderated LEU (low enriched uranium) systems to determine core reactivity, flux and power profiles, reaction-rate ratios, the worth of control rods (both in-core and reflector based), the worth of burnable poisons, kinetic parameters, and the effects of moisture ingress on these parameters. Fuel for the experiments was provided by the KFA Research Center in Jülich, Germany. Initial criticality was achieved on July 7, 1992. These experiments were conducted over a range of experimental parameters such as carbon-to-uranium ratio $(\mathrm{C} / \mathrm{U})$, core height-to-diameter ratio, and simulated moisture concentration (Ref. 3).

In any PBR, the fuel elements are spherical "pebbles" roughly the size of billiard balls, composed of a graphite matrix in which thousands of tiny $(\sim 1 \mathrm{~mm}$ diameter) coated fuel particles are embedded. These particles are known as tristructural-isotropic (TRISO) and are composed of a central $\mathrm{UO}_{2}$ kernel surrounded by thin layers of graphite and silicon-carbide.

In the PROTEUS set of experiments, ten different core configurations were constructed and studied. Several cores had more than one reference state either to test reproducibility or further simplify or improve upon the core configuration from the previous reference state. This means that there are slight changes but the basic core configuration remains the same. Core 4 is the only configuration using randomly placed pebbles in the core barrel. All other configurations used hand-stacked pebbles in known packing configurations. The experimenters used the term "deterministic" to denote these regular core lattices. These lattices were either hexagonal close-packed (HCP) or columnar hexagonal point-on-point (CHPOP) configurations. The former arrangement can be visualized as oranges placed in a crate (Figure 1.0-2). In the latter configuration, the pebbles in successive layers form columns without any relative lateral displacement (Figure 1.0-3). The deterministic arrangements are considered much more useful for benchmarking reactor physics computer codes.

Theoretical pebble packing fractions for the HCP and CHPOP configurations are 0.7405 and 0.6046 , respectively. A reference value for the random packing of pebbles in the HTR-PROTEUS assembly is

Revision: 1

Date: March 31, 2014

Page 2 of 375 
NEA/NSC/DOC(2006)1

\section{Gas Cooled (Thermal) Reactor - GCR \\ PROTEUS-GCR-EXP-004 \\ CRIT-REAC}

0.61. ${ }^{\mathrm{a}}$ The packing fraction of the CHPOP configuration is very close to that of a PBR, as a value of 0.61 is a good approximation for the inner part of a PBR, whereas the packing fraction decreases at the core/reflector interface. ${ }^{\mathrm{b}}$

Table 1.0-1 provides a brief explanation of the cores and their reference states. Additional descriptions of each core and reference state will appear throughout the reports.

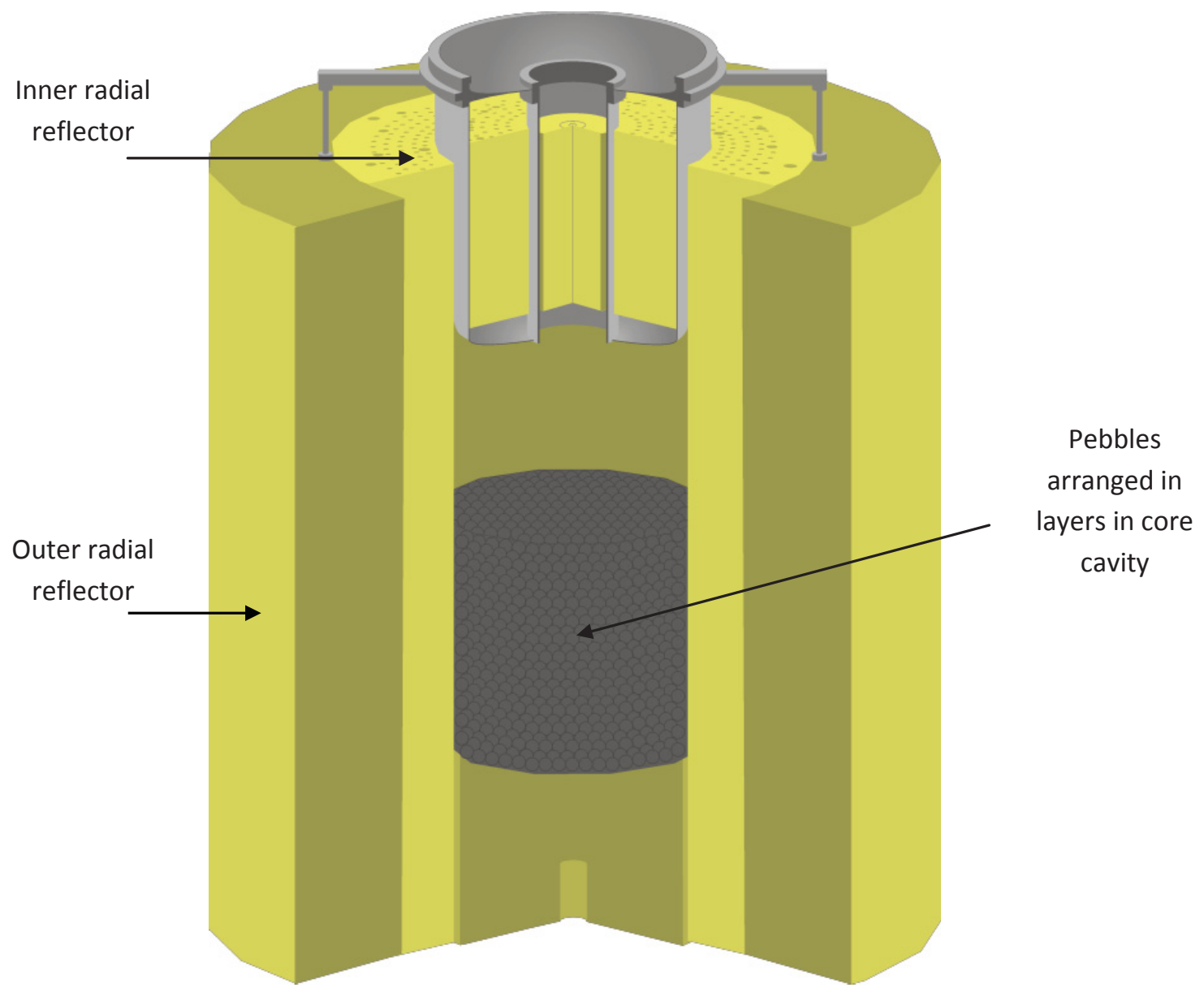

Figure 1.0-1. Generic HTR-PROTEUS Configuration (derived from Ref. 2).

a Difilippo, F. C., "Monte Carlo Calculations of Pebble Bed Benchmark Configurations of the PROTEUS Facility," Nucl. Sci. Eng., 143, 240-253 (2003).

${ }^{\mathrm{b}}$ Personal communication with Oliver Köberl at PSI (September 2, 2011).

Revision: 1

Date: March 31, 2014

Page 3 of 375 
NEA/NSC/DOC(2006)1

Gas Cooled (Thermal) Reactor - GCR

PROTEUS-GCR-EXP-004

CRIT-REAC

\section{Layer 1} (odd layer)

Layer 2 (even layer)
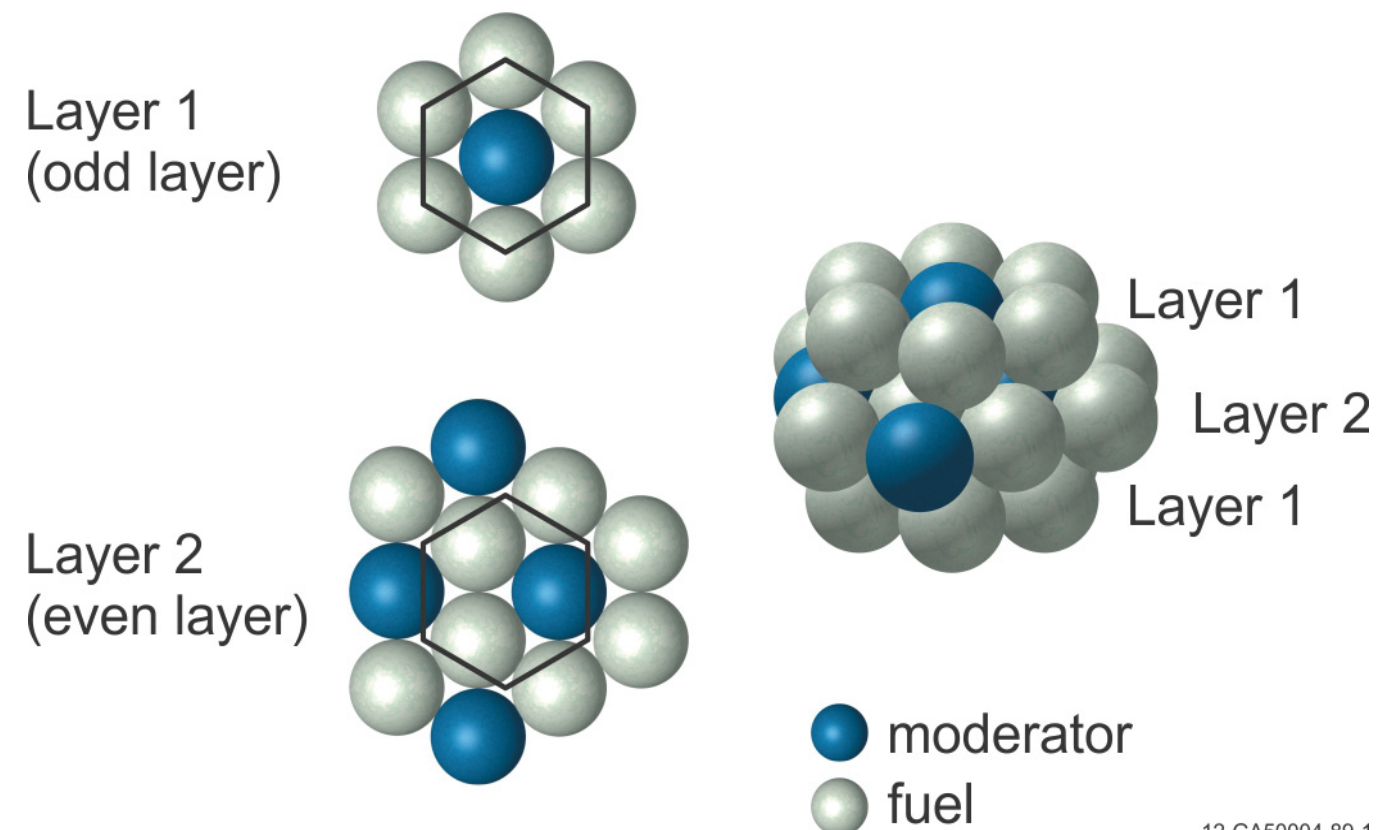

12-GA50004-89-1

Figure 1.0-2. Subunit for Construction of the Hexagonal Close-Packed (HCP) Cell.

\section{Layer 1}

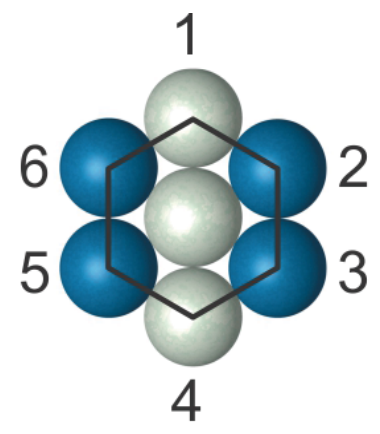

Layer 2
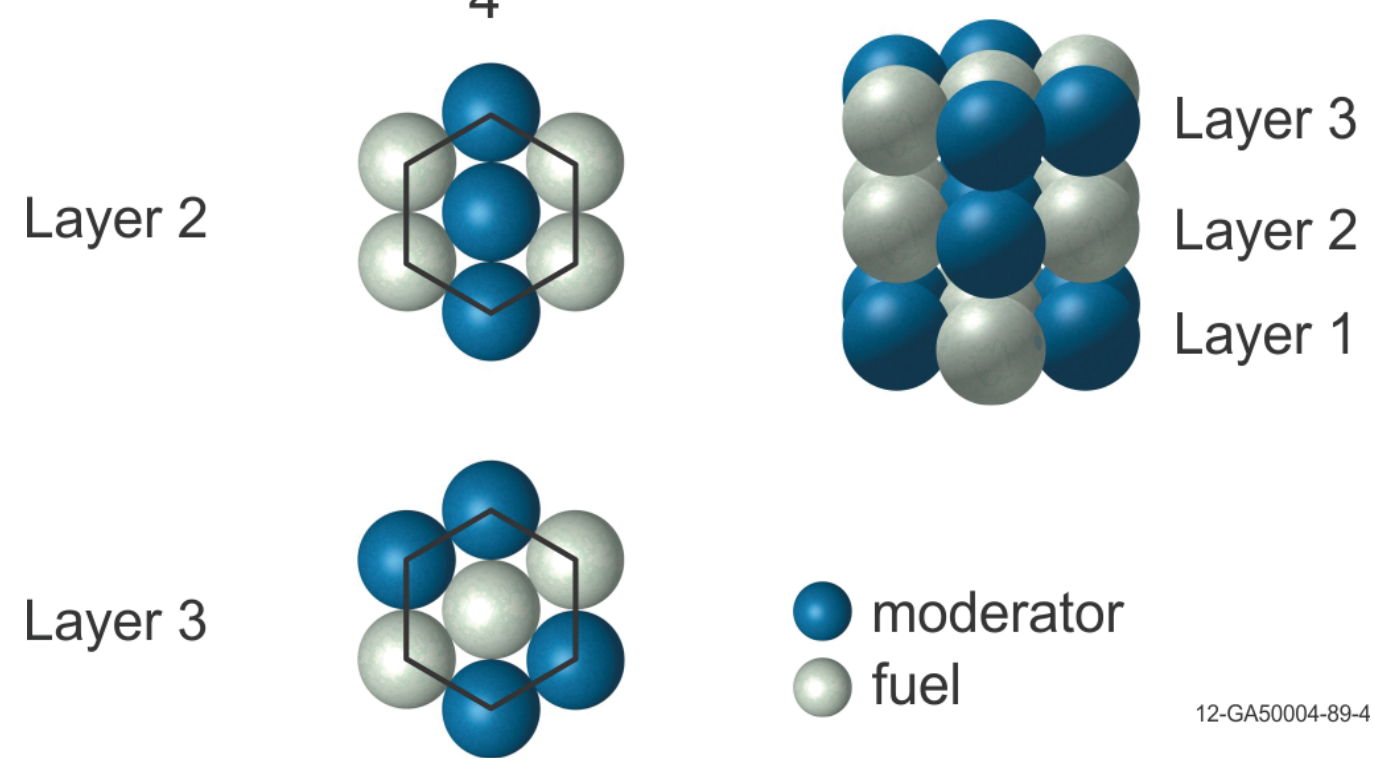

12-GA50004-89-4

Figure 1.0-3. Subunit for Construction of the Columnar Hexagonal Point-On-Point (CHPOP) Cell. 
NEA/NSC/DOC(2006)1

\section{Gas Cooled (Thermal) Reactor - GCR \\ PROTEUS-GCR-EXP-004 \\ CRIT-REAC}

Table 1.0-1. HTR-PROTEUS Core Configurations (Ref. 1 and 3).

\begin{tabular}{|c|c|c|}
\hline Core & State & Notes \\
\hline 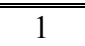 & 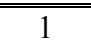 & Only configuration that used ZEBRA control rods. Hexagonal close-packed pebbles. \\
\hline $1 \mathrm{~A}$ & $\begin{array}{l}1 \\
2\end{array}$ & $\begin{array}{l}\text { Equivalent to Core 1, ZEBRA control rods replaced with withdrawable control rods. } \\
\text { Repeat of State \#1 to check reproducibility with minor configuration changes. }\end{array}$ \\
\hline 2 & 1 & $\begin{array}{l}\text { Similar to Core } 1 \text { A with decreased core height and increased upper graphite reflection. Used } \\
\text { to investigate "cavity effect". }\end{array}$ \\
\hline 3 & 1 & $\begin{array}{l}\text { Similar to Core } 1 \mathrm{~A} \text { with polyethylene rods added to simulate water ingress. Every available } \\
\text { vertical channel between pebbles contained an } 8.9-\mathrm{mm} \text {-diameter polyethylene rod. }\end{array}$ \\
\hline 4.1 & 1 & Random pebble loading using separate fuel and moderator pebble delivery tubes. \\
\hline 4.2 & 1 & Random pebble loading using a single pebble delivery tube. \\
\hline 4.3 & 1 & Random pebble loading using a single pebble delivery tube (core reload for reproducibility). \\
\hline 5 & $\begin{array}{l}1 \\
2 \\
3\end{array}$ & $\begin{array}{l}\text { Columnar hexagonal point-on-point packing implemented to improve homogeneity of core. } \\
\text { Coolant channels in bottom reflector open. } \\
\text { Equivalent to Core } 5 \text {, State \#1, with coolant channels in bottom reflector filled with graphite. } \\
\text { Repeat of State \#2 to check reproducibility and complete some additional reactor physics } \\
\text { measurements. }\end{array}$ \\
\hline 6 & 1 & $\begin{array}{l}\text { Similar to Core } 5 \text { with hollow polyethylene rods added to simulate water ingress. Copper } \\
\text { wire absorbers were placed within the polyethylene rods to compensate for the positive } \\
\text { reactivity addition. Maximum polyethylene loading. }\end{array}$ \\
\hline 7 & 1 & $\begin{array}{l}\text { Similar to Core } 5 \text { with polyethylene rods added to simulate water ingress. Maximum } \\
\text { polyethylene loading compensated by reduced core height. }\end{array}$ \\
\hline 8 & 1 & $\begin{array}{l}\text { Similar to Core } 5 \text { with short polyethylene rods added to simulate water ingress in lower core } \\
\text { region. Every vertical channel contained a } 15 \mathrm{~cm} \text { long triangular polyethylene rod. }\end{array}$ \\
\hline 9 & $\begin{array}{l}1 \\
2\end{array}$ & $\begin{array}{l}\text { Columnar hexagonal point-on-point packing with increased moderator pebble content. } \\
\text { Essentially Core } 5 \text { with an equal number of fuel and moderator pebbles } \\
\text { Repeat of State \#1 with additional layer of moderator pebbles. }\end{array}$ \\
\hline 10 & 1 & $\begin{array}{l}\text { Similar to Core } 9 \text { with polyethylene rods added to simulate water ingress. Maximum } \\
\text { polyethylene loading compensated by reduced core height. }\end{array}$ \\
\hline
\end{tabular}

Acceptable benchmark experiments evaluated in this report include the following:

- $\quad$ Core 9

- Critical configuration

- Control rod worths (4)

- Control rod bank worth (full and partial)

- Autorod insertion

- Individual and combined safety/shutdown rod worths (10)

- Core 10
- Critical configuration
- Control rod worths (4)
- Control rod bank worth (full and partial)
- Autorod insertion
- Individual and combined safety/shutdown rod worths (10) 
NEA/NSC/DOC(2006)1

Gas Cooled (Thermal) Reactor - GCR

PROTEUS-GCR-EXP-004

CRIT-REAC

\subsection{Description of the Critical and / or Subcritical Configuration}

\subsubsection{Overview of Experiment}

Only Cores 9 and 10 are evaluated in this benchmark report due to similarities in their construction. The other core configurations of the HTR-PROTEUS program are evaluated in their respective reports as outlined in Section 1.0.

Cores 9 and 10 were evaluated and determined to be acceptable benchmark experiments.

\subsubsection{Geometry of the Experiment Configuration and Measurement Procedure}

The PROTEUS assembly can basically be described as a graphite cylinder with an outer diameter of $3262 \mathrm{~mm}$ and a height of $3304 \mathrm{~mm}$. It has a central cavity that sits $780 \mathrm{~mm}$ above the bottom of the radial and lower axial reflectors and consists of a 22 -sided polygon with a flat-to-flat separation distance of $1250 \mathrm{~mm}$. Random or deterministic lattices of pure graphite moderator pebbles and fuel (16.7 wt.\% enriched in ${ }^{235} \mathrm{U}$ ) pebbles were arranged within this cavity. Additional graphite filler pieces were utilized to provide support for the irregular outer surface of the deterministic pebble arrangements, providing a 12 -sided core cavity region with a flat-to-flat separation distance of $\sim 1205 \mathrm{~mm}$. A removable, 1235-mm-high, upper axial reflector assembly consisted of an aluminum tank containing a 780-mm-high graphite reflector; normally an air gap was between the upper reflector and the topmost layer of the pebble bed. An aluminum safety ring is located $1764 \mathrm{~mm}$ above the floor of the cavity to prevent the upper reflector from falling onto the pebble bed. Reactor shutdown was achieved using four boron-steel rods placed at a radius of $680 \mathrm{~mm}$; reactor control was typically performed using four fine control rods placed at a radius of $900 \mathrm{~mm}$. In Core 1, however, Cd Shutter, or ZEBRA type, rods were used in place of the fine control rods. Water ingress was simulated by using polyethylene rods introduced axially into vertical channels of the deterministic cores (Ref. 2). Schematic representations of the PROTEUS assembly are shown in Figures 1.1-1 and 1.1-2.

While there are many components of the PROTEUS that remain unchanged throughout the course of the HTR-PROTEUS experiments, many parameters did change between experiments, such as the use of graphite filler pieces, control rod types and locations, the presence of polyethylene rods to simulate water ingress, core pebble packing, and conditions at criticality. Section 1.1.2.1 provides information regarding general components common to all HTR-PROTEUS configurations. Section 1.1.2.2 provides information specific to the core configurations evaluated in this report. 
NEA/NSC/DOC(2006)1

Gas Cooled (Thermal) Reactor - GCR

PROTEUS-GCR-EXP-004

CRIT-REAC

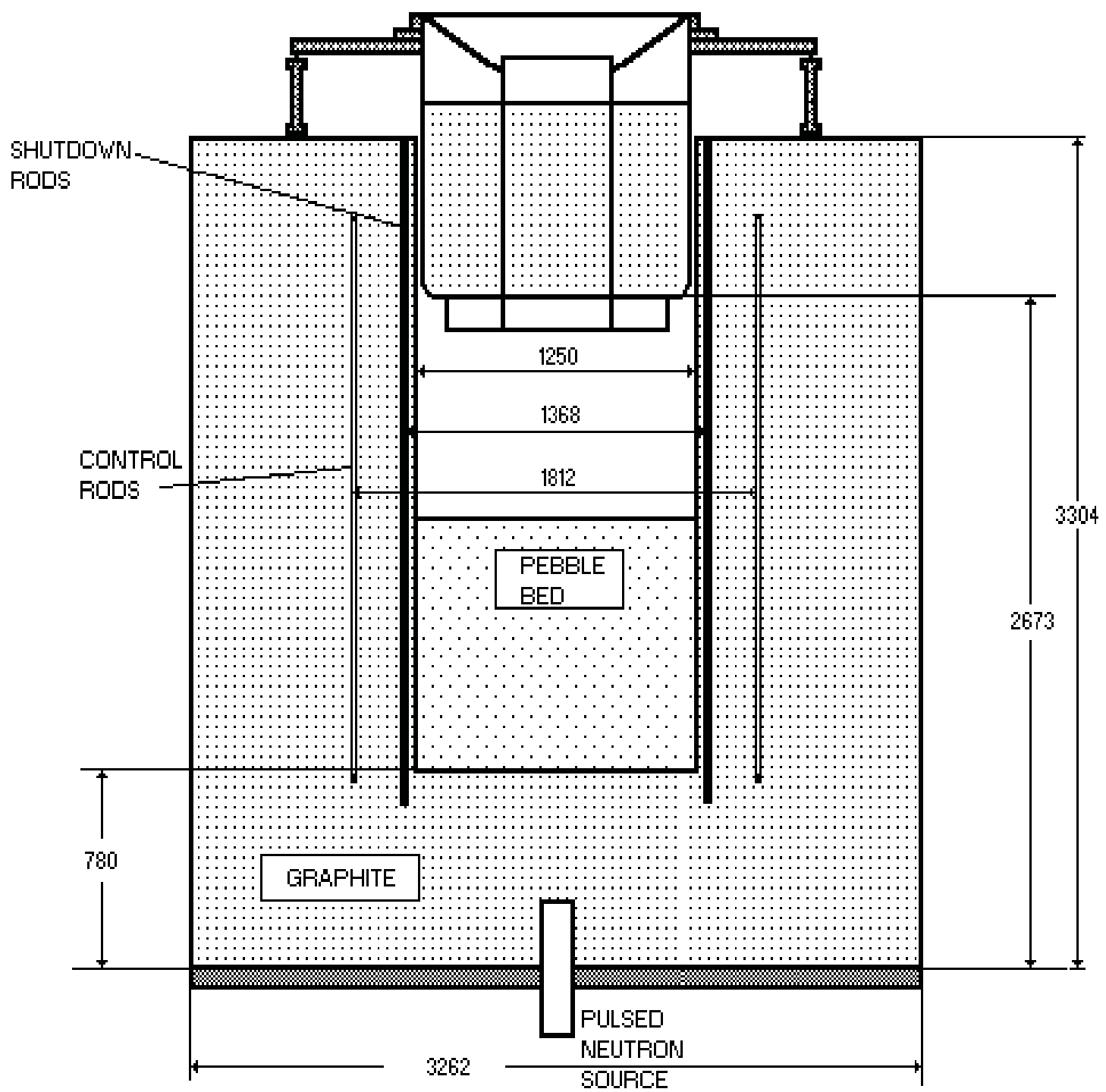

Figure 1.1-1. Schematic Side View of the HTR-PROTEUS Facility (dimensions in mm), (Ref. 2 and 3). 
NEA/NSC/DOC(2006)1

Gas Cooled (Thermal) Reactor - GCR

PROTEUS-GCR-EXP-004

CRIT-REAC

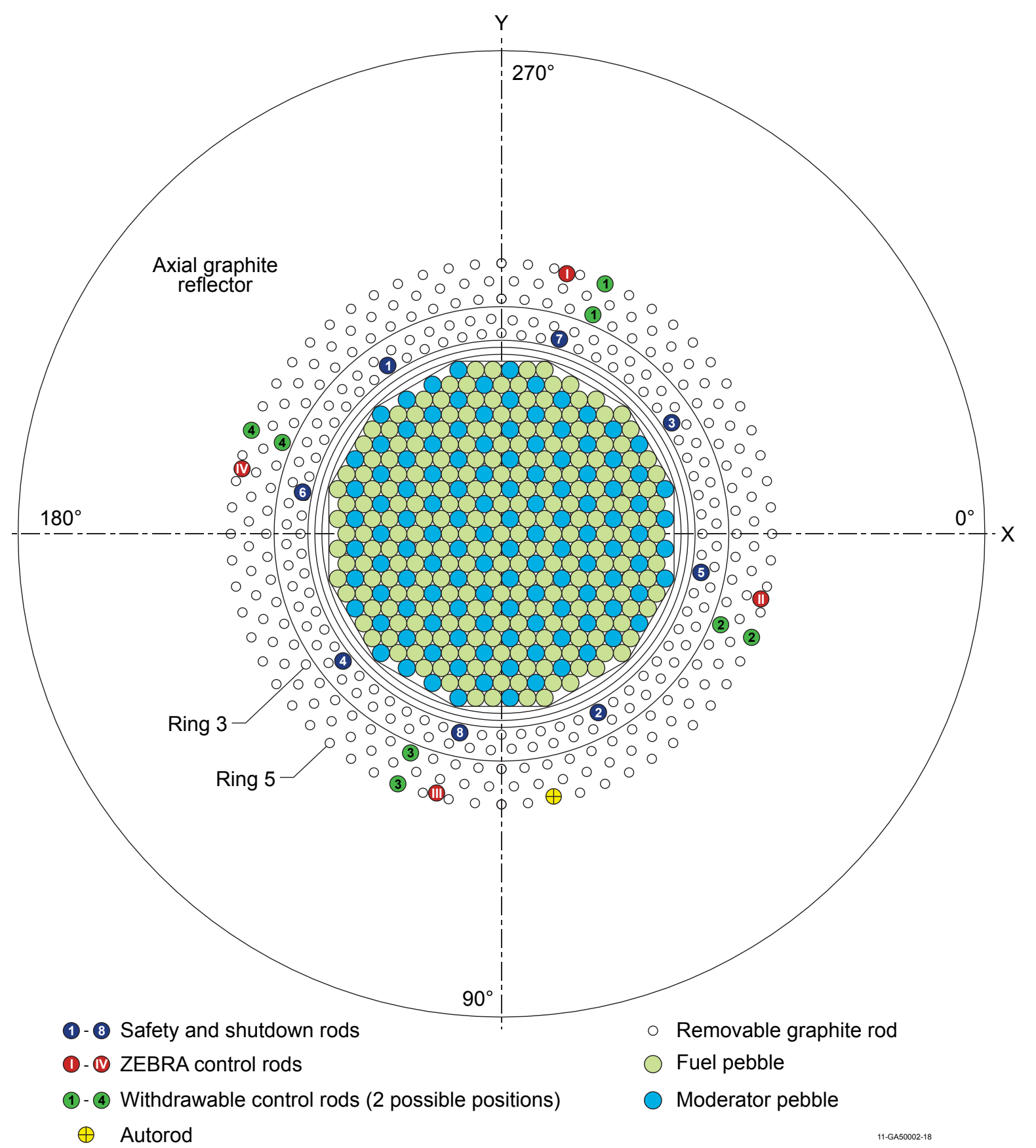

Figure 1.1-2a. HTR-PROTEUS Control Rod Positions and Bore Hole Locations (derived from Ref. 2). 
NEA/NSC/DOC(2006)1

Gas Cooled (Thermal) Reactor - GCR

PROTEUS-GCR-EXP-004

CRIT-REAC

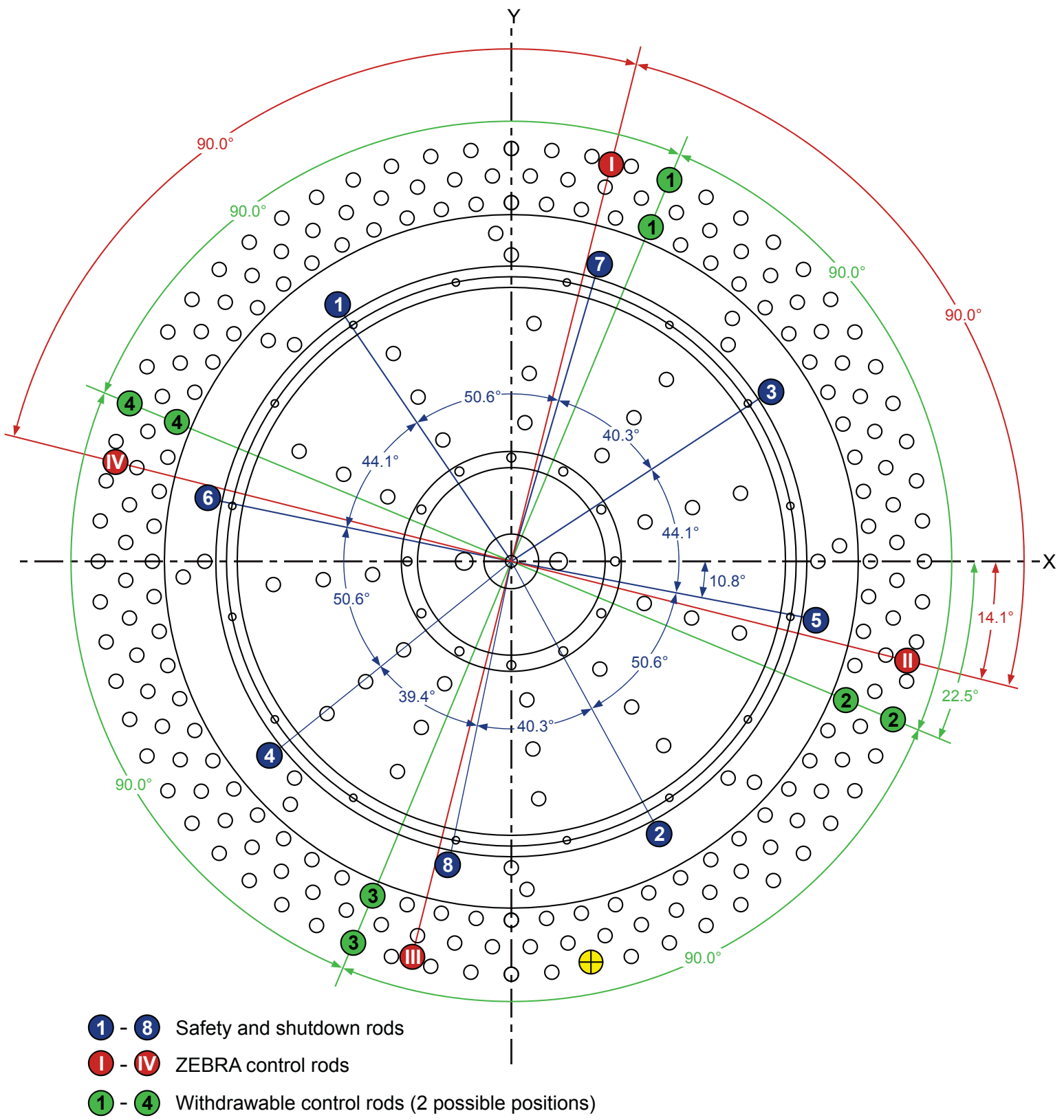

$\oplus$ Autorod

11-GA50002-17

Figure 1.1-2b. HTR-PROTEUS Control Rod Positions and Bore Hole Locations (Ref. 2). 
NEA/NSC/DOC(2006)1

\section{Gas Cooled (Thermal) Reactor - GCR \\ PROTEUS-GCR-EXP-004 \\ CRIT-REAC}

\subsubsection{General HTR-PROTEUS Components}

The following components are common to all HTR-PROTEUS core configurations.

\section{Concrete}

Concrete shielding surrounds the reactor system entirely (Ref. 2). The reactor is surrounded by $800 \mathrm{~mm}$ of concrete shielding. No significant room return effects from neutron streaming were measured. ${ }^{\text {a }}$

\section{Steel Plate Pedestal}

The PROTEUS assembly rests upon a stainless steel plate pedestal. ${ }^{\mathrm{b}}$

\section{Radial Reflector}

The radial reflector was a 22-sided polygon with a height of $3304 \mathrm{~mm}$ and outer diameter of $3262 \mathrm{~mm}$ (see Figures 1.1-1 and 1.1-3). A central cavity sat with its base $780 \mathrm{~mm}$ above the reflector base and had a flat-to-flat separation distance of $1250 \mathrm{~mm}$ (Ref. 2 and 3). The central cavity contained fuel (16.7 wt.\% enriched in ${ }^{235} \mathrm{U}$ ) and moderator (pure graphite) pebbles either deterministically or randomly arranged in one of several different geometrical arrangements. Graphite filler pieces were placed at the core-reflector boundary to support the stacked pebble structures (Ref. 3).

The external boundary of the 22-sided polygon had sides located $1631.6 \mathrm{~mm}$ from the center, which would be an equivalent area cylinder of $1637.7 \mathrm{~mm}$ radius. The internal cavity was a 22-sided polygon with sides $626 \mathrm{~mm}$ from the center, which would be an equivalent area cylinder of $628.4 \mathrm{~mm}$ radius. In summary, the cavity had an average radial thickness $\sim 1029 \mathrm{~mm}$ of graphite, and lower and upper axial thicknesses $780 \mathrm{~mm}$ of graphite. ${ }^{\mathrm{b}}$

A cylindrical version of the radial reflector would have the following radius (the first value represents an equal perimeter, and the second value represents an equal area): external radius, 1643.6 and $1637.7 \mathrm{~mm}$, respectively; internal radius for the 22-sided cavity, 630.6 and $628.4 \mathrm{~mm}$, respectively. ${ }^{\mathrm{c}}$

The radial reflector contains various minor penetrations serving as control rod and instrumentation channels. The reflector contained 308 C-Driver channels (see Figure 1.1-3), which were vertical channels of $27.43 \mathrm{~mm}$ diameter running the full height of the radial reflector and were left over from previous PROTEUS experiments. These channels were arranged in five concentric rings. Unless otherwise stated, these channels were filled with $26.5 \mathrm{~mm}$ diameter graphite rods (Ref. 2). These rods were relatively easy to remove and useful in estimating the effect of missing graphite (Ref. 3).

Attached to one side of the radial reflector was a reactor thermal column, which was a quasi-rectangular structure with a height and width of $1200 \mathrm{~mm}$ and a depth of $\sim 500 \mathrm{~mm}$. Its top surface was situated 1120 $\mathrm{mm}$ from the upper surface of the radial reflector (Ref. 2).

A safety ring was included in the design as an additional safety measure in the unlikely event that the upper axial reflector should fall into the cavity. It was comprised of a Peraluman ring $10 \mathrm{~mm}$ thick with inner and outer radii of 604 and $700 \mathrm{~mm}$, respectively. It was situated $1764 \mathrm{~mm}$ above the floor of the cavity, as depicted in Figure 1.1-4 (Ref. 2).

\footnotetext{
a Williams, T., "HTR PROTEUS CORE 1: Reactivity Corrections for the Critical Balance," TM-41-93-20, Paul Scherrer Institut, Villigen, October 7, 1993.

b Difilippo, F. C., "Monte Carlo Calculations of Pebble Bed Benchmark Configurations of the PROTEUS Facility," Nucl. Sci. Eng., 143, 240-253 (2003).

${ }^{c}$ Difilippo, F. C., "Applications of Monte Carlo Simulations of Thermalization Processes to the Nondestructive Assay of Graphite,” Nucl. Sci. Eng., 133, 163-177 (1999).
}

Revision: 1

Date: March 31, 2014

Page 10 of 375 


\section{NEA/NSC/DOC(2006)1}

Gas Cooled (Thermal) Reactor - GCR

PROTEUS-GCR-EXP-004

CRIT-REAC

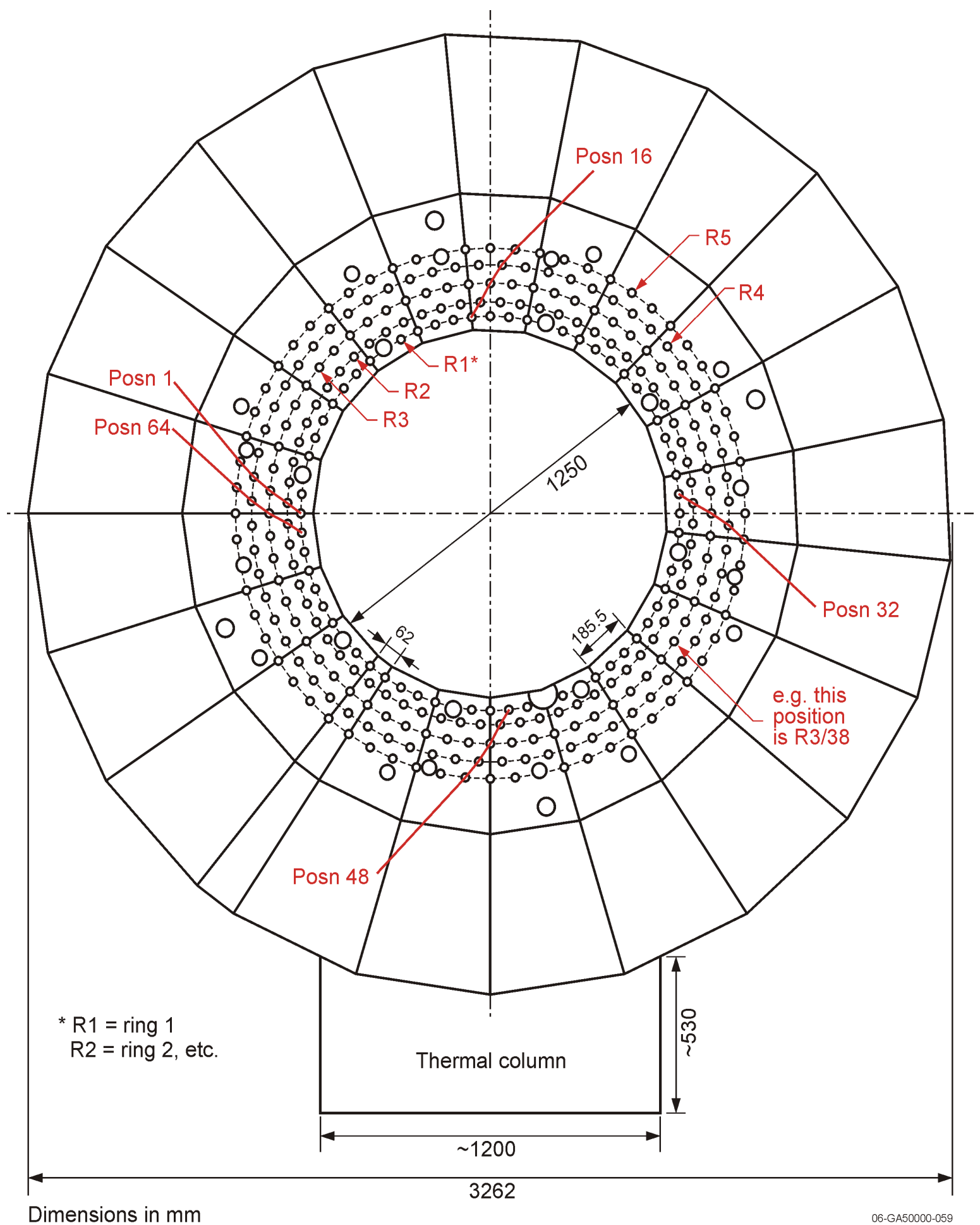

Figure 1.1-3. Cross Section View of the Radial Reflector (Ref. 2).

The radial reflector contained various minor penetrations for the introduction of instrumentation and sources. Explicit geometries and descriptions are unavailable. When not in use, the penetrations were filled with graphite plugs. 
NEA/NSC/DOC(2006)1

\author{
Gas Cooled (Thermal) Reactor - GCR \\ PROTEUS-GCR-EXP-004 \\ CRIT-REAC
}

\title{
Upper Axial Reflector
}

Detailed drawings of the upper axial reflector and its aluminum housing are shown in Figures 1.1-4 through 1.1-6. The graphite has two components; the first component is a central cylinder of $394 \mathrm{~mm}$ diameter with a central, open, $27.43 \mathrm{~mm}$ diameter channel, surrounded by the second component, an annulus with an inner diameter of $418.6 \mathrm{~mm}$ and an outer diameter of $1234 \mathrm{~mm}$. The annulus contains 33 coolant channels corresponding with those found in the lower axial reflector. All 34 channels are always open. The outer graphite annulus includes a separate outer shell consisting of 36 smaller, individual rectangular pieces that do not fit exactly flush with the bulk graphite. The upper axial reflector graphite had a height of $780 \mathrm{~mm}$ (Ref. 2).

The upper reflector tank is a complex structure that supports the upper axial graphite reflector in place above the cavity. It was comprised of two main parts, an inner and an outer tank. The inner tank, which contained the graphite cylinder, was removable, and it had to be removed before the outer tank could be removed. The outer tank contained the graphite annulus. The dimensions and layout of the upper reflector are shown in Figures 1.1-4 through 1.1-6. A steel lid and flanges, external to the core reflector, were used to hold the upper reflector above the core cavity (Ref. 2).

The upper axial reflector closed the cavity at a height of $1863 \mathrm{~mm}$ from the bottom of the cavity. ${ }^{\text {a }}$

\footnotetext{
a Difilippo, F. C., "Monte Carlo Calculations of Pebble Bed Benchmark Configurations of the PROTEUS Facility," Nucl. Sci. Eng., 143, 240-253 (2003).

Revision: 1 


\section{NEA/NSC/DOC(2006)1}

\section{Gas Cooled (Thermal) Reactor - GCR \\ PROTEUS-GCR-EXP-004 \\ CRIT-REAC}

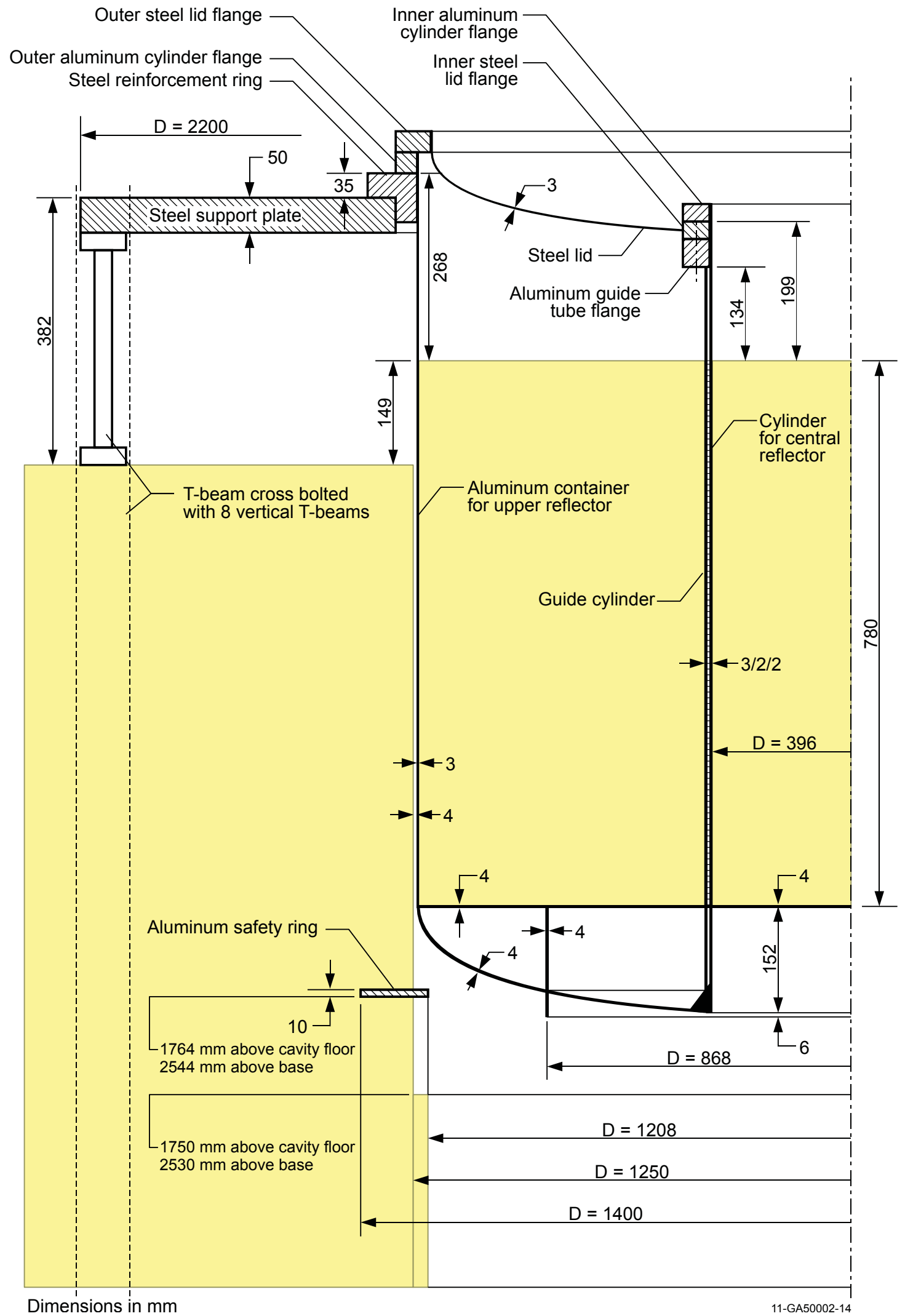

Figure 1.1-4. Placement of the Upper Axial Reflector (Ref. 2).

Revision: 1 
NEA/NSC/DOC(2006)1

Gas Cooled (Thermal) Reactor - GCR

PROTEUS-GCR-EXP-004

CRIT-REAC
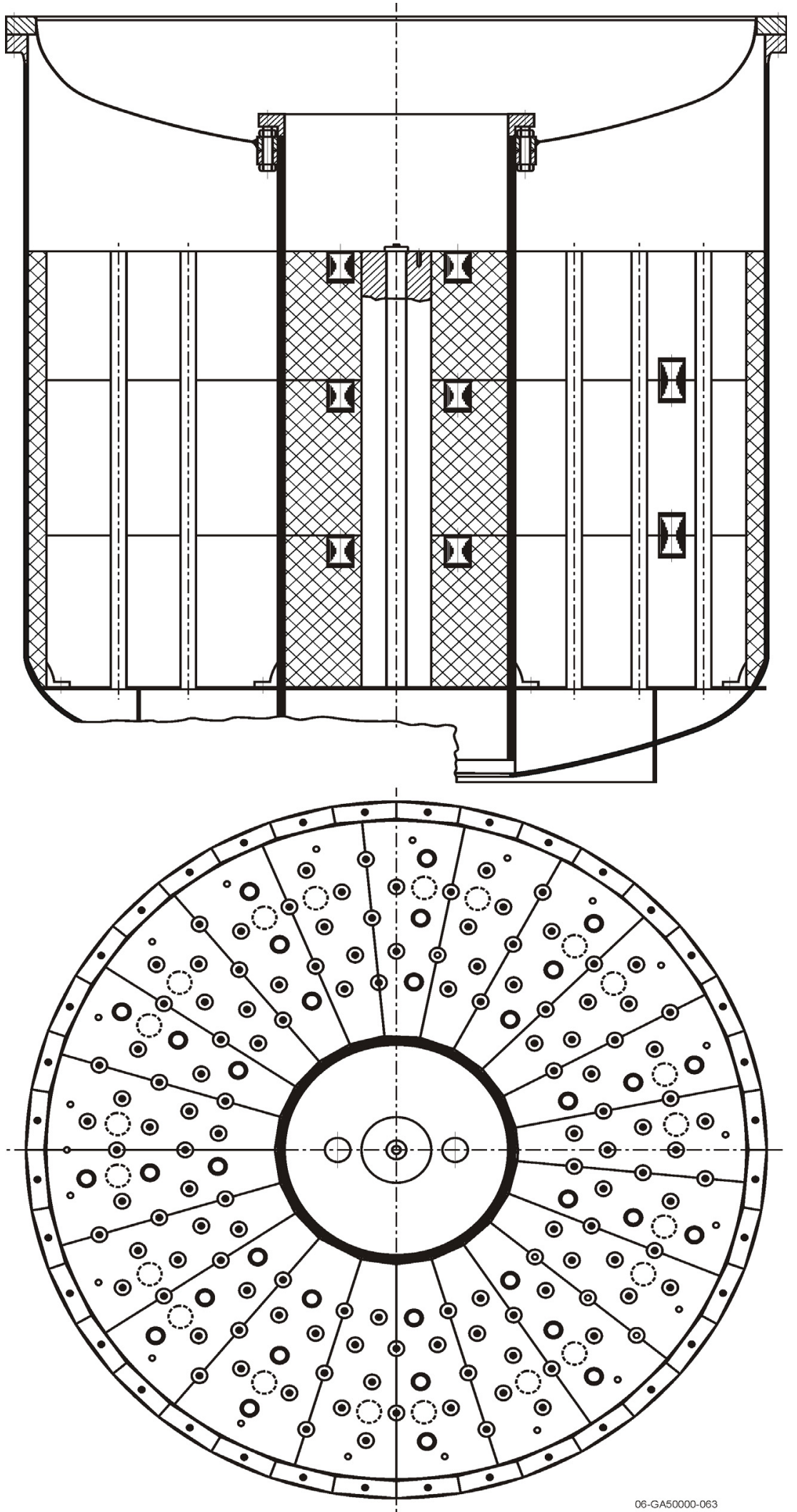

Figure 1.1-5. Non-dimensional Cross Sections of the Upper Axial Reflector (Ref. 2). 
NEA/NSC/DOC(2006)1

Gas Cooled (Thermal) Reactor - GCR

PROTEUS-GCR-EXP-004

CRIT-REAC
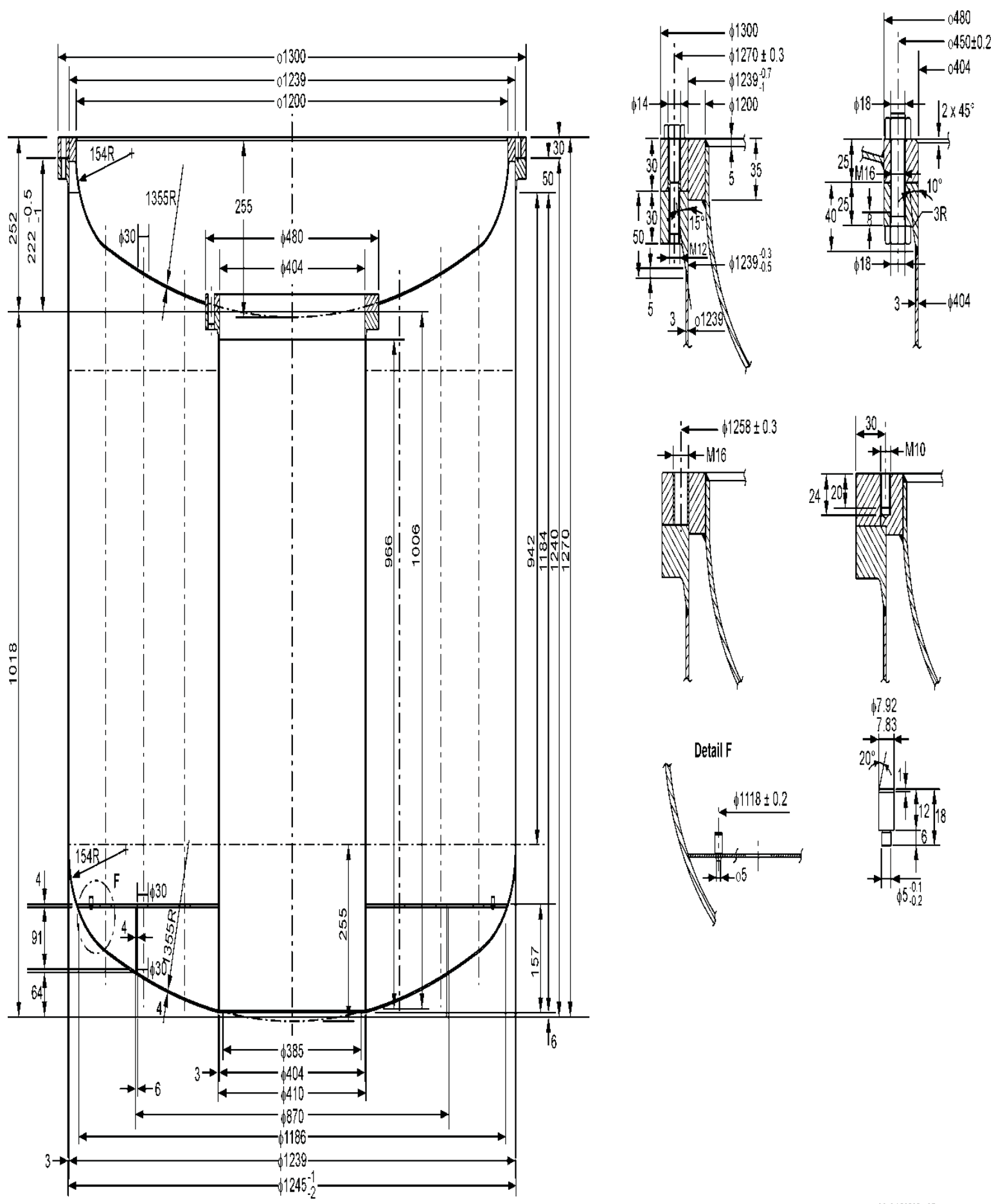

$06.6450000 \cdot 187$

Figure 1.1-6. Details of the Main Aluminum Structure of the Upper Axial Reflector (Ref. 2). Units are in millimeters. 
NEA/NSC/DOC(2006)1

\author{
Gas Cooled (Thermal) Reactor - GCR \\ PROTEUS-GCR-EXP-004 \\ CRIT-REAC
}

\title{
Lower Axial Reflector
}

The lower axial reflector is $780 \mathrm{~mm}$ thick and contains, for historical reasons, 160 symmetrically positioned $27.42 \mathrm{~mm}$ diameter channels. At least 127 of these channels were filled with $780 \mathrm{~mm}$ long, $26.5 \mathrm{~mm}$ diameter graphite rods. The dimensions of the lower axial reflector are shown in Figure 1.1-7; the positions of the 33 (typically open) coolant channels are also indicated. The open channels are arranged in three concentric rings of radii 300,410 , and $515 \mathrm{~mm}$, with each ring containing eleven channels. The channels in each ring are positioned at azimuthal angles of $16.875,50.625,84.375$, $118.125,140.625,174.375,208.125,241.875,275.625,309.375$, and $343.125^{\circ}$, as measured in the clockwise direction from the $+x$-axis, as shown in Figure 1.1-2 (Ref. 2). In some of the core configurations all of the coolant channels in the lower axial reflector were filled with graphite plugs (Ref. 3). In all the deterministic cores, $\sim 12$ pebbles were directly over one of the 33 cooling channels in the lower axial reflector. To avoid pebble displacement in these cases, special aluminum plugs were developed to support the pebbles in Core 1. In later cores, simple graphite rods were used (Ref. 3).

A special, $121 \mathrm{~mm}$ diameter, channel was provided in the center of the lower axial reflector with approximately $500 \mathrm{~mm}$ of graphite separating it from the core. This channel could be used for measurements using the pulsed neutron source. The pulsed neutron source, when used for subcriticality measurements, was partially inserted into the lower axial reflector. When not in use, it was replaced with a plug of graphite of dimensions $250 \mathrm{~mm}$ in height and $120 \mathrm{~mm}$ in diameter (Ref. 2 and 3). 


\section{NEA/NSC/DOC(2006)1}

Gas Cooled (Thermal) Reactor - GCR

PROTEUS-GCR-EXP-004

CRIT-REAC
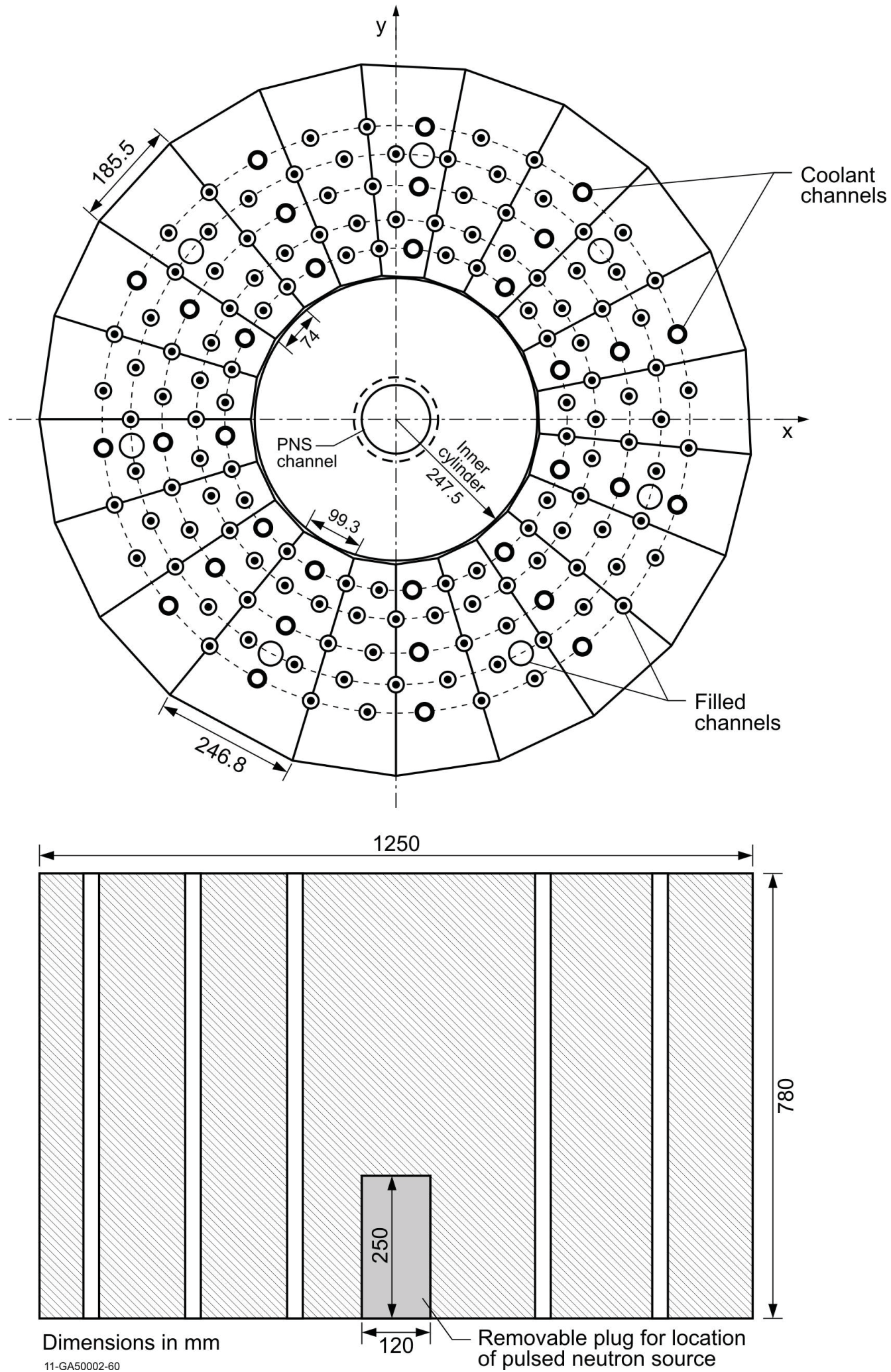

Figure 1.1-7. Details of the Lower Axial Reflector. Note the 33 coolant channels, the small air gap between outer and inner parts, and the position of the pulsed source channel (Ref. 2). 
NEA/NSC/DOC(2006)1

\author{
Gas Cooled (Thermal) Reactor - GCR \\ PROTEUS-GCR-EXP-004 \\ CRIT-REAC
}

\title{
Safety/Shutdown Rods
}

There were eight, identical, borated-steel safety/shutdown rods located adjacent to the core in the radial reflector (see Figure 1.1-2). These rods were separated into two groups of four rods (rods 1-4 and rods 5-8). One of these groups was selected as the "safety rod" group and the other as the "shutdown rod" group. These rods were not used as control rods, such as the four ZEBRA type rods used in Core 1 or the withdrawable stainless steel control rods used in Cores 1A through 10 (Ref. 2 and 3).

Rods numbered 1 through 4 are the shutdown rods and rods numbered 5 through 8 are the safety rods. ${ }^{a}$

The safety/shutdown rods consisted of $35 \mathrm{~mm}$ diameter borated steel rod sections enclosed in 18/8 stainless steel tubes with an inside diameter of $36 \mathrm{~mm}$ and outside diameter of $40 \mathrm{~mm}$. The rods were located in $45 \mathrm{~mm}$ inner diameter graphite guide tubes within the radial reflector. The centers of the guide tubes were $684 \mathrm{~mm}$ from the center of the core, or about $59 \mathrm{~mm}$ from the inner surface of the radial reflector (without filler pieces). The azimuthal positions of the eight rods are shown in Figure 1.1-2, in which the slight azimuthal asymmetry of the rod positions should be noted (Ref. 2 and 3).

A diagram of a safety/shutdown rod is shown in Figure 1.1-8; the borated steel portion of the rods was $2100 \mathrm{~mm}$ in length. The fully in and out positions of the rods are shown in Figure 1.1-9; the rods traveled a total distance of $2900 \mathrm{~mm}$ ( $2530 \mathrm{~mm}$ free fall plus $370 \mathrm{~mm}$ braking distance) from fully withdrawn to fully inserted positions. When fully inserted, the bottom of the borated steel region is located $350 \mathrm{~mm}$ below the bottom of the reactor cavity with the top of the borated steel region slightly above the top of the $1730 \mathrm{~mm}$ high cavity. When fully withdrawn, the bottom of the borated steel region is $26 \mathrm{~mm}$ below the top surface of the radial reflector (Ref. 2).

Each rod contains six, $35 \mathrm{~mm}$ diameter, $350 \mathrm{~mm}$ long, cylindrical pieces of borated steel. Aluminum and steel shock dampers were located under each of the safety/shutdown rods, as shown in Figure 1.1-9, to prevent damage in case one of the rod cables should fail. A gap of approximately $30 \mathrm{~mm}$ separated the bottom of the safety rod from the upper, aluminum part of the shock damper. The aluminum parts of the shock damper was comprised of a $280.5 \mathrm{~mm}$ long hollow tube with $29 \mathrm{~mm}$ inner diameter, $40 \mathrm{~mm}$ outer diameter, and capped at both ends with aluminum of $2 \mathrm{~mm}$ thickness. The steel parts of the shock dampers (end caps, springs, and damper chamber) were affixed to the underside of the lower support plate, which itself is $\sim 75 \mathrm{~mm}$ thick; only a fraction of the total mass of these components resided within the graphite reflector (Ref. 2).

The safety rods were always maintained in withdrawn positions, i.e., out of the reflector. Criticality was achieved when the four shutdown rods were also fully withdrawn and only the four control rods and the autorod were partially inserted for fine control. ${ }^{\mathrm{b}, \mathrm{c}}$

\footnotetext{
${ }^{a}$ Köberl, O., and Seiler, R., "Detailed Analysis of Pebble-Bed HTR PROTEUS Experiments with the Monte Carlo Code TRIPOLI4,” Proc. 2nd Int. Topical Mtg. on High Temperature Reactor Technology, Beijing, China, September 22-24, 2004.

${ }^{\mathrm{b}}$ Chawla, R., Joneja, O. P., Rosselet, M., and Williams, T., "Definition and Analysis of an Experimental Benchmark on Shutdown Rod Worths in LEU-HTR Configurations," Nucl. Technol., 139, 50-60 (2002).

${ }^{c}$ Köberl, O., Seiler, R., and Chawla, R., "Experimental Determination of the Ratio of 238U Capture to 235U Fission in LEU-HTR Pebble-Bed Configurations,” Nucl. Sci. Eng., 146, 1-12 (2004).
}

Revision: 1

Date: March 31, 2014

Page 18 of 375 
NEA/NSC/DOC(2006)1

Gas Cooled (Thermal) Reactor - GCR

PROTEUS-GCR-EXP-004

CRIT-REAC
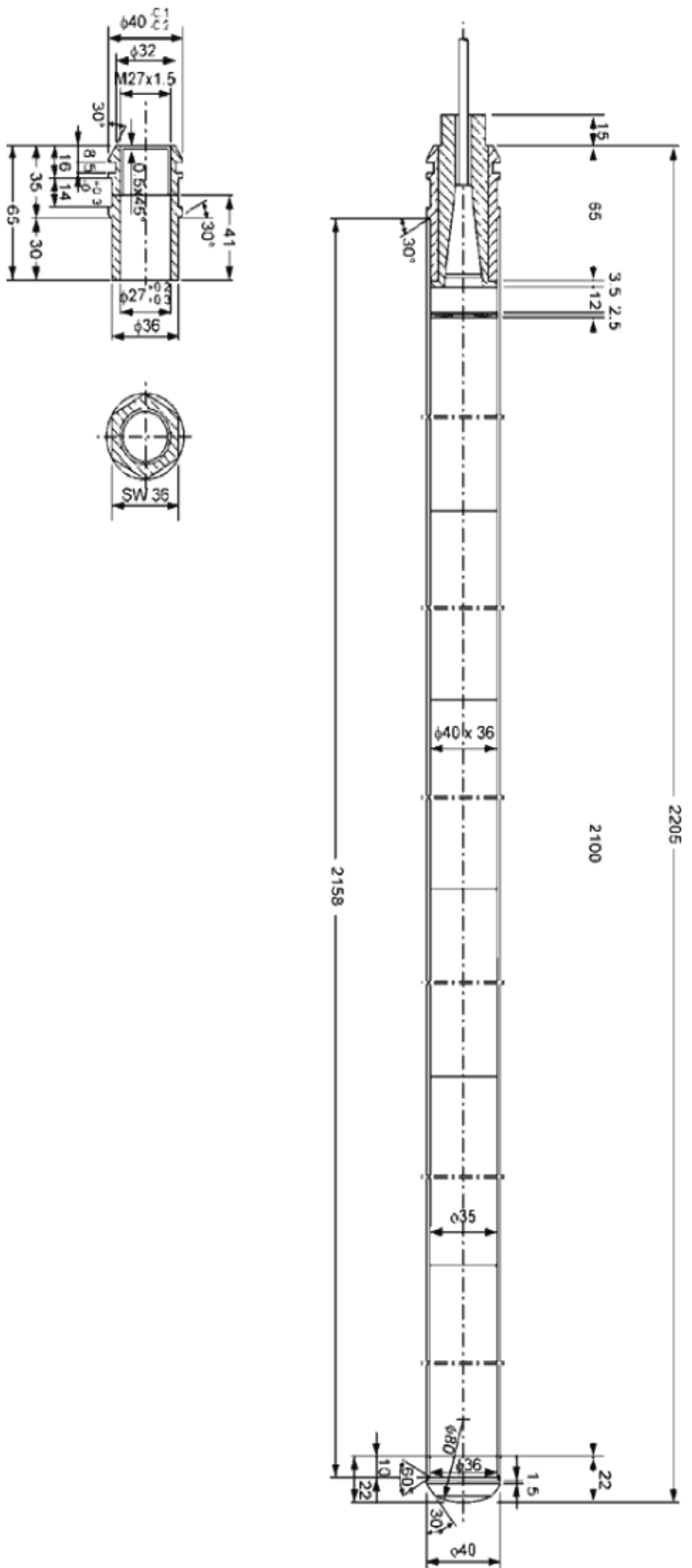

Figure 1.1-8. Details of Safety/Shutdown Rods (Ref. 2). Units are in millimeters.

Revision: 1

Date: March 31, 2014

Page 19 of 375 
NEA/NSC/DOC(2006)1

Gas Cooled (Thermal) Reactor - GCR

PROTEUS-GCR-EXP-004

CRIT-REAC

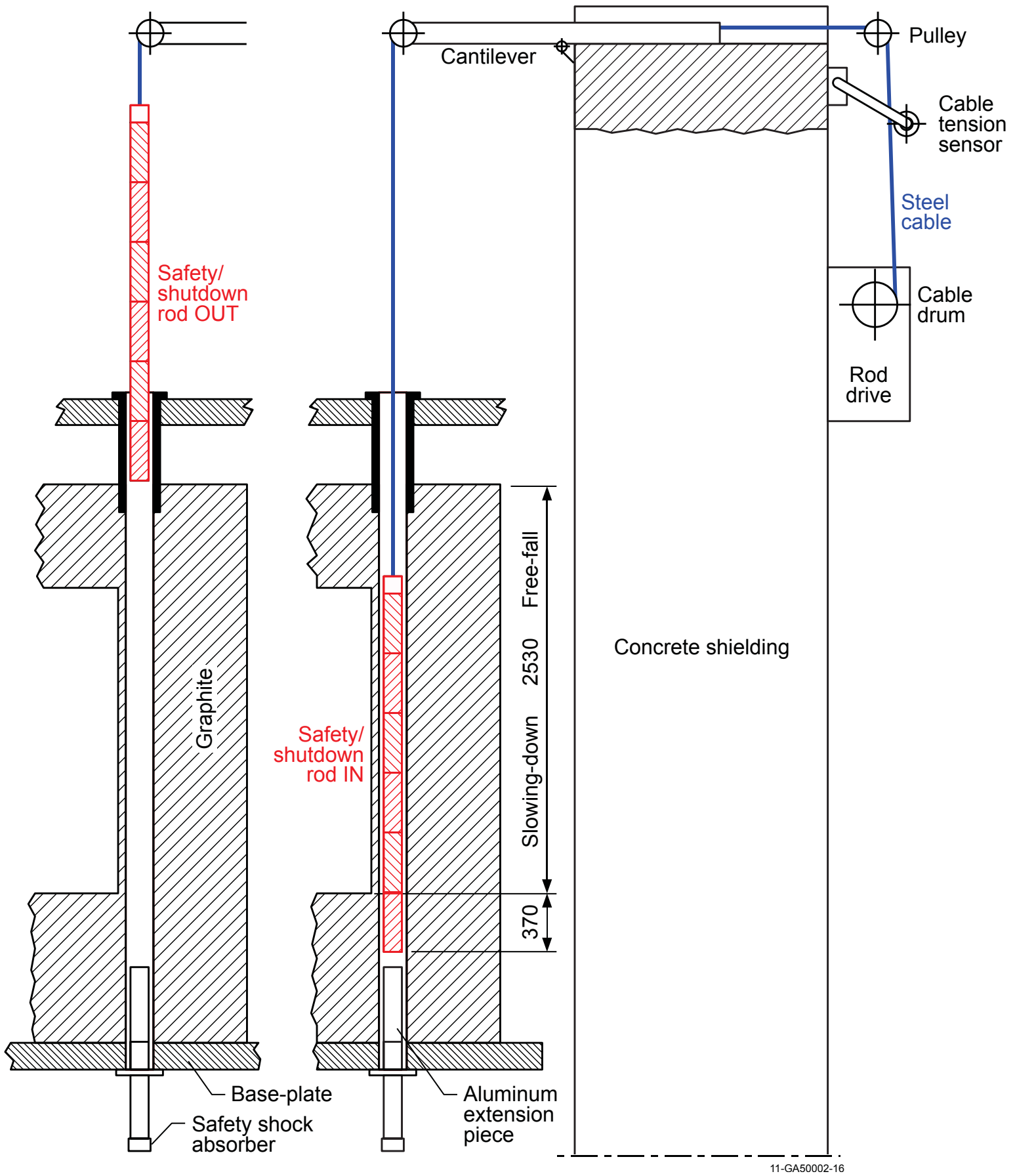

Figure 1.1-9. Safety/shutdown Rod Movement (Ref. 2). Units are in millimeters.

Revision: 1

Date: March 31, 2014

Page 20 of 375 
NEA/NSC/DOC(2006)1

\author{
Gas Cooled (Thermal) Reactor - GCR \\ PROTEUS-GCR-EXP-004 \\ CRIT-REAC
}

\title{
Automatic Control Rod (Autorod)
}

A single, fine control rod (Figure 1.1-10) was utilized to automatically maintain reactor criticality at a nominal required power. It responded to signals from a single ionization chamber (deviation channel) located in the radial reflector $810 \mathrm{~mm}$ above the cavity floor and $\sim 500 \mathrm{~mm}$ from the outer radial boundary of the core. The rod itself is located in a vertical channel with an inside diameter of $55 \mathrm{~mm}$ situated $890 \mathrm{~mm}$ from the radial center of the system; it was located azimuthally $\sim 80^{\circ}$ from the $\mathrm{x}$-direction in a clockwise direction (see Figure 1.1-2). The rod was comprised of a wedge shaped copper plate supported within an aluminum tube with an outer diameter of $44 \mathrm{~mm}$. The copper plate was $3 \mathrm{~mm}$ thick, $2300 \mathrm{~mm}$ long, and $39 \mathrm{~mm}$ at its wide end with a reduction in width along its length of $17 \mathrm{~mm}$ per meter. The rod was fully inserted when the position display showed $0 \mathrm{~mm}$ and the pointed end of the copper plate was flush with the underside of the steel plate upon which the reactor stands. The complete withdrawal of the autorod was indicated by a display of $1000 \mathrm{~mm}$ when the pointed end of the copper plate was $\sim 200 \mathrm{~mm}$ above the base of the core cavity and the blunt end was $79 \mathrm{~mm}$ below the top of the radial reflector graphite. Because the rod remains within the system even when fully "withdrawn" it has a significant rest worth that is larger than the total max-min worth of the rod.

The worth of the autorod exhibits a linear response over the range of 200 to $800 \mathrm{~mm}$ with a differential control rod worth of $6.3 \times 10^{-3} \phi / \mathrm{mm}\left(\beta_{\mathrm{eff}}=0.00723\right)$ and an uncertainty of around $5 \%$. The autorod response was intercalibrated with the ZEBRA (and later withdrawable) control rods. ${ }^{a}$

${ }^{a}$ Williams, T., "HTR PROTEUS CORE 1: Reactivity Corrections for the Critical Balance," TM-41-93-20, Paul Scherrer Institut, Villigen, October 7, 1993.

Revision: 1

Date: March 31, 2014 
NEA/NSC/DOC(2006)1

Gas Cooled (Thermal) Reactor - GCR

PROTEUS-GCR-EXP-004

CRIT-REAC

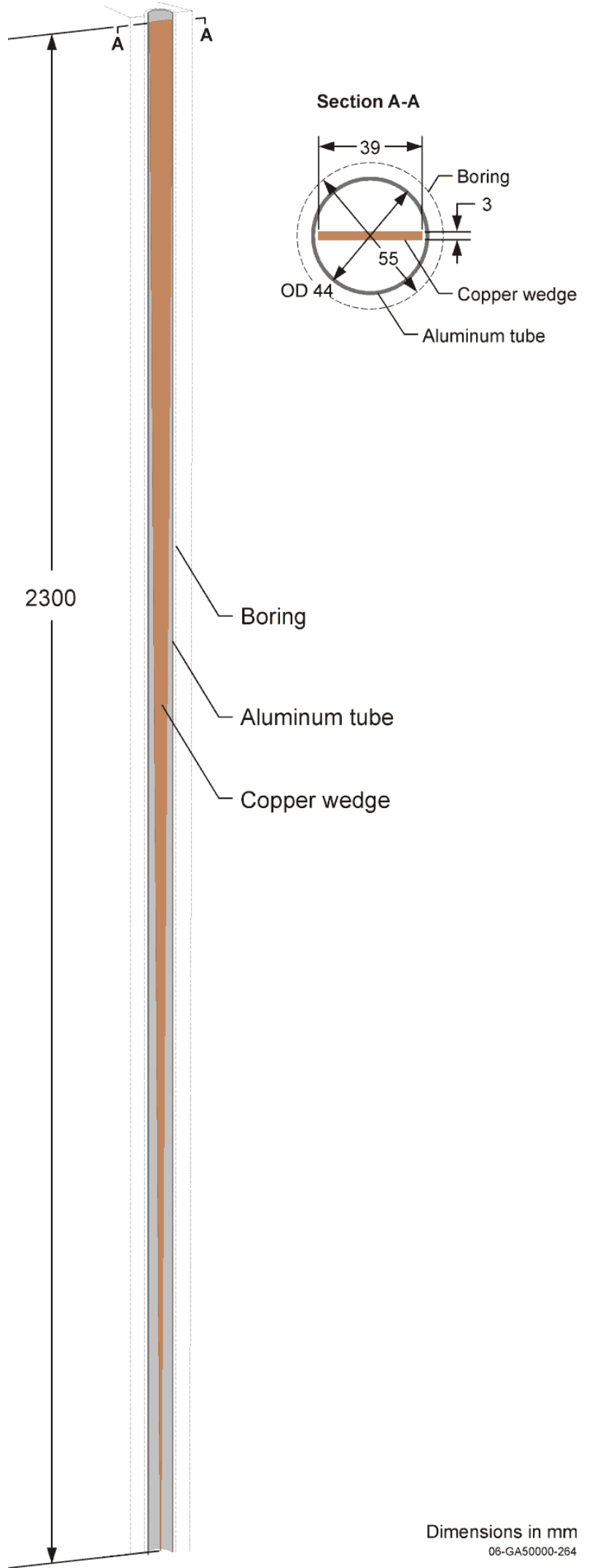

Figure 1.1-10. Automatic Control Rod (derived from Ref. 2).

Revision: 1

Date: March 31, 2014

Page 22 of 375 
NEA/NSC/DOC(2006)1

\author{
Gas Cooled (Thermal) Reactor - GCR \\ PROTEUS-GCR-EXP-004 \\ CRIT-REAC
}

\title{
Static Measurement Rods
}

Simulated control rods were manufactured for these experiments to investigate the spatial dependence of control rod worths in a particular configuration; this was necessary because the operational control rods were very restricted in their locational possibilities. These rods were designed to be inserted in either CDriver channels in the radial reflector or into a specially designed graphite sleeve which replaced a column of pebbles in the columnar hexagonal cores. Because the core and radial reflectors were of significantly different heights, two pairs of rods were produced; apart from the axial dimensions, they were nominally identical (Ref. 2 and 3).

The rods consisted of cylindrical assemblies with an outer diameter of $26 \mathrm{~mm}$ and $2 \mathrm{~mm}$ thick Peraluman R-257 wall. The shorter pair of tubes contained eleven, $22 \mathrm{~mm}$ diameter, borated steel pieces of various lengths between 120 and $180 \mathrm{~mm}$, totaling $1581 \pm 1 \mathrm{~mm}$ in each assembly. The longer pair contained a total of $1711 \pm 1 \mathrm{~mm}$ of borated steel pieces. The longer rods also contained a graphite filler piece, above the borated steel section, with a length of $1414 \mathrm{~mm}$. Figure 1.1-11 and 1.1-12 show the long and short variations of the static measurement rods, respectively. The dimensions of both pairs of rods were arranged such that the borated steel regions were similarly located with respect to the axial position of the fuelled region. When the longer rods were resident in the radial reflector, the bottom of the hole in the upper hanger was flush with the upper surface of the upper steel support plate. When the shorter rod was inserted in the core region, it rested on the cavity floor. The graphite sleeve for the shorter rods (shown in Figure 1.1-13) had a length of $1730 \mathrm{~mm}$, an inner diameter of $27 \mathrm{~mm}$, and an outer diameter of $60 \mathrm{~mm}$ (Ref. 2). 
NEA/NSC/DOC(2006)1

Gas Cooled (Thermal) Reactor - GCR

PROTEUS-GCR-EXP-004

CRIT-REAC
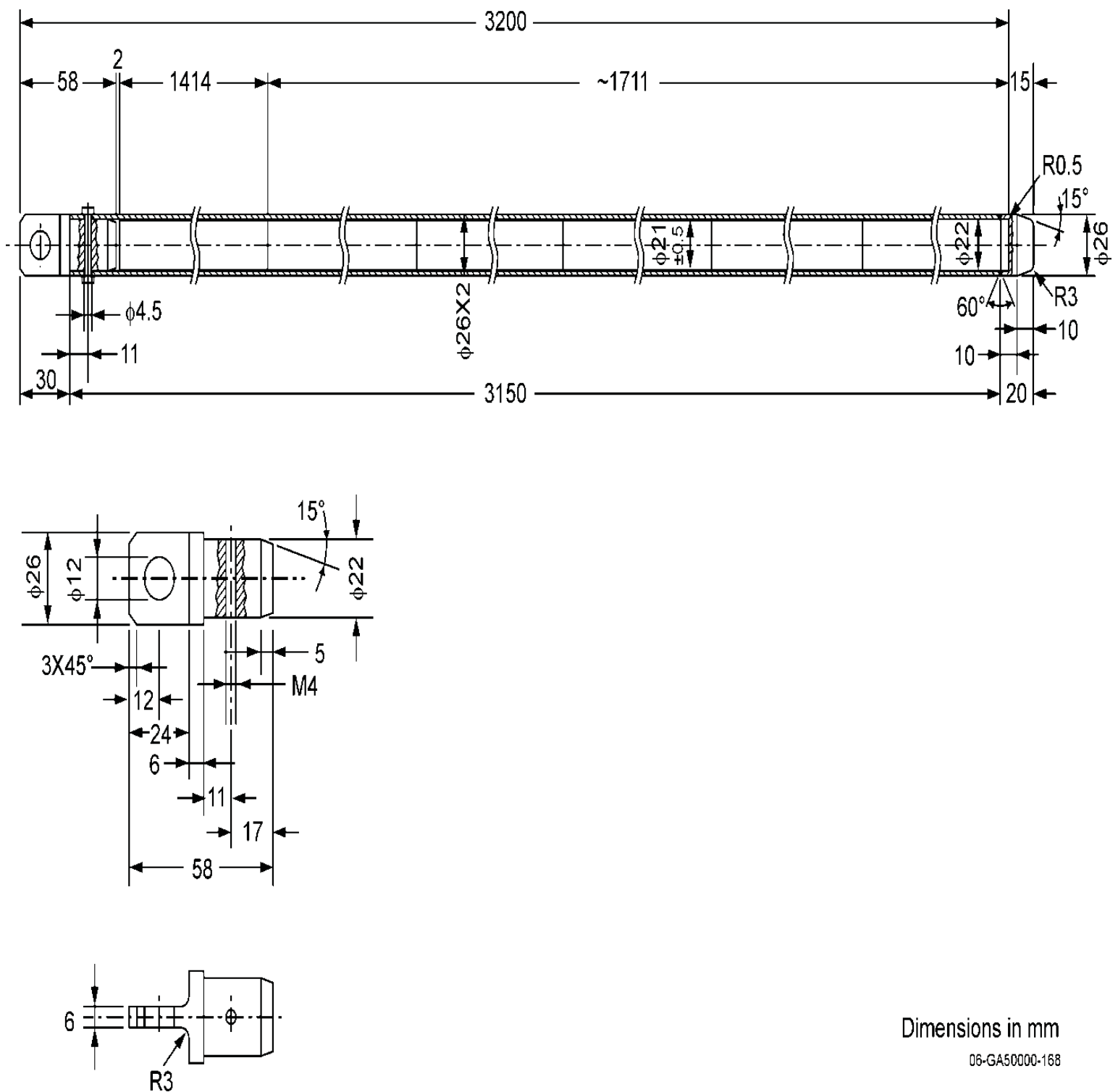

Dimensions in $\mathrm{mm}$

06-GA50000-168

Figure 1.1-11. Details of the Long Static Measurement Rod (Ref. 2). 
NEA/NSC/DOC(2006)1

Gas Cooled (Thermal) Reactor - GCR

PROTEUS-GCR-EXP-004

CRIT-REAC

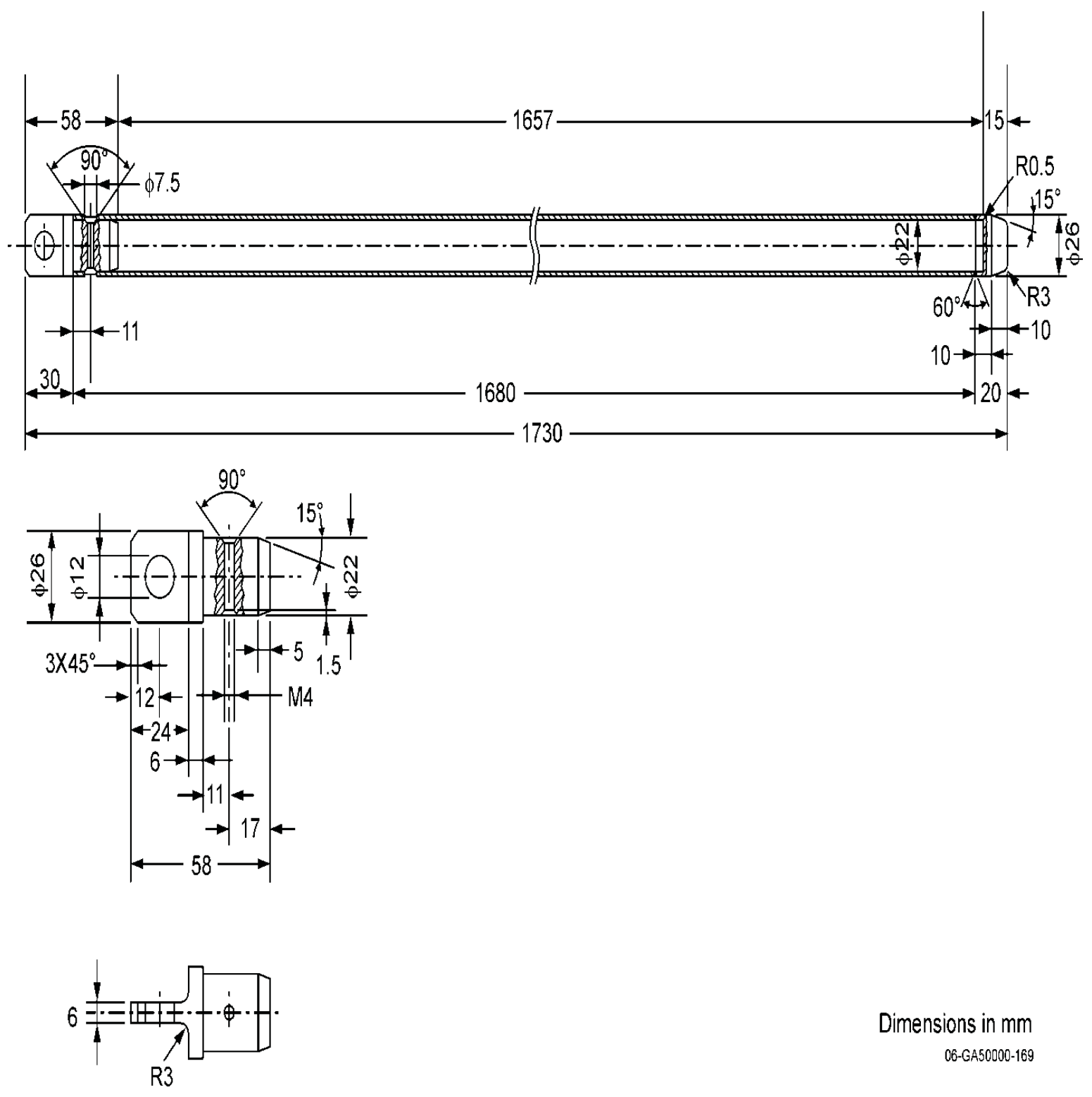

Figure 1.1-12. Details of the Short Static Measurement Rod (Ref. 2). 
NEA/NSC/DOC(2006)1

Gas Cooled (Thermal) Reactor - GCR

PROTEUS-GCR-EXP-004

CRIT-REAC

Boron steel control rod

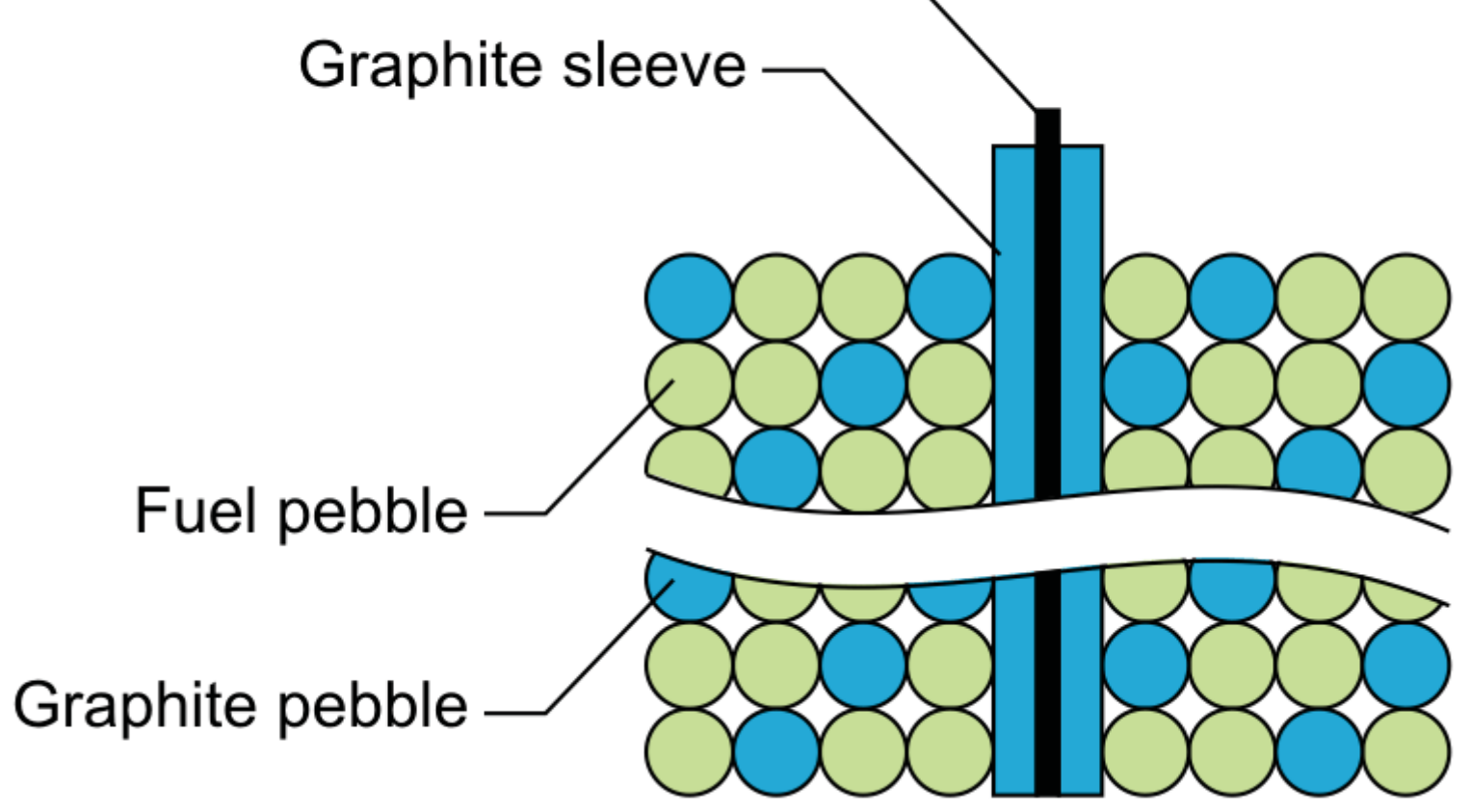

11-GA50002-12-3

Figure 1.1-13. Graphite Sleeve for Short Static Measurement Rod (Ref. 2). 
NEA/NSC/DOC(2006)1

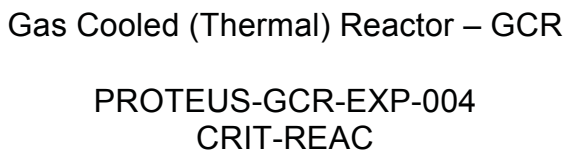

\section{Fuel Pebbles}

The fuel pebble physical properties are provided in Table 1.1-1. Unless otherwise noted, these properties were obtained from the original quality control records. The specified values are averages with their corresponding $1 \sigma$ standard deviations. The diameter and mass of the fuel pebbles were measured at PSI on August 17, 1992, and again on October 30, 1995. The masses of the fuel pebbles did not change significantly over the $>3$ year time period. However, there was a slight reduction in the fuel pebble diameter, presumably due to slight indentations of the surface caused during the loading process, and is considered insignificant. ${ }^{a}$ Measurements performed on August 17, 1992, are recommended by PSI for use in modeling these experiments (Ref. 2 and 3). The construction and dimensions of the fuel pebble are shown in Figure 1.1-14.

Fuel for the experiments was provided by the KFA Research Center in Jülich, Germany (Ref. 3).

Arbeitsgemeinschaft Versuchsreaktor (AVR)-type fuel pebbles were employed in the HTR-PROTEUS experiments. Fuel particles were distributed randomly throughout the graphite matrix of the fuel pebbles. ${ }^{b}$

Some 5460 LEU AVR fuel pebbles were transferred from the LEU HTR experimental program in the AVR test facility to the PROTEUS facility in March and April of 1992. ${ }^{\mathrm{c}}$

There are 9394 fuel kernels in the fuel region of each fuel pebble. ${ }^{\mathrm{d}}$

\footnotetext{
${ }^{a}$ The HTR-PROTEUS Core 5 had been loaded three times over the course of 1.5 years; the variation in the reactivity was insignificant, which is a strong indication that the change in mass was negligible.

${ }^{\mathrm{b}}$ Chawla, R., Joneja, O. P., Rosselet, M., and Williams, T., "Definition and Analysis of an Experimental Benchmark on Shutdown Rod Worths in LEU-HTR Configurations," Nucl. Technol., 139, 50-60 (2002).

c Brogli, R., Mathews, D., and Seiler, R., "HTR Roteus Experiments," Proc. 2nd JAERI Symposium on HTGR Technologies, Oarai, Japan, October 21-23, 1992, p. 233-239, JAERI-M 92-215 (1993).

'Difilippo, F. C., "Monte Carlo Calculations of Pebble Bed Benchmark Configurations of the PROTEUS Facility," Nucl. Sci. Eng., 143, 240-253 (2003).
}

Revision: 1

Date: March 31, 2014 
NEA/NSC/DOC(2006)1

\section{Gas Cooled (Thermal) Reactor - GCR \\ PROTEUS-GCR-EXP-004 \\ CRIT-REAC}

Table 1.1-1. Fuel Pebble Physical Specifications (Ref. 2 and 3). ${ }^{(a)}$

\begin{tabular}{|c|c|c|c|c|}
\hline${ }^{234} \mathrm{U}$ mass per fuel pebble & 0.008 & \pm & 0.001 & gram \\
\hline${ }^{235} \mathrm{U}$ mass per fuel pebble & 1.000 & \pm & 0.010 & gram \\
\hline${ }^{236} \mathrm{U}$ mass per fuel pebble & 0.005 & \pm & 0.001 & gram \\
\hline${ }^{238} \mathrm{U}$ mass per fuel pebble & 4.953 & \pm & 0.050 & gram \\
\hline Total uranium mass per fuel pebble & 5.966 & \pm & 0.060 & gram \\
\hline Carbon mass per fuel pebble & 193.1 & \pm & 0.2 & gram \\
\hline Total mass per fuel pebble ${ }^{(\mathrm{b}),(\mathrm{c})}$ & 202.22 & \pm & 0.18 & gram \\
\hline Fuel pebble inner (fueled) zone radius ${ }^{(\mathrm{d})}$ & $2.350^{(\mathrm{f})}$ & \pm & 0.025 & $\mathrm{~cm}$ \\
\hline Fuel pebble outer radius & 3.0006 & \pm & 0.002 & $\mathrm{~cm}$ \\
\hline Radius of fuel particles $\left(\mathrm{UO}_{2} \text { substrates }\right)^{(\mathrm{e})}$ & $0.02510^{(\mathrm{f})}$ & \pm & $0.0010^{(\mathrm{g})}$ & $\mathrm{cm}$ \\
\hline Thickness of particle buffer coatings $(\mathrm{C})$ & 0.00915 & \pm & $0.0025^{(\mathrm{h})}$ & $\mathrm{cm}$ \\
\hline Thickness of particle inner PyC coatings ${ }^{(e)}$ & 0.00399 & \pm & $0.0010^{(\mathrm{h})}$ & $\mathrm{cm}$ \\
\hline Thickness of particle SiC coatings & 0.00353 & \pm & $0.0004^{(\mathrm{h})}$ & $\mathrm{cm}$ \\
\hline Thickness of particle outer PyC coatings ${ }^{(\mathrm{e})}$ & 0.00400 & \pm & $0.0008^{(h)}$ & $\mathrm{cm}$ \\
\hline Density of fuel particles ( $\mathrm{UO}_{2}$ substrates) & 10.88 & \pm & 0.04 & $\mathrm{~g} / \mathrm{cm}^{3}$ \\
\hline Density of fuel particle buffer coatings (C) & 1.10 & +0 & $-0.11^{(\mathrm{i})}$ & $\mathrm{g} / \mathrm{cm}^{3}$ \\
\hline Density of fuel particle inner PyC coatings & 1.90 & \pm & 0.05 & $\mathrm{~g} / \mathrm{cm}^{3}$ \\
\hline Density of fuel particle $\mathrm{SiC}$ coatings & 3.20 & \pm & 0.02 & $\mathrm{~g} / \mathrm{cm}^{3}$ \\
\hline Density of fuel particle outer PyC coatings & 1.89 & \pm & 0.05 & $\mathrm{~g} / \mathrm{cm}^{3}$ \\
\hline
\end{tabular}

(a) The fuel pebble masses and outer diameters were measured at PSI on August 17, 1992, and October 30, 1995. The second series of measurements indicated a significant reduction of the pebble diameter over the 3 years of operation; however, since the mass measurements indicated no such decrease it was assumed that the apparent diameter reduction was due to indentations in the pebbles caused during handling and not from a general loss of material.

(b) The total mass of oxygen and silicon in the fuel pebbles was not reported.

(c) There is a discrepancy of $0.86 \mathrm{~g}(0.43 \%)$ in the total fuel pebble mass of $201.4 \mathrm{~g}$ computed from the individual components provided in the table as compared with the measured fuel mass of $202.22 \pm 0.18 \mathrm{~g}$ on August 17, 1992 .

(d) The $47 \pm 0.5 \mathrm{~mm}$ diameter of the fuelled region obtained from neutron radiographs made by E. Lehmann at the PSI Saphir reactor corresponds with the $47 \mathrm{~mm}$ diameter fuelled region given by Gontard et al. (KFA Jülich report HBK-IB-10/86).

(e) There are slight differences in the reported radius/thickness between this table and Figure 1.1-14; the differences are within their reported $1 \sigma$ uncertainties.

(f) The last significant digit on these two values, zero, is not reported in Reference 3 but is reported in Reference 2 .

(g) The uncertainty in the $\mathrm{UO}_{2}$ particle radius is a $90 \%$ confidence value.

(h) The uncertainties in the particle coating thicknesses are $95 \%(2 \sigma)$ confidence values.

(i) The density of the fuel particle buffer coatings is stated to be $\leq 1.1 \mathrm{~g} / \mathrm{cm}^{3}$. The onesided $10 \%$ uncertainty $(1 \sigma)$ was assumed by the authors of the reference reports in the absence of measured data. 
NEA/NSC/DOC(2006)1

Gas Cooled (Thermal) Reactor - GCR

PROTEUS-GCR-EXP-004

CRIT-REAC

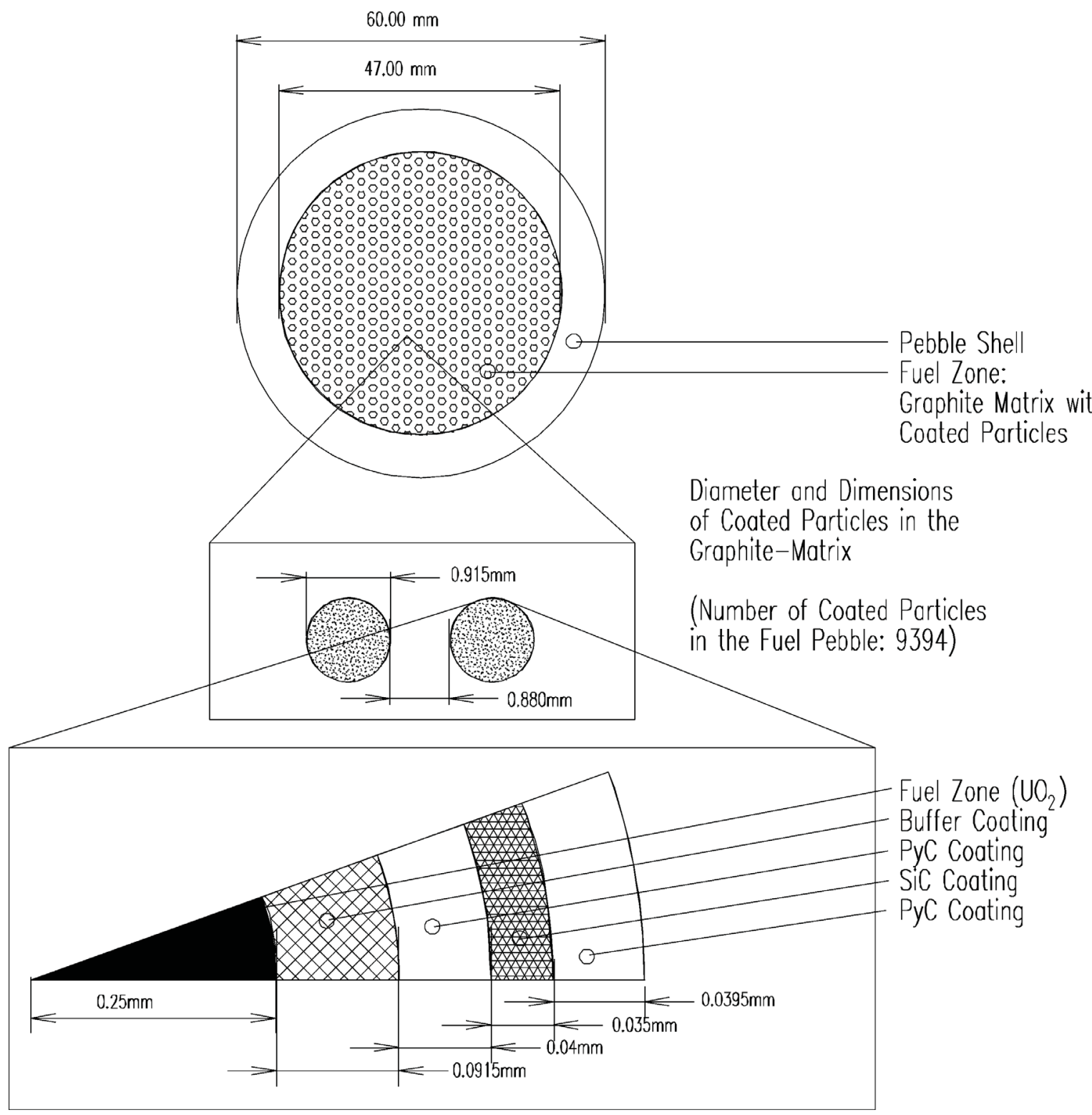

Figure 1.1-14. HTR-PROTEUS Fuel Pebble and Coated Fuel Particle (Ref. 2 and 3). 
NEA/NSC/DOC(2006)1

\author{
Gas Cooled (Thermal) Reactor - GCR \\ PROTEUS-GCR-EXP-004 \\ CRIT-REAC
}

\title{
Moderator Pebbles
}

The physical properties of the moderator pebbles (Table 1.1-2) were obtained from measurements performed at PSI on August 17, 1992, May 3, 1995, and October 30, 1995. These values correspond well with those provided in relevant quality control records. The specified values are averages with a $1 \sigma$ standard deviation. There were no significant changes noted in the properties of the moderator pebbles throughout the course of these experiments (Ref. 2).

Table 1.1-2. Moderator Pebble Physical Specifications (Ref. 2 and 3).

\begin{tabular}{||l|rrrr||}
\hline \hline Moderator Pebble Mass & 190.54 & \pm & 1.44 & $\mathrm{~g}$ \\
\hline Moderator Pebble Outer Radius & 2.9979 & \pm & 0.0015 & $\mathrm{~cm}$ \\
\hline
\end{tabular}

\section{Start-Up Source}

The reactor start-up sources were normally in their "in" position during reactor operation. At low fluxes their reactivity effect is positive by virtue of the apparent enhanced neutron multiplication; at normal operating fluxes of $>10^{7} \mathrm{n} / \mathrm{cm}^{2} / \mathrm{s}$, their effect was negative due to parasitic neutron absorption in the source and casing. The start-up sources pass through horizontal aluminum guide tubes situated in the radial reflector at about the level of the cavity floor (Ref. 3).

\section{Detectors}

There are a total of eight detection channels used for nuclear instrumentation: three safety channels, two impulse channels, one logarithmic channel, one linear channel, and one deviation channel. Apart from the two impulse channels, which were fission chambers, all the instrumentation consisted of large ionization chambers $(220 \mathrm{~mm} \times 90 \mathrm{~mm} \varnothing)$ situated in horizontal channels in the reflector at a radius of $\sim 1000 \mathrm{~mm}$ (Ref. 3).

\section{Temperature Sensors}

There are typically four separate temperature sensors in the system: two in the core and two in the radial reflector (Ref. 3).

\subsubsection{Components Unique to Cores 9 and 10}

The following components are unique to core configurations 9 and 10 .

\section{Graphite Fillers}

Graphite filler pieces were utilized to support the outer surfaces of the various deterministic configurations and to modify the shape of the cavity floor to avoid ordering effects in random core configurations (Ref. 2). The graphite filler pieces used to modify the cavity floor were not used for Cores 9 and 10.

For the hexagonal close packed cores, the 22 -sided cavity is converted to a 12 -sided one using twelve graphite pieces running from the bottom of the cavity to just beneath the aluminum safety ring $(1750 \mathrm{~mm})$. Each graphite piece is unique, as shown in Figures 1.1-15 (Ref. 2). These graphite filler pieces serve as axial modifiers to the core cavity. 


\section{Gas Cooled (Thermal) Reactor - GCR \\ PROTEUS-GCR-EXP-004 \\ CRIT-REAC}

The 12-sided polygon cavity developed with the graphite fillers had a height of $1729 \mathrm{~mm}$ with sides at alternated distances of 601.5 and $603 \mathrm{~mm}$ from the center. The equivalent area cylinder would have a radius of $608.3 \mathrm{~mm} .^{\text {a }}$

A cylindrical version of the 12-sided polygon cavity would have a radius (the first value represents an equal perimeter, and the second value represents an equal area) of 615.4 and $608.3 \mathrm{~mm}$, respectively. ${ }^{\mathrm{b}}$

The graphite filler pieces numbered as pieces 13 and 14 in Figure 1.1-16 were not used in Cores 9 and 10 (Ref. 2).

\footnotetext{
a Difilippo, F. C., "Monte Carlo Calculations of Pebble Bed Benchmark Configurations of the PROTEUS Facility," Nucl. Sci. Eng., 143, 240-253 (2003).

${ }^{\mathrm{b}}$ Difilippo, F. C., "Applications of Monte Carlo Simulations of Thermalization Processes to the Nondestructive Assay of Graphite,” Nucl. Sci. Eng., 133, 163-177 (1999).

Revision: 1

Date: March 31, 2014

Page 31 of 375
} 
NEA/NSC/DOC(2006)1

Gas Cooled (Thermal) Reactor - GCR

PROTEUS-GCR-EXP-004

CRIT-REAC

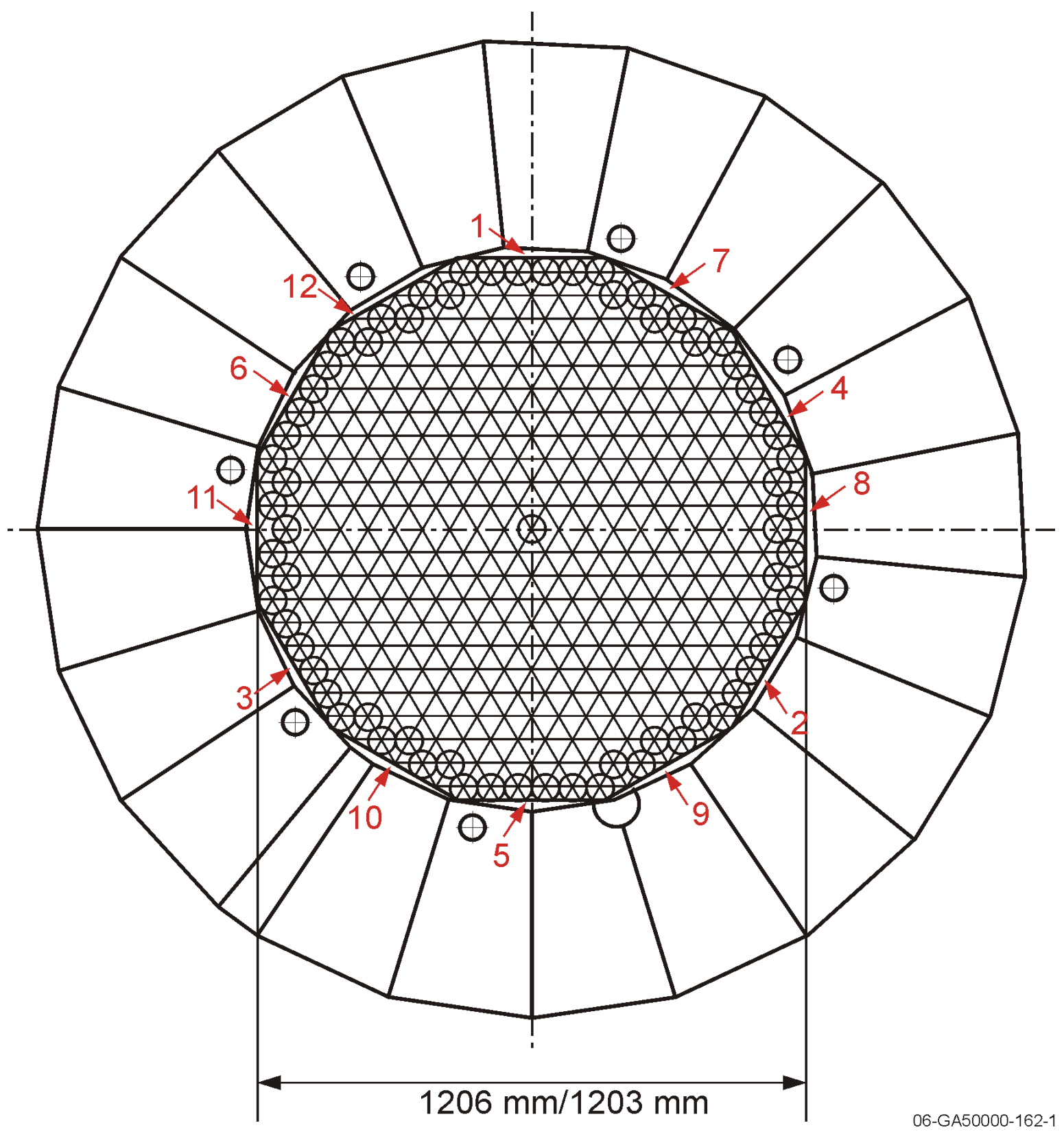

Figure 1.1-15. Positioning of the Graphite Filler Pieces used in the Deterministic Cores (Ref. 2). 
NEA/NSC/DOC(2006)1

Gas Cooled (Thermal) Reactor - GCR

PROTEUS-GCR-EXP-004

CRIT-REAC
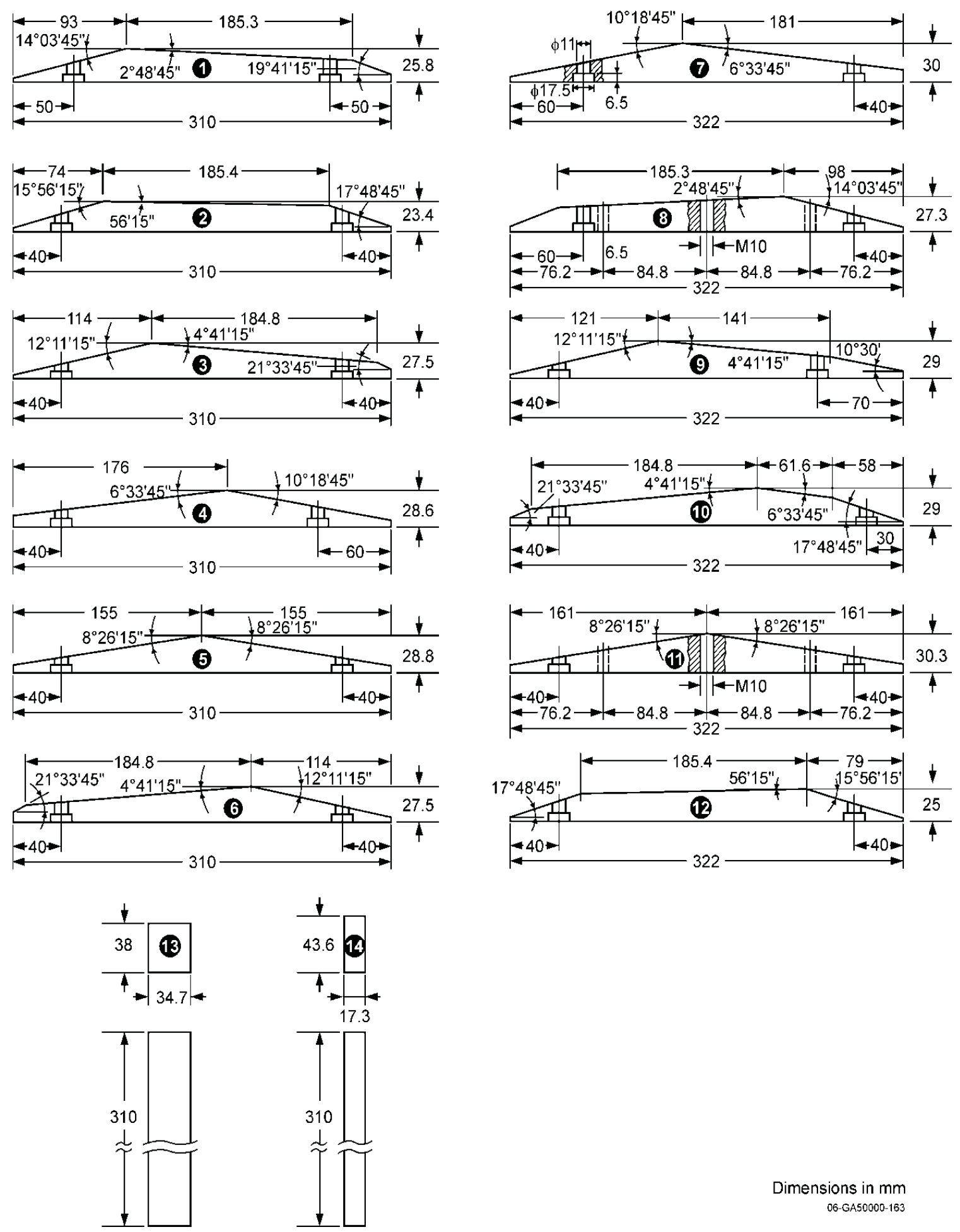

Figure 1.1-16. Graphite Filler Pieces used in the Deterministic Cores (Ref. 2). The position numbers are engraved into the top of each graphite filler piece. 
NEA/NSC/DOC(2006)1

\author{
Gas Cooled (Thermal) Reactor - GCR \\ PROTEUS-GCR-EXP-004 \\ CRIT-REAC
}

\title{
ZEBRA Control Rods
}

The ZEBRA control rods were not used in the experiments with Cores 9 and 10.

\section{Withdrawable Stainless Steel Control Rods}

The ZEBRA type control rods used in Core 1were replaced with four withdrawable stainless steel control rods for Cores $1 \mathrm{~A}$ through 10. The stainless steel rods were placed in four C-Driver channels, instead of the channels used for the ZEBRA rods, but close to the original ZEBRA positions (see Figure 1.1-2). These rods were intended to increase operational flexibility and were designed to operate at two radii: $789 \mathrm{~mm}$ (ring 3) or $906 \mathrm{~mm}$ (ring 5). They were exclusively used in ring 5 throughout the measurements due to the thermal flux gradient in the radial reflector at these positions (Ref. 2 and 3).

Each rod was comprised of two concentric stainless steel tubes. The inner stainless steel (type St1.4301) tube had an inner diameter of $9.5 \mathrm{~mm}$, outer diameter of $13.5 \mathrm{~mm}$, and length of $2150 \mathrm{~mm}$; this tube could contain various materials, such as $\mathrm{B}_{4} \mathrm{C}$ pellets, to further adjust the rods' worth. The outer stainless steel (type St1.4541) rod had an inner diameter of $14 \mathrm{~mm}$, outer diameter of $22 \mathrm{~mm}$, and length of 2149 $\mathrm{mm}$; this rod was added as a means of increasing rod mass to achieve a satisfactory cable tension. Stainless steel plugs were used to seal both ends of the tubes. The total rod length, including end-stops, was $2200 \mathrm{~mm}$. Technical drawings of these rods are provided in Figure 1.1-17. The rods are fully inserted when the base of the cavity in the inner tube corresponded to the core cavity floor with the tips of the rods lying $25 \mathrm{~mm}$ below this; the indicated rod position on the control panel was $2500 \mathrm{~mm}$. The rods are fully withdrawn when the control panel indicated $\sim 6 \mathrm{~mm}$ and the rod tips were just $49 \mathrm{~mm}$ below the upper surface of the radial reflector. The total rod range was $2494 \mathrm{~mm}$. The bottom of each control rod channel was filled with a $26.5 \mathrm{~mm}$ diameter, $730 \mathrm{~mm}$ long graphite plug, leaving an air gap of $25 \mathrm{~mm}$ below the rod tip (Ref. 2).

No inserts were placed within these stainless steel control rods (Ref. 2). 
NEA/NSC/DOC(2006)1

Gas Cooled (Thermal) Reactor - GCR

PROTEUS-GCR-EXP-004

CRIT-REAC
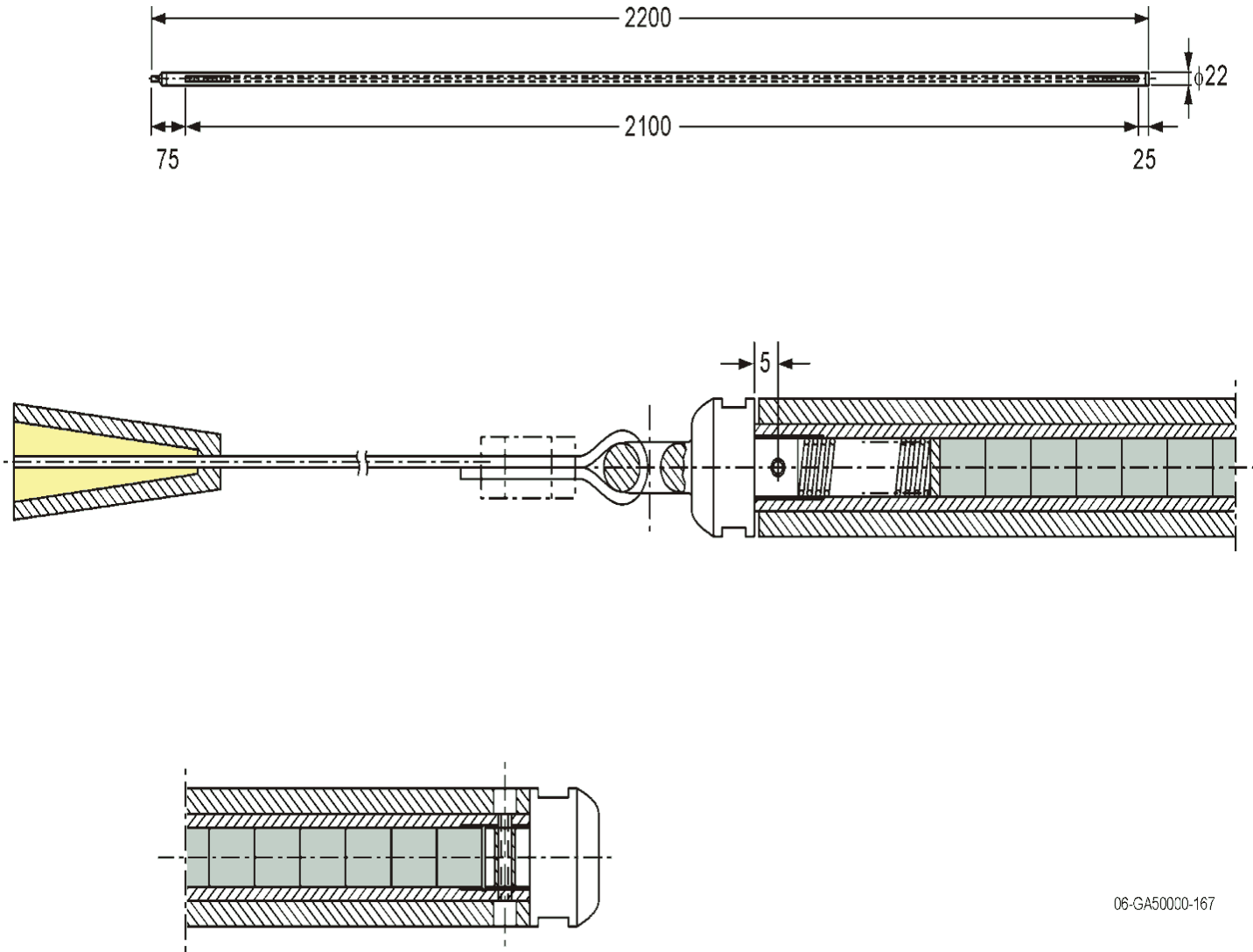

06-GA50000-167

Figure 1.1-17. Details of the Withdrawable Stainless Steel Control Rods (Ref. 2). Units are in millimeters.

Revision: 1 


\section{Gas Cooled (Thermal) Reactor - GCR \\ PROTEUS-GCR-EXP-004 \\ CRIT-REAC}

\section{Polyethylene Rods}

One of the primary goals of the HTR-PROTEUS project was to measure the effect of accidental water ingress into the core. The use of water in the experiments was forbidden and impractical; the presence of moisture was simulated with polyethylene $\left(\mathrm{CH}_{2}\right)$ rods. Different shapes and sizes of polyethylene rods were introduced into the cores to simulate a range of water densities in the void spaces between the pebbles. The dimensions and specific linear densities of the various polyethylene rods are shown in Figure 1.1-18. Most of the rods were either unmachined, or machined down to the reported diameter from a larger diameter rod. Measurements at PSI showed that the 6 and $9 \mathrm{~mm}$ diameter unmachined rods demonstrated higher homogeneity than the machined versions. Additionally, the unmachined rods had not been exposed to extra 'impurity hazardous' machining environments (Ref. 2 and 3). Only the 6.5-, 8.3-, and 8.9-mm-diameter rods, and the triangular rods, were used to establish the critical core configurations of the HTR-PROTEUS experiments.

Polyethylene rods were not used in the experiments with Core 9 . A total of 654 polyethylene rods were placed in the inter-pebble channels (no edge channels) of Core 10. Each rod had a diameter of $6.5 \mathrm{~mm}$ (see Figure 1.1-18) and a total length of $1450(60 \mathrm{~mm} \times 24$ layers $+10 \mathrm{~mm}) \pm 5 \mathrm{~mm}$ (Ref. 1). 
NEA/NSC/DOC(2006)1

Gas Cooled (Thermal) Reactor - GCR

PROTEUS-GCR-EXP-004

CRIT-REAC

$2.96 \mathrm{~mm}$ diameter (machined)

$3 \mathrm{~mm}$ diameter (un-machined)

$5.9 \mathrm{~mm}$ diameter (machined)

$6.5 \mathrm{~mm}$ diameter (un-machined)

$8.3 \mathrm{~mm}$ diameter (un-machined)

$8.9 \mathrm{~mm}$ diameter (machined)

$15.0 \mathrm{~mm}$ sides $6 \mathrm{~mm}$ hole

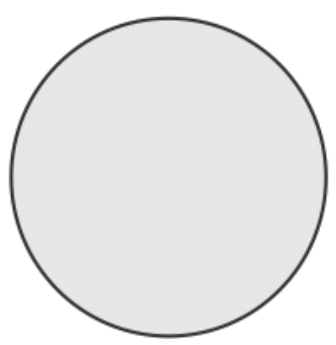

\section{$4.808 \pm 0.001 \mathrm{~g} / \mathrm{cm}$}

Figure 1.1-18. Physical Properties of Available Polyethylene Rods (Ref. 2 and 3).

Reference 3 states that the sides of the triangular rod are $13.5 \mathrm{~mm}$; however, the resultant mass density calculates to be $\sim 1.3 \mathrm{~g} / \mathrm{cm}^{3}$, much greater than a typical density of $0.94 \mathrm{~g} / \mathrm{cm}^{3}$. 
NEA/NSC/DOC(2006)1

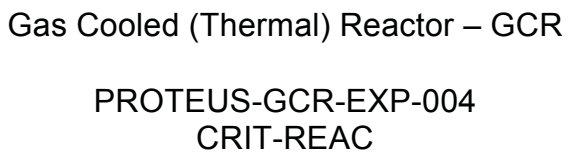

\section{Copper Wire}

Copper wire was not used in the experiments with Cores 9 and 10.

\section{Core Pebble Packing}

Cores 9 and 10 were deterministically stacked in Columnar Hexagonal Point-On-Point (CHPOP) cells, as shown in Figure 1.0-3.

The deterministic configurations were loaded by hand; the fueling machine was used to deliver pebbles to the loading personnel. To facilitate access to the pebble bed, a specially constructed, shielded "loadingbasket" was used. Special anodized aluminum "tripods" were constructed to facilitate with the loading of the point-on-point cores; the tripods were then removed when the layer was complete (Ref. 3).

\section{Core Configurations}

Tables 1.1-3 through 1.1-5 provide detailed summaries of the core description and critical balance information for Cores 9 and 10. Figures 1.1-19 through 1.1-31 provide scale drawings for each different pebble layer, indicating the exact type and position of every pebble, polyethylene rod, and graphite filler piece in the system. Some cores had more than one reference state, indicating either that one or more critical configurations was constructed for this core, for instance with and without the coolant channels being filled in the lower axial reflector, or, that the core was unloaded and loaded again at a later date (Ref. 1).

- Core 9 (reference state \#1): Table 1.1-3 and Figures 1.1-19 through 1.1-24

- Core 9 (reference state \#2): Table 1.1-4 and Figures 1.1-19 through 1.1-25

- Core 10 (reference state \#1): Table 1.1-5 and Figures 1.1-26 through 1.1-31

Where possible, experimental conditions had been measured directly (indicated by $\mathbf{M}$ in the tables) but in a few cases the values were estimated $(\mathbf{E})$.

Excess reactivity worths for individual components in each core configuration are discussed in Section 1.1.5. 
NEA/NSC/DOC(2006)1

\section{Gas Cooled (Thermal) Reactor - GCR \\ PROTEUS-GCR-EXP-004 \\ CRIT-REAC}

Table 1.1-3. Core 9 (Reference State \#1) Critical Information (Ref. 1 and 3). ${ }^{(a)}$

\begin{tabular}{|c|c|c|c|}
\hline \multicolumn{4}{|c|}{ Core Description } \\
\hline $1^{\text {st }}$ Criticality & February 22, 1996 & & \\
\hline Unloaded & May 8, 1996 & & \\
\hline Nominal Pebble Ratio & 1:1 moderator:fuel & & \\
\hline Pebble Count & \multicolumn{3}{|l|}{27 layers $^{(\mathrm{b})}$} \\
\hline Pebble Packing & \multicolumn{3}{|c|}{ Columnar Hexagonal Point-on-Point } \\
\hline Polyethylene Loading & None & & \\
\hline \multicolumn{4}{|l|}{ Critical Balance } \\
\hline Date & February 22, 1996 & & \\
\hline Critical Loading & 27 layers & $\mathbf{M}^{(\mathrm{c})}$ & See Figure 1.1-19 through 1.1-24 \\
\hline Critical Height & $1.62 \mathrm{~m}$ & $\mathbf{M}$ & $27 \times 6 \mathrm{~cm}^{(\mathrm{d})}$ \\
\hline $\begin{array}{l}\begin{array}{l}\text { Rod Positions } \\
\text { (Control/Autorod) }\end{array} \\
\end{array}$ & $0 / 258 \mathrm{~mm}$ & $\mathbf{M}$ & $0 / 1000 \mathrm{~mm}=$ fully out ${ }^{(\mathrm{e})}$ \\
\hline Nominal Flux & $5 \times 10^{7} \mathrm{n} / \mathrm{cm}^{2} / \mathrm{s}$ & $\mathbf{M}$ & \\
\hline Hall Temperature & $19.6^{\circ} \mathrm{C}$ & $\mathbf{M}$ & \\
\hline $\begin{array}{l}\begin{array}{l}\text { Core Temperatures } \\
\text { (Center/Edge) }\end{array} \\
\end{array}$ & $\mathrm{N} / \mathrm{A}$ & $\mathbf{M}$ & \\
\hline $\begin{array}{l}\text { Reflector Temperatures } \\
\left(\mathbf{R 2 , 4 7 / R 2 , 1 5 )}{ }^{(\mathrm{f})}\right.\end{array}$ & N/A & $\mathbf{M}$ & \\
\hline Air Pressure & $980 \mathrm{mbar}$ & $\mathbf{M}$ & \\
\hline Air Humidity & $25 \%$ & $\mathbf{M}$ & \\
\hline
\end{tabular}

(a) Core 9 was, apart from the random cores $4(1,2,3)$, the first loading of a 1:1 (moderator:fuel pebble ratio) core in HTR-PROTEUS. The individual layer loading patterns shown in Figure 1.1-20 through 1.1-25 were chosen both as a result of consideration of the homogeneity of the system, in both the horizontal and vertical planes, and upon consideration of the ease of loading. Channels in the lower axial reflector were filled.

(b) With 27 layers loaded, the system was just critical with all control rods fully withdrawn and the channels in the lower axial reflector filled. For the operational loading, an extra layer of moderator pebbles was added to bring the critical control rod positions into a convenient range (see Core 9, reference state \#2).

(c) Directly measured experimental measurements are indicated with an $\mathbf{M}$; sometimes a few values were estimated, and indicated with an $\mathbf{E}$.

(d) In the columnar hexagonal point-on-point packed cores the height of each layer is $\sim 6 \mathrm{~cm}$, the approximate diameter of the pebbles. See Figure 1.0-3.

(e) The withdrawable control rods and autorod are considered fully withdrawn when their positions indicate 0 and $1000 \mathrm{~mm}$, respectively.

(f) The nomenclature for the channels in the radial reflector is described in Figure 1.1-3. 
NEA/NSC/DOC(2006)1

Gas Cooled (Thermal) Reactor - GCR

PROTEUS-GCR-EXP-004

CRIT-REAC

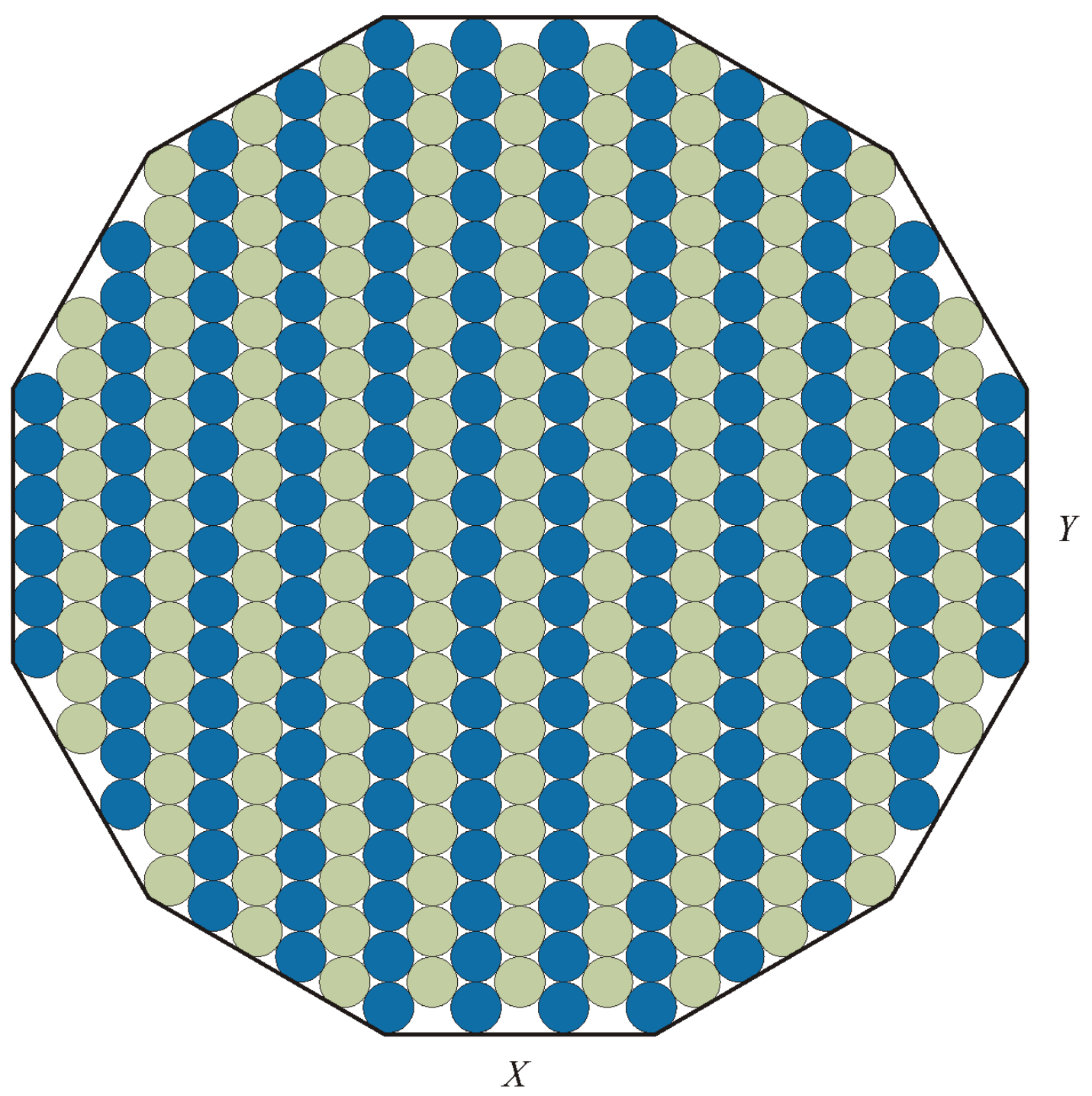

Fuel pebbles:

Moderator pebbles:

Total pebbles: $\quad \overline{361}$

Figure 1.1-19. Layers 1, 7, 13, 19, and 25 of Core 9 (Ref. 1). 
NEA/NSC/DOC(2006)1

Gas Cooled (Thermal) Reactor - GCR

PROTEUS-GCR-EXP-004

CRIT-REAC

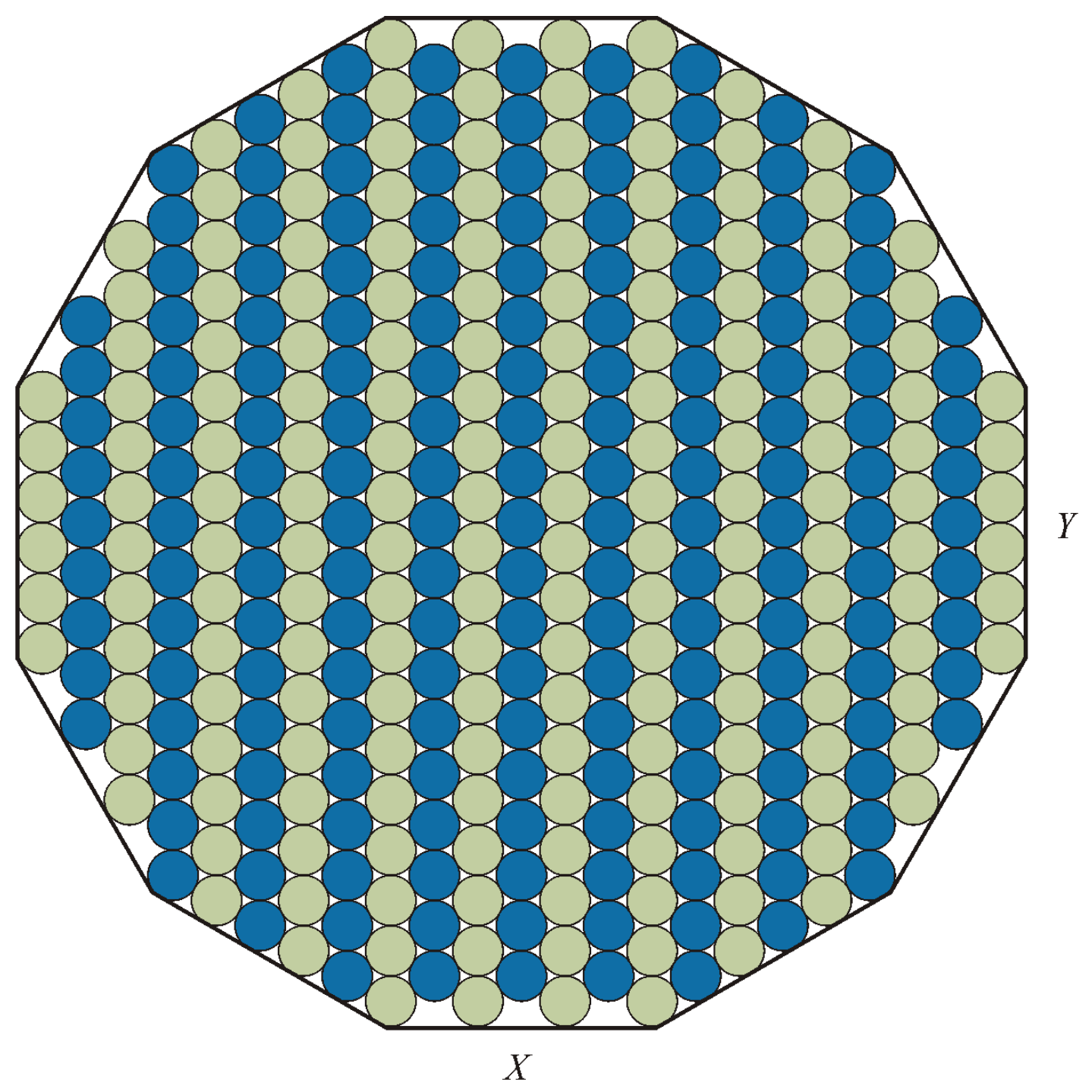

Fuel pebbles: $\quad 184$

Moderator pebbles: $\frac{177}{361}$
Total pebbles:

Figure 1.1-20. Layers 2, 8, 14, 20, and 26 of Core 9 (Ref. 1). 
NEA/NSC/DOC(2006)1

Gas Cooled (Thermal) Reactor - GCR

PROTEUS-GCR-EXP-004

CRIT-REAC

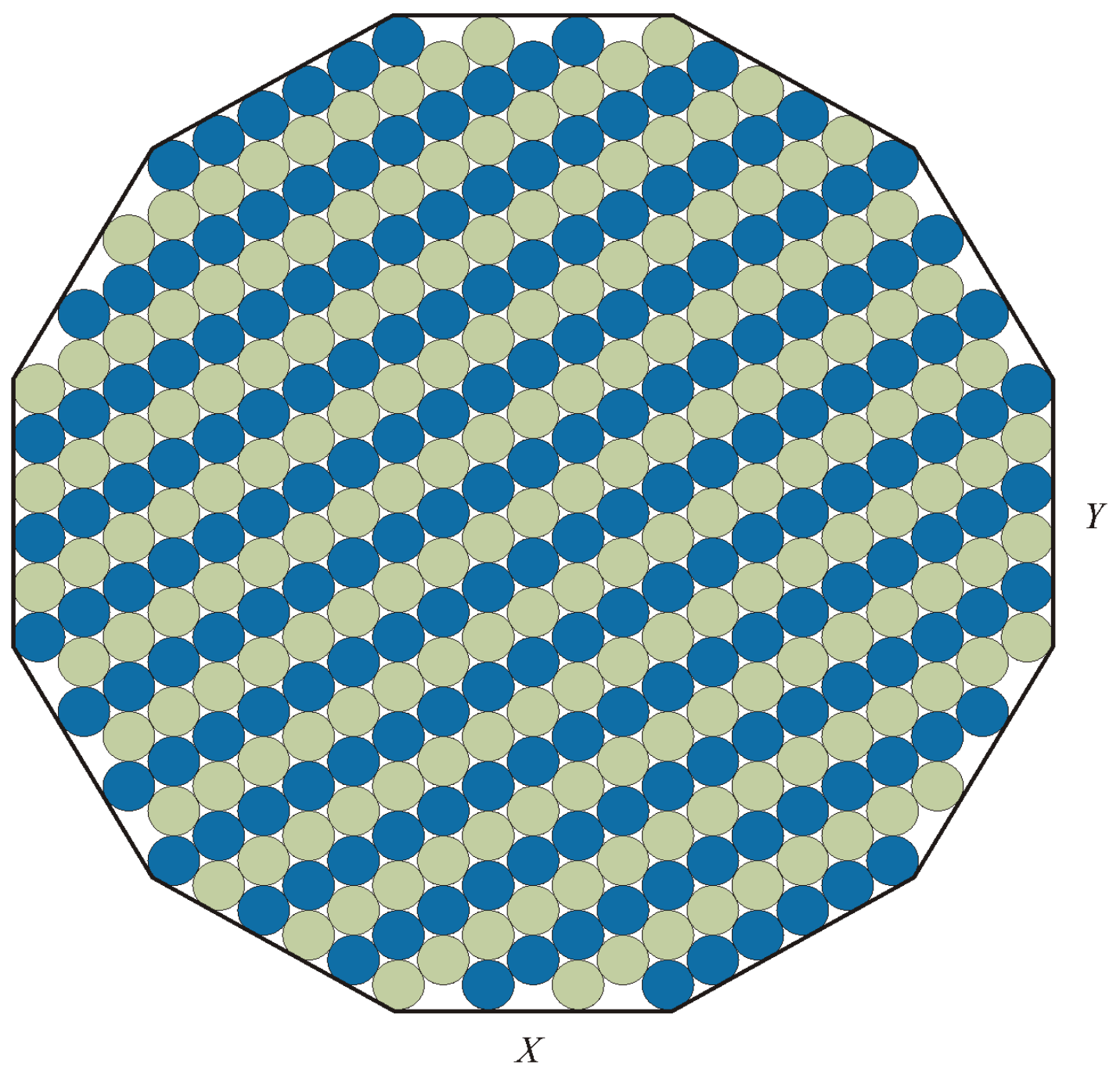

\section{Fuel pebbles: $\quad 177$}

Moderator pebbles: $\frac{184}{361}$

Total pebbles: $\quad 361$

Figure 1.1-21. Layers 3, 9, 15, 21, and 27 of Core 9 (Ref. 1). 
NEA/NSC/DOC(2006)1

Gas Cooled (Thermal) Reactor - GCR

PROTEUS-GCR-EXP-004

CRIT-REAC

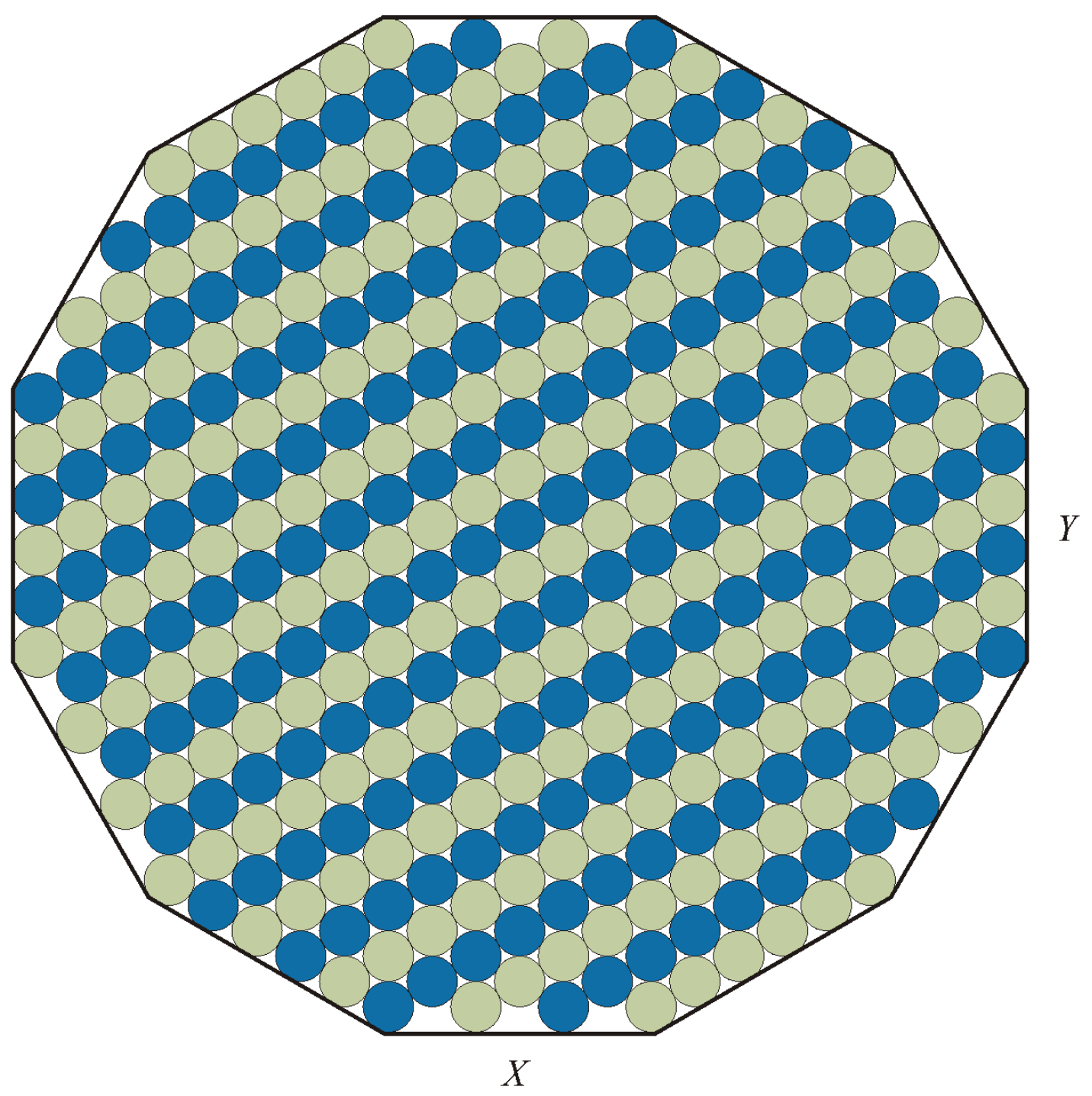

Fuel pebbles:

184

Moderator pebbles: $\frac{177}{361}$
Total pebbles:

Figure 1.1-22. Layers 4, 10, 16, and 22 of Core 9 (Ref. 1). 
NEA/NSC/DOC(2006)1

Gas Cooled (Thermal) Reactor - GCR

PROTEUS-GCR-EXP-004

CRIT-REAC

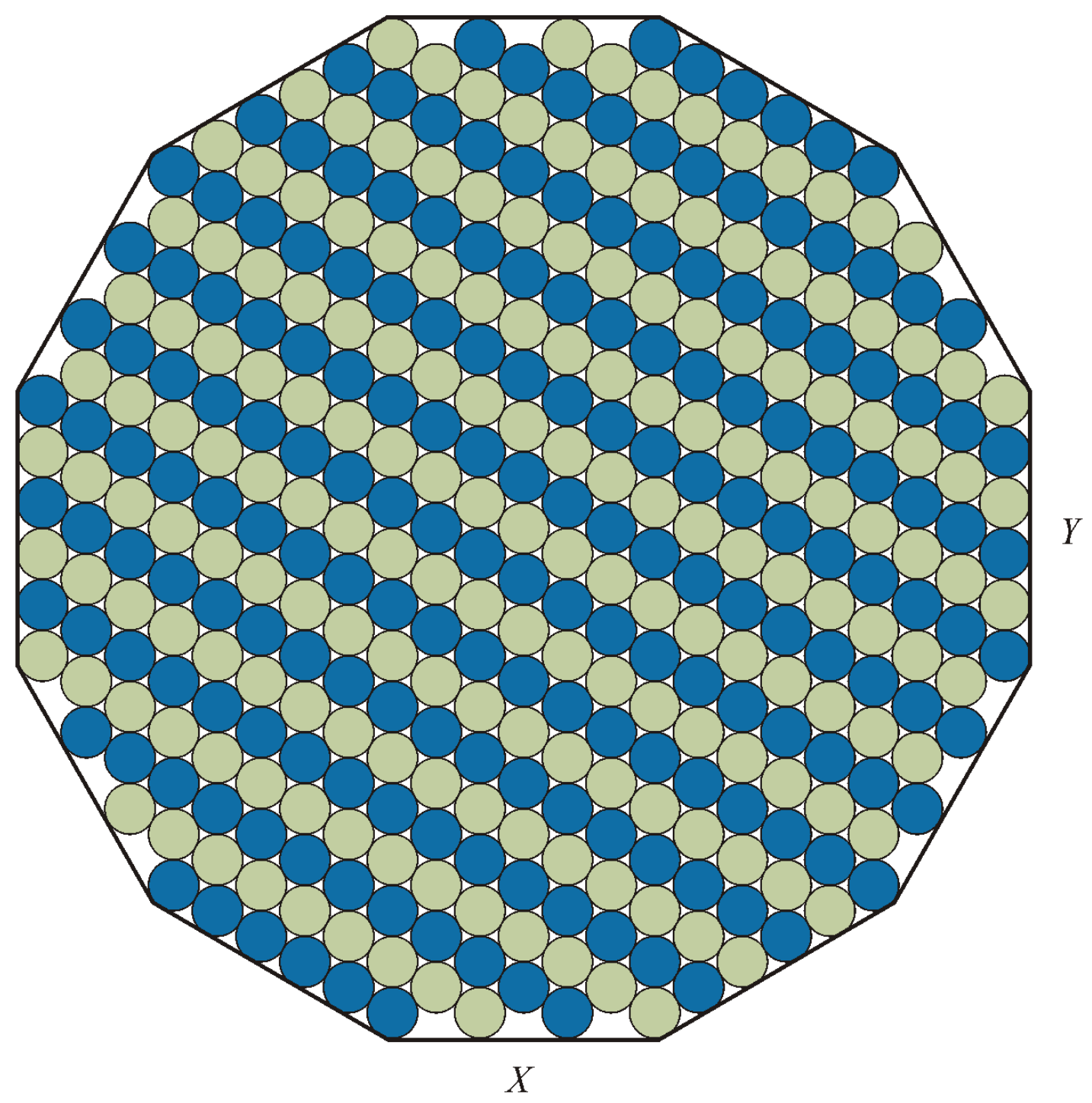

Fuel pebbles: $\quad 177$

Moderator pebbles: 184 Total pebbles: $\quad \overline{361}$

Figure 1.1-23. Layers 5, 11, 17, and 23 of Core 9 (Ref. 1). 
NEA/NSC/DOC(2006)1

Gas Cooled (Thermal) Reactor - GCR

PROTEUS-GCR-EXP-004

CRIT-REAC

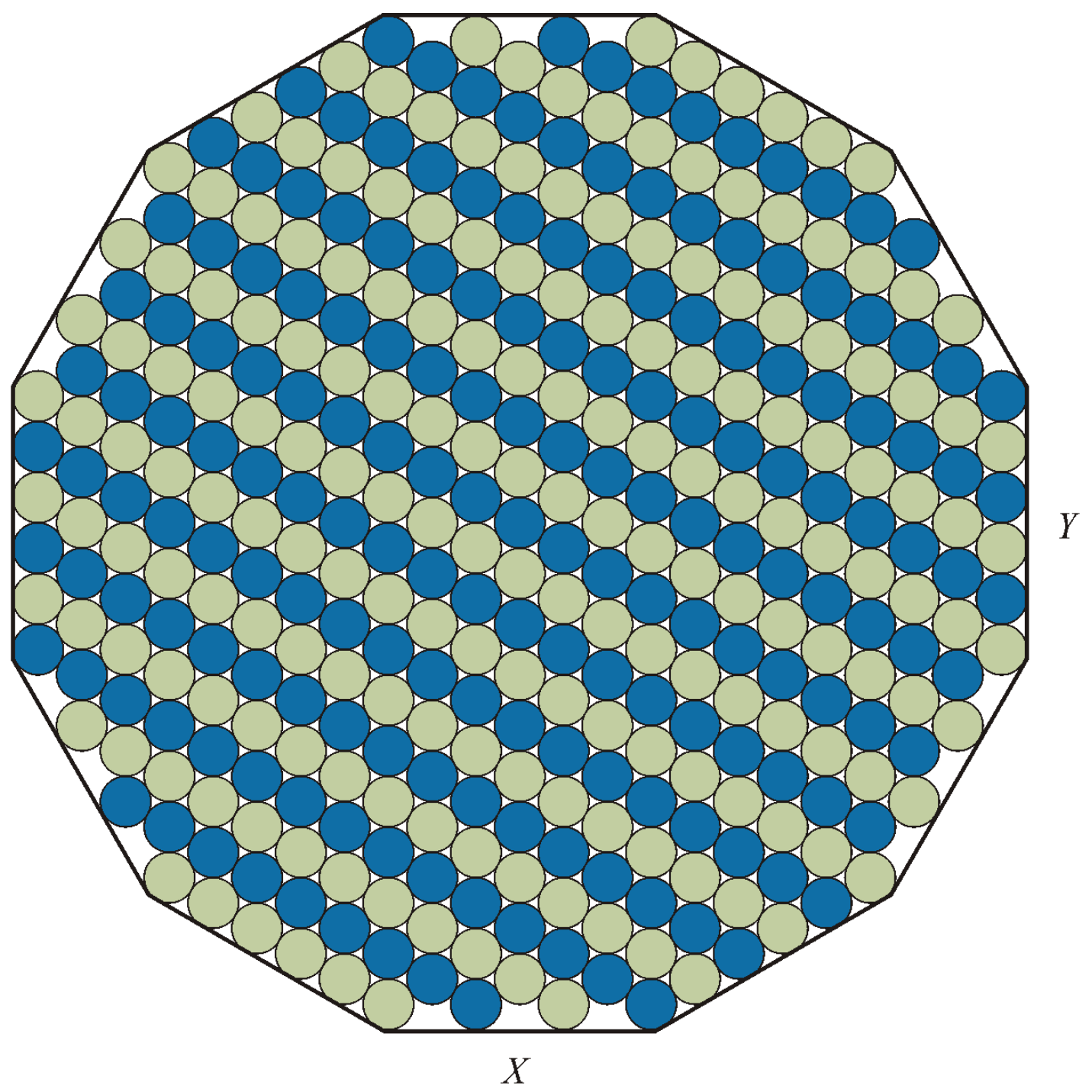

Fuel pebbles: $\quad 184$

Moderator pebbles: $\frac{177}{361}$
Total pebbles:

Figure 1.1-24. Layers 6, 12, 18, and 24 of Core 9 (Ref. 1). 
NEA/NSC/DOC(2006)1

\section{Gas Cooled (Thermal) Reactor - GCR \\ PROTEUS-GCR-EXP-004 \\ CRIT-REAC}

Table 1.1-4. Core 9 (Reference State \#2) Critical Information (Ref. 1 and 3). ${ }^{(a)}$

\begin{tabular}{|c|c|c|c|}
\hline \multicolumn{4}{|c|}{ Core Description } \\
\hline $1^{\text {st }}$ Criticality & February 22, 1996 & & \\
\hline Unloaded & May 8,1996 & & \\
\hline Nominal Pebble Ratio & 1:1 moderator:fuel & & \\
\hline Pebble Count & \multicolumn{3}{|c|}{27 layers +1 pure moderator } \\
\hline Pebble Packing & \multicolumn{3}{|c|}{ Columnar Hexagonal Point-on-Point } \\
\hline Polyethylene Loading & None & & \\
\hline \multicolumn{4}{|l|}{ Critical Balance } \\
\hline Date & February 23, 1996 & & \\
\hline Critical Loading & $27+1$ layers & $\mathbf{M}^{(\mathrm{b})}$ & See Figures 1.1-19 through 1.1-25 \\
\hline Critical Height & $1.68 \mathrm{~m}$ & $\mathbf{M}$ & $28 \times 6 \mathrm{~cm}^{(\mathrm{c})}$ \\
\hline $\begin{array}{l}\text { Rod Positions } \\
\text { (Control/Autorod) }\end{array}$ & $1620 / 25 \mathrm{~mm}$ & $\mathbf{M}$ & $0 / 1000 \mathrm{~mm}=$ fully out ${ }^{(\mathrm{d})}$ \\
\hline Nominal Flux & $5 \times 10^{7} \mathrm{n} / \mathrm{cm}^{2} / \mathrm{s}$ & M & \\
\hline Hall Temperature & $19.2^{\circ} \mathrm{C}$ & $\mathbf{M}$ & \\
\hline $\begin{array}{l}\text { Core Temperatures } \\
\text { (Center/Edge) }\end{array}$ & $\mathrm{N} / \mathrm{A}$ & $\mathbf{M}$ & \\
\hline $\begin{array}{l}\text { Reflector Temperatures } \\
(\mathrm{R} 2,47 / \mathrm{R} 2,15)^{(\mathrm{e})}\end{array}$ & $\mathrm{N} / \mathrm{A}$ & $\mathbf{M}$ & \\
\hline Air Pressure & $981 \mathrm{mbar}$ & $\mathbf{E}$ & \\
\hline Air Humidity & $25 \%$ & $\mathbf{E}$ & \\
\hline
\end{tabular}

(a) This configuration differs from reference state \#1 only in the fact that an extra layer of moderator pebbles was loaded to make the configuration slightly more reactive and thus bring the control rods into an operationally convenient position. It is included here as it provides a clean comparison between control rod worths and core height.

(b) Directly measured experimental measurements are indicated with an $\mathbf{M}$; sometimes a few values were estimated, and indicated with an $\mathbf{E}$.

(c) In the columnar hexagonal point-on-point packed cores the height of each layer is $\sim 6$ $\mathrm{cm}$, the approximate diameter of the pebbles. See Figure 1.0-3.

(d) The withdrawable control rods and autorod are considered fully withdrawn when their positions indicate 0 and $1000 \mathrm{~mm}$, respectively.

(e) The nomenclature for the channels in the radial reflector is described in Figure 1.1-3. 
NEA/NSC/DOC(2006)1

Gas Cooled (Thermal) Reactor - GCR

PROTEUS-GCR-EXP-004

CRIT-REAC

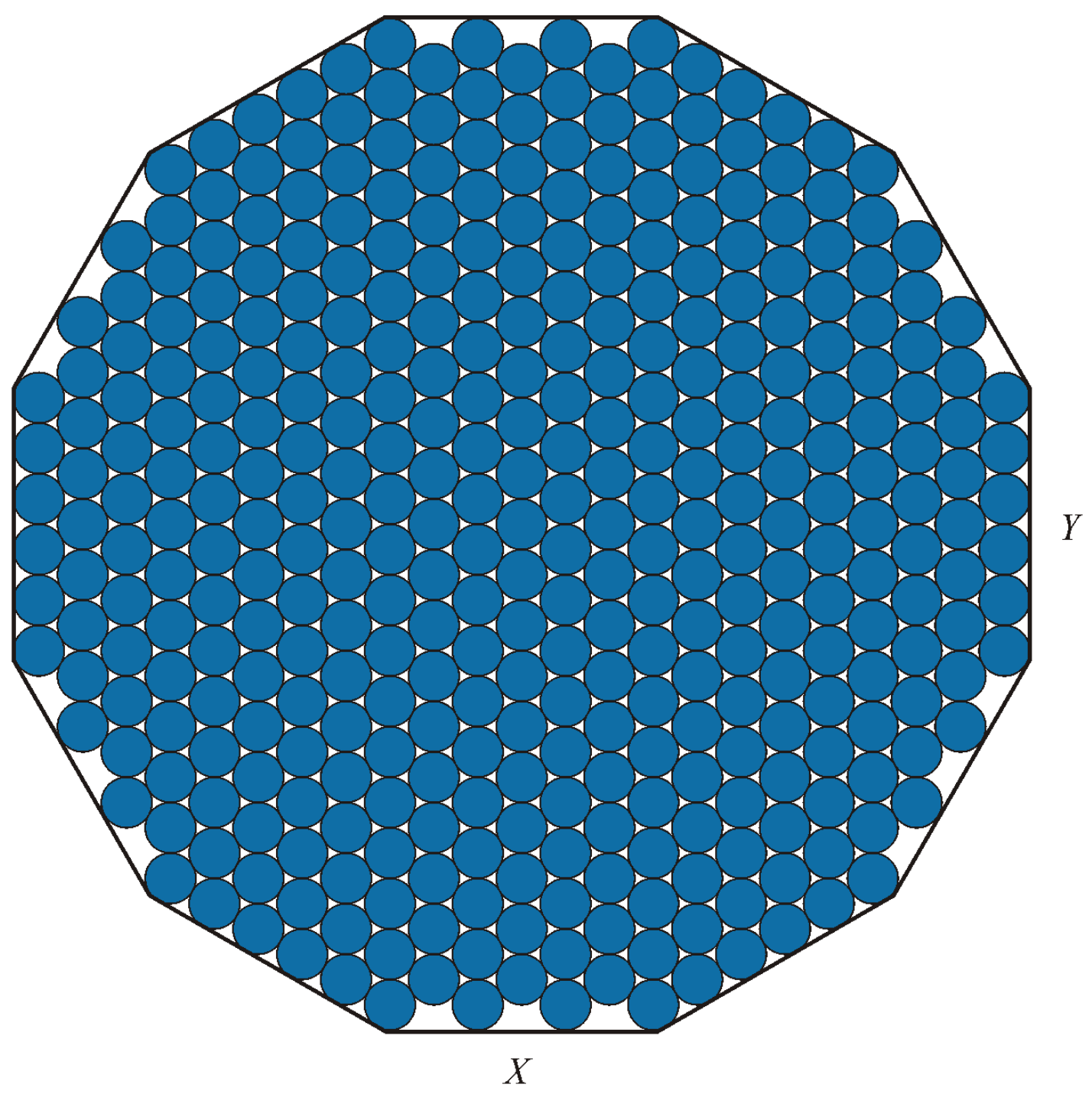

Moderator pebbles: $\frac{361}{361}$
Total pebbles:

Figure 1.1-25. Additional Moderator Layer $\left(28^{\text {th }}\right)$ of Core 9, Reference State \#2 (Ref. 1). 
NEA/NSC/DOC(2006)1

\section{Gas Cooled (Thermal) Reactor - GCR \\ PROTEUS-GCR-EXP-004 \\ CRIT-REAC}

Table 1.1-5. Core 10 (Reference State \#1) Critical Information (Ref. 1 and 3). ${ }^{(\mathrm{a})}$

\begin{tabular}{|c|c|c|c|}
\hline \multicolumn{4}{|c|}{ Core Description } \\
\hline $1^{\text {st }}$ Criticality & May 10,1996 & & \\
\hline Unloaded & October 1996 & & \\
\hline Nominal Pebble Ratio & $1: 1$ moderator:fuel & & \\
\hline Pebble Count & \multicolumn{3}{|l|}{24 layers } \\
\hline Pebble Packing & \multicolumn{3}{|c|}{ Columnar Hexagonal Point-on-Point } \\
\hline Polyethylene Loading & \multicolumn{3}{|c|}{$6546.5 \mathrm{~mm}$ rods having a length of $1450 \mathrm{~mm}$} \\
\hline \multicolumn{4}{|l|}{ Critical Balance } \\
\hline Date & July 5,1996 & & \\
\hline Critical Loading & 24 layers & $\mathbf{M}^{(\mathrm{b})}$ & See Figure 1.1-26 through 1.1-31 \\
\hline Critical Height & $1.44 \mathrm{~m}$ & $\mathbf{M}$ & $24 \times 6 \mathrm{~cm}^{(\mathrm{c})}$ \\
\hline $\begin{array}{l}\text { Rod Positions } \\
\text { (Control/Autorod) }\end{array}$ & $1540 / 15 \mathrm{~mm}$ & M & $0 / 1000 \mathrm{~mm}=$ fully out ${ }^{(\mathrm{d})}$ \\
\hline Nominal Flux & $5 \times 10^{7} \mathrm{n} / \mathrm{cm}^{2} / \mathrm{s}$ & M & \\
\hline Hall Temperature & $21.7^{\circ} \mathrm{C}$ & M & \\
\hline $\begin{array}{l}\text { Core Temperatures } \\
\text { (Center/Edge) }\end{array}$ & $\mathrm{N} / \mathrm{A}$ & $\mathbf{M}$ & \\
\hline $\begin{array}{l}\text { Reflector Temperatures } \\
(\mathrm{R} 2,47 / \mathrm{R} 2,15 / \mathrm{R} 2,63)^{(\mathrm{e})}\end{array}$ & N/A & $\mathbf{M}$ & \\
\hline Air Pressure & $975.9 \mathrm{mbar}$ & $\mathbf{E}$ & \\
\hline Air Humidity & $74 \%$ & $\mathbf{E}$ & \\
\hline
\end{tabular}

(a) Core 10 is a repeat of the Core 9 geometry with the addition of $654,6.5-\mathrm{mm}$ diameter polyethylene rods and a correspondingly reduced core height. The polyethylene rods had a length of $1450(60 \mathrm{~mm} \times 24$ layers $+10 \mathrm{~mm}) \pm 5 \mathrm{~mm}$. The channels in the lower axial reflector were filled.

(b) Directly measured experimental measurements are indicated with an $\mathbf{M}$; sometimes a few values were estimated, and indicated with an $\mathbf{E}$.

(c) In the columnar hexagonal point-on-point packed cores the height of each layer is $\sim 6 \mathrm{~cm}$, the approximate diameter of the pebbles. See Figure 1.0-3.

(d) The withdrawable control rods and autorod are considered fully withdrawn when their positions indicate 0 and $1000 \mathrm{~mm}$, respectively.

(e) The nomenclature for the channels in the radial reflector is described in Figure 1.1-3. 
NEA/NSC/DOC(2006)1

Gas Cooled (Thermal) Reactor - GCR

PROTEUS-GCR-EXP-004

CRIT-REAC

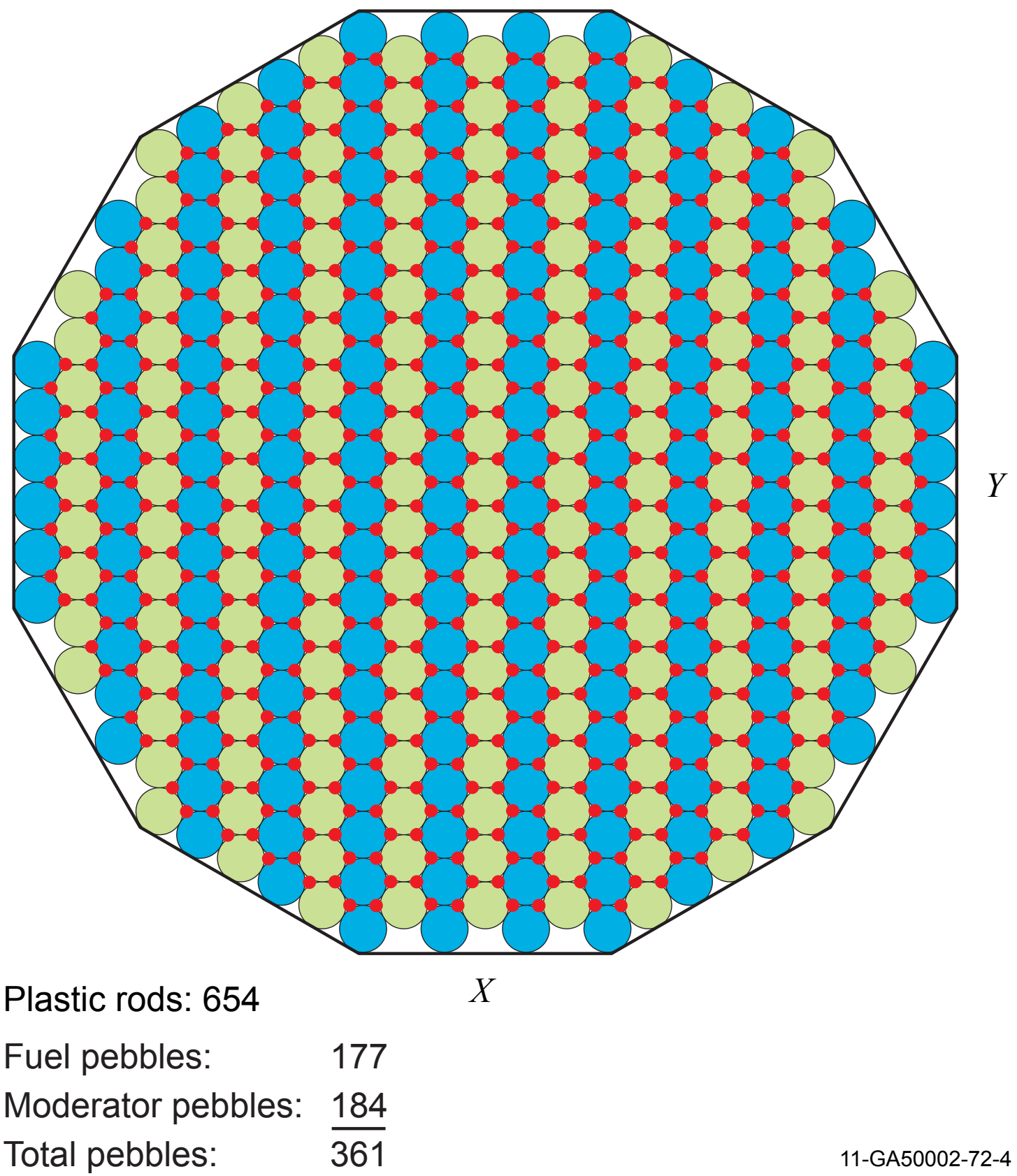

Figure 1.1-26. Layers 1, 7, 13, 19, and 25 of Core 10 (Ref. 1). 
NEA/NSC/DOC(2006)1

Gas Cooled (Thermal) Reactor - GCR

PROTEUS-GCR-EXP-004

CRIT-REAC

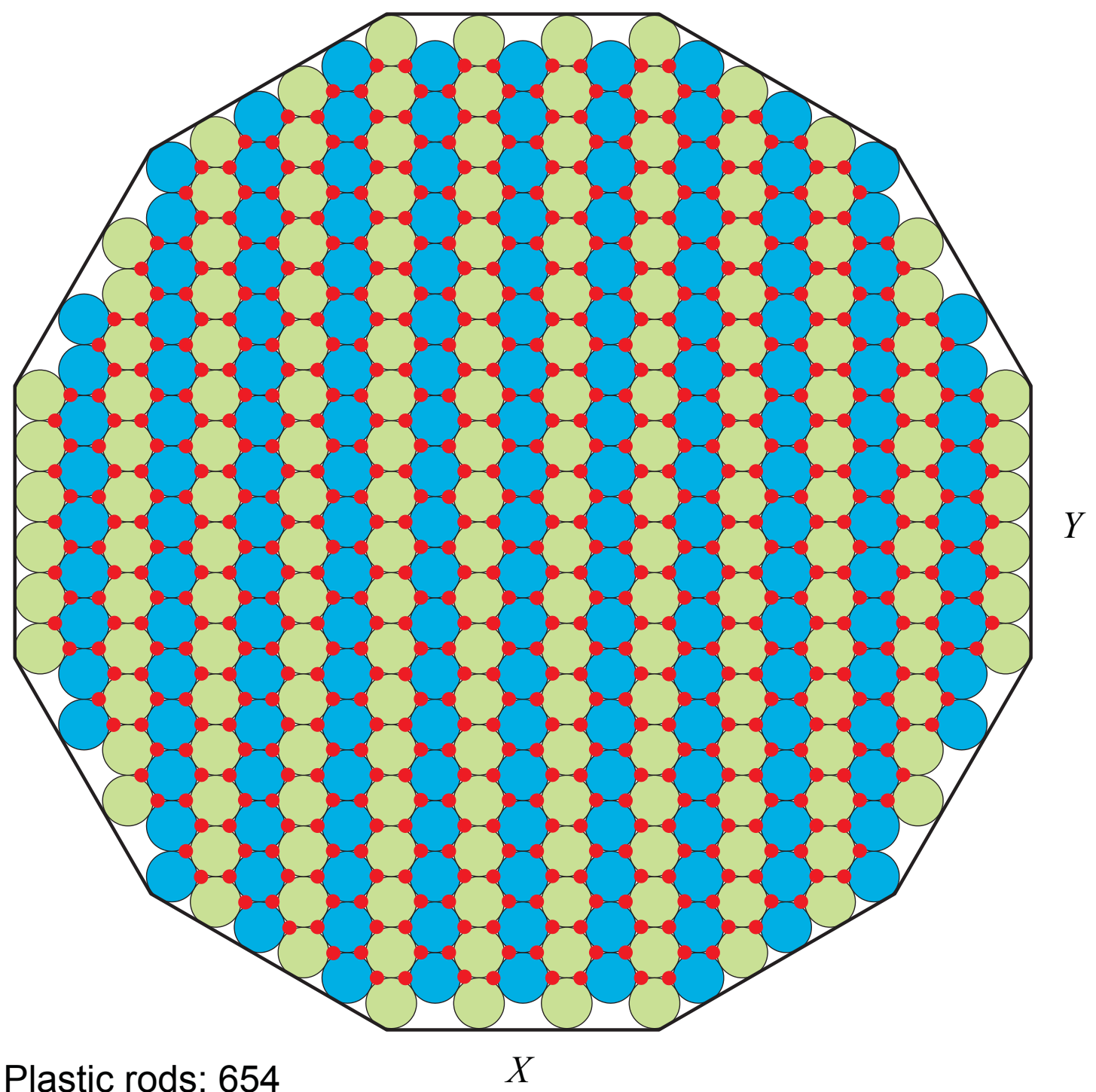

- Plastic rods: 654

184

Moderator pebbles: $\frac{177}{361}$
Total pebbles:

Figure 1.1-27. Layers 2, 8, 14, 20, and 26 of Core 10 (Ref. 1). 
NEA/NSC/DOC(2006)1

Gas Cooled (Thermal) Reactor - GCR

PROTEUS-GCR-EXP-004

CRIT-REAC

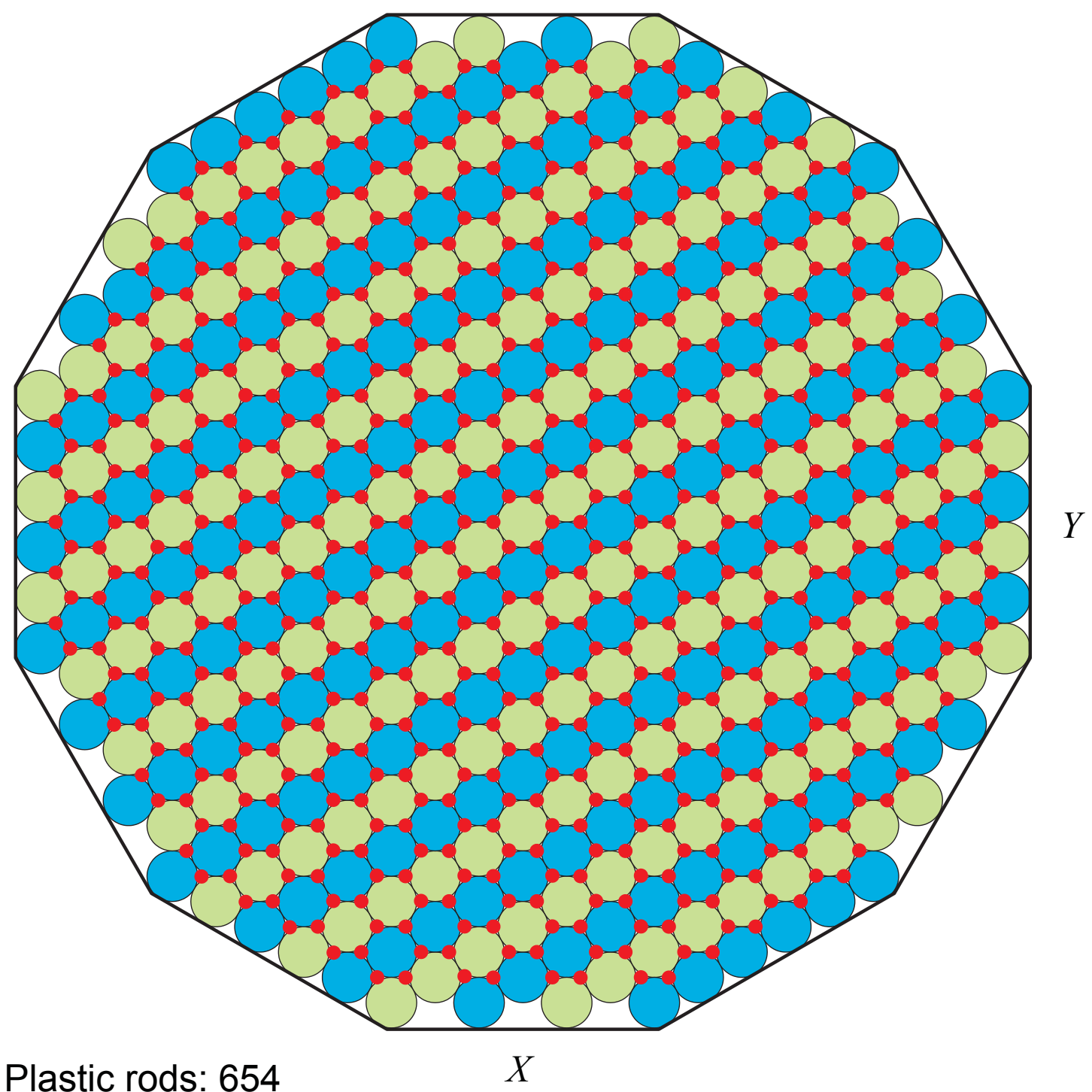

- Plastic rods: 654

Moderator pebbles: $\frac{184}{361}$
Total pebbles:

Figure 1.1-28. Layers 3, 9, 15, 21, and 27 of Core 10 (Ref. 1). 
NEA/NSC/DOC(2006)1

Gas Cooled (Thermal) Reactor - GCR

PROTEUS-GCR-EXP-004

CRIT-REAC

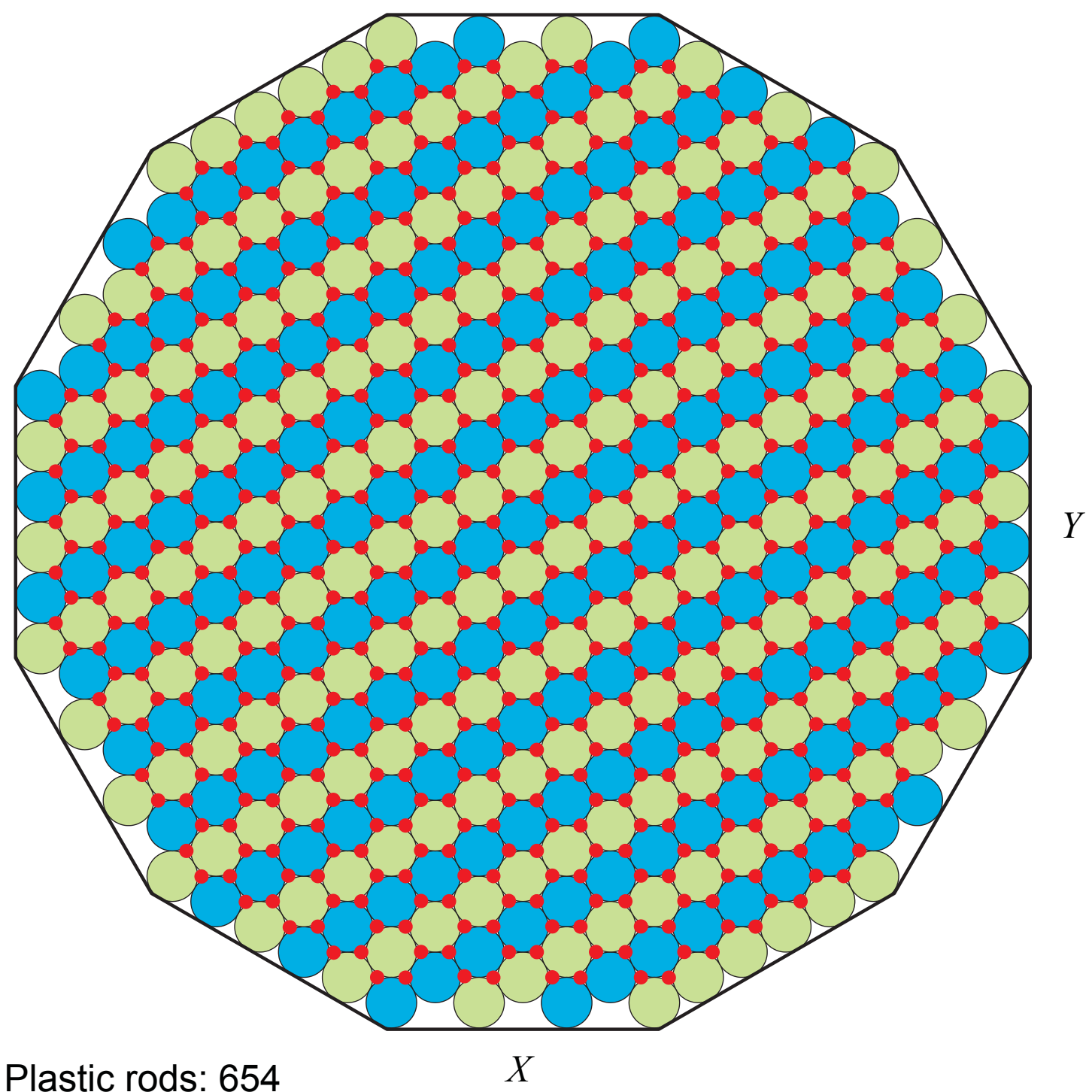

- Plastic rods: 654

184

Moderator pebbles: $\frac{177}{361}$
Total pebbles:

Figure 1.1-29. Layers 4, 10, 16, and 22 of Core 10 (Ref. 1). 
NEA/NSC/DOC(2006)1

Gas Cooled (Thermal) Reactor - GCR

PROTEUS-GCR-EXP-004 CRIT-REAC

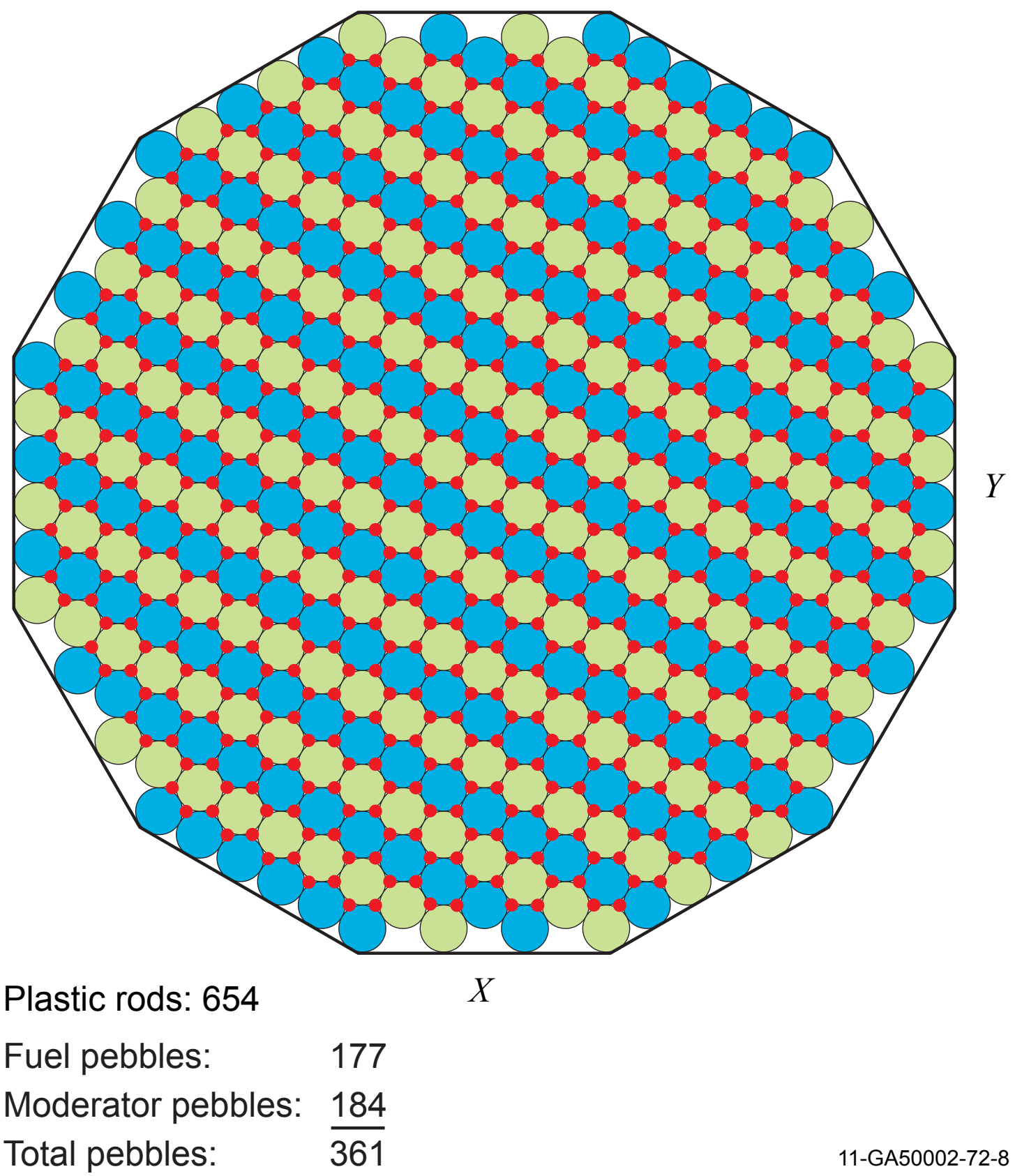

Figure 1.1-30. Layers 5, 11, 17, and 23 of Core 10 (Ref. 1). 
NEA/NSC/DOC(2006)1

Gas Cooled (Thermal) Reactor - GCR

PROTEUS-GCR-EXP-004

CRIT-REAC

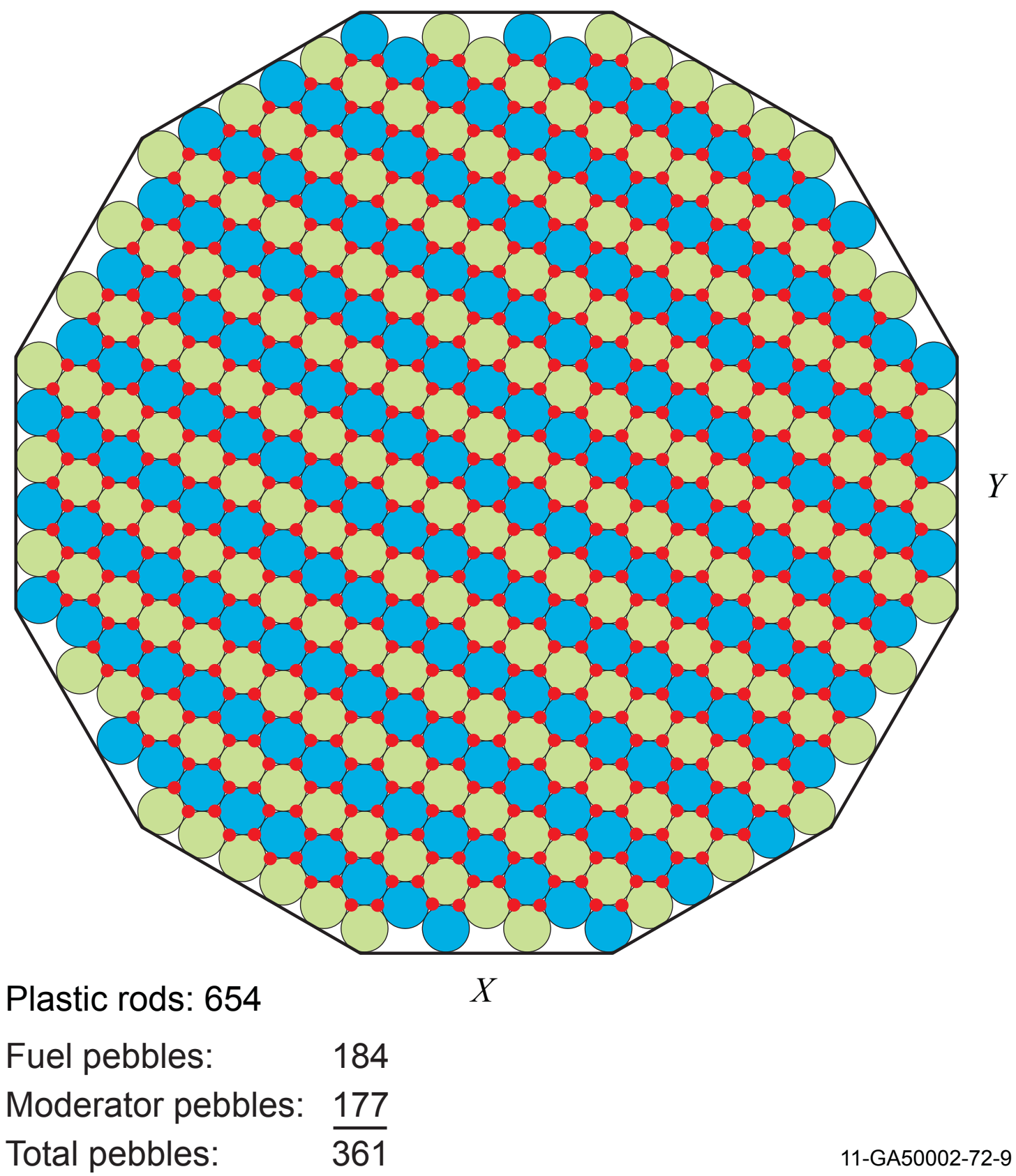

Figure 1.1-31. Layers 6, 12, 18, and 24 of Core 10 (Ref. 1). 
NEA/NSC/DOC(2006)1

\author{
Gas Cooled (Thermal) Reactor - GCR \\ PROTEUS-GCR-EXP-004 \\ CRIT-REAC
}

\title{
1.1.2.3 Experimental Procedure
}

The approach to critical for each configuration was accompanied by the usual "inverse counts versus core loading" plot with an extrapolation to $1 /$ counts $=0$ being made after each pebble loading step to give the predicted critical loading. After the first two loading steps, which were administratively limited to 1/3 and $1 / 6$ of the number of pebbles predicted for the critical loading respectively, the remaining steps were limited to one half of the predicted additional number of pebbles required to achieve criticality, or the worth of the control rod bank, whichever was the larger value. The count rates were measured using neutron detectors situated in the radial reflector. Because the loading of a pebble bed involves a continuous core height and thus core-detector geometry change, it was expected that the approach curves would show considerable spatial dependence. For this reason, early loadings were monitored with additional detectors. The approach curves showed considerable non-linearity for detectors close to the core, with a noticeable effect as the core upper surface reached the axial position of the detector. For this reason, all subsequent approaches were performed with detectors situated further out in the radial reflector (Ref. 3).

Criticality is established and power is raised by means of control rod movements. Criticality is maintained via the autorod, which is a single, radial-reflector-based rod driven automatically by the signal from a "deviation channel", to maintain reactor power and thus criticality. Since the deviation channel was comprised of an ionization chamber situated in the radial reflector, the signal noise, and hence accuracy of the determination of a critical configuration, was determined by the flux level in the reactor. The autorod itself was typically worth a total of less than $0.1 \$$ and the uncertainty in its position represented much less than $\pm 5 \%$ of this range, even at relatively low fluxes. An uncertainty of $< \pm 0.005 \$$ was typically regarded as negligible (Ref. 3 ).

\subsubsection{Material Data}

While there are many components of the PROTEUS that remain unchanged throughout the course of the HTR-PROTEUS experiments, many parameters did change between experiments, such as the use of graphite filler pieces, control rod types and locations, the presence of polyethylene rods to simulate water ingress, core pebble packing, and conditions at criticality. Section 1.1.3.1 provides information regarding general components common to all HTR-PROTEUS configurations. Section 1.1.3.2 provides information specific to the core configurations evaluated in this report.

The PROTEUS was a zero-power critical facility. It was operated at low power and temperatures; therefore, burnup of the fuel, activation of the graphite, and heating effects were negligible.

\subsubsection{General HTR-PROTEUS Components}

The following components are common to all HTR-PROTEUS core configurations.

\section{Concrete}

Concrete shielding material properties were not provided in the references. It is indicated elsewhere that barium concrete walls surrounded the experimental facility. ${ }^{\mathrm{a}}$

\section{Steel Plate Pedestal}

The stainless steel plate pedestal material properties were not available.

\footnotetext{
${ }^{a}$ Difilippo, F. C., "Monte Carlo Calculations of Pebble Bed Benchmark Configurations of the PROTEUS Facility," Nucl. Sci. Eng., 143, 240-253 (2003).
}

Revision: 1

Date: March 31, 2014

Page 55 of 375 
NEA/NSC/DOC(2006)1

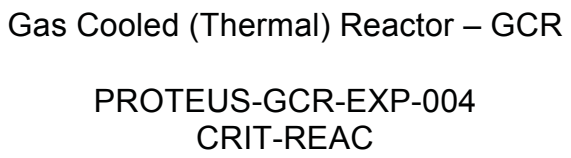

\section{Radial Reflector}

The HTR-PROTEUS reflectors consist of graphite of various ages from several different sources. The older graphite is mainly of type "Reactor Grade A" and made by British Achesons Electrodes Ltd., of Sheffield, England, in about 1968. Some less important sections, away from the core region, were made from a similar grade material from stock material at the facility. The new graphite was manufactured in Chedde, France, by the Sociéte des Electrodes et Réfractaires Savoie in several batches over the period 1991 to 1993 . The location, densities, and nominal, "as delivered", impurity contents for the graphite are summarized in Table 1.1-6 (Ref. 2).

No attempt was made to describe the impurity content of individual reflector components. A recommended global value was measured and reported, an equivalent boron content of $4.09 \pm 0.05$ mbarn, which includes absorbed moisture and intergranular nitrogen from air (Ref. 3). ${ }^{\mathrm{a}}$

Pulsed neutron measurements were performed in the empty PROTEUS graphite reflectors (lower axial and radial) to determine the effective impurity content. The corrected measurements provide a nominal ${ }^{10} \mathrm{~B}$ absorption cross section in the cavity of $2.69 \pm 0.16 \mathrm{mbarn}$, which is equivalent to a concentration of 0.2696 and $0.2591 \mathrm{ppm}$ for the radial and axial graphite reflectors, respectively.

\footnotetext{
${ }^{a}$ Williams, T., Mathews, D., and Yamane, T., "Measurement of the Absorption Properties of the HTR-PROTEUS Reflector Graphite by Means of a Pulsed-Neutron Technique," TM-41-93-34, Paul Scherrer Institut, Villigen, October 3, 1995.

${ }^{\mathrm{b}}$ Difilippo, F. C., “Applications of Monte Carlo Simulations of Thermalization Processes to the Nondestructive Assay of Graphite,” Nucl. Sci. Eng., 133, 163-177 (1999).

Revision: 1

Date: March 31, 2014

Page 56 of 375
} 
NEA/NSC/DOC(2006)1

\section{Gas Cooled (Thermal) Reactor - GCR \\ PROTEUS-GCR-EXP-004 \\ CRIT-REAC}

Table 1.1-6. Summary of Reactor Graphite in HTR-PROTEUS (Ref. 2 and 3).

\begin{tabular}{|c|c|c|c|}
\hline Graphite Type & Occurrence & $\begin{array}{l}\text { Density } \\
\left(\mathrm{g} / \mathrm{cm}^{3}\right)\end{array}$ & $\underset{(\text { mbarn/atom) })^{(a)}}{\text { Nominal } \sigma_{\mathrm{a}}}$ \\
\hline $\begin{array}{l}\text { Old graphite } \\
\text { remaining from } \\
\text { previous } \\
\text { experiments } \\
(\sim 1968) \\
\end{array}$ & Majority of system & $1.76 \pm 0.01^{(\mathrm{b})}$ & $3.785 \pm 0.3^{(b)}$ \\
\hline $\begin{array}{l}\text { New graphite for } \\
\text { HTR PROTEUS } \\
\text { - Batch } 1(\sim 1991) \\
\\
\text { PSI Order } \\
\text { Numbers } \\
34618,37129\end{array}$ & $\begin{array}{l}\text { 1. Central part bottom axial reflector } \\
\text { 2. Central part top axial reflector } \\
\text { 3. Filler rods for } \approx 50 \% \text { "C-Driver" channels } \\
\text { (inner channels) } \\
\text { 4. Top } 12 \mathrm{~cm} \text { of radial reflector } \\
\text { 5. Filler pieces to adjust cavity shape for required } \\
\text { geometry }\end{array}$ & $\begin{array}{l}1.75 \pm \\
0.007^{(\mathrm{c})}\end{array}$ & $3.77 \pm 0.09^{(\mathrm{c})}$ \\
\hline $\begin{array}{l}\text { New graphite for } \\
\text { HTR PROTEUS } \\
\text { - Batch } 2(\sim 1993) \\
\text { PSI Order } \\
\text { Numbers } \\
40442,40901 \\
\end{array}$ & $\begin{array}{ll}\text { 1. } & \text { Filler rods for } \approx 50 \% \text { "C-Driver" channels } \\
\text { (outer channels) }\end{array}$ & $1.78^{(\mathrm{d})}$ & $4.08^{(\mathrm{d})}$ \\
\hline $\begin{array}{l}\text { Moderator } \\
\text { pebbles }\end{array}$ & Core & $1.68 \pm 0.03^{(\mathrm{e})}$ & $4.79^{(\mathrm{e})}$ \\
\hline Fuel pebbles & Core & $1.73^{(\mathrm{e})}$ & $0.3829^{(\mathrm{e})} \mathrm{ppm} \mathrm{B}$ \\
\hline
\end{tabular}

(a) $\sigma_{\mathrm{a}}$ is the neutron absorption cross section of the graphite.

(b) Reactor-based measurements reported in N.R.E. PROTEUS Construction Manual Section A.

(c) Reactor-based measurements SERS Test Certificates January 25, 1991, and October 10, 1991.

(d) Reactor-based measurements SERS Test Certificates January 7, 1993.

(e) Chemical analyses HOBEG GmbH Test Certificates for fuel and moderator pebbles.

The apparent density of seven samples from each of the four separate graphitizing heats (batches) of the Achesons graphite were measured (twenty-eight samples altogether). An average density of $1.763 \pm$ $0.012 \mathrm{~g} / \mathrm{cm}^{3}$ was obtained ( $1 \sigma$ standard deviation based on the twenty-eight reported results). Quality control documentation for the new graphite claimed densities between 1.75 and $1.78 \mathrm{~g} / \mathrm{cm}^{3}$, consistent with the older graphite value. The old graphite comprises the majority of the reflector system (Ref. 2).

Four samples of reflector graphite were heated to $500{ }^{\circ} \mathrm{C}$ under vacuum for five hours at PSI on May 14, 1993. The results are shown in Table 1.1-7. Sample number three was from new graphite manufactured in $1990 ;{ }^{\mathrm{a}}$ the other three samples were from the older 1968 graphite. The average weight loss of the older samples was $0.0241 \mathrm{wt} . \%$, compared to a loss of $0.0156 \mathrm{wt} . \%$ for the newer graphite. The weight loss was assumed to be primarily due to the removal of absorbed moisture (Ref. 2).

\footnotetext{
${ }^{a}$ It is unclear how a piece of new graphite manufactured in 1990 was used in this analysis when the new graphite was delivered in batches over the course of 1991 to 1993.
}

Revision: 1

Date: March 31, 2014 
NEA/NSC/DOC(2006)1

\section{Gas Cooled (Thermal) Reactor - GCR \\ PROTEUS-GCR-EXP-004 \\ CRIT-REAC}

Table 1.1-7. Reflector Graphite Weight Loss During Heating in a Vacuum (Ref. 2).

\begin{tabular}{||c|c|c|c|c|c||}
\hline \hline \multirow{2}{*}{$\begin{array}{l}\text { Sample Number } \\
\text { (Graphite Type) }\end{array}$} & Diameter (cm) & Length (cm) & Original Mass (g) & \multicolumn{2}{|c||}{ Mass Loss } \\
\hline \hline 1 (gold) & 4.4 & 6.0 & 150.742385 & 0.02033 & 0.0135 \\
2 (wt.\%) \\
3 (neld) & 4.0 & 4.1 & 85.523130 & 0.02866 & 0.0335 \\
4 (old) & 2.65 & 6.0 & 57.980115 & 0.009055 & 0.0156 \\
\hline
\end{tabular}

The safety ring was comprised of Peraluman-300 (Table 1.1-8) and had a total mass of $10.42 \mathrm{~kg}$ (Ref. 2).

Table 1.1-8. Peraluman-300 (Ref. 2).

\begin{tabular}{||c|c||}
\hline \hline Element & Composition (wt.\%) \\
\hline \hline $\mathrm{B}$ & $<0.001$ \\
\hline $\mathrm{Mg}$ & $<3.1$ \\
\hline $\mathrm{Al}$ & 95.55 \\
\hline $\mathrm{Si}$ & 0.4 \\
\hline $\mathrm{Mn}$ & $<0.5$ \\
\hline $\mathrm{Fe}$ & 0.3 \\
\hline $\mathrm{Cu}$ & 0.05 \\
\hline $\mathrm{Zn}$ & 0.1 \\
\hline $\mathrm{Ga}$ & $<0.01$ \\
\hline $\mathrm{Cd}$ & $<0.001$ \\
\hline
\end{tabular}

\section{Upper Axial Reflector}

The total mass of the graphite contained in the upper axial reflector was $1585.64 \mathrm{~kg}$ (Ref. 2).

The location of old and new graphite in the upper axial reflector is shown in Table 1.1-6.

The aluminum housing consisted of Peraluman-300, shown in Table 1.1-8. The total mass of Peraluman contained in this structure, below the upper surface of the graphite, was $71.48 \mathrm{~kg}$ (Ref. 2).

\section{Lower Axial Reflector}

The total mass of the graphite contained in the lower axial reflector was not reported.

The location of old and new graphite in the lower axial reflector is shown in Table 1.1-6.

\section{Graphite Plugs}

New graphite was used for the graphite plugs placed into holes in the reflectors (Table 1.1-6).

\section{Safety/Shutdown Rods}

The borated steel rod sections contain nominally $5 \mathrm{wt} . \%$ boron and are enclosed in $18 / 8$ stainless steel tubes. The borated steel used in the HTR-PROTEUS experiments was similar to those used in previous PROTEUS experiments but was manufactured in 1991 by Böhler AG, Edelstahlwerke, Düsseldorf,

Revision: 1

Date: March 31, 2014

Page 58 of 375 
NEA/NSC/DOC(2006)1

\section{Gas Cooled (Thermal) Reactor - GCR \\ PROTEUS-GCR-EXP-004 \\ CRIT-REAC}

Germany for the HTR-PROTEUS experiments. The steel was chemically analyzed by the manufacturer and by PSI. The Böhler measurements, performed on June 14, 1991, indicated a boron content of $4.95 \%$; the PSI measurements, performed on January, 8, 1992, indicated a boron content of $4.70 \%$. Böhler indicated that their chemical analyses were performed prior to the final casting and machining steps and that some boron could have been lost during these steps. It was not originally reported whether these measurements were performed in at.\% or wt.\%; the measurements were believed to be in wt.\% (Ref. 2). ${ }^{\text {a }}$

The borated steel density, $6.878 \mathrm{~g} / \mathrm{cc}$, was measured at PSI on December 15, 1993, and has the composition shown in Table 1.1-9. The 18/8 stainless steel cladding material (Table 1.1-10) had specified elemental compositions and density, $7.92 \mathrm{~g} / \mathrm{cc}$ (Ref. 2).

The aluminum parts of the shock damper were pure aluminum alloy with a measured mass of $633.65 \mathrm{~g}$ (Ref. 2).

Table 1.1-9. Borated Steel (Ref. 2). ${ }^{(a)}$

\begin{tabular}{|c|c|}
\hline \hline Element & Composition (wt.\%) \\
\hline \hline${ }^{10} \mathrm{~B}$ & 0.94 \\
\hline${ }^{11} \mathrm{~B}$ & 3.76 \\
\hline $\mathrm{Si}$ & 1.02 \\
\hline $\mathrm{Cr}$ & 40.4 \\
\hline $\mathrm{Mn}$ & 1.30 \\
\hline $\mathrm{Fe}$ & 41.8 \\
\hline $\mathrm{Ni}$ & 9.83 \\
\hline \hline Total & 99.05 \\
\hline
\end{tabular}

a. Measurement performed on January 8, 1992, by R. Keil of PSI.

Table 1.1-10. 18/8 Stainless Steel (Ref. 2).

\begin{tabular}{|c|c|}
\hline Element & Composition (wt.\%) \\
\hline \hline $\mathrm{Cr}$ & 18 \\
\hline $\mathrm{Fe}$ & 74 \\
\hline $\mathrm{Ni}$ & 8 \\
\hline
\end{tabular}

\section{Automatic Control Rod (Autorod)}

The autorod is comprised of a copper plate within an aluminum tube. Detailed material properties were not available in the reference reports.

\section{Static Measurement Rods}

The static measurement rods were comprised of a Peraluman R-257 tube containing borated steel pieces. The Peraluman R-257 density was $2.65 \mathrm{~g} / \mathrm{cm}^{3}$ with the specified composition shown in Table 1.1-11. Peraluman R-257 has lower neutron absorption than Peraluman-300 due to the reduced manganese content. The borated steel had a nominal boron content of $5 \mathrm{wt} . \%$. Some borated steel sections were analyzed separately at PSI on January 8, 1992 (see Table 1.1-9). The borated steel density was measured on December 17, 1993, using three as-built pieces; the density was $7.199 \pm 0.029 \mathrm{~g} / \mathrm{cc}$. The long pair of

\footnotetext{
${ }^{\mathrm{a}}$ A boron content of $\sim 5 \mathrm{wt} . \%$ is equal to $\sim 20$ at. $\%$; therefore, the assumption that the original measurements were reported in wt. $\%$ is correct.
}

Revision: 1

Date: March 31, 2014 
NEA/NSC/DOC(2006)1

\section{Gas Cooled (Thermal) Reactor - GCR \\ PROTEUS-GCR-EXP-004 \\ CRIT-REAC}

rods also contained a graphite filler piece. The short pair of rods was placed within a graphite sleeve, which had a mass of $6.80 \mathrm{~kg}$ (Ref. 2).

Table 1.1-11. Peraluman R-257 (Ref. 2).

\begin{tabular}{|c|c|}
\hline Element & Composition (wt.\%) \\
\hline \hline $\mathrm{B}$ & $<0.001$ \\
\hline $\mathrm{Mg}$ & $<2.8$ \\
\hline $\mathrm{Al}$ & 96.658 \\
\hline $\mathrm{Si}$ & 0.2 \\
\hline $\mathrm{Mn}$ & $<0.01$ \\
\hline $\mathrm{Fe}$ & 0.2 \\
\hline $\mathrm{Cu}$ & 0.02 \\
\hline $\mathrm{Zn}$ & 0.1 \\
\hline $\mathrm{Ga}$ & $<0.01$ \\
\hline $\mathrm{Cd}$ & $<0.001$ \\
\hline
\end{tabular}

\section{Fuel Pebbles}

Fuel masses are shown in Table 1.1-1.

Impurities in the $\mathrm{UO}_{2}$ used in the TRISO fuel particles are provided in Table 1.1-12. The specified values are averages taken from the fuel pebble quality control records. Impurity estimates for five elements contributing less than $1 \%$ of the total boron equivalent were not given (Ref. 2).

The graphite impurities in the assembled fuel pebbles are provided in Table 1.1-13. The specified values are averages taken from the fuel pebble quality control records. Impurity estimates for five elements contributing less than $1 \%$ of the total boron equivalent were not given (Ref. 2). 
NEA/NSC/DOC(2006)1

Gas Cooled (Thermal) Reactor - GCR

PROTEUS-GCR-EXP-004

CRIT-REAC

Table 1.1-12. $\mathrm{UO}_{2}$ Impurities (Ref. 2).

\begin{tabular}{|c|c|}
\hline Element & Concentration (ppm by wt.) \\
\hline \hline $\mathrm{Ag}$ & $<0.2$ \\
\hline $\mathrm{B}$ & 0.085 \\
\hline $\mathrm{Ca}$ & 51 \\
\hline $\mathrm{Cd}$ & $<0.2$ \\
\hline $\mathrm{Cl}$ & $<3$ \\
\hline $\mathrm{Co}$ & $<1$ \\
\hline $\mathrm{Cr}$ & 23 \\
\hline $\mathrm{Dy}$ & $<0.02$ \\
\hline $\mathrm{Eu}$ & $<0.02$ \\
\hline $\mathrm{Fe}$ & 28 \\
\hline $\mathrm{Gd}$ & $<0.02$ \\
\hline $\mathrm{Li}$ & $<1$ \\
\hline $\mathrm{Mn}$ & 7.5 \\
\hline $\mathrm{Mo}$ & $<3$ \\
\hline $\mathrm{Ni}$ & 2.5 \\
\hline $\mathrm{S}$ & $<0.04$ \\
\hline $\mathrm{Ti}$ & $<10$ \\
\hline $\mathrm{V}$ & $<10$ \\
\hline & \\
\hline
\end{tabular}

Table 1.1-13. Fuel Pebble Graphite Impurities (Ref. 2).

\begin{tabular}{|c|c||}
\hline Element & Concentration (ppm by wt.) \\
\hline \hline $\mathrm{Ag}$ & $<0.2$ \\
\hline $\mathrm{B}$ & 0.101 \\
\hline $\mathrm{Ca}$ & 9.28 \\
\hline $\mathrm{Cd}$ & $<0.103$ \\
\hline $\mathrm{Cl}$ & $<3$ \\
\hline $\mathrm{Co}$ & $<0.13$ \\
\hline $\mathrm{Cr}$ & 1.81 \\
\hline $\mathrm{Dy}$ & $<0.01$ \\
\hline $\mathrm{Eu}$ & $<0.01$ \\
\hline $\mathrm{Fe}$ & 2.95 \\
\hline $\mathrm{Gd}$ & $<0.01$ \\
\hline $\mathrm{Li}$ & $<1$ \\
\hline $\mathrm{Mn}$ & 0.43 \\
\hline $\mathrm{Ni}$ & $<1$ \\
\hline $\mathrm{S}$ & $<0.011$ \\
\hline $\mathrm{Ti}$ & 0.497 \\
\hline $\mathrm{V}$ & $<0.433$ \\
\hline \multicolumn{2}{|c|}{} \\
\hline
\end{tabular}

Revision: 1

Date: March 31, 2014 
NEA/NSC/DOC(2006)1

\author{
Gas Cooled (Thermal) Reactor - GCR \\ PROTEUS-GCR-EXP-004 \\ CRIT-REAC
}

\title{
Moderator Pebbles
}

Moderator pebble impurities are given in Table 1.1-14, and were obtained from the moderator pebble quality control records. Uncertainties for the moderator pebble impurities were not available, and values for fourteen elements contributing less than $0.1 \%$ to the total boron equivalent were not given. The table does not include values for absorbed moisture in the pebbles. The quantity of moisture contained in the pebbles was measured at PSI by randomly selecting two moderator pebbles and heating them to $500{ }^{\circ} \mathrm{C}$ under vacuum for five hours. Each pebble showed a weight loss of $0.02 \mathrm{~g}, 0.01$ wt.\% (Ref. 2).

Table 1.1-14. Moderator Pebble Impurities (Ref. 2).

\begin{tabular}{||c|c||}
\hline Element & Concentration (ppm by wt.) \\
\hline \hline $\mathrm{B}$ & 0.76 \\
\hline $\mathrm{Ca}$ & 129 \\
\hline $\mathrm{Cd}$ & $<0.6$ \\
\hline $\mathrm{Cl}$ & 18.64 \\
\hline $\mathrm{Dy}$ & 0.065 \\
\hline $\mathrm{Eu}$ & 0.13 \\
\hline $\mathrm{Fe}$ & 5.9 \\
\hline $\mathrm{Gd}$ & 0.040 \\
\hline $\mathrm{Li}$ & 0.88 \\
\hline $\mathrm{Ni}$ & 0.78 \\
\hline $\mathrm{S}$ & 140 \\
\hline $\mathrm{Si}$ & 35 \\
\hline $\mathrm{Sm}$ & 0.086 \\
\hline $\mathrm{Ti}$ & 10 \\
\hline $\mathrm{V}$ & 13 \\
\hline
\end{tabular}

\section{Start-Up Source}

The material properties of the start-up source were not available in the reference reports.

\section{Detectors}

The material properties of the detectors (six ionization chambers and two fission chambers) were not available in the reference reports.

\section{Temperature Sensors}

The material properties of the temperature sensors were not available in the reference reports.

\subsubsection{Components Unique to Cores 9 and 10}

The following components are unique to core configurations 9 and 10 .

\section{Graphite Fillers}

The total mass of the twelve filler pieces used to convert the 22-sided cavity into a 12 -sided one was $211.2 \mathrm{~kg}$ (Ref. 2). 
NEA/NSC/DOC(2006)1

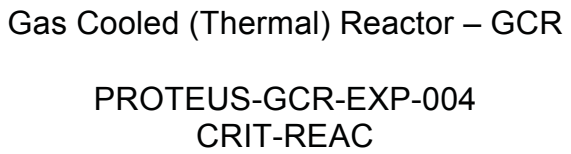

\section{ZEBRA Control Rods}

The ZEBRA control rods were not used in the experiments with Cores 9 and 10 .

\section{Withdrawable Stainless Steel Control Rods}

The inner tube of the withdrawable stainless steel control rods was St1.4301 (Table 1.1-15) and the outer tube was St1.4541 (Table 1.1-16). Both steels had a density of $7.9 \mathrm{~g} / \mathrm{cm}^{3}$ (Ref. 2).

Table 1.1-15. St1.4301 (Ref. 2).

\begin{tabular}{|c|c|}
\hline Element & Composition (wt.\%) \\
\hline \hline $\mathrm{C}$ & $\leq 0.07$ \\
\hline $\mathrm{Si}$ & $\leq 1.0$ \\
\hline $\mathrm{Mn}$ & $\leq 2.0$ \\
\hline $\mathrm{Cr}$ & $17.0-20.0$ \\
\hline $\mathrm{Ni}$ & $9.0-11.5$ \\
\hline
\end{tabular}

Table 1.1-16. St1.4541 (Ref. 2).

\begin{tabular}{|c|c|}
\hline Element & Composition (wt.\%) \\
\hline \hline $\mathrm{C}$ & $\leq 0.10$ \\
\hline $\mathrm{Si}$ & $\leq 1.0$ \\
\hline $\mathrm{Mn}$ & $\leq 2.0$ \\
\hline $\mathrm{Cr}$ & $17.0-19.0$ \\
\hline $\mathrm{Ni}$ & $9.0-11.5$ \\
\hline $\mathrm{Ti}$ & $\geq \mathrm{x} \% \mathrm{C}$ \\
\hline
\end{tabular}

\section{Polyethylene Rods}

Great care was taken during the manufacture of the plastic to avoid contamination with foreign isotopes. Samples of the polyethylene were sent for an elemental analysis to three independent organic chemistry laboratories; unfortunately, the results from one laboratory were inconsistent with the others, leading to additional uncertainty in the $\mathrm{CH}_{2}$ stoichiometry. The recommended value is $\mathrm{CH}_{2.03 \pm 0.03}$, which is consistent with the elemental analyses and experimental comparisons between $\mathrm{CH}_{2}$ and $\mathrm{H}_{2} \mathrm{O}$ worths in Cores 5, 7, and 9. The linear densities of the polyethylene rods are shown in Figure 1.1-18 (Ref. 2).

\section{Copper Wire}

Copper wire was not used in the experiments with Cores 9 and 10. 
NEA/NSC/DOC(2006)1

\section{Gas Cooled (Thermal) Reactor - GCR \\ PROTEUS-GCR-EXP-004 \\ CRIT-REAC}

\section{Ambient Air}

Ambient (hall) temperatures, air pressure, and humidity for HTR-PROTEUS critical experiments, Cores 9 and 10 , are provided in the following tables:

- $\quad$ Core 9 (reference state \#1): Table 1.1-3

- Core 9 (reference state \#2): Table 1.1-4

- Core 10 (reference state \#1): Table 1.1-5

\subsubsection{Temperature Data}

Room (hall) temperatures for HTR-PROTEUS critical experiments, Cores 9 and 10, are provided in the following tables (core and reflector temperatures were not measured):

- Core 9 (reference state \#1): Table 1.1-3

- Core 9 (reference state \#2): Table 1.1-4

- Core 10 (reference state \#1): Table 1.1-5

The reactor was operated at room temperature with the power limited to $1 \mathrm{~kW}$ so that no active cooling systems were required. ${ }^{\mathrm{a}}$

\subsubsection{Additional Information Relevant to Critical and Subcritical Measurements}

An estimate of excess reactivity, in units of dollars, was provided for each of the core configurations. The value of $\beta_{\text {eff }}$ is provided for each case. The excess reactivity was provided in terms of individual component worths such that users could pick and choose which simplifications to incorporate into their models. Where possible, the component worths had been measured directly in the relevant configurations (indicated by $\mathbf{M}$ in the tables) but in many cases the values had to be calculated $(\mathbf{C})$, estimated $(\mathbf{E})$, or scaled from another configuration (S). Most reference component worth measurements were performed in Cores 1 and 5 (Ref. 1). These measurements represent deviations of the real-life assembly from an ideal, clean core configuration. The effects of these deviations are quantified; an example of how these measurements were performed was provided elsewhere for Core $1 .^{\mathrm{b}}$ Reactivity corrections for Cores 9 and 10 , provided in the original references, are summarized in the following tables:

- Core 9 (reference state \#1): Table 1.1-17

- Core 9 (reference state \#2): Table 1.1-18

- Core 10 (reference state \#1): Table 1.1-19

The worth of various core components was provided to allow for the development of simplified models for calculation of the HTR-PROTEUS experiments. The measured worths of the individual components are normally evaluated against the worths of the ZEBRA/control rods, which were carefully calibrated using the stable period technique, or against the autorod worth, which had been subsequently intercalibrated with the ZEBRA/control rods (Ref. 3).

A small degree of inhomogeneity in the radial graphite reflector was inevitable. Axial holes were required for control and shutdown rod insertion and radial and axial holes for nuclear instrumentation. The C-Driver holes in the inner radial reflector, left over from the previous experiments, had to be filled with graphite rods. These rods were relatively easy to remove and useful in estimating the effect of missing graphite. Correction for the air gaps between the $27.5 \mathrm{~mm}$ ID C-Driver channels and the

\footnotetext{
${ }^{a}$ Köberl, O., Seiler, R., and Chawla, R., "Experimental Determination of the Ratio of 238U Capture to 235U Fission in LEU-HTR Pebble-Bed Configurations," Nucl. Sci. Eng., 146, 1-12 (2004).

${ }^{\mathrm{b}}$ Williams, T., "HTR PROTEUS CORE 1: Reactivity Corrections for the Critical Balance," TM-41-93-20, Paul Scherrer Institut, Villigen, October 7, 1993.
}

Revision: 1

Date: March 31, 2014

Page 64 of 375 
NEA/NSC/DOC(2006)1

\section{Gas Cooled (Thermal) Reactor - GCR \\ PROTEUS-GCR-EXP-004 \\ CRIT-REAC}

$26.5 \mathrm{~mm}$ OD graphite filler rods were calculated by V. D. Davidenko of the Kurchatov Institute using the Cristall code system (Ref. 3).

No explicit measurements were performed to determine the worth of the four empty ZEBRA/control rod channels. The values reported in the tables were made on the basis of the results of the C-Driver hole measurements. For safety reasons, the worth of the eight safety and shutdown rod channels cannot be measured and their values were calculated at PSI using the TWODANT code. It was considered reasonable to include them in the calculational model, removing them from the reactivity excess list (Ref. 3).

The upper and lower axial reflectors were furnished with 33 "ventilation holes" to enable air-cooling of the core. The axial thermal flux peak is strongly shifted downwards and graphite density variations in the upper part of the lower axial reflector were of greater significance than those above. Unfortunately, for practical reasons, it was difficult to measure the effect in the lower reflector and satisfactory measurements could only be made in the upper axial reflector. In the upper reflector, measurements were performed with 11 of the 33 holes plugged with graphite. Because full access to the ventilation holes in the lower axial reflector is impeded from below, it was not possible to measure their worth in the usual manner. At best, it was possible to partially fill some of the channels with graphite and linearly scale the effect to 33 filled channels. In some of the core configurations all of the coolant channels in the lower axial reflector were filled with graphite plugs (Ref. 3).

In all the deterministic cores, $\sim 12$ pebbles were directly over one of the 33 cooling channels in the lower axial reflector. To avoid pebble displacement in these cases, special aluminum plugs were developed to support the pebbles in Core 1. In later cores, simple graphite rods were used (Ref. 3).

The reactor start-up sources were normally in their "in" position during reactor operation. At low fluxes their reactivity effect is positive by virtue of the apparent enhanced neutron multiplication; at normal operating fluxes of $>10^{7} \mathrm{n} / \mathrm{cm}^{2} / \mathrm{s}$, their effect was negative due to parasitic neutron absorption in the source and casing. The start-up sources pass through horizontal aluminum guide tubes situated in the radial reflector at about the level of the cavity floor. The worth of these penetrations were also measured (Ref. 3).

The pulsed neutron source, when used for subcriticality measurements, was partially inserted into the lower axial reflector. Its reactivity worth was measured by replacing it with a plug of graphite of dimensions $250 \mathrm{~mm} \times 120 \mathrm{~mm} \varnothing$ (Ref. 3).

The worth of one of the six ionization chambers compared with a graphite plug was measured by opening a plugged channel and inserting a spare ionization chamber. The worth of one of the two impulse channels in the outer radial reflector was also measured by means of filling a similar channel first with a replacement detector and then with a graphite plug (Ref. 3).

The temperature sensors were systematically removed from the system in order to assess their reactivity worths (Ref. 3).

The value of $\beta_{\text {eff }}$ was calculated for each of the cores (Ref. 3). 
NEA/NSC/DOC(2006)1

\section{Gas Cooled (Thermal) Reactor - GCR \\ PROTEUS-GCR-EXP-004 \\ CRIT-REAC}

Table 1.1-17. Core 9 (Reference State \#1) Reactivity Corrections (Ref. 1 and 3).

\begin{tabular}{|c|c|c|c|c|c|c|}
\hline Reactivity Corrections to Critical Loading & No. & & Total $\not$ & & & Comments \\
\hline Control Rod Insertion $(0 \mathrm{~mm})^{(\mathrm{a})}$ & 4 & M & 0 & & & No Control Rod Insertion \\
\hline Control Rod Channels ${ }^{(\mathrm{b})}$ & 4 & $\mathrm{~S}$ & -2.5 & \pm & 0.3 & Scaled from Core 5 \\
\hline Autorod Rest Worth ${ }^{(\mathrm{b})}$ & 1 & $\mathrm{~S}$ & -12.5 & \pm & 0.5 & Scaled from Core 5 \\
\hline Autorod Insertion $(258 \mathrm{~mm})^{(\mathrm{b})}$ & 1 & $\mathrm{~S}$ & -7.5 & \pm & 0.5 & Scaled from Core 5 \\
\hline Autorod Channel $^{(\mathrm{b})}$ & 1 & $\mathrm{~S}$ & -0.7 & \pm & 0.3 & Scaled from Core 5 \\
\hline Safety and Shutdown Rod Channels ${ }^{(\mathrm{b})}$ & 8 & $\mathrm{~S}$ & -32 & \pm & 8 & Scaled from Core 5 \\
\hline Empty Channels R2 ${ }^{(\mathrm{b}, \mathrm{c})}$ & 3 & $\mathrm{~S}$ & -5 & \pm & 1 & Scaled from Core 5 \\
\hline Air Gaps in C-Driver Holes ${ }^{(b, d)}$ & 320 & $\mathrm{~S}$ & -10.5 & & & Scaled from Core 5 \\
\hline Channels in Upper Reflector $^{(\mathrm{e})}$ & 34 & & & & & No Estimate \\
\hline Channels in Lower Reflector & 0 & & & & & Channels Filled \\
\hline Start-up Sources ${ }^{(b)}$ & 2 & M & -4 & \pm & 1 & \\
\hline Start-up Source Penetrations ${ }^{(b)}$ & 2 & $\mathrm{~S}$ & -1 & \pm & 0.2 & Core 5 Value \\
\hline Nuclear Instrumentation (Ionization) ${ }^{(b)}$ & 6 & $\mathrm{~S}$ & -9.0 & \pm & 1.5 & Scaled from Core 5 \\
\hline Nuclear Instrumentation (Fission) $^{(\mathrm{b})}$ & 2 & $\mathrm{~S}$ & -1.0 & \pm & 0.7 & Scaled from Core 5 \\
\hline \multicolumn{3}{|l|}{ Total Correction } & 86 & \pm & 9 & \\
\hline \multicolumn{3}{|l|}{ Corrected $\mathrm{k}_{\mathrm{eff}}\left(\beta_{\mathrm{eff}}=0.00720\right)$} & 1.0062 & \pm & 0.0007 & \\
\hline
\end{tabular}

(a) The control rods were fully withdrawn for this critical balance. The control rods were however calibrated in Core 9 reference state \#2 and found to have a total worth of $152 \pm 2$ cents. This value is significantly larger than the Core 5 value, not due to the increased core moderation in Core 9, which would tend to reduce rod worths, but rather from the increased core height and corresponding active length of the rods.

(b) Only a few individual component worths were measured in Core 9. Therefore, in order to obtain an estimate of the reactivity excess, the component worths measured in Core 5 were scaled by the ratio of the control rod bank worths in the two cores, namely Core $9 /$ Core $5=152 \phi / 134 \phi=1.14$. The same comment applies also to the autorod, which was measured in Core 5 to have a max-min worth of -8.9 cents and scaled in Core 9 to a value of 10.1 cents.

(c) R2 and R3 indicate the second and third rings, respectively, of the C-Driver channels.

(d) Corrects for the air gaps between the $27.5 \mathrm{~mm}$ ID C-Driver channels and the $26.5 \mathrm{~mm}$ OD graphite filler rods. The value here was calculated by V. D. Davidenko of the Kurchatov Institute using the Cristall code system.

(e) No measurement was made of this effect and no justifiable basis could be found for its estimate. The channels in the upper reflector must therefore be included in any Core 9 model. 
NEA/NSC/DOC(2006)1

\section{Gas Cooled (Thermal) Reactor - GCR \\ PROTEUS-GCR-EXP-004 \\ CRIT-REAC}

Table 1.1-18. Core 9 (Reference State \#2) Reactivity Corrections (Ref. 1 and 3).

\begin{tabular}{|c|c|c|c|c|c|c|}
\hline $\begin{array}{c}\text { Reactivity Corrections to Critical } \\
\text { Loading }\end{array}$ & No. & & \multicolumn{3}{|c|}{ Total $\propto$} & Comments \\
\hline Control Rod Insertion $(1620 \mathrm{~mm})^{(\mathrm{a})}$ & 4 & M & -70.4 & \pm & 1.0 & $\begin{array}{l}\text { Calibrated via Stable } \\
\text { Period }\end{array}$ \\
\hline Control Rod Channels ${ }^{(b)}$ & 4 & $\mathrm{~S}$ & -2.5 & \pm & 0.3 & Scaled from Core 5 \\
\hline Autorod Rest Worth & 1 & $\mathrm{~S}$ & -12.5 & \pm & 0.5 & Scaled from Core 5 \\
\hline Autorod Insertion $(25 \mathrm{~mm})^{(\mathrm{c})}$ & 1 & $\mathrm{~S}$ & -10.0 & \pm & 0.5 & Scaled from Core 5 \\
\hline Autorod Channel & 1 & $\mathrm{~S}$ & -0.7 & \pm & 0.3 & Scaled from Core 5 \\
\hline Safety and Shutdown Rod Channels ${ }^{(\mathrm{d})}$ & 8 & $\mathrm{~S}$ & -32 & \pm & 8 & Scaled from Core 5 \\
\hline Empty Channels R2 $2^{(\mathrm{e})}$ & 3 & $\mathrm{~S}$ & -5 & \pm & 1 & Scaled from Core 5 \\
\hline Air Gaps in C-Driver Holes ${ }^{(\mathrm{f})}$ & 320 & $\mathrm{~S}$ & -10.5 & & & Scaled from Core 5 \\
\hline Channels in Upper Reflector & 34 & & & & & No Estimate \\
\hline Channels in Lower Reflector & 0 & & & & & Channels Filled \\
\hline Start-up Sources & 2 & M & -4 & \pm & 1 & \\
\hline Start-up Source Penetrations & 2 & $\mathrm{~S}$ & -1 & \pm & 0.2 & Core 5 Value \\
\hline Nuclear Instrumentation (Ionization) & 6 & $\mathrm{~S}$ & -9.0 & \pm & 1.5 & Scaled from Core 5 \\
\hline Nuclear Instrumentation (Fission) & 2 & $\mathrm{~S}$ & -1.0 & \pm & 0.7 & Scaled from Core 5 \\
\hline \multicolumn{3}{|l|}{ Total Correction } & 159 & \pm & 10 & \\
\hline \multicolumn{3}{|l|}{ Corrected $\mathrm{k}_{\mathrm{eff}}\left(\beta_{\mathrm{eff}}=0.00720\right)$} & $1.01142^{(\mathrm{g})}$ & \pm & 0.0007 & \\
\hline
\end{tabular}

(a) The control rods are fully inserted when $2500 \mathrm{~mm}$ is indicated. Control rod bank calibrations in this configuration were found to be $152 \pm 2$ cents.

(b) The worth of the new control rod channels was assumed to be the same as that of the ZEBRA rod channels in Core 1. Although the ZEBRA rod channels are somewhat larger than the new control rod channels, it is considered that the small size of the correction and its associated uncertainty justifies this approximation. The uncertainty was slightly increased.

(c) Calibrated in Core 5 and scaled by 1.14 for Core 9 (see footnote $b$ in Table 1.1-17).

(d) For safety reasons the worth of these eight channels cannot be measured and the values were calculated at PSI using the TWODANT code. Independent calculations by V. D. Davidenko of the Kurchatov Institute yielded a value of 16.6 cents for this core.

(e) R2 indicates the second ring of the C-Driver channels.

(f) Corrects for the air gaps between the $27.5 \mathrm{~mm}$ ID C-Driver channels and the $26.5 \mathrm{~mm}$ OD graphite filler rods. The value here was calculated by V. D. Davidenko of the Kurchatov Institute using the Cristall code system.

(g) This value had been originally reported as 1.0142, which did not agree with a total correction of $158.6 \varnothing$, the sum of the individual reactivity corrections, converted to $\Delta \mathrm{k}_{\text {eff }}$ with the reported $\beta_{\text {eff }}$ of 0.00720 . It was later corrected in Köberl, O., Seiler, R., "Detailed Analysis of Pebble-Bed HTR PROTEUS Experiments with the Monte Carlo Code TRIPOLI4, $2^{\text {nd }}$ Intern. Topical Meeting on High Tempertaure Reactor Technology, Beijing, China, September 22-24 (2004). 
NEA/NSC/DOC(2006)1

\section{Gas Cooled (Thermal) Reactor - GCR \\ PROTEUS-GCR-EXP-004 \\ CRIT-REAC}

Table 1.1-19. Core 10 (Reference State \#1) Reactivity Corrections (Ref. 1 and 3).

\begin{tabular}{|c|c|c|c|c|c|c|}
\hline Reactivity Corrections to Critical Loading & No. & & \multicolumn{3}{|c|}{ Total $\not$} & Comments \\
\hline Control Rod Insertion $(1540 \mathrm{~mm})^{(\mathrm{a})}$ & 4 & M & -36.8 & \pm & 1 & Measured Core 10 \\
\hline Control Rod Channels ${ }^{(b)}$ & 4 & $\mathrm{~S}$ & -2.0 & \pm & 0.2 & Core 1A Value \\
\hline Autorod Rest Worth ${ }^{(\mathrm{b})}$ & 1 & $\mathrm{~S}$ & -7.7 & \pm & 0.5 & Core 1A Value \\
\hline Autorod Insertion (15 mm) & 1 & M & -7 & \pm & 0.4 & Measured Core 10 \\
\hline Autorod Channel $^{(b)}$ & 1 & $\mathrm{~S}$ & -0.5 & \pm & 0.3 & Core 1A Value \\
\hline Safety and Shutdown Rod Channels ${ }^{(b, c)}$ & 8 & $\mathrm{C}, \mathrm{S}$ & -24 & \pm & 6 & Core 1A Value \\
\hline Empty Channels R2 $2^{(\mathrm{d})}$ & 3 & $\mathrm{~S}$ & -4 & \pm & 1 & Scaled from Core $1 \mathrm{~A}$ \\
\hline Air Gaps in C-Driver Holes ${ }^{(b, e)}$ & 320 & $\mathrm{C}, \mathrm{S}$ & -8.3 & & & Core $1 \mathrm{~A}$ Value \\
\hline Channels in Upper Reflector ${ }^{(b)}$ & 34 & $\mathrm{~S}$ & -3.6 & \pm & 2.0 & Core 1A Value \\
\hline Channels in Lower Reflector & 0 & & & & & \\
\hline Start-up Source Penetrations ${ }^{(b)}$ & 2 & $\mathrm{~S}$ & -1 & \pm & 0.2 & Core 1A Value \\
\hline Nuclear Instrumentation (Ionization) ${ }^{(b)}$ & 6 & $\mathrm{~S}$ & -8.4 & \pm & 1.2 & \\
\hline Nuclear Instrumentation (Fission) $^{(\mathrm{b})}$ & 2 & $\mathrm{~S}$ & -0.8 & \pm & 0.6 & Core 1A Value \\
\hline \multicolumn{3}{|l|}{ Total Correction } & 104 & \pm & 7 & \\
\hline \multicolumn{3}{|l|}{ Corrected $\mathrm{k}_{\mathrm{eff}}\left(\beta_{\mathrm{eff}}=0.00720\right)$} & 1.0075 & \pm & 0.0001 & \\
\hline
\end{tabular}

(a) The control rods are fully inserted when $2500 \mathrm{~mm}$ is indicated. The control rod bank was calibrated via a stable period technique and found to be 109.6 cents.

(b) Since the core height and control bank worths in Core 10 are similar to those in Core 1A, it was considered to be justified to use some of the component worths measured in Core 1A directly in Core 10 with a small arbitrary increase in the uncertainties.

(c) For safety reasons the worth of these eight channels cannot be measured and the values were calculated at PSI using the TWODANT code. Independent calculations by V. D. Davidenko of the Kurchatov Institute yielded a value of 16.6 cents for this core.

(d) R2 indicates the second ring of the C-Driver channels.

(e) Corrects for the air gaps between the $27.5 \mathrm{~mm}$ ID C-Driver channels and the $26.5 \mathrm{~mm}$ OD graphite filler rods. The value here was calculated by V. D. Davidenko of the Kurchatov Institute using the Cristall code system.

(f) No information was provided in either reference for the source of this bias; it is most likely derived from the Core $1 \mathrm{~A}$ value, as discussed in footnote (b).

\subsection{Description of Buckling and Extrapolation Length Measurements}

Buckling and extrapolation length measurements were performed but have not yet been evaluated.

\subsection{Description of Spectral Characteristics Measurements}

Spectral characteristics measurements were performed but have not yet been evaluated. 
NEA/NSC/DOC(2006)1

\section{Gas Cooled (Thermal) Reactor - GCR \\ PROTEUS-GCR-EXP-004 \\ CRIT-REAC}

\section{$1.4 \quad$ Description of Reactivity Effects Measurements}

\subsubsection{Overview of Experiment}

Only Cores 9 and 10 are evaluated in this benchmark report due to similarities in their construction. The other core configurations of the HTR-PROTEUS program are evaluated in their respective reports as outlined in Section 1.0. An overview of the general timeline for each core configuration and the associated test matrix can be found in Appendix D.

Experimental data in this section include rod worth measurements performed for the safety/shutdown rods, withdrawable control rods, and the autorod. Additional measurements for the worth of graphiteplugged holes in the reflectors were also evaluated. Measured worth corrections for the start-up source, with associated graphite penetrations, and nuclear instrumentation were not evaluated because insufficient data were available to model them.

Sixteen reactivity effects measurements apiece for both Cores 9 and 10 (total of 32 measurements) were evaluated and determined to be acceptable benchmark experiments.

\subsubsection{Geometry of the Experiment Configuration and Measurement Procedure}

The geometry of the core configurations and individual reactor components is provided in Section 1.1.2. Changes from the nominal configurations of the critical core configurations and a description of the measurement procedures are provided below.

\subsubsection{Reactivity Measurements in the HTR-PROTEUS}

An important aspect of the HTR-PROTEUS experimental program was to maintain the accurate measurement of the reactivity worth of absorber rods in the core and reflector across various configurations with a range of moderation properties. Requirements included utilization of a method that would: be compatible with small, highly reflected thermal systems; be applicable to highly subcritical cores; have limited dependence upon calculations; have complimentary techniques to other methods with characterizable uncertainties; and be economically feasible. The methods ultimately selected for the HTR-PROTEUS experiments were the pulsed neutron source (PNS) and inverse kinetics (IK) techniques (Ref. 3).

The accurate, unambiguous measurement of reactivity values in graphite-moderated, highly-reflected systems such as the HTR-PROTEUS is a difficult task. Relatively long characteristic timescales render most methods (e.g., PNS, noise, and source jerk) problematic due to the inherent difficulty in separating prompt and delayed decay modes. The long diffusion lengths transport local effects far into the system, challenging the limits of the point reactor approximation techniques (i.e. IK, SP, etc.). The distinct twozone nature of the system leads to additional complications associated with spectral effects (kinetic distortion) that require intuitive detector placement, correction factors, or both (Ref. 6).

A more thorough discussion of the theory and methods for these techniques can be found in Section 6 of Reference 3, Chapter 2 of Reference 4, as well as various technical reports from PSI.,

\footnotetext{
${ }^{a}$ Rosselet, M., "Reactivity Measurement on HTR-PROTEUS Core 5 with the PNS Technique and Preliminary Investigations for the Use of an Epithermal Neutron Detector," TM-41-94-23, Paul Sherrer Institut, Villigen, November 21, 1994.

${ }^{\mathrm{b}}$ Rosselet, M., "PNS Measurements using Epithermal Neutron Detectors in HTR-PROTEUS Core 7," TM-41-9517, Paul Sherrer Institut, Villigen, October 16, 1995.
}

Revision: 1

Date: March 31, 2014

Page 69 of 375 
NEA/NSC/DOC(2006)1

\section{Gas Cooled (Thermal) Reactor - GCR \\ PROTEUS-GCR-EXP-004 \\ CRIT-REAC}

For each measurement of a particular subcritical state, the following procedure was implemented:

1. The detection system was switched on and allowed to stabilize.

2. A critical balance was established with the PNS and neutron detectors in place, and the reactor start-up sources withdrawn (to avoid unnecessary background interference).

3. The autorod (and control rod) positions are frozen.

4. The subcritical state of interest is established, this may involve the insertion of the shutdown rods, the removal of the upper reflector, the insertion of a dummy control rod, etc.

5. The PNS is switched on and the multichannel-analyzer system channel width, pulse-rate, and number of channels (invariably 512) adjusted as required. The system is pulsed for $\sim 15$ minutes, without measuring, to allow an equilibrium state of the delayed neutron background to develop.

6. When a stable equilibrium has been achieved, the multichannel-analyzer system is triggered and data is accumulated until satisfactory statistics are obtained.

7. The accumulation is stopped, the PNS is switched off, the total number of measured pulses and the total measurement time are recorded.

8. After a suitable delay, to allow the flux to stabilize, the measurement is repeated, without pulsing, to establish the background contribution to the measurement.

9. The stored raw data is then processed to calculate the desired reactivity worth measurement (Ref. 3)

Uncertainties in PNS measurements normally comprise statistical uncertainties in the measured data and systematic uncertainties associated with the data utilized to convert the measured parameter to reactivity. Statistical uncertainties are reduced by increasing the count rates and measuring times of individual measurements or repeating measurements. The first method is limited by the properties of the counting system such as the dead time and detector efficiency. The second method is effective, but more costly in time and effort. The experimenters recommended that to reduce the uncertainty associated with use of a particular set of delayed neutron data would require the use of a better data set. A summary of typical uncertainties in three PNS techniques over a range of reactivities is shown in Table 1.4-1. There are large uncertainties for measurements with an absolute value of reactivity less than $1 \$$; for the inhour technique this is due to the prompt and delayed terms in the inhour equation being very similar leading to a small value with a large uncertainty. The other two, area ratio, methods have a large uncertainty in the correction factor. For larger measured worths, the uncertainties rapidly decrease. The generation time dominates the prompt approximation of the inhour method and the minimum uncertainty limit is $3 \%$ for the area ratio methods. The statistical uncertainties in all three methods are insignificant compared with those associated with the delayed neutron data (Ref. 3).

Table 1.4-1. Typical Uncertainties for Three PNS Techniques (Ref. 3).

\begin{tabular}{||c|c|c|c|c|c||}
\hline \hline Technique & Nominal Reactivity (\$) & $\mathbf{- 0 . 1 5}$ & $\mathbf{- 1 . 0}$ & $\mathbf{- 5 . 6}$ & $\mathbf{- 1 2 . 0}$ \\
\hline \hline \multirow{2}{*}{ Inhour } & \multirow{2}{*}{$\begin{array}{c}\text { Measured Reactivity } \\
\pm \text { Total Uncertainty }\end{array}$} & $\begin{array}{c}-0.15 \pm 68 \% \\
( \pm 7.5 \%)\end{array}$ & $\begin{array}{c}-1.01 \pm 11 \% \\
( \pm 0.8 \%)\end{array}$ & $\begin{array}{c}-5.52 \pm 6 \% \\
( \pm 0.5 \%)\end{array}$ & $\begin{array}{c}-11.5 \pm 5.5 \% \\
( \pm 0.3 \%)\end{array}$ \\
\cline { 4 - 6 } & \multirow{2}{*}{\begin{tabular}{c}
$( \pm$ Statistical Uncertainty $)$ \\
\cline { 3 - 6 }
\end{tabular}} & $-0.156 \pm 27 \%$ & $-0.987 \pm 3.6 \%$ & $-5.879 \pm 3.6 \%$ & $-11.77 \pm 3.0 \%$ \\
\cline { 3 - 6 } Gozani & $-0.163 \pm 27 \%$ & $-1.01 \pm 3.7 \%$ & $-5.84 \pm 3.3 \%$ & $-11.59 \pm 3.1 \%$ \\
\hline
\end{tabular}

For negative reactivities rod-drop measurements needed a small detection dead time. In order to have good statistics a high count rate at critical before the rod drop is necessary. Two approaches were utilized:

1. Initially low dead-time detectors were unavailable and the detectors for the PNS measurements, with dead times of $1.4 \pm 0.1 \mu \mathrm{sec}$ were used. Use of a single detector gave unacceptably large uncertainties on the derived reactivities. A method was developed using two detectors, with different sensitivities, situated close together in the system (near the outer surface of the system because of their high efficiencies). The responses of these detectors were fitted over a small overlap range directly following the rod drop to give a composite response with the effect of a 
NEA/NSC/DOC(2006)1

\section{Gas Cooled (Thermal) Reactor - GCR \\ PROTEUS-GCR-EXP-004 \\ CRIT-REAC}

time-dependent sensitivity. This approach was considered somewhat messy and time consuming but unavoidable.

2. From Core 5 onwards, a new measuring system was available that had been previously used for IK measurements on the SAPHIR reactor. It had the advantage of a very small dead time with each amplified pulse having a width of only a few nanoseconds. Count rates approached some 800,000 counts per second without significant dead time effects (Ref. 3).

All rod-drop measurements were carried out in a similar manner:

1. Establish a critical state with the reactor start-up sources withdrawn and the detectors in place. When stable, freeze all control absorbers.

2. Trigger the multichannel-analyzer system with a channel width of 0.1 seconds and at least 2048 measurement channels. Channel widths greater than 0.1 seconds were shown in simulations to lead to systematic errors in the estimated reactivity due to an inability to resolve the "dropregion". Narrower channel widths led to very poor statistics and significant "rounding-down" effects.

3. After a nominal 20 seconds, to establish the initial critical flux level and to measure the initial reactivity (nominally 0 ), the required shutdown rod configuration (normally 1, 2, 3 or 4 rods, occasionally 8) is dropped.

4. The same measurement is repeated to check for reproducibility and to reduce uncertainties.

5. The same configuration is measured with the detectors in a different position in the system, to provide measurements of the same parameter with different spatial correction factors.

6. The stored raw data are then processed to calculate the desired reactivity worth measurement (Ref. 3).

For small positive reactivities, such as differential calibration of control-rods in HTR-PROTEUS, the stable period (SP) technique was exclusively used. The experimental setup for the SP measurements was very similar to that used for the PNS measurements. The experimental procedure was as follows:

1. Establish a critical state with the required detectors in place. When stable, freeze all control absorbers.

2. Trigger the multichannel-analyzer system, which has been configured with a channel width of 1 second and 4096 measurement channels.

3. After a nominal 20 seconds (to establish a start reactivity, nominally $=0.0$, but cannot be judged exactly due to drift, statistical fluctuations of the autorod position, etc.) the control rods are driven out the required amount (corresponding to a few cents, maximum 10 cents).

4. The measurement is ceased when the count-rate becomes too high (dead-time consideration).

5. The stored raw data are then processed to calculate the desired reactivity worth measurement (Ref. 3).

The uncertainty in the reactivity obtained via SP measurements arises from the statistical uncertainties in the measured data and systematic uncertainties associated with the data used in the inhour equation used to derive reactivity. The statistical uncertainties can be reduced by increasing count rates and measuring times in individual measurements or by repeating measurements. The former method is limited by the properties particular to the counting system, namely dead-time and detector efficiency, and the latter method, although effective, is expensive in time and effort. Reductions in the uncertainties associated with the use of delayed neutron data can only be achieved by using a better data set. A sample measurement of the worth of Control Rod 4 in Core 5 inserted from 2500 to $2100 \mathrm{~mm}$ demonstrated an uncertainty of $3.7 \%$ in the measured worth. The statistical uncertainty was $0.17 \%$ of the measured worth, or $\sim 5 \%$ of the total uncertainty. Further evaluation of the uncertainties indicated that the contribution of the prompt term of the reactivity calculation was only $\sim 2 \%$ while the largest contributor to this uncertainty ( $>50 \%$ for the second delayed group) had relatively low uncertainties and thus did not contribute more significantly to the total uncertainty in the reactivity measurement (Ref. 3).

For the reactivity measurements reported in Ref. 3, the uncertainties are associated mainly with the statistical uncertainties inherent in the measurement itself. Uncertainties were not applied to the calculated delayed neutron parameters. In general, the delayed neutron data were based upon the JEF-1.1

Revision: 1

Date: March 31, 2014

Page 71 of 375 
NEA/NSC/DOC(2006)1

\section{Gas Cooled (Thermal) Reactor - GCR \\ PROTEUS-GCR-EXP-004 \\ CRIT-REAC}

evaluation. While the slight energy dependence of the total yield was ignored, the energy dependence of the delayed neutron spectra was not. It was demonstrated that the delayed neutron data available in ENDF/B-VI and JEF-2.2 were not acceptable for predicting the behavior of control rods in the HTRPROTEUS experiments. ${ }^{\mathrm{a}}$

\subsubsection{Control Rod Worth Measurements}

Individual integral control rod worth measurements were reported for the four withdrawable stainless steel control rods described in Section 1.1.2.2.

The reactivity worth of the withdrawable control rods was measured using IK techniques. Two experimental approaches were tested in HTR-PROTEUS:

1. The reactor was in a critical state with the rod of interest completely inserted. Then, the rod was completely withdrawn in a few (typically three or four) steps. After each step, the reactor was made critical with the other rods. The positive reactivity of each step was determined with the IK equation and the stable reactor period technique.

2. The reactor was in a critical state with the rod of interest completely withdrawn. Then the rod was driven in completely, which takes $156 \mathrm{~s}$. The reactivity was determined via the inverse kinetics equation.

With the first approach, only the integral worth was obtained, whereas with the second approach, both the integral and the differential rod worth could be obtained. While only the first approach was used in Core 5 and both approaches in Core 7, only the second approach was utilized in Cores 9 and 10 (Ref. 3).

The results of the SP and IK techniques for the positive reactivity steps were seen to agree within $0.7 \%$. The comparison to the results of the second approach showed that they agreed within $1.7 \%$. All results agree within $2 \sigma$ and only the average of all techniques was reported. It should be noted that there is an observable asymmetry in the worths of the control rods caused by the shadowing effect of the relatively low-worth autorod. The worth of the control rods in Positions 2 and 3 are slightly lower than those in Positions 1 and 4. Reported control rods worths for Cores 9 and 10 are in Table 1.4-2 (Ref. 3 and 4).

Table 1.4-2. The Integral Worth of the Control Rods (dollarcents). $[1 \$=720 \mathrm{pcm}]$ (Ref. 3 and 4). ${ }^{(\mathrm{a})}$

\begin{tabular}{|c|c|c|}
\hline Rod & Core 9 & Core 10 \\
\hline \hline 1 & $39.69 \pm 0.09$ & $28.19 \pm 0.070$ \\
\hline 2 & $39.04 \pm 0.09$ & $27.85 \pm 0.084$ \\
\hline 3 & $39.07 \pm 0.09$ & $27.64 \pm 0.074$ \\
\hline 4 & $39.61 \pm 0.09$ & $28.15 \pm 0.071$ \\
\hline \hline Average of Rods 1 and 4 & $39.65 \pm 0.06^{(b)}$ & $28.17 \pm 0.05$ \\
\hline \hline Bank Worth (Sum of the Rods) & $157.41 \pm 0.18$ & $111.83 \pm 0.15$ \\
\hline \hline
\end{tabular}

(a) Evaluator's Note: Reported uncertainty is statistical and does not include additional sources of uncertainty such as from delayed neutron data.

(b) This value was incorrectly reported as 36.65 in Ref. 3.

\footnotetext{
${ }^{a}$ Williams, T., "On the Choice of Delayed Neutron Parameters for the Analysis of Kinetics Experiments in ${ }^{235} \mathrm{U}$ Systems," Ann. Nucl. Energy, 23, 1261-1265 (1996).

Revision: 1 
NEA/NSC/DOC(2006)1

\section{Gas Cooled (Thermal) Reactor - GCR \\ PROTEUS-GCR-EXP-004 \\ CRIT-REAC}

Additional reactivity corrections were measured for the critical core loadings to account for insertion of the control rods (Ref. 2 and 3). Worth corrections related to direct measurements of control rod bank insertions for Cores 9 (State \#2) and 10 are as follows:

1. Core 9 (State \#2) partial control rod bank insertion of $1620 \mathrm{~mm}$, measured via SP, $-70.4 \pm 1.0 \notin$,

2. Core 9 full control rod bank worth, or worth of inserting all control rods at once, is $152 \pm 2 \phi$,

3. Core 10 partial control rod insertion of $1540 \mathrm{~mm},-36.8 \pm 1 \notin$, and

4. Core 10 full control rod bank worth, or worth of inserting all control rods at once, is $109.6 \phi$, calibrated via SP technique.

The control rods were fully withdrawn for Core 9 (State \#1). The reported value of $\beta_{\text {eff }}$ is 0.00720 for each case.

Reference 5 indicates a partial control rod bank insertion worth of $-70.4 \notin(-507 \mathrm{pcm})$ worth for Core 9 , with a reported value of $\beta_{\text {eff }}$ of $720 \mathrm{pcm}$.

\subsubsection{Autorod Worth Measurements}

Autorod worth measurements were reported for the autorod described in Section 1.1.2.1.

Additional reactivity corrections were measured for the critical core loadings to account for the presence of the autorod (Ref. 2 and 3). Worth corrections related to the presence of the autorod for Cores 9 (States $\# 1$ and 2) and 10 are as follows:

1. Core 9 autorod rest worth (i.e. the worth of removing the absorber rod after it has been fully withdrawn), scaled from Core 5 worth measurement, $-12.5 \pm 0.5 \phi$,

2. Core 9 (State \#1) partial autorod insertion of $258 \mathrm{~mm}$, scaled from Core 5 worth measurement, $-7.5 \pm 0.5 \phi$,

3. Core 9 (State \#2) partial autorod insertion of $25 \mathrm{~mm}$, scaled from Core 5 worth measurement, $-10.0 \pm 0.5 \phi,{ }^{\mathrm{a}}$

4. Core 9 max-min worth of $10.1 \notin$, scaled from Core 5 worth measurement,

5. Core 10 autorod rest worth, scaled from Core $1 \mathrm{~A}$ worth measurement, $-7.7 \pm 0.5 \phi$, and

6. Core 10 partial autorod insertion of $15 \mathrm{~mm}$, directly measured in Core $10,-7 \pm 0.4 \not$. $^{\mathrm{b}}$

The reported value of $\beta_{\text {eff }}$ of 0.00720 for each case.

The worth of the autorod was determined by hand control while measuring reactivity via the inverse kinetics technique and simultaneously measuring the position of the autorod. The measured integral reactivity worths, i.e. the reactivity differences between the fully inserted and fully withdrawn states, is reported in Table 1.4-3 (Ref. 4).

\footnotetext{
${ }^{\text {a }}$ Note that State \#2 of Core 9 had an additional layer of moderator pebbles placed on top of the pebble configuration of State \#1. Hence the apparent inconsistency in autorod worths reported for the two configurations of Core 9.

${ }^{\mathrm{b}}$ Comparison of autorod worth measurements between Cores 9 and 10 is difficult due to the change in spectra from the addition of polyethylene rods, to modification in number of stacked pebble layers, and the change in control rod insertion depths.
}

Revision: 1

Date: March 31, 2014

Page 73 of 375 
NEA/NSC/DOC(2006)1

\section{Gas Cooled (Thermal) Reactor - GCR \\ PROTEUS-GCR-EXP-004 \\ CRIT-REAC}

Table 1.4-3. The Integral Worth of the Autorod (dollarcents). $[1 \$=720 \mathrm{pcm}]$ (Ref. 4). ${ }^{(a)}$

\begin{tabular}{|c|c|c|}
\hline Core & 9 & 10 \\
\hline \hline Autorod Worth & $9.18 \pm 0.06$ & $6.96 \pm 0.044$ \\
\hline \hline
\end{tabular}

(a) Evaluator's Note: Reported uncertainty is statistical and does not include additional sources of uncertainty such as from delayed neutron data.

\subsubsection{Safety/Shutdown Rod Worth Measurements}

Individual integral safety/shutdown rod worth measurements were reported for the shutdown rods described in Section 1.1.2.1.

In their fully withdrawn state the tips of the eight boron-steel safety/shutdown rods are slightly dipping into the radial reflector; it was demonstrated in Core 1 that there was no reactivity worth associated with their presence. ${ }^{\mathrm{a}}$

Figure 1.4-1 shows an example of a typical detector response, with corresponding reactivity results, during a rod-drop experiment. At the time $\mathrm{t}=11 \mathrm{~s}$, three shutdown rods, having a total worth of $\sim 8 \$$, were inserted in HTR-PROTEUS Core 10. The reactivity change, as a function of time, was plotted with each point having its properly derived $1 \sigma$ statistical uncertainty. Since only the integral rod worth is being measured, the average values deduced for reactivity both before and after the drop can be used, which correspondingly reduces the uncertainty. The IK technique is only applicable to transient system measurements that normally start from a critical or near-critical state. Measurement of a constant subcritical system requires the PNS method (Ref. 10).

\footnotetext{
${ }^{a}$ Williams, T., Bourguin, P, and Chawla, R. "HTR PROTEUS CORE 1: Reactivity Corrections for the Critical Balance,” TM-41-93-20, Paul Sherrer Institut, Villigen, October 7, 1993.

Revision: 1

Date: March 31, 2014

Page 74 of 375
} 
NEA/NSC/DOC(2006)1

Gas Cooled (Thermal) Reactor - GCR

PROTEUS-GCR-EXP-004

CRIT-REAC

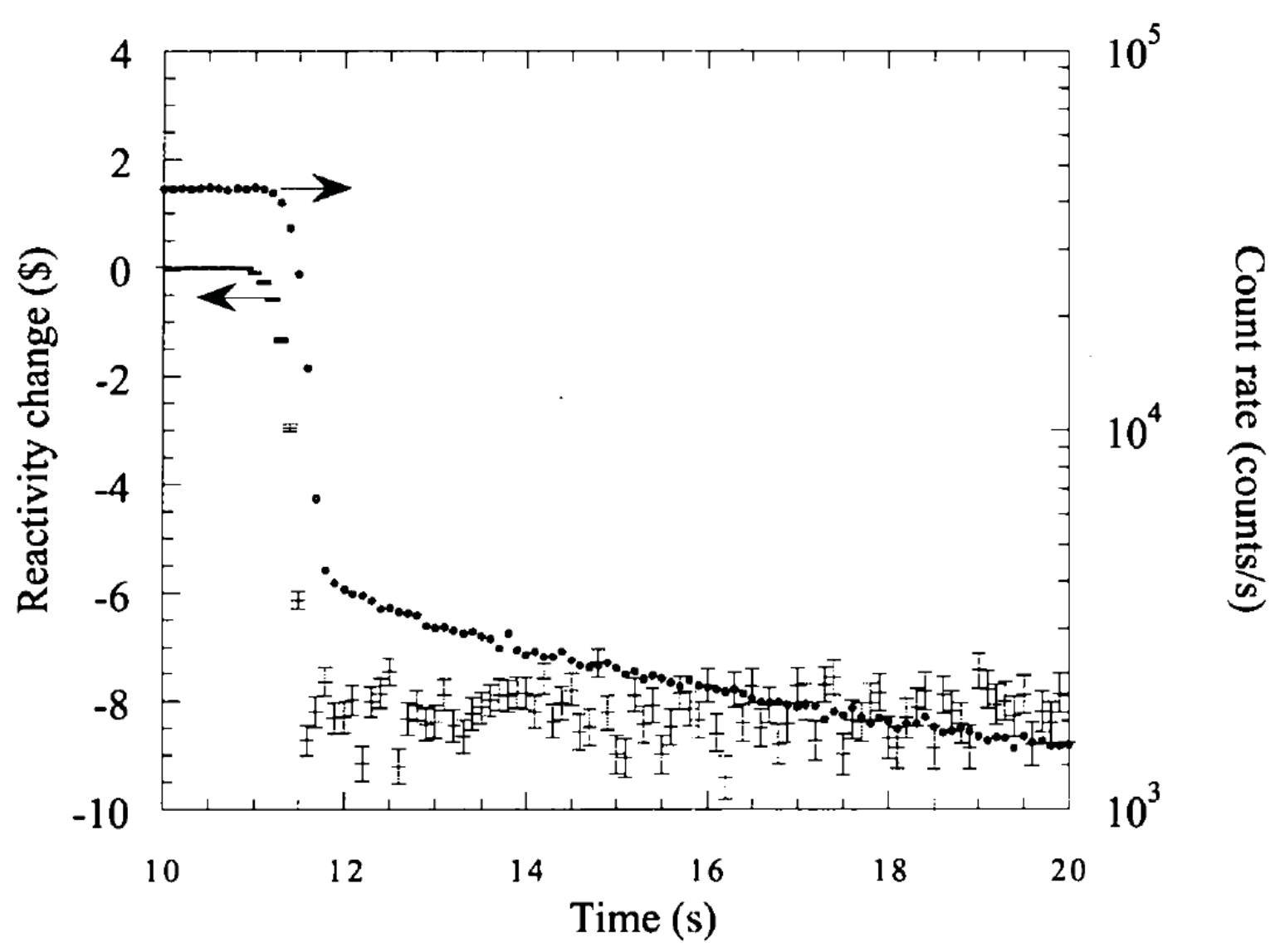

Figure 1.4-1. Example Count Rate and Reactivity Change Following Absorber Rod Insertion in Core 10 (Ref. 10).

Figure 1.4-2 shows a typical detector response for a PNS measurements of a subcritical Core 10 with four shutdown rods inserted. After the perturbations from the spatial harmonics, the first part of the response can be approximated by a single exponential corresponding to the prompt neutron decay; the second half is due to the delayed neutrons. A common criticism of the PNS method is that measured reactivity values depend upon correction factors and/or kinetics parameters that need to be calculated using the same neutronics codes as those being validated by said measurements. For example, the area-ratio method must be corrected for kinetic distortion, which arises from spatial and spectral differences of the prompt and delayed neutron fluxes during a transient. The inhour (Simmons-King) method calculates a reactivity value independent of kinetic distortion, but the results is strongly dependent upon a calculation of the prompt neutron generation time (Ref. 10). 
NEA/NSC/DOC(2006)1

Gas Cooled (Thermal) Reactor - GCR

PROTEUS-GCR-EXP-004

CRIT-REAC

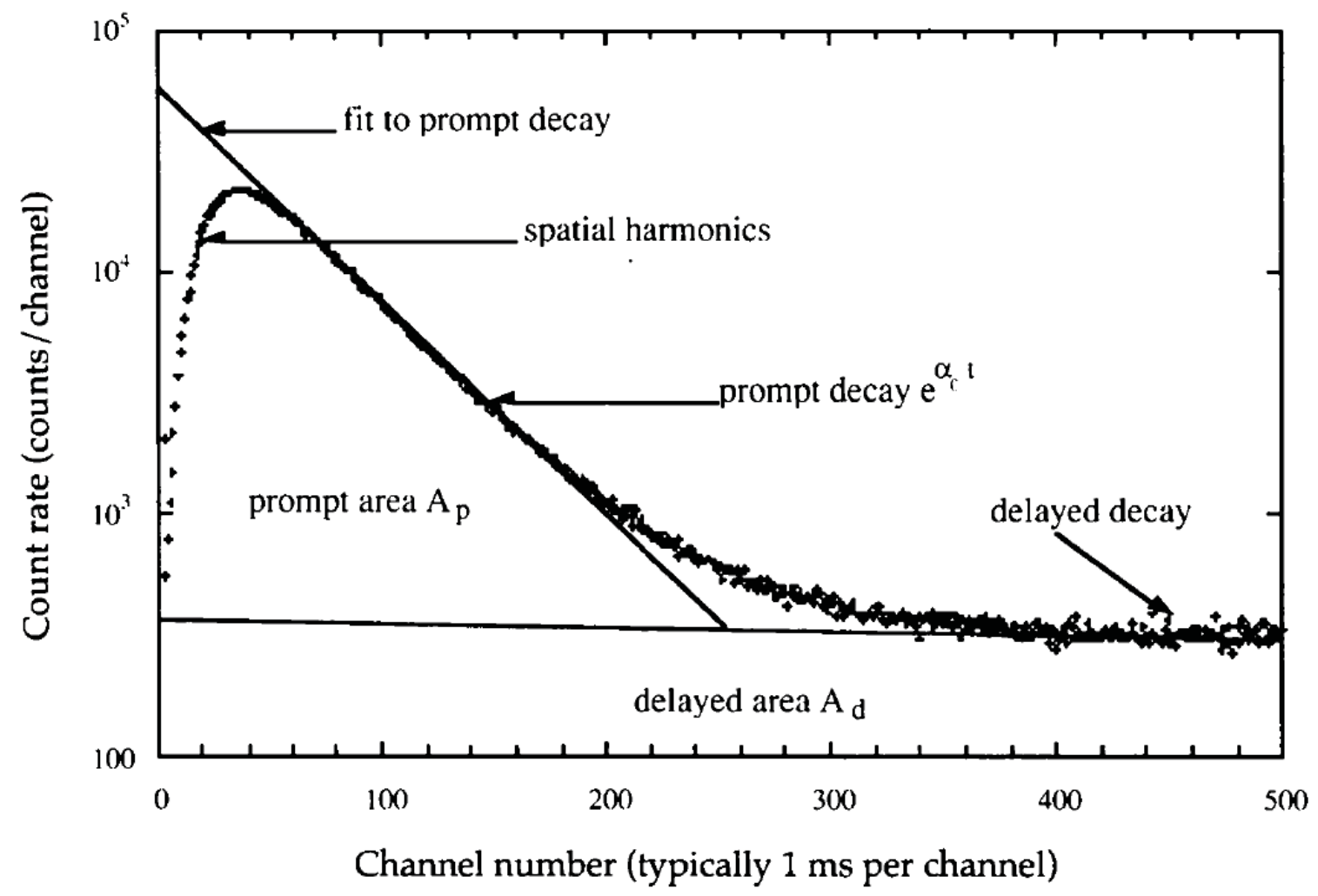

Figure 1.4-2. Example Count Rate Response for a Pulsed Neutron Source Measurement (Ref. 10).

The combination of an undermoderated core and strongly interacting reflector zones renders accurate subcriticality measurements particularly difficult in a small-sized pebble-bed HTR. This is due to the strong spatial effects in the system requiring relatively large calculated correction factors in the interpretation of both IK and PNS measurements. To reduce this dependence of conventional experimental techniques on calculational results, new techniques based on the use of epithermal neutron detectors were developed and applied in the HTR-PROTEUS program. The sensitivity to calculated correction factors and/or kinetic parameters was shown to be considerably reduced for both types of measurements (Ref. 3 and 10). The experimental procedures and extended analytical methodology required for the interpretation of the epithermal measurement techniques is provided in Ref. 7 and 8.

Different combinations of shutdown rods were measured applying in each case a variety of experimental methods. These ranged, from conventional (thermal) and newly developed (epithermal) IK and PNS techniques, using both Simmons-King and Gozani theories to analyze the PNS measurements. Generally statistical uncertainties were smaller in experiments using thermal detectors. However the lower sensitivity to correction factors in epithermal measurements yielded more reliable results. A parallel application of several different techniques was used to check for any systematic errors. The spatial dependence of the IK measurements and PNS measurements using Gozani theory were corrected; the uncertainties correspond to the $1 \sigma$ statistical error for cases where only one measurement was made and in the other cases the uncertainties are standard deviations on the average values. A variance-weighted average was also calculated; however the uncertainty was tabulated as the square-root of the sum of the squares instead of the inverse of the squared uncertainty because of the small experimental sample size. Reactivity worth measurements for various shutdown rod combinations are shown in Table 1.4-4 for Core 9 and 1.4-5 for Core 10 (Ref. 3 and 6). 
NEA/NSC/DOC(2006)1

\section{Gas Cooled (Thermal) Reactor - GCR \\ PROTEUS-GCR-EXP-004 \\ CRIT-REAC}

It should be noted that there is an observable asymmetry in the worths of the shutdown rods caused by the shadowing effect of the relatively low-worth autorod, albeit slightly smaller in effect than in the control rods (Ref. 3).

Comparison of Figures 1.4-3 and 1.4-4 demonstrate how use of epithermal measurements can effectively reduce the magnitude of the correction factors; furthermore, the perturbation of the epithermal flux is appreciably smaller compared to the thermal flux perturbation (Ref. 10).

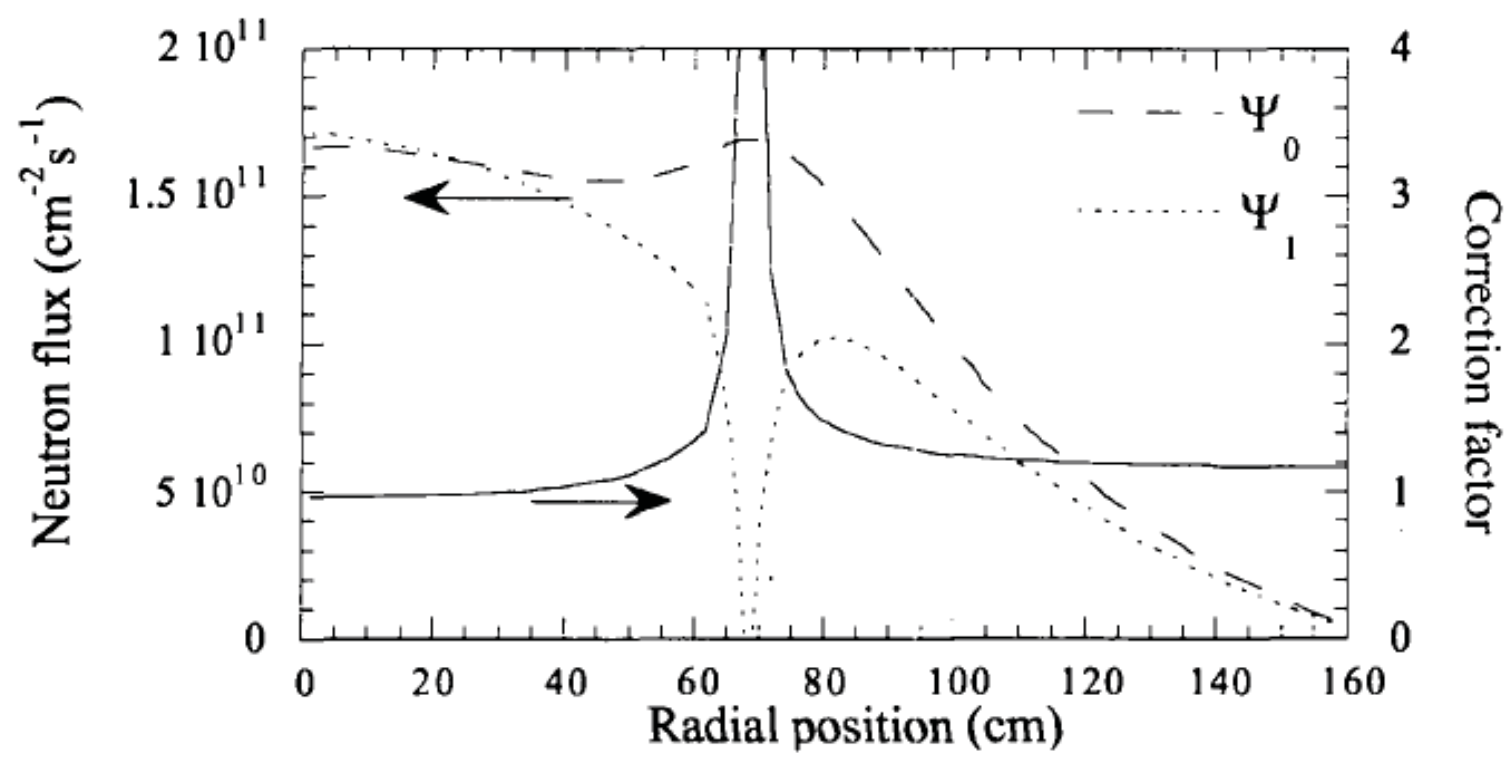

Figure 1.4-3. Calculated Thermal $(\mathrm{E}<0.625 \mathrm{eV})$ Fluxes Before $\left(\Psi_{0}\right)$ and After $\left(\Psi_{1}\right)$ the Insertion of a Shutdown Rod in Core 10, and Correction Factor for the Reactivity Measured with the IK Technique (Ref. 10). 
NEA/NSC/DOC(2006)1

\section{Gas Cooled (Thermal) Reactor - GCR \\ PROTEUS-GCR-EXP-004 \\ CRIT-REAC}

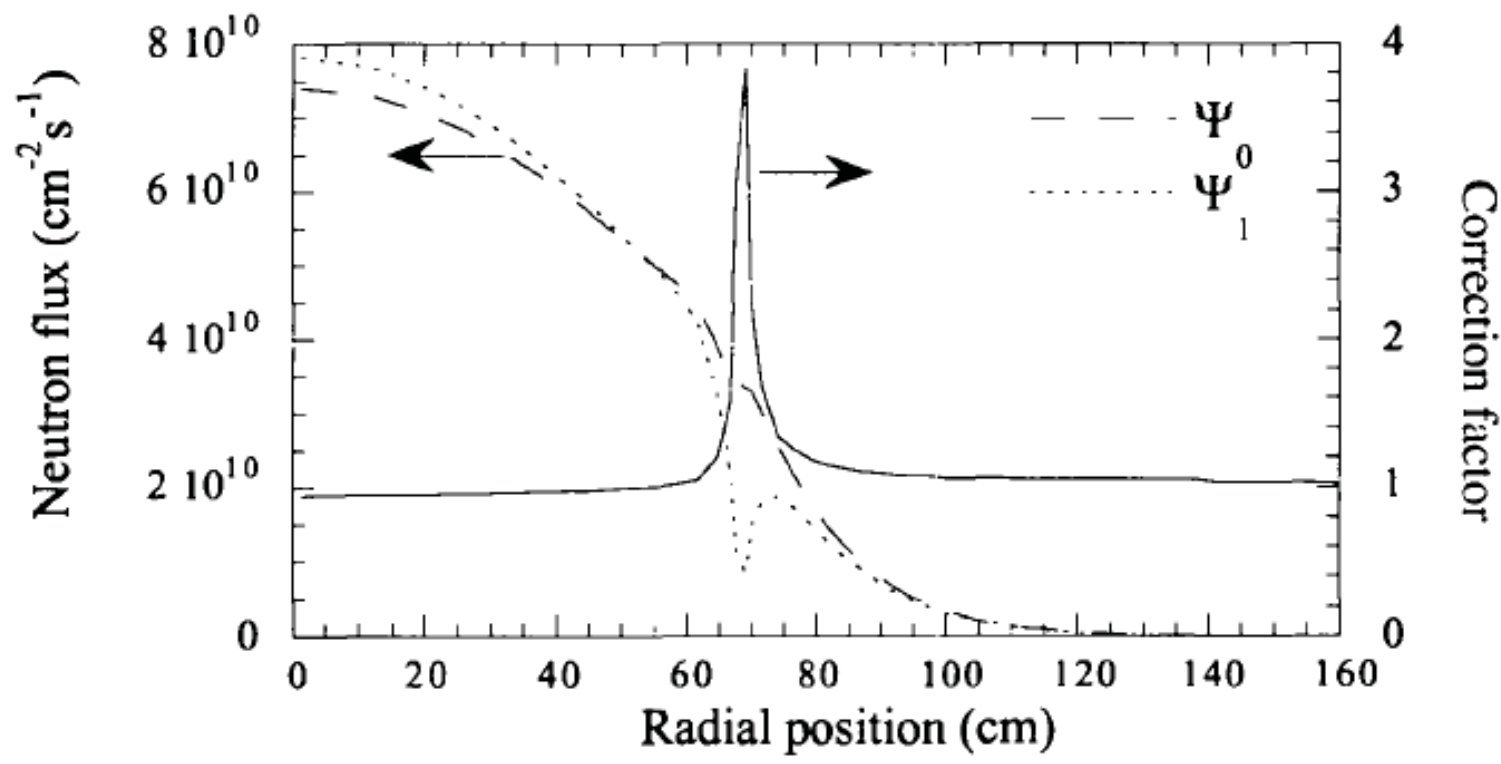

Figure 1.4-4. Calculated Epithermal $(0.625 \mathrm{eV}<\mathrm{E}<750 \mathrm{eV})$ Fluxes Before $\left(\Psi_{0}\right)$ and After $\left(\Psi_{1}\right)$ the Insertion of a Shutdown Rod in Core 10, and Correction Factor for the Reactivity Measured with the IK Technique (Ref. 10).

Table 1.4-4. Reactivity Worth Measurements for Various Combinations of the Shutdown Rods in Core 9 [The values in square brackets represent the number of measurements made for a given configuration] (Ref. 3, 6, 9, and 10). ${ }^{(a)}$

\begin{tabular}{|c|c|c|c|c|}
\hline Rods Inserted & $\mathbf{6}^{(\mathbf{b})}$ & $5-6$ & $5-6-7$ & $5-6-7-8$ \\
\hline Thermal IK measurement (\$) & $\begin{array}{c}-3.73 \pm 0.02 \\
{[2]}\end{array}$ & $\begin{array}{c}-7.71 \pm 0.09 \\
{[2]}\end{array}$ & $\begin{array}{c}-11.69 \pm 0.23 \\
{[2]} \\
\end{array}$ & $\begin{array}{c}-16.00 \pm 0.41 \\
{[2]} \\
\end{array}$ \\
\hline Epithermal IK measurement (\$) & $\begin{array}{c}-3.63 \pm 0.08 \\
{[1]}\end{array}$ & -- & $\begin{array}{c}-11.36 \pm 0.20 \\
{[2]}\end{array}$ & - \\
\hline $\begin{array}{c}\text { Thermal Simmons-King PNS } \\
\text { measurement }(\$)\end{array}$ & $\begin{array}{c}-3.69 \pm 0.06 \\
{[3]}\end{array}$ & $\begin{array}{c}-7.74 \pm 0.19 \\
{[5]}\end{array}$ & $\begin{array}{c}-11.63 \pm 0.33 \\
{[3]}\end{array}$ & $\begin{array}{c}-15.63 \pm 0.53 \\
{[6]}\end{array}$ \\
\hline $\begin{array}{c}\text { Thermal Gozani PNS } \\
\text { measurement }(\$)\end{array}$ & $\begin{array}{c}-3.77 \pm 0.01 \\
{[2]}\end{array}$ & $\begin{array}{c}-7.88 \pm 0.05 \\
{[3]}\end{array}$ & $\begin{array}{c}-12.25 \pm 0.11 \\
{[2]}\end{array}$ & $\begin{array}{c}-16.39 \pm 0.36 \\
{[4]}\end{array}$ \\
\hline $\begin{array}{c}\text { Epithermal Gozani PNS } \\
\text { measurement }(\$)\end{array}$ & $\begin{array}{c}-3.73 \pm 0.08 \\
{[4]}\end{array}$ & $\begin{array}{c}-7.85 \pm 0.14 \\
{[5]}\end{array}$ & $\begin{array}{c}-11.85 \pm 0.24 \\
{[4]}\end{array}$ & $\begin{array}{c}-16.43 \pm 0.45 \\
{[8]} \\
\end{array}$ \\
\hline $\begin{array}{l}\text { Weighted Average (\$) } \\
\text { (worth per rod) }\end{array}$ & 1 & $\begin{array}{l}-7.82 \pm 0.06 \\
\quad(-3.91)\end{array}$ & $\begin{array}{c}-11.83 \pm 0.10 \\
(-3.94)\end{array}$ & $\begin{array}{c}-16.17 \pm 0.24 \\
(-4.04)\end{array}$ \\
\hline
\end{tabular}

(a) Evaluator's Note: Reported uncertainty is statistical and does not include additional sources of uncertainty such as from delayed neutron data.

(b) This rod number is reported as 6 in Ref. 3 and 5 in Ref. 5 and 6. Comparison with results reported for Rods 5 and 6 in Ref. 7 support that the actual rod number is 6. This is also confirmed with Ref. 10. 
NEA/NSC/DOC(2006)1

\author{
Gas Cooled (Thermal) Reactor - GCR \\ PROTEUS-GCR-EXP-004 \\ CRIT-REAC
}

Table 1.4-5. Reactivity Worth Measurements for Various Combinations of the Shutdown Rods in Core 10 [The values in square brackets represent the number of measurements made for a given configuration] (Ref. 3, 6, 9, and 10). ${ }^{\text {(a) }}$

\begin{tabular}{|c|c|c|c|c|}
\hline Rods Inserted & 6 $^{(\mathbf{b})}$ & $5-6$ & 5-6-7 & 5-6-7-8 \\
\hline Thermal IK measurement (\$) & $\begin{array}{c}-2.75 \pm 0.02 \\
{[1]} \\
\end{array}$ & $\begin{array}{c}-6.17 \pm 0.07 \\
{[2]} \\
\end{array}$ & $\begin{array}{c}-9.38 \pm 0.60 \\
{[3]} \\
\end{array}$ & $\begin{array}{c}-12.99 \pm 1.3 \\
{[2]} \\
\end{array}$ \\
\hline Epithermal IK measurement (\$) & $\begin{array}{c}-2.63 \pm 0.06 \\
{[1]}\end{array}$ & $\begin{array}{c}-5.56 \pm 0.10 \\
{[1]}\end{array}$ & $\begin{array}{c}-8.61 \pm 0.34 \\
{[6]}\end{array}$ & $\begin{array}{c}-11.80 \pm 0.19 \\
{[3]}\end{array}$ \\
\hline $\begin{array}{c}\text { Thermal Simmons-King PNS } \\
\text { measurement (\$) }\end{array}$ & $\begin{array}{c}-2.65 \pm 0.05 \\
{[4]}\end{array}$ & $\begin{array}{c}-5.48 \pm 0.12 \\
{[4]}\end{array}$ & $\begin{array}{c}-8.42 \pm 0.33 \\
{[4]}\end{array}$ & $\begin{array}{c}-11.42 \pm 0.32 \\
{[8]}\end{array}$ \\
\hline $\begin{array}{c}\text { Thermal Gozani PNS measurement } \\
(\$)\end{array}$ & $\begin{array}{c}-2.69 \pm 0.06 \\
{[4]}\end{array}$ & $\begin{array}{c}-5.72 \pm 0.30 \\
{[4]}\end{array}$ & $\begin{array}{c}-9.38 \pm 0.15 \\
{[4]}\end{array}$ & $\begin{array}{c}-12.12 \pm 0.37 \\
{[8]}\end{array}$ \\
\hline $\begin{array}{c}\text { Epithermal Gozani PNS } \\
\text { measurement }(\$)\end{array}$ & $\begin{array}{c}-2.59 \pm 0.05 \\
{[1]}\end{array}$ & $\begin{array}{c}-5.47 \pm 0.16 \\
{[1]}\end{array}$ & $\begin{array}{c}-8.64 \pm 0.18 \\
{[1]}\end{array}$ & $\begin{array}{c}-11.71 \pm 0.28 \\
{[9]} \\
\end{array}$ \\
\hline $\begin{array}{l}\text { Weighted Average }^{(\mathrm{c})}(\$) \\
\text { (worth per rod) }\end{array}$ & $-2.66 \pm 0.03$ & $\begin{array}{c}-5.54 \pm 0.09 \\
(-2.77)\end{array}$ & $\begin{array}{l}-8.91 \pm 0.13 \\
\quad(-2.97)\end{array}$ & $\begin{array}{c}-11.74 \pm 0.16 \\
(-2.94)\end{array}$ \\
\hline
\end{tabular}

(a) Evaluator's Note: Reported uncertainty is statistical and does not include additional sources of uncertainty such as from delayed neutron data.

(b) This rod number is reported as 6 in Ref. 3 and 5 in Ref. 5 and 6 for Core 9. Comparison with results reported for Rods 5 and 6 in Ref. 7 support that the actual rod number is 6 . This rod should similarly be labeled as number 6 in Core 10. This is also confirmed with Ref. 10.

(c) The results obtained from the thermal IK measurements were not considered while deducing the weighted-average values. The large discrepancy as regard to the other techniques was probably due to an inadequacy of the Core $10 \mathrm{r}-\Theta$ TWODANT model used for the calculation of the correction factors.

The different methods generally show good agreement for Cores 9 and 10 with one exception. In Core 10, the results obtained with the thermal IK technique generally show large discrepancies with respect to the others, possibly due to an inadequacy of the model used to calculate the correction factor. The epithermal IK measurements are very consistent; this emphasizes the reduced dependence on calculations. The same $r-\Theta$ model was used to correct the epithermal measurements but the corrections were much smaller than for the thermal measurements; epithermal results were not significantly impacted by the inadequacy of the model (Ref. 3 and 10).

The individual rod worth increases slightly with the number of rods inserted. The reactivity worth of the four shutdown rods inserted is always bigger than four times the worth on an individual rod. This arises from positive shadowing effects reported for HTR-PROTEUS measurements, which is less pronounced in Cores 9 and 10 than in earlier HTR-PROTEUS configurations. Also, the worth of the shutdown rods decreases when the effect of water ingress was simulated. This is seen by comparing rod worths between Cores 9 and 10 (Ref. 3 and 6).

Asymmetry effects on the early HTR-PROTEUS cores were investigated. It was shown that antishadowing effects for absorber rods in the radial reflector were significant. The presence of a cavity above the core reduced cross-core anti-shadowing effects. ${ }^{a}$

The relative quality of the thermal and epithermal measurements was evaluated by grouping the measurements separately and taking an equally-weighted average of the experimental measurements. As indicated previously, the erratic IK thermal measurement for Core 10 was neglected. There was no significant difference between thermal or epithermal measurements for Core 9 . Up to a $10 \%$

\footnotetext{
${ }^{a}$ Williams, T., Chawla, R., Hager, H., Mathews, D., Seiler, R., “Absorber-Rod Interaction and Asymmetry Effects in Experimental LEU-HTR Configurations," Proc. 1994 ANS Topical Mtg. on Advances in Reactor Physics, Knoxville, TN, April 11-15, 1994.
}

Revision: 1

Date: March 31, 2014

Page 79 of 375 
NEA/NSC/DOC(2006)1

\section{Gas Cooled (Thermal) Reactor - GCR \\ PROTEUS-GCR-EXP-004 \\ CRIT-REAC}

discrepancy was identified for Core 10 between the thermal and epithermal measurements. The comparison of the average epithermal and thermal worths for Cores 9 and 10 are shown in Tables 1.4-6 and $1.4-7$, respectively.

Table 1.4-6. Averaged Reactivity Worth Measurements the Shutdown Rods in Core 9 (Ref. 6). ${ }^{(a)}$

\begin{tabular}{|c|c|c|c|c|}
\hline Rods Inserted & $\mathbf{6}^{(\mathbf{b})}$ & $\mathbf{5 - 6}$ & $\mathbf{5 - 6 - 7}$ & $\mathbf{5 - 6 - 7 - 8}$ \\
\hline \hline Thermal measurements $(\$)$ & $-3.73 \pm 0.02$ & $-7.78 \pm 0.07$ & $-11.86 \pm 0.14$ & $-16.01 \pm 0.25$ \\
\hline Epithermal measurements $(\$)$ & $-3.68 \pm 0.06$ & $-7.85 \pm 0.14$ & $-11.61 \pm 0.16$ & $-16.43 \pm 0.45$ \\
\hline
\end{tabular}

(a) Evaluator's Note: Reported uncertainty is statistical and does not include additional sources of uncertainty such as from delayed neutron data.

(b) This rod number is reported as 6 in Ref. 3 and 5 in Ref. 5 and 6. Comparison with results reported for Rods 5 and 6 in Ref. 7 support that the actual rod number is 6.

Table 1.4-7. Averaged Reactivity Worth Measurements the Shutdown Rods in Core 10 (Ref. 6). ${ }^{\text {(a) }}$

\begin{tabular}{|c|c|c|c|c||}
\hline Rods Inserted & $\mathbf{6}^{(\mathbf{b})}$ & $\mathbf{5 - 6}$ & $\mathbf{5 - 6 - 7}$ & $\mathbf{5 - 6 - 7 - 8}$ \\
\hline \hline Thermal measurements (\$) & $-2.70 \pm 0.03$ & $-5.79 \pm 0.11$ & $-9.06 \pm 0.23$ & $-12.18 \pm 0.46$ \\
\hline Epithermal measurements (\$) & $-2.61 \pm 0.04$ & $-5.52 \pm 0.09$ & $-8.63 \pm 0.19$ & $-11.76 \pm 0.17$ \\
\hline
\end{tabular}

(a) Evaluator's Note: Reported uncertainty is statistical and does not include additional sources of uncertainty such as from delayed neutron data.

(b) This rod number is reported as 6 in Ref. 3 and 5 in Ref. 5 and 6 for Core 9. Comparison with results reported for Rods 5 and 6 in Ref. 7 support that the actual rod number is 6 . This rod should similarly be labeled as number 6 in Core 10.

Corrected IK measurements of the reactivity worth of various combinations of shutdown rods in Core 9 and 10 are reported for individual detector positions in Tables 1.4-8 and 1.4-9, respectively (Ref. 7 and $10)$. 
NEA/NSC/DOC(2006)1

Gas Cooled (Thermal) Reactor - GCR

PROTEUS-GCR-EXP-004

CRIT-REAC

Table 1.4-8. Corrected Results of IK Measurements of the Reactivity Worth of Various Combinations of the Shutdown Rods in Core 9 (Ref. 7 and 10). ${ }^{(\text {a) }}$

\begin{tabular}{|c|c|c|c|}
\hline $\begin{array}{c}\text { Shutdown Rods } \\
\text { Inserted in the } \\
\text { Reactor }\end{array}$ & Detector Position & $\begin{array}{c}\text { Corrected Reactivity } \\
\text { Measured with } \\
\text { Thermal Detectors (\$) }\end{array}$ & $\begin{array}{c}\text { Corrected Reactivity } \\
\text { Measured with } \\
\text { Epithermal Detectors } \\
\text { (\$) }\end{array}$ \\
\hline 5 & $1^{(\mathrm{b})}$ & $-3.69 \pm 0.02$ & \\
\hline $2^{(\mathrm{c})}$ & $-3.61 \pm 0.03$ & \\
\hline 6 & 1 & $-3.72 \pm 0.01$ & $-3.63 \pm 0.08$ \\
\hline 5,6 & 2 & $-3.73 \pm 0.02$ & \\
\hline & 1 & $-7.69 \pm 0.05$ & \\
\hline $5,6,7$ & 2 & $-7.72 \pm 0.07$ & $-11.23 \pm 0.34$ \\
\hline & 1 & $-11.61 \pm 0.12$ & $-11.49 \pm 0.37$ \\
\hline $5,6,7,8$ & 2 & $-11.76 \pm 0.13$ & \\
\hline & 1 & $-16.10 \pm 0.17$ & \\
\hline & 2 & $-15.89 \pm 0.19$ & \\
\hline
\end{tabular}

(a) Evaluator's Note: Reported uncertainty is statistical and does not include additional sources of uncertainty such as from delayed neutron data.

(b) Position 1: detector in the radial reflector close to Rod 7.

(c) Position 2: detector in the radial reflector close to Rod 8. 
NEA/NSC/DOC(2006)1

\section{Gas Cooled (Thermal) Reactor - GCR \\ PROTEUS-GCR-EXP-004 \\ CRIT-REAC}

Table 1.4-9. Corrected Results of IK Measurements of the Reactivity Worth of Various Combinations of the Shutdown Rods in Core 10 (Ref. 7 and 10). ${ }^{(a)}$

\begin{tabular}{|c|c|c|c|c|}
\hline $\begin{array}{c}\text { Shutdown Rods } \\
\text { Inserted in the } \\
\text { Reactor }\end{array}$ & Detector Position & $\begin{array}{c}\text { Corrected } \\
\text { Reactivity } \\
\text { Measured with } \\
\text { Thermal } \\
\text { Detectors (\$) }\end{array}$ & $\begin{array}{c}\text { Corrected } \\
\text { Reactivity } \\
\text { Measured with } \\
\text { Epithermal } \\
\text { Detectors (Cd } \\
\text { Shielding) (\$) }\end{array}$ & $\begin{array}{c}\text { Corrected } \\
\text { Reactivity } \\
\text { Measured with } \\
\text { Epithermal } \\
\text { Detectors (Cd + In } \\
\text { Shielding) (\$) }\end{array}$ \\
\hline \hline 5 & $3^{(\mathrm{b})}$ & $-2.75 \pm 0.02$ & & $-2.63 \pm 0.06$ \\
\hline 6 & 3 & $-2.79 \pm 0.02$ & & \\
\hline 5,6 & 3 & $-6.17 \pm 0.04$ & & $-5.56 \pm 0.10$ \\
\hline $5,6,7$ & $1^{(\mathrm{c})}$ & $-8.72 \pm 0.08$ & $-8.26 \pm 0.21$ & $-8.18 \pm 0.15$ \\
\hline & $2^{(\mathrm{d})}$ & $-9.60 \pm 0.08$ & $-8.99 \pm 0.20$ & $-8.94 \pm 0.13$ \\
\hline & 3 & $-9.83 \pm 0.06$ & $-8.68 \pm 0.26$ & $-8.60 \pm 0.15$ \\
\hline $5,6,7,8$ & 1 & $-12.04 \pm 0.13$ & & $-11.70 \pm 0.30$ \\
\hline & 2 & & & $-11.71 \pm 0.40$ \\
\hline & 3 & $-13.94 \pm 0.13$ & & $-11.98 \pm 0.35$ \\
\hline
\end{tabular}

(a) Evaluator's Note: Reported uncertainty is statistical and does not include additional sources of uncertainty such as from delayed neutron data.

(b) Position 3: detector at the radial center of the core.

(c) Position 1: detector in the radial reflector close to Rod 7.

(d) Position 2: detector in the radial reflector close to Rod 8.

The measurements on Cores 9 and 10 were performed for various subcritical configurations with the detectors at several different positions that corresponded to significantly different spatial perturbations. This was done so that a group of measurements representing the same measured parameter could be assessed to obtain an indication of the uncertainty and reliability of the calculational correction factors being applied. Sufficient measurements were performed to show the important differences between the different techniques (Ref. 7 and 10).

Insertion of absorber rods induced local flux perturbations that had a more significant impact on thermal measurements. It was reported that the discrepancy on the reactivity measured at the same two positions, with three shutdown rods inserted into Core 9 , is only $1.4 \pm 2.9 \%$ with epithermal detectors and $2.3 \pm$ $4.5 \%$ in the thermal detectors; this demonstrates the different magnitudes of spatial perturbations with their impact on the derived correction factors. ${ }^{a}$ The difference between thermal results for corrected detector measurement worths during the 4-rod insertion in Core 10 is $\sim 16 \%$ while it is $<2.5 \%$ for the epithermal detectors. There is much better agreement between thermal and epithermal results in Core 9 than in Core 10; the experimenters believed this was due to modeling weaknesses of the r- $\Theta$ model when calculating the correction factors for the water-moderated core. The calculated $\beta_{\text {eff }}$ values were 0.00717 and 0.00720 for Cores 9 and 10, respectively (Ref. 7 and 10).

Further discussion of the IK and PNS method using thermal and epithermal measurements, and calculation of correction factors for Cores 9 and 10 can be found in Reference 10.

\footnotetext{
${ }^{a}$ Regardless of what was reported here in Reference 7, the differences are within the statistics of the measurements.

Revision: 1

Date: March 31, 2014

Page 82 of 375
} 
NEA/NSC/DOC(2006)1

\section{Gas Cooled (Thermal) Reactor - GCR \\ PROTEUS-GCR-EXP-004 \\ CRIT-REAC}

Reference 5 reports the shutdown rod worths for Core 9, as shown in Table 1.4-10, and indicates that Ref. 6 is the initial source of these data. Comparison with Table 1.4-6 confirms that Ref. 5 repeats the measurements reported for the averaged reactivity worth of just the epithermal measurements.

Table 1.4-10. Reactivity Worth Measurements for Various Combinations of the Shutdown Rods in Core 9 (Ref. 5). ${ }^{(a)}$

\begin{tabular}{|c|c|c|c|c|}
\hline Rods Inserted & $6^{(\mathrm{b})}$ & $5-6$ & $5-6-7$ & $5-6-7-8$ \\
\hline Experimental Worth (\$) & $-3.68(6)$ & $-7.85(14)$ & $-11.61(16)$ & $-16.43(45)$ \\
\hline
\end{tabular}

(a) Evaluator's Note: Reported uncertainty is statistical and does not include additional sources of uncertainty such as from delayed neutron data.

(b) This rod number is reported as 6 in Ref. 3 and 5 in Ref. 5 and 6. Comparison with results reported for Rods 5 and 6 in Ref. 7 support that the actual rod number is 6 .

An earlier references reported slightly different values for PNS shutdown rod worth measurements in Core 9. Results are shown in Table 1.4-11 (Ref. 8).

Table 1.4-11. Reactivity Worth Measurements for Combinations of Shutdown Rods in Core 9 (Ref. 8). ${ }^{(a)}$

\begin{tabular}{|c|c|c|c|}
\hline $\begin{array}{c}\text { Number of Rods } \\
\text { Inserted }^{(\mathbf{b})}\end{array}$ & $\begin{array}{c}\text { Simmons-King } \\
\text { Reactivity (\$) }\end{array}$ & $\begin{array}{c}\text { Corrected Thermal } \\
\text { Gozani Reactivity (\$) }\end{array}$ & $\begin{array}{c}\text { Corrected Epithermal } \\
\text { Gozani Reactivity (\$) }\end{array}$ \\
\hline 1 & $-3.64 \pm 0.03$ & $-3.70 \pm 0.05$ & $-3.62 \pm 0.02$ \\
\hline 2 & $-7.74 \pm 0.09$ & $-7.83 \pm 0.42$ & $-7.89 \pm 0.14$ \\
\hline 3 & $-11.63 \pm 0.08$ & $-11.91 \pm 0.78$ & $-12.03 \pm 0.21$ \\
\hline 4 & $-15.63 \pm 0.12$ & $-15.52 \pm 1.72$ & $-16.81 \pm 0.54$ \\
\hline
\end{tabular}

(a) Evaluator's Note: Reported uncertainty is statistical and does not include additional sources of uncertainty such as from delayed neutron data.

(b) The rod positions that correspond with each measurement were not reported.

Additional IK measurement rod worths (corrected for spatial dependence and neutron spectrum change) were reported for Cores 9 and 10 in Ref. 4. For both sets of measurements, the results from the second detector were reported as "suspiciously high" and thus results from this detector were not deemed acceptable by the experimenters, as the same problem existed when taking measurements with the autorod. Measured shutdown rod worths for Core 9 are in Table 1.4-12 and Core 10 in Table 1.4-13 (Ref. 4). 
NEA/NSC/DOC(2006)1

Gas Cooled (Thermal) Reactor - GCR

PROTEUS-GCR-EXP-004

CRIT-REAC

Table 1.4-12. Measured Shutdown Rod Worths (in \$) in Core 9 (Ref. 4). ${ }^{(a)}$

\begin{tabular}{|c|c|c||}
\hline Rods Inserted & Detector 1 & Detector 2 $^{(\mathbf{b})}$ \\
\hline \hline 5 & $3.558 \pm 0.008$ & $4.880 \pm 0.040$ \\
\hline 6 & $3.594 \pm 0.005$ & $4.185 \pm 0.019$ \\
\hline 7 & $3.578 \pm 0.006$ & $4.040 \pm 0.016$ \\
\hline 8 & $3.482 \pm 0.007$ & - \\
\hline $5+6$ & $7.48 \pm 0.013$ & $8.71 \pm 0.042$ \\
\hline $5+7$ & $7.19 \pm 0.017$ & - \\
\hline $5+8$ & $7.15 \pm 0.019$ & - \\
\hline $5+6+7+8$ & $15.25 \pm 0.050$ & - \\
\hline \hline
\end{tabular}

(a) Evaluator's Note: Reported uncertainty is statistical and does not include additional sources of uncertainty such as from delayed neutron data.

(b) Results from Detector 2 deemed unacceptable by experimenters.

Table 1.4-13. Measured Shutdown Rod Worths (in \$) in Core 10 (Ref. 4). ${ }^{(a)}$

\begin{tabular}{|c|c|c|c|c|}
\hline Rods Inserted & Detector 1 & Detector 2 & Detector 3 & $\begin{array}{c}\text { Weighted } \\
\text { Average }^{(\mathbf{c})}\end{array}$ \\
\hline 5 & $2.72 \pm 0.006$ & $3.10 \pm 0.010$ & $2.63 \pm 0.007$ & $2.75 \pm 0.12$ \\
\hline 6 & $2.74 \pm 0.007$ & $2.92 \pm 0.010$ & $2.71 \pm 0.009$ & $2.77 \pm 0.06$ \\
\hline 7 & $2.73 \pm 0.049$ & $2.92 \pm 0.010$ & $2.60 \pm 0.009$ & $2.74 \pm 0.09$ \\
\hline 8 & $2.67 \pm 0.008$ & $2.56 \pm 0.010$ & $2.51 \pm 0.026$ & $2.62 \pm 0.04$ \\
\hline $5+6$ & $5.79 \pm 0.012$ & $6.43 \pm 0.028$ & $5.52 \pm 0.015$ & $5.76 \pm 0.18$ \\
\hline $5+7$ & $5.65 \pm 0.015$ & $6.17 \pm 0.021$ & $5.26 \pm 0.012$ & $5.54 \pm 0.23$ \\
\hline $5+8$ & $5.59 \pm 0.013$ & $6.25 \pm 0.022$ & $5.35 \pm 0.017$ & $5.64 \pm 0.22$ \\
\hline $5+6+7+8$ & $12.63 \pm 0.025$ & $12.93 \pm 0.066$ & $11.57 \pm 0.045$ & $12.43 \pm 0.32$ \\
\hline \hline
\end{tabular}

(a) Evaluator's Note: Reported uncertainty is statistical and does not include additional sources of uncertainty such as from delayed neutron data.

(b) Results from Detector 2 deemed unacceptable by experimenters.

(c) The reported weighted average appears to include Detector 2 measurements. 
NEA/NSC/DOC(2006)1

\section{Gas Cooled (Thermal) Reactor - GCR \\ PROTEUS-GCR-EXP-004 \\ CRIT-REAC}

Information regarding the thermal neutron detectors was unavailable.

The epithermal neutron detectors consisted of high-efficiency $\mathrm{BF}_{3}$ proportional counters shielded with $0.5 \mathrm{~mm}$ of $\mathrm{Cd}$, which suppresses $\sim 99.9 \%$ of the thermal flux $(<0.3 \mathrm{eV})$ and only $1.2 \%$ of the epithermal $(>1 \mathrm{eV})$. However, the overall efficiency of the detector is drastically reduced. A test in the thermal column of the PROTEUS demonstrated that the Cd-shielded detector had a count rate of about $0.15 \%$ of that of an unshielded one. Polyethylene was introduced ( $8 \mathrm{~mm}$ thick) to thermalize the epithermal neutrons that passed through the Cd shielding, thus increasing the efficiency by a factor of 2 (Ref. 10).

Figure 1.4-5 shows a schematic of the two types of $\mathrm{BF}_{3}$ epithermal detectors typically utilized. Some measurements were tested with an additional layer of $0.5 \mathrm{~mm}$ of indium to increase the detection threshold energy. The efficiency was reduced to the point that the detectors were useless for PNS measurements but still usable for IK measurements in Core 10 (Ref. 10).
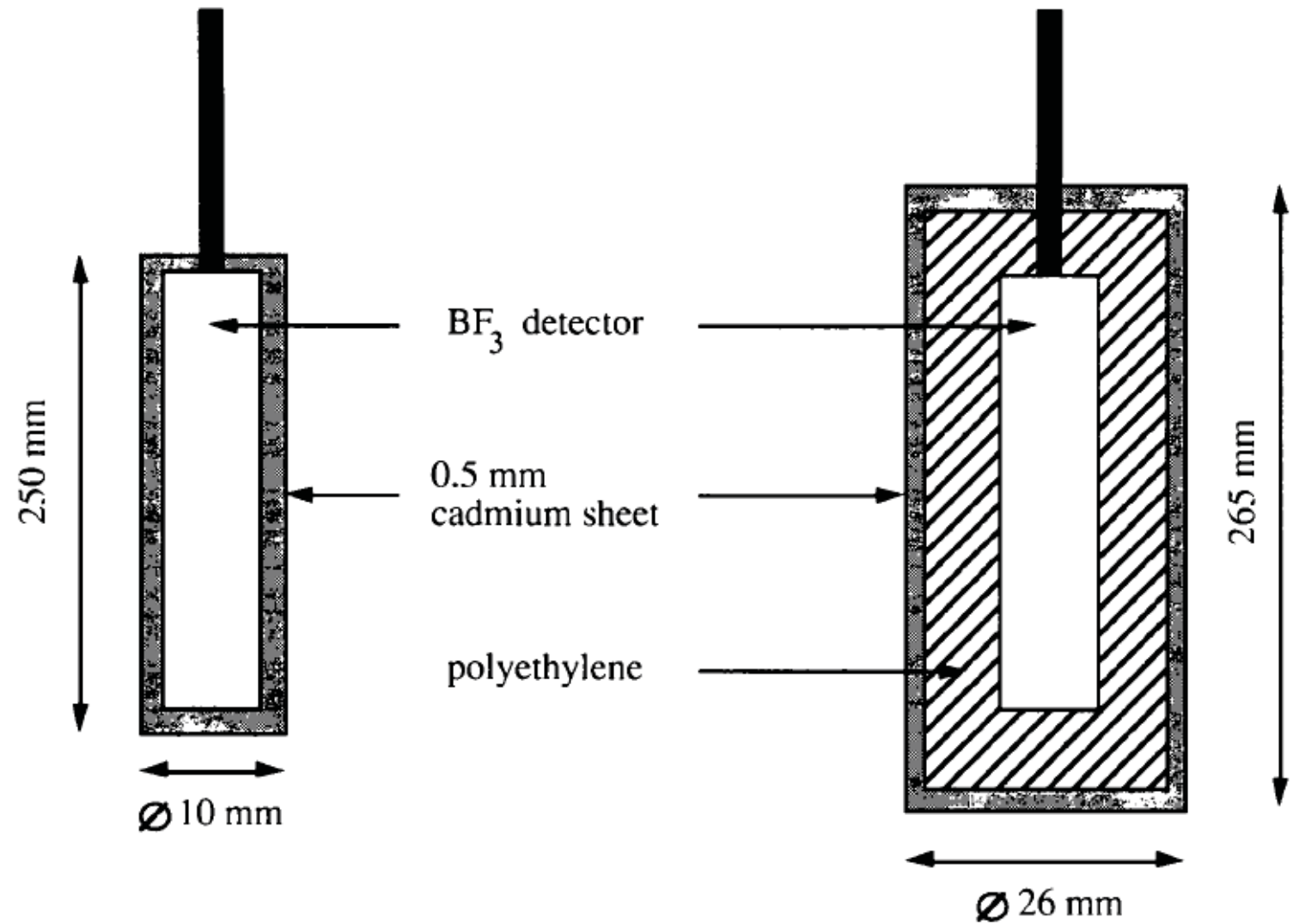

Figure 1.4-5. Schematic View of $\mathrm{BF}_{3}$ Detectors Shielded with Cd (Ref. 10). 
NEA/NSC/DOC(2006)1

\section{Gas Cooled (Thermal) Reactor - GCR \\ PROTEUS-GCR-EXP-004 \\ CRIT-REAC}

Epithermal measurements for the IK measurements were performed in three different positions, with the radial and azimuthal locations displayed in Figure 1.4-6. In Core 10, the epithermal measurements were performed with detectors shielded by cadmium and indium, as well as detectors shielded only by cadmium. The detectors containing polyethylene were not used for the IK measurements because the count rates were sufficiently high without it. As seen in Table 1.4-9, there was excellent agreement between the results obtained with the two types of shielding, which confirmed that the small difference in threshold energy did not influence the epithermal results (Ref. 10).

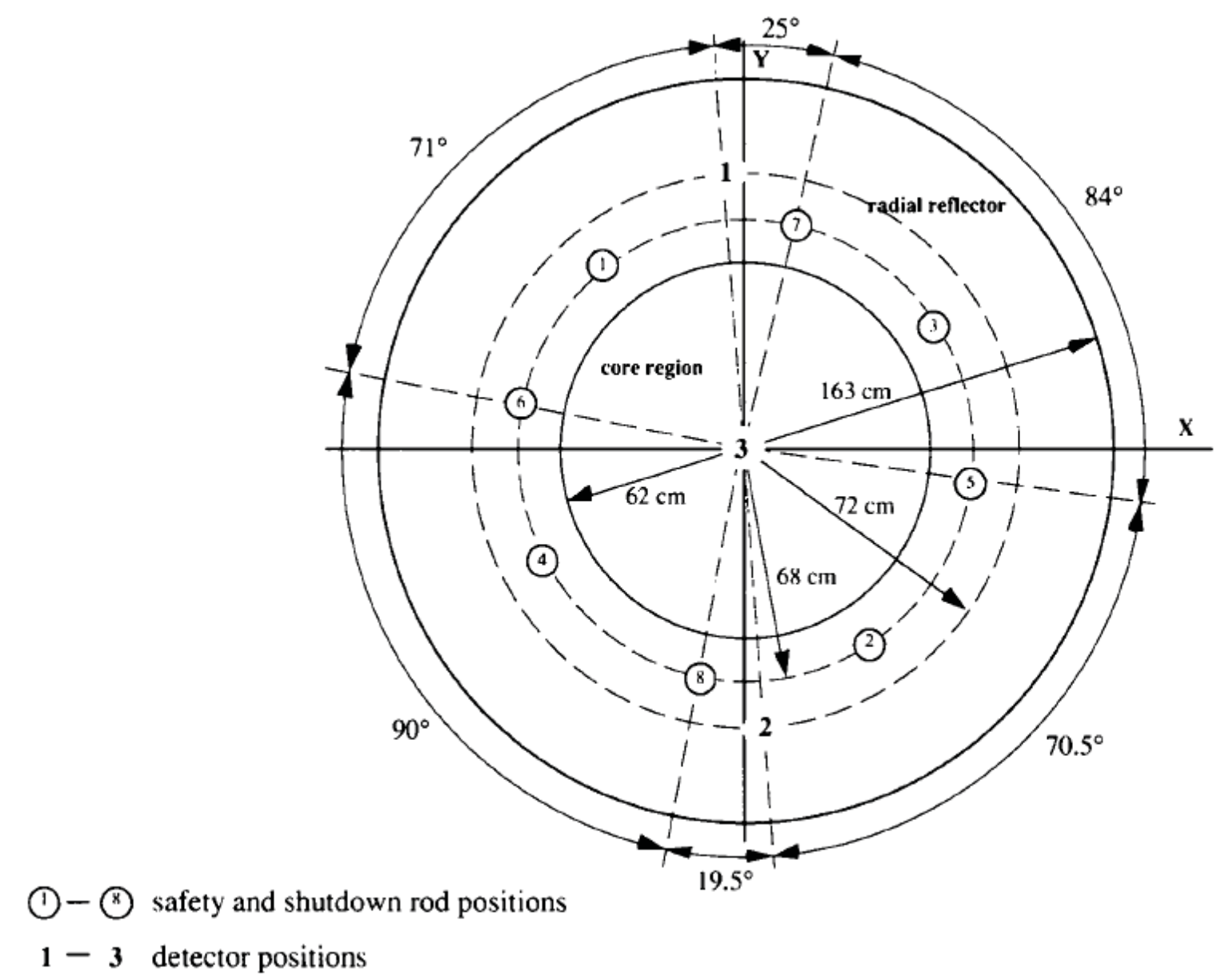

Figure 1.4-6. Horizontal Cross Section Indicating Radial and Azimuthal Locations of the Shutdown Rods and Detectors in the Various Cores (Ref. 10). 
NEA/NSC/DOC(2006)1

\section{Gas Cooled (Thermal) Reactor - GCR \\ PROTEUS-GCR-EXP-004 \\ CRIT-REAC}

The uncertainties ( $1 \sigma$ statistical errors) on the epithermal measurements are seen to be somewhat larger than those on the thermal measurements. The conventional (thermal) IK measurements utilized a special detector (and associated electronics) with a very small dead time of $0.5 \mu$ s to enable the measurement of high count rates at critical with improved statistics after the rod drop. This detector had a lower efficiency than the $\mathrm{BF}_{3}$ detectors used in the PNS measurements, resulting in poor statistics for epithermal measurements. Therefore epithermal IK measurements were performed using the same detectors as those utilized in the epithermal PNS measurements. The dead time of these detectors was $1.4 \mu \mathrm{s}$, which was the limiting factor for obtaining count rates at critical. The statistical uncertainty is thus effectively a limitation imposed by the reactor conditions and the detector used for the experiments, and not of the epithermal technique itself. Equivalent accuracies should be achievable with the epithermal and thermal techniques under appropriate operational conditions (Ref. 10).

I general schematic showing positions of various detectors and the Pulsed Neutron Source for Core 5 is shown in Figure 1.4-7. A similar diagram for Cores 9 and 10 was unavailable.

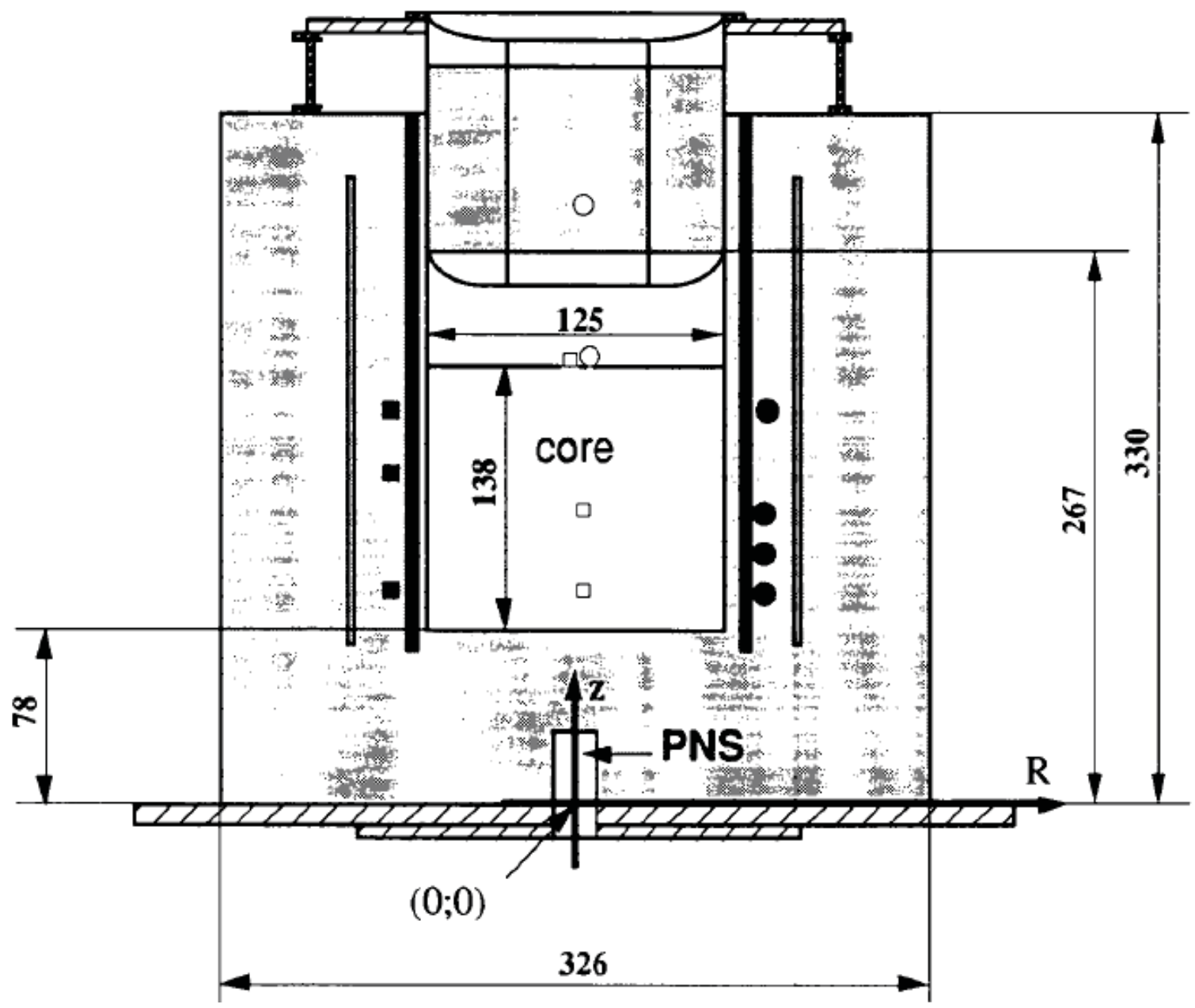

Figure 1.4-7. Schematic View Showing Positions of the Detectors and the PNS in Core 5. Circles and squares denote epithermal and thermal detector positions, respectively. Dimensions are in $\mathrm{cm}$ (Ref. 10). 
NEA/NSC/DOC(2006)1

\section{Gas Cooled (Thermal) Reactor - GCR \\ PROTEUS-GCR-EXP-004 \\ CRIT-REAC}

\subsubsection{Graphite Plug Worth Measurements}

Additional reactivity corrections were measured for the critical core loadings to account for holes and penetrations in the graphite reflectors. Worth corrections related to holes and penetrations in the graphite reflectors that can be filled with plugs for Cores 9 and 10 are in Tables 1.4-14 and 1.4-15, respectively. Where possible, the component worths had been measured directly in the relevant configurations (indicated by $\mathbf{M}$ in the tables), but in many cases the values had to be scaled from another configuration (S). The reported value of $\beta_{\text {eff }}$ is 0.00720 for each case.

The effective worths of penetrations in the graphite reflectors were effectively measured by comparing core reactivity for conditions where the holes contain graphite rods/plugs (i.e. the holes were filled) and conditions where the graphite has been removed. ${ }^{\mathrm{a}}$

Table 1.4-14. Reactivity Worths for Graphite Holes and Penetrations in Core 9 (Ref. 1 and 3).

\begin{tabular}{|c|c|c|c|c|c|c|}
\hline Reactivity Component & No. & & \multicolumn{3}{|c|}{ Total $\mathfrak{c}^{(\mathbf{a})}$} & Comments \\
\hline Control Rod Channels ${ }^{(\mathrm{b}, \mathrm{c})}$ & 4 & $\mathrm{~S}$ & -2.5 & \pm & 0.3 & Scaled from Core 5 \\
\hline Autorod Channel $^{(b)}$ & 1 & $\mathrm{~S}$ & -0.7 & \pm & 0.3 & Scaled from Core 5 \\
\hline Empty Channels R2 $2^{(\mathrm{b}, \mathrm{d})}$ & 3 & $\mathrm{~S}$ & -5 & \pm & 1 & Scaled from Core 5 \\
\hline Channels in Upper Reflector ${ }^{(\mathrm{e})}$ & 34 & & & & & No Estimate \\
\hline Channels in Lower Reflector & 0 & & & & & Channels Filled \\
\hline
\end{tabular}

(a) Evaluator's Note: Reported uncertainty is statistical and does not include additional sources of uncertainty such as from delayed neutron data.

(b) Only a few individual component worths were measured in Core 9. Therefore, in order to obtain an estimate of the reactivity excess, the component worths measured in Core 5 were scaled by the ratio of the control rod bank worths in the two cores, namely Core $9 /$ Core $5=$ $152 \phi / 134 \phi=1.14$.

(c) The worth of the new control rod channels was assumed to be the same as that of the ZEBRA rod channels in Core 1. Although the ZEBRA rod channels are somewhat larger than the new control rod channels, it is considered that the small size of the correction and its associated uncertainty justifies this approximation. The uncertainty was slightly increased.

(d) R2 indicates the second ring of the C-Driver channels.

(e) No measurement was made of this effect and no justifiable basis could be found for its estimate.

Table 1.4-15. Reactivity Worths for Graphite Holes and Penetrations in Core 10 (Ref. 1 and 3).

\begin{tabular}{|c|c|c|c|c|c|}
\hline Reactivity Corrections to Critical Loading & No. & & \multicolumn{2}{|c|}{ Total $e^{(\mathbf{a})}$} & Comments \\
\hline Control Rod Channels ${ }^{(b)}$ & 4 & $\mathrm{~S}$ & $-2.0 \pm$ & 0.2 & Core 1A Value \\
\hline Autorod Channel $^{(\mathrm{b})}$ & 1 & $\mathrm{~S}$ & $-0.5 \pm$ & 0.3 & Core 1A Value \\
\hline Empty Channels R2 ${ }^{(\mathrm{c})}$ & 3 & $\mathrm{~S}$ & -4 & 1 & Scaled from Core $1 \mathrm{~A}$ \\
\hline Channels in Upper Reflector ${ }^{(b)}$ & 34 & $\mathrm{~S}$ & $-3.6 \pm$ & 2.0 & Core 1A Value \\
\hline Channels in Lower Reflector & 0 & & & & \\
\hline
\end{tabular}

(a) Evaluator's Note: Reported uncertainty is statistical and does not include additional sources of uncertainty such as from delayed neutron data.

(b) Since the core height and control bank worths in Core 10 are similar to those in Core 1A, it was considered to be justified to use some of the component worths measured in Core 1A directly in Core 10 with a small arbitrary increase in the uncertainties.

(c) R2 indicates the second ring of the C-Driver channels.

\footnotetext{
${ }^{a}$ Williams, T., Bourguin, P, and Chawla, R. "HTR PROTEUS CORE 1: Reactivity Corrections for the Critical Balance,” TM-41-93-20, Paul Sherrer Institut, Villigen, October 7, 1993.
}

Revision: 1

Date: March 31, 2014

Page 88 of 375 
NEA/NSC/DOC(2006)1

\section{Gas Cooled (Thermal) Reactor - GCR \\ PROTEUS-GCR-EXP-004 \\ CRIT-REAC}

Reference 5 indicates a control rod channel worth of $-2.5 ф(-18 \mathrm{pcm})$ worth for Core 9 , with a reported value of $\beta_{\text {eff }}$ of $720 \mathrm{pcm}$.

The three empty channels in the radial reflector, R2, were positions 15,47 , and 63 , which were used for temperature measurements in the reflector. See Figure 1.1-3 for the location of these channels.

\subsubsection{Source/Instrumentation Worth Measurements}

Additional reactivity corrections were measured for the critical core loadings for the start-up sources, with associated penetrations, and nuclear instrumentation. No further details are available beyond their measured worth and comments regarding how the worth values were obtained. Worth corrections related to source/instrumentation measurements for Cores 9 and 10 are in Tables 1.4-16 and 1.4-17, respectively. Where possible, the component worths had been measured directly in the relevant configurations (indicated by $\mathbf{M}$ in the tables) but in many cases the values had to be scaled from another configuration (S). The reported value of $\beta_{\text {eff }}$ is 0.00720 for each case.

Table 1.4-16. Reactivity Worths for Source/Instrumentation Components of Core 9 (Ref. 1 and 3).

\begin{tabular}{|l|r|r|rrr|l||}
\hline \multicolumn{1}{|c|}{ Reactivity Component } & No. & & \multicolumn{3}{|c||}{${\text { Total } \boldsymbol{c}^{(\mathbf{a})}}$ Comments } \\
\hline \hline Start-up Sources & 2 & $\mathrm{M}$ & -4 & \pm & 1 & \\
Start-up Source Penetrations & 2 & $\mathrm{~S}$ & -1 & \pm & 0.2 & Core 5 Value \\
Nuclear Instrumentation (Ionization) & 6 & $\mathrm{~S}$ & -9.0 & \pm & 1.5 & Scaled from Core 5 \\
Nuclear Instrumentation (Fission) & 2 & $\mathrm{~S}$ & -1.0 & \pm & 0.7 & Scaled from Core 5 \\
\hline
\end{tabular}

(a) Evaluator's Note: Reported uncertainty is statistical and does not include additional sources of uncertainty such as from delayed neutron data.

Table 1.4-17. Reactivity Worths for Source/Instrumentation Components of Core 10 (Ref. 1 and 3).

\begin{tabular}{|l|r|r|rrr|c||}
\hline \multicolumn{1}{|c|}{ Reactivity Component } & No. & & \multicolumn{3}{|c||}{${\text { Total } \boldsymbol{c}^{(\mathbf{a})}}^{\text {Comments }}$} \\
\hline \hline Start-up Source Penetrations & 2 & $\mathrm{~S}$ & -1 & \pm & 0.2 & Core 1A Value \\
Nuclear Instrumentation (Ionization) & 6 & $\mathrm{~S}$ & -8.4 & \pm & 1.2 & \\
Nuclear Instrumentation (Fission) & 2 & $\mathrm{~S}$ & -0.8 & \pm & 0.6 & Core 1A Value \\
\hline \hline
\end{tabular}

(a) Evaluator's Note: Reported uncertainty is statistical and does not include additional sources of uncertainty such as from delayed neutron data.

\subsubsection{Material Data}

The materials in the core were those described in Section 1.1.3.

\subsubsection{Temperature Data}

Room (hall) temperatures for HTR-PROTEUS critical experiments, Cores 9 and 10, are provided in the following tables (core and reflector temperatures were not measured):

- Core 9 (Reference State \#1): Table 1.1-3

- Core 9 (Reference State \#2): Table 1.1-4

- Core 10 (Reference State \#1): Table 1.1-5 


\section{Gas Cooled (Thermal) Reactor - GCR \\ PROTEUS-GCR-EXP-004 \\ CRIT-REAC}

The reactor was operated at room temperature with the power limited to $1 \mathrm{~kW}$ so that no active cooling systems were required. ${ }^{\mathrm{a}}$

Environmental conditions when additional reactor physics measurements were performed would be very similar to those recorded for the critical configurations.

\subsubsection{Additional Information Relevant to Reactivity Effects Measurements}

Additional information is not available.

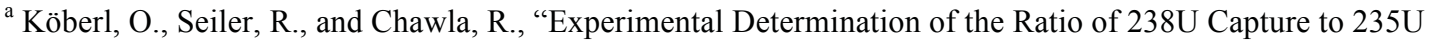
Fission in LEU-HTR Pebble-Bed Configurations," Nucl. Sci. Eng., 146, 1-12 (2004).

Revision: 1

Date: March 31, 2014 


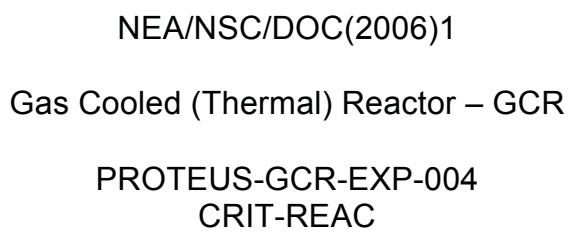

\subsection{Description of Reactivity Coefficient Measurements}

Reactivity coefficient measurements were performed but have not yet been evaluated.

\subsection{Description of Kinetics Measurements}

Kinetics measurements were performed but have not yet been evaluated.

\subsection{Description of Reaction-Rate Distribution Measurements}

Reaction-rate distribution measurements were performed but have not yet been evaluated.

\subsection{Description of Power Distribution Measurements}

Power distribution measurements were not performed.

\subsection{Description of Isotopic Measurements}

Isotopic measurements were not performed.

\subsection{Description of Other Miscellaneous Types of Measurements}

Other miscellaneous types of measurements were not performed. 
NEA/NSC/DOC(2006)1

\section{Gas Cooled (Thermal) Reactor - GCR \\ PROTEUS-GCR-EXP-004 \\ CRIT-REAC}

\subsection{EVALUATION OF EXPERIMENTAL DATA}

Two benchmark experiments were evaluated in this report: Cores 9 and 10. These core configurations represent the columnar hexagonal point-on-point (CHPOP) configurations of the HTR-PROTEUS experiment with a moderator-to-fuel pebble ratio of 1:1. Cores 9 and 10 use withdrawable, hollow, stainless steel control rods. Core 9 has 27 pebble layers; a second configuration, or state, of Core 9 that included a $28^{\text {th }}$ layer of just moderator pebbles was not evaluated as it was very similar in core design and implemented to perform core operations after initial criticality was attained. Core 10 retains the same pebble loading as Core 9, but to a height of 24 layers. It has polyethylene rods inserted between pebbles to simulate water ingress.

Monte Carlo n-Particle (MCNP) version 5-1.60 calculations were utilized to estimate the biases and uncertainties associated with the experimental results in this evaluation. MCNP is a general-purpose, continuous-energy, generalized-geometry, time-dependent, coupled n-particle Monte Carlo transport code. ${ }^{\mathrm{a}}$ The Evaluated Neutron Data File library, ENDF/B-VII.0, ${ }^{\mathrm{b}}$ nuclear data was also used in this evaluation. The statistical uncertainty in $\mathrm{k}_{\text {eff }}$ and $\Delta \mathrm{k}_{\text {eff }}$ is $\leq 0.00007$ and $\leq 0.00010$, respectively. Calculations were performed with 1,650 generations with 100,000 neutrons per generation. The $\mathrm{k}_{\text {eff }}$ estimates (with the first 150 generations skipped) are the result of 150,000,000 neutron histories.

\subsection{Evaluation of Critical and / or Subcritical Configuration Data}

The benchmark critical configurations for Cores 9 and 10 will be referred to as Cases 1 and 2, respectively. Both methods of identification are utilized throughout the rest of this report to facilitate users with differing familiarities with HTR-PROTEUS and IRPhEP benchmark format.

Variations of the benchmark model provided in Section 3 were utilized with perturbations of the model parameters to estimate uncertainties in $\mathrm{k}_{\mathrm{eff}}$ due to uncertainties in parameter values defining the benchmark experiment. Some perturbations required more detail than that retained in the benchmark model. More detailed models (Appendix C) were utilized to evaluate these uncertainties. Transformation from the detailed model to the benchmark model is described in Section 3.1.1.1. Where applicable, comparison of the upper and lower perturbation $\mathrm{k}_{\text {eff }}$ values to evaluate the uncertainty in the eigenvalue were utilized to minimize correlation effects, if any, induced by comparing all perturbations to the original benchmark model configuration, as discussed elsewhere. ${ }^{\mathrm{c}}$

Unless specifically stated otherwise, all uncertainty values in this section correspond to $1 \sigma$. When the change in $\mathrm{k}_{\text {eff }}$ between the base case and the perturbed model (single-sided perturbation), or two perturbed models (double-sided perturbation directly comparing an upper and a lower perturbation from the base case), is less than the statistical uncertainty of the Monte Carlo results, the changes in the variable are amplified, if possible, and the calculations repeated. The resulting calculated change is then scaled back, using a scaling factor, corresponding to the actual uncertainty, assuming that it is linear, which should be adequate for these changes in $\mathrm{k}_{\text {eff. }}$ Throughout Section 2, the difference in eigenvalues computed using the perturbation method described is denoted with $\Delta \mathrm{k}_{\mathrm{p}}$; the scaled $1 \sigma$ uncertainty is denoted as $\Delta \mathrm{k}_{\text {eff. }}$. All $\Delta \mathrm{k}_{\text {eff }}$ uncertainties are considered to be absolute values whose magnitude applies both positively and negatively to the experimental $\mathrm{k}_{\text {eff }}$, as shown in Tables 2.1-45 and 2.1-46. Negative signs are retained in other tables in Section 2, where the effective uncertainty is reported for a given uncertainty perturbation, to demonstrate whether the effect in $\mathrm{k}_{\text {eff }}$ was directly or indirectly proportional to the uncertainty.

\footnotetext{
a X-5 Monte Carlo Team, "MCNP - a General Monte Carlo n-Particle Transport Code, version 5," LA-UR-031987, Los Alamos National Laboratory (2003).

${ }^{b}$ M. B. Chadwick, et al., "ENDF/B-VII.0: Next Generation Evaluated Nuclear Data Library for Nuclear Science and Technology," Nucl. Data Sheets, 107: 2931-3060 (2006).

c D. Mennerdahl, "Statistical Noise for Nuclear Criticality Safety Specialists," Trans. Am. Nucl. Soc., 101: 465-466 (2009).
}

Revision: 1

Date: March 31, 2014

Page 92 of 375 
NEA/NSC/DOC(2006)1

\section{Gas Cooled (Thermal) Reactor - GCR \\ PROTEUS-GCR-EXP-004 \\ CRIT-REAC}

Evaluated uncertainties $\leq 0.00010$ are considered negligible because their calculated worth is within the statistical uncertainty of the Monte Carlo approach being utilized.

Elemental data such as molecular weights and isotopic abundances were taken from the $16^{\text {th }}$ edition of the Chart of the Nuclides. ${ }^{\text {a }}$ These values are summarized in Appendix E.

Milling and finishing of the graphite components to tight tolerances would be necessary to fit all the components of this assembly together. Small dimensional inconsistencies would result in increased void fractions between graphite components. The effect of these void fractions would be minor compared to the uncertainty in graphite density. The dimensions of some of the graphite parts used in this experiment series are often recorded with many significant digits. While the number of significant digits may not always represent the accuracy or precision of their respective measured value, it is assumed by the evaluator that an uncertainty of \pm 1 in the last reported significant digit should be adequate in evaluating the uncertainty in reported graphite dimensions. Similar discussion of tight manufacturing tolerances and the resultant small or negligible uncertainties can be found in other gas-cooled thermal reactor benchmarks (HTTR-GCR-RESR-001, -002, -003, and HTR10-GCR-RESR-001).

The total evaluated uncertainty in $\mathrm{k}_{\mathrm{eff}}$ for this experiment is provided in Section 2.1.11; individual uncertainties are summed under quadrature to obtain the total uncertainty in the experimental $\mathrm{k}_{\mathrm{eff}}$.

When evaluating parameters such as measured diameters, heights, and mass, all parts of a given type are perturbed at the same time: e.g., the uranium mass in all fuel pebbles is simultaneously increased or decreased. Then the calculated uncertainty is reduced by the square root of the number of components perturbed, representative of a random uncertainty. For many of these uncertainties, there is insufficient information available to evaluate what portion of the total evaluated uncertainty is systematic instead of random. All uncertainties involving the perturbation of multiple assembly components are treated as $15 \%$ systematic in this evaluation, unless otherwise specified.

This assumption provides a basic prediction of the effect on $\mathrm{k}_{\mathrm{eff}}$. Most systematic uncertainties should be below $50 \%$ of the total uncertainty and above the historic approach of ignoring the unknown systematic components (i.e., treat it with a $0 \%$ probability). In actuality, careful experimenters may have an unknown systematic uncertainty that is approximately $10-15 \%$ of their total reported uncertainty. Because significant effort had gone into the development of benchmark quality HTR-PROTEUS experiments, a systematic uncertainty of $15 \%$ is assumed. Evaluated uncertainties are listed as calculated, such that the readers may themselves adjust results according to some desired systematic-torandom uncertainty ratio.

The following evaluated uncertainties would have both systematic and random uncertainties (Table 2.11). Many of these uncertainties are negligible without adjusting the computed value to account for multiple assembly components (i.e., treating the uncertainty as $100 \%$ systematic is still negligible). The systematic and random components are only evaluated in more detail when the evaluated uncertainty (assuming $100 \%$ systematic) is not negligible (>0.00010).

\footnotetext{
${ }^{a}$ E. M. Baum, H. D. Knox, and T. R. Miller, Nuclides and Isotopes: 16th Edition, Knolls Atomic Power Laboratory (2002).
}

Revision: 1

Date: March 31, 2014

Page 93 of 375 
NEA/NSC/DOC(2006)1

\section{Gas Cooled (Thermal) Reactor - GCR \\ PROTEUS-GCR-EXP-004 \\ CRIT-REAC}

Table 2.1-1. Summary of Uncertainties with Systematic and Random Components.

\begin{tabular}{|c|c|}
\hline 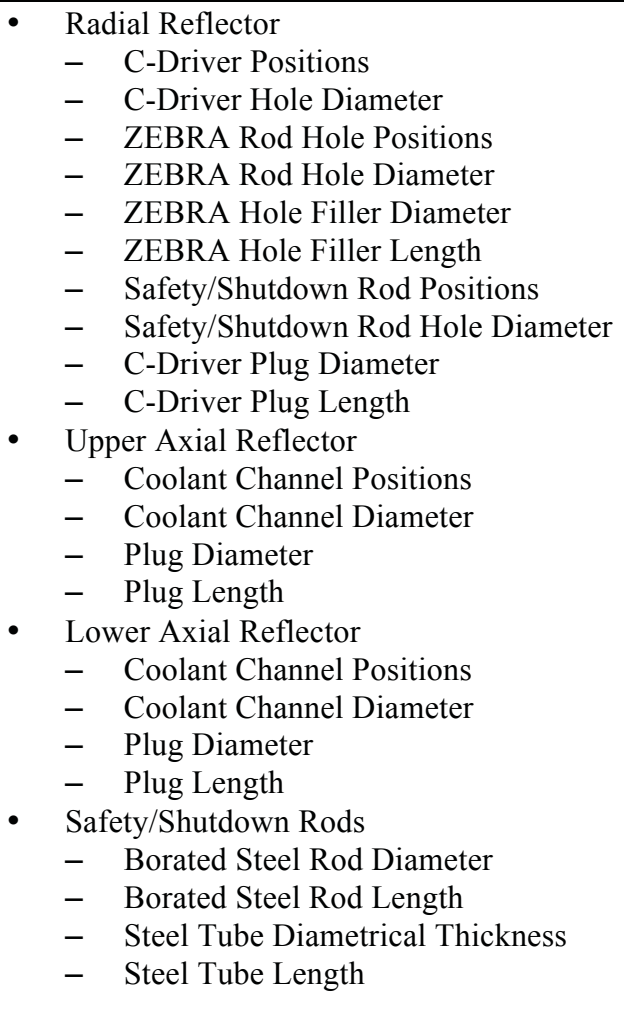 & 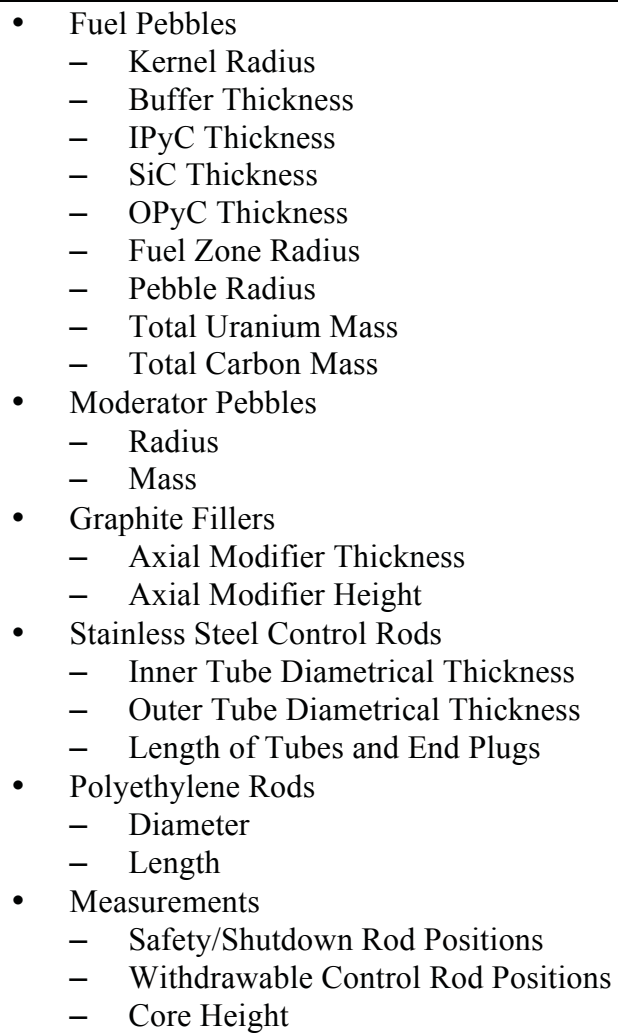 \\
\hline
\end{tabular}

\subsubsection{Streamlining the Uncertainty Analysis}

A comprehensive uncertainty analysis was performed for the initial HTR-PROTEUS configurations, Cores 1, 1A, 2, and 3 (PROTEUS-GCR-EXP-001). The evaluated uncertainty for many of the perturbed parameters were determined to be negligible $(\leq 0.00010 \Delta \mathrm{k})$, resulting in a much shorter list of uncertainties actually contributing to the total uncertainty (see Section 2.1.22 of

PROTEUS-GCR-EXP-001). A summary of negligible uncertainties pertinent to the current benchmark configurations is provided in Table 2.1-2; these uncertainties were not evaluated as their contribution to the total uncertainty in the benchmark configurations is judged to be negligible. Table 2.1-3 contains a list of uncertainties that are individually evaluated in this report. Uncertainties relating to the ZEBRA control rods and associated holes were not evaluated as they were only pertinent in Core 1. Uncertainties in the polyethylene rod diameter, length, and impurity content were included in this analysis because Core 10 contains significantly more polyethylene rods than Core 3. 
NEA/NSC/DOC(2006)1

\section{Gas Cooled (Thermal) Reactor - GCR \\ PROTEUS-GCR-EXP-004 \\ CRIT-REAC}

Table 2.1-2. Summary of Negligible Uncertainties Not Evaluated for Cores 9 and 10.

\begin{tabular}{|c|c|}
\hline 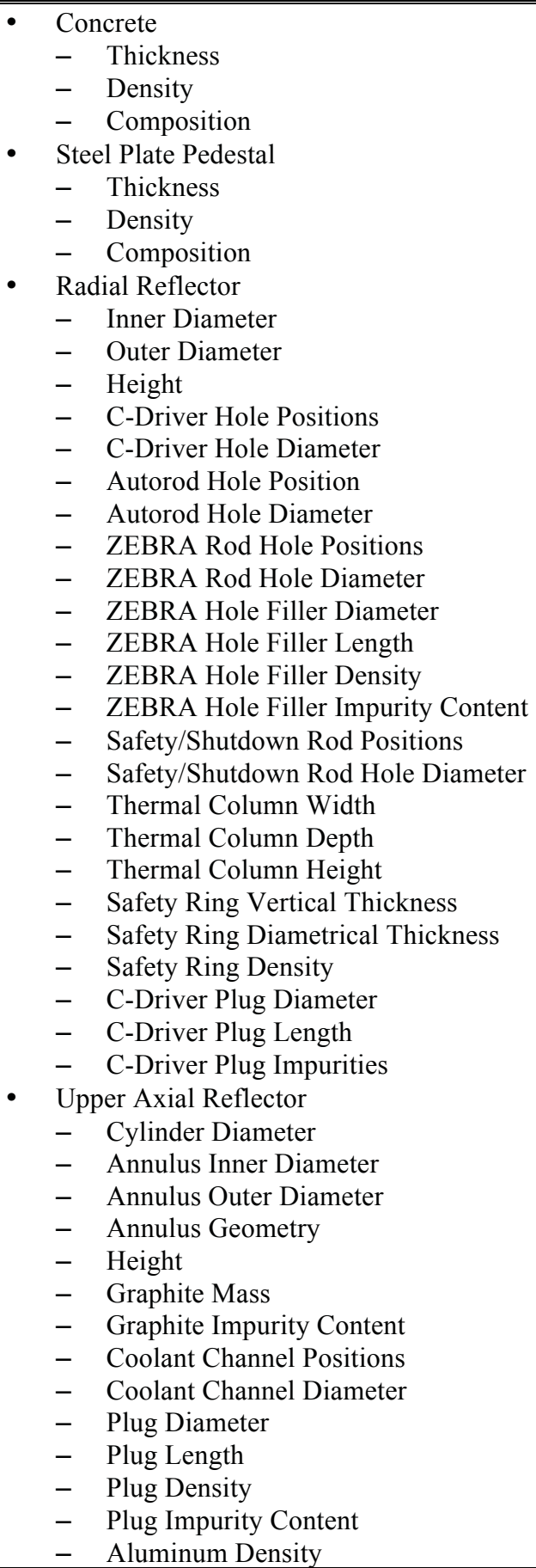 & 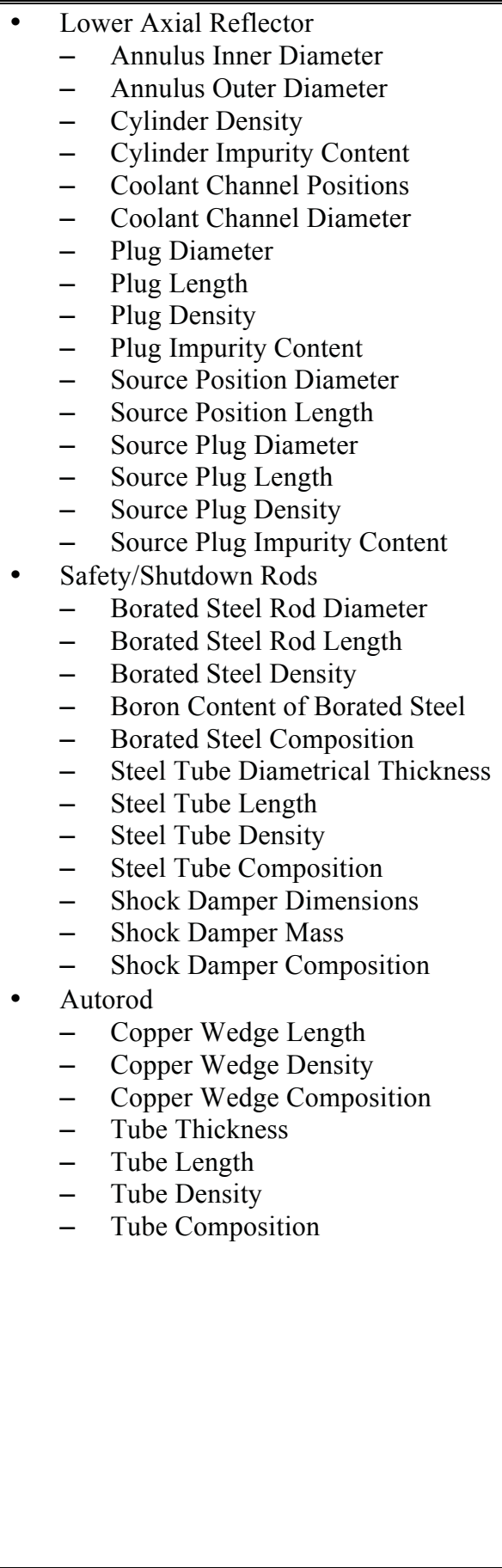 \\
\hline
\end{tabular}


NEA/NSC/DOC(2006)1

\section{Gas Cooled (Thermal) Reactor - GCR \\ PROTEUS-GCR-EXP-004 \\ CRIT-REAC}

Table 2.1-2. (cont'd.). Summary of Negligible Uncertainties Not Evaluated for Cores 9 and 10.

\begin{tabular}{|c|c|}
\hline \multicolumn{2}{|c|}{ Fuel Pebbles } \\
\hline- & Quantity of Pebbles \\
\hline- & Pebble Packing Fraction \\
\hline- & Pebble Random Packing \\
\hline- & Buffer Thickness \\
\hline - & IPyC Thickness \\
\hline- & SiC Thickness \\
\hline - & OPyC Thickness \\
\hline - & Fuel Zone Radius \\
\hline- & Pebble Radius \\
\hline & ${ }^{236}$ U Isotopic Content \\
\hline & ${ }^{238} \mathrm{U}$ Isotopic Content \\
\hline- & Total Carbon Mass \\
\hline & Total Pebble Mass \\
\hline & Kernel Density \\
\hline & Buffer Density \\
\hline & IPyC Density \\
\hline & SiC Density \\
\hline & OPyC Density \\
\hline & Kernel Impurity Content \\
\hline & Buffer Impurity Content \\
\hline & IPyC Impurity Content \\
\hline & SiC Impurity Content \\
\hline & OPyC Impurity Content \\
\hline
\end{tabular}

- $\quad$ Moderator Pebbles

- Quantity of Pebbles

- Radius

- Graphite Fillers

- Axial Modifier Thickness

- Axial Modifier Height

- Axial Modifier Mass

- Stainless Steel Control Rods

- Inner Tube Diametrical Thickness

- Outer Tube Diametrical Thickness

- Length of Tubes and End Plugs

- Inner Tube Density

- Outer Tube Density

- Inner Tube Composition

- Measurements

- Measurement of $\mathrm{k}_{\text {eff }}$

- Autorod Position

- Safety/Shutdown Rod Positions

- Withdrawable Control Rod Positions

- Temperature

- Ambient Air

- Temperature

- Pressure

- Humidity

- Isotopic Abundance of Boron

Revision: 1 
NEA/NSC/DOC(2006)1

\section{Gas Cooled (Thermal) Reactor - GCR \\ PROTEUS-GCR-EXP-004 \\ CRIT-REAC}

Table 2.1-3. Summary of Uncertainties Evaluated for Cores 9 and 10.

\begin{tabular}{|c|c|}
\hline 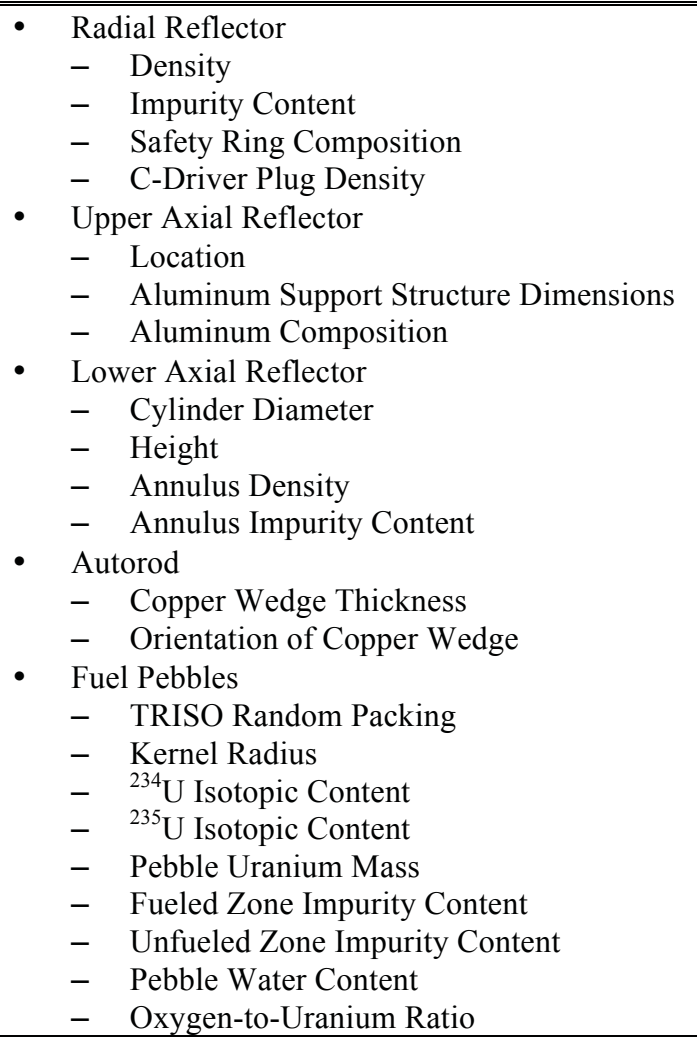 & $\begin{array}{ll}\text { - } & \text { Moderator Pebbles } \\
- & \text { Mass } \\
- & \text { Impurity Content } \\
- & \text { Water Content } \\
\text { - Stainless Steel Control Rods } \\
-\quad \text { Outer Tube Composition } \\
\text { - Polyethylene Rods } \\
-\quad \text { Diameter } \\
-\quad \text { Length } \\
- \text { Density } \\
- \text { H:C Ratio } \\
- \text { Impurity Content } \\
\text { Measurements } \\
-\quad \text { Stacked Pebble Height }\end{array}$ \\
\hline
\end{tabular}

\subsubsection{Radial Reflector}

\subsubsection{Graphite Density}

The graphite for the majority of the system, which includes much of the radial reflector and thermal column, was reported to have a density of $1.76 \pm 0.01 \mathrm{~g} / \mathrm{cm}^{3}$ (Table 1.1-6), obtained from reactor-based measurements. Measurement of 28 graphite samples resulted in an apparent average density of $1.763 \pm$ $0.012 \mathrm{~g} / \mathrm{cm}^{3}$. A value of $1.76 \pm 0.012 \mathrm{~g} / \mathrm{cm}^{3}(1 \sigma)$ was selected to represent the graphite utilized in the radial reflector and thermal column, using the reported average density from the construction of the assembly and the larger uncertainty obtained from apparent density measurements. All graphite (excluding pebbles) used in the HTR-PROTEUS experiments are assumed to have the same density uncertainty unless otherwise specified.

The density of the radial reflector surrounding the core, and the thermal column, was $1.76 \mathrm{~g} / \mathrm{cm}^{3}$. The uncertainty in the density was $0.012 \mathrm{~g} / \mathrm{cm}^{3}(1 \sigma)$. A double-sided perturbation was performed in which the density was perturbed by $\pm 0.036 \mathrm{~g} / \mathrm{cm}^{3}$ to estimate the uncertainty in $\mathrm{k}_{\text {eff }}$ due to the uncertainty in the density of the radial reflector. Half of the differences between the calculated upper and lower perturbed values were then scaled to obtain the $1 \sigma$ uncertainty. Results are shown in Table 2.1-4. 
NEA/NSC/DOC(2006)1

\section{Gas Cooled (Thermal) Reactor - GCR \\ PROTEUS-GCR-EXP-004 \\ CRIT-REAC}

Table 2.1-4. Effect of Uncertainty in Graphite Density.

\begin{tabular}{||c|c|ccc|c|ccc||}
\hline $\begin{array}{c}\text { Case } \\
\text { (Core) }\end{array}$ & Deviation & $\Delta \mathbf{k}_{\mathbf{p}}$ & \pm & $\boldsymbol{\sigma}_{\text {akp }}$ & $\begin{array}{c}\text { Scaling } \\
\text { Factor }\end{array}$ & $\boldsymbol{\Delta} \mathbf{k}_{\text {eff }}(\mathbf{1 \sigma})$ & \pm & $\boldsymbol{\sigma}_{\text {akeff }}$ \\
\hline \hline $1(9)$ & $\pm 0.036 \mathrm{~g} / \mathrm{cm}^{3}$ & 0.00306 & \pm & 0.00005 & 3 & 0.00102 & \pm & 0.00002 \\
\hline $2(10)$ & $\pm 0.036 \mathrm{~g} / \mathrm{cm}^{3}$ & 0.00244 & \pm & 0.00004 & 3 & 0.00081 & \pm & 0.00001 \\
\hline
\end{tabular}

\subsubsection{Graphite Impurities}

Various values were reported for the nominal absorption cross section or boron content for the graphite material used in the core (Table 1.1-6). Subtraction of the absorption cross section of graphite $(\sim 3.5 \mathrm{mbarn} / \mathrm{atom})$ allows for estimation of the equivalent boron content (EBC) using nominal boron data $\left(3,840,000\right.$ mbarn/atom ${ }^{10} \mathrm{~B}, 19.9 \%{ }^{10} \mathrm{~B}$ in $\left.\mathrm{B}_{\text {nat }}\right){ }^{\text {a }}{ }^{\mathrm{a}}$ These values, however, are low since they do not account for the water or air content absorbed into the graphite. Table 1.1-7 with its accompanying text provides some insight into the evaluated water content. Pulsed neutron source measurements were performed to obtain global impurity measurements for the entire core that included moisture content and intergranular nitrogen from the air. These measurements were performed in the empty PROTEUS graphite reflectors and were initially evaluated using diffusion theory. ${ }^{\mathrm{b}}$ Later Monte Carlo methods were used to evaluate the measured data to provide a nominal ${ }^{10} \mathrm{~B}$ concentration of $2.69 \pm 0.16$ (assumed units of mbarn/atom), which corresponds to 0.2696 and $0.2591 \mathrm{ppma}$ in the radial and axial reflectors, respectively. ${ }^{\mathrm{c}}$ The average EBC is $1.33 \mathrm{ppm}$ (by at.\%). The uncertainty in the initial reported concentration ( $\pm 0.16 \mathrm{mbarn} /$ atom $)$ is propagated to obtain an uncertainty in the $\mathrm{EBC}$ of \pm 0.08 ppma $(1 \sigma)$. All graphite (excluding pebbles) used in the HTR-PROTEUS experiments are assumed to have the same impurity content and uncertainty unless otherwise specified.

The impurity content of the radial reflector surrounding the core and the thermal column was $1.33 \mathrm{ppm}$ (EBC by atom percent). The uncertainty in the impurity content was 0.08 ppma $(1 \sigma)$. A double-sided perturbation was performed in which the impurity content was perturbed by \pm 0.24 ppma to estimate the uncertainty in $\mathrm{k}_{\text {eff }}$ due to the uncertainty in the impurity content of the radial reflector. Half of the differences between the calculated upper and lower perturbed values were then scaled to obtain the $1 \sigma$ uncertainty. Results are shown in Table 2.1-5.

Table 2.1-5. Effect of Uncertainty in Graphite Impurity Content.

\begin{tabular}{||c|c|ccc|c|ccc||}
\hline \hline $\begin{array}{c}\text { Case } \\
\text { (Core) }\end{array}$ & Deviation & $\mathbf{\Delta k}_{\mathbf{p}}$ & \pm & $\boldsymbol{\sigma}_{\text {skp }}$ & $\begin{array}{c}\text { Scaling } \\
\text { Factor }\end{array}$ & $\Delta_{\mathbf{k}_{\text {eff }}}(\mathbf{1} \boldsymbol{\sigma})$ & \pm & $\boldsymbol{\sigma}_{\text {skeff }}$ \\
\hline \hline $1(9)$ & $\pm 0.24 \mathrm{ppma}$ & -0.00340 & \pm & 0.00005 & 3 & -0.00113 & \pm & 0.00002 \\
\hline $2(10)$ & $\pm 0.24 \mathrm{ppma}$ & -0.00240 & \pm & 0.00004 & 3 & -0.00080 & \pm & 0.00001 \\
\hline \hline
\end{tabular}

\footnotetext{
${ }^{a}$ E. M. Baum, H. D. Knox, and T. R. Miller, Nuclides and Isotopes: 16th Edition, Knolls Atomic Power Laboratory (2002).

${ }^{b}$ Williams, T., Mathews, D., and Yamane, T., "Measurement of the Absorption Properties of the HTR-PROTEUS Reflector Graphite by Means of a Pulsed-Neutron Technique," TM-41-93-34, Paul Scherrer Institut, Villigen, October 3, 1995.

${ }^{c}$ Difilippo, F. C., “Applications of Monte Carlo Simulations of Thermalization Processes to the Nondestructive Assay of Graphite,” Nucl. Sci. Eng., 133, 163-177 (1999).
}

Revision: 1

Date: March 31, 2014

Page 98 of 375 
NEA/NSC/DOC(2006)1

\section{Gas Cooled (Thermal) Reactor - GCR \\ PROTEUS-GCR-EXP-004 \\ CRIT-REAC}

\subsubsection{Safety Ring Composition}

The composition specifications for Peraluman-300 is provided in Table 1.1-8. The composition values listed as less than a given value are taken at half this maximum value in the nominal material composition. The aluminum content is adjusted such that the total composition adds up to $100 \%$. The nominal composition used for evaluation of the uncertainty in the composition of the safety ring is in Table 2.1-6.

A double-sided perturbation was performed in which the plate composition was perturbed by minimizing and maximizing the aluminum content in the Peraluman, while simultaneously maximizing or minimizing the other elemental constituents within the specified limits, to estimate the uncertainty in $\mathrm{k}_{\text {eff }}$ due to the uncertainty in the safety ring composition. Half of the differences between the calculated upper and lower perturbed values were then scaled to obtain the $1 \sigma$ uncertainty assuming a bounding limit with uniform probability distribution. Results are shown in Table 2.1-7.

Table 2.1-6. Composition of the Peraluman-300.

\begin{tabular}{|c|c|c|c|c||}
\hline \hline Element & Minimum wt.\% & Maximum wt.\% & Nominal wt.\% & Nominal Atoms/barn-cm \\
\hline \hline $\mathrm{B}$ & -- & 0.001 & 0.0005 & $7.3807 \mathrm{E}-07$ \\
\hline $\mathrm{Mg}$ & -- & 3.1 & 1.55 & $1.0177 \mathrm{E}-03$ \\
\hline $\mathrm{Al}$ & \multicolumn{2}{|c|}{ Balance } & 97.344 & $5.7575 \mathrm{E}-02$ \\
\hline $\mathrm{Si}$ & 0.4 & 0.4 & 0.4 & $2.2729 \mathrm{E}-04$ \\
\hline $\mathrm{Mn}$ & -- & 0.5 & 0.25 & $7.2621 \mathrm{E}-05$ \\
\hline $\mathrm{Fe}$ & 0.3 & 0.3 & 0.3 & $8.5730 \mathrm{E}-05$ \\
\hline $\mathrm{Cu}$ & 0.05 & 0.05 & 0.05 & $1.2557 \mathrm{E}-05$ \\
\hline $\mathrm{Zn}$ & 0.1 & 0.1 & 0.1 & $2.4398 \mathrm{E}-05$ \\
\hline $\mathrm{Ga}$ & -- & 0.01 & 0.005 & $1.1444 \mathrm{E}-06$ \\
\hline $\mathrm{Cd}$ & -- & 0.001 & 0.0005 & $7.0983 \mathrm{E}-08$ \\
\hline \hline $\mathrm{Total}$ & -- & -- & 100 & $5.9018 \mathrm{E}-02$ \\
\hline \hline
\end{tabular}

Table 2.1-7. Effect of Uncertainty in Safety Ring Composition.

\begin{tabular}{||l|c|ccc|c|ccc||}
\hline $\begin{array}{c}\text { Case } \\
\text { (Core) }\end{array}$ & Deviation & $\boldsymbol{\Delta}_{\mathbf{p}}$ & \pm & $\boldsymbol{\sigma}_{\text {skp }}$ & $\begin{array}{c}\text { Scaling } \\
\text { Factor }\end{array}$ & $\mathbf{\Delta}_{\text {eff }}(\mathbf{1} \boldsymbol{\sigma})$ & \pm & $\boldsymbol{\sigma}_{\text {skeff }}$ \\
\hline \hline $1(9)$ & Min/Max Al & 0.00021 & \pm & 0.00005 & $\sqrt{ } 3$ & 0.00012 & \pm & 0.00003 \\
\hline $2(10)$ & Min/Max Al & 0.00004 & \pm & 0.00004 & $\sqrt{ } 3$ & 0.00002 & \pm & 0.00002 \\
\hline \hline
\end{tabular}

\subsubsection{C-Driver Plug Density}

The C-Driver plugs were reported in Table 1.1-6 to have a density of $1.75 \pm 0.007 \mathrm{~g} / \mathrm{cm}^{3}$ in $\sim 50 \%$ of the channels (inner rings) and $1.78 \mathrm{~g} / \mathrm{cm}^{3}$ in $\sim 50 \%$ of the channels (outer rings). There is no clear designation how a 50:50 split is managed for an odd number of rings. Therefore an average density of $1.765 \mathrm{~g} / \mathrm{cm}^{3}$ is used for all $311 \mathrm{C}$-Driver plugs. A total of 308 plugs were reported as being placed in the reflector since some were removed for core instrumentation; however, the exact location of the removed plugs is unknown. Additional uncertainty due to the evaluation of the density of three additional plugs is assumed to be negligible. It is assumed that the measured density uncertainty of $0.012 \mathrm{~g} / \mathrm{cm}^{3}(1 \sigma)$, discussed in Section 2.1.2.1, sufficiently encompasses the uncertainty in the new graphite density. The reported density range of 1.75 to $1.78 \mathrm{~g} / \mathrm{cm}^{3}$ for the new graphite is reported to be consistent with the old graphite. This range is encompassed by the $3 \sigma$ uncertainty.

Revision: 1 
NEA/NSC/DOC(2006)1

\section{Gas Cooled (Thermal) Reactor - GCR \\ PROTEUS-GCR-EXP-004 \\ CRIT-REAC}

The density of the C-Driver plugs within the radial reflector surrounding the core was $1.765 \mathrm{~g} / \mathrm{cm}^{3}$. The uncertainty in the density was $0.012 \mathrm{~g} / \mathrm{cm}^{3}(1 \sigma)$. A double-sided perturbation was performed in which the density was perturbed by $\pm 0.036 \mathrm{~g} / \mathrm{cm}^{3}$ to estimate the uncertainty in $k_{\text {eff }}$ due to the uncertainty in the density of the C-Driver plugs. Half of the differences between the calculated upper and lower perturbed values were then scaled to obtain the $1 \sigma$ uncertainty. Results are shown in Table 2.1-8. The calculated uncertainty is negligible $(\leq 0.00010)$.

Table 2.1-8. Effect of Uncertainty in C-Driver Plug Density.

\begin{tabular}{||l|c|ccc|c|ccc||}
\hline $\begin{array}{l}\text { Case } \\
\text { (Core) }\end{array}$ & Deviation & $\boldsymbol{\Delta} \mathbf{k}_{\mathbf{p}}$ & \pm & $\boldsymbol{\sigma}_{\text {skp }}$ & $\begin{array}{c}\text { Scaling } \\
\text { Factor }\end{array}$ & $\Delta \mathbf{k}_{\text {eff }}(\mathbf{1 \sigma})$ & \pm & $\boldsymbol{\sigma}_{\text {skeff }}$ \\
\hline \hline $1(9)$ & $\pm 0.036 \mathrm{~g} / \mathrm{cm}^{3}$ & 0.00028 & \pm & 0.00005 & 3 & 0.00009 & \pm & 0.00002 \\
\hline $2(10)$ & $\pm 0.036 \mathrm{~g} / \mathrm{cm}^{3}$ & 0.00023 & \pm & 0.00004 & 3 & 0.00008 & \pm & 0.00001 \\
\hline
\end{tabular}

\subsubsection{Upper Axial Reflector}

\subsubsection{Location above Core}

The bottom surface of the graphite in the upper axial reflector is located $1893 \mathrm{~mm}$ above the top surface of the lower axial reflector, creating a core cavity with a height of $1893 \mathrm{~mm}$. This value is obtained by calculating the difference between reported heights in Figure 1.1-1. Elsewhere it has been reported that this height is $1863 \mathrm{~mm} .{ }^{a}$ It is believed that this latter value was reported incorrectly. The suspended position of the upper axial reflector was measured to within 3 to $5 \mathrm{~mm}$.

The location of the upper axial reflector above the inside bottom of the core cavity is $1893 \mathrm{~mm}$. The uncertainty in this location was assumed to be $5 \mathrm{~mm}$ (bounding limit with uniform probability distribution). A double-sided perturbation was performed in which the location was perturbed by $\pm 15 \mathrm{~mm}$ to estimate the uncertainty in $\mathrm{k}_{\text {eff }}$ due to the uncertainty in the location of the upper axial reflector. Half of the differences between the calculated upper and lower perturbed values were then scaled to obtain the $1 \sigma$ uncertainty. Results are shown in Table 2.1-9.

Table 2.1-9. Effect of Uncertainty in the Location of the Upper Axial Reflector.

\begin{tabular}{||c|c|ccc|c|ccc||}
\hline $\begin{array}{c}\text { Case } \\
\text { (Core) }\end{array}$ & Deviation & $\boldsymbol{\Delta}_{\mathbf{p}}$ & \pm & $\boldsymbol{\sigma}_{\mathbf{s k}}$ & $\begin{array}{c}\text { Scaling } \\
\text { Factor }\end{array}$ & $\mathbf{\Delta} \mathbf{k}_{\text {eff }}(\mathbf{1 \sigma})$ & \pm & $\boldsymbol{\sigma}_{\Delta \mathbf{k e f f}}$ \\
\hline \hline $1(9)$ & $\pm 15 \mathrm{~mm}$ & -0.00078 & \pm & 0.00005 & $3 \sqrt{ } 3$ & -0.00015 & \pm & 0.00001 \\
\hline $2(10)$ & $\pm 15 \mathrm{~mm}$ & -0.00040 & \pm & 0.00004 & $3 \sqrt{ } 3$ & -0.00008 & \pm & 0.00001 \\
\hline \hline
\end{tabular}

\footnotetext{
a Difilippo, F. C., "Monte Carlo Calculations of Pebble Bed Benchmark Configurations of the PROTEUS Facility," Nucl. Sci. Eng., 143, 240-253 (2003).

${ }^{\mathrm{b}}$ Personal communication with Oliver Köberl at PSI (October 26, 2011).

Revision: 1

Date: March 31, 2014

Page 100 of 375
} 
NEA/NSC/DOC(2006)1

\author{
Gas Cooled (Thermal) Reactor - GCR \\ PROTEUS-GCR-EXP-004 \\ CRIT-REAC
}

\title{
2.1.3.2 Aluminum Dimensions
}

A detailed model was prepared (see Appendix C) where the aluminum support structure for the upper axial reflector (see Figures 1.1-4 and 1.1-6) was included with the geometry and dimensions modeled as identical as possible to those provided in the figures. Components of the aluminum support structure were included below the upper surface of the upper axial reflector. Uncertainty in the exact geometry is assumed to be negligible since the effective bias for compacting the curved surface below the graphite components of the reflector was negligible (see Section 3.1.1.1). An uncertainty was assumed of $1 \mathrm{~mm}$ in the thickness of all aluminum sheet material used to manufacture the structural support for the upper axial reflector. Due to the difficulty in exactly modeling the dimensions of all aluminum components, this uncertainty is treated as systematic and total aluminum mass was not conserved.

The uncertainty in dimensions of the aluminum support structure was assumed to be $1 \mathrm{~mm}$ (bounding limit with uniform probability distribution). A single-sided perturbation was performed in which all thicknesses were simultaneously decreased by $2 \mathrm{~mm}$ (material replaced by void) to estimate the uncertainty in $\mathrm{k}_{\mathrm{eff}}$ due to the uncertainty in the dimensions of the aluminum support structure. The calculated results were then scaled to obtain the $1 \sigma$ uncertainty. The total mass of the aluminum was not conserved. Results are shown in Table 2.1-10.

Table 2.1-10. Effect of Uncertainty in Aluminum Dimensions.

\begin{tabular}{||c|c|ccc|c|ccc||}
\hline $\begin{array}{c}\text { Case } \\
\text { (Core) }\end{array}$ & Deviation & $\boldsymbol{\Delta} \mathbf{k}_{\mathbf{p}}$ & \pm & $\boldsymbol{\sigma}_{\text {skp }}$ & $\begin{array}{c}\text { Scaling } \\
\text { Factor }\end{array}$ & $\boldsymbol{\Delta}_{\text {eff }}(\mathbf{1} \boldsymbol{\sigma})$ & \pm & $\boldsymbol{\sigma}_{\text {skeff }}$ \\
\hline \hline $1(9)$ & $-2 \mathrm{~mm}$ & -0.00250 & \pm & 0.00010 & $2 \sqrt{ } 3$ & -0.00072 & \pm & 0.00003 \\
\hline $2(10)$ & $-2 \mathrm{~mm}$ & -0.00159 & \pm & 0.00008 & $2 \sqrt{ } 3$ & -0.00046 & \pm & 0.00002 \\
\hline
\end{tabular}

\subsubsection{Aluminum Composition}

The composition specifications for Peraluman-300 is provided in Table 1.1-8. The composition values listed as less than a given value are taken at half this maximum value in the nominal material composition. The aluminum content is adjusted such that the total composition adds up to $100 \%$. The nominal composition used for evaluation of the uncertainty in the composition of the safety ring is in Table 2.1-6.

A double-sided perturbation was performed in which the plate composition was perturbed by minimizing and maximizing the aluminum content in the Peraluman, while simultaneously maximizing or minimizing the other elemental constituents within the specified limits, to estimate the uncertainty in $\mathrm{k}_{\text {eff }}$ due to the uncertainty in the composition of the aluminum support structure for the upper axial reflector. Half of the differences between the calculated upper and lower perturbed values were then scaled to obtain the $1 \sigma$ uncertainty assuming a bounding limit with uniform probability distribution. Results are shown in Table 2.1-11.

Table 2.1-11. Effect of Uncertainty in Support Structure Composition.

\begin{tabular}{||l|c|ccc|c|ccc||}
\hline $\begin{array}{c}\text { Case } \\
\text { (Core) }\end{array}$ & Deviation & $\boldsymbol{\Delta} \mathbf{k}_{\mathbf{p}}$ & \pm & $\boldsymbol{\sigma}_{\text {skp }}$ & $\begin{array}{c}\text { Scaling } \\
\text { Factor }\end{array}$ & $\mathbf{\Delta k}_{\text {eff }}(\mathbf{1} \boldsymbol{\sigma})$ & \pm & $\boldsymbol{\sigma}_{\Delta \mathbf{k e f f}}$ \\
\hline \hline $1(9)$ & Min/Max Al & 0.00067 & \pm & 0.00005 & $\sqrt{ } 3$ & 0.00039 & \pm & 0.00003 \\
\hline $2(10)$ & Min/Max Al & 0.00042 & \pm & 0.00004 & $\sqrt{ } 3$ & 0.00024 & \pm & 0.00002 \\
\hline \hline
\end{tabular}

Revision: 1 
NEA/NSC/DOC(2006)1

\section{Gas Cooled (Thermal) Reactor - GCR \\ PROTEUS-GCR-EXP-004 \\ CRIT-REAC}

\subsubsection{Lower Axial Reflector}

\subsubsection{Central Cylinder Diameter}

The diameter of the central graphite cylinder of the lower axial reflector was $495 \mathrm{~mm}$. The uncertainty in the diameter was assumed to be $1 \mathrm{~mm}$ (bounding limit with uniform probability distribution). A onesided perturbation was performed in which the diameter was perturbed by $-2 \mathrm{~mm}$ to estimate the uncertainty in $\mathrm{k}_{\mathrm{eff}}$ due to the uncertainty in diameter of the graphite cylinder. The calculated results were then scaled to obtain the $1 \sigma$ uncertainty. Results are shown in Table 2.1-12. The calculated uncertainty is negligible $(\leq 0.00010)$.

Table 2.1-12. Effect of Uncertainty in Central Cylinder Diameter.

\begin{tabular}{|c|c|c|c|c|c|c|c|c|}
\hline $\begin{array}{c}\text { Case } \\
\text { (Core) } \\
\end{array}$ & Deviation & $\Delta \mathbf{k}_{\mathrm{p}}$ & \pm & $\sigma_{\Delta \mathrm{kp}}$ & $\begin{array}{l}\text { Scaling } \\
\text { Factor } \\
\end{array}$ & $\Delta \mathbf{k}_{\mathrm{eff}}(1 \sigma)$ & \pm & $\sigma_{\text {skeff }}$ \\
\hline $1(9)$ & $-2 \mathrm{~mm}$ & 0.00017 & \pm & 0.00010 & $2 \sqrt{3}$ & 0.00005 & \pm & 0.00003 \\
\hline $2(10)$ & $-2 \mathrm{~mm}$ & -0.00005 & \pm & 0.00008 & $2 \sqrt{3}$ & -0.00001 & \pm & 0.00002 \\
\hline
\end{tabular}

\subsubsection{Height}

The lower axial reflector (see Figure 1.1-7) has penetrations, many of which filled with graphite plugs, that penetrate axially the full length of the graphite. All holes and associated plugs are perturbed along with the perturbation in the total height of the radial reflector. Any additional uncertainty or correlation effects are assumed to be negligible.

The height of the lower axial reflector was $780 \mathrm{~mm}$. The uncertainty in the height was assumed to be $1 \mathrm{~mm}$ (bounding limit with uniform probability distribution). A double-sided perturbation was performed in which the height was perturbed by $\pm 3 \mathrm{~mm}$ to estimate the uncertainty in $\mathrm{k}_{\text {eff }}$ due to the uncertainty in the height of the lower axial reflector. Half of the differences between the calculated upper and lower perturbed values were then scaled to obtain the $1 \sigma$ uncertainty. Results are shown in Table 2.1-13. The calculated uncertainty is negligible $(\leq 0.00010)$.

Table 2.1-13. Effect of Uncertainty in Height.

\begin{tabular}{||l|c|ccc|c|ccc||}
\hline $\begin{array}{c}\text { Case } \\
\text { (Core) }\end{array}$ & Deviation & $\boldsymbol{\Delta} \mathbf{k}_{\mathbf{p}}$ & \pm & $\boldsymbol{\sigma}_{\text {akp }}$ & $\begin{array}{c}\text { Scaling } \\
\text { Factor }\end{array}$ & $\Delta \mathbf{k}_{\text {eff }}(\mathbf{1 \sigma})$ & \pm & $\boldsymbol{\sigma}_{\Delta \mathbf{k e f f}}$ \\
\hline \hline $1(9)$ & $\pm 3 \mathrm{~mm}$ & -0.00001 & \pm & 0.00005 & $3 \sqrt{ } 3$ & $<0.00001$ & \pm & 0.00001 \\
\hline $2(10)$ & $\pm 3 \mathrm{~mm}$ & 0.00007 & \pm & 0.00004 & $3 \sqrt{ } 3$ & 0.00001 & \pm & 0.00001 \\
\hline
\end{tabular}

\subsubsection{Annulus Density}

Old graphite was used for the annulus of the lower axial reflector (Table 1.1-6) with a reported density of $1.76 \pm 0.01 \mathrm{~g} / \mathrm{cm}^{3}$. It is assumed that the measured density uncertainty of $0.012 \mathrm{~g} / \mathrm{cm}^{3}(1 \sigma)$, discussed in Section 2.1.2.1, sufficiently describes the uncertainty in the graphite density of the lower axial reflector.

The density of the annulus of the lower axial reflector was $1.76 \mathrm{~g} / \mathrm{cm}^{3}$. The uncertainty in the density was $0.012 \mathrm{~g} / \mathrm{cm}^{3}(1 \sigma)$. A double-sided perturbation was performed in which the density was perturbed by $\pm 0.036 \mathrm{~g} / \mathrm{cm}^{3}$ to estimate the uncertainty in $k_{\text {eff }}$ due to the uncertainty in the density of the annulus of the lower axial reflector. Half of the differences between the calculated upper and lower perturbed values were then scaled to obtain the $1 \sigma$ uncertainty. Results are shown in Table 2.1-14. 
NEA/NSC/DOC(2006)1

\section{Gas Cooled (Thermal) Reactor - GCR \\ PROTEUS-GCR-EXP-004 \\ CRIT-REAC}

Table 2.1-14. Effect of Uncertainty in Annulus Density.

\begin{tabular}{||c|c|ccc|c|ccc||}
\hline $\begin{array}{c}\text { Case } \\
(\text { Core) }\end{array}$ & Deviation & $\boldsymbol{\Delta} \mathbf{k}_{\mathbf{p}}$ & \pm & $\boldsymbol{\sigma}_{\text {skp }}$ & Factor & $\Delta \mathbf{k}_{\text {eff }}(\mathbf{1} \boldsymbol{\sigma})$ & \pm & $\boldsymbol{\sigma}_{\text {akeff }}$ \\
\hline \hline $1(9)$ & $\pm 0.036 \mathrm{~g} / \mathrm{cm}^{3}$ & 0.00038 & \pm & 0.00005 & 3 & 0.00013 & \pm & 0.00002 \\
\hline $2(10)$ & $\pm 0.036 \mathrm{~g} / \mathrm{cm}^{3}$ & 0.00028 & \pm & 0.00004 & 3 & 0.00009 & \pm & 0.00001 \\
\hline
\end{tabular}

\subsubsection{Annulus Impurities}

It is assumed that the EBC of $1.33 \pm 0.08$ ppm (by wt.\%), discussed in Section 2.1.2.2, sufficiently described the impurity content in the central annulus of the lower axial reflector.

The impurity content of the annulus of the lower axial reflector was $1.33 \mathrm{ppm}$ (EBC by atom percent). The uncertainty in the impurity content was $0.08 \mathrm{ppma}(1 \sigma)$. A double-sided perturbation was performed in which the impurity content was perturbed by \pm 0.24 ppma to estimate the uncertainty in $k_{\text {eff }}$ due to the uncertainty in the impurity content of the annulus. Half of the differences between the calculated upper and lower perturbed values were then scaled to obtain the $1 \sigma$ uncertainty. Results are shown in Table 2.115.

Table 2.1-15. Effect of Uncertainty in Annulus Impurity Content.

\begin{tabular}{||l|c|ccc|c|ccc||}
\hline \hline $\begin{array}{l}\text { Case } \\
\text { (Core) }\end{array}$ & Deviation & $\boldsymbol{\Delta}_{\mathbf{p}}$ & \pm & $\boldsymbol{\sigma}_{\text {akp }}$ & Factor & $\boldsymbol{\Delta k}_{\text {eff }}(\mathbf{1} \boldsymbol{\sigma})$ & \pm & $\boldsymbol{\sigma}_{\text {akeff }}$ \\
\hline \hline $1(9)$ & $\pm 0.24 \mathrm{ppma}$ & -0.00038 & \pm & 0.00005 & 3 & -0.00013 & \pm & 0.00002 \\
\hline $2(10)$ & $\pm 0.24 \mathrm{ppma}$ & -0.00032 & \pm & 0.00004 & 3 & -0.00011 & \pm & 0.00001 \\
\hline \hline
\end{tabular}

\subsubsection{Autorod}

\subsubsection{Copper Thickness}

The thickness of the copper was $3 \mathrm{~mm}$ (Figure 1.1-10). The uncertainty in the thickness was assumed to be $1 \mathrm{~mm}$ (bounding limit with uniform probability distribution). A double-sided perturbation was performed in which the thickness was perturbed by $\pm 3 \mathrm{~mm}$ (where $-3 \mathrm{~mm}$ would represent the condition of no autorod copper being present in the core) to estimate the uncertainty in $k_{\text {eff }}$ due to the uncertainty in the thickness of the copper. Half of the differences between the calculated upper and lower perturbed values were then scaled to obtain the $1 \sigma$ uncertainty. Results are shown in Table 2.1-16. The calculated uncertainty is negligible $(\leq 0.00010)$.

Table 2.1-16. Effect of Uncertainty in Copper Thickness.

\begin{tabular}{||c|c|ccc|c|ccc||}
\hline $\begin{array}{c}\text { Case } \\
\text { (Core) }\end{array}$ & Deviation & $\boldsymbol{\Delta} \mathbf{k}_{\mathbf{p}}$ & \pm & $\boldsymbol{\sigma}_{\text {skp }}$ & $\begin{array}{c}\text { Scaling } \\
\text { Factor }\end{array}$ & $\boldsymbol{\Delta}_{\text {eff }}(\mathbf{1 \sigma})$ & \pm & $\boldsymbol{\sigma}_{\text {skeff }}$ \\
\hline \hline $1(9)$ & $\pm 3 \mathrm{~mm}$ & -0.00048 & \pm & 0.00005 & $3 \sqrt{ } 3$ & -0.00009 & \pm & 0.00001 \\
\hline $2(10)$ & $\pm 3 \mathrm{~mm}$ & -0.00034 & \pm & 0.00004 & $3 \sqrt{ } 3$ & -0.00006 & \pm & 0.00001 \\
\hline \hline
\end{tabular}


NEA/NSC/DOC(2006)1

\author{
Gas Cooled (Thermal) Reactor - GCR \\ PROTEUS-GCR-EXP-004 \\ CRIT-REAC
}

\title{
2.1.5.2 Orientation
}

The orientation of the copper wedge in the autorod compared to the core center was not reported. It is assumed to be parallel to the y-axis in the benchmark model. A single-sided perturbation was performed in which the orientation of the autorod was rotated by $90^{\circ}$ (parallel to the $\mathrm{x}$-axis) to estimate the uncertainty in $\mathrm{k}_{\mathrm{eff}}$ due to the uncertainty in the orientation of the copper. The calculated results were then scaled to obtain the $1 \sigma$ uncertainty assuming a bounding limit with uniform probability distribution. Results are shown in Table 2.1-17.

Table 2.1-17. Effect of Uncertainty in Copper Orientation.

\begin{tabular}{||c|c|ccc|c|ccc||}
\hline \hline $\begin{array}{c}\text { Case } \\
\text { (Core) }\end{array}$ & Deviation & $\boldsymbol{\Delta} \mathbf{k}_{\mathbf{p}}$ & \pm & $\boldsymbol{\sigma}_{\text {skp }}$ & $\begin{array}{c}\text { Scaling } \\
\text { Factor }\end{array}$ & $\mathbf{\Delta k}_{\text {eff }}(\mathbf{1} \boldsymbol{\sigma})$ & \pm & $\boldsymbol{\sigma}_{\text {skeff }}$ \\
\hline \hline $1(9)$ & Rotate $90^{\circ}$ & 0.00019 & \pm & 0.00010 & $\sqrt{ } 3$ & 0.00011 & \pm & 0.00006 \\
\hline $2(10)$ & Rotate $90^{\circ}$ & -0.00003 & \pm & 0.00008 & $\sqrt{ } 3$ & -0.00002 & \pm & 0.00005 \\
\hline \hline
\end{tabular}

\subsubsection{Fuel Pebbles}

\subsubsection{Quantity of Pebbles}

Exact quantities of fuel and moderator pebbles were placed in the cores. There is no associated uncertainty. The number of fuel pebbles reported for each core configuration is summarized in Table 2.1-18.

Table 2.1-18. Number of Fuel Pebbles.

\begin{tabular}{||c|c||}
\hline $\begin{array}{c}\text { Case } \\
\text { (Core) }\end{array}$ & $\begin{array}{c}\text { \# Fuel } \\
\text { Pebbles }\end{array}$ \\
\hline \hline $1(9)$ & 4870 \\
$2(10)$ & 4332 \\
\hline
\end{tabular}

\subsubsection{Pebble Packing Fraction}

The theoretical packing fraction for an infinite columnar hexagonal point-on-point (CHPOP) packed configuration is $0.6046 .{ }^{\text {a }}$ The packing fraction for each core configuration was computed by taking the total volume of pebbles within the core cavity (assumed diameter of $6.000 \mathrm{~cm}$ apiece) and dividing by the total core volume within the 12 -sided region of the cavity and the pebble stack height. The packing fraction is approximately $58 \mathrm{vol} \%$, due to additional void space at the core/reflector interface. The pebbles were placed in exact positions, there is small uncertainty in the packing fraction due to the small uncertainties in the diameters of the pebbles and dimensions of the graphite reflector. This uncertainty is evaluated as part of Section 2.1.10.1.

\footnotetext{
a Difilippo, F. C., "Monte Carlo Calculations of Pebble Bed Benchmark Configurations of the PROTEUS Facility," Nucl. Sci. Eng., 143, 240-253 (2003).
}

Revision: 1 
NEA/NSC/DOC(2006)1

\section{Gas Cooled (Thermal) Reactor - GCR \\ PROTEUS-GCR-EXP-004 \\ CRIT-REAC}

Table 2.1-19. Pebble Packing Fraction.

\begin{tabular}{|c|c|c|c|c|c|}
\hline $\begin{array}{l}\text { Case } \\
\text { (Core) } \\
\end{array}$ & $\begin{array}{l}\text { Total \# } \\
\text { Pebbles } \\
\end{array}$ & $\begin{array}{c}\text { Pebble } \\
\text { Volume }\left(\mathrm{m}^{3}\right) \\
\end{array}$ & $\begin{array}{c}\text { Pebble Stack } \\
\text { Height }(\mathbf{m}) \\
\end{array}$ & $\begin{array}{c}\text { Core } \\
\text { Volume }\left(\mathbf{m}^{3}\right) \\
\end{array}$ & $\begin{array}{c}\text { Packing } \\
\text { Fraction }\left(\text { vol. \%) }^{(\mathbf{a})}\right. \\
\end{array}$ \\
\hline $1(9)$ & 9747 & $\sim 1.1024$ & 1.62 & $\sim 1.8893$ & $\sim 58.35$ \\
\hline $2(10)$ & 8664 & $\sim 0.9799$ & 1.44 & $\sim 1.6793$ & $\sim 58.35$ \\
\hline
\end{tabular}

(a) An infinite columnar hexagonal point-on-point packed lattice has a theoretical filling factor of 0.6046 .

\subsubsection{TRISO Random Packing}

Each fuel pebble was reported to contain 9394 TRISO particles (Figure 1.1-14); this value was most probably derived from knowledge of the total mass of uranium per fuel pebble. The exact quantity of TRISO particles may vary between pebbles; however, the uncertainty in the exact quantity of TRISO particles is directly correlated with the uncertainty in fuel mass per pebble, and evaluated in Section 2.1.6.7.

The TRISO particles are randomly located within the fueled zone of each fuel pebble. To investigate the effective uncertainty in TRISO particle placement within the fuel pebbles, TRISO particles were modeled within a lattice structure (see Figure 4.1-1 with associated textual discussion) and the URAN card in MCNP5 was used to simulate the random particle displacement. ${ }^{a}$ Care was taken to ensure that the fuel kernels of the TRISO particles (but not the graphite layers) were not truncated by the boundary of the fueled region. The resultant $\mathrm{k}_{\text {eff }}$ value calculated in this manner was compared against a similar case in which the random positioning of particles was not enabled. Results are shown in Table 2.1-20. The uncertainty is small and approximately at the $2 \sigma$ statistical uncertainty for Core 10 and negligible in Core 9 .

Table 2.1-20. Effect of Uncertainty in TRISO Random Packing.

\begin{tabular}{||c|c|ccc|c|ccc||}
\hline \hline $\begin{array}{c}\text { Case } \\
\text { (Core) }\end{array}$ & Deviation & $\mathbf{\Delta k}_{\mathbf{p}}$ & \pm & $\boldsymbol{\sigma}_{\text {skp }}$ & $\begin{array}{c}\text { Scaling } \\
\text { Factor }\end{array}$ & $\mathbf{\Delta k}_{\text {eff }}(\mathbf{1} \boldsymbol{\sigma})$ & \pm & $\boldsymbol{\sigma}_{\text {akeff }}$ \\
\hline \hline $1(9)$ & Random Particle Placement & -0.00002 & \pm & 0.00010 & 1 & -0.00002 & \pm & 0.00010 \\
\hline $2(10)$ & Random Particle Placement & -0.00015 & \pm & 0.00008 & 1 & -0.00015 & \pm & 0.00008 \\
\hline
\end{tabular}

\subsubsection{Kernel Radius}

The radius of the TRISO kernel was $0.02510 \mathrm{~cm}$ (Table 1.1-1). The uncertainty in the radius was $0.0006 \mathrm{~cm}(1 \sigma)$. A double-sided perturbation was performed in which the radius was perturbed by $\pm 0.0015 \mathrm{~cm}$ to estimate the uncertainty in $\mathrm{k}_{\text {eff }}$ due to the uncertainty in the radius of the TRISO kernel. Uranium mass was conserved. Half of the differences between the calculated upper and lower perturbed values were then scaled to obtain the $1 \sigma$ uncertainty. Results are shown in Table 2.1-21.

The calculated $\Delta \mathrm{k}_{\text {eff }}$ uncertainty was adjusted to account for random and systematic components of the total uncertainty. The systematic component is assumed to represent $15 \%$ of the total uncertainty; the random component is negligible due to the perturbation of a large quantity of objects. The final adjusted $\Delta \mathrm{k}_{\text {eff }}$ uncertainty is therefore only the preserved systematic uncertainty. The calculated uncertainty is negligible $(\leq 0.00010)$.

\footnotetext{
${ }^{a}$ X-5 Monte Carlo Team, "MCNP - a General Monte Carlo n-Particle Transport Code, version 5, Volume II: User's Guide" LA-CP-03-0245, Los Alamos National Laboratory (2003).

Revision: 1

Date: March 31, 2014

Page 105 of 375
} 
NEA/NSC/DOC(2006)1

\section{Gas Cooled (Thermal) Reactor - GCR \\ PROTEUS-GCR-EXP-004 \\ CRIT-REAC}

Table 2.1-21. Effect of Uncertainty in $\mathrm{UO}_{2}$ Kernel Radius.

\begin{tabular}{|c|c|c|c|c|c|c|c|c|c|}
\hline $\begin{array}{c}\text { Case } \\
\text { (Core) } \\
\end{array}$ & Deviation & $\Delta \mathbf{k}_{\mathbf{p}}$ & \pm & $\sigma_{\mathrm{skp}}$ & $\begin{array}{l}\text { Scaling } \\
\text { Factor } \\
\end{array}$ & $\Delta k_{\text {eff }}(1 \sigma)$ & \pm & $\sigma_{\Delta \text { keff }}$ & $\begin{array}{c}\text { Systematic } \\
\text { Component of } \\
\Delta \mathrm{k}_{\mathrm{eff}}(1 \sigma) \\
\end{array}$ \\
\hline $1(9)$ & $\pm 0.0015 \mathrm{~cm}$ & -0.00079 & \pm & 0.00005 & 2.5 & -0.00032 & \pm & 0.00002 & -0.00005 \\
\hline $2(10)$ & $\pm 0.0015 \mathrm{~cm}$ & 0.00030 & \pm & 0.00004 & 2.5 & 0.00012 & \pm & 0.00002 & 0.00002 \\
\hline
\end{tabular}

\subsubsection{Isotopic Content (Mass) ${ }^{234} \mathrm{U}$}

The mass and uncertainty of each uranium isotope in a fuel pebble was reported in Table 1.1-1. The isotopic content of the fuel would have been measured and the mass of each isotope calculated based upon the total uranium mass within each pebble. The isotopic content (in wt.\%) of each isotope was computed for both the uranium metal and $\mathrm{UO}_{2}$ fuel kernel (see Table 2.1-22).

The mass of ${ }^{234} \mathrm{U}$ reported was $0.008 \mathrm{~g}$ per pebble (Table 1.1-1). The uncertainty in the ${ }^{234} \mathrm{U}$ mass was $0.001 \mathrm{~g}(\sim 0.017 \mathrm{wt} . \%, 1 \sigma)$. A double-sided perturbation was performed in which the ${ }^{234} \mathrm{U}$ mass was perturbed by $\pm 0.003 \mathrm{~g}(\sim 0.050 \mathrm{wt} . \%)$ to estimate the uncertainty in $\mathrm{k}_{\text {eff }}$ due to the uncertainty in the isotopic content of ${ }^{234} \mathrm{U}$. To conserve total uranium mass, the ${ }^{238} \mathrm{U}$ mass was adjusted. Half of the differences between the calculated upper and lower perturbed values were then scaled to obtain the $1 \sigma$ uncertainty. Results are shown in Table 2.1-23.

Table 2.1-22. Isotopic Composition of Uranium.

\begin{tabular}{|c|c|c|c||}
\hline Isotope/Element & Mass (g) & $\begin{array}{c}\text { Uranium Metal } \\
\text { Composition (wt.\%) }\end{array}$ & UO Composition (wt.\%) \\
\hline \hline${ }^{234} \mathrm{U}$ & 0.008 & 0.134 & 0.118 \\
${ }^{235} \mathrm{U}$ & 1.000 & 16.762 & 14.77 \\
${ }^{236} \mathrm{U}$ & 0.005 & 0.084 & 0.074 \\
${ }^{238} \mathrm{U}$ & 4.953 & 83.020 & 73.155 \\
$\mathrm{O}$ & -- & -- & 11.87 \\
Impurities & -- & -- & 0.013 \\
\hline \hline \multicolumn{2}{|r|}{} & 100.000 & 100.000 \\
\hline
\end{tabular}

Table 2.1-23. Effect of Uncertainty in the ${ }^{234} \mathrm{U}$ content.

\begin{tabular}{||c|c|ccc|c|ccc||}
\hline $\begin{array}{c}\text { Case } \\
\text { (Core) }\end{array}$ & Deviation & $\Delta \mathbf{k}_{\mathbf{p}}$ & \pm & $\boldsymbol{\sigma}_{\text {skp }}$ & $\begin{array}{c}\text { Scaling } \\
\text { Factor }\end{array}$ & $\Delta \mathbf{k}_{\text {eff }}(\mathbf{1 \sigma})$ & \pm & $\boldsymbol{\sigma}_{\text {skeff }}$ \\
\hline \hline $1(9)$ & $\pm 0.003 \mathrm{~g}(\sim 0.050 \mathrm{wt} . \%)$ & -0.00056 & \pm & 0.00005 & 3 & -0.00019 & \pm & 0.00002 \\
\hline $2(10)$ & $\pm 0.003 \mathrm{~g}(\sim 0.050 \mathrm{wt} . \%)$ & -0.00041 & \pm & 0.00004 & 3 & -0.00014 & \pm & 0.00001 \\
\hline
\end{tabular}


NEA/NSC/DOC(2006)1

\section{Gas Cooled (Thermal) Reactor - GCR \\ PROTEUS-GCR-EXP-004 \\ CRIT-REAC}

\subsubsection{Isotopic Content (Mass) ${ }^{235} \mathrm{U}$}

See discussion in Section 2.1.6.5 regarding the isotopic content of the uranium fuel.

The mass of ${ }^{235} \mathrm{U}$ reported was $1.000 \mathrm{~g}$ per pebble (Table 1.1-1). The uncertainty in the ${ }^{235} \mathrm{U}$ mass was $0.010 \mathrm{~g}(\sim 0.17 \mathrm{wt} . \%, 1 \sigma)$. A double-sided perturbation was performed in which the ${ }^{235} \mathrm{U}$ mass was perturbed by $\pm 0.030 \mathrm{~g}(\sim 0.50 \mathrm{wt} . \%)$ to estimate the uncertainty in $\mathrm{k}_{\text {eff }}$ due to the uncertainty in the isotopic content of ${ }^{235} \mathrm{U}$. To conserve total uranium mass, the ${ }^{238} \mathrm{U}$ mass was adjusted. Half of the differences between the calculated upper and lower perturbed values were then scaled to obtain the $1 \sigma$ uncertainty. Results are shown in Table 2.1-24.

Table 2.1-24. Effect of Uncertainty in the ${ }^{235} \mathrm{U}$ Content.

\begin{tabular}{||c|c|ccc|c|ccc||}
\hline \hline $\begin{array}{c}\text { Case } \\
\text { (Core) }\end{array}$ & Deviation & $\Delta \mathbf{k}_{\mathbf{p}}$ & \pm & $\boldsymbol{\sigma}_{\text {akp }}$ & $\begin{array}{c}\text { Scaling } \\
\text { Factor }\end{array}$ & $\Delta \mathbf{k}_{\text {eff }}(\mathbf{1 \sigma})$ & \pm & $\boldsymbol{\sigma}_{\Delta k e f f}$ \\
\hline \hline $1(9)$ & $\pm 0.030 \mathrm{~g}(\sim 0.50 \mathrm{wt} . \%)$ & 0.00786 & \pm & 0.00005 & 3 & 0.00262 & \pm & 0.00002 \\
\hline $2(10)$ & $\pm 0.030 \mathrm{~g}(\sim 0.50 \mathrm{wt} . \%)$ & 0.00937 & \pm & 0.00004 & 3 & 0.00312 & \pm & 0.00001 \\
\hline \hline
\end{tabular}

\subsubsection{Uranium Mass}

Table 1.1-1 reports a mass uncertainty in the fuel of $\pm 0.060 \mathrm{~g}$, which appears to be a sum of the uncertainties in the ${ }^{235} \mathrm{U}$ and ${ }^{238} \mathrm{U}$ masses and equates to a mass density uncertainty in the $\mathrm{UO}_{2}$ fuel of approximately $0.11 \mathrm{~g} / \mathrm{cm}^{3}$. However, this table also reports the uncertainty in the $\mathrm{UO}_{2}$ density as $\pm 0.04 \mathrm{~g} / \mathrm{cm}^{3}$, almost a factor of 3 smaller. The table has footnotes for some of the uncertainties to explain the confidence level of the measured parameters; however, no additional information is provided for the uranium fuel mass or $\mathrm{UO}_{2}$ density. A fuel mass of $5.966 \mathrm{~g}\left(\mathrm{UO}_{2}\right.$ density of $\left.10.88 \mathrm{~g} / \mathrm{cm}^{3}\right)$ was selected for the fuel kernels and the larger uncertainty of $0.060 \mathrm{~g}\left(0.11 \mathrm{~g} / \mathrm{cm}^{3}\right)$ selected to represent the $1 \sigma$ uncertainty in the uranium mass.

The total mass of uranium per fuel pebble was $5.966 \mathrm{~g}$ (Table 1.1-1). The uncertainty in the mass was $0.060 \mathrm{~g}\left(0.068 \mathrm{~g} \mathrm{UO}_{2}, 0.11 \mathrm{~g} / \mathrm{cm}^{3}, 1 \sigma\right)$. A double-sided perturbation was performed in which the uranium dioxide density was perturbed by $\pm 0.12 \mathrm{~g} / \mathrm{cm}^{3}$ to estimate the uncertainty in $\mathrm{k}_{\text {eff }}$ due to the uncertainty in the uranium mass per fuel pebble. Half of the differences between the calculated upper and lower perturbed values were then scaled to obtain the $1 \sigma$ uncertainty. The radius of the $\mathrm{UO}_{2}$ kernels and the oxygen-to-uranium ratio was held constant. Results are shown in Table 2.1-25.

The calculated $\Delta \mathrm{k}_{\text {eff }}$ uncertainty was adjusted to account for random and systematic components of the total uncertainty. The systematic component is assumed to represent $15 \%$ of the total uncertainty; the random component is negligible due to the perturbation of a large quantity of objects. The final adjusted $\Delta \mathrm{k}_{\text {eff }}$ uncertainty is therefore only the preserved systematic uncertainty. 
NEA/NSC/DOC(2006)1

\section{Gas Cooled (Thermal) Reactor - GCR \\ PROTEUS-GCR-EXP-004 \\ CRIT-REAC}

Table 2.1-25. Effect of Uncertainty in the Fuel Pebble Uranium Mass.

\begin{tabular}{|c|c|c|c|c|c|c|c|c|c|}
\hline $\begin{array}{c}\text { Case } \\
\text { (Core) } \\
\end{array}$ & Deviation & $\Delta k_{p}$ & \pm & $\sigma_{\text {akp }}$ & $\begin{array}{c}\text { Scaling } \\
\text { Factor }^{(\mathbf{a})} \\
\end{array}$ & $\Delta k_{\text {eff }}(1 \sigma)$ & \pm & $\sigma_{\text {akeff }}$ & $\begin{array}{c}\text { Systematic } \\
\text { Component of } \\
\Delta \mathrm{k}_{\mathrm{eff}}(1 \sigma) \\
\end{array}$ \\
\hline $1(9)$ & $\pm 0.065 \mathrm{~g}\left(0.12 \mathrm{~g} / \mathrm{cm}^{3}\right)$ & 0.00245 & \pm & 0.00005 & $12 / 11$ & 0.00225 & \pm & 0.00005 & 0.00034 \\
\hline $2(10)$ & $\pm 0.065 \mathrm{~g}\left(0.12 \mathrm{~g} / \mathrm{cm}^{3}\right)$ & 0.00308 & \pm & 0.00004 & $12 / 11$ & 0.00282 & \pm & 0.00004 & 0.00042 \\
\hline
\end{tabular}

(a) The scaling factor converts the perturbation uncertainty of $0.12 \mathrm{~g} / \mathrm{cm}^{3}$, which represents the reported $3 \sigma$ uncertainty in the $\mathrm{UO}_{2}$ mass density to the $0.11 \mathrm{~g} / \mathrm{cm}^{3} 1 \sigma$ uncertainty in the mass density based upon the reported uncertainty in the uranium mass measurements.

\subsubsection{Fueled Zone Impurities}

The reported impurity content for the fuel pebbles is listed in Table 1.1-13. The composition values listed as less than a given value are taken at half this maximum value in the nominal material composition. The fueled zone composition (graphite region within the pebble surrounding the TRISO particles) is adjusted such that the total composition adds up to $100 \%$. The nominal impurity content used for evaluation of the uncertainty in the fueled zone impurities is in Table 2.1-26.

The nominal fueled zone impurity content is shown in Table 2.1-26. The selected uncertainty in each impurity was $50 \%$ of the nominal value $(1 \sigma)$. A double-sided perturbation was performed in which all impurities were simultaneously perturbed by $\pm 50 \%$ to estimate the uncertainty in $k_{\text {eff }}$ due to the uncertainty in the fueled zone impurity content. Half of the differences between the calculated upper and lower perturbed values were then scaled to obtain the $1 \sigma$ uncertainty.

The scaling factor was obtained by first determining the equivalent boron content (EBC) of each impurity based upon their concentration in the graphite and their respective ASTM EBC factor. ${ }^{\text {a }}$ The ratio of the equivalent boron content for each individual impurity to the total $\mathrm{EBC}$ was calculated; most ratios were small compared to the dominant impurities of boron $(\sim 41 \%)$ and lithium $(\sim 30 \%)$. Sample perturbations were performed to confirm that perturbations of the dominant impurities produced uncertainties in $\mathrm{k}_{\text {eff, }}$, divided by the total uncertainty obtained by perturbing all impurities simultaneously, would produce ratios approximately equal to the $\mathrm{EBC}$ ratios. The $\mathrm{EBC}$ ratios for all the graphite impurities were combined taking the square root of the sum of the squares to obtain a scaling factor of $53 \%$, which is needed to convert the additive perturbation of impurity content into one representing the quadrative summation expected for perturbing each impurity individually by $50 \%$. Results are shown in Table 2.127.

\footnotetext{
a ASTM C1233-03, "Standard Practice for Determining Equivalent Boron Contents of Nuclear Materials," ASTM International, West Conshohocken, PA (2009).
}

Revision: 1

Date: March 31, 2014 
NEA/NSC/DOC(2006)1

Gas Cooled (Thermal) Reactor - GCR

PROTEUS-GCR-EXP-004

CRIT-REAC

Table 2.1-26. Fuel Pebble Impurities.

\begin{tabular}{|c|c|c|c||}
\hline Element & $\begin{array}{l}\text { Minimum } \\
\text { ppm (wt.\%) }\end{array}$ & $\begin{array}{l}\text { Maximum } \\
\text { ppm (wt.\%) }\end{array}$ & $\begin{array}{l}\text { Nominal } \\
\text { ppm (wt.\%) }\end{array}$ \\
\hline \hline $\mathrm{Ag}$ & -- & 0.2 & 0.1 \\
\hline $\mathrm{B}$ & 0.101 & 0.101 & 0.101 \\
\hline $\mathrm{Ca}$ & 9.28 & 9.28 & 9.28 \\
\hline $\mathrm{Cd}$ & -- & 0.103 & 0.0515 \\
\hline $\mathrm{Cl}$ & -- & 3 & 1.5 \\
\hline $\mathrm{Co}$ & -- & 0.13 & 0.065 \\
\hline $\mathrm{Cr}$ & 1.81 & 1.81 & 1.81 \\
\hline $\mathrm{Dy}$ & -- & 0.01 & 0.005 \\
\hline $\mathrm{Eu}$ & -- & 0.01 & 0.005 \\
\hline $\mathrm{Fe}$ & 2.95 & 2.95 & 2.95 \\
\hline $\mathrm{Gd}$ & -- & 0.01 & 0.005 \\
\hline $\mathrm{Li}$ & -- & 1 & 0.5 \\
\hline $\mathrm{Mn}$ & 0.43 & 0.43 & 0.43 \\
\hline $\mathrm{Ni}$ & -- & 1 & 0.5 \\
\hline $\mathrm{S}$ & -- & 0.011 & 0.0055 \\
\hline $\mathrm{Ti}$ & 0.497 & 0.497 & 0.497 \\
\hline $\mathrm{V}$ & -- & 0.433 & 0.2165 \\
\hline \hline $\mathrm{Total}$ & -- & -- & 18.0215 \\
\hline \hline
\end{tabular}

Table 2.1-27. Effect of Uncertainty in the Fueled Zone Impurities.

\begin{tabular}{||c|c|ccc|c|ccc||}
\hline \hline $\begin{array}{c}\text { Case } \\
\text { (Core) }\end{array}$ & Deviation & $\boldsymbol{\Delta} \mathbf{k}_{\mathbf{p}}$ & \pm & $\boldsymbol{\sigma}_{\text {skp }}$ & $\begin{array}{c}\text { Scaling } \\
\text { Factor }\end{array}$ & $\Delta \mathbf{k}_{\text {eff }}(\mathbf{1 \sigma})$ & \pm & $\boldsymbol{\sigma}_{\text {skeff }}$ \\
\hline \hline $1(9)$ & $\pm 50 \%$ & -0.00023 & \pm & 0.00005 & $1 / 0.53$ & -0.00012 & \pm & 0.00003 \\
\hline $2(10)$ & $\pm 50 \%$ & -0.00017 & \pm & 0.00004 & $1 / 0.53$ & -0.00009 & \pm & 0.00002 \\
\hline \hline
\end{tabular}

Revision: 1 
NEA/NSC/DOC(2006)1

\author{
Gas Cooled (Thermal) Reactor - GCR \\ PROTEUS-GCR-EXP-004 \\ CRIT-REAC
}

\title{
2.1.6.9 Unfueled Zone Impurities
}

The reported impurity content for the fuel pebbles is listed in Table 1.1-13. It is assumed that the unfueled zone impurities are the same as the fueled zone impurities in Section 2.1.6.8. The composition values listed as less than a given value are taken at half this maximum value in the nominal material composition. The unfueled zone composition (graphite shell surrounding the fueled zone of the pebble) is adjusted such that the total composition adds up to $100 \%$. The nominal impurity content used for evaluation of the uncertainty in the unfueled zone impurities is in Table 2.1-26.

The nominal unfueled zone impurity content is shown in Table 2.1-26. The selected uncertainty in each impurity was $50 \%$ of the nominal value $(1 \sigma)$. A double-sided perturbation was performed in which all impurities were simultaneously perturbed by $\pm 50 \%$ to estimate the uncertainty in $\mathrm{k}_{\text {eff }}$ due to the uncertainty in the unfueled zone impurity content. Half of the differences between the calculated upper and lower perturbed values were then scaled to obtain the $1 \sigma$ uncertainty.

The scaling factor was obtained by first determining the equivalent boron content (EBC) of each impurity based upon their concentration in the graphite and their respective ASTM EBC factor. ${ }^{\text {a }}$ The ratio of the equivalent boron content for each individual impurity to the total $\mathrm{EBC}$ was calculated; most ratios were small compared to the dominant impurities of boron $(\sim 41 \%)$ and lithium $(\sim 30 \%)$. Sample perturbations were performed to confirm that perturbations of the dominant impurities produced uncertainties in $\mathrm{k}_{\text {eff }}$, divided by the total uncertainty obtained by perturbing all impurities simultaneously, would produce ratios approximately equal to the $\mathrm{EBC}$ ratios. The $\mathrm{EBC}$ ratios for all the graphite impurities were combined taking the square root of the sum of the squares to obtain a scaling factor of $53 \%$, which is needed to convert the additive perturbation of impurity content into one representing the quadrative summation expected for perturbing each impurity individually by $50 \%$. Results are shown in Table 2.128.

Table 2.1-28. Effect of Uncertainty in the Unfueled Zone Impurities.

\begin{tabular}{||c|c|ccc|c|ccc||}
\hline $\begin{array}{c}\text { Case } \\
\text { (Core) }\end{array}$ & Deviation & $\Delta \mathbf{k}_{\mathbf{p}}$ & \pm & $\boldsymbol{\sigma}_{\text {skp }}$ & $\begin{array}{c}\text { Scaling } \\
\text { Factor }\end{array}$ & $\Delta \mathbf{k}_{\text {eff }}(\mathbf{1 \sigma})$ & \pm & $\boldsymbol{\sigma}_{\text {skeff }}$ \\
\hline \hline $1(9)$ & $\pm 50 \%$ & -0.00023 & \pm & 0.00005 & $1 / 0.53$ & -0.00012 & \pm & 0.00003 \\
\hline $2(10)$ & $\pm 50 \%$ & -0.00032 & \pm & 0.00004 & $1 / 0.53$ & -0.00017 & \pm & 0.00002 \\
\hline \hline
\end{tabular}

\subsubsection{Water Content}

The water content of the moderator pebbles was reported to be $0.01 \mathrm{wt} . \%$. It is assumed that the water content of the fuel pebbles was also $0.01 \mathrm{wt} . \%$. The uncertainty in the water content is also assumed to be $0.01 \mathrm{wt} . \%$ (bounding limit with uniform probability distribution). A double-sided perturbation was performed in which the water content was perturbed by $\pm 0.01 \mathrm{wt} . \%$ to estimate the uncertainty in $\mathrm{k}_{\text {eff }}$ due to the uncertainty in the fuel pebble water content. Half of the differences between the calculated upper and lower perturbed values were then scaled to obtain the $1 \sigma$ uncertainty. Results are shown in Table 2.129.

A second perturbation analysis was performed in PROTEUS-GCR-EXP-001 assessing the uncertainty in the fuel pebble water content if the water was absorbed only within the outer surface of the pebble (depth of $3.5 \mathrm{~mm}$ ) because dry graphite will obtain water from the surrounding atmosphere. The results from that perturbation analysis were negligible and is assumed to also be negligible for other HTR-PROTEUS core configurations. Because it is unclear how or when water was retained within the pebbles, the larger

\footnotetext{
a ASTM C1233-03, "Standard Practice for Determining Equivalent Boron Contents of Nuclear Materials," ASTM International, West Conshohocken, PA (2009).
}

Revision: 1

Date: March 31, 2014

Page 110 of 375 
NEA/NSC/DOC(2006)1

\section{Gas Cooled (Thermal) Reactor - GCR \\ PROTEUS-GCR-EXP-004 \\ CRIT-REAC}

uncertainty, obtained using uniform distribution of water content throughout the pebble, is utilized to represent the total uncertainty in the fuel pebble water content.

Table 2.1-29. Effect of Uncertainty in the Water Content.

\begin{tabular}{||c|c|ccc|c|ccc||}
\hline $\begin{array}{c}\text { Case } \\
\text { (Core) }\end{array}$ & Deviation & $\Delta_{\mathbf{k}_{\mathbf{p}}}$ & \pm & $\boldsymbol{\sigma}_{\text {akp }}$ & $\begin{array}{c}\text { Scaling } \\
\text { Factor }\end{array}$ & $\mathbf{\Delta k}_{\text {eff }}(\mathbf{1 \sigma})$ & \pm & $\boldsymbol{\sigma}_{\text {akeff }}$ \\
\hline \hline $1(9)$ & \pm 0.01 wt. $\%$ & 0.00018 & \pm & 0.00005 & $\sqrt{ } 3$ & 0.00011 & \pm & 0.00003 \\
\hline $2(10)$ & \pm 0.01 wt. $\%$ & 0.00003 & \pm & 0.00004 & $\sqrt{ } 3$ & 0.00001 & \pm & 0.00002 \\
\hline
\end{tabular}

\subsubsection{Oxygen-to-Uranium Ratio}

A nominal oxygen-to-uranium ratio is 2.00 . The uncertainty in the ratio was assumed to be $0.01(1 \sigma)$. A double-sided perturbation was performed in which the ratio was perturbed by \pm 0.03 to estimate the uncertainty in $k_{\text {eff }}$ due to the uncertainty in the oxygen-to-uranium ratio. Half of the differences between the calculated upper and lower perturbed values were then scaled to obtain the $1 \sigma$ uncertainty. Results are shown in Table 2.1-30.

Table 2.1-30. Effect of Uncertainty in the Oxygen-to-Uranium Ratio.

\begin{tabular}{||c|c|ccc|c|ccc||}
\hline $\begin{array}{c}\text { Case } \\
\text { (Core) }\end{array}$ & Deviation & $\boldsymbol{\Delta} \mathbf{k}_{\mathbf{p}}$ & \pm & $\boldsymbol{\sigma}_{\text {skp }}$ & $\begin{array}{c}\text { Scaling } \\
\text { Factor }\end{array}$ & $\mathbf{\Delta k}_{\text {eff }}(\mathbf{1} \boldsymbol{\sigma})$ & \pm & $\boldsymbol{\sigma}_{\text {akeff }}$ \\
\hline \hline $1(9)$ & \pm 0.03 & -0.00039 & \pm & 0.00005 & 3 & -0.00013 & \pm & 0.00002 \\
\hline $2(10)$ & \pm 0.03 & -0.00048 & \pm & 0.00004 & 3 & -0.00016 & \pm & 0.00001 \\
\hline
\end{tabular}

\subsubsection{Moderator Pebbles}

\subsubsection{Quantity of Pebbles}

Exact quantities of fuel and moderator pebbles were placed in the cores. There is no associated uncertainty. The number of moderator pebbles reported for each core configuration is given in Table 2.1-31.

Table 2.1-31. Number of Moderator Pebbles.

\begin{tabular}{||c|c||}
\hline $\begin{array}{c}\text { Case } \\
\text { (Core) }\end{array}$ & $\begin{array}{c}\text { \# Moderator } \\
\text { Pebbles }\end{array}$ \\
\hline \hline $1(9)$ & 4877 \\
$2(10)$ & 4332 \\
\hline
\end{tabular}

\subsubsection{Mass}

The total mass per moderator pebble was $190.54 \mathrm{~g}$ (Table 1.1-2). The uncertainty in the mass was $1.44 \mathrm{~g}$ $\left(0.013 \mathrm{~g} / \mathrm{cm}^{3}, 1 \sigma\right)$. A double-sided perturbation was performed in which the mass was perturbed by $\pm 4.32 \mathrm{~g}\left(0.038 \mathrm{~g} / \mathrm{cm}^{3}\right)$ to estimate the uncertainty in $\mathrm{k}_{\text {eff }}$ due to the uncertainty in the mass per moderator pebble. The radius of the moderator pebbles was conserved. Half of the differences between the 
NEA/NSC/DOC(2006)1

\section{Gas Cooled (Thermal) Reactor - GCR \\ PROTEUS-GCR-EXP-004 \\ CRIT-REAC}

calculated upper and lower perturbed values were then scaled to obtain the $1 \sigma$ uncertainty. Results are shown in Table 2.1-32.

The calculated $\Delta \mathrm{k}_{\text {eff }}$ uncertainty was adjusted to account for random and systematic components of the total uncertainty. The systematic component is assumed to represent $15 \%$ of the total uncertainty; the random component is negligible due to the perturbation of a large quantity of objects. The final adjusted $\Delta \mathrm{k}_{\text {eff }}$ uncertainty is therefore only the preserved systematic uncertainty.

Table 2.1-32. Effect of Uncertainty in the Moderator Pebble Mass.

\begin{tabular}{||c|c|ccc|c|ccc|c||}
\hline $\begin{array}{c}\text { Case } \\
\text { (Core) }\end{array}$ & Deviation & $\Delta \mathbf{k}_{\mathbf{p}}$ & \pm & $\boldsymbol{\sigma}_{\text {akp }}$ & $\begin{array}{c}\text { Scaling } \\
\text { Factor }\end{array}$ & $\Delta \mathbf{k}_{\text {eff }}(\mathbf{1 \sigma})$ & \pm & $\boldsymbol{\sigma}_{\text {akeff }}$ & $\begin{array}{c}\text { Systematic } \\
\text { Component of } \\
\Delta \mathbf{k}_{\text {eff }}(\mathbf{1 \sigma})\end{array}$ \\
\hline \hline $1(9)$ & $\pm 4.32 \mathrm{~g}\left(0.038 \mathrm{~g} / \mathrm{cm}^{3}\right)$ & 0.00309 & \pm & 0.00005 & 3 & 0.00103 & \pm 0.00002 & 0.00015 \\
\hline $2(10)$ & $\pm 4.32 \mathrm{~g}\left(0.038 \mathrm{~g} / \mathrm{cm}^{3}\right)$ & 0.00200 & \pm & 0.00004 & 3 & 0.00067 & \pm 0.00001 & 0.00010 \\
\hline \hline
\end{tabular}

\subsubsection{Impurities}

The reported impurity content for the moderator pebbles is listed in Table 1.1-14. The composition values listed as less than a given value are taken at half this maximum value in the nominal material composition. The moderator pebble composition is adjusted such that the total composition adds up to $100 \%$. The nominal impurity content used for evaluation of the uncertainty in the unfueled zone impurities is in Table 2.1-33.

The nominal moderator pebble impurity content is shown in Table 2.1-33. The selected uncertainty in each impurity was $50 \%$ of the nominal value $(1 \sigma)$. A double-sided perturbation was performed in which all impurities were simultaneously perturbed by $\pm 50 \%$ to estimate the uncertainty in $k_{\text {eff }}$ due to the uncertainty in the moderator pebble impurity content. Half of the differences between the calculated upper and lower perturbed values were then scaled to obtain the $1 \sigma$ uncertainty.

The scaling factor was obtained by first determining the equivalent boron content (EBC) of each impurity based upon their concentration in the graphite and their respective ASTM EBC factor. ${ }^{\text {a }}$ The ratio of the equivalent boron content for each individual impurity to the total $\mathrm{EBC}$ was calculated; most ratios were small compared to the dominant impurities of boron $(\sim 47 \%)$, chlorine $(\sim 16 \%)$ and gadolinium $(\sim 11 \%)$. Sample perturbations were performed to confirm that perturbations of the dominant impurities produced uncertainties in $\mathrm{k}_{\mathrm{eff}}$, divided by the total uncertainty obtained by perturbing all impurities simultaneously, would produce ratios approximately equal to the EBC ratios. The EBC ratios for all the graphite impurities were combined taking the square root of the sum of the squares to obtain a scaling factor of $52 \%$, which is needed to convert the additive perturbation of impurity content into one representing the quadrative summation expected for perturbing each impurity individually by $50 \%$. Results are shown in Table 2.1-34.

\footnotetext{
a ASTM C1233-03, "Standard Practice for Determining Equivalent Boron Contents of Nuclear Materials," ASTM International, West Conshohocken, PA (2009).
}

Revision: 1 
NEA/NSC/DOC(2006)1

\section{Gas Cooled (Thermal) Reactor - GCR \\ PROTEUS-GCR-EXP-004 \\ CRIT-REAC}

Table 2.1-33. Moderator Pebble Impurities.

\begin{tabular}{|c|c|c|c|}
\hline Element & $\begin{array}{c}\text { Minimum } \\
\text { ppm (wt.\%) }\end{array}$ & $\begin{array}{c}\text { Maximum } \\
\text { ppm (wt.\%) }\end{array}$ & $\begin{array}{c}\text { Nominal } \\
\text { ppm (wt.\%) }\end{array}$ \\
\hline B & 0.76 & 0.76 & 0.76 \\
\hline $\mathrm{Ca}$ & 129 & 129 & 129 \\
\hline $\mathrm{Cd}$ & -- & 0.6 & 0.3 \\
\hline $\mathrm{Cl}$ & 18.64 & 18.64 & 18.64 \\
\hline Dy & 0.065 & 0.065 & 0.065 \\
\hline $\mathrm{Eu}$ & 0.13 & 0.13 & 0.13 \\
\hline $\mathrm{Fe}$ & 5.9 & 5.9 & 5.9 \\
\hline $\mathrm{Gd}$ & 0.040 & 0.040 & 0.040 \\
\hline $\mathrm{Li}$ & 0.88 & 0.88 & 0.88 \\
\hline $\mathrm{Ni}$ & 0.78 & 0.78 & 0.78 \\
\hline $\mathrm{S}$ & 140 & 140 & 140 \\
\hline $\mathrm{Si}$ & 35 & 35 & 35 \\
\hline $\mathrm{Sm}$ & 0.086 & 0.086 & 0.086 \\
\hline $\mathrm{Ti}$ & 10 & 10 & 10 \\
\hline $\mathrm{V}$ & 13 & 13 & 13 \\
\hline Total & -- & -- & 354.581 \\
\hline
\end{tabular}

Table 2.1-34. Effect of Uncertainty in the Moderator Pebble Impurities.

\begin{tabular}{||c|c|ccc|c|ccc||}
\hline $\begin{array}{c}\text { Case } \\
\text { (Core) }\end{array}$ & Deviation & $\Delta \mathbf{k}_{\mathbf{p}}$ & \pm & $\boldsymbol{\sigma}_{\text {skp }}$ & $\begin{array}{c}\text { Scaling } \\
\text { Factor }\end{array}$ & $\Delta \mathbf{k}_{\text {eff }}(\mathbf{1 \sigma})$ & \pm & $\boldsymbol{\sigma}_{\text {skeff }}$ \\
\hline \hline $1(9)$ & $50 \%$ & -0.00318 & \pm & 0.00005 & $1 / 0.52$ & -0.00166 & \pm & 0.00003 \\
\hline $2(10)$ & $50 \%$ & -0.00296 & \pm & 0.00004 & $1 / 0.52$ & -0.00154 & \pm & 0.00002 \\
\hline \hline
\end{tabular}

\subsubsection{Water Content}

The water content of the moderator pebbles was reported to be $0.01 \mathrm{wt} . \%$. The uncertainty in the water content was assumed to be $0.01 \mathrm{wt} . \%$ (bounding limit with uniform probability distribution). A doublesided perturbation was performed in which the water content was perturbed by $\pm 0.01 \mathrm{wt} . \%$ to estimate the uncertainty in $\mathrm{k}_{\mathrm{eff}}$ due to the uncertainty in the moderator pebble water content. Half of the differences between the calculated upper and lower perturbed values were then scaled to obtain the $1 \sigma$ uncertainty. Results are shown in Table 2.1-35. The calculated uncertainty is negligible $(\leq 0.00010)$.

A second perturbation analysis was performed in PROTEUS-GCR-EXP-001 assessing the uncertainty in the moderator pebble water content if the water was absorbed only within the outer surface of the pebble (depth of $3.5 \mathrm{~mm}$ ) because dry graphite will obtain water from the surrounding atmosphere. The results from that perturbation analysis were negligible and is assumed to also be negligible for other HTR-

PROTEUS core configurations. 
NEA/NSC/DOC(2006)1

\section{Gas Cooled (Thermal) Reactor - GCR \\ PROTEUS-GCR-EXP-004 \\ CRIT-REAC}

Table 2.1-35. Effect of Uncertainty in the Water Content.

\begin{tabular}{||l|c|ccc|c|ccc||}
\hline $\begin{array}{c}\text { Case } \\
\text { (Core) }\end{array}$ & Deviation & $\Delta \mathbf{k}_{\mathbf{p}}$ & \pm & $\boldsymbol{\sigma}_{\text {skp }}$ & $\begin{array}{c}\text { Scaling } \\
\text { Factor }\end{array}$ & $\Delta \mathbf{k}_{\text {eff }}(\mathbf{1 \sigma})$ & \pm & $\boldsymbol{\sigma}_{\text {skeff }}$ \\
\hline \hline $1(9)$ & \pm 0.01 wt.\% & 0.00012 & \pm & 0.00005 & $\sqrt{ } 3$ & 0.00007 & \pm & 0.00003 \\
\hline $2(10)$ & \pm 0.01 wt.\% & -0.00002 & \pm & 0.00004 & $\sqrt{ } 3$ & -0.00001 & \pm & 0.00002 \\
\hline
\end{tabular}

\subsubsection{Withdrawable Control Rods}

\subsubsection{Outer Tube Composition}

The end plugs of the stainless steel control rods are assumed to be manufactured from the same material as the outer tube (St1.4541). There is no significant difference between using this material or the composition of the inner tube (St1.4301).

The composition of the outer steel tube and end plugs was stainless steel Type St1.4541 (see Table 2.136). A double-sided perturbation was performed in which the composition was perturbed by minimizing and maximizing the iron content in the steel, while simultaneously maximizing or minimizing the other elemental constituents within the specified limits, to estimate the uncertainty in $\mathrm{k}_{\text {eff }}$ due to the uncertainty in steel tube composition. Half of the differences between the calculated upper and lower perturbed values were then scaled to obtain the $1 \sigma$ uncertainty assuming a bounding limit with uniform probability distribution. Results are shown in Table 2.1-37. The calculated uncertainty is negligible $(\leq 0.00010)$.

Table 2.1-36. Composition of Stainless Steel St1.4541.

\begin{tabular}{|c|c|c|c||}
\hline \hline Element & Minimum wt.\% & Maximum wt.\% & Nominal wt.\% \\
\hline \hline $\mathrm{C}$ & -- & 0.1 & 0.05 \\
\hline $\mathrm{Si}$ & -- & 1 & 0.5 \\
\hline $\mathrm{Mn}$ & -- & 2 & 1 \\
\hline $\mathrm{Cr}$ & 17 & 19 & 18 \\
\hline $\mathrm{Ni}$ & 9 & 11.5 & 10.25 \\
\hline $\mathrm{Ti}$ & -- & $\geq \mathrm{wt} . \% \mathrm{C}$ & 0.05 \\
\hline $\mathrm{Fe}$ & \multicolumn{2}{|c|}{ Balance } & 70.15 \\
\hline \hline Total & -- & - & 100 \\
\hline
\end{tabular}

Table 2.1-37. Effect of Uncertainty in Outer Steel Tube Composition.

\begin{tabular}{||c|c|ccc|c|ccc||}
\hline $\begin{array}{c}\text { Case } \\
\text { (Core) }\end{array}$ & Deviation & $\boldsymbol{\Delta} \mathbf{k}_{\mathbf{p}}$ & \pm & $\boldsymbol{\sigma}_{\text {skp }}$ & $\begin{array}{c}\text { Scaling } \\
\text { Factor }\end{array}$ & $\mathbf{\Delta k}_{\text {eff }}(\mathbf{1} \boldsymbol{\sigma})$ & \pm & $\boldsymbol{\sigma}_{\text {skeff }}$ \\
\hline \hline $1(9)$ & $\mathrm{Min} / \mathrm{Max} \mathrm{Fe}$ & -0.00005 & \pm & 0.00005 & $\sqrt{ } 3$ & -0.00003 & \pm & 0.00003 \\
\hline $2(10)$ & $\mathrm{Min} / \mathrm{Max} \mathrm{Fe}$ & 0.00003 & \pm & 0.00004 & $\sqrt{ } 3$ & 0.00001 & \pm & 0.00002 \\
\hline
\end{tabular}


NEA/NSC/DOC(2006)1

\section{Gas Cooled (Thermal) Reactor - GCR \\ PROTEUS-GCR-EXP-004 \\ CRIT-REAC}

\subsubsection{Polyethylene Rods}

\subsubsection{Diameter}

The diameter of the polyethylene rods was $6.5 \mathrm{~mm}$ (Figure 1.1-18). The uncertainty in diameter was assumed to be $0.1 \mathrm{~mm}$ (bounding limit with uniform probability distribution). A double-sided perturbation was performed in which the diameter was perturbed by $\pm 0.3 \mathrm{~mm}$ to estimate the uncertainty in $k_{\text {eff }}$ due to the uncertainty in the diameter of the polyethylene rods. Linear density, which is the unit mass per length of rod, was conserved. Half of the differences between the calculated upper and lower perturbed values were then scaled to obtain the $1 \sigma$ uncertainty. Results are shown in Table 2.1-38. The calculated uncertainty is negligible $(\leq 0.00010)$.

Table 2.1-38. Effect of Uncertainty in the Polyethylene Rod Diameter.

\begin{tabular}{|c|c|c|c|c|c|c|c|c|}
\hline $\begin{array}{c}\text { Case } \\
\text { (Core) } \\
\end{array}$ & Deviation & $\Delta \mathbf{k}_{\mathrm{p}}$ & \pm & $\sigma_{\text {okp }}$ & $\begin{array}{l}\text { Scaling } \\
\text { Factor } \\
\end{array}$ & $\Delta \mathrm{k}_{\mathrm{eff}}(1 \sigma)$ & \pm & $\sigma_{\text {skeff }}$ \\
\hline $1(9)$ & NA & & NA & & NA & & NA & \\
\hline $2(10)$ & $\pm 0.3 \mathrm{~mm}$ & 0.00045 & + & 0.00004 & $3 \sqrt{3}$ & 0.00009 & \pm & 0.00001 \\
\hline
\end{tabular}

\subsubsection{Length}

The length of the polyethylene rods was $1450 \mathrm{~mm}$. The uncertainty in length was reported to be $5 \mathrm{~mm}$ (assumed to be $1 \sigma$ ). A double-sided perturbation was performed in which the length was perturbed by $\pm 15 \mathrm{~mm}$ to estimate the uncertainty in $\mathrm{k}_{\mathrm{eff}}$ due to the uncertainty in the length of the polyethylene rods. Half of the differences between the calculated upper and lower perturbed values were then scaled to obtain the $1 \sigma$ uncertainty. Results are shown in Table 2.1-39. The calculated uncertainty is negligible $(\leq 0.00010)$.

Table 2.1-39. Effect of Uncertainty in the Polyethylene Rod Length.

\begin{tabular}{||l|c|ccc|c|ccc||}
\hline \hline $\begin{array}{c}\text { Case } \\
\text { (Core) }\end{array}$ & Deviation & $\Delta \mathbf{k}_{\mathbf{p}}$ & \pm & $\boldsymbol{\sigma}_{\Delta \mathbf{k p}}$ & $\begin{array}{c}\text { Scaling } \\
\text { Factor }\end{array}$ & $\boldsymbol{\Delta} \mathbf{k}_{\text {eff }}(\mathbf{1} \boldsymbol{\sigma})$ & \pm & $\boldsymbol{\sigma}_{\Delta \mathbf{k e f f}}$ \\
\hline \hline $1(9)$ & NA & \multicolumn{3}{|c|}{ NA } & NA & \multicolumn{3}{|c|}{ NA } \\
\hline $2(10)$ & $\pm 15 \mathrm{~mm}$ & -0.00007 & \pm & 0.00004 & 3 & -0.00002 & \pm & 0.00001 \\
\hline \hline
\end{tabular}

\subsubsection{Density}

The linear density of the polyethylene rods was $0.3161 \mathrm{~g} / \mathrm{cm}$ (mass density: $0.9526 \mathrm{~g} / \mathrm{cm}^{3}$, Figure 1.1-21). The uncertainty in the linear density was $0.0001 \mathrm{~g} / \mathrm{cm}\left(0.0003 \mathrm{~g} / \mathrm{cm}^{3}, 1 \sigma\right)$. A double-sided perturbation was performed in which the density was perturbed by $\pm 0.0003 \mathrm{~g} / \mathrm{cm}\left(0.0009 \mathrm{~g} / \mathrm{cm}^{3}\right)$ to estimate the uncertainty in $\mathrm{k}_{\mathrm{eff}}$ due to the uncertainty in the density of the polyethylene rods. Half of the differences between the calculated upper and lower perturbed values were then scaled to obtain the $1 \sigma$ uncertainty. Results are shown in Table 2.1-40. The calculated uncertainty is negligible $(\leq 0.00010)$. 
NEA/NSC/DOC(2006)1

\section{Gas Cooled (Thermal) Reactor - GCR \\ PROTEUS-GCR-EXP-004 \\ CRIT-REAC}

Table 2.1-40. Effect of Uncertainty in the Polyethylene Rod Density.

\begin{tabular}{|c|c|c|c|c|c|c|c|c|}
\hline $\begin{array}{c}\text { Case } \\
\text { (Core) } \\
\end{array}$ & Deviation & $\Delta \mathbf{k}_{\mathbf{p}}$ & \pm & $\sigma_{\Delta \mathrm{kp}}$ & $\begin{array}{l}\text { Scaling } \\
\text { Factor } \\
\end{array}$ & $\Delta k_{\text {eff }}(1 \sigma)$ & \pm & $\sigma_{\text {skeff }}$ \\
\hline $1(9)$ & NA & & NA & & NA & & NA & \\
\hline $2(10)$ & $\pm 0.0003 \mathrm{~g} / \mathrm{cm}\left(0.0009 \mathrm{~g} / \mathrm{cm}^{3}\right)$ & -0.00001 & \pm & 0.00004 & 3 & $<0.00001$ & \pm & 0.00001 \\
\hline
\end{tabular}

\subsubsection{Hydrogen-to-Carbon Ratio}

The hydrogen-to-carbon ratio of the polyethylene rods was 2.03 . The uncertainty in the ratio was 0.03 $(1 \sigma)$. A double-sided perturbation was performed in which the ratio was perturbed by \pm 0.09 to estimate the uncertainty in $\mathrm{k}_{\text {eff }}$ due to the uncertainty in the hydrogen-to-carbon ratio of the polyethylene rods. The bulk density of the polyethylene rods was conserved. Half of the differences between the calculated upper and lower perturbed values were then scaled to obtain the $1 \sigma$ uncertainty. Results are shown in Table 2.1-41.

Table 2.1-41. Effect of Uncertainty in the Hydrogen-to-Carbon Ratio.

\begin{tabular}{|c|c|c|c|c|c|c|c|c|}
\hline $\begin{array}{c}\text { Case } \\
\text { (Core) } \\
\end{array}$ & Deviation & $\Delta \mathbf{k}_{\mathrm{p}}$ & \pm & $\sigma_{\text {skp }}$ & $\begin{array}{c}\text { Scaling } \\
\text { Factor } \\
\end{array}$ & $\Delta k_{\text {eff }}(1 \sigma)$ & \pm & $\sigma_{\text {skeff }}$ \\
\hline $1(9)$ & NA & & NA & & NA & & NA & \\
\hline $2(10)$ & \pm 0.09 & -0.00037 & \pm & 0.00004 & 3 & -0.00012 & \pm & 0.00001 \\
\hline
\end{tabular}

\subsubsection{Impurities}

The impurity content of the polyethylene rods was not reported. An EBC of $0.5 \pm 0.5 \mathrm{ppm}$ (by wt.\%) was assumed to represent a reasonable estimate for the impurity content with its respective uncertainty, and is included in the composition of the polyethylene rods. The effect of impurities in these rods are insignificant compared to the impurity content of the graphite blocks and pebbles, therefore larger contents and uncertainties were not investigated further.

The impurity content of the polyethylene rods was $0.5 \mathrm{ppm}$ (EBC by weight). The uncertainty in the impurity content was $0.5 \mathrm{ppm}$ (bounding limit with uniform probability distribution). A double-sided perturbation was performed in which the impurity content was perturbed by $\pm 0.5 \mathrm{ppm}$ to estimate the uncertainty in $\mathrm{k}_{\text {eff }}$ due to the uncertainty in the impurity content of the polyethylene rods. Half of the differences between the calculated upper and lower perturbed values were then scaled to obtain the $1 \sigma$ uncertainty. Results are shown in Table 2.1-42. The calculated uncertainty is negligible $(\leq 0.00010)$.

Table 2.1-42. Effect of Uncertainty in the Polyethylene Impurities.

\begin{tabular}{|c|c|c|c|c|c|c|c|c|}
\hline $\begin{array}{c}\text { Case } \\
\text { (Core) } \\
\end{array}$ & Deviation & $\Delta \mathbf{k}_{\mathrm{p}}$ & \pm & $\sigma_{\Delta \mathrm{kp}}$ & $\begin{array}{l}\text { Scaling } \\
\text { Factor } \\
\end{array}$ & $\Delta k_{\text {eff }}(1 \sigma)$ & \pm & $\sigma_{\Delta \mathrm{keff}}$ \\
\hline $1(9)$ & NA & \multicolumn{3}{|c|}{ NA } & NA & \multicolumn{3}{|c|}{ NA } \\
\hline $2(10)$ & $\pm 0.5 \mathrm{ppm}$ & 0.00001 & \pm & 0.00004 & 1 & 0.00001 & \pm & 0.00004 \\
\hline
\end{tabular}


NEA/NSC/DOC(2006)1

\section{Gas Cooled (Thermal) Reactor - GCR \\ PROTEUS-GCR-EXP-004 \\ CRIT-REAC}

\subsubsection{Experimental Measurements}

\subsubsection{Stacked Pebble Height}

The pebbles in Cores 9 and 10 were stacked in CHPOP cells (see Figure 1.0-3) and thus the core height is determined by calculating the height of pebbles stacked directly on top of each other (as reported in Tables 1.1-3 and 1.1-5). The total stack height for each core configuration is provided in Table 2.1-43.

Table 2.1-43. Stacked Pebble Height.

\begin{tabular}{||c|c|c||}
\hline $\begin{array}{c}\text { Case } \\
\text { (Core) }\end{array}$ & $\begin{array}{c}\text { \# Pebble } \\
\text { Layers }\end{array}$ & $\begin{array}{c}\text { Stack } \\
\text { Height (m) }\end{array}$ \\
\hline \hline $1(9)$ & 27 & 1.62 \\
$2(10)$ & 24 & 1.44 \\
\hline
\end{tabular}

The uncertainty in the core height is a function of the uncertainty in the diameter of the individual pebbles. The uncertainty in the pebble radii were evaluated separately (see Section 2.1.9.11 and 2.1.10.2 of PROTEUS-GCR-EXP-001) while maintaining the total pebble stack height, effectively increasing or decreasing the packing fraction of each core. The uncertainty in diameter of the pebbles was determined to be negligible. A separate uncertainty analysis was performed in which the core stack height was allowed to vary with the perturbation in pebble diameter to investigate the effect of perturbing the pebble packing fraction.

The uncertainty in core height was $\sim 0.06 \%$ (based on a pebble radius uncertainty of $0.00175 \mathrm{~cm}$ ). A single-sided perturbation was performed in which the height was increased by $\pm 0.06 \%$ (pebble radii increased by $0.00175 \mathrm{~cm}$ ) to estimate the uncertainty in $\mathrm{k}_{\mathrm{eff}}$ due to the uncertainty in the stacked pebble height. Results are shown in Table 2.1-44.

The calculated $\Delta \mathrm{k}_{\text {eff }}$ uncertainty was adjusted to account for random and systematic components of the total uncertainty. The systematic component is assumed to represent $15 \%$ of the total uncertainty; the random component is negligible due to the perturbation of a large quantity of objects. The final adjusted $\Delta \mathrm{k}_{\text {eff }}$ uncertainty is therefore only the preserved systematic uncertainty. The calculated uncertainty is negligible $(\leq 0.00010)$.

Table 2.1-44. Effect of Uncertainty in the Stacked Pebble Height.

\begin{tabular}{|c|c|c|c|c|c|c|c|c|c|}
\hline $\begin{array}{c}\text { Case } \\
\text { (Core) } \\
\end{array}$ & Deviation & $\Delta \mathbf{k}_{\mathrm{p}}$ & \pm & $\sigma_{\Delta \mathrm{kp}}$ & $\begin{array}{l}\text { Scaling } \\
\text { Factor } \\
\end{array}$ & $\Delta \mathbf{k}_{\mathrm{eff}}(1 \sigma)$ & \pm & $\sigma_{\text {skeff }}$ & $\begin{array}{c}\begin{array}{c}\text { Systematic } \\
\text { Component } \\
\text { of }\end{array} \\
\Delta \mathrm{k}_{\mathrm{eff}}(1 \sigma) \\
\end{array}$ \\
\hline $1(9)$ & $\begin{array}{c} \pm 0.06 \% \text { (pebble } \\
\text { radii increased by } \\
0.00175 \mathrm{~cm} \text { ) }\end{array}$ & -0.00006 & \pm & 0.00005 & 1 & -0.00006 & \pm & 0.00005 & -0.00001 \\
\hline $2(10)$ & $\begin{array}{c} \pm 0.06 \% \text { (pebble } \\
\text { radii increased by } \\
0.00175 \mathrm{~cm} \text { ) }\end{array}$ & -0.00007 & \pm & 0.00004 & 1 & -0.00007 & \pm & 0.00004 & -0.00001 \\
\hline
\end{tabular}


NEA/NSC/DOC(2006)1

\section{Gas Cooled (Thermal) Reactor - GCR \\ PROTEUS-GCR-EXP-004 \\ CRIT-REAC}

\subsubsection{Total Experimental Uncertainty}

A compilation of the total evaluated uncertainty in the critical configurations of Cores 9 and 10 (Cases 1 and 2, respectively) of the HTR-PROTEUS experiments is provided in Tables 2.1-45 and 2.1-46, respectively. As discussed earlier, uncertainties that are not treated as $100 \%$ systematic, because perturbation analyses were simultaneously applied to multiple components are treated as $15 \%$ systematic (to preserve some uncertainty due to possible, yet unknown, systematic effects) and $85 \%$ random. The random portion of the uncertainty is then divided by the square root of the number of perturbed components, and is negligible for most uncertainties. The total evaluated uncertainty is the root-sum square of all individual uncertainties. A graphical representation of the primary sources of uncertainty is shown in Figure 2.1-1.

Uncertainties $\leq 0.00010$ are reported as negligible (neg) and those that do not apply to a given configuration because they are not used or included as part of the evaluation of a different uncertainty are marked as not applicable (NA). The most significant contribution to the overall uncertainty is the fuel enrichment and the impurity content of the moderator pebbles. All uncertainties providing at least 0.05 $\% \Delta \mathrm{k}_{\text {eff }}$ are highlighted in Tables 2.1-45 and 2.1-46. The uncertainties in the experimental critical configurations for Cores 9 and 10 were evaluated and determined to be acceptable. 
NEA/NSC/DOC(2006)1

\section{Gas Cooled (Thermal) Reactor - GCR \\ PROTEUS-GCR-EXP-004 \\ CRIT-REAC}

Table 2.1-45. Summary of Evaluated Uncertainties in HTR-PROTEUS Case 1 (Core 9).

\begin{tabular}{|c|c|c|c|}
\hline Perturbed Parameter & $\begin{array}{c}\text { Parameter } \\
\text { Value } \\
\end{array}$ & $\begin{array}{c}\sigma \\
\text { Uncertainty } \\
\end{array}$ & $\Delta k_{\text {eff }}(1 \sigma)$ \\
\hline Radial Reflector Density $\left(\mathrm{g} / \mathrm{cm}^{3}\right)$ & 1.76 & 0.012 & 0.00102 \\
\hline Radial Reflector Impurities (ppma EBC) & 1.33 & 0.08 & 0.00113 \\
\hline Safety Ring Composition (wt.\%) & Table 2.1-6 & $1 / \sqrt{ } 3$ & 0.00012 \\
\hline C-Driver Plug Density $\left(\mathrm{g} / \mathrm{cm}^{3}\right)$ & 1.765 & 0.012 & neg \\
\hline Location of Upper Axial Reflector (mm) & 1893 & $5 / \sqrt{ } 3$ & 0.00015 \\
\hline Upper Axial Aluminum Dimensions (mm) & Figure 1.1-4 & $1 / \sqrt{ } 3$ & 0.00072 \\
\hline Upper Axial Aluminum Composition & Table 2.1-6 & $1 / \sqrt{ } 3$ & 0.00039 \\
\hline Lower Axial Cylinder Diameter (mm) & 495 & $1 / \sqrt{ } 3$ & neg \\
\hline Lower Axial Reflector Height (mm) & 780 & $1 / \sqrt{3}$ & neg \\
\hline Lower Axial Annulus Density $\left(\mathrm{g} / \mathrm{cm}^{3}\right)$ & 1.76 & 0.012 & 0.00013 \\
\hline Lower Axial Annulus Impurities (ppma EBC) & 1.33 & 0.08 & 0.00013 \\
\hline Autorod Copper Wedge Thickness (mm) & 3 & $1 / \sqrt{ } 3$ & neg \\
\hline Orientation of Autorod Copper Wedge $\left(^{\circ}\right)$ & Unknown & $90^{\circ} / \sqrt{ } 3$ & 0.00011 \\
\hline TRISO Random Packing & \multicolumn{2}{|c|}{ See Section 2.1.6.3 } & neg \\
\hline Kernel Radius $(\mathrm{cm})$ & 0.02510 & 0.0006 & neg \\
\hline${ }^{234}$ U Isotopic Content (wt.\%) & $\sim 0.134$ & $\sim 0.017$ & 0.00019 \\
\hline${ }^{235} \mathrm{U}$ Isotopic Content (wt.\%) & $\sim 16.762$ & $\sim 0.17$ & 0.00262 \\
\hline Fuel Pebble Uranium Mass (g) & 5.966 & 0.060 & 0.00034 \\
\hline Fueled Zone Impurities (ppm) & Table 2.1-26 & $50 \%$ & 0.00012 \\
\hline Unfueled Zone Impurities (ppm) & Table 2.1-26 & $50 \%$ & 0.00012 \\
\hline Fuel Pebble Water Content (wt.\%) & 0.01 & $0.01 / \sqrt{ } 3$ & 0.00011 \\
\hline Oxygen-to-Uranium Ratio & 2.00 & 0.01 & 0.00013 \\
\hline Moderator Pebble Mass (g) & 190.54 & 1.44 & 0.00015 \\
\hline Moderator Pebble Impurities (ppm) & Table 2.1-33 & $50 \%$ & 0.00166 \\
\hline Moderator Pebble Water Content (wt.\%) & 0.01 & $0.01 / \sqrt{ } 3$ & neg \\
\hline Control Rod Outer Tube Composition (wt.\%) & Table 2.1-36 & $1 / \sqrt{ } 3$ & neg \\
\hline Polyethylene Rod Diameter (mm) & -- & -- & NA \\
\hline Polyethylene Rod Length (mm) & -- & -- & NA \\
\hline Polyethylene Rod Linear Density $(\mathrm{g} / \mathrm{cm})$ & -- & -- & NA \\
\hline Polyethylene Rod H:C Ratio & -- & -- & NA \\
\hline Polyethylene Rod Impurities (ppm EBC) & -- & -- & NA \\
\hline Stacked Pebble Height (mm) & 1.62 & $0.06 \%$ & neg \\
\hline Total Experimental Uncertainty & -- & - & 0.00359 \\
\hline
\end{tabular}


NEA/NSC/DOC(2006)1

\section{Gas Cooled (Thermal) Reactor - GCR \\ PROTEUS-GCR-EXP-004 \\ CRIT-REAC}

Table 2.1-46. Summary of Evaluated Uncertainties in HTR-PROTEUS Case 2 (Core 10).

\begin{tabular}{|c|c|c|c|}
\hline Perturbed Parameter & $\begin{array}{c}\text { Parameter } \\
\text { Value } \\
\end{array}$ & $\begin{array}{c}1 \sigma \\
\text { Uncertainty } \\
\end{array}$ & $\Delta \mathbf{k}_{\text {eff }}(1 \sigma)$ \\
\hline Radial Reflector Density $\left(\mathrm{g} / \mathrm{cm}^{3}\right)$ & 1.76 & 0.012 & 0.00081 \\
\hline Radial Reflector Impurities (ppma EBC) & 1.33 & 0.08 & 0.00080 \\
\hline Safety Ring Composition (wt.\%) & Table 2.1-6 & $1 / \sqrt{ } 3$ & neg \\
\hline C-Driver Plug Density $\left(\mathrm{g} / \mathrm{cm}^{3}\right)$ & 1.765 & 0.012 & neg \\
\hline Location of Upper Axial Reflector (mm) & 1893 & $5 / \sqrt{ } 3$ & neg \\
\hline Upper Axial Aluminum Dimensions (mm) & Figure 1.1-4 & $1 / \sqrt{ } 3$ & 0.00046 \\
\hline Upper Axial Aluminum Composition & Table 2.1-6 & $1 / \sqrt{ } 3$ & 0.00024 \\
\hline Lower Axial Cylinder Diameter (mm) & 495 & $1 / \sqrt{ } 3$ & neg \\
\hline Lower Axial Reflector Height (mm) & 780 & $1 / \sqrt{ } 3$ & neg \\
\hline Lower Axial Annulus Density $\left(\mathrm{g} / \mathrm{cm}^{3}\right)$ & 1.76 & 0.012 & neg \\
\hline Lower Axial Cylinder Impurities (ppma EBC) & 1.33 & 0.08 & 0.00011 \\
\hline Autorod Copper Wedge Thickness (mm) & 3 & $1 / \sqrt{3}$ & neg \\
\hline Orientation of Autorod Copper Wedge $\left(^{\circ}\right)$ & Unknown & $90^{\circ} / \sqrt{ } 3$ & neg \\
\hline TRISO Random Packing & \multicolumn{2}{|c|}{ See Section 2.1.6.3 } & 0.00015 \\
\hline Kernel Radius (cm) & 0.02510 & 0.0006 & neg \\
\hline${ }^{234}$ U Isotopic Content (wt.\%) & $\sim 0.134$ & $\sim 0.017$ & 0.00014 \\
\hline${ }^{235} \mathrm{U}$ Isotopic Content (wt.\%) & $\sim 16.762$ & $\sim 0.17$ & 0.00312 \\
\hline Fuel Pebble Uranium Mass (g) & 5.966 & 0.060 & 0.00042 \\
\hline Fueled Zone Impurities (ppm) & Table 2.1-26 & $50 \%$ & neg \\
\hline Unfueled Zone Impurities (ppm) & Table 2.1-26 & $50 \%$ & 0.00017 \\
\hline Fuel Pebble Water Content (wt.\%) & 0.01 & $0.01 / \sqrt{ } 3$ & neg \\
\hline Oxygen-to-Uranium Ratio & 2.00 & 0.01 & 0.00016 \\
\hline Moderator Pebble Mass (g) & 190.54 & 1.44 & neg \\
\hline Moderator Pebble Impurities (ppm) & Table $2.1-33$ & $50 \%$ & 0.00154 \\
\hline Moderator Pebble Water Content (wt.\%) & 0.01 & $0.01 / \sqrt{ } 3$ & neg \\
\hline Control Rod Outer Tube Composition (wt.\%) & Table 2.1-36 & $1 / \sqrt{3}$ & neg \\
\hline Polyethylene Rod Diameter (mm) & 6.5 & $0.1 / \sqrt{ } 3$ & neg \\
\hline Polyethylene Rod Length (mm) & 1450 & $10 / \sqrt{ } 3$ & neg \\
\hline Polyethylene Rod Linear Density (g/cm) & 0.3161 & 0.0019 & neg \\
\hline Polyethylene Rod H:C Ratio & 2.03 & 0.03 & 0.00012 \\
\hline Polyethylene Rod Impurities (ppm EBC) & 0.5 & 0.5 & neg \\
\hline Stacked Pebble Height (mm) & 1.44 & $0.06 \%$ & neg \\
\hline Total Experimental Uncertainty & -- & -- & 0.00374 \\
\hline
\end{tabular}

Revision: 1 
NEA/NSC/DOC(2006)1

Gas Cooled (Thermal) Reactor - GCR

PROTEUS-GCR-EXP-004

CRIT-REAC

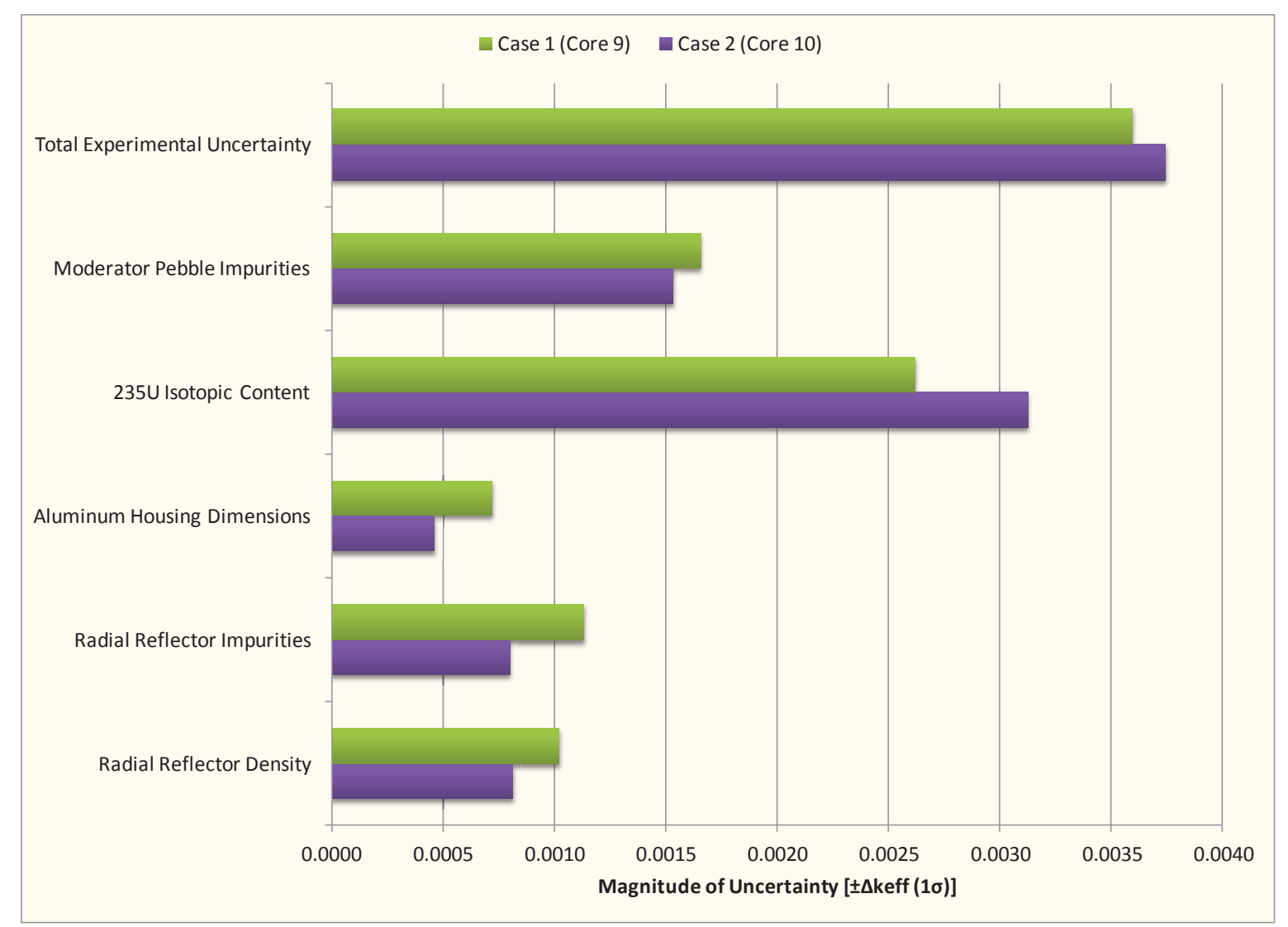

Figure 2.1-1. Graphical Representation of Primary Uncertainties in HTR-PROTEUS.

\subsection{Evaluation of Buckling and Extrapolation Length Data}

Buckling and extrapolation length measurements were performed but have not yet been evaluated.

\subsection{Evaluation of Spectral Characteristics Data}

Spectral characteristics measurements were performed but have not yet been evaluated. 
NEA/NSC/DOC(2006)1

\section{Gas Cooled (Thermal) Reactor - GCR \\ PROTEUS-GCR-EXP-004 \\ CRIT-REAC}

\subsection{Evaluation of Reactivity Effects Data}

Models based upon the benchmark description provided in Section 3.1, for Cores 9 and 10, were utilized with model perturbations simulating control rod movements, as discussed in Section 3.4, to calculate the worths reported in Section 1.4.

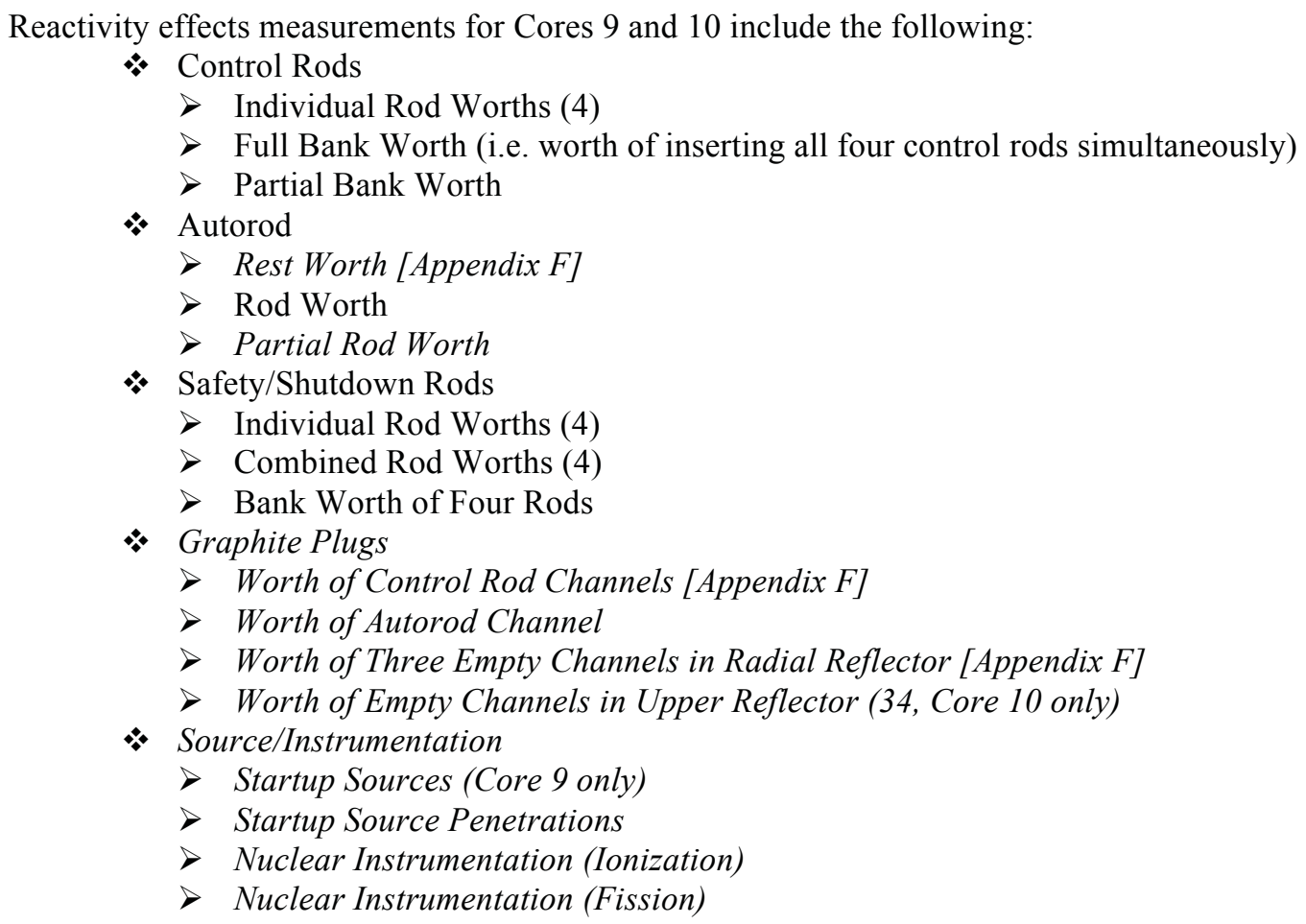

The reactivity measurements were evaluated and found to be acceptable as benchmark experiment measurements except for those indicated in italics in the above list. Some of the rejected measurements include reported worth measurements obtained by scaling measured parameters on other HTRPROTEUS core configuration using the ratio of control rod bank worths. Because these scaled measurements were not directly performed on the current core, they were rejected as benchmark measurements. However, as sufficient information was available to evaluate them, an evaluation is provided in this section of the report with modeling specifications provided in Appendix F. Further discussion of the evaluation process and unacceptability of some of the measurements is discussed in this section. A total of 32 reactivity effects measurements were determined to be acceptable benchmark experiments for both Cores 9 and 10 (16 apiece).

\subsubsection{Delayed Neutron Fraction, $\beta_{\text {eff }}$}

Reactivity worths for HTR-PROTEUS measurements were typically reported in $\$$ or $\phi$. Staff typically used TWODANT to calculate a $\beta_{\text {eff }}$ value for each of the core configurations, which was approximately $720 \mathrm{pcm}$ for most measurements, especially in Cores 9 and 10, which was then used to convert reactivity measurements to and from $\Delta \mathrm{k}$.

Calculations were performed using the adjoint-weighted point kinetics capabilities of MCNP5 ${ }^{\mathrm{a}, \mathrm{b}}$ with ENDF/B-VII.0 nuclear data to determine a $\beta_{\text {eff }}$ for Cores 9 and 10. The calculated $\beta_{\text {eff }}$ for Core 9 , using

\footnotetext{
${ }^{a}$ B. C. Kiedrowski, T. E. Booth, F. B. Brown, J. S. Bull, J. A. Favorite, R. A. Forster, and R. L. Martz, "MCNP51.60 Feature Enhancements \& Manual Clarifications," LA-UR-10-06217, Los Alamos National Laboratory (2010).

${ }^{\mathrm{b}}$ R. K. Meulekamp and S. C. van der Marck, "Calculating the Effective Delayed Neutron Fraction with Monte Carlo,” Nucl. Sci. Eng., 152, 142-148 (2006).
}

Revision: 1 
NEA/NSC/DOC(2006)1

\section{Gas Cooled (Thermal) Reactor - GCR \\ PROTEUS-GCR-EXP-004 \\ CRIT-REAC}

the benchmark model in Section 3.1 and sample input deck in Appendix A, is $693 \mathrm{pcm}$. An uncertainty of $5 \%$, which is typically applied to account for the uncertainty in nuclear data, was applied, providing an uncertainty in $\beta_{\text {eff }}$ of $35 \mathrm{pcm}$. Core 10 was calculated to have a $\beta_{\text {eff }}$ of $685 \pm 34 \mathrm{pcm}$. Because all reported reactivity measurement worths were reported in units of $\rho(\$)$ using a $\beta_{\text {eff }}$ value of 0.00720 for both Cores 9 and 10 (except for the safety/shutdown rods in Core 9, which had a reported $\beta_{\text {eff }}$ value of 0.00717 ), they were adjusted to new calculated $\beta_{\text {eff }}$ values to enable direct comparison between the calculated and benchmark worths. While the statistical uncertainty calculated by MCNP in $\beta_{\text {eff }}$ is $<10$ $\mathrm{pcm}$, the larger uncertainty of $5 \%$ is utilized to address the uncertainty in $\beta_{\text {eff }}$ due to the nuclear data parameters themselves; this was evaluated in more detail for a TRIGA-type reactor in NRAD-FUND-RESR-001 (Appendix I), has been similarly seen when evaluating other reactor types using different nuclear data libraries, and is assumed to apply herein as well.

It is assumed that use of the MCNP5-calculated values for $\beta_{\text {eff }}$ are more appropriate since they were generated using a 3D representation of the core. The difference between the MCNP5-generated values and those reported in the references as being obtained using TWODANT $\mathrm{r}-\Theta$ models is approximately $1 \sigma$. Original HTR-PROTEUS experimentalists found that 2D calculations were limited in applications requiring axial positioning of detectors and anomalous sensitivities for some measurement configurations. Both the JEF-1.1 and ENDF/B-VII.0 delayed neutron data for ${ }^{235} \mathrm{U}$ are based upon the original Keepin data; ${ }^{\mathrm{a}}$ the JEF-1.1 data was reported with a slightly greater uncertainty in the individual parameters (Ref. 10).

\subsubsection{Uncertainties in Rod Worth Measurements}

Typically the uncertainty in the measurement method is much greater than any uncertainties obtained via computational analysis of geometry and composition perturbations in the benchmark models, such as the comprehensive evaluation performed in Section 2.1 for the critical configurations.

Information is limited regarding a comprehensive analysis of the uncertainties in the HTR-PROTEUS absorber rod worth measurements, where often the reported uncertainty pertains to the statistical uncertainty in the measurements and not the additional uncertainties introduced due to techniques, methods, and rod shadowing effects. These uncertainties have been addressed in other thermal research reactors, such as TRIGA ${ }^{\circledR}$ (Training, Research, Isotopes, General Atomics) reactors, ${ }^{\text {b,c }}$ and have been utilized in this evaluation to supplement evaluation of the uncertainty in absorber worth measurements performed in the HTR-PROTEUS.

To evaluate the uncertainty in the methods utilized to measure reactivity worth, a literature survey was performed regarding other types of research reactors. While the fundamental physics behind each reactor type is different and impacts typical operations and characteristic uncertainties, the uncertainty in the measurement methods, as discussed below, are relatively similar. Therefore, it is judged to be acceptable to utilize method uncertainties for the measurement of reactivity effects from different research reactors to estimate an uncertainty in the methods utilized to measure the control rod worths in the PROTEUS reactor.

In the evaluation herein, methods and their respective uncertainties in TRIGA-type reactors have been applied to the HTR-PROTEUS experimental measurements. The HTR-PROTEUS operates in a more epithermal spectra than a TRIGA reactor, which is highly moderated by $\mathrm{ZrH}$ and water; therefore, the diffusion length is much longer in the HTR-PROTEUS. Control rod movement in a TRIGA reactor core tend to cause shadowing effects, effectively reducing the effective worth of an adjacent control rod. The PROTEUS absorber rods are located in the radial graphite reflector; hence control rod movement create

\footnotetext{
${ }^{\text {a }}$ G. R. Keepin, T. F. Wimett, R. K. Zeigler, "Delayed Neutrons from Fissionable Isotopes of Uranium, Plutonium and Thorium," J. Nucl. Energy, 6, 1-21 (1957).

${ }^{\mathrm{b}}$ TRIGA $^{\circledR}$ Nuclear Reactors, General Atomics, http://triga.ga.com/ (Accessed October 15, 2009).

${ }^{c}$ D. M. Fouquet, J. Razvi, W. L. Whittemore, "TRIGA Research Reactors: A Pathway to the Peaceful Applications of Nuclear Energy," Nuclear News, 46(12), 46-56 (2003).
}

Revision: 1

Date: March 31, 2014

Page 123 of 375 
NEA/NSC/DOC(2006)1

\section{Gas Cooled (Thermal) Reactor - GCR \\ PROTEUS-GCR-EXP-004 \\ CRIT-REAC}

anti-shadowing effects (i.e. control rod worth is increased because to localized neutron flux has increased) when the neutron flux is redistributed.

As evaluated in Section 2.1.19 of PROTEUS-GCR-EXP-001, and discussed elsewhere in a separate analysis of a TRIGA research reactor, ${ }^{a}$ the slight vertical movements and changes in position of the control rods is minor for most worth calculations, $<1 \notin$. The uncertainty in the control rod positions is assumed to be included within the uncertainty in the method.

Comparison of the measurements for the safety/shutdown rods (see Section 1.4.2.4) indicates that the $1 \sigma$ standard deviation of the mean value of multiple rod worth measurements is $\leq 11 \phi$, or $5 \%$ of a single rod worth. The standard deviation of the mean increases to $<6 \%$ for measurements of combined rod drop worths. However, no direct repeatability measurements were performed. In NRAD-FUND-RESR-001, the repeatability in a rod worth measurement was shown to be $<1 \not$.

Insufficient reactivity effects measurements were performed to support qualitative assignation of a systematic and random component to each measurement. All evaluated uncertainty components contributing to the total uncertainty in the reactivity effects measurements are assumed to be systematic and combined in quadrature to obtain the total estimated uncertainty in each experimental value.

Expert judgment was used to assign uncertainty. In the evaluation of rod worths measurements and their respective uncertainties, static conditions are utilized to simulate these dynamic measurements.

\subsubsection{Rod Drop Method and Rod Shadowing Effects}

The excitation of a multiplying system, such as a critical reactor, by a rapid change of state, such as a rod drop, will generate short-lived flux modes that are not characteristic of the fundamental mode of the system. However, on the time scales of minutes, the typical period over which rod drop measurements are performed and measured, these harmonics are generally negligible (Ref. 3).

The prompt rod drop method is the easiest way to estimate the reactivity change in a reactor core; this method is based on the prompt flux adjustment that occurs directly after a perturbation, and assumes that the delayed neutron source is constant compared to the initial state. This method is sensitive to spatial effects and less accurate than other methods. Typical uncertainties are on the order of 5-6\% for a TRIGA research reactor. ${ }^{\mathrm{b}}$, The dominant systematic uncertainty is in the kinetic constants and flux perturbations with the statistical component of the uncertainty $<1 \%$.

Common uncertainties for rod insertion methods include uncertainties in the delayed neutron data, which is systematic and common to all measuring methods. Another uncertainty source is in the flux redistribution in the subcritical core. As mentioned previously, the changes in flux are dominated by the long-lived delayed neutron precursors, whose distribution closely resembled that of the critical reactor shortly after the perturbation of the core. The most significant uncertainty source is the flux redistribution in the presence of control rods, i.e., rod shadowing effects. The positions of the control rods in the reactor impact the worth of the rod being measured. The uncertainty in this method is reported to be $3 \%-5 \%$ for individual control rod worths in a TRIGA research reactor, with a statistical

\footnotetext{
a I. Mele, M. Ravnik, and A. Trkov, "TRIGA Mark II Benchmark Experiment, Part I: Steady-State Operation," Nucl. Technol., 105, 37-51 (1994).

${ }^{\mathrm{b}}$ C. Jammes, B. Geslot, R. Rosa, G. Imel, and P. Fougeras, "Comparison of Reactivity Estimations Obtained from Rod-Drop and Pulsed Neutron Source Experiments," Ann. Nucl. Energy, 32, 1131-1145 (2005).

${ }^{\mathrm{c}}$ G. Perret, C. Jammes, G. Imel, C. Destouches, P. Chaussonnet, J. M. Laurens, R. Soule, G. M. Thomas, W. Assal, P. Fougeras, P. Blaise, J-P. Hudelot, H. Philibert, and G. Bignan, "Determination of Reactivity by a Revised RodDrop Technique in the MUSE-4 Programme - Comparison with Dynamic Measurements," $7^{\text {th }}$ Information Exchange Meeting on Actinide and Fission Product Partitioning and Transmutation, Juja, Korea, October 14-16 (2002).
}

Revision: 1

Date: March 31, 2014

Page 124 of 375 
NEA/NSC/DOC(2006)1

\section{Gas Cooled (Thermal) Reactor - GCR \\ PROTEUS-GCR-EXP-004 \\ CRIT-REAC}

error of $<0.2 \%$. However, the impact of the interference due to the presence of other control rods increases the estimated uncertainty to $\sim 10 \%$. ${ }^{\text {a }}$

The rod insertion method is very similar to the rod drop method, except that the control rods are driven into the core instead of dropped. It has been shown that flux perturbation effects due to control rods present in the core (rod shadowing) can impact the measured worth of a control rod by more than $30 \%$. On average, however, the reported values for this analysis of a TRIGA research reactor varied $\sim 8 \%$ and the core operated with most of the control rods partially inserted into the core. ${ }^{b}$

Insufficient information is available regarding the exact positions, sizes, and compositions of the various detectors utilized throughout the HTR-PROTEUS experimental program. Should sufficient information have been available, calculation of flux form factors to account for the redistribution of the flux could be performed to very accurately assess the uncertainty contribution from rod shadowing effects. ${ }^{a, c}$ As such, this uncertainty was estimated as discussed in the next paragraph.

Comparison of the difference between the sum of the individual control rod worths (see Table 1.4-2) with the measured control rod bank worth (see Section 1.4.2.2), indicates a difference of $\sim 4 \%$ in Core 9 and $\sim 2 \%$ in Core 10. Then comparison of the sum of the average measured worth of Shutdown Rods 5 through 8 and the measured worth for dropping all rods together (see Tables 1.4-8 and 1.4-9 for Cores 9 and 10 , respectively) indicates a difference of $\sim 11 \%$ in Core 9 and $\sim 13 \%$ in Core 10 . It is assumed that these differences can be utilized to approximate an additional bounding uncertainty (i.e. $\div \sqrt{3}$ ) that can be applied to the method uncertainty to account for shadowing effects in the HTR-PROTEUS absorber rod measurements.

\subsubsection{Stable Period Method}

The positive period method is also referred to as the rod exchange method or stable period method, where the rod worth is measured relative to another, calibrated, control rod, or stepwise movements of the rod are measured with a reactivity meter. Calibration curve measurements in a TRIGA research reactor have shown that the uncertainty in rod exchange measurements are slightly $<10 \%$. The uncertainty includes uncertainties in control rod positions, interference effects from other control rods during the measurement (rod shadowing), and statistical errors from reactivity calculated by the reactivity meter; the latter of which are negligible. ${ }^{\text {d }}$

Due to the severe local flux distribution deformation in rod exchange measurements, simulations need to model the effects correctly for each measurement step. An uncertainty of $10 \%$ is typical for this type of measurement, ${ }^{\mathrm{e}}$ and has become the standard for treating the uncertainty in reactivity measurements in other TRIGA research reactors. ${ }^{\mathrm{f}}$

\footnotetext{
${ }^{\text {a } I . ~ M e l e, ~ M . ~ R a v n i k, ~ a n d ~ A . ~ T r k o v, ~ “ T R I G A ~ M a r k ~ I I ~ B e n c h m a r k ~ E x p e r i m e n t, ~ P a r t ~ I I: ~ P u l s e ~ O p e r a t i o n, ” ~ N u c l . ~}$ Technol., 105, 52-58 (1994).

${ }^{\mathrm{b}}$ A. Trkov, M. Ravnik, H. Wimmer, B. Glumac, and H. Böck, “Application of the Rod-Insertion Method for Control Rod Worth Measurements in Research Reactors," Kerntechnik, 60, 255-261 (1995).

${ }^{c}$ M. Pdvratnik, L. Snoj, A. Trkov, G. Žerovnik, "Calculations to Support Absolute Thermal Power Calibration of the Slovenian TRIGA Mark II Reactor,” $20^{\text {th }}$ Int. Conf. Nuclear Energy for New Europe 2011, Bovec, Slovenia, September 12-15, 2011.

d I. Mele, M. Ravnik, and A. Trkov, "TRIGA Mark II Benchmark Experiment, Part II: Pulse Operation,” Nucl. Technol., 105, 52-58 (1994).

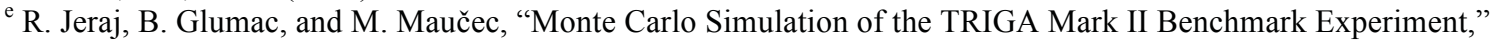
Nucl. Technol., 120, 179-187 (1997).

${ }^{\mathrm{f}} \mathrm{T}$. Matsumoto and N. Hayakawa, "Benchmark Analysis of TRIGA Mark II Reactivity Experiment Using a Continuous Energy Monte Carlo Code MCNP,” J. Nucl. Sci. Technol., 37, 1082-1087 (2000).
}

Revision: 1

Date: March 31, 2014

Page 125 of 375 
NEA/NSC/DOC(2006)1

\author{
Gas Cooled (Thermal) Reactor - GCR \\ PROTEUS-GCR-EXP-004 \\ CRIT-REAC
}

\title{
2.4.2.3 Pulsed Neutron Source Method
}

Uncertainties have been reported for pulse neutron source measurements as low as $1 \%$. $^{\mathrm{a}}$ However, the typical uncertainties are reported in Table 1.4-1 for the three PNS techniques. The Sjöstrand and Gozani techniques are utilized in the HTR-PROTEUS safety/shutdown rod worth measurements. As the worth of those rods (individually or in combination) is $>1 \$$, the uncertainty in the measurement technique is between $3 \%$ and $4 \%$.

\subsubsection{Uncertainties Applied to Worth Measurements in HTR-PROTEUS}

An uncertainty of $6 \%$ is selected to represent the uncertainty in the inverse kinetics and stable period techniques utilized to obtain a single experimental worth measurement pertaining to the control rods, autorod, and graphite plugs; this uncertainty includes the small contributions due to statistical and repeatability uncertainties $(<1 \%)$, some minor contribution due to rod shadowing effects $(\sim 1 \%-2 \%)$, and the dominant contribution from the measurement techniques $(\sim 5 \%-6 \%)$. The statistical and repeatability uncertainties are known to be small, as discussed in Sections 2.4.2 and 2.4.2.1. The rod shadowing effect uncertainty was derived for these HTR-PROTEUS control rod measurements at the end of Section 2.4.2.1. The uncertainties in rod drop measurements were derived from the uncertainty inverse kinetics methods of TRIGA reactors, also discussed in Section 2.4.2.1. The reported uncertainty of a typical stable period measurement of $<10 \%$ (see Section 2.4.2.2) included rod shadowing effects; it is assumed that the estimated total uncertainty of $6 \%$ adequately represents a $1 \sigma$ uncertainty in the control rod worth measurements for HTR-PROTEUS Cores 9 and 10.

An uncertainty of $8 \%$ is selected to represent the uncertainty in the pulsed neutron source techniques utilized to obtain a single experimental worth measurement pertaining to safety/shutdown rods; this uncertainty includes the small contributions due to statistical and repeatability uncertainties $(<1 \%)$, some minor contribution due to the measurement techniques $(\sim 3 \%-4 \%)$, and the dominant contribution due to rod shadowing effects $(\sim 6 \%-8 \%)$. Although safety/shutdown rod measurements were performed using IK and/or PNS measurements, the larger uncertainty of $8 \%$ was selected instead of $6 \%$ as there is possibly a trade off between technique uncertainty and the uncertainty in shadowing effects impacting the measurement technique. The statistical and repeatability uncertainties are known to be small, as discussed in Sections 2.4.2 and 2.4.2.1. The rod shadowing effect uncertainty was derived for these HTR-PROTEUS safety/shutdown rod measurements at the end of Section 2.4.2.1. The uncertainty in PNS measurements were discussed in Section 2.4.2.3. It is assumed that the estimated total uncertainty of $8 \%$ adequately represents a $1 \sigma$ uncertainty in the safey/shutdown rod worth measurements for HTRPROTEUS Cores 9 and 10.

The worths of the graphite plugs were estimated based on measurements performed on similar HTRPROTEUS core configurations; the reported uncertainties for Cores 9 and 10 are greater than the estimated $6 \%$. The larger reported uncertainties will be utilized as the uncertainties in the graphite plug worths.

\subsubsection{Control Rod Worth Measurements}

Individual and bank worth measurements were performed for the four withdrawable stainless steel control rods. Radial positions in the core are shown in Figure 1.1-2 (outermost radial positions). The individual rod worths are found in Table 1.4-2. Due to rod shadowing effects, the summation of the individual rod worths incorrectly reflects the worth of the control rod bank. Measured control rod bank insertions (partial and full) are recorded in Section 1.4.2.2. The reported values were adjusted to use the same $\beta_{\text {eff }}$ value (see Section 2.4.1) as used in the MCNP calculations. The evaluated experimental values are provided in Tables 2.4-1 and 2.4-2 for Cores 9 and 10, respectively.

\footnotetext{
${ }^{a}$ C. Jammes, B. Geslot, R. Rosa, G. Imel, and P. Fougeras, "Comparison of Reactivity Estimations Obtained from Rod-Drop and Pulsed Neutron Source Experiments," Ann. Nucl. Energy, 32, 1131-1145 (2005).

Revision: 1

Date: March 31, 2014

Page 126 of 375
} 
NEA/NSC/DOC(2006)1

\section{Gas Cooled (Thermal) Reactor - GCR \\ PROTEUS-GCR-EXP-004 \\ CRIT-REAC}

Table 2.4-1. Evaluated Control Rod Worth Measurements (Core 9).

\begin{tabular}{|c|c|c|c|c|c|c|c|}
\hline \multirow{2}{*}{$\begin{array}{c}\operatorname{Rod}(s) \\
\text { Inserted }\end{array}$} & \multirow{2}{*}{$\begin{array}{l}\text { Measurement } \\
\text { Technique }^{(a)}\end{array}$} & \multicolumn{3}{|c|}{ Measured Worth } & \multicolumn{3}{|c|}{ Adjusted Worth ${ }^{(b)}$} \\
\hline & & $\rho(\$)$ & \pm & $\sigma$ & $\rho(\$)$ & \pm & $\sigma^{(\mathbf{c})}$ \\
\hline 1 & IK & -0.3969 & \pm & 0.0009 & -0.41 & \pm & 0.02 \\
\hline 2 & $\mathrm{IK}$ & -0.3904 & \pm & 0.0009 & -0.41 & \pm & 0.02 \\
\hline 3 & IK & -0.3907 & \pm & 0.0009 & -0.41 & \pm & 0.02 \\
\hline 4 & $\mathrm{IK}$ & -0.3961 & \pm & 0.0009 & -0.41 & \pm & 0.02 \\
\hline Full Bank & SP & -1.52 & \pm & 0.02 & -1.58 & \pm & 0.09 \\
\hline Partial Bank $^{(\mathrm{d})}$ & SP & -0.704 & \pm & 0.01 & -0.73 & \pm & 0.04 \\
\hline
\end{tabular}

(a) $\mathrm{IK}=$ Inverse Kinetics; SP = Stable Period

(b) The measured worth is adjusted per the discussion in Section 2.4.1 from the reported calculated $\beta_{\text {eff }}$ of $720 \mathrm{pcm}$ to the currently calculated value of $693 \mathrm{pcm}$. The number of significant digits is reduced due to the increased uncertainty in the adjusted worths.

(c) The uncertainty in the measured worth is increased to at least $6 \%$ when the reported uncertainty in the measurement is less than this minimum uncertainty value.

(d) Partial insertion was performed from a control rod bank position of 1620 $\mathrm{mm}$.

Table 2.4-2. Evaluated Control Rod Worth Measurements (Core 10).

\begin{tabular}{|c|c|c|c|c|c|c|c|}
\hline \multirow{2}{*}{$\begin{array}{c}\operatorname{Rod}(s) \\
\text { Inserted }\end{array}$} & \multirow{2}{*}{$\begin{array}{l}\text { Measurement } \\
\text { Technique }^{(a)}\end{array}$} & \multicolumn{3}{|c|}{ Measured Worth } & \multicolumn{3}{|c|}{ Adjusted Worth ${ }^{(b)}$} \\
\hline & & $\rho(\$)$ & \pm & $\boldsymbol{\sigma}$ & $\rho(\$)$ & \pm & $\sigma^{(\mathrm{c})}$ \\
\hline 1 & $\mathrm{IK}$ & -0.2819 & \pm & 0.0007 & -0.30 & \pm & 0.02 \\
\hline 2 & $\mathrm{IK}$ & -0.2785 & \pm & 0.00084 & -0.29 & \pm & 0.02 \\
\hline 3 & IK & -0.2764 & \pm & 0.00074 & -0.29 & \pm & 0.02 \\
\hline 4 & $\mathrm{IK}$ & -0.2815 & \pm & 0.00071 & -0.30 & \pm & 0.02 \\
\hline Full Bank & SP & -1.096 & \pm & -- & -1.15 & \pm & 0.07 \\
\hline Partial Bank $^{(\mathrm{d})}$ & SP & -0.368 & \pm & 0.01 & -0.39 & \pm & 0.02 \\
\hline
\end{tabular}

(a) $\mathrm{IK}=$ Inverse Kinetics; $\mathrm{SP}=$ Stable Period

(b) The measured worth is adjusted per the discussion in Section 2.4.1 from the reported calculated $\beta_{\text {eff }}$ of $720 \mathrm{pcm}$ to the currently calculated value of $685 \mathrm{pcm}$. The number of significant digits is reduced due to the increased uncertainty in the adjusted worths.

(c) The uncertainty in the measured worth is increased to at least $6 \%$ when the reported uncertainty in the measurement is less than this minimum uncertainty value.

(d) Partial insertion was performed from a control rod bank position of 1540 $\mathrm{mm}$. 
NEA/NSC/DOC(2006)1

\section{Gas Cooled (Thermal) Reactor - GCR \\ PROTEUS-GCR-EXP-004 \\ CRIT-REAC}

\subsubsection{Autorod Worth Measurements}

Worth measurements were performed for the single autorod. The radial position of the autorod is shown in Figure 1.1-2. The rest worth (i.e. the worth of removing the absorber rod after it has been fully withdrawn), the full rod insertion worth, and partial rod insertion worths are reported for both Cores 9 and 10 in Section 1.4.2.3. For Core 9, the total insertion worth of the autorod (reported in Table 1.4-3) was selected over the worth reported to scaling Core 5 worth measurements because the former was physically measured. Partial autorod worth measurements were not evaluated as their respective worths were almost identical to the full insertion worth of the autorod within experimental measurements uncertainty. The reported values were adjusted to use the same $\beta_{\text {eff }}$ value (see Section 2.4.1) as used in the MCNP calculations. The evaluated experimental values are provided in Tables 2.4-3 and 2.4-4 for Cores 9 and 10, respectively.

The autorod rest worth measurements were scaled measurements and not considered as a benchmark measurements.

Table 2.4-3. Evaluated Autorod Worth Measurements (Core 9).

\begin{tabular}{|c|c|cccc|ccc||}
\hline \multirow{2}{*}{$\begin{array}{c}\text { Measured } \\
\text { Value }\end{array}$} & \multirow{2}{*}{ Measurement $^{\text {Technique }^{(\mathbf{a})}}$} & \multicolumn{2}{|c|}{ Measured Worth } & \multicolumn{3}{|c|}{ Adjusted Worth } \\
& $\mathbf{\rho}(\mathbf{\$})$ & \pm & $\boldsymbol{\sigma}$ & $\boldsymbol{\rho}(\mathbf{\$})$ & \pm & $\boldsymbol{\sigma}^{(\mathbf{c})}$ \\
\hline Rest Worth & $\begin{array}{c}\text { Scaled from } \\
\text { Core 5 Value }\end{array}$ & -0.125 & \pm & 0.005 & -0.13 & \pm & 0.01 \\
\hline Insertion Worth & IK & -0.0918 & \pm & 0.0006 & -0.10 & \pm & 0.01 \\
\hline
\end{tabular}

(a) $\mathrm{IK}=$ Inverse Kinetics

(b) The measured worth is adjusted per the discussion in Section 2.4.1 from the reported calculated $\beta_{\text {eff }}$ of $720 \mathrm{pcm}$ to the currently calculated value of $693 \mathrm{pcm}$. The number of significant digits is reduced due to the increased uncertainty in the adjusted worths.

(c) The uncertainty in the measured worth is increased to at least $6 \%$ when the reported uncertainty in the measurement is less than this minimum uncertainty value.

Table 2.4-4. Evaluated Autorod Worth Measurements (Core 10).

\begin{tabular}{|c|c|c|c|c|c|c|c|}
\hline \multirow{2}{*}{$\begin{array}{l}\text { Measured } \\
\text { Value }\end{array}$} & \multirow{2}{*}{$\underset{\text { Technique }^{(\mathrm{a})}}{\text { Measurement }}$} & \multicolumn{3}{|c|}{ Measured Worth } & \multicolumn{3}{|c|}{ Adjusted Worth ${ }^{(b)}$} \\
\hline & & & + & 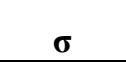 & & 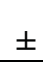 & $\sigma^{(\mathrm{c})}$ \\
\hline & & & & & -0.081 & \pm & 0. \\
\hline 100 & IK & -0.0696 & \pm & 0.00044 & -0.073 & \pm & 0.004 \\
\hline
\end{tabular}

(a) $\mathrm{IK}=$ Inverse Kinetics

(b) The measured worth is adjusted per the discussion in Section 2.4.1 from the reported calculated $\beta_{\text {eff }}$ of $720 \mathrm{pcm}$ to the currently calculated value of $685 \mathrm{pcm}$.

(c) The uncertainty in the measured worth is increased to at least $6 \%$ when the reported uncertainty in the measurement is less than this minimum uncertainty value. 
NEA/NSC/DOC(2006)1

\section{Gas Cooled (Thermal) Reactor - GCR \\ PROTEUS-GCR-EXP-004 \\ CRIT-REAC}

\subsubsection{Safety/Shutdown Rod Worth Measurements}

\subsubsection{Tube Compositions}

The information provided in this section is necessary to develop the benchmark model atom densities in Section 3.4.3 for the safety/shutdown absorber rods, whereas insufficient information was provided in Section 1.4.3.

The safety/shutdown absorber rods consisted of borated steel rod section ( $\sim 5 \mathrm{wt} . \%$ boron) enclosed within $18 / 8$ stainless steel tubes. The borated steel was reported to have a density of $6.878 \mathrm{~g} / \mathrm{cm}^{3}$ and $7.92 \mathrm{~g} / \mathrm{cm}^{3}$ for the stainless steel. The borated steel composition, as reported, is in Table 1.1-9. The stainless steel composition, as reported, is in Table 1.1-10. Because the borated steel composition does not total to $100 \mathrm{wt} . \%$, it was renormalized, as provided in Table 2.4-5.

Table 2.4-5. Composition of Borated Steel.

\begin{tabular}{||c|c||c||}
\hline Element & $\begin{array}{c}\text { Reported } \\
\text { Composition } \\
\text { (wt.\%) }\end{array}$ & $\begin{array}{c}\text { Renormalized } \\
\text { Composition } \\
\text { (wt.\%) }\end{array}$ \\
\hline \hline${ }^{10} \mathrm{~B}$ & 0.94 & 0.94902 \\
\hline${ }^{11} \mathrm{~B}$ & 3.76 & 3.79606 \\
\hline $\mathrm{Si}$ & 1.02 & 1.02978 \\
\hline $\mathrm{Cr}$ & 40.4 & 40.78748 \\
\hline $\mathrm{Mn}$ & 1.30 & 1.31247 \\
\hline $\mathrm{Fe}$ & 41.8 & 42.20091 \\
\hline $\mathrm{Ni}$ & 9.83 & 9.92428 \\
\hline \hline Total & 99.05 & 100.00000 \\
\hline
\end{tabular}

The 18/8 stainless steel has a comparable composition to Type 301/302/304 stainless steel, which include the additional constituents carbon, silicon, and manganese. ${ }^{\text {a }}$ The iron content in the 18/8 stainless steel composition was slightly reduced to include these additional elements. The revised stainless steel tube composition for the safety/shutdown rods is provide in Table 2.4-6.

Table 2.4-6. Composition of 18/8 Stainless Steel.

\begin{tabular}{|c|c||c||}
\hline Element & $\begin{array}{c}\text { Reported } \\
\text { Composition } \\
\text { (wt.\%) }\end{array}$ & $\begin{array}{c}\text { Revised } \\
\text { Composition } \\
\text { (wt.\%) }\end{array}$ \\
\hline \hline $\mathrm{Cr}$ & 18 & 18 \\
\hline $\mathrm{Fe}$ & 74 & 72.425 \\
\hline $\mathrm{Ni}$ & 8 & 8 \\
\hline $\mathrm{C}$ & -- & 0.075 \\
\hline $\mathrm{Si}$ & -- & 0.5 \\
\hline $\mathrm{Mn}$ & -- & 1 \\
\hline \hline Total & 100 & 100 \\
\hline
\end{tabular}

The aluminum shock dampers were not included in this analysis; the impact of their removal on reactivity measurements is negligible.

\footnotetext{
${ }^{\text {a }}$ R. H. Perry and Don W. Green, Perry's Chemical Engineers' Handbook, $7^{\text {th }}$ edition, McGraw-Hill, New York, NY (1997).
}

Revision: 1 
NEA/NSC/DOC(2006)1

\section{Gas Cooled (Thermal) Reactor - GCR \\ PROTEUS-GCR-EXP-004 \\ CRIT-REAC}

\subsubsection{Consolidation of Worth Measurements}

Worth measurements were performed for four of the safety/shutdown absorber rods (identified as Rods 5, 6, 7, and 8). The radial positions in the core are shown in Figure 1.1-2. Various individual rod worths and combined rod drop worths were measured, including a bank worth with all four rods, as provided in Section 1.4.2.4. An average worth for each measurement was determined after removing repetitive measurements from the literature and "bad measurements", as indicated by the experimenters. A summary of the various measurements reported in Tables 1.4-4 through 1.4-13 is provided below in Tables 2.4-7 and 2.4-8 for Cores 9 and 10, respectively. The summary tables indicate which measurements were included in determination of the benchmark experiment absorber rod worths.

Mean values were computed using the non-excluded measurements by computing a non-weighted average and standard deviation for each set of worth measurements. The average values were then adjusted to use the same $\beta_{\text {eff }}$ value (see Section 2.4.1) as used in the MCNP calculations. The standard deviation in each average value was less than $8 \%$; therefore, the uncertainty was increased to $8 \%$ for each measurement. Finally the uncertainty was divided by the square-root of the number of individual measurements performed for each mean worth value to obtain the uncertainty for each experimental worth. The evaluated experimental values are provided in Tables 2.4-9 and 2.4-10 for Cores 9 and 10, respectively.

Table 2.4-7. Summary of Safety/Shutdown Rod Worth Measurements (Core 9).

\begin{tabular}{|c|c|c|c|}
\hline $\begin{array}{l}\text { Rods(s) } \\
\text { Inserted }\end{array}$ & Table & $\begin{array}{c}\text { Reported Worth } \\
\rho(\$)\end{array}$ & Reason for Exclusion \\
\hline \multirow[t]{3}{*}{$?$} & $1.4-11$ & -3.64 & Unclear which single rod was inserted \\
\hline & & -3.70 & Unclear which single rod was inserted \\
\hline & & -3.62 & Unclear which single rod was inserted \\
\hline \multirow[t]{4}{*}{5} & $1.4-8$ & -3.69 & \\
\hline & & -3.61 & \\
\hline & $1.4-12$ & -3.558 & \\
\hline & & -4.880 & Reported as a bad detector \\
\hline \multirow[t]{14}{*}{6} & $1.4-4$ & -3.73 & \\
\hline & & -3.63 & \\
\hline & & -3.69 & \\
\hline & & -3.77 & \\
\hline & & -3.73 & \\
\hline & & -3.74 & Average of previous measurements \\
\hline & $1.4-6$ & -3.73 & Average of previous measurements \\
\hline & & -3.68 & Average of previous measurements \\
\hline & $1.4-8$ & -3.72 & \\
\hline & & -3.73 & \\
\hline & & -3.63 & \\
\hline & $1.4-10$ & -3.68 & Same average value as found in Table 1.4-6 \\
\hline & $1.4-12$ & -3.594 & \\
\hline & & -4.185 & Reported as a bad detector \\
\hline \multirow[t]{2}{*}{7} & $1.4-12$ & -3.578 & \\
\hline & & -4.040 & Reported as a bad detector \\
\hline 8 & $1.4-12$ & -3.482 & \\
\hline
\end{tabular}

Revision: 1 
NEA/NSC/DOC(2006)1

\section{Gas Cooled (Thermal) Reactor - GCR \\ PROTEUS-GCR-EXP-004 \\ CRIT-REAC}

Table 2.4-7. (cont'd.) Summary of Safety/Shutdown Rod Worth Measurements (Core 9).

\begin{tabular}{|c|c|c|c|}
\hline $\begin{array}{l}\text { Rods(s) } \\
\text { Inserted }\end{array}$ & Table & $\begin{array}{c}\text { Reported Worth } \\
\rho(\$)\end{array}$ & Reason for Exclusion \\
\hline \multirow[t]{15}{*}{$5+6$} & $1.4-4$ & -7.71 & \\
\hline & & -7.74 & \\
\hline & & -7.88 & \\
\hline & & -7.85 & \\
\hline & & -7.82 & Average of previous measurements \\
\hline & $1.4-6$ & -7.78 & Average of previous measurements \\
\hline & & -7.85 & Average of previous measurements \\
\hline & $1.4-8$ & -7.69 & \\
\hline & & -7.72 & \\
\hline & $1.4-10$ & -7.85 & Same average value as found in Table 1.4-6 \\
\hline & $1.4-11^{(\mathrm{a})}$ & -7.74 & \\
\hline & & -7.83 & \\
\hline & & -7.89 & \\
\hline & $1.4-12$ & -7.48 & \\
\hline & & -8.71 & Reported as a bad detector \\
\hline $5+7$ & $1.4-12$ & -7.19 & \\
\hline $5+8$ & $1.4-12$ & -7.15 & \\
\hline \multirow[t]{16}{*}{$5+6+7$} & $1.4-4$ & -11.69 & \\
\hline & & -11.36 & \\
\hline & & -11.63 & \\
\hline & & -12.25 & \\
\hline & & -11.85 & \\
\hline & & -11.83 & Average of previous measurements \\
\hline & $1.4-6$ & -11.86 & Average of previous measurements \\
\hline & & -11.61 & Average of previous measurements \\
\hline & $1.4-8$ & -11.61 & \\
\hline & & -11.76 & \\
\hline & & -11.23 & \\
\hline & & -11.49 & \\
\hline & $1.4-10$ & -11.61 & Same average value as found in Table 1.4-6 \\
\hline & $1.4-11^{(b)}$ & -11.63 & \\
\hline & & -11.91 & \\
\hline & & -12.03 & \\
\hline \multirow[t]{14}{*}{$5+6+7+8$} & $1.4-4$ & -16.00 & \\
\hline & & -15.63 & \\
\hline & & -16.39 & \\
\hline & & -16.43 & \\
\hline & & -16.17 & Average of previous measurements \\
\hline & $1.4-6$ & -16.01 & Average of previous measurements \\
\hline & & -16.43 & Average of previous measurements \\
\hline & $1.4-8$ & -16.10 & \\
\hline & & -15.89 & \\
\hline & $1.4-10$ & -16.43 & Same average value as found in Table 1.4-6 \\
\hline & $1.4-11^{(\mathrm{b})}$ & -15.63 & \\
\hline & & -15.52 & \\
\hline & & -16.81 & \\
\hline & $1.4-12$ & -15.25 & \\
\hline
\end{tabular}

(a) Due to the similarity in worth measurement magnitudes for Table 1.4-11 dual-rod insertions, and the typical insertion of both Rods 5 and 6 concurrently, these three measurements are assumed to pertain to this measurement.

(b) Similar argument for footnote (a) also applies for the triple- and quad-rod insertions in Table 1.4-11.

Revision: 1 
NEA/NSC/DOC(2006)1

Gas Cooled (Thermal) Reactor - GCR

PROTEUS-GCR-EXP-004

CRIT-REAC

Table 2.4-8. Summary of Safety/Shutdown Rod Worth Measurements (Core 10).

\begin{tabular}{|c|c|c|c|}
\hline $\begin{array}{l}\text { Rods(s) } \\
\text { Inserted }\end{array}$ & Table & $\begin{array}{c}\text { Reported Worth } \\
\rho(\$)\end{array}$ & Reason for Exclusion \\
\hline \multirow[t]{6}{*}{5} & $1.4-9$ & -2.75 & \\
\hline & & -2.63 & \\
\hline & $1.4-13$ & -2.72 & \\
\hline & & -3.10 & Reported as a bad detector \\
\hline & & -2.63 & \\
\hline & & -2.75 & Average of previous measurements \\
\hline \multirow[t]{13}{*}{6} & $1.4-5$ & -2.75 & Rejected by experimenter \\
\hline & & -2.63 & \\
\hline & & -2.65 & \\
\hline & & -2.69 & \\
\hline & & -2.59 & \\
\hline & & -2.66 & Average of previous measurements \\
\hline & $1.4-7$ & -2.70 & Average of previous measurements \\
\hline & & -2.61 & Average of previous measurements \\
\hline & $1.4-9$ & -2.79 & \\
\hline & $1.4-13$ & -2.74 & \\
\hline & & -2.92 & Reported as a bad detector \\
\hline & & -2.71 & \\
\hline & & -2.77 & Average of previous measurements \\
\hline \multirow[t]{4}{*}{7} & $1.4-13$ & -2.73 & \\
\hline & & -2.92 & Reported as a bad detector \\
\hline & & -2.60 & \\
\hline & & -2.74 & Average of previous measurements \\
\hline \multirow[t]{4}{*}{8} & $1.4-13$ & -2.67 & \\
\hline & & -2.56 & Reported as a bad detector \\
\hline & & -2.51 & \\
\hline & & -2.62 & Average of previous measurements \\
\hline
\end{tabular}

Revision: 1 
NEA/NSC/DOC(2006)1

\section{Gas Cooled (Thermal) Reactor - GCR \\ PROTEUS-GCR-EXP-004 \\ CRIT-REAC}

Table 2.4-8. (cont'd.) Summary of Safety/Shutdown Rod Worth Measurements (Core 10).

\begin{tabular}{|c|c|c|c|}
\hline $\begin{array}{l}\text { Rods(s) } \\
\text { Inserted }\end{array}$ & Table & $\begin{array}{c}\text { Reported Worth } \\
\rho(\$) \\
\end{array}$ & Reason for Exclusion \\
\hline \multirow[t]{14}{*}{$5+6$} & $1.4-5$ & -6.17 & Rejected by experimenter \\
\hline & & -5.56 & \\
\hline & & -5.48 & \\
\hline & & -5.72 & \\
\hline & & -5.47 & \\
\hline & & -5.54 & Average of previous measurements \\
\hline & $1.4-7$ & -5.79 & Average of previous measurements \\
\hline & & -5.52 & Average of previous measurements \\
\hline & $1.4-9$ & -6.17 & \\
\hline & & -5.56 & \\
\hline & $1.4-13$ & -5.79 & \\
\hline & & -6.43 & Reported as a bad detector \\
\hline & & -5.52 & \\
\hline & & -5.76 & Average of previous measurements \\
\hline \multirow[t]{4}{*}{$5+7$} & $1.4-13$ & -5.65 & \\
\hline & & -6.17 & Reported as a bad detector \\
\hline & & -5.26 & \\
\hline & & -5.54 & Average of previous measurements \\
\hline \multirow[t]{4}{*}{$5+8$} & $1.4-13$ & -5.59 & \\
\hline & & -6.25 & Reported as a bad detector \\
\hline & & -5.35 & \\
\hline & & -5.64 & Average of previous measurements \\
\hline \multirow[t]{17}{*}{$5+6+7$} & $1.4-5$ & -9.38 & Rejected by experimenter \\
\hline & & -8.61 & \\
\hline & & -8.42 & \\
\hline & & -9.38 & \\
\hline & & -8.64 & \\
\hline & & -8.91 & Average of previous measurements \\
\hline & $1.4-7$ & -9.06 & Average of previous measurements \\
\hline & & -8.63 & Average of previous measurements \\
\hline & $1.4-9$ & -8.72 & \\
\hline & & -9.60 & \\
\hline & & -9.83 & \\
\hline & & -8.26 & \\
\hline & & -8.99 & \\
\hline & & -8.68 & \\
\hline & & -8.18 & \\
\hline & & -8.94 & \\
\hline & & -8.60 & \\
\hline \multirow[t]{17}{*}{$5+6+7+8$} & $1.4-5$ & -12.99 & Rejected by experimenter \\
\hline & & -11.80 & \\
\hline & & -11.42 & \\
\hline & & -12.12 & \\
\hline & & -11.71 & \\
\hline & & -11.74 & Average of previous measurements \\
\hline & $1.4-7$ & -12.18 & Average of previous measurements \\
\hline & & -11.76 & Average of previous measurements \\
\hline & $1.4-9$ & -12.04 & \\
\hline & & -13.94 & \\
\hline & & -11.70 & \\
\hline & & -11.71 & \\
\hline & & -11.98 & \\
\hline & $1.4-13$ & -12.63 & \\
\hline & & -12.93 & Reported as a bad detector \\
\hline & & -11.57 & \\
\hline & & -12.43 & Average of previous measurements \\
\hline
\end{tabular}

Revision: 1 
NEA/NSC/DOC(2006)1

\section{Gas Cooled (Thermal) Reactor - GCR \\ PROTEUS-GCR-EXP-004 \\ CRIT-REAC}

Table 2.4-9. Evaluated Safety/Shutdown Rod Worth Measurements (Core 9).

\begin{tabular}{|c|c|c|c|c|c|c|c|}
\hline $\begin{array}{c}\operatorname{Rod}(\mathbf{s}) \\
\text { Inserted }\end{array}$ & $\begin{array}{c}\text { Number } \\
\text { Unique } \\
\text { Values (N) } \\
\end{array}$ & $\begin{array}{l}\text { Measurement } \\
\text { Technique(s) }\end{array}$ & $\begin{array}{l}\text { Averag } \\
\rho(\$)\end{array}$ & e & $\begin{array}{l}\text { orth } \\
\sigma^{(\mathrm{c})} \\
\end{array}$ & 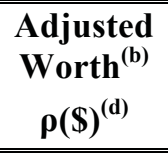 & $\begin{array}{c}\text { Adjusted } \\
\text { Uncertainty }^{(\mathbf{b})} \\
\pm \boldsymbol{\sigma} / \sqrt{ } \mathbf{N}^{(\mathbf{d}, \mathrm{e})} \\
\end{array}$ \\
\hline 5 & 3 & IK & -3.62 & \pm & 0.07 & -3.74 & 0.17 \\
\hline 6 & 9 & IK, PNS & -3.69 & \pm & 0.06 & -3.82 & 0.10 \\
\hline 7 & 1 & IK & -3.58 & \pm & 0.01 & -3.70 & 0.30 \\
\hline 8 & 1 & IK & -3.48 & \pm & 0.01 & -3.60 & 0.29 \\
\hline $5+6$ & 10 & IK, PNS & -7.75 & \pm & 0.12 & -8.02 & 0.20 \\
\hline $5+7$ & 1 & $\mathrm{IK}$ & -7.19 & 王 & 0.02 & -7.44 & 0.60 \\
\hline $5+8$ & 1 & IK & -7.15 & \pm & 0.02 & -7.40 & 0.59 \\
\hline $5+6+7$ & 12 & IK, PNS & -11.70 & \pm & 0.28 & -12.11 & 0.28 \\
\hline $5+6+7+8$ & 10 & IK, PNS & -15.97 & \pm & 0.48 & -16.52 & 0.42 \\
\hline
\end{tabular}

(a) $\mathrm{IK}=$ Inverse Kinetics; PNS = Pulsed Neutron Source

(b) The measured worth is adjusted per the discussion in Section 2.4.1 from the reported calculated $\beta_{\text {eff }}$ of $717 \mathrm{pcm}$ to the currently calculated value of $693 \mathrm{pcm}$.

(c) This uncertainty is the standard deviation of the average when multiple measurements are combined. For a single measurement, it is the reported uncertainty in that measurement.

(d) The uncertainty in the adjusted worth is increased to $8 \%$.

(e) The uncertainty in the adjusted worth is adjusted according to the number of unique measurements incorporated in determining the mean adjusted worth. 
NEA/NSC/DOC(2006)1

Gas Cooled (Thermal) Reactor - GCR

PROTEUS-GCR-EXP-004

CRIT-REAC

Table 2.4-10. Evaluated Safety/Shutdown Rod Worth Measurements (Core 10).

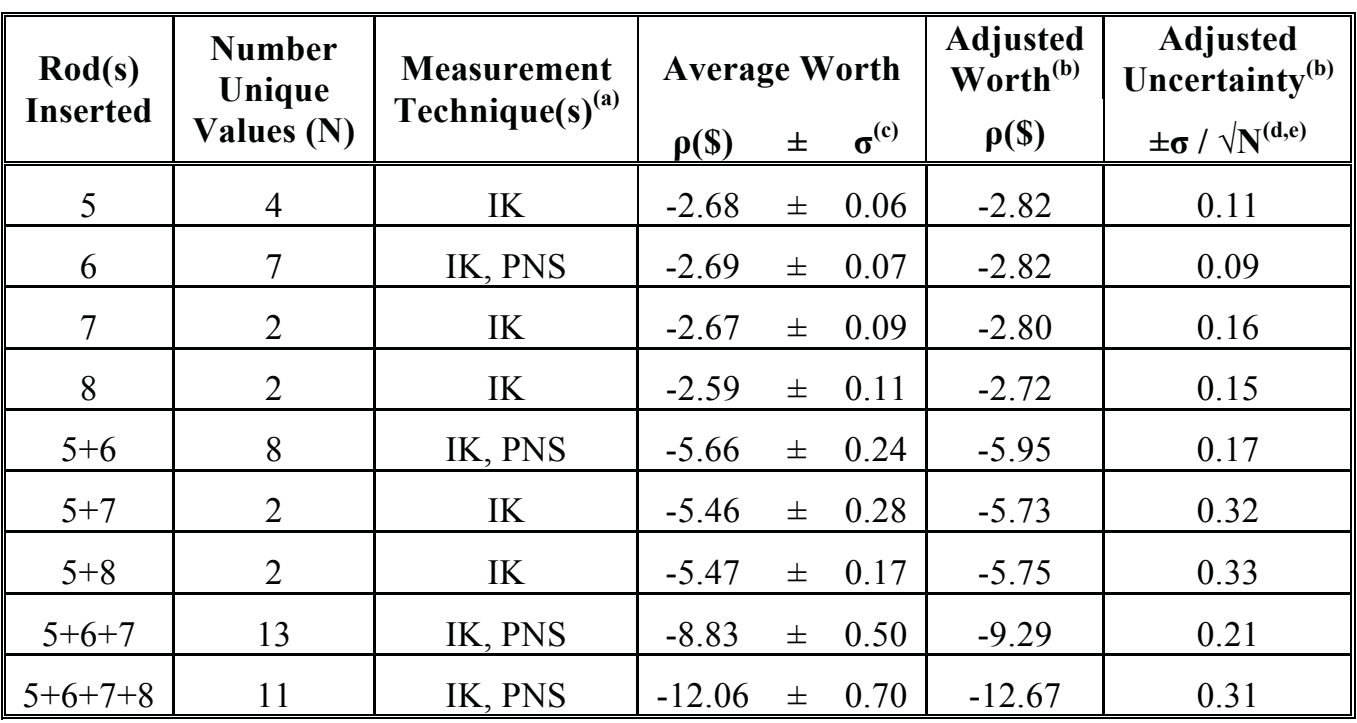

(a) $\mathrm{IK}=$ Inverse Kinetics; PNS = Pulsed Neutron Source

(b) The measured worth is adjusted per the discussion in Section 2.4.1 from the reported calculated $\beta_{\text {eff }}$ of $720 \mathrm{pcm}$ to the currently calculated value of $685 \mathrm{pcm}$.

(c) This uncertainty is the standard deviation of the average when multiple measurements are combined. For a single measurement, it is the reported uncertainty in that measurement.

(d) The uncertainty in the adjusted worth is increased to $8 \%$.

(e) The uncertainty in the adjusted worth is adjusted according to the number of unique measurements incorporated in determining the mean adjusted worth.

\subsubsection{Graphite Plug Worth Measurements}

Graphite plug worth measurements were reported for Cores 9 and 10 (see Tables 1.4-14 and 1.4-15, respectively) and include the worth of graphite in the control rod channels, autorod channel, empty channels in three $\mathrm{R} 2$ positions of the radial reflector, and 34 channels in the upper reflector. Calculations of the worth of the autorod channel are more than three times greater than the reported "measured" worth obtained by scaling measurements from other core configurations. It is unclear why there is such a large discrepancy. The small estimated uncertainty of the "measured" worth with a large estimated experimental uncertainty contributes to this discrepancy. The worth of the autorod channel was determined to not represent an acceptable benchmark experiment. A similar discrepancy is seen for calculations of the worth of the 34 graphite plugs in the upper axial reflector for Core 10; in this instance, however, calculations are approximately three times smaller than the reported "measured" worth obtained by scaling measurements from another core configuration. The uncertainty in this measurement is also large. The worth of the empty channels in the upper axial reflector for Core 10 was also determined to not represent an acceptable benchmark experiment. The reported values were adjusted to use the same $\beta_{\text {eff }}$ value (see Section 2.4.1) as used in the MCNP calculations. The evaluated experimental values are provided in Tables 2.4-11 and 2.4-12 for Cores 9 and 10, respectively.

The graphite plug worths are evaluated simply by filling the voided channel volume with the graphite plug composition. The plugs were designed to minimize air channel volume in the radial and axial graphite reflectors. Minor variation in the diameter of the simulated plug material is assumed to introduce a negligible computational bias or uncertainty due to the large experimental uncertainty associated with these measurements. The locations of the control rod channels and empty R2 channels are shown in Figure 3.4-4. 
NEA/NSC/DOC(2006)1

\section{Gas Cooled (Thermal) Reactor - GCR \\ PROTEUS-GCR-EXP-004 \\ CRIT-REAC}

The material properties and dimensions of the graphite plugs, as well as their respective uncertainties, are discussed in detail in Section 2.1 of PROTEUS-GCR-EXP-001 for both the radial and axial reflectors. Uncertainties in the graphite plug properties are assumed to be negligible compared to the uncertainty in the total experiment uncertainty, which is dominated by the uncertainty in the measurement techniques employed in evaluating the worth of the graphite plugs.

The graphite plug worth measurements were scaled measurements and not considered as a benchmark measurements.

Table 2.4-11. Evaluated Graphite Plug Worth Measurements (Core 9).

\begin{tabular}{||c|c|ccc|ccc||}
\hline \multirow{2}{*}{$\begin{array}{c}\text { Measured } \\
\text { Value }\end{array}$} & Measurement & \multicolumn{3}{|c||}{ Measured Worth } & \multicolumn{3}{|c||}{ Adjusted Worth } \\
& Technique & $\boldsymbol{\rho}(\mathbf{\$})$ & \pm & $\boldsymbol{\sigma}$ & $\boldsymbol{\rho}(\mathbf{\$})$ & \pm & $\boldsymbol{\sigma}$ \\
\hline \hline Control Rod Channels & $\begin{array}{c}\text { Scaled from } \\
\text { Core 5 Value }\end{array}$ & -0.025 & \pm & 0.003 & -0.026 & \pm & 0.003 \\
\hline Autorod Channel ${ }^{(\mathrm{b})}$ & $\begin{array}{c}\text { Scaled from } \\
\text { Core 5 Value }\end{array}$ & -0.007 & \pm & 0.003 & -0.007 & \pm & 0.003 \\
\hline $\begin{array}{c}\text { Empty Channels: } \\
\text { R2-15, -47, \& -63 }\end{array}$ & $\begin{array}{c}\text { Scaled from } \\
\text { Core 5 Value }\end{array}$ & -0.05 & \pm & 0.01 & -0.05 & \pm & 0.01 \\
\hline
\end{tabular}

(a) The measured worth is adjusted per the discussion in Section 2.4.1 from the reported calculated $\beta_{\text {eff }}$ of $720 \mathrm{pcm}$ to the currently calculated value of $693 \mathrm{pcm}$.

(b) This worth value is calculated to be $-0.024 \pm 0.014 \rho(\$)$ using MCNP5 and ENDF/B-VII.0 neutron data. The (C-E)/E values is $232 \% \pm 240 \%$. This experimental measurement is judged to be unacceptable.

Table 2.4-12. Evaluated Graphite Plug Worth Measurements (Core 10).

\begin{tabular}{|c|c|c|c|c|c|c|c|}
\hline \multirow{2}{*}{$\begin{array}{l}\text { Measured } \\
\text { Value }\end{array}$} & \multirow{2}{*}{$\begin{array}{c}\text { Measurement } \\
\text { Technique }\end{array}$} & \multicolumn{3}{|c|}{ Measured Worth } & \multicolumn{3}{|c|}{ Adjusted Worth ${ }^{(a)}$} \\
\hline & & $\rho(\$)$ & \pm & 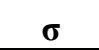 & $\rho(\$)$ & \pm & $\boldsymbol{\sigma}$ \\
\hline Control Rod Channels & Core 1A Value & -0.02 & \pm & 0.002 & -0.021 & \pm & 0.002 \\
\hline Autorod Cha & 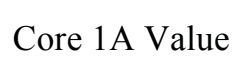 & 0005 & + & 0.003 & -0.022 & + & 0012 \\
\hline $\begin{array}{l}\text { Empty Channels: } \\
\text { R2-15, -47, \& -63 }\end{array}$ & $\mathrm{Co}$ & -0.04 & \pm & 0.01 & -0 & \pm & 1 \\
\hline 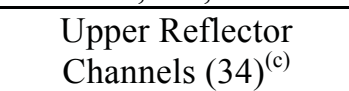 & $\begin{array}{c}\text { Scaled from } \\
\text { Core } 1 \mathrm{~A} \text { Value }\end{array}$ & -0.036 & \pm & 0.02 & -0.038 & \pm & 0.38 \\
\hline
\end{tabular}

(a) The measured worth is adjusted per the discussion in Section 2.4.1 from the reported calculated $\beta_{\text {eff }}$ of $720 \mathrm{pcm}$ to the currently calculated value of $685 \mathrm{pcm}$.

(b) This worth value is calculated to be $-0.022 \pm 0.014 \rho(\$)$ using MCNP5 and ENDF/B-VII.0 neutron data. The (C-E)/E values is $310 \% \pm 340 \%$. This experimental measurement is judged to be unacceptable.

(c) This worth value is calculated to be $-0.014 \pm 0.012 \rho(\$)$ using MCNP5 and ENDF/B-VII.0 neutron data. The (C-E)/E values is $-62 \% \pm 39 \%$. This experimental measurement is judged to be unacceptable. 


\section{Gas Cooled (Thermal) Reactor - GCR \\ PROTEUS-GCR-EXP-004 \\ CRIT-REAC}

\subsubsection{Source/Instrumentation Worth Measurements}

Worths were reported in Tables 1.4-16 and 1.4-17 for the removal of the start-up sources, start-up source penetrations, nuclear instrumentation (ionization), and nuclear instrumentation (fission), for Cores 9 and 10, respectively. However, there was insufficient information available to sufficiently model and evaluate these measurements. Therefore, all measurements pertaining to the worth of the sources and instrumentation were deemed unacceptable for use as benchmark experiments.

\subsubsection{Summary of Reactivity Effects Measurements}

A summary of the adjusted worth measurements, as described and evaluated in Section 2.4, is provided in Tables 2.4-13 and 2.4-14 for Cores 9 and 10, respectively. Measurements scaled from another core configuration were evaluated but deemed not acceptable as benchmark data; further information for modeling these data is provided in Appendix F. Case numbers are assigned as follows, X.Y-Z, where X represents the critical core case number, $Y$ indicates the measurement type (in this case 4 for reactivity effect measurement), and $\mathrm{Z}$ represents the ordering of individual measurements for the main core configuration, $\mathrm{X}$. 
NEA/NSC/DOC(2006)1

\section{Gas Cooled (Thermal) Reactor - GCR \\ PROTEUS-GCR-EXP-004 \\ CRIT-REAC}

Table 2.4-13. Adjusted Experimental Reactivity Effects Measurements (Core 9).

\begin{tabular}{|c|c|c|c|c|c|}
\hline \multirow{2}{*}{ Case } & \multirow{2}{*}{ Measured Parameter } & \multirow{2}{*}{$\begin{array}{c}\text { Benchmark } \\
\text { Measurement? }\end{array}$} & \multicolumn{3}{|c|}{ Experimental Worth } \\
\hline & & & $\rho(\$)$ & \pm & $\sigma$ \\
\hline $1.4-1$ & Control Rod 1 & Yes & -0.41 & \pm & 0.02 \\
\hline $1.4-2$ & Control Rod 2 & Yes & -0.41 & \pm & 0.02 \\
\hline $1.4-3$ & Control Rod 3 & Yes & -0.41 & \pm & 0.02 \\
\hline $1.4-4$ & Control Rod 4 & Yes & -0.41 & \pm & 0.02 \\
\hline $1.4-5$ & $\begin{array}{l}\text { Control Rod Bank } \\
\text { Full Insertion }\end{array}$ & Yes & -1.58 & \pm & 0.09 \\
\hline $1.4-6$ & $\begin{array}{l}\text { Control Rod Bank } \\
\text { Partial Insertion }\end{array}$ & Yes & -0.73 & \pm & 0.04 \\
\hline-- & Autorod Rest Worth & No & -0.13 & \pm & 0.01 \\
\hline $1.4-7$ & Autorod Insertion & Yes & -0.10 & \pm & 0.01 \\
\hline $1.4-8$ & Safety/Shutdown Rod 5 & Yes & -3.74 & \pm & 0.17 \\
\hline $1.4-9$ & Safety/Shutdown Rod 6 & Yes & -3.82 & \pm & 0.10 \\
\hline $1.4-10$ & Safety/Shutdown Rod 7 & Yes & -3.70 & \pm & 0.30 \\
\hline $1.4-11$ & Safety/Shutdown Rod 8 & Yes & -3.60 & \pm & 0.29 \\
\hline $1.4-12$ & Safety/Shutdown Rods $5+6$ & Yes & -8.02 & \pm & 0.20 \\
\hline $1.4-13$ & Safety/Shutdown Rods $5+7$ & Yes & -7.44 & \pm & 0.60 \\
\hline $1.4-14$ & Safety/Shutdown Rods $5+8$ & Yes & -7.40 & \pm & 0.59 \\
\hline $1.4-15$ & Safety/Shutdown Rods $5+6+7$ & Yes & -12.11 & \pm & 0.28 \\
\hline $1.4-16$ & Safety/Shutdown Rods $5+6+7+8$ & Yes & -16.52 & \pm & 0.42 \\
\hline-- & Graphite in Control Rod Channels & No & -0.026 & \pm & 0.003 \\
\hline -- & $\begin{array}{c}\text { Graphite in Empty Channels: } \\
\text { R2-15,-47, \& }-63\end{array}$ & No & -0.05 & \pm & 0.01 \\
\hline
\end{tabular}


NEA/NSC/DOC(2006)1

Gas Cooled (Thermal) Reactor - GCR

PROTEUS-GCR-EXP-004

CRIT-REAC

Table 2.4-14. Adjusted Experimental Reactivity Effects Measurements (Core 10).

\begin{tabular}{|c|c|c|ccc||}
\hline \multirow{2}{*}{ Case } & Measured Parameter & Benchmark & \multicolumn{3}{|c|}{ Experimental Worth } \\
& & Measurement? & p(\$) & $\boldsymbol{\sigma}$ \\
\hline $2.4-1$ & Control Rod 1 & Yes & -0.30 & \pm & 0.02 \\
\hline $2.4-2$ & Control Rod 2 & Yes & -0.29 & \pm & 0.02 \\
\hline $2.4-3$ & Control Rod 3 & Yes & -0.29 & \pm & 0.02 \\
\hline $2.4-4$ & Control Rod 4 & Yes & -0.30 & \pm & 0.02 \\
\hline $2.4-5$ & Control Rod Bank & Yes & -1.15 & \pm & 0.07 \\
\hline $2.4-6$ & $\begin{array}{c}\text { Control Rod Bank } \\
\text { Partial Insertion }\end{array}$ & Yes & -0.39 & \pm & 0.02 \\
\hline-- & Autorod Rest Worth & No & -0.081 & \pm & 0.005 \\
\hline $2.4-7$ & Autorod Insertion & Yes & -0.073 & \pm & 0.004 \\
\hline $2.4-8$ & Safety/Shutdown Rod 5 & Yes & -2.82 & \pm & 0.11 \\
\hline $2.4-9$ & Safety/Shutdown Rod 6 & Yes & -2.82 & \pm & 0.09 \\
\hline $2.4-10$ & Safety/Shutdown Rod 7 & Yes & -2.80 & \pm & 0.16 \\
\hline $2.4-11$ & Safety/Shutdown Rod 8 & Yes & -2.72 & \pm & 0.15 \\
\hline $2.4-12$ & Safety/Shutdown Rods 5+6 & Yes & -5.95 & \pm & 0.17 \\
\hline $2.4-13$ & Safety/Shutdown Rods 5+7 & Yes & -5.73 & \pm & 0.32 \\
\hline $2.4-14$ & Safety/Shutdown Rods 5+8 & Yes & -5.75 & \pm & 0.33 \\
\hline $2.4-15$ & Safety/Shutdown Rods 5+6+7 & Yes & -9.29 & \pm & 0.21 \\
\hline $2.4-16$ & Safety/Shutdown Rods 5+6+7+8 & Yes & -12.67 & \pm & 0.31 \\
\hline-- & Graphite in Control Rod Channels & No & -0.021 & \pm & 0.002 \\
\hline-- & $\begin{array}{c}\text { Graphite in Empty Channels: } \\
\text { R2-15, -47, \& -63 }\end{array}$ & No & -0.04 & \pm & 0.01 \\
\hline
\end{tabular}




\section{NEA/NSC/DOC(2006)1 \\ Gas Cooled (Thermal) Reactor - GCR \\ PROTEUS-GCR-EXP-004 \\ CRIT-REAC}

\subsection{Evaluation of Reactivity Coefficient Data}

Reactivity coefficient measurements were performed but have not yet been evaluated.

\subsection{Evaluation of Kinetics Measurements Data}

Kinetics measurements were performed but have not yet been evaluated.

\subsection{Evaluation of Reaction-Rate Distributions}

Reaction-rate distribution measurements were performed but have not yet been evaluated.

\subsection{Evaluation of Power Distribution Data}

Power distribution measurements were not performed.

\section{$2.9 \quad$ Evaluation of Isotopic Measurements}

Isotopic measurements were not performed.

\subsection{Evaluation of Other Miscellaneous Types of Measurements}

Other miscellaneous types of measurements were not performed. 
NEA/NSC/DOC(2006)1

\author{
Gas Cooled (Thermal) Reactor - GCR \\ PROTEUS-GCR-EXP-004 \\ CRIT-REAC
}

\title{
3.0 BENCHMARK SPECIFICATIONS
}

Two benchmark experiments were evaluated in this report: Cores 9 and 10. These core configurations represent the columnar hexagonal point-on-point (CHPOP) configurations of the HTR-PROTEUS experiment with a moderator-to-fuel pebble ratio of $1: 1$. Cores 9 and 10 use withdrawable, hollow, stainless steel control rods. Core 9 has 27 pebble layers; a second configuration, or state, of Core 9 that included a $28^{\text {th }}$ layer of just moderator pebbles was not evaluated as it was very similar in core design and implemented to perform core operations after initial criticality was attained. Core 10 retains the same pebble loading as Core 9, but to a height of 24 layers. It has polyethylene rods inserted between pebbles to simulate water ingress.

\subsection{Benchmark-Model Specifications for Critical and / or Subcritical Measurements}

The benchmark critical configurations for Cores 9 and 10 will be referred to as Cases 1 and 2, respectively. Both methods of identification are utilized throughout the rest of this report to facilitate users with differing familiarities with HTR-PROTEUS and IRPhEP benchmark format.

The HTR-PROTEUS configurations consist of a thick annular graphite reflector surrounding a pair of thick axial graphite reflectors that sandwich a core cavity region containing fuel and moderator pebbles (see Figures 3.1-16 and 3.1-23). Most core configurations in the HTR-PROTEUS experimental series included exact placement of the pebbles, as is the case with Cases 1 and 2 evaluated in this benchmark report. Penetrations in the graphite reflectors were provided for control rods and instrumentation; typically these holes were filled with graphite plugs or filler pieces when not in use.

Case 1 (Core 9) represented the initial critical experiment, or "base case", against which Case 2 (Core 10), which included polyethylene rods placed in channels between the pebbles, was constructed to simulate water ingress effects within pebble bed systems. Both cores could be compared with the randomly stacked core configuration (Core 4) with the same moderator to fuel pebble ratio of 1:1 (see PROTEUS-GCR-EXP-002).

\subsubsection{Description of the Benchmark Model Simplifications}

Various simplifications were necessary to prepare benchmark model specifications for the critical core configurations. Experimental measurements were performed or estimated based on experimental measurements for a variety of simplifications (see Section 1.1.5), since the original intent of this experimental series was to provide benchmark quality experiments that could be easily modeled. Only a selection of the measured simplifications was retained as biases to be applied to the benchmark models (see Tables 3.1-1 and 3.1-2). Some of the core features were retained in the models to reduce the total effective bias, since they could be modeled easily. The retained measured biases generally represent simplifications of the benchmark models where insufficient information existed to reproduce the measurement with a calculation or reverse the simplification by adding more detail to the benchmark model. Simplifications that were simulated in the original reference reports (also reported in Section 1.1.5) were not retained, but instead recalculated.

Significant simplifications in assembly geometries and compositions were investigated for the first HTRPROTEUS cores: 1, 1A, 2, and 3 (PROTEUS-GCR-EXP-001). Those simplifications that yielded small $(\leq 0.00100 \Delta \mathrm{k})$ or negligible $(\leq 0.00010 \Delta \mathrm{k})$ biases that were incorporated into the other benchmark models are now also included in these benchmark models (see Table 3.1-3). Biases calculated for the removal of control rods, coolant channels in the axial reflectors, removal of upper axial reflector aluminum support structure, and voiding of air were large and considered unacceptable for the benchmark models of Cores $1,1 \mathrm{~A}, 2$, and 3. Therefore, these simplifications were not performed and the features were retained in the benchmark models of Cores 9 and 10 . 
NEA/NSC/DOC(2006)1

\section{Gas Cooled (Thermal) Reactor - GCR \\ PROTEUS-GCR-EXP-004 \\ CRIT-REAC}

\subsubsection{Evaluation of Benchmark Model Biases}

A summary of the experimentally measured reactivity corrections utilized for the benchmark models is provided in Tables 3.1-1 and 3.1-2 for Cases 1 and 2, respectively. The values for Cases 1 and 2 were obtained from Tables 1.1-17 and 1.1-19, respectively. The reported $\beta_{\text {eff }}$ values were used to convert the reactivity corrections and their associated uncertainties from their original measured reactivities in units of $\notin$ into $\Delta \mathrm{k}$; it was assumed that there was an additional bias uncertainty due to the use of the reported $\beta_{\text {eff }}$ values of $5 \%(1 \sigma)$ of the reported value $\left(\sim 0.00036 \Delta \beta_{\text {eff }}\right)$. Many of the measurement biases were used directly, since sufficient information was not available to include most of them in the models.

Some of the C-Driver channels in the $2^{\text {nd }}$ and $3^{\text {rd }}$ rings of the radial reflector contained instrumentation instead of graphite rods. The effect of filling these empty positions with graphite rods was measured.

Start-up sources with associated penetrations were used in HTR-PROTEUS. The effect of removing these sources and filling the penetrations with graphite was measured.

Instrumentation and detectors in the core were removed and the effect was measured.

Typically 33 coolant channels in the lower axial reflector and 34 coolant channels in the upper axial reflector were empty during many of the HTR-PROTEUS experiments. However, for Cores 9 and 10, the channels in the lower axial reflector were filled. Both core configurations were modeled with the open coolant channels in the upper reflector and filled coolant channels in the lower reflector.

Table 3.1-1. Experimentally Determined Reactivity Corrections for Case 1 (Core 9).

\begin{tabular}{|c|c|c|c|c|c|c|}
\hline \multirow[b]{2}{*}{ Measured Effect } & \multicolumn{3}{|c|}{ Reactivity Correction } & \multicolumn{3}{|c|}{ Reactivity Correction } \\
\hline & $\rho c$ & \pm & $\boldsymbol{\sigma}$ & $\Delta \mathbf{k}$ & \pm & $\boldsymbol{\sigma}$ \\
\hline Empty Channels in Ring 2 of Radial Reflector & 5 & \pm & 1 & 0.00036 & \pm & 0.00007 \\
\hline Start-Up Sources & 4 & \pm & 1 & 0.00029 & \pm & 0.00007 \\
\hline Start-Up Source Penetrations & 1 & \pm & 0.2 & 0.00007 & \pm & 0.00001 \\
\hline Nuclear Instrumentation (Ionization) & 9 & \pm & 1.5 & 0.00065 & \pm & 0.00011 \\
\hline Nuclear Instrumentation (Fission) & 1 & \pm & 0.7 & 0.00007 & \pm & 0.00005 \\
\hline Total (Reported $\left.\beta_{\text {eff }}=0.00720\right)^{(a)}$ & 20 & \pm & 2.19 & 0.00144 & \pm & 0.00016 \\
\hline
\end{tabular}

(a) Assumed uncertainty in $\beta_{\text {eff }}$ of $5 \%(1 \sigma)$.

Table 3.1-2. Experimentally Determined Reactivity Corrections for Case 2 (Core 10).

\begin{tabular}{||l|ccc|ccc||}
\hline \multirow{2}{*}{ Measured Effect } & \multicolumn{3}{|c|}{ Reactivity Correction } & \multicolumn{3}{|c||}{ Reactivity Correction } \\
& $\boldsymbol{\rho} \mathbf{c}$ & \pm & $\boldsymbol{\sigma}$ & $\boldsymbol{\Delta} \mathbf{k}$ & \pm & $\boldsymbol{\sigma}$ \\
\hline \hline Empty Channels in Ring 2 of Radial Reflector & 4 & \pm & 1 & 0.00029 & \pm & 0.00007 \\
Start-Up Sources & -- & \pm & -- & -- & \pm & -- \\
Start-Up Source Penetrations & 1 & \pm & 0.2 & 0.00007 & \pm & 0.00001 \\
Nuclear Instrumentation (Ionization) & 8.4 & \pm & 1.2 & 0.00060 & \pm & 0.00009 \\
Nuclear Instrumentation (Fission) & 0.8 & \pm & 0.6 & 0.00006 & \pm & 0.00004 \\
\hline Total (Reported $\left.\beta_{\text {eff }}=0.00723\right)^{(a)}$ & 14.2 & \pm & 1.69 & 0.00102 & \pm & 0.00013 \\
\hline
\end{tabular}

(a) Assumed uncertainty in $\beta_{\text {eff }}$ of $5 \%(1 \sigma)$. 
NEA/NSC/DOC(2006)1

\author{
Gas Cooled (Thermal) Reactor - GCR \\ PROTEUS-GCR-EXP-004 \\ CRIT-REAC
}

Additional biases were evaluated for the benchmark simplifications of Cores 1, 1A, 2, and 3

(PROTEUS-GCR-EXP-001); a summary of the biases is listed in Table 3.1-3. The effective bias for most of the individually calculated biases were negligible compared to the statistical uncertainty for Cores 1 , $1 \mathrm{~A}, 2$, and 3, except for the bias for homogenizing the radial reflector; therefore, individual calculations were not performed for Cores 9 and 10 and only a summary of the simplifications is provided with the total effective bias for incorporation of these simplifications in the benchmark models. The effective simplification bias was computed by comparing calculated eigenvalues obtained with MCNP5 input decks (Appendix A) of the benchmark models described in Section 3 and detailed models (Appendix C).

Simplifications to the benchmark models include the removal of many of the assembly components external to the large radial reflector, such as the concrete walls, steel support pedestal, and thermal column (Figures 1.1-1, 1.1-3, and 1.1-9). Experimental measurements confirmed that room return effects were negligible for this series of experiments and therefore deemed unnecessary in the benchmark models.

The safety/shutdown rods and the aluminum shock dampers (Figure 1.1-9) were removed from the benchmark models. The eight channels for these rods were retained in the models (Figure 1.1-2b). Since the safety/shutdown rods were fully withdrawn from the core, their removal from the benchmark models was effectively negligible.

The radial reflector was homogenized with the axial modifiers in the core cavity, C-Driver channels, and graphite plugs in the C-Driver channels (Figures 1.1-2 and 1.1-3). Only penetrations for control rod use were retained: withdrawable control rods, safety/shutdown rods, and autorod. The withdrawable control rods were placed in four of the C-Driver channels in ring 5 of the radial reflector. The ZEBRA rod channels from the initial core, Core 1, were filled with graphite plugs. Radial reflector simplifications facilitate ease of modeling these benchmark configurations. The outer and inner 22-sided polygon surfaces of the radial reflector were converted to cylindrical surfaces; the core cavity region retained its 12 -sided polygon surface.

The safety ring (Figure 1.1-4) is removed from the benchmark model and the aluminum support structure of the upper axial reflector was simplified such that the aluminum spherical surfaces (Figures 1.1-4 and 1.1-6) are modeled as an aluminum disc, 1-cm-thick, retaining the outer diameter of the aluminum structure $(104.2 \mathrm{~cm})$. The aluminum support structure was a complex entity and very difficult to model with exact detail.

The lower axial reflector was simplified by cylinderizing the graphite annulus and filling the small source gap with graphite (Figure 1.1-7). As with simplification of the radial reflector, removal of the exact location of vertices for the multifaceted polygons used to generate this core by using cylindrical representations greatly simplifies modeling of these benchmark configurations.

All pebbles in the models have a radius of $3.000 \mathrm{~cm}$. The mass of the pebbles was conserved and the resultant bias is negligible.

Impurities in the TRISO particles are removed from the models.

A standard air composition was used for all models with a temperature of $20^{\circ} \mathrm{C}$, pressure of $980 \mathrm{mbar}$, and $50 \%$ humidity. Neon, helium, and krypton are not included in the benchmark model; the bias for their removal is negligible. 
NEA/NSC/DOC(2006)1

\section{Gas Cooled (Thermal) Reactor - GCR \\ PROTEUS-GCR-EXP-004 \\ CRIT-REAC}

Table 3.1-3. Calculated Simplification Biases.

- Removal of Concrete Walls

- Removal of Steel Support Pedestal

- Removal of Thermal Column

- Removal of Safety/Shutdown Rods

- Includes Shock Dampers

- Cylinderization of Radial Reflector

- Outer and inner 22-sided polygon surfaces converted to cylindrical surfaces; the core cavity region retained its 12-sided polygon surface

- Removal of Safety Ring

- Homogenization of Radial Reflector

- Remove All Penetrations Except Those for Control Rods

- Simplify Aluminum Support Structure of Upper Axial Reflector

- Cylinderize Lower Axial Reflector Annulus

- Fill Source Gap with Graphite

- Model All Pebbles with a Radius of $3.000 \mathrm{~cm}$

- Remove $\mathrm{UO}_{2}$ Impurities in the TRISO Kernels

- Remove Impurities in the TRISO Layers

- Use a Standard Air Composition for All Models - Remove Ne, He, and Kr from Air Composition

\begin{tabular}{|l|ll|l||}
\hline Case (Core) & \multicolumn{2}{|c|}{$1(9)$} & \multicolumn{2}{c|}{$2(10)$} \\
\hline $\operatorname{Bias}(\Delta \mathbf{k})$ & $0.00147 \pm 0.00010$ & $0.00096 \pm 0.00008$ \\
\hline
\end{tabular}

The total bias for each benchmark configuration (Table 3.1-4) is obtained by summation of the experimentally measured corrections (Tables 3.1-1 and 3.1-2) with the computed simplification bias (Table 3.1-3). The total bias uncertainties are obtained by summing under quadrature the individual bias uncertainties. For example, for Case 1 (Core 9), the measured correction of $0.00144 \pm 0.00016 \Delta \mathrm{k}$ (Table 3.1-1) is added to the calculated simplification bias of $0.00147 \pm 0.00010 \Delta \mathrm{k}$ (Table 3.1-3) to obtain a total simplification bias for the benchmark model of $0.00291 \pm 0.00019 \Delta \mathrm{k}(3.1-4)$.

Table 3.1-4. Total Benchmark Biases $(\Delta \mathrm{k})$.

\begin{tabular}{|l||lll|ll||}
\hline \hline Case (Core) & \multicolumn{2}{|c|}{$1(9)$} & \multicolumn{2}{c|}{$2(10)$} \\
\hline \hline Measured Corrections & 0.00144 & \pm & 0.00016 & 0.00102 & \pm 0.00013 \\
\hline Calculated Simplifications & 0.00147 & \pm & 0.00010 & 0.00096 & \pm 0.00008 \\
\hline Total Bias & $\mathbf{0 . 0 0 2 9 1}$ & $\pm \mathbf{0 . 0 0 0 1 9}$ & $\mathbf{0 . 0 0 1 9 8}$ & $\pm \mathbf{0 . 0 0 0 1 5}$ \\
\hline
\end{tabular}


NEA/NSC/DOC(2006)1

\section{Gas Cooled (Thermal) Reactor - GCR \\ PROTEUS-GCR-EXP-004 \\ CRIT-REAC}

\subsubsection{Dimensions}

\subsubsection{Radial Reflector}

The graphite radial reflector (Figure 3.1-1) is an annulus with an equivalent inner radius of $62.83398 \mathrm{~cm}$, an equivalent outer radius of $163.76986 \mathrm{~cm}$, and a height of $330.4 \mathrm{~cm}$. The portion of the radial reflector surrounding the core cavity region can be described as a cylinder with a dodecagon (12-sided polygon) cross section; the distance between the midpoint of each side alternates between 60.15 and $60.3 \mathrm{~cm}$ from the core center. This region of the radial reflector extends $172.9 \mathrm{~cm}$ upward from the base of the cavity region, located $78.0 \mathrm{~cm}$ above the bottom of the radial reflector. Penetrations in the radial reflector are provided for eight safety/shutdown rods, an autorod, and four withdrawable control rods. These holes axially penetrate completely through the radial reflector with the $\mathrm{x}, \mathrm{y}$ positions provided in Table 3.1-5 and shown in Figures 3.1-2. While the penetrations for the safety/shutdown rods are preserved in the benchmark model, the rods themselves are not included.

Table 3.1-5. Penetrations in Radial Reflector (dimensions in $\mathrm{cm}$ ).

\begin{tabular}{|l|c|c|c|}
\hline \multicolumn{1}{|c|}{ Penetration Purpose } & x-Coordinate & y-Coordinate & Hole Diameter \\
\hline \hline Safety/Shutdown Rod Hole 1 & -38.45 & 56.57 & 4.5 \\
Safety/Shutdown Rod Hole 2 & 32.74 & -60.05 & 4.5 \\
Safety/Shutdown Rod Hole 3 & 57.17 & 37.55 & 4.5 \\
Safety/Shutdown Rod Hole 4 & -53.23 & -42.95 & 4.5 \\
Safety/Shutdown Rod Hole 5 & 67.19 & -12.82 & 4.5 \\
Safety/Shutdown Rod Hole 6 & -66.98 & 13.87 & 4.5 \\
Safety/Shutdown Rod Hole 7 & 19.31 & 65.62 & 4.5 \\
Safety/Shutdown Rod Hole 8 & -13.87 & -66.98 & 4.5 \\
\hline Autorod Hole & 17.36 & -87.29 & 5.5 \\
\hline Withdrawable Control Rod Hole 1 & -83.70 & 34.67 & 2.743 \\
Withdrawable Control Rod Hole 2 & 34.67 & 83.70 & 2.743 \\
Withdrawable Control Rod Hole 3 & 83.70 & -34.67 & 2.743 \\
Withdrawable Control Rod Hole 4 & -34.67 & -83.70 & 2.743 \\
\hline
\end{tabular}

Revision: 1 
NEA/NSC/DOC(2006)1

Gas Cooled (Thermal) Reactor - GCR

PROTEUS-GCR-EXP-004

CRIT-REAC

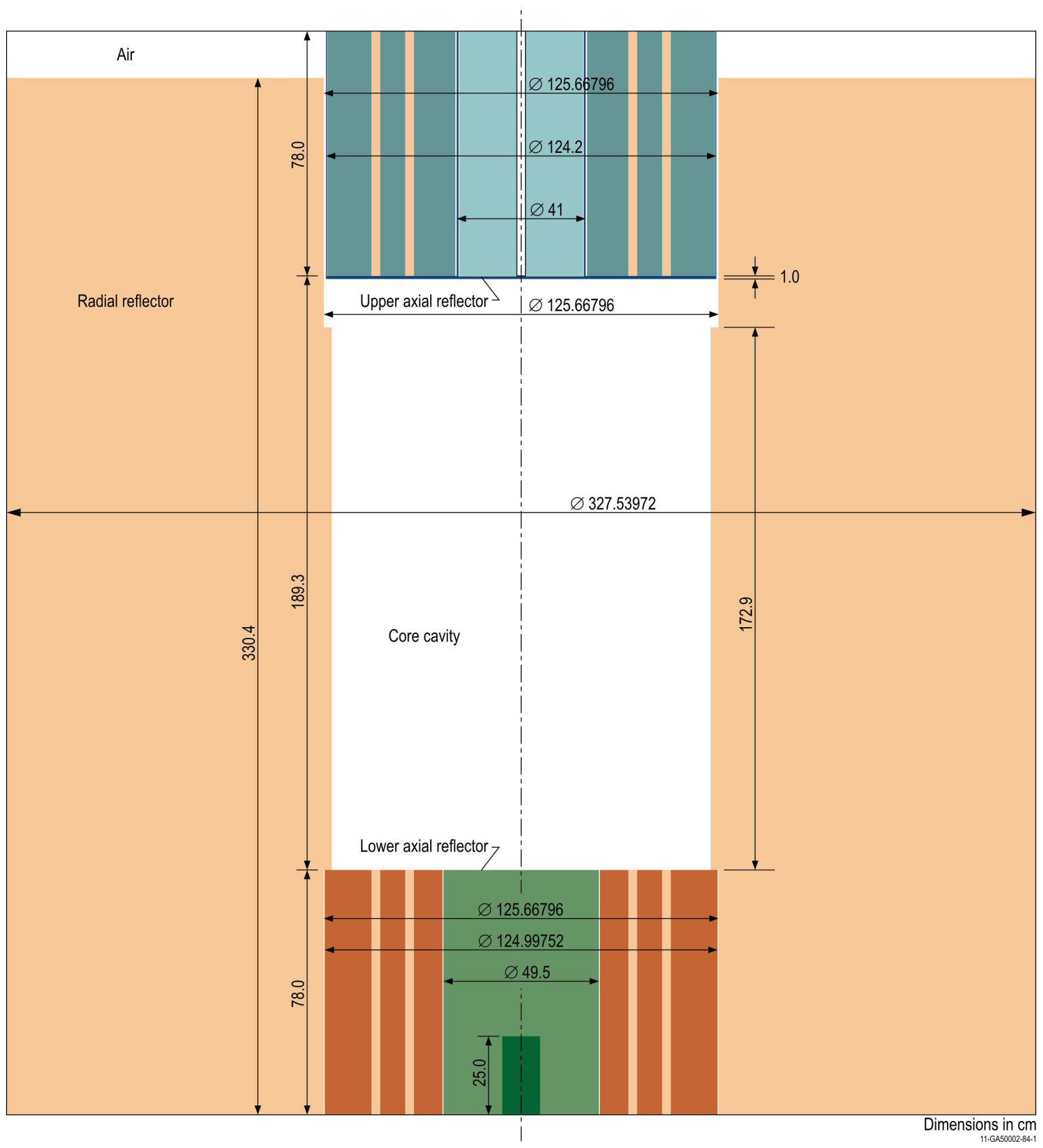

Figure 3.1-1. Radial and Axial Reflectors Surrounding Core Cavity Region.

Revision: 1

Date: March 31, 2014 
NEA/NSC/DOC(2006)1

Gas Cooled (Thermal) Reactor - GCR

PROTEUS-GCR-EXP-004

CRIT-REAC

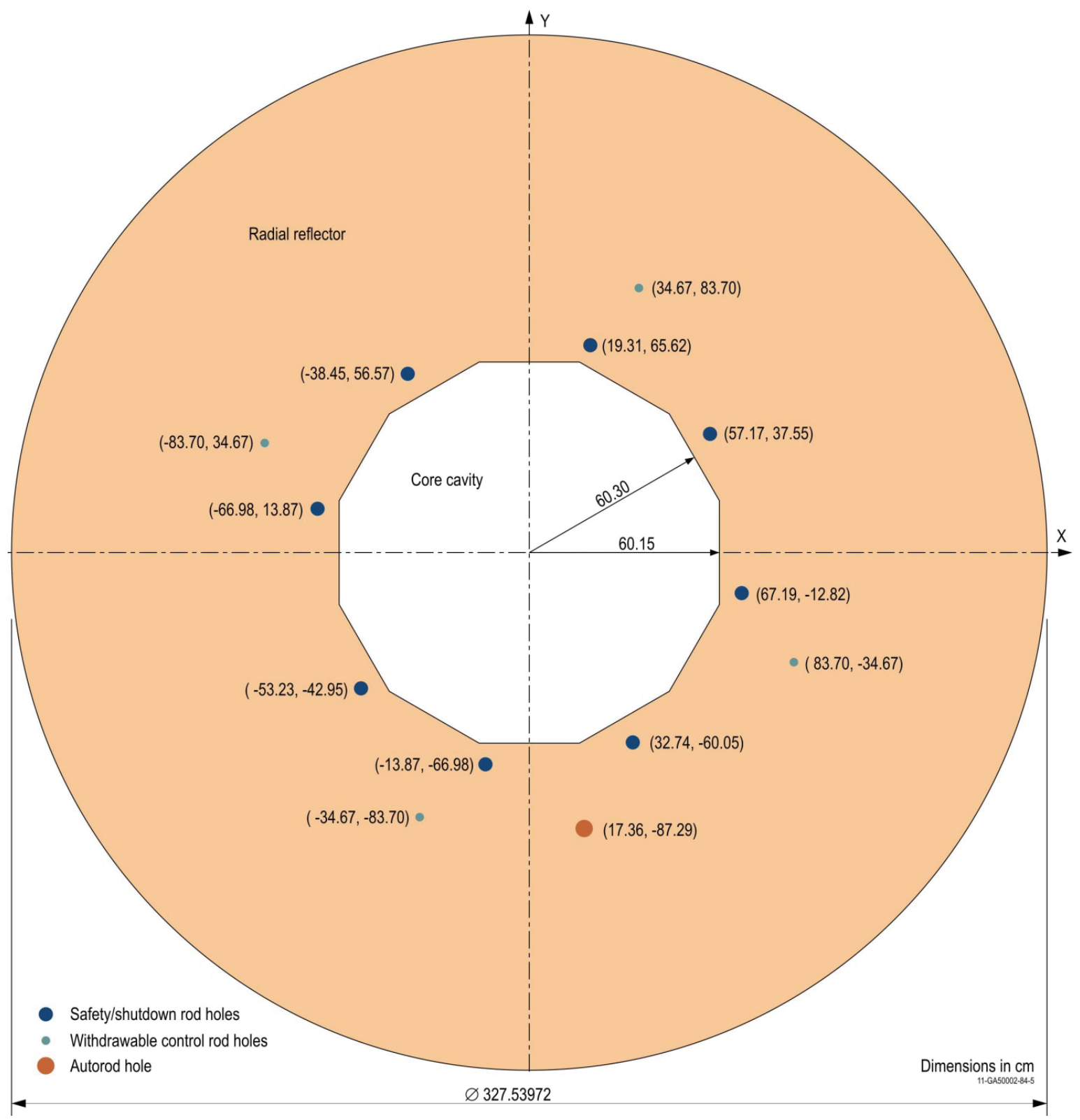

Figure 3.1-2. Radial Reflector Surrounding Core Cavity Region.

Revision: 1

Date: March 31, 2014 
NEA/NSC/DOC(2006)1

\section{Gas Cooled (Thermal) Reactor - GCR \\ PROTEUS-GCR-EXP-004 \\ CRIT-REAC}

\subsubsection{Upper Axial Reflector}

The upper axial reflector consists of a graphite cylinder (radius of $19.7 \mathrm{~cm}$ ) with a single coolant channel (diameter of $2.743 \mathrm{~cm}$ ) and a graphite annulus (inner radius of $20.93 \mathrm{~cm}$ and outer radius of $61.7 \mathrm{~cm}$ ) with 160 coolant channels (diameters of $2.743 \mathrm{~cm}$ ) distributed equally and uniformly spaced within 5 annular locations with distances of $30.0,35.5,41.0,46.25$, and $51.5 \mathrm{~cm}$ radially from the center of the reflector (see Figure 3.1-4 and Table 3.1-6). Of the 161 channels, 127 are filled with graphite plugs (diameter of $2.65 \mathrm{~cm}$ ), as noted in Table 3.1-6 with a "Y". The height of all graphite components is 78.0 $\mathrm{cm}$. An aluminum structure supports the graphite components of the upper axial reflector with an inner annular sheet (19.8 cm inner radius and $20.5 \mathrm{~cm}$ outer radius) separating the graphite annulus and cylinder and another outer annular sheet $(61.8 \mathrm{~cm}$ inner radius and $62.1 \mathrm{~cm}$ outer radius) surrounding the entire axial reflector. Air gaps exist between the graphite and aluminum portions of the reflector. The thickness of the aluminum structure below the graphite is $1.0 \mathrm{~cm}$. The bottom of the graphite in the upper axial reflector rests $189.3 \mathrm{~cm}$ above the top of the lower axial reflector. The inside radius of the radial reflector surrounding the upper axial reflector is $62.83398 \mathrm{~cm}$.

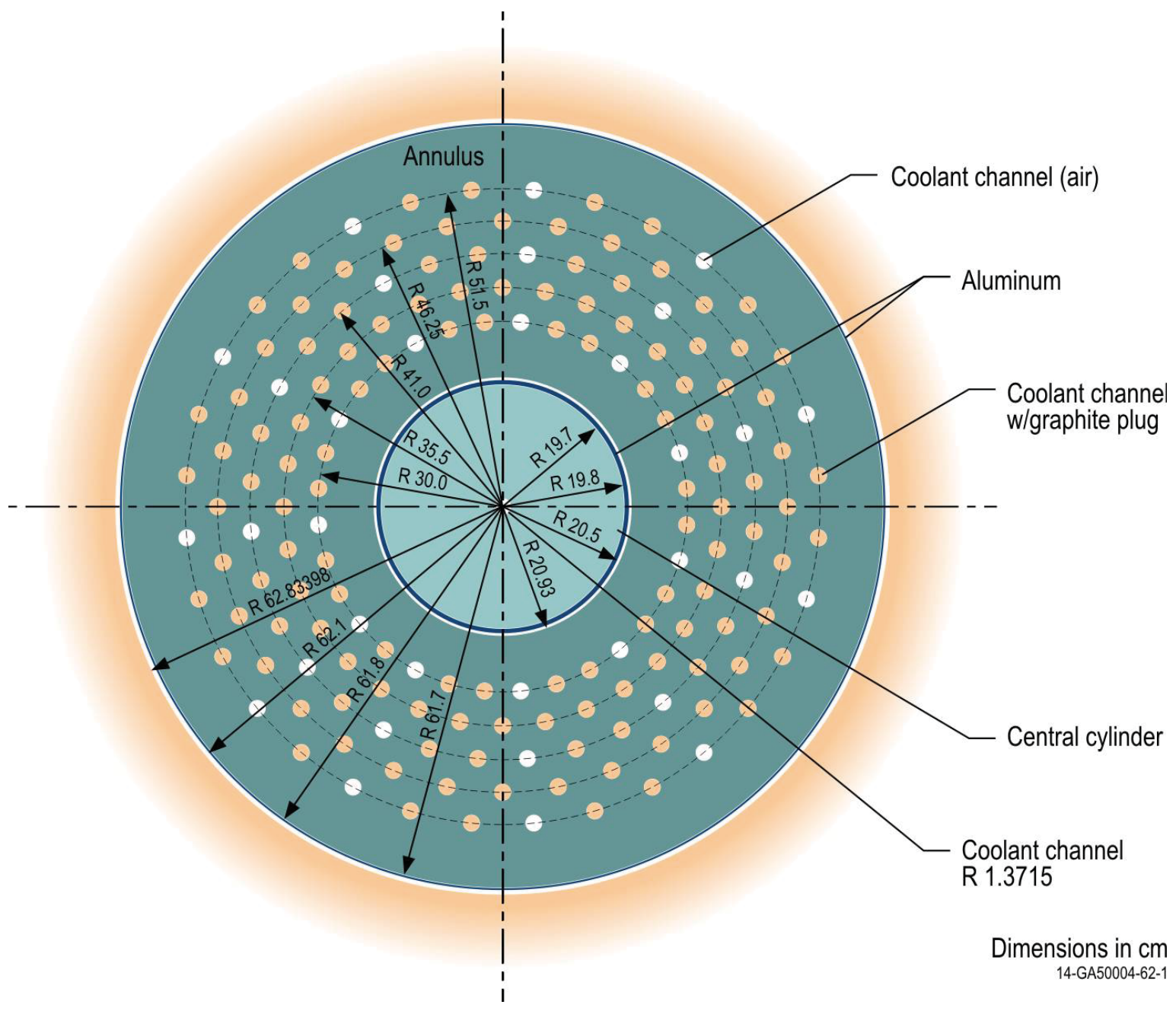

Figure 3.1-4. Upper Axial Reflector. 
NEA/NSC/DOC(2006)1

\section{Gas Cooled (Thermal) Reactor - GCR \\ PROTEUS-GCR-EXP-004 \\ CRIT-REAC}

Table 3.1-6. Penetration Coordinates in the Axial Reflectors (dimensions in $\mathrm{cm}$ ).

\begin{tabular}{|c|c|c|c|c|c|c|c|c|c|}
\hline Ring & & 1 & & & 2 & & & 3 & \\
\hline Position & $\mathbf{x}$ & $\mathbf{y}$ & Plug? ${ }^{(a)}$ & $\mathbf{x}$ & $\mathbf{y}$ & Plug? ${ }^{(a)}$ & $\mathbf{x}$ & $\mathbf{y}$ & Plug? ${ }^{\text {(a) }}$ \\
\hline 1 & -29.86 & 2.94 & $\mathrm{Y}$ & -34.82 & 6.93 & $\mathrm{Y}$ & -39.23 & 11.90 & $\mathrm{Y}$ \\
\hline 2 & -28.71 & 8.71 & Y & -32.80 & 13.59 & $\mathrm{Y}$ & -36.16 & 19.33 & $\mathrm{~N}$ \\
\hline 3 & -26.46 & 14.14 & $\mathrm{~N}$ & -29.52 & 19.72 & $\mathrm{Y}$ & -31.69 & 26.01 & $\mathrm{Y}$ \\
\hline 4 & -23.19 & 19.03 & $\mathrm{Y}$ & -25.10 & 25.10 & $\mathrm{Y}$ & -26.01 & 31.69 & $\mathrm{Y}$ \\
\hline 5 & -19.03 & 23.19 & $\mathrm{Y}$ & -19.72 & 29.52 & $\mathrm{Y}$ & -19.33 & 36.16 & $\mathrm{~N}$ \\
\hline 6 & -14.14 & 26.46 & $\mathrm{~N}$ & -13.59 & 32.80 & $\mathrm{Y}$ & -11.90 & 39.23 & $\mathrm{Y}$ \\
\hline 7 & -8.71 & 28.71 & Y & -6.93 & 34.82 & $\mathrm{Y}$ & -4.02 & 40.80 & $\mathrm{Y}$ \\
\hline 8 & -2.94 & 29.86 & $\mathrm{Y}$ & 0.00 & 35.50 & $\mathrm{Y}$ & 4.02 & 40.80 & $\mathrm{~N}$ \\
\hline 9 & 2.94 & 29.86 & $\mathrm{~N}$ & 6.93 & 34.82 & $\mathrm{Y}$ & 11.90 & 39.23 & $\mathrm{Y}$ \\
\hline 10 & 8.71 & 28.71 & $\mathrm{Y}$ & 13.59 & 32.80 & $\mathrm{Y}$ & 19.33 & 36.16 & $\mathrm{Y}$ \\
\hline 11 & 14.14 & 26.46 & $\mathrm{Y}$ & 19.72 & 29.52 & $\mathrm{Y}$ & 26.01 & 31.69 & $\mathrm{~N}$ \\
\hline 12 & 19.03 & 23.19 & $\mathrm{~N}$ & 25.10 & 25.10 & Y & 31.69 & 26.01 & Y \\
\hline 13 & 23.19 & 19.03 & Y & 29.52 & 19.72 & $\mathrm{Y}$ & 36.16 & 19.33 & $\mathrm{Y}$ \\
\hline 14 & 26.46 & 14.14 & Y & 32.80 & 13.59 & $\mathrm{Y}$ & 39.23 & 11.90 & $\mathrm{~N}$ \\
\hline 15 & 28.71 & 8.71 & $\mathrm{~N}$ & 34.82 & 6.93 & $\mathrm{Y}$ & 40.80 & 4.02 & $\mathrm{Y}$ \\
\hline 16 & 29.86 & 2.94 & $\mathrm{Y}$ & 35.50 & 0.00 & $\mathrm{Y}$ & 40.80 & -4.02 & $\mathrm{Y}$ \\
\hline 17 & 29.86 & -2.94 & $\mathrm{Y}$ & 34.82 & -6.93 & $\mathrm{Y}$ & 39.23 & -11.90 & $\mathrm{~N}$ \\
\hline 18 & 28.71 & -8.71 & $\mathrm{~N}$ & 32.80 & -13.59 & $\mathrm{Y}$ & 36.16 & -19.33 & $\mathrm{Y}$ \\
\hline 19 & 26.46 & -14.14 & $\mathrm{Y}$ & 29.52 & -19.72 & $\mathrm{Y}$ & 31.69 & -26.01 & $\mathrm{Y}$ \\
\hline 20 & 23.19 & -19.03 & $\mathrm{Y}$ & 25.10 & -25.10 & $\mathrm{Y}$ & 26.01 & -31.69 & $\mathrm{~N}$ \\
\hline 21 & 19.03 & -23.19 & $\mathrm{~N}$ & 19.72 & -29.52 & $\mathrm{Y}$ & 19.33 & -36.16 & $\mathrm{Y}$ \\
\hline 22 & 14.14 & -26.46 & $\mathrm{Y}$ & 13.59 & -32.80 & $\mathrm{Y}$ & 11.90 & -39.23 & $\mathrm{Y}$ \\
\hline 23 & 8.71 & -28.71 & $\mathrm{Y}$ & 6.93 & -34.82 & $\mathrm{Y}$ & 4.02 & -40.80 & $\mathrm{~N}$ \\
\hline 24 & 2.94 & -29.86 & $\mathrm{~N}$ & 0.00 & -35.50 & $\mathrm{Y}$ & -4.02 & -40.80 & $\mathrm{Y}$ \\
\hline 25 & -2.94 & -29.86 & $\mathrm{Y}$ & -6.93 & -34.82 & $\mathrm{Y}$ & -11.90 & -39.23 & $\mathrm{Y}$ \\
\hline 26 & -8.71 & -28.71 & Y & -13.59 & -32.80 & Y & -19.33 & -36.16 & $\mathrm{~N}$ \\
\hline 27 & -14.14 & -26.46 & $\mathrm{~N}$ & -19.72 & -29.52 & $\mathrm{Y}$ & -26.01 & -31.69 & $\mathrm{Y}$ \\
\hline 28 & -19.03 & -23.19 & Y & -25.10 & -25.10 & $\mathrm{Y}$ & -31.69 & -26.01 & $\mathrm{~N}$ \\
\hline 29 & -23.19 & -19.03 & $\mathrm{~N}$ & -29.52 & -19.72 & $\mathrm{Y}$ & -36.16 & -19.33 & $\mathrm{Y}$ \\
\hline 30 & -26.46 & -14.14 & $\mathrm{Y}$ & -32.80 & -13.59 & $\mathrm{Y}$ & -39.23 & -11.90 & $\mathrm{Y}$ \\
\hline 31 & -28.71 & -8.71 & $\mathrm{Y}$ & -34.82 & -6.93 & $\mathrm{Y}$ & -40.80 & -4.02 & $\mathrm{~N}$ \\
\hline 32 & -29.86 & -2.94 & $\mathrm{~N}$ & -35.50 & 0.00 & $\mathrm{Y}$ & -40.80 & 4.02 & $\mathrm{Y}$ \\
\hline
\end{tabular}

Revision: 1 
NEA/NSC/DOC(2006)1

\section{Gas Cooled (Thermal) Reactor - GCR \\ PROTEUS-GCR-EXP-004 \\ CRIT-REAC}

Table 3.1-6. (cont'd.). Penetration Coordinates in the Axial Reflectors (dimensions in cm).

\begin{tabular}{|c|c|c|c|c|c|c|}
\hline Ring & & 4 & & & 5 & \\
\hline Position & $\mathbf{x}$ & $\mathbf{y}$ & Plug? ${ }^{\text {(a) }}$ & $\mathbf{x}$ & $\mathbf{y}$ & Plug? ${ }^{(a)}$ \\
\hline 1 & -42.73 & 17.70 & $\mathrm{Y}$ & -45.42 & 24.28 & $\mathrm{~N}$ \\
\hline 2 & -38.46 & 25.70 & $\mathrm{Y}$ & -39.81 & 32.67 & $\mathrm{Y}$ \\
\hline 3 & -32.70 & 32.70 & $\mathrm{Y}$ & -32.67 & 39.81 & $\mathrm{Y}$ \\
\hline 4 & -25.70 & 38.46 & $\mathrm{Y}$ & -24.28 & 45.42 & $\mathrm{~N}$ \\
\hline 5 & -17.70 & 42.73 & $\mathrm{Y}$ & -14.95 & 49.28 & $\mathrm{Y}$ \\
\hline 6 & -9.02 & 45.36 & $\mathrm{Y}$ & -5.05 & 51.25 & $\mathrm{Y}$ \\
\hline 7 & 0.00 & 46.25 & $\mathrm{Y}$ & 5.05 & 51.25 & $\mathrm{~N}$ \\
\hline 8 & 9.02 & 45.36 & $\mathrm{Y}$ & 14.95 & 49.28 & $\mathrm{Y}$ \\
\hline 9 & 17.70 & 42.73 & $\mathrm{Y}$ & 24.28 & 45.42 & $\mathrm{Y}$ \\
\hline 10 & 25.70 & 38.46 & $\mathrm{Y}$ & 32.67 & 39.81 & $\mathrm{~N}$ \\
\hline 11 & 32.70 & 32.70 & $\mathrm{Y}$ & 39.81 & 32.67 & $\mathrm{Y}$ \\
\hline 12 & 38.46 & 25.70 & $\mathrm{Y}$ & 45.42 & 24.28 & $\mathrm{Y}$ \\
\hline 13 & 42.73 & 17.70 & $\mathrm{Y}$ & 49.28 & 14.95 & $\mathrm{~N}$ \\
\hline 14 & 45.36 & 9.02 & $\mathrm{Y}$ & 51.25 & 5.05 & $\mathrm{Y}$ \\
\hline 15 & 46.25 & 0.00 & $\mathrm{Y}$ & 51.25 & -5.05 & $\mathrm{Y}$ \\
\hline 16 & 45.36 & -9.02 & $\mathrm{Y}$ & 49.28 & -14.95 & $\mathrm{~N}$ \\
\hline 17 & 42.73 & -17.70 & $\mathrm{Y}$ & 45.42 & -24.28 & $\mathrm{Y}$ \\
\hline 18 & 38.46 & -25.70 & $\mathrm{Y}$ & 39.81 & -32.67 & $\mathrm{Y}$ \\
\hline 19 & 32.70 & -32.70 & $\mathrm{Y}$ & 32.67 & -39.81 & $\mathrm{~N}$ \\
\hline 20 & 25.70 & -38.46 & $\mathrm{Y}$ & 24.28 & -45.42 & $\mathrm{Y}$ \\
\hline 21 & 17.70 & -42.73 & $\mathrm{Y}$ & 14.95 & -49.28 & $\mathrm{Y}$ \\
\hline 22 & 9.02 & -45.36 & $\mathrm{Y}$ & 5.05 & -51.25 & $\mathrm{~N}$ \\
\hline 23 & 0.00 & -46.25 & $\mathrm{Y}$ & -5.05 & -51.25 & $\mathrm{Y}$ \\
\hline 24 & -9.02 & -45.36 & $\mathrm{Y}$ & -14.95 & -49.28 & $\mathrm{Y}$ \\
\hline 25 & -17.70 & -42.73 & $\mathrm{Y}$ & -24.28 & -45.42 & $\mathrm{~N}$ \\
\hline 26 & -25.70 & -38.46 & $\mathrm{Y}$ & -32.67 & -39.81 & $\mathrm{Y}$ \\
\hline 27 & -32.70 & -32.70 & $\mathrm{Y}$ & -39.81 & -32.67 & $\mathrm{~N}$ \\
\hline 28 & -38.46 & -25.70 & $\mathrm{Y}$ & -45.42 & -24.28 & $\mathrm{Y}$ \\
\hline 29 & -42.73 & -17.70 & $\mathrm{Y}$ & -49.28 & -14.95 & $\mathrm{Y}$ \\
\hline 30 & -45.36 & -9.02 & $\mathrm{Y}$ & -51.25 & -5.05 & $\mathrm{~N}$ \\
\hline 31 & -46.25 & 0.00 & $\mathrm{Y}$ & -51.25 & 5.05 & $\mathrm{Y}$ \\
\hline 32 & -45.36 & 9.02 & $\mathrm{Y}$ & -49.28 & 14.95 & $\mathrm{Y}$ \\
\hline
\end{tabular}

(a) This column notes whether a graphite plug is (marked by "Y") or is not (marked by " $\mathrm{N}$ ") located within the coolant channel. 
NEA/NSC/DOC(2006)1

\section{Gas Cooled (Thermal) Reactor - GCR \\ PROTEUS-GCR-EXP-004 \\ CRIT-REAC}

\subsubsection{Lower Axial Reflector}

The lower axial reflector consists of a graphite cylinder (radius of $24.75 \mathrm{~cm}$ ) containing a removable source plug and a graphite annulus (equivalent inner radius of $25.05171 \mathrm{~cm}$ and equivalent outer radius of $62.71754 \mathrm{~cm}$ ) with 160 coolant channels (diameter of $2.742 \mathrm{~cm}$ ) with the same XY positions as the upper axial reflector (see Figure 3.1-5 and Table 3.1-6). Graphite plugs (diameter of $2.65 \mathrm{~cm}$ ) fill all 160 coolant channel positions. The height of all graphite components, except the source plug, is $78.0 \mathrm{~cm}$. The source plug is located at the bottom of the graphite cylinder along its axis and has a radius of $6.0 \mathrm{~cm}$ and height of $25.0 \mathrm{~cm}$, located within a hole in the graphite cylinder with the same dimensions. The inside radius of the radial reflector surrounding the lower axial reflector is $62.83398 \mathrm{~cm}$.

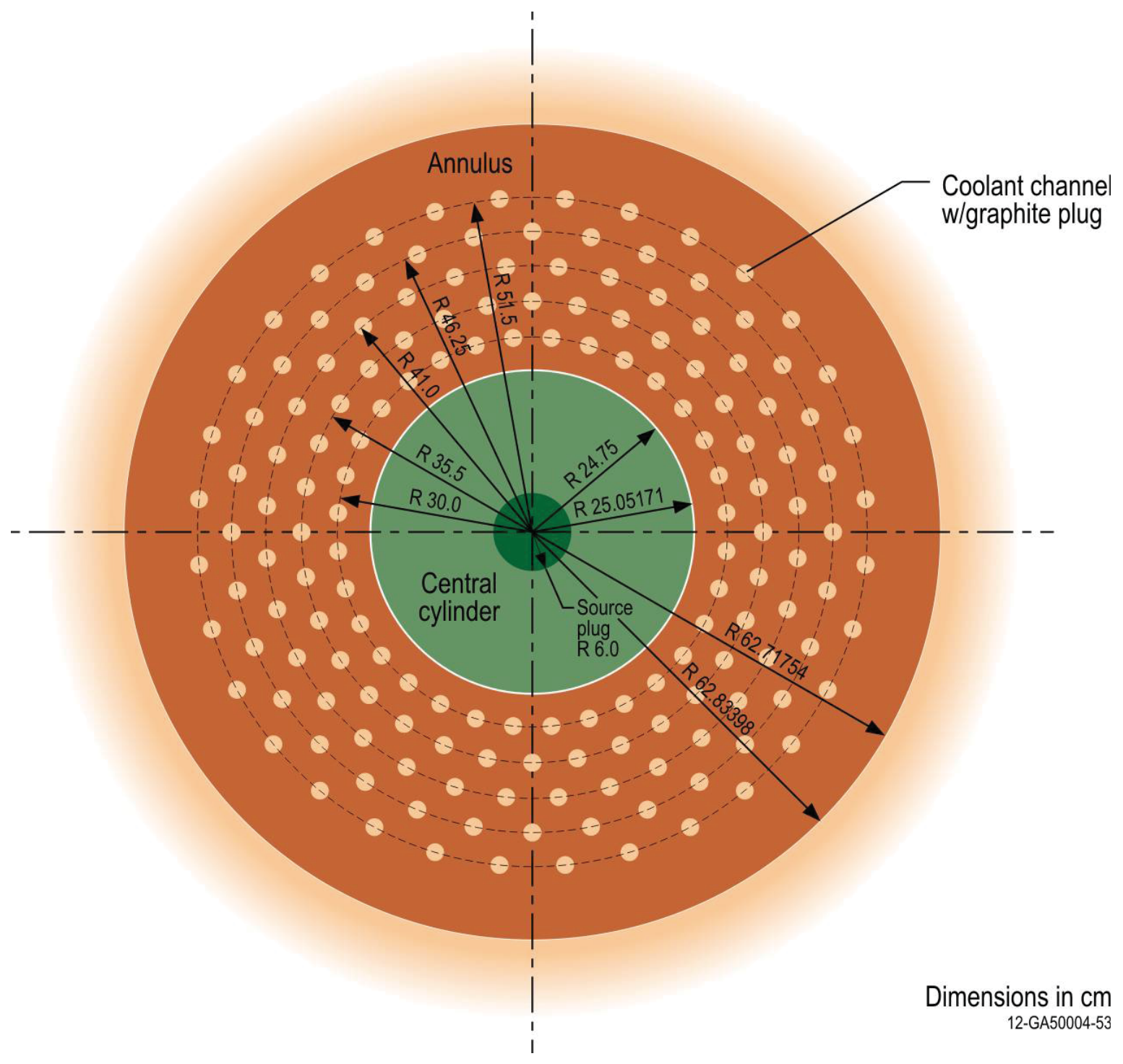

Figure 3.1-5. Lower Axial Reflector. 
NEA/NSC/DOC(2006)1

\section{Gas Cooled (Thermal) Reactor - GCR \\ PROTEUS-GCR-EXP-004 \\ CRIT-REAC}

\subsubsection{Autorod}

The autorod (Figures 3.1-6 and 3.1-7) consists of an aluminum guide tube (inner diameter of $4 \mathrm{~cm}$ and outer diameter of $4.4 \mathrm{~cm}$ ) running the full length of its penetration in the radial reflector. A copper wedge can be raised or lowered within the tube for fine reactivity control of the assembly. The copper wedge has a thickness of $0.3 \mathrm{~cm}$ and a length of $230 \mathrm{~cm}$. The top of the wedge has a width of $3.9 \mathrm{~cm}$ and tapers to a point at the bottom of the wedge. The XY position of the autorod compared to the core is shown in Figure 3.1-2 with the orientation shown in Figure 3.1-6. When fully inserted, the tip of the autorod is located $7.5 \mathrm{~cm}$ below the bottom of the radial reflector. The autorod is considered fully "withdrawn" in its uppermost position of $100.0 \mathrm{~cm}$ above its fully inserted position (see Figure 3.1-7). The distance the autorod is withdrawn from the fully inserted position for each core configuration is provided in Table $3.1-7$.

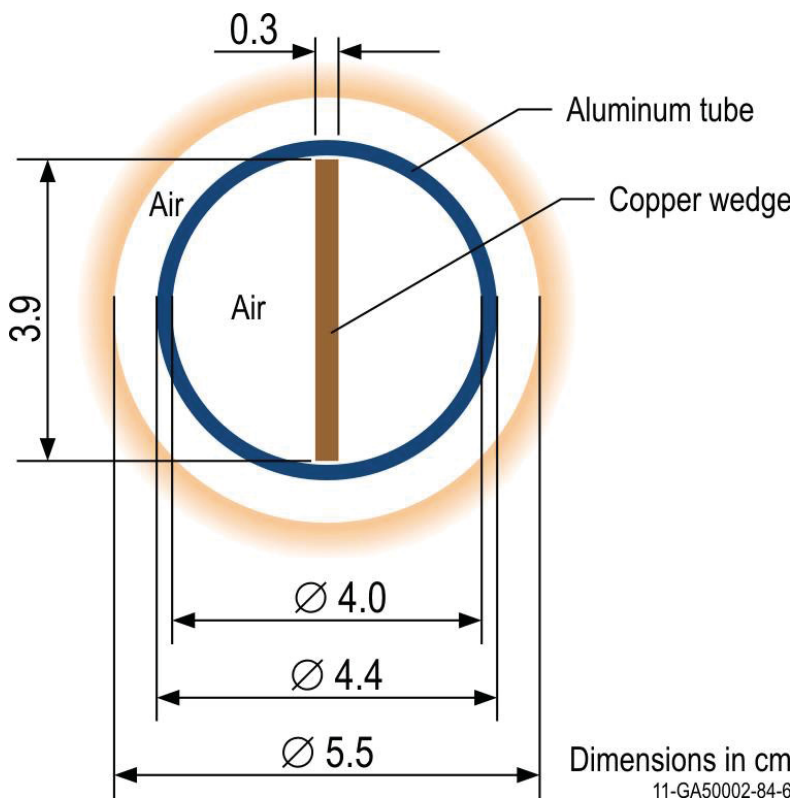

Figure 3.1-6. Top View of Autorod. 
NEA/NSC/DOC(2006)1

Gas Cooled (Thermal) Reactor - GCR

PROTEUS-GCR-EXP-004

CRIT-REAC

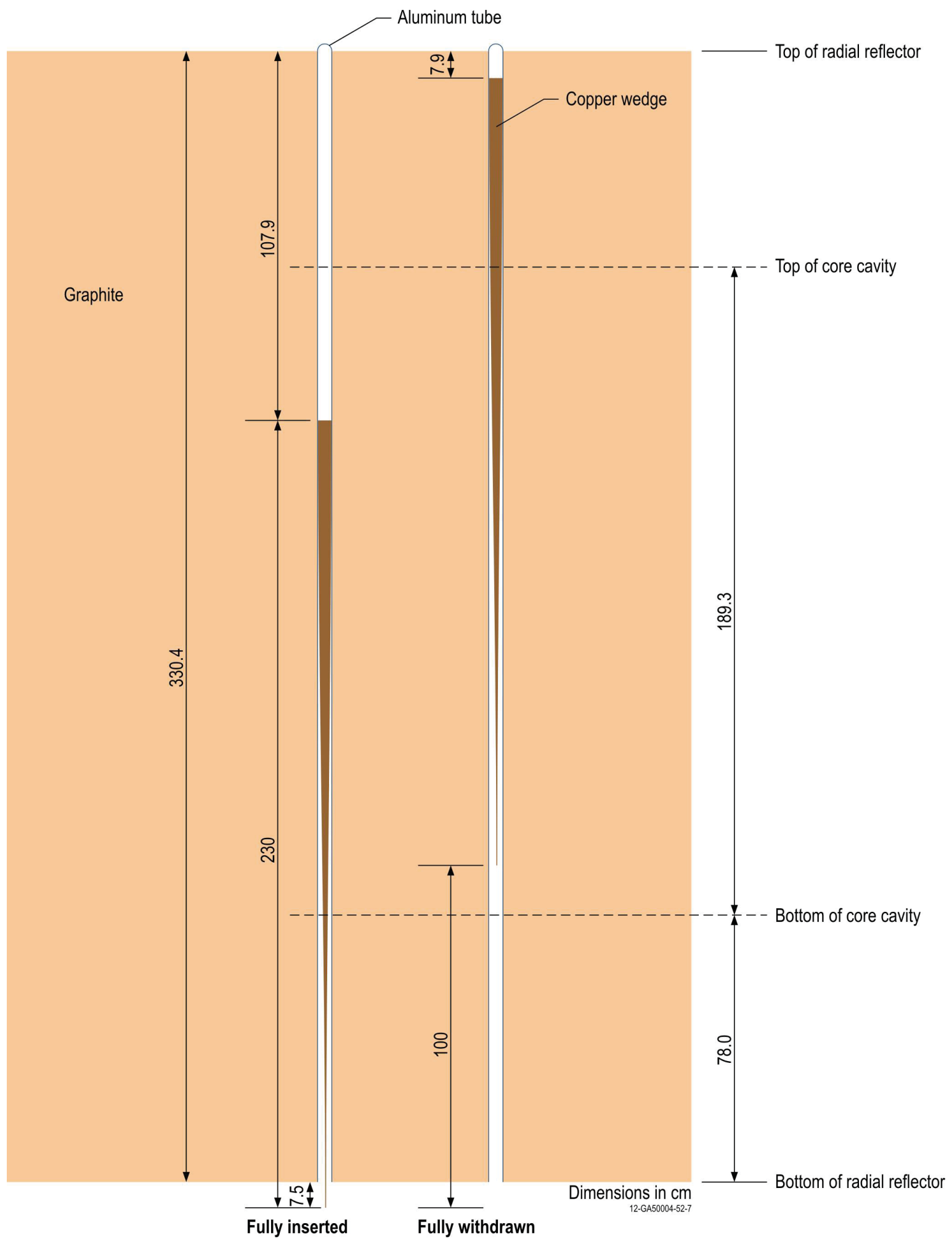

Figure 3.1-7. Autorod Vertical Position within Radial Reflector.

Revision: 1

Date: March 31, 2014 
NEA/NSC/DOC(2006)1

Gas Cooled (Thermal) Reactor - GCR

PROTEUS-GCR-EXP-004

CRIT-REAC

Table 3.1-7. Control Rod Positions (distance in cm).

\begin{tabular}{||l|c|c||}
\hline \multicolumn{1}{||c|}{ Case (Core) } & 1 (9) & 2 (10) \\
\hline \hline Safety/Shutdown Rod & $\begin{array}{c}\text { Withdrawn } \\
\text { Distance }\end{array}$ & $\begin{array}{c}\text { Withdrawn } \\
\text { Distance }\end{array}$ \\
Safety/Shutdown Rod 2 & NA & NA \\
Safety/Shutdown Rod 3 & NA & NA \\
Safety/Shutdown Rod 4 & NA & NA \\
Safety/Shutdown Rod 5 & NA & NA \\
Safety/Shutdown Rod 6 & NA & NA \\
Safety/Shutdown Rod 7 & NA & NA \\
Safety/Shutdown Rod 8 & NA & NA \\
\hline Autorod & 25.8 & 1.5 \\
\hline Withdrawable Control Rod 1 & 249.4 & 96.0 \\
Withdrawable Control Rod 2 & 249.4 & 96.0 \\
Withdrawable Control Rod 3 & 249.4 & 96.0 \\
Withdrawable Control Rod 4 & 249.4 & 96.0 \\
\hline \hline
\end{tabular}

Revision: 1

Date: March 31, 2014

Page 154 of 375 
NEA/NSC/DOC(2006)1

\author{
Gas Cooled (Thermal) Reactor - GCR \\ PROTEUS-GCR-EXP-004 \\ CRIT-REAC
}

\title{
3.1.2.5 Fuel Pebbles
}

The graphite fuel pebbles have a diameter of $6.000 \mathrm{~cm}$. A total of 9394 TRISO particles are randomly distributed within the graphite matrix of the fueled zone (diameter of $4.700 \mathrm{~cm}$ ) of each fuel pebble (Figure 3.1-8). The fuel pebbles are located in the core cavity; their positions in each core configuration are described in more detail in Section 3.1.2.10. Each TRISO particle consists of four layers surrounding a $\mathrm{UO}_{2}$ kernel. The fuel kernel has a diameter of $0.0502 \mathrm{~cm}$. A graphite buffer layer (thickness of 0.00915 $\mathrm{cm}$ ) surrounds the fuel kernel. An inner pyrolytic carbon (IPyC) layer (thickness of $0.00399 \mathrm{~cm}$ ), $\mathrm{SiC}$ layer (thickness of $0.00353 \mathrm{~cm}$ ), and outer pyrolytic carbon (OPyC) layer (thickness of $0.00400 \mathrm{~cm}$ ) then each, in succession, surround the growing TRISO particle, as shown in Figure 3.1-8.

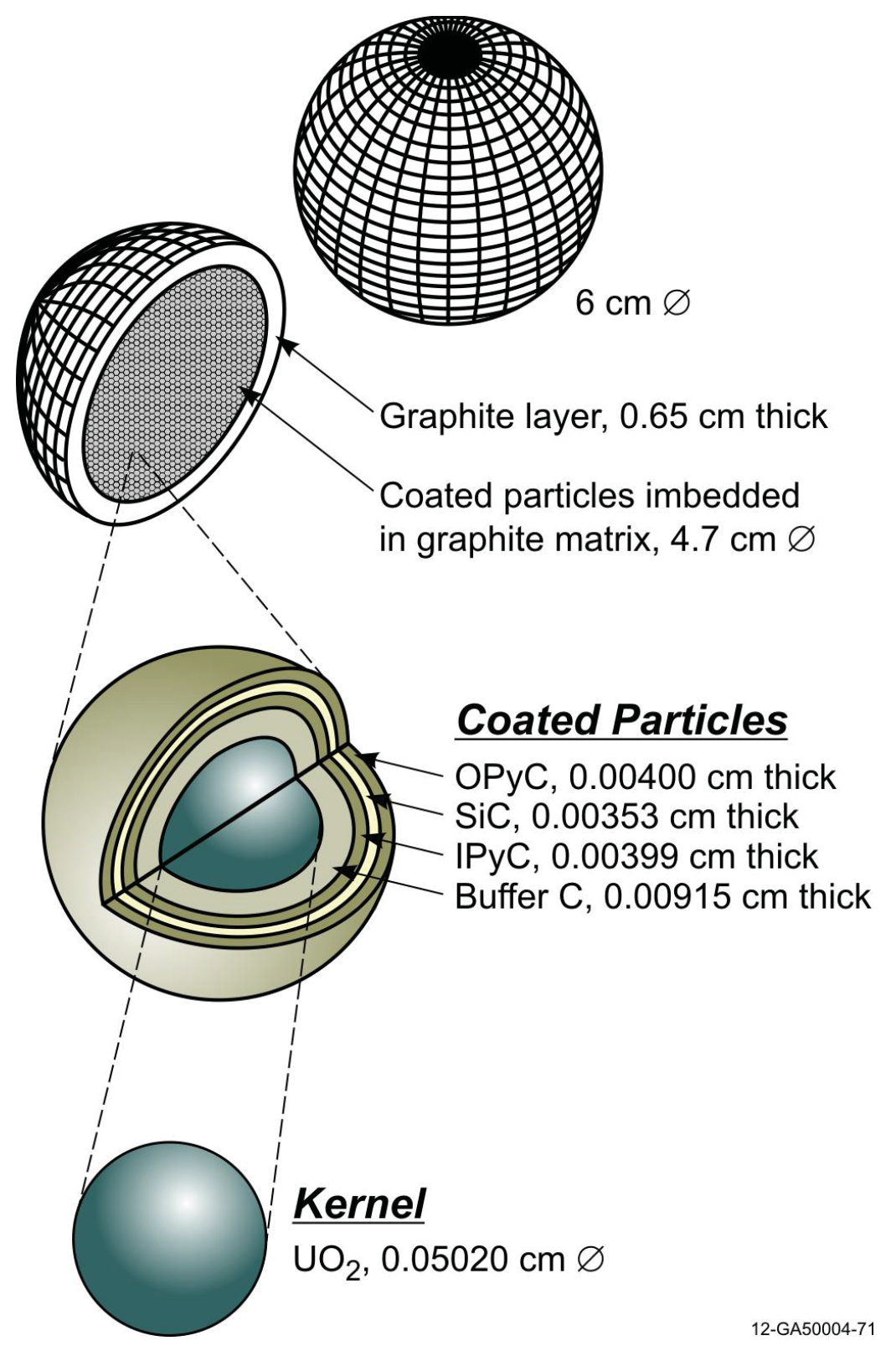

Figure 3.1-8. Fuel Pebble and TRISO Particle. 
NEA/NSC/DOC(2006)1

\section{Gas Cooled (Thermal) Reactor - GCR \\ PROTEUS-GCR-EXP-004 \\ CRIT-REAC}

\subsubsection{Moderator Pebbles}

The graphite moderator pebbles have a diameter of $6.000 \mathrm{~cm}$. They are located in the core cavity; their positions in each core configuration are described in more detail in Section 3.1.2.10.

\subsubsection{Withdrawable Stainless Steel Control Rods}

The withdrawable control rods (Figures 3.1-9 through 3.1-11) are comprised of two concentric stainless steel tubes with end plugs. The inner tube has an inner diameter of $0.95 \mathrm{~cm}$ and an outer diameter of 1.35 $\mathrm{cm}$. The outer tube has an inner diameter of $1.4 \mathrm{~cm}$ and an outer diameter of $2.2 \mathrm{~cm}$. Both tubes have a total length of $215.0 \mathrm{~cm}$. The dimensions for the end plugs are shown in Figure 3.1-10. The stainless steel control rods are completely inserted into the core when the bottom surface of the bottom end plug is located $75.5 \mathrm{~cm}$ above the bottom of the radial reflector; they are completely withdrawn when raised $249.4 \mathrm{~cm}$ from the fully inserted position (see Figure 3.1-11). A graphite plug (diameter of $2.65 \mathrm{~cm}$ and height of $73.0 \mathrm{~cm}$ ) is located in the bottom of each penetration for the withdrawable control rods. The withdrawn positions of the withdrawable control rods are provided in Table 3.1-7.

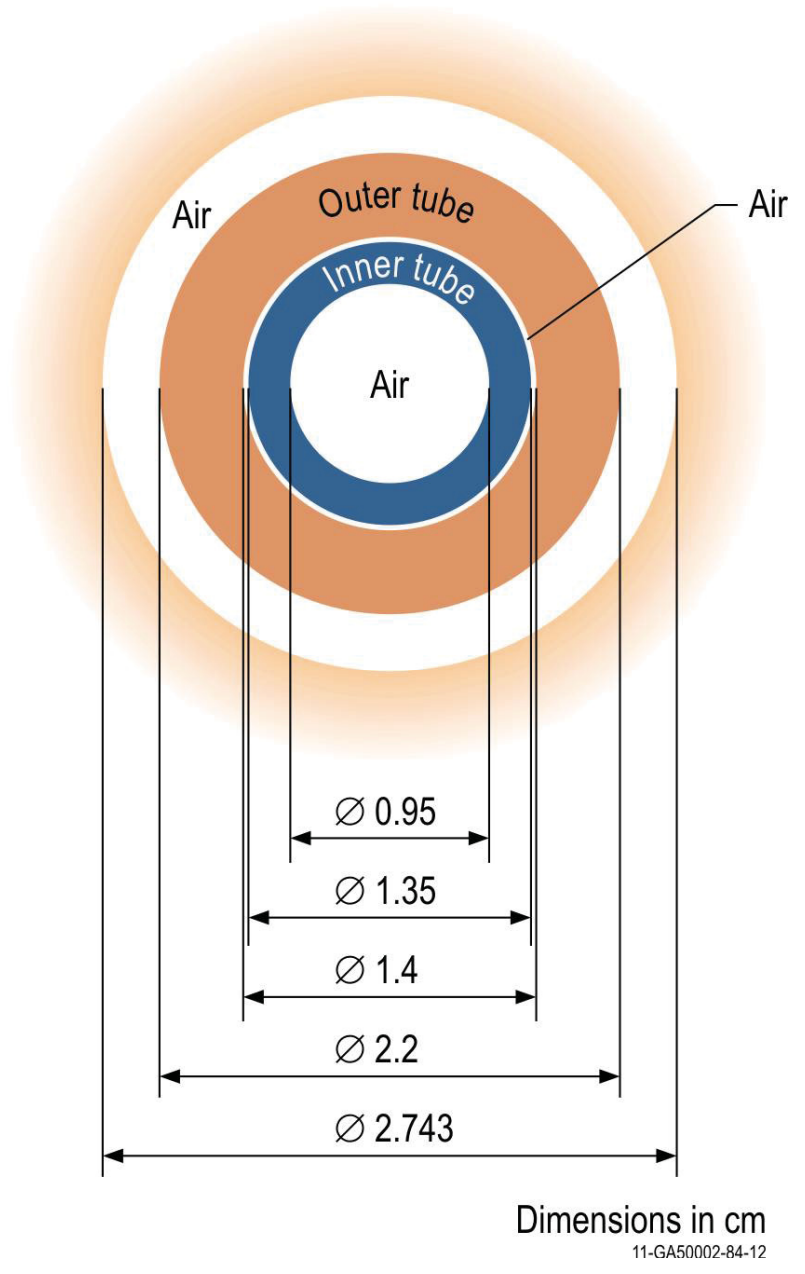

Figure 3.1-9. Top View of Withdrawable Control Rod. 
NEA/NSC/DOC(2006)1

Gas Cooled (Thermal) Reactor - GCR

PROTEUS-GCR-EXP-004

CRIT-REAC

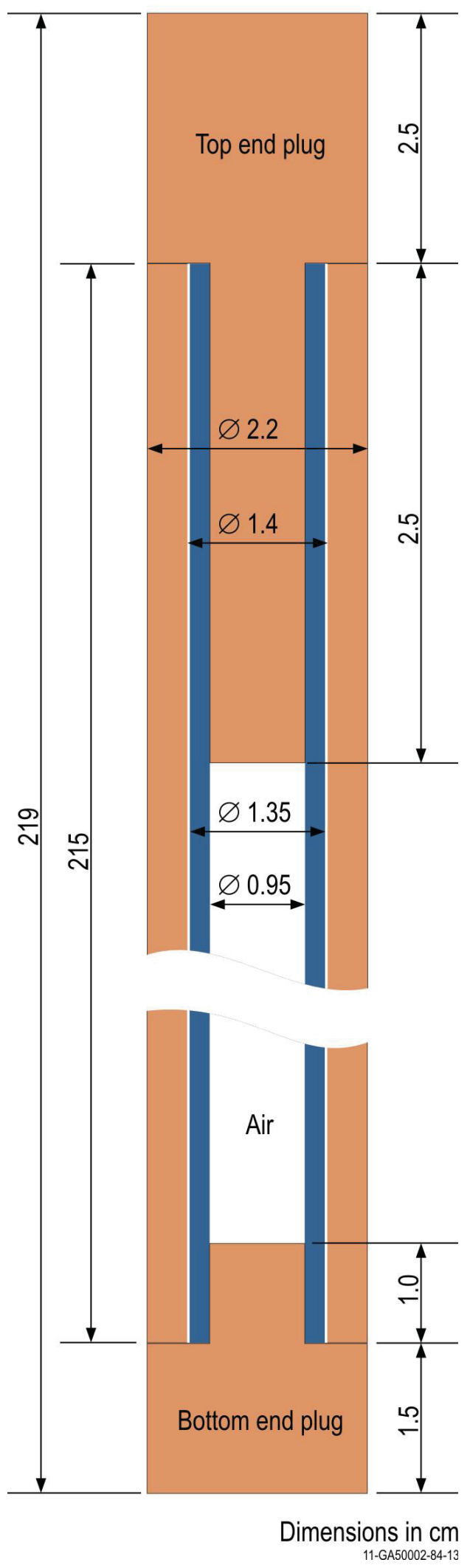

Figure 3.1-10. Axial View of Withdrawable Control Rod.

Revision: 1

Date: March 31, 2014

Page 157 of 375 
NEA/NSC/DOC(2006)1

Gas Cooled (Thermal) Reactor - GCR

PROTEUS-GCR-EXP-004

CRIT-REAC

Air

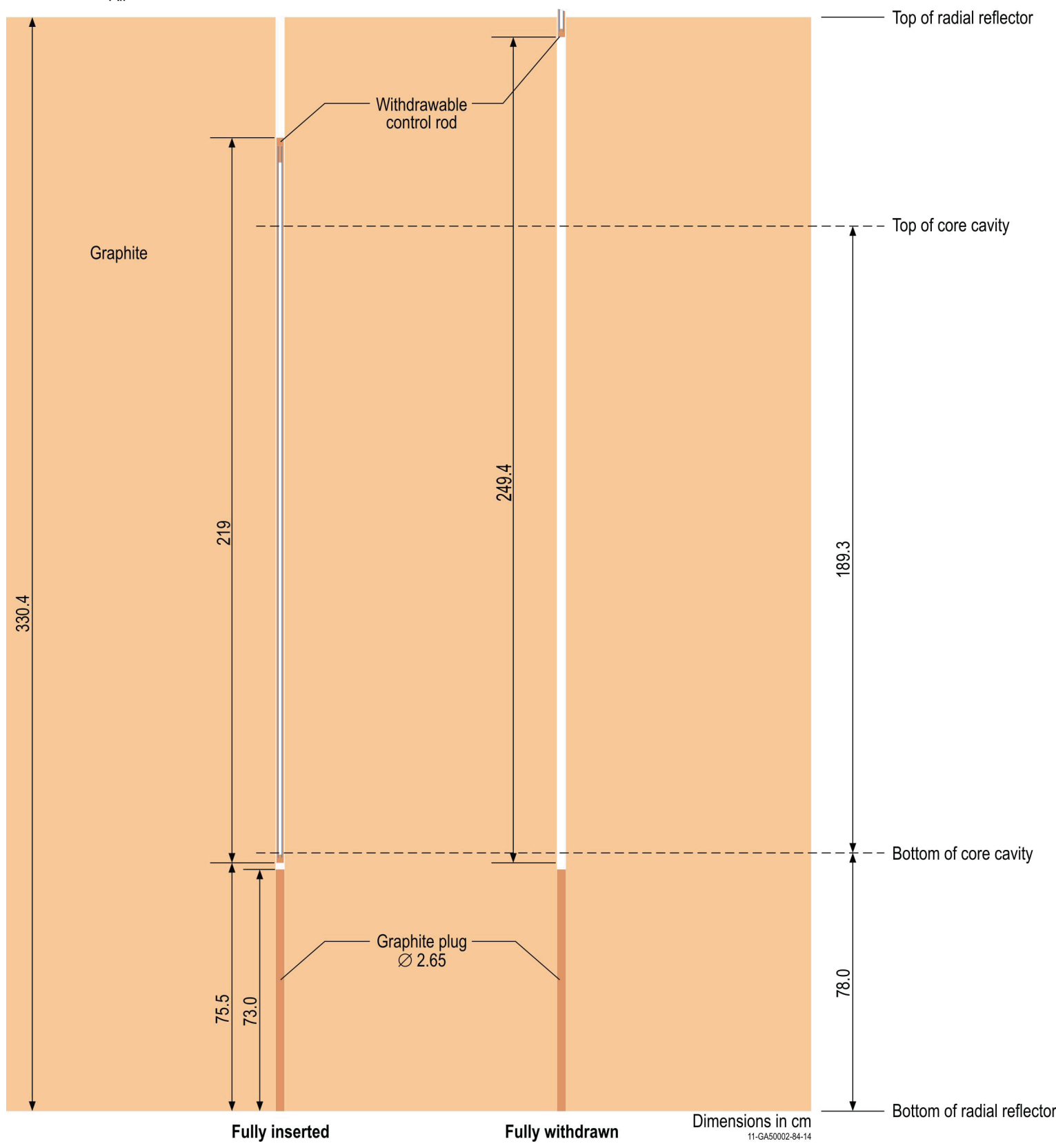

Figure 3.1-11. Withdrawable Control Rod Vertical Position within Radial Reflector.

\subsubsection{Polyethylene Rods}

Polyethylene (sometimes referred to as plastic) rods are used to simulate water ingress in Case 2 (Core 10) only. Each rod has a diameter of $0.65 \mathrm{~cm}$ and length of $145.0 \mathrm{~cm}$. There are a total of 654 rods located in the core; they are placed vertically in interstitial pebble channels (see Figures 3.1-21 and 3.126).

Revision: 1

Date: March 31, 2014 
NEA/NSC/DOC(2006)1

\section{Gas Cooled (Thermal) Reactor - GCR \\ PROTEUS-GCR-EXP-004 \\ CRIT-REAC}

\subsubsection{Ambient Air}

Air is located in any gaps, holes, or penetrations within the benchmark model that does not contain the graphite reflectors, graphite plugs, aluminum support structure, pebbles, lattice supports, or control rods.

\subsubsection{Core Configurations}

Each core has a unique configuration of fuel and moderator pebbles stacked within the core cavity. The position of each pebble is known since the pebbles are deterministically placed in columnar hexagonal point-on-point packed lattices, as shown in Figure 3.1-12. Information corresponding to the loading of each configuration is provided in Table 3.1-8 with additional visualization of the core descriptions in Figures 3.1-13 through 3.1-26.

\section{Layer 1}

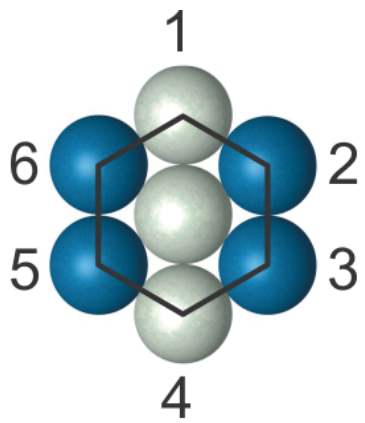

Layer 2
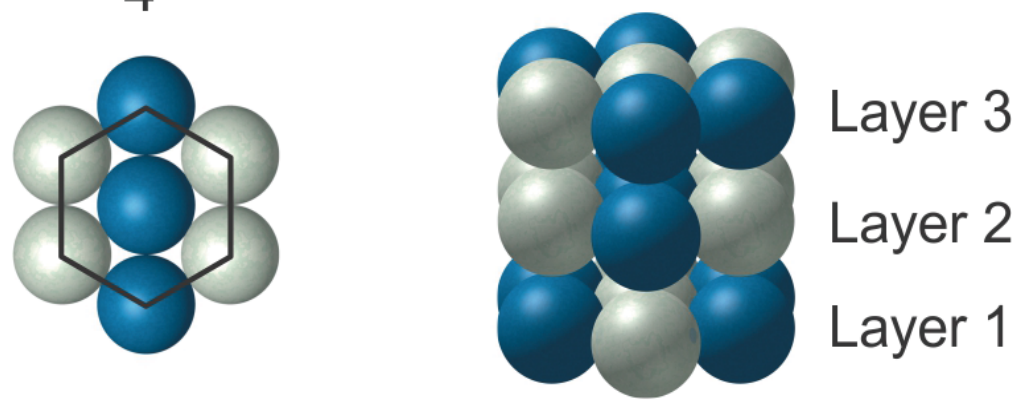

Layer 3
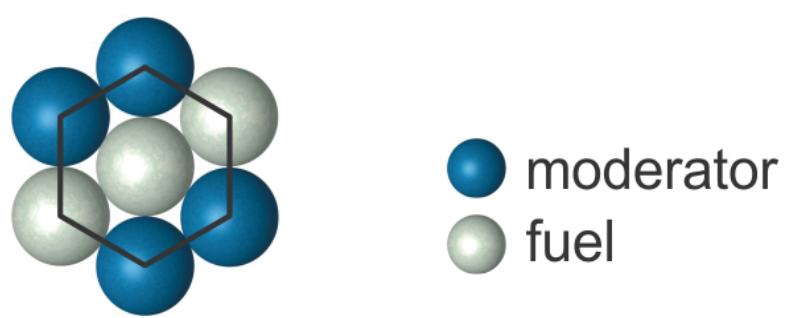

12-GA50004-89-4

Figure 3.1-12. Subunit for Construction of the Columnar Hexagonal Point-On-Point (CHPOP) Cell.

Table 3.1-8. Additional Core Configuration Parameters.

\begin{tabular}{|c|c|c|c|c|c|c|c||}
\hline \hline Case & Core & $\begin{array}{c}\text { \# Fuel } \\
\text { Pebbles }\end{array}$ & $\begin{array}{c}\text { \# Moderator } \\
\text { Pebbles }\end{array}$ & $\begin{array}{c}\text { \# Pebble } \\
\text { Layers }\end{array}$ & $\begin{array}{c}\text { Core } \\
\text { Height (m) }\end{array}$ & $\begin{array}{c}\text { \# Polyethylene } \\
\text { Rods }\end{array}$ & $\begin{array}{c}\text { Associated } \\
\text { Figures }\end{array}$ \\
\hline \hline 1 & 9 & 4870 & 4877 & 27 & 1.62 & 0 & $3.1-13$ to 3.1-19 \\
\hline 2 & 10 & 4332 & 4332 & 24 & 1.44 & 654 & $3.1-20$ to 3.1-26 \\
\hline
\end{tabular}


NEA/NSC/DOC(2006)1

Gas Cooled (Thermal) Reactor - GCR

PROTEUS-GCR-EXP-004

CRIT-REAC

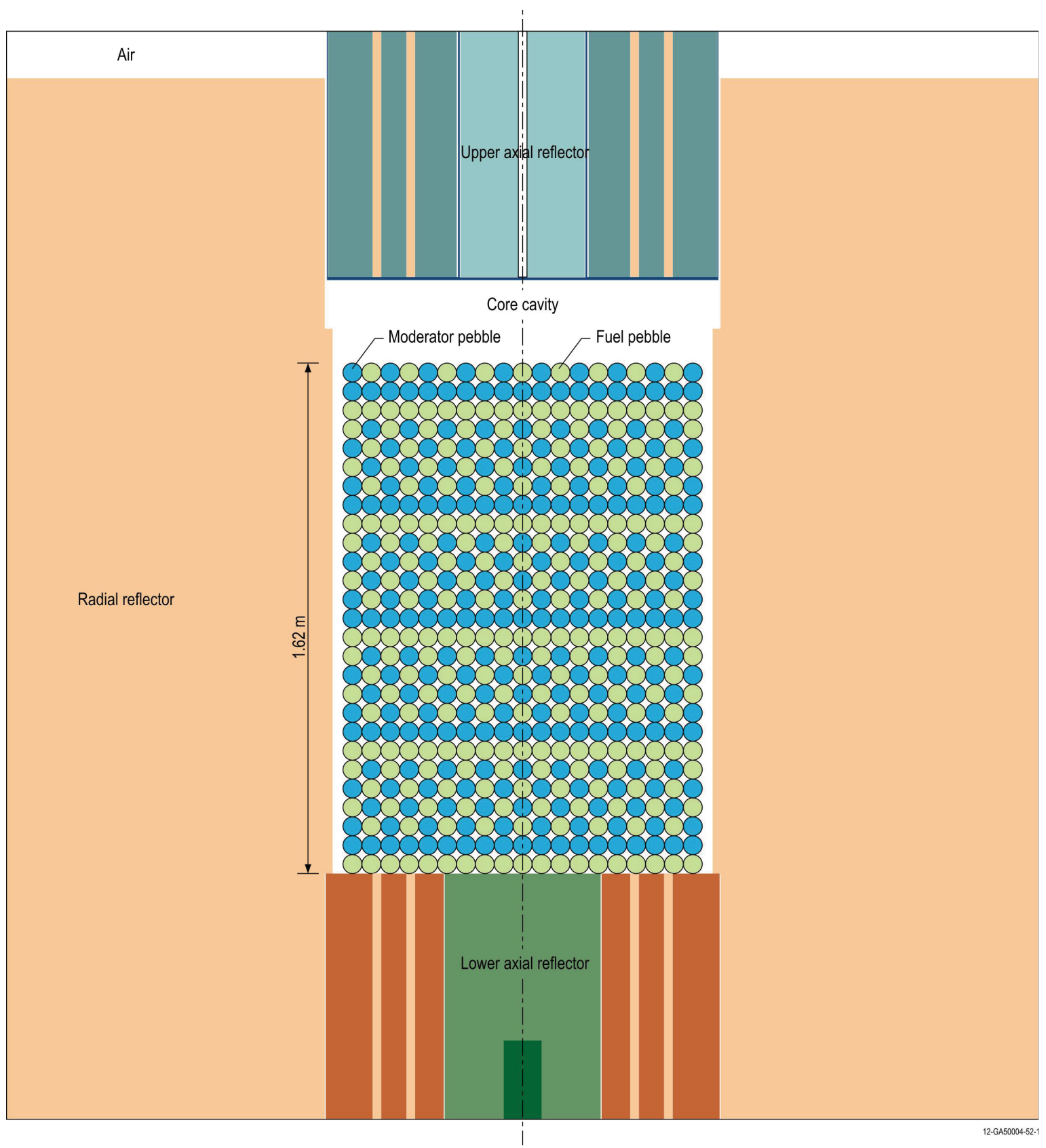

Figure 3.1-13. Vertical Profile of Case 1 (Core 9).

Revision: 1

Date: March 31, 2014 
NEA/NSC/DOC(2006)1

Gas Cooled (Thermal) Reactor - GCR

PROTEUS-GCR-EXP-004

CRIT-REAC

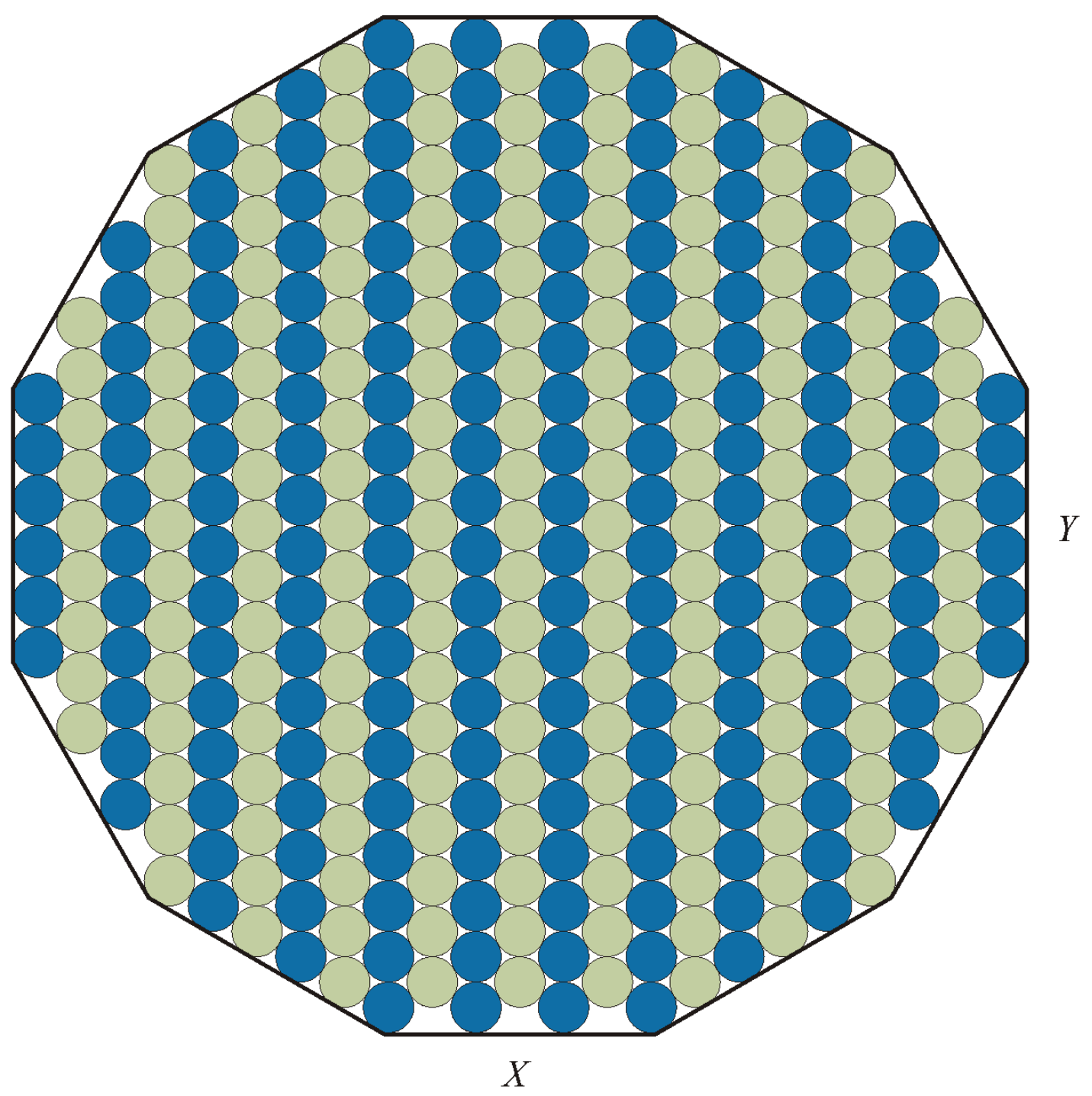

Fuel pebbles:

Moderator pebbles:

Total pebbles: $\quad \overline{361}$

Figure 3.1-14. Layers 1, 7, 13, 19, and 25 of Case 1 (Core 9). 
NEA/NSC/DOC(2006)1

Gas Cooled (Thermal) Reactor - GCR

PROTEUS-GCR-EXP-004

CRIT-REAC

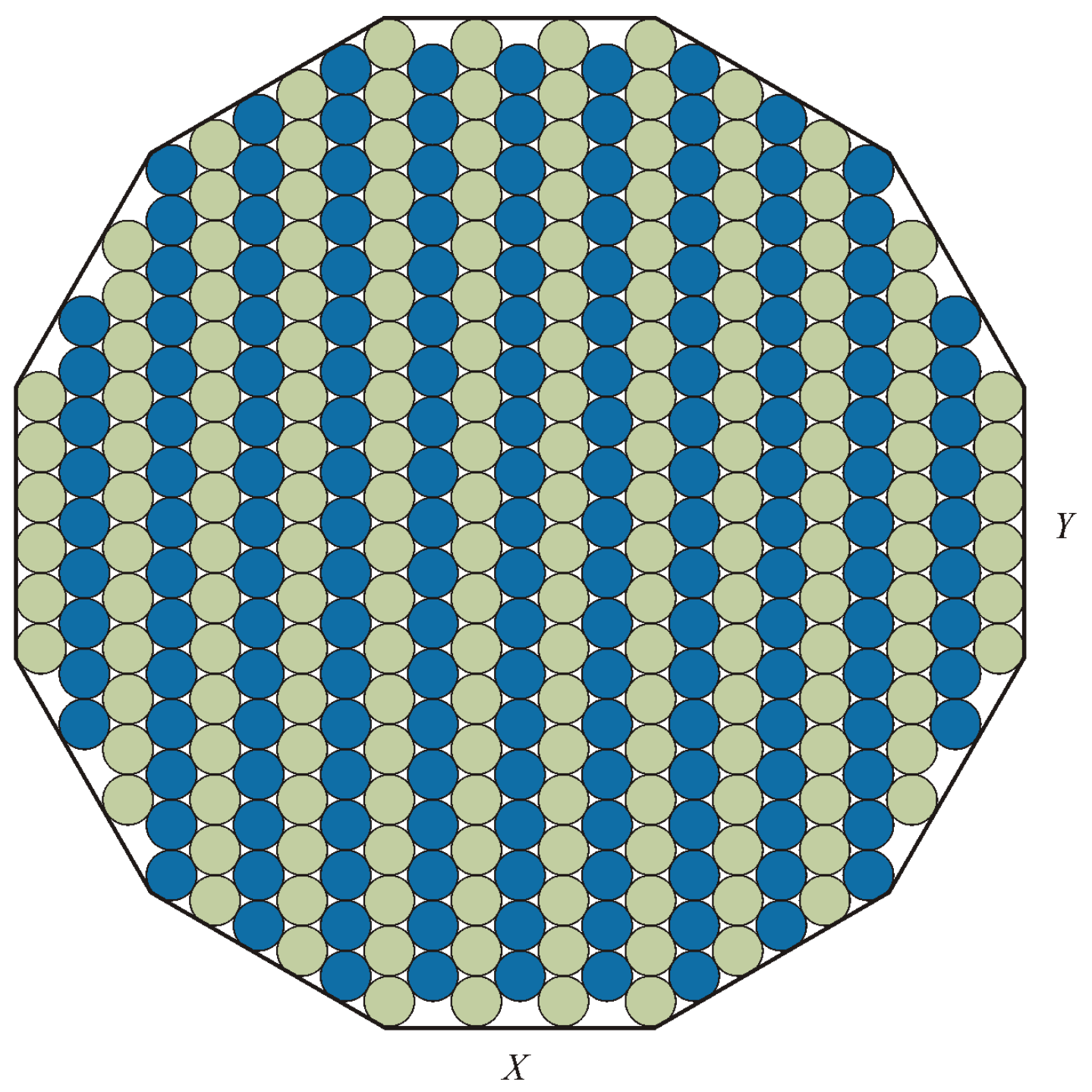

Fuel pebbles: $\quad 184$

Moderator pebbles: $\frac{177}{361}$
Total pebbles:

Figure 3.1-15. Layers 2, 8, 14, 20, and 26 of Case 1 (Core 9). 
NEA/NSC/DOC(2006)1

Gas Cooled (Thermal) Reactor - GCR

PROTEUS-GCR-EXP-004

CRIT-REAC

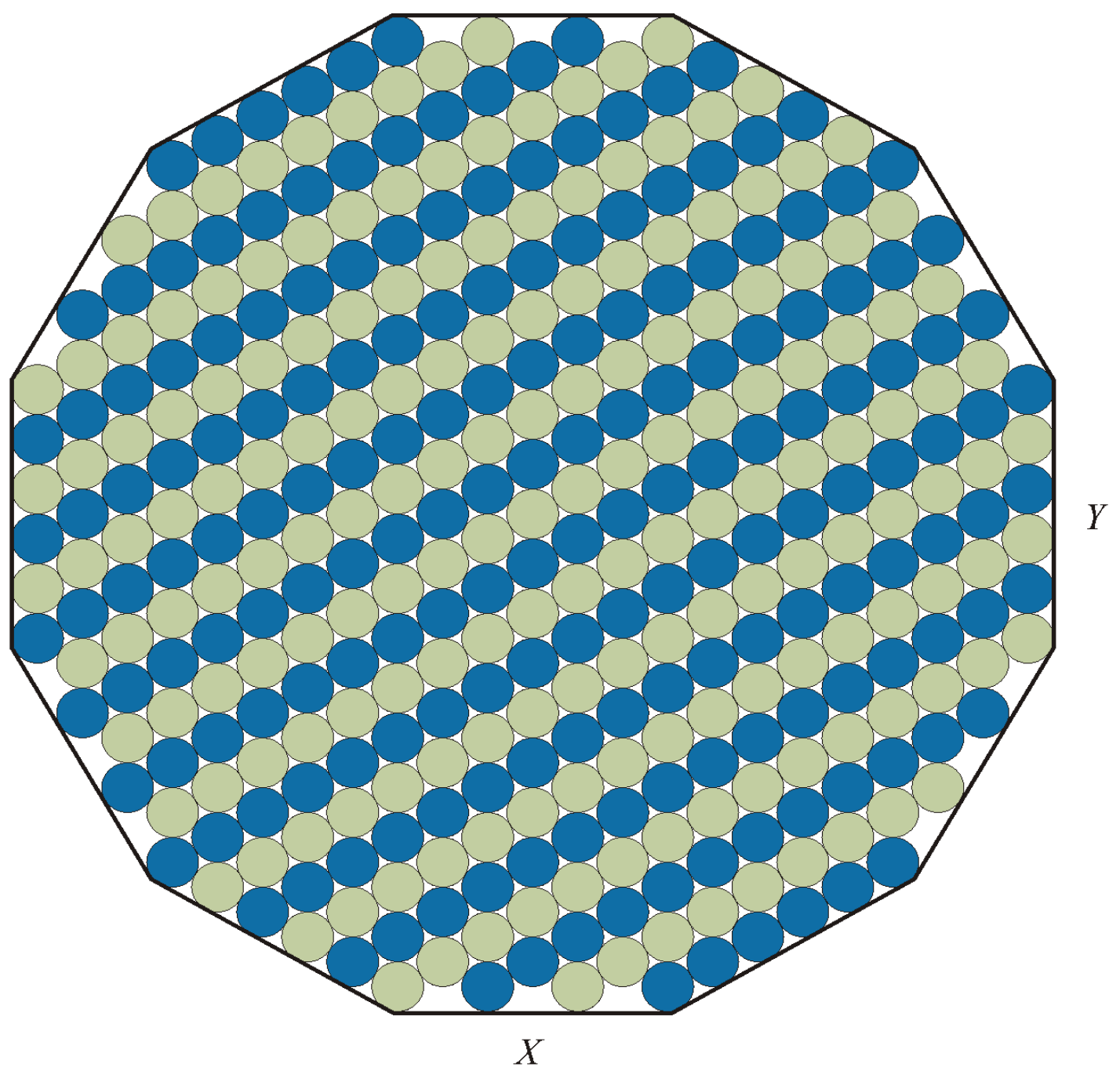

Fuel pebbles:

Moderator pebbles: $\frac{184}{361}$ Total pebbles: $\quad 361$

Figure 3.1-16. Layers 3, 9, 15, 21, and 27 of Case 1 (Core 9). 
NEA/NSC/DOC(2006)1

Gas Cooled (Thermal) Reactor - GCR

PROTEUS-GCR-EXP-004

CRIT-REAC

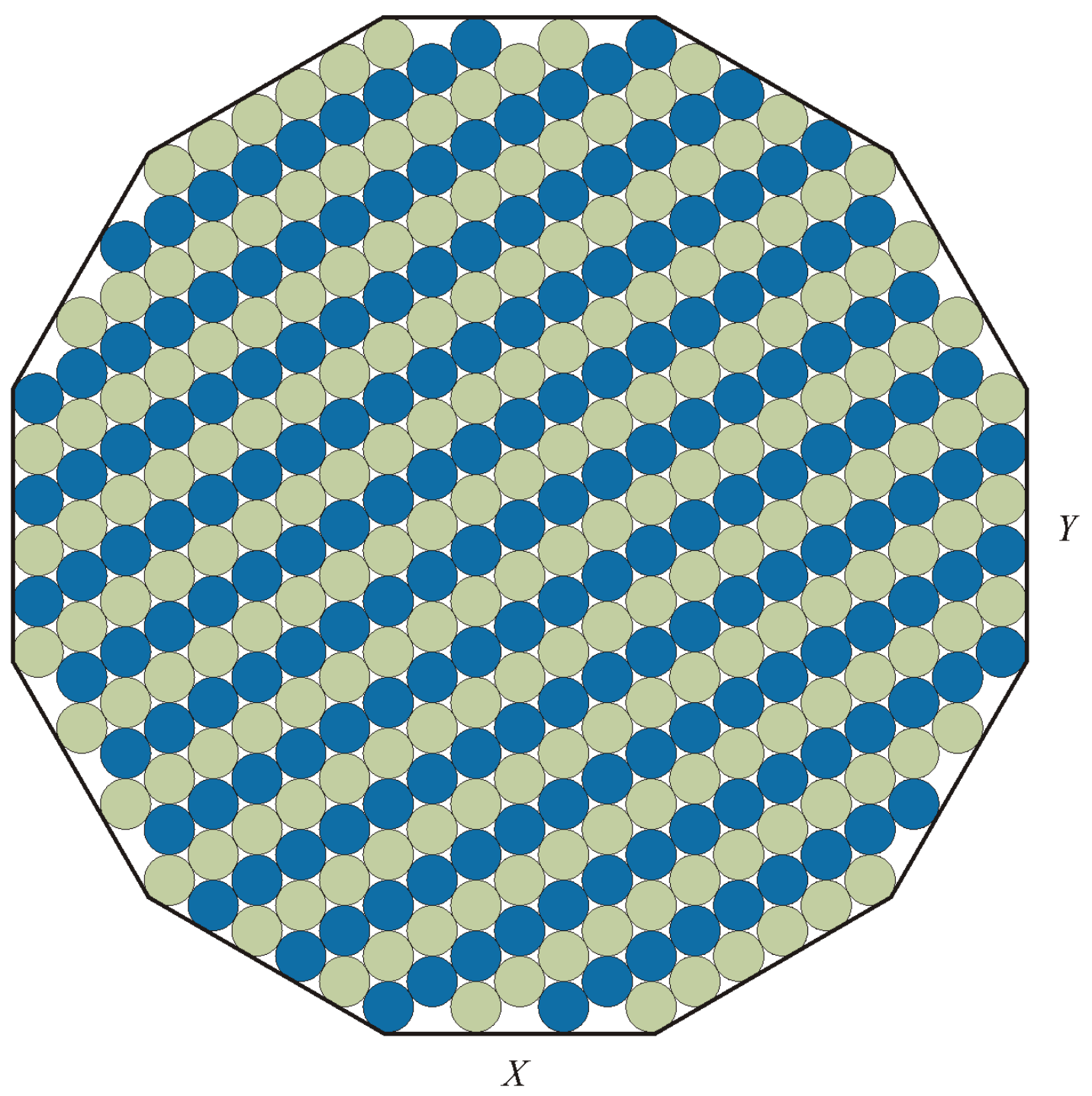

Fuel pebbles:

184

Moderator pebbles: $\frac{177}{361}$
Total pebbles:

Figure 3.1-17. Layers 4, 10, 16, and 22 of Case 1 (Core 9). 
NEA/NSC/DOC(2006)1

Gas Cooled (Thermal) Reactor - GCR

PROTEUS-GCR-EXP-004

CRIT-REAC

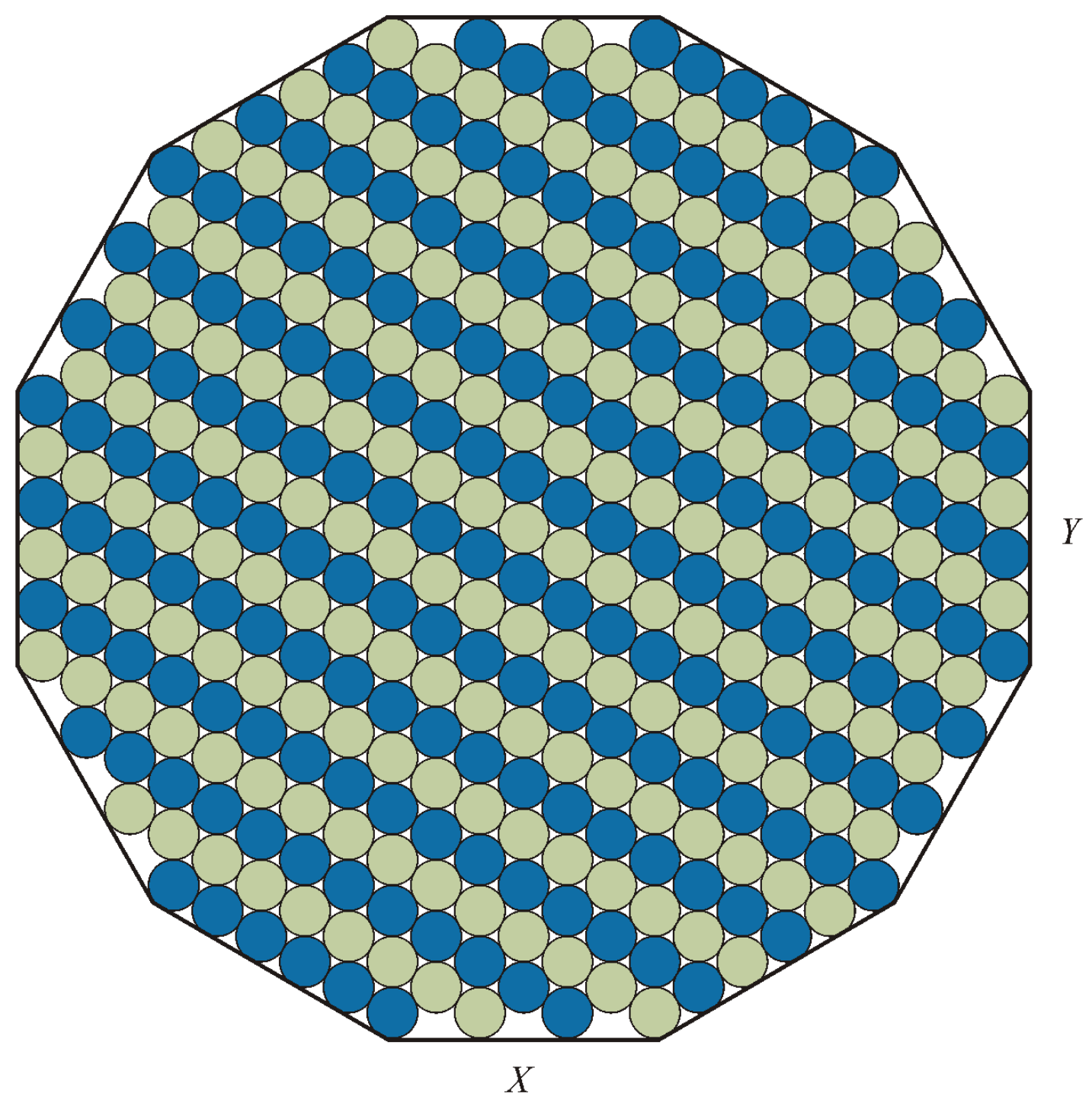

Fuel pebbles: $\quad 177$

Moderator pebbles: 184 Total pebbles: $\quad \overline{361}$

Figure 3.1-18. Layers 5, 11, 17, and 23 of Case 1 (Core 9). 
NEA/NSC/DOC(2006)1

Gas Cooled (Thermal) Reactor - GCR

PROTEUS-GCR-EXP-004

CRIT-REAC

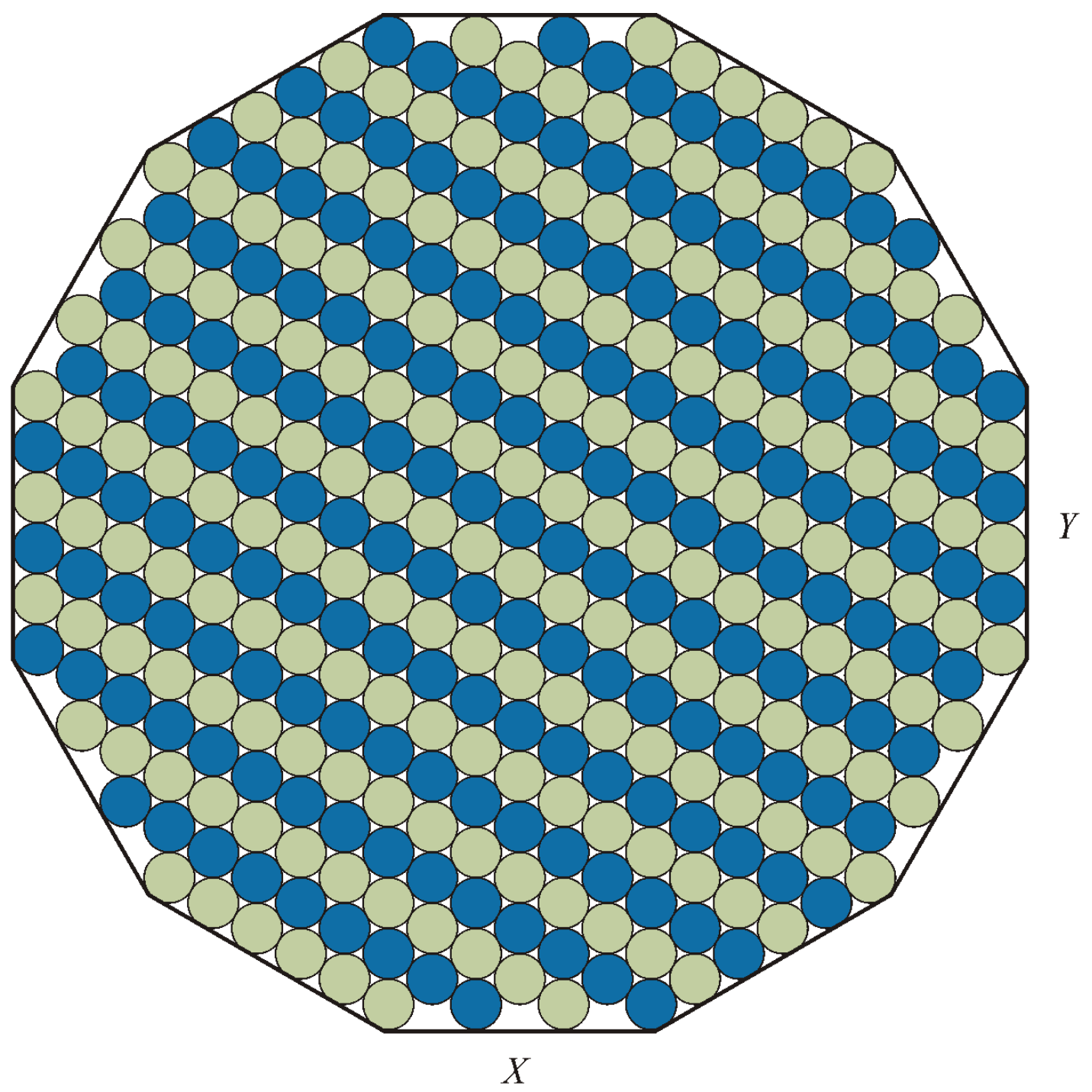

Fuel pebbles: $\quad 184$

Moderator pebbles: $\frac{177}{361}$
Total pebbles:

Figure 3.1-19. Layers 6, 12, 18, and 24 of Case 1 (Core 9). 
NEA/NSC/DOC(2006)1

Gas Cooled (Thermal) Reactor - GCR

PROTEUS-GCR-EXP-004

CRIT-REAC

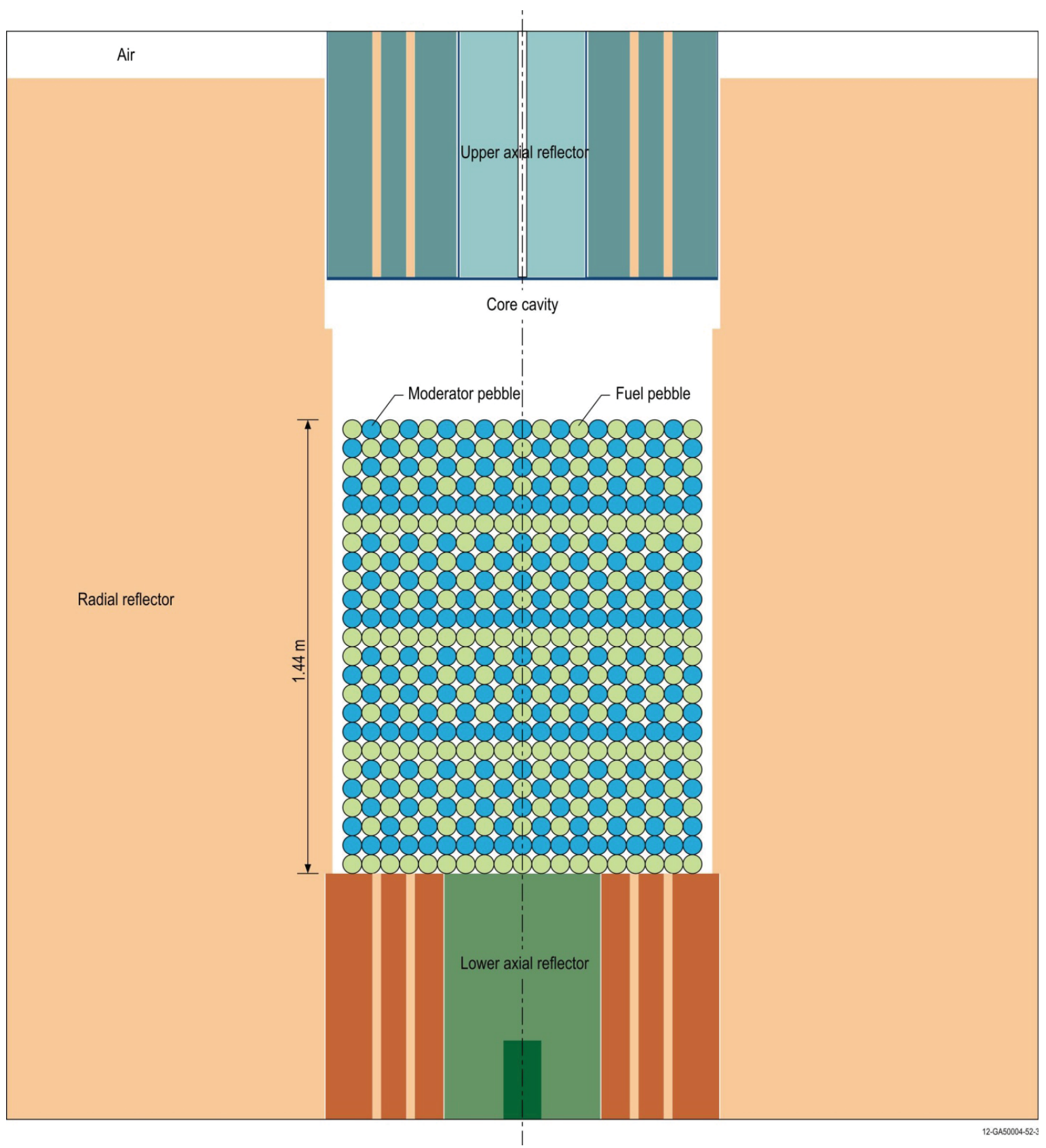

Figure 3.1-20. Vertical Profile of Case 2 (Core 10).

Revision: 1

Date: March 31, 2014 
NEA/NSC/DOC(2006)1

Gas Cooled (Thermal) Reactor - GCR

PROTEUS-GCR-EXP-004

CRIT-REAC

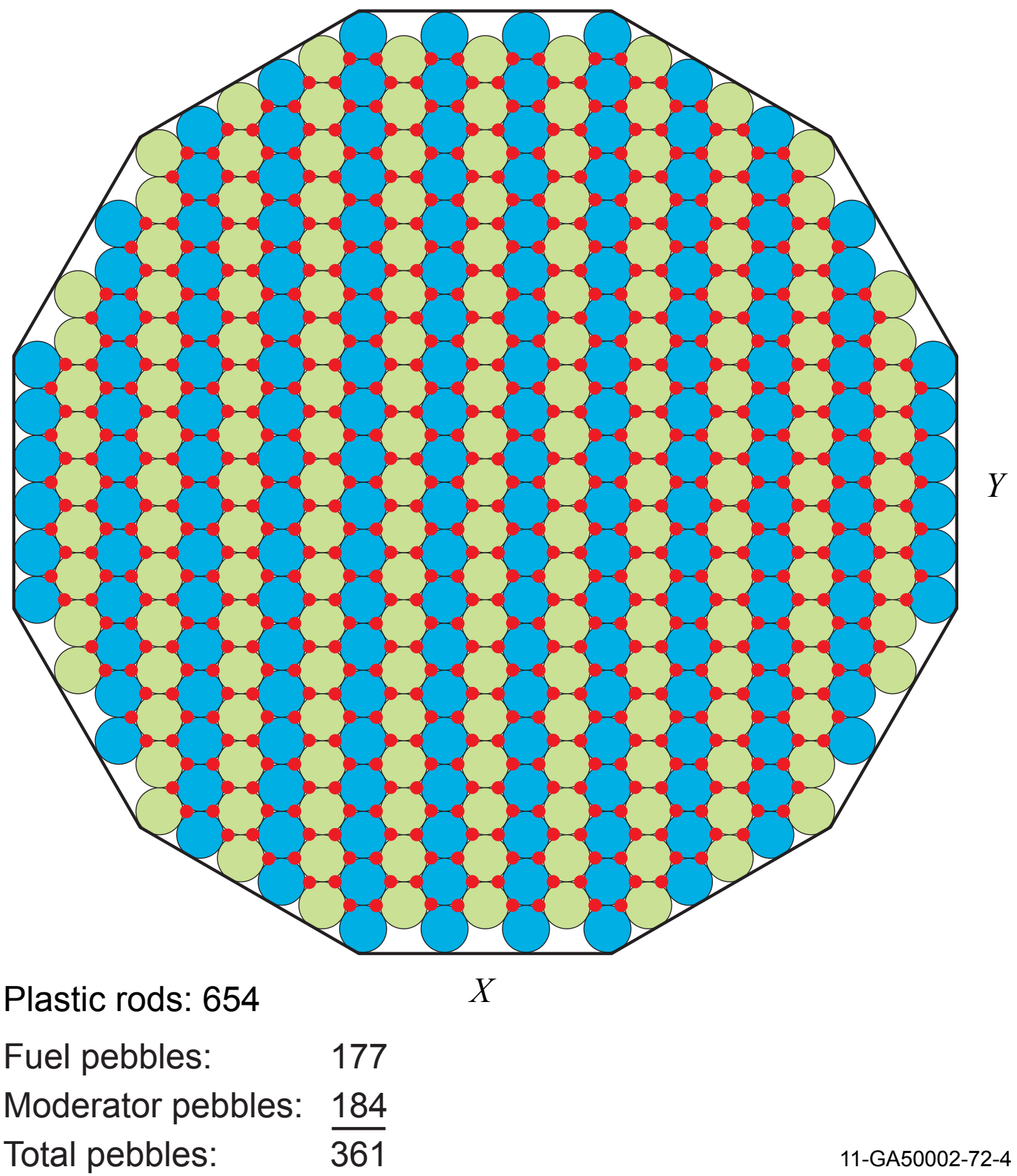

Figure 3.1-21. Layers 1, 7, 13, 19, and 25 of Case 2 (Core 10). 
NEA/NSC/DOC(2006)1

Gas Cooled (Thermal) Reactor - GCR

PROTEUS-GCR-EXP-004

CRIT-REAC

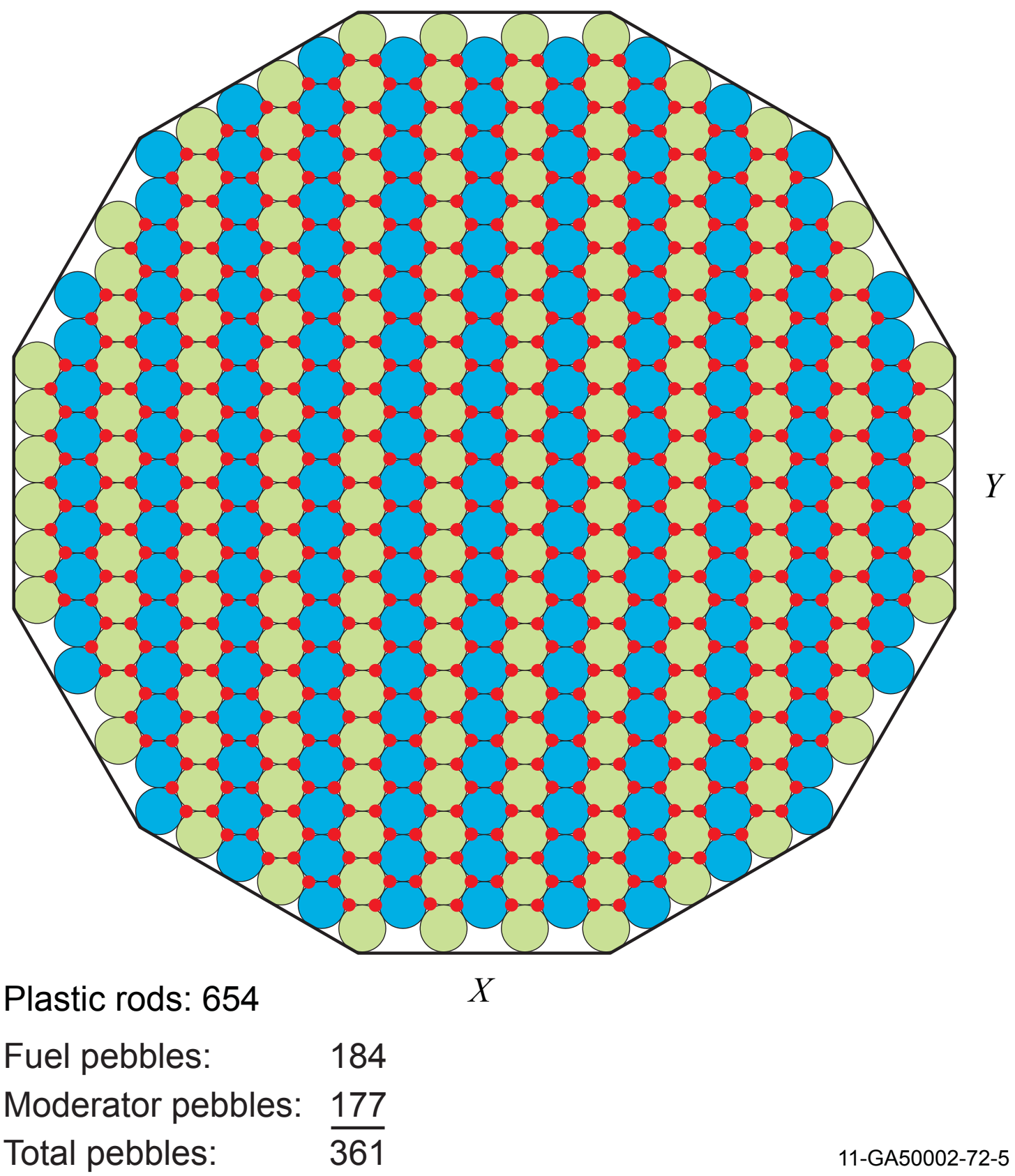

Figure 3.1-22. Layers 2, 8, 14, 20, and 26 of Case 2 (Core 10). 
NEA/NSC/DOC(2006)1

Gas Cooled (Thermal) Reactor - GCR

PROTEUS-GCR-EXP-004

CRIT-REAC

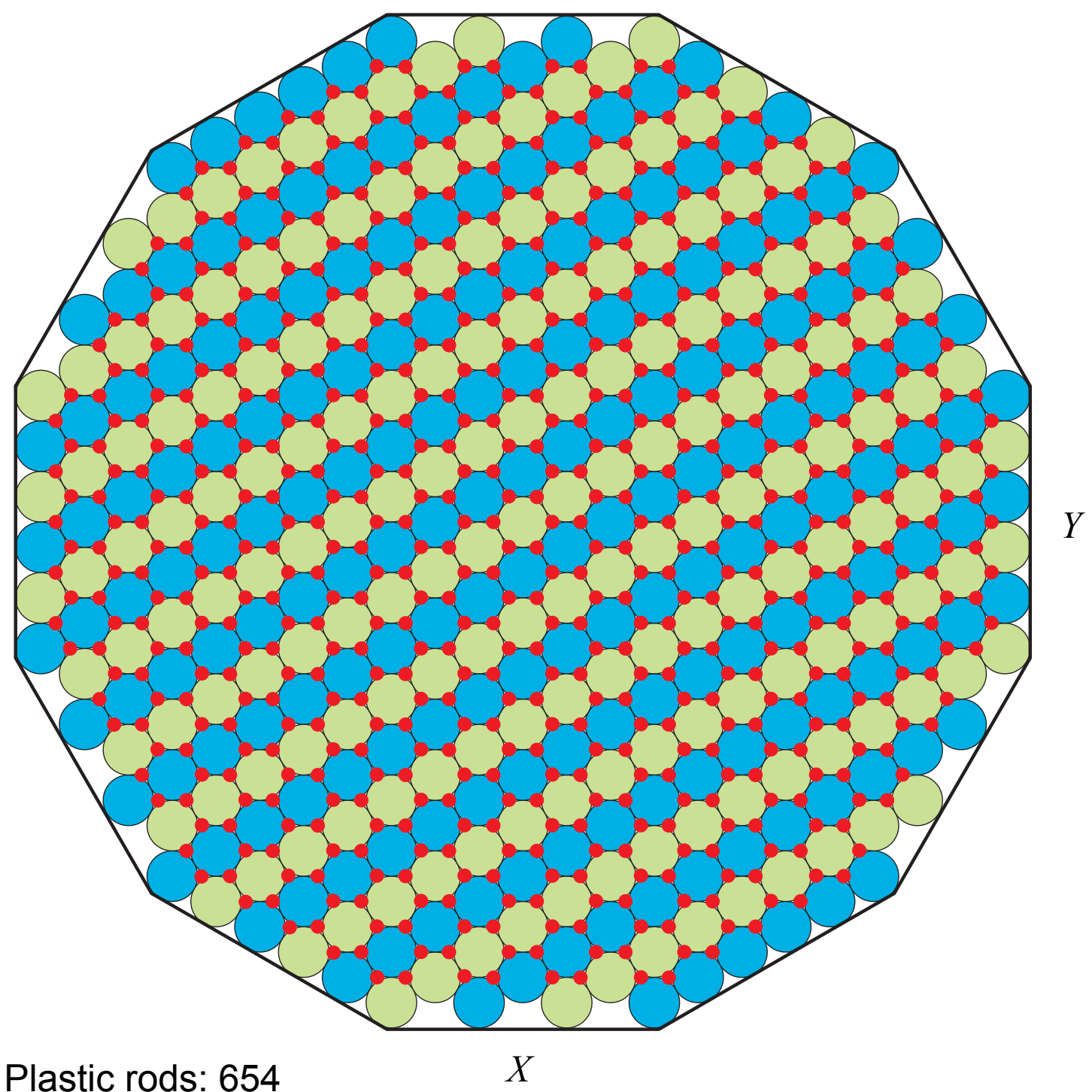

- Plastic rods: 654

Moderator pebbles: $\frac{184}{361}$
Total pebbles:

Figure 3.1-23. Layers 3, 9, 15, 21, and 27 of Case 2 (Core 10). 
NEA/NSC/DOC(2006)1

Gas Cooled (Thermal) Reactor - GCR

PROTEUS-GCR-EXP-004

CRIT-REAC

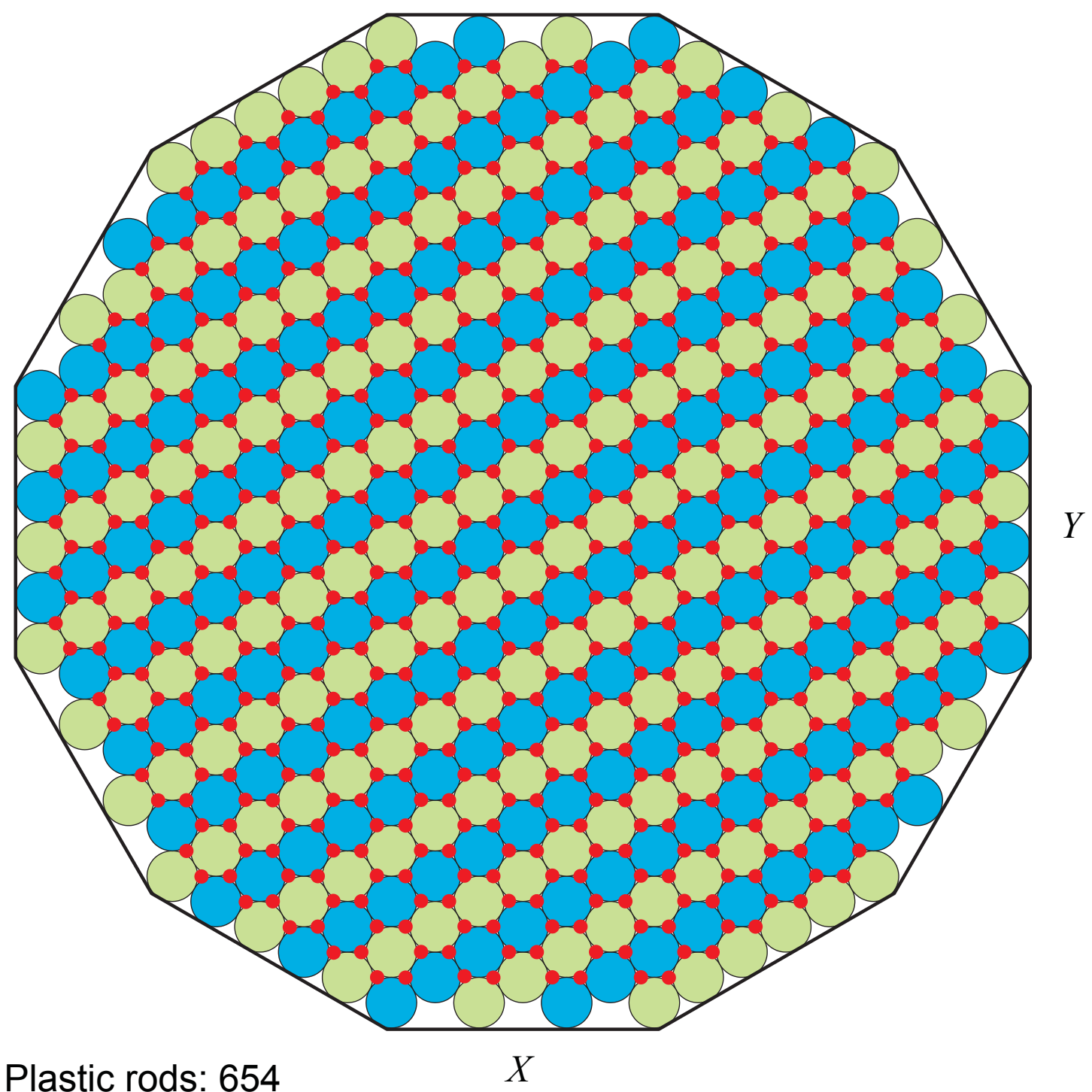

- Plastic rods: 654

184

Moderator pebbles: $\frac{177}{361}$
Total pebbles:

Figure 3.1-24. Layers 4, 10, 16, and 22 of Case 2 (Core 10). 
NEA/NSC/DOC(2006)1

Gas Cooled (Thermal) Reactor - GCR

PROTEUS-GCR-EXP-004 CRIT-REAC

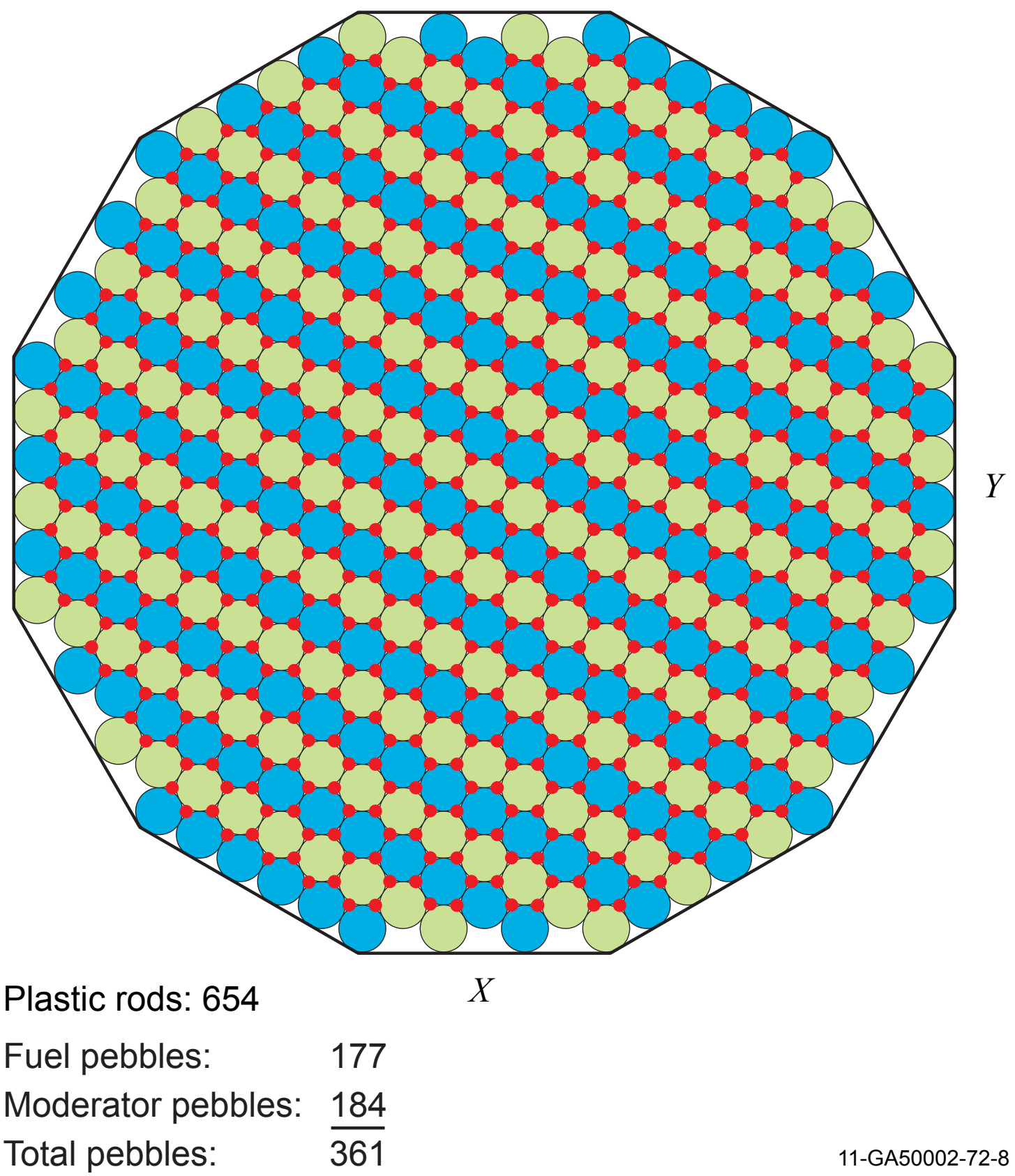

Figure 3.1-25. Layers 5, 11, 17, and 23 of Case 2 (Core 10). 
NEA/NSC/DOC(2006)1

Gas Cooled (Thermal) Reactor - GCR

PROTEUS-GCR-EXP-004

CRIT-REAC

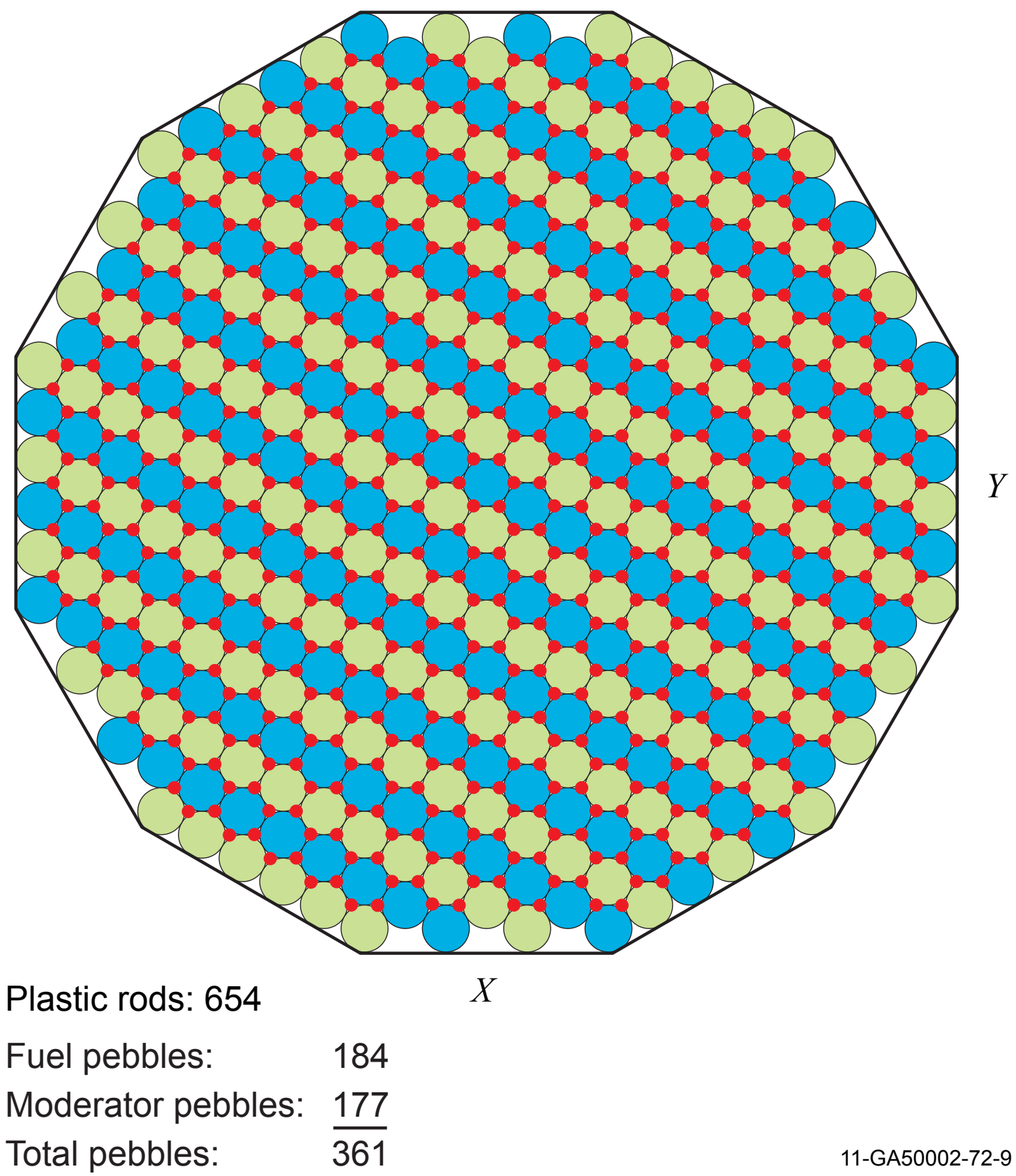

Figure 3.1-26. Layers 6, 12, 18, and 24 of Case 2 (Core 10). 
NEA/NSC/DOC(2006)1

\section{Gas Cooled (Thermal) Reactor - GCR \\ PROTEUS-GCR-EXP-004 \\ CRIT-REAC}

\subsubsection{Material Data}

\subsubsection{Radial Reflector}

The homogenized (see Section 3.1.1.1) graphite radial reflector has the compositions in Table 3.1-9. The graphite in the radial reflector has $1.33 \mathrm{ppm} \mathrm{EBC} \mathrm{(by} \mathrm{at. \% ),} \mathrm{which} \mathrm{equates} \mathrm{to} \mathrm{a} \mathrm{nominal}{ }^{10} \mathrm{~B}$ concentration of $2.69 \mathrm{mbarn} /$ atom.

Table 3.1-9. Radial Reflector Graphite Composition.

\begin{tabular}{|c|c|}
\hline Isotope/Element & Atoms/barn-cm \\
\hline \hline${ }^{10} \mathrm{~B}$ & $2.3253 \mathrm{E}-08$ \\
${ }^{11} \mathrm{~B}$ & $9.3597 \mathrm{E}-08$ \\
$\mathrm{C}$ & $8.7857 \mathrm{E}-02$ \\
\hline \hline Total & $8.7857 \mathrm{E}-02$ \\
\hline \hline Mass Density $\left(\mathrm{g} / \mathbf{c m}^{\mathbf{3}}\right)$ & 1.752264 \\
\hline
\end{tabular}

\subsubsection{Upper Axial Reflector}

The upper axial reflector graphite is comprised of three compositions, depending on the component of the assembly (see Table 3.1-10). The support structure into which the graphite material is placed is Peraluman-300 (Table 3.1-11).

Table 3.1-10. Upper Axial Reflector Graphite Composition (see Figure 3.1-4).

\begin{tabular}{|c|c|c|c|}
\hline Component & Cylinder & Annulus & Plugs \\
\hline \hline Isotope/Element & Atoms/barn-cm & Atoms/barn-cm & Atoms/barn-cm \\
\hline \hline${ }^{10} \mathrm{~B}$ & $2.3235 \mathrm{E}-08$ & $2.3368 \mathrm{E}-08$ & $2.3356 \mathrm{E}-08$ \\
${ }^{11} \mathrm{~B}$ & $9.3524 \mathrm{E}-08$ & $9.4059 \mathrm{E}-08$ & $9.4011 \mathrm{E}-08$ \\
$\mathrm{C}$ & $8.7789 \mathrm{E}-02$ & $8.8291 \mathrm{E}-02$ & $8.8245 \mathrm{E}-02$ \\
\hline \hline Total & $8.7789 \mathrm{E}-02$ & $8.8291 \mathrm{E}-02$ & $8.8245 \mathrm{E}-02$ \\
\hline \hline Mass Density $\left(\mathbf{g} / \mathbf{c m}^{\mathbf{3}}\right)$ & 1.750896 & 1.760901 & 1.76 \\
\hline
\end{tabular}




\section{NEA/NSC/DOC(2006)1 \\ Gas Cooled (Thermal) Reactor - GCR \\ PROTEUS-GCR-EXP-004 \\ CRIT-REAC}

Table 3.1-11. Upper Axial Reflector Peraluman-300 Support Structure Composition.

\begin{tabular}{|c|c||}
\hline Isotope/Element & Atoms/barn-cm \\
\hline${ }^{10} \mathrm{~B}$ & $1.4688 \mathrm{E}-07$ \\
${ }^{11} \mathrm{~B}$ & $5.9119 \mathrm{E}-07$ \\
$\mathrm{Mg}$ & $1.0177 \mathrm{E}-03$ \\
$\mathrm{Al}$ & $5.7575 \mathrm{E}-02$ \\
$\mathrm{Si}$ & $2.2729 \mathrm{E}-04$ \\
$\mathrm{Mn}$ & $7.2621 \mathrm{E}-05$ \\
$\mathrm{Fe}$ & $8.5730 \mathrm{E}-05$ \\
$\mathrm{Cu}$ & $1.2557 \mathrm{E}-05$ \\
$\mathrm{Zn}$ & $2.4398 \mathrm{E}-05$ \\
$\mathrm{Ga}$ & $1.1444 \mathrm{E}-06$ \\
$\mathrm{Cd}$ & $7.0983 \mathrm{E}-08$ \\
\hline Total & $5.9018 \mathrm{E}-02$ \\
\hline \hline Mass Density $\left(\mathbf{g} / \mathbf{c m}^{3}\right)$ & 2.65 \\
\hline \hline
\end{tabular}

\subsubsection{Lower Axial Reflector}

The lower axial reflector graphite is comprised of two compositions, depending on the component of the assembly (see Table 3.1-12).

Table 3.1-12. Lower Axial Reflector Graphite Composition.

\begin{tabular}{|c|c|c||}
\hline Component & Cylinder & $\begin{array}{c}\text { Annulus / } \\
\text { Source Plug }\end{array}$ \\
\hline \hline Isotope/Element & Atoms/barn-cm & Atoms/barn-cm \\
\hline \hline${ }^{10} \mathrm{~B}$ & $2.3223 \mathrm{E}-08$ & $2.3356 \mathrm{E}-08$ \\
${ }^{11} \mathrm{~B}$ & $9.3476 \mathrm{E}-08$ & $9.4011 \mathrm{E}-08$ \\
$\mathrm{C}$ & $8.7744 \mathrm{E}-02$ & $8.8245 \mathrm{E}-02$ \\
\hline \hline Total & $8.7744 \mathrm{E}-02$ & $8.8245 \mathrm{E}-02$ \\
\hline \hline Mass Density $\left(\mathbf{g} / \mathbf{c m}^{\mathbf{3}}\right)$ & 1.75 & 1.76 \\
\hline
\end{tabular}

Revision: 1 
NEA/NSC/DOC(2006)1

Gas Cooled (Thermal) Reactor - GCR

PROTEUS-GCR-EXP-004

CRIT-REAC

\subsubsection{Autorod}

The autorod consists of copper wedge (Table 3.1-13) within an aluminum guide tube (Table 3.1-14).

Table 3.1-13. Autorod Copper (Type C110) Wedge Composition.

\begin{tabular}{|c|c||}
\hline Element & Atoms/barn-cm \\
\hline \hline $\mathrm{Cu}$ & $8.4206 \mathrm{E}-02$ \\
$\mathrm{O}$ & $6.6923 \mathrm{E}-05$ \\
$\mathrm{Ag}$ & $3.7224 \mathrm{E}-06$ \\
$\mathrm{~S}$ & $1.2522 \mathrm{E}-05$ \\
$\mathrm{Ni}$ & $6.8410 \mathrm{E}-06$ \\
$\mathrm{Fe}$ & $7.1900 \mathrm{E}-06$ \\
\hline \hline Total & $8.4303 \mathrm{E}-02$ \\
\hline \hline Mass Density $\left(\mathrm{g} / \mathrm{cm}^{\mathbf{3}}\right)$ & 8.89 \\
\hline
\end{tabular}

Table 3.1-14. Autorod Aluminum (Type 1100) Tube Composition.

\begin{tabular}{|c|c|}
\hline Element & Atoms/barn-cm \\
\hline \hline $\mathrm{Si}$ & $2.8947 \mathrm{E}-04$ \\
$\mathrm{Fe}$ & $1.4558 \mathrm{E}-04$ \\
$\mathrm{Cu}$ & $3.1984 \mathrm{E}-05$ \\
$\mathrm{Mn}$ & $7 . .661 \mathrm{E}-06$ \\
$\mathrm{Zn}$ & $1.2429 \mathrm{E}-05$ \\
$\mathrm{Co}$ & $6.8975 \mathrm{E}-05$ \\
$\mathrm{Ni}$ & $6.9257 \mathrm{E}-05$ \\
$\mathrm{Sn}$ & $3.4242 \mathrm{E}-05$ \\
$\mathrm{Al}$ & $5.9087 \mathrm{E}-02$ \\
\hline Total & $5.9746 \mathrm{E}-02$ \\
\hline \hline Mass Density (g/cm & 2.70 \\
\hline \hline
\end{tabular}

Revision: 1

Date: March 31, 2014 
NEA/NSC/DOC(2006)1

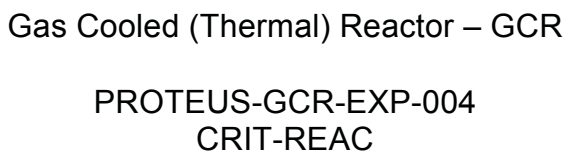

\subsubsection{Fuel Pebbles}

The $\mathrm{UO}_{2}$ fuel used for the TRISO kernels has the composition provided in Table 3.1-15. The compositions of the additional $\mathrm{SiC}$ and graphite layers surrounding the kernel to form the TRISO particle are in Table 3.1-16. The fuel pebble graphite matrix surrounding the TRISO particles in the fueled zone and forming the outer unfueled layer has the composition shown in Table 3.1-17.

Table 3.1-15. $\mathrm{UO}_{2}$ Fuel Kernel Composition.

\begin{tabular}{|c|c||}
\hline Isotope/Element & Atoms/barn-cm \\
\hline \hline $\mathrm{O}$ & $4.8612 \mathrm{E}-02$ \\
${ }^{234} \mathrm{U}$ & $3.3079 \mathrm{E}-05$ \\
${ }^{235} \mathrm{U}$ & $4.1172 \mathrm{E}-03$ \\
${ }^{236} \mathrm{U}$ & $2.0499 \mathrm{E}-05$ \\
${ }^{238} \mathrm{U}$ & $2.0135 \mathrm{E}-02$ \\
\hline \hline Total & $7.2917 \mathrm{E}-02$ \\
\hline \hline Mass Density $\left(\mathbf{g} / \mathbf{c m}^{\mathbf{3}}\right)$ & 10.88 \\
\hline \hline
\end{tabular}

Table 3.1-16. TRISO SiC and Graphite Layer Compositions.

\begin{tabular}{|c|c|c|c|c|}
\hline Layer & Buffer & IPyC & $\mathrm{SiC}$ & OPyC \\
\hline Isotope/Element & Atoms/barn-cm & Atoms/barn-cm & Atoms/barn-cm & Atoms/barn-cm \\
\hline $\mathrm{C}$ & $5.2640 \mathrm{E}-02$ & $9.5254 \mathrm{E}-02$ & $4.8055 \mathrm{E}-02$ & $9.4752 \mathrm{E}-02$ \\
\hline $\mathrm{Si}$ & -- & -- & $4.8055 \mathrm{E}-02$ & -- \\
\hline Total & $5.2640 \mathrm{E}-02$ & $9.5254 \mathrm{E}-02$ & $9.6110 \mathrm{E}-02$ & $9.4752 \mathrm{E}-02$ \\
\hline Mass Density $\left(\mathrm{g} / \mathrm{cm}^{3}\right)$ & 1.05 & 1.90 & 3.20 & 1.89 \\
\hline
\end{tabular}


NEA/NSC/DOC(2006)1

Gas Cooled (Thermal) Reactor - GCR

PROTEUS-GCR-EXP-004

CRIT-REAC

Table 3.1-17. Fuel Pebble Graphite Composition.

\begin{tabular}{|c|c|}
\hline \hline Isotope/Element & Atoms/barn-cm \\
\hline \hline $\mathrm{C}$ & $8.6842 \mathrm{E}-02$ \\
$\mathrm{Ag}$ & $9.6706 \mathrm{E}-10$ \\
${ }^{10} \mathrm{~B}$ & $1.9393 \mathrm{E}-09$ \\
${ }^{11} \mathrm{~B}$ & $7.8061 \mathrm{E}-09$ \\
$\mathrm{Ca}$ & $2.4154 \mathrm{E}-07$ \\
$\mathrm{Cd}$ & $4.7791 \mathrm{E}-10$ \\
$\mathrm{Cl}$ & $4.4135 \mathrm{E}-08$ \\
$\mathrm{Co}$ & $1.1505 \mathrm{E}-09$ \\
$\mathrm{Cr}$ & $3.6312 \mathrm{E}-08$ \\
$\mathrm{Dy}$ & $3.2097 \mathrm{E}-11$ \\
$\mathrm{Eu}$ & $3.4322 \mathrm{E}-11$ \\
$\mathrm{Fe}$ & $5.5104 \mathrm{E}-08$ \\
$\mathrm{Gd}$ & $3.3169 \mathrm{E}-11$ \\
${ }^{6} \mathrm{Li}$ & $5.7034 \mathrm{E}-09$ \\
${ }^{7} \mathrm{Li}$ & $6.9441 \mathrm{E}-08$ \\
$\mathrm{Mn}$ & $8.1647 \mathrm{E}-09$ \\
$\mathrm{Ni}$ & $8.8864 \mathrm{E}-09$ \\
$\mathrm{~S}$ & $1.7893 \mathrm{E}-10$ \\
$\mathrm{Ti}$ & $1.0831 \mathrm{E}-08$ \\
$\mathrm{~V}$ & $4.4334 \mathrm{E}-09$ \\
$\mathrm{H}$ & $1.1581 \mathrm{E}-05$ \\
$\mathrm{O}$ & $5.7904 \mathrm{E}-06$ \\
\hline \hline Total & $8.6859 \mathrm{E}-02$ \\
\hline${ }^{7}$ & 1.732204 \\
\hline
\end{tabular}

Revision: 1

Date: March 31, 2014

Page 178 of 375 


\section{NEA/NSC/DOC(2006)1 \\ Gas Cooled (Thermal) Reactor - GCR \\ PROTEUS-GCR-EXP-004 \\ CRIT-REAC}

\subsubsection{Moderator Pebbles}

The composition of the graphite moderator pebbles is in Table 3.1-18.

Table 3.1-18. Moderator Pebble Graphite Composition.

\begin{tabular}{|c|c|}
\hline \hline Isotope/Element & Atoms/barn-cm \\
\hline \hline $\mathrm{C}$ & $8.4434 \mathrm{E}-02$ \\
${ }^{10} \mathrm{~B}$ & $1.4193 \mathrm{E}-08$ \\
${ }^{11} \mathrm{~B}$ & $5.7130 \mathrm{E}-08$ \\
$\mathrm{Ca}$ & $3.2656 \mathrm{E}-06$ \\
$\mathrm{Cd}$ & $2.7077 \mathrm{E}-09$ \\
$\mathrm{Cl}$ & $5.3343 \mathrm{E}-07$ \\
$\mathrm{Dy}$ & $4.0583 \mathrm{E}-10$ \\
$\mathrm{Eu}$ & $8.6793 \mathrm{E}-10$ \\
$\mathrm{Fe}$ & $1.0719 \mathrm{E}-07$ \\
$\mathrm{Gd}$ & $2.5808 \mathrm{E}-10$ \\
${ }^{6} \mathrm{Li}$ & $9.7630 \mathrm{E}-09$ \\
${ }^{7} \mathrm{Li}$ & $1.1887 \mathrm{E}-07$ \\
$\mathrm{Ni}$ & $1.3483 \mathrm{E}-08$ \\
$\mathrm{~S}$ & $4.4297 \mathrm{E}-06$ \\
$\mathrm{Si}$ & $1.2644 \mathrm{E}-06$ \\
$\mathrm{Sm}$ & $5.8029 \mathrm{E}-10$ \\
$\mathrm{Ti}$ & $2.1196 \mathrm{E}-07$ \\
$\mathrm{~V}$ & $2.5891 \mathrm{E}-07$ \\
$\mathrm{H}$ & $1.1263 \mathrm{E}-05$ \\
$\mathrm{O}$ & $5.6317 \mathrm{E}-06$ \\
\hline \hline Total & $8.4461 \mathrm{E}-02$ \\
\hline \hline Mass Density (g/cm $\left.{ }^{3}\right)$ & 1.684743 \\
\hline
\end{tabular}

Revision: 1

Date: March 31, 2014 
NEA/NSC/DOC(2006)1

\section{Gas Cooled (Thermal) Reactor - GCR \\ PROTEUS-GCR-EXP-004 \\ CRIT-REAC}

\subsubsection{Withdrawable Control Rods}

The withdrawable control rods consist of an inner stainless steel tube (Table 3.1-19) held within an outer stainless steel tube with end plugs (Table 3.1-20).

Table 3.1-19. Control Rod Stainless Steel (Type St1.4301) Tube Composition.

\begin{tabular}{|c|c|}
\hline Element & Atoms/barn-cm \\
\hline \hline $\mathrm{C}$ & $1.3864 \mathrm{E}-04$ \\
$\mathrm{Si}$ & $8.4696 \mathrm{E}-04$ \\
$\mathrm{Mn}$ & $8.6597 \mathrm{E}-04$ \\
$\mathrm{Cr}$ & $1.6927 \mathrm{E}-02$ \\
$\mathrm{Ni}$ & $8.3083 \mathrm{E}-03$ \\
$\mathrm{Fe}$ & $5.9391 \mathrm{E}-02$ \\
\hline Total & $8.6477 \mathrm{E}-02$ \\
\hline \hline Mass Density (g/cm & \\
\hline
\end{tabular}

Table 3.1-20. Control Rod Stainless Steel (Type St1.4541) Tube and End Plug Composition.

\begin{tabular}{|c|c|}
\hline Element & Atoms/barn-cm \\
\hline \hline $\mathrm{C}$ & $1.9805 \mathrm{E}-04$ \\
$\mathrm{Si}$ & $8.4696 \mathrm{E}-04$ \\
$\mathrm{Mn}$ & $8.6597 \mathrm{E}-04$ \\
$\mathrm{Cr}$ & $1.6469 \mathrm{E}-02$ \\
$\mathrm{Ni}$ & $8.3083 \mathrm{E}-03$ \\
$\mathrm{Ti}$ & $4.9695 \mathrm{E}-05$ \\
$\mathrm{Fe}$ & $5.9761 \mathrm{E}-02$ \\
\hline \hline Total & $8.6499 \mathrm{E}-02$ \\
\hline \hline Mass Density $\left(\mathrm{g} / \mathbf{c m}^{\mathbf{3}}\right)$ & 7.9 \\
\hline
\end{tabular}


NEA/NSC/DOC(2006)1

Gas Cooled (Thermal) Reactor - GCR
PROTEUS-GCR-EXP-004
CRIT-REAC

\subsubsection{Polyethylene Rods}

The composition of the polyethylene (sometimes referred to as plastic) rods is in Table 3.1-21.

Table 3.1-21. Polyethylene Rod Composition.

\begin{tabular}{|c|c||}
\hline Isotope/Element & Atoms/barn-cm \\
\hline \hline${ }^{10} \mathrm{~B}$ & $5.2797 \mathrm{E}-09$ \\
${ }^{11} \mathrm{~B}$ & $2.1252 \mathrm{E}-08$ \\
$\mathrm{H}$ & $8.2845 \mathrm{E}-02$ \\
$\mathrm{C}$ & $4.0810 \mathrm{E}-02$ \\
\hline \hline Total & $1.2365 \mathrm{E}-01$ \\
\hline \hline Mass Density $\left(\mathbf{g} / \mathbf{c m}^{3}\right)$ & 0.95259 \\
\hline \hline
\end{tabular}

\subsubsection{Ambient Air}

The composition of the ambient air is in Table 3.1-22. The air has a temperature of $293 \mathrm{~K}$, pressure of 980 mbar, and $50 \%$ humidity.

Table 3.1-22. Ambient Air Composition.

\begin{tabular}{|c|c|}
\hline Element & Atoms/barn-cm \\
\hline \hline $\mathrm{H}$ & $5.7098 \mathrm{E}-07$ \\
$\mathrm{~N}$ & $3.7362 \mathrm{E}-05$ \\
$\mathrm{O}$ & $1.0326 \mathrm{E}-05$ \\
$\mathrm{Ar}$ & $2.2345 \mathrm{E}-07$ \\
$\mathrm{C}$ & $9.1319 \mathrm{E}-09$ \\
\hline \hline Total & $4.8492 \mathrm{E}-05$ \\
\hline \hline Mass Density $\left(\mathbf{g} / \mathbf{c m}^{3}\right)$ & 0.00115932 \\
\hline
\end{tabular}

\subsubsection{Temperature Data}

The benchmark model temperature is $293 \mathrm{~K}$. 
NEA/NSC/DOC(2006)1

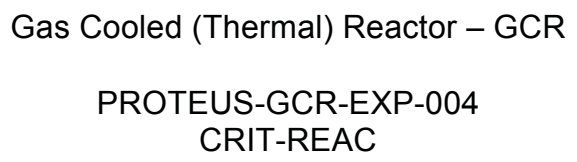

\subsubsection{Experimental and Benchmark-Model $k_{\text {eff }}$ and / or Subcritical Parameters}

The experimental $k_{\text {eff }}$ was approximately at unity, maintained at delayed critical with the $1 \sigma$ uncertainty summarized in Section 2.1.11 for each of the two configurations. Simplification biases and uncertainties, as discussed in Section 3.1.1.1 were applied to the benchmark model. The benchmark $k_{\text {eff }}$ is shown in Table 3.1-23 for each of the two cases. The uncertainty in the benchmark $k_{\text {eff }}$ value is obtained by summing under quadrature the total experimental uncertainty (Tables 2.1-45 and 2.1-46) and the total bias uncertainty (Table 3.1-4).

Table 3.1-23. Experimental and Benchmark Eigenvalues, Biases, and Uncertainties.

\begin{tabular}{|c|c|c|c|c|c|c|c|c|c|c|}
\hline \multirow{2}{*}{ Case } & \multirow{2}{*}{$\begin{array}{c}\text { Core } \\
\text { (state) }\end{array}$} & \multicolumn{3}{|c|}{ Experimental } & \multicolumn{3}{|c|}{ Bias } & \multicolumn{3}{|c|}{ Benchmark } \\
\hline & & $\mathbf{k}_{\text {eff }}$ & \pm & $\sigma$ & $\Delta \mathbf{k}$ & \pm & $\sigma$ & $\mathbf{k}_{\text {eff }}$ & \pm & $\sigma$ \\
\hline 1 & $9(\# 1)$ & 1.0000 & \pm & 0.0036 & 0.0029 & \pm & 0.0002 & 1.0029 & \pm & 0.0036 \\
\hline 2 & 10 & 1.0000 & \pm & 0.0037 & 0.0020 & \pm & 0.0002 & 1.0020 & \pm & 0.0037 \\
\hline
\end{tabular}

\subsection{Benchmark-Model Specifications for Buckling and Extrapolation-Length Measurements}

Buckling and extrapolation length measurements were performed but have not yet been evaluated.

\subsection{Benchmark-Model Specifications for Spectral Characteristics Measurements}

Spectral characteristics measurements were performed but have not yet been evaluated. 
NEA/NSC/DOC(2006)1

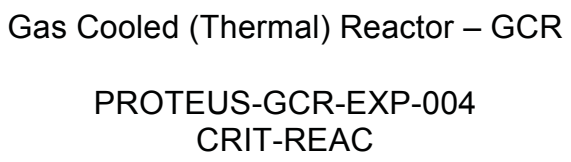

\subsection{Benchmark-Model Specifications for Reactivity Effects Measurements}

A total of 32 reactivity effects measurements were determined to be acceptable benchmark experiments for both Cores 9 and 10 (16 apiece).

\subsubsection{Description of the Benchmark Model Simplifications}

Detailed models (see Appendix C) of the PROTEUS reactor core configurations were prepared to evaluate biases in the benchmark models for the critical configurations. Sample calculations performed using the benchmark models provided in Section 3.1 with the model simplifications described in Section 3.1.1.1 yielded results similar to, within the statistical uncertainty, results calculated using the detailed models. Therefore, no bias is applied to the benchmark values.

The reactivity effects measurements were reported in units of $\$$ using the reported, calculated $\beta_{\text {eff }}$ values of 0.00720 for both Cores 9 and 10 (except for the safety/shutdown rods in Core 9, which had a reported $\beta_{\text {eff }}$ value of 0.00717); they were adjusted to the MCNP-calculated $\beta_{\text {eff }}$ values of 0.00693 for Core 9 and 0.00685 for Core 10 for comparison with calculations and for use as benchmark measurements. For further discussion regarding the selection of $\beta_{\text {eff }}$, see Section 2.4.1.

\subsubsection{Dimensions}

The dimensions of the benchmark models for determination of the reactivity effects measurements in the HTR-PROTEUS Cores 9 and 10 are identical to those of the critical core configurations described in Section 3.1.2, with exceptions discussed below.

\subsubsection{Control Rod Worths}

The radial positions of the control rods are shown in Figure 3.1-2 with the maximum and minimum range of vertical placement shown in Figure 3.1-11. The x-y positions of the four control rods are listed in Table 3.4-1. The individual control rod worth is obtained taking the benchmark critical configurations (Section 3.1) and comparing the condition with a single control rod fully withdrawn and then fully inserted. The control rod bank worth is obtained by comparison of the condition with all control rods fully withdrawn and then fully inserted. The partial bank insertion worth is obtained by comparison of the cores with the control rod bank partially withdrawn and then fully inserted. The distance the control rod bank is withdrawn upward from full insertion is $88.0 \mathrm{~cm}$ for Core 9 and $96.0 \mathrm{~cm}$ for Core 10 . The geometric description of the control rods is provided in Section 3.1.2.7. 
NEA/NSC/DOC(2006)1

\section{Gas Cooled (Thermal) Reactor - GCR \\ PROTEUS-GCR-EXP-004 \\ CRIT-REAC}

Table 3.4-1. Absorber Rod x-y Positions (distance in cm).

\begin{tabular}{||c|c|c||}
\hline \multicolumn{1}{|c|}{ Absorber Rod } & X & Y \\
\hline \hline Safety/Shutdown Rod 1 & -38.45 & 56.57 \\
Safety/Shutdown Rod 2 & 32.74 & -60.05 \\
Safety/Shutdown Rod 3 & 57.17 & 37.55 \\
Safety/Shutdown Rod 4 & -53.23 & -42.95 \\
Safety/Shutdown Rod 5 & 67.19 & -12.82 \\
Safety/Shutdown Rod 6 & -66.98 & 13.87 \\
Safety/Shutdown Rod 7 & 19.31 & 65.62 \\
Safety/Shutdown Rod 8 & -13.87 & -66.98 \\
\hline Autorod & 17.36 & -87.29 \\
\hline Withdrawable Control Rod 1 & 34.67 & 83.70 \\
Withdrawable Control Rod 2 & 83.70 & -34.67 \\
Withdrawable Control Rod 3 & -34.67 & -83.70 \\
Withdrawable Control Rod 4 & -83.70 & 34.67 \\
\hline \hline
\end{tabular}

\subsubsection{Autorod Worths}

The radial location of the autorod is shown in Figure 3.1-2 with the maximum and minimum range of vertical placement shown in Figure 3.1-7. The x-y position of the autorod is listed in Table 3.4-1. The autorod worth is obtained taking the benchmark critical configurations (Section 3.1) and comparing the condition with the autorod fully withdrawn and then fully inserted. The geometric description of the autorod is provided in Section 3.1.2.4.

\subsubsection{Safety/Shutdown Rod Worths}

The radial positions of the safety/shutdown rods are shown in Figure 3.1-2. The safety/shutdown rods (Figures 3.4-1 through 3.4-3) are comprised of borated steel rod contained within a stainless steel tube with end plugs. The borated steel rod has a diameter of $3.5 \mathrm{~cm}$ and length of $210 \mathrm{~cm}$. The stainless steel tube has an inner diameter of $3.6 \mathrm{~cm}$ and outer diameter of $4.0 \mathrm{~cm}$. The dimensions for the end plugs are shown in Figure 3.4-2. The safety/shutdown rods are completely inserted into the core when the bottom surface of the borated steel is located $35 \mathrm{~cm}$ below the floor of the core cavity; they are completely withdrawn when raised $290 \mathrm{~cm}$ from the fully inserted position (see Figure 3.4-3). The x-y positions of the eight safety/shutdown rods is listed in Table 3.4-1.

The individual or combined safety/shutdown rod worths are obtained taking the benchmark critical configurations (Section 3.1), modifying them to include the safety/shutdown rods as described in this section, and comparing the condition with a the safety/shutdown rods fully withdrawn and then fully inserted. 
NEA/NSC/DOC(2006)1

Gas Cooled (Thermal) Reactor - GCR

PROTEUS-GCR-EXP-004

CRIT-REAC

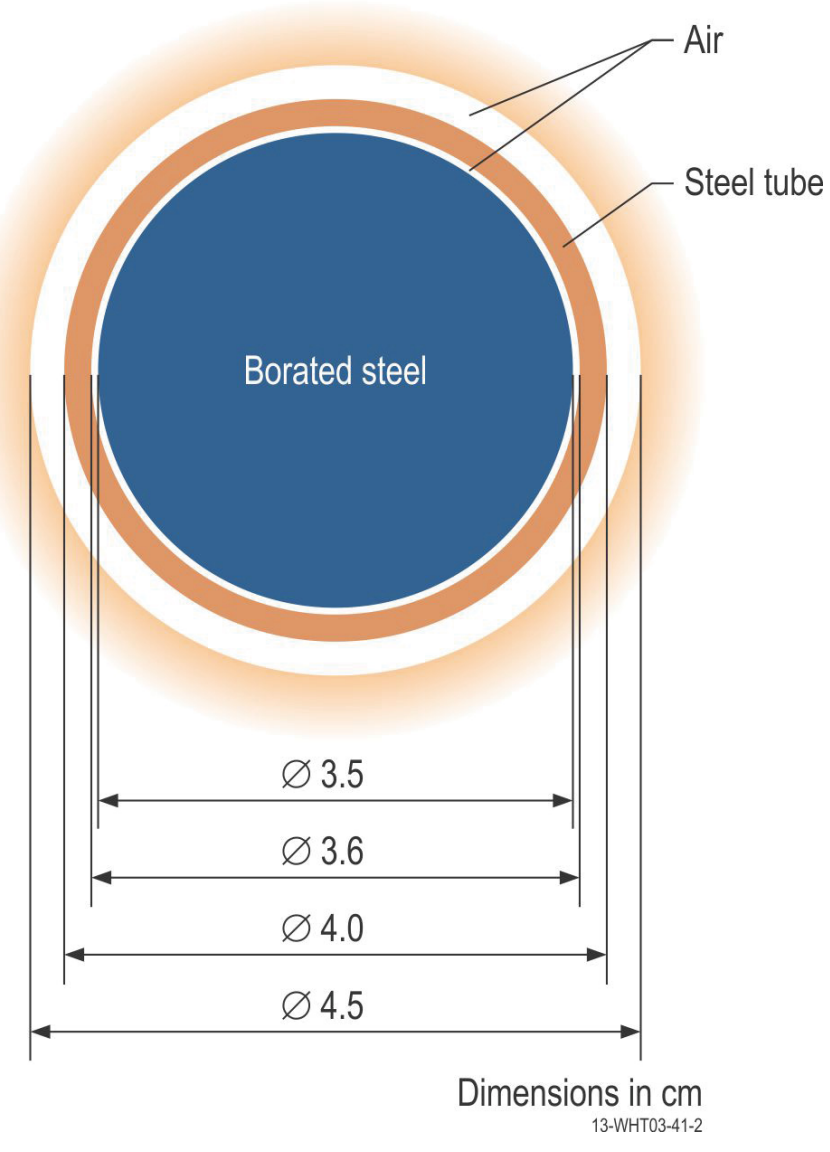

Figure 3.4-1. Top View of Safety/Shutdown Rod.

Revision: 1

Date: March 31, 2014 
NEA/NSC/DOC(2006)1

Gas Cooled (Thermal) Reactor - GCR

PROTEUS-GCR-EXP-004

CRIT-REAC

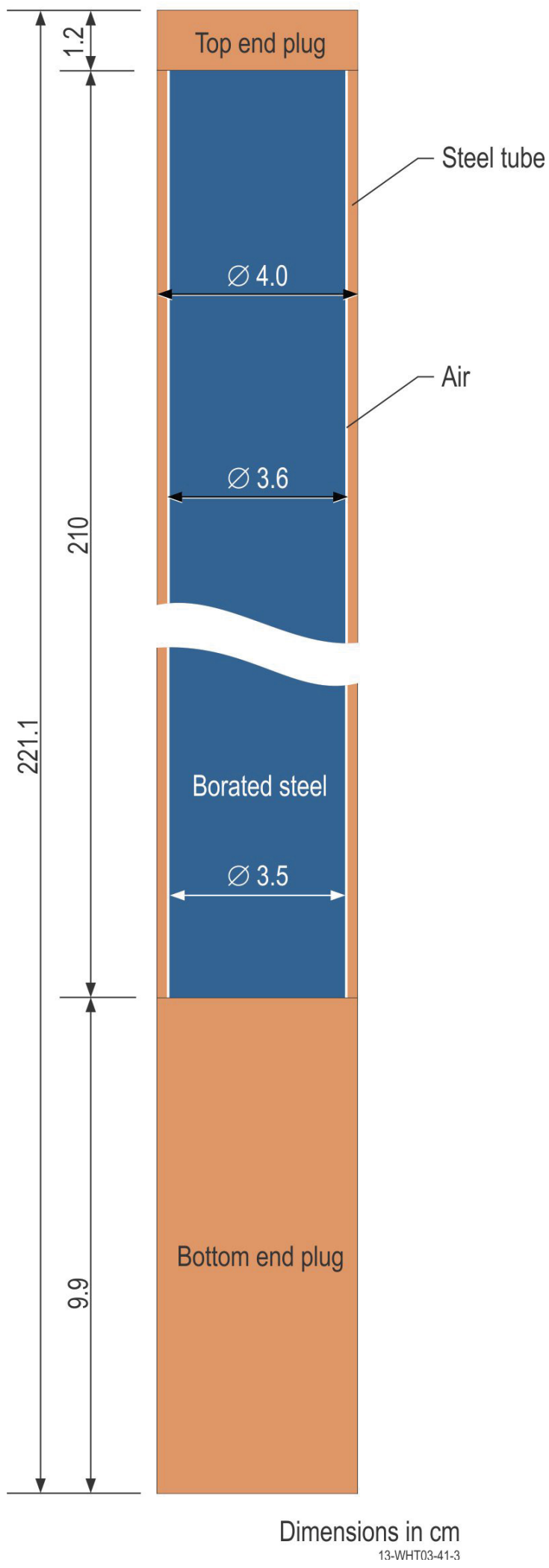

Figure 3.4-2. Axial View of Safety/Shutdown Rod.

Revision: 1

Date: March 31, 2014

Page 186 of 375 
NEA/NSC/DOC(2006)1

Gas Cooled (Thermal) Reactor - GCR

PROTEUS-GCR-EXP-004

CRIT-REAC

Air

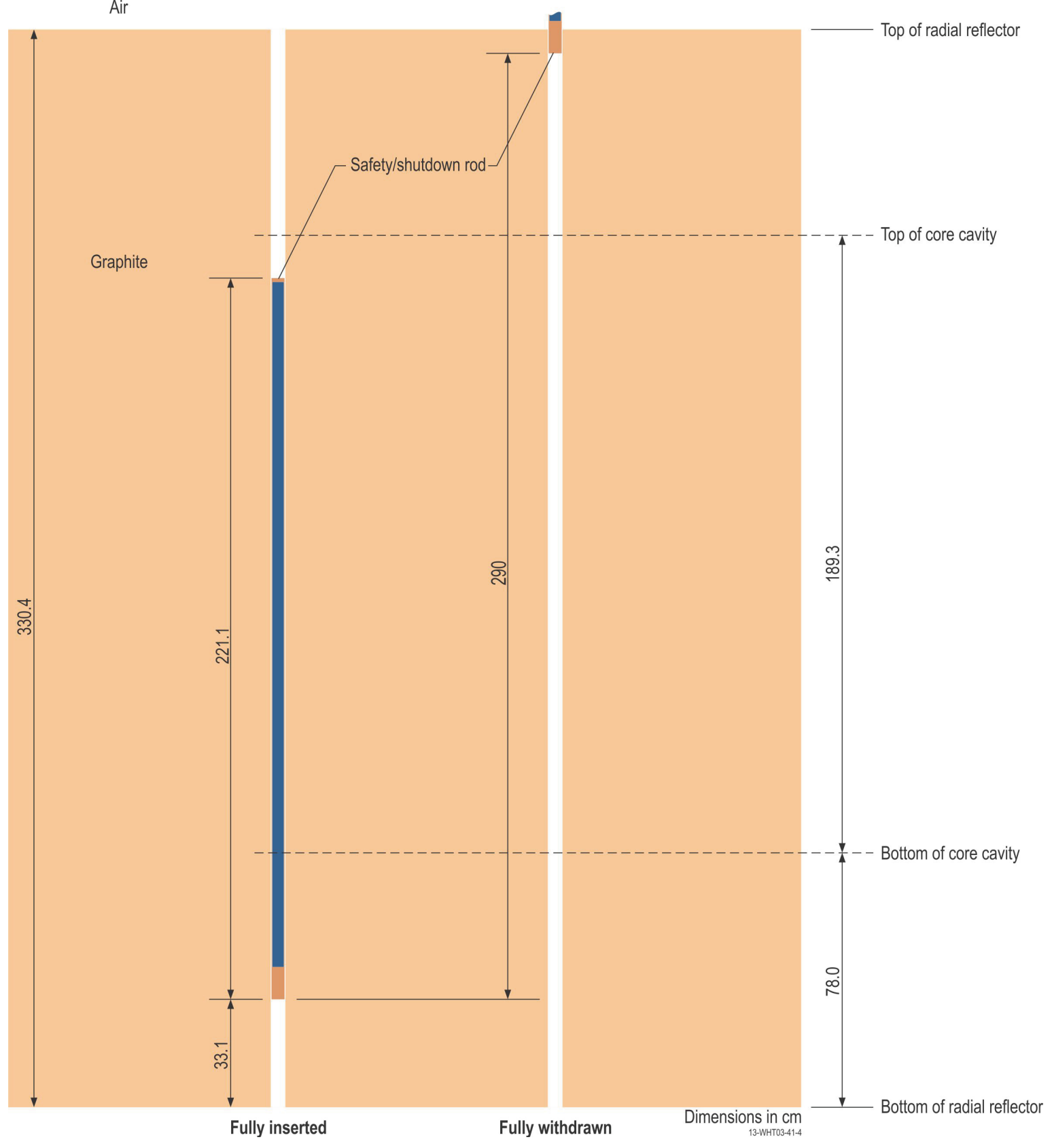

Figure 3.4-3. Safety/Shutdown Rod Vertical Position within Radial Reflector.

Revision: 1

Date: March 31, 2014

Page 187 of 375 


\section{NEA/NSC/DOC(2006)1}

\section{Gas Cooled (Thermal) Reactor - GCR \\ PROTEUS-GCR-EXP-004 \\ CRIT-REAC}

\subsubsection{Material Data}

The materials of the benchmark models for determination of the reactivity effects measurements in the HTR-PROTEUS Cores 9 and 10 are identical to those of the critical core configurations described in Section 3.1.3.

\subsubsection{Control Rod Worths}

No additional information is necessary for these benchmark measurements. The material description of the control rods is provided in Section 3.1.3.7.

\subsubsection{Autorod Worths}

No additional information is necessary for these benchmark measurements. The material description of the autorod is provided in Section 3.1.3.4.

\subsubsection{Safety/Shutdown Rod Worths}

The safety/shutdown rods consist of an inner borated steel rod (Table 3.4-2) held within an outer stainless steel tube with end plugs (Table 3.4-3).

Table 3.4-2. Safety/Shutdown Rod Borated Steel ( $\sim 5$ wt.\%) Rod Composition.

\begin{tabular}{|c|c|}
\hline Element & Atoms/barn-cm \\
\hline \hline${ }^{10} \mathrm{~B}$ & $3.9257 \mathrm{E}-03$ \\
${ }^{11} \mathrm{~B}$ & $1.4282 \mathrm{E}-02$ \\
$\mathrm{Si}$ & $1.5187 \mathrm{E}-03$ \\
$\mathrm{Cr}$ & $3.2491 \mathrm{E}-02$ \\
$\mathrm{Mn}$ & $9.8952 \mathrm{E}-04$ \\
$\mathrm{Fe}$ & $3.1300 \mathrm{E}-02$ \\
$\mathrm{Ni}$ & $7.0036 \mathrm{E}-03$ \\
\hline \hline Total & $9.1511 \mathrm{E}-02$ \\
\hline \hline
\end{tabular}


NEA/NSC/DOC(2006)1

\section{Gas Cooled (Thermal) Reactor - GCR \\ PROTEUS-GCR-EXP-004 \\ CRIT-REAC}

Table 3.4-3. Safety/Shutdown Rod Stainless Steel (Type 18/8) Tube and End Plug Composition.

\begin{tabular}{|c|c|}
\hline Element & Atoms/barn-cm \\
\hline $\mathrm{Cr}$ & $1.6511 \mathrm{E}-02$ \\
$\mathrm{Fe}$ & $6.1855 \mathrm{E}-02$ \\
$\mathrm{Ni}$ & $6.5009 \mathrm{E}-03$ \\
$\mathrm{C}$ & $2.9783 \mathrm{E}-04$ \\
$\mathrm{Si}$ & $8.4910 \mathrm{E}-04$ \\
$\mathrm{Mn}$ & $8.6816 \mathrm{E}-04$ \\
\hline Total & $8.6882 \mathrm{E}-02$ \\
\hline \hline Mass Density $\left(\mathbf{g} / \mathbf{c m}^{3}\right)$ & 7.92 \\
\hline
\end{tabular}

\subsubsection{Temperature Data}

The benchmark model temperature is $300 \mathrm{~K}$.

\subsubsection{Benchmark-Model Specification for Reactivity Effects Parameters}

The experimental measurements are evaluated in Section 2.4 and summarized in Tables 2.4-13 and 2.414. These values represent the benchmark experiment worths, and are repeated in Table 3.4-4 for Core 9 and Table 3.4-5 for Core 10. 
NEA/NSC/DOC(2006)1

Gas Cooled (Thermal) Reactor - GCR

PROTEUS-GCR-EXP-004

CRIT-REAC

Table 3.4-4. Benchmark Reactivity Effects Measurements (Core 9).

\begin{tabular}{|c|c|ccc||}
\hline \multirow{2}{*}{ Case } & Measured Parameter & \multicolumn{3}{|c|}{ Benchmark Worth } \\
& & $\mathbf{\rho}(\mathbf{\$})$ & $\mathbf{\sigma}$ \\
\hline $1.4-1$ & Control Rod 1 & -0.41 & \pm & 0.02 \\
\hline $1.4-2$ & Control Rod 2 & -0.41 & \pm & 0.02 \\
\hline $1.4-3$ & Control Rod 3 & -0.41 & \pm & 0.02 \\
\hline $1.4-4$ & Control Rod 4 & -0.41 & \pm & 0.02 \\
\hline $1.4-5$ & Control Rod Bank & -1.58 & \pm & 0.09 \\
\hline $1.4-6$ & Cull Insertion & -0.73 & \pm & 0.04 \\
\hline $1.4-8$ & Partial Insertion & -0.10 & \pm & 0.01 \\
\hline $1.4-8$ & Autorod Insertion & -3.75 & \pm & 0.17 \\
\hline $1.4-9$ & Safety/Shutdown Rod 5 & -3.82 & \pm & 0.10 \\
\hline $1.4-10$ & Safety/Shutdown Rod 7 & -3.70 & \pm & 0.30 \\
\hline $1.4-11$ & Safety/Shutdown Rod 8 & -3.60 & \pm & 0.29 \\
\hline $1.4-12$ & Safety/Shutdown Rods 5+6 & -8.02 & \pm & 0.20 \\
\hline $1.4-13$ & Safety/Shutdown Rods 5+7 & -7.44 & \pm & 0.60 \\
\hline $1.4-14$ & Safety/Shutdown Rods 5+8 & -7.40 & \pm & 0.59 \\
\hline $1.4-15$ & Safety/Shutdown Rods 5+6+7 & -12.11 & \pm & 0.28 \\
\hline $1.4-16$ & Safety/Shutdown Rods 5+6+7+8 & -16.52 & \pm & 0.47 \\
\hline & & & & \\
\hline
\end{tabular}


NEA/NSC/DOC(2006)1

Gas Cooled (Thermal) Reactor - GCR

PROTEUS-GCR-EXP-004

CRIT-REAC

Table 3.4-5. Benchmark Reactivity Effects Measurements (Core 10).

\begin{tabular}{|c|c|ccc|}
\hline \multirow{2}{*}{ Case } & Measured Parameter & \multicolumn{4}{|c|}{ Benchmark Worth } \\
& & $\boldsymbol{\rho}(\$)$ & $\boldsymbol{\sigma}$ \\
\hline \hline $2.4-1$ & Control Rod 1 & -0.30 & \pm & 0.02 \\
\hline $2.4-2$ & Control Rod 2 & -0.29 & \pm & 0.02 \\
\hline $2.4-3$ & Control Rod 3 & -0.29 & \pm & 0.02 \\
\hline $2.4-4$ & Control Rod 4 & -0.30 & \pm & 0.02 \\
\hline $2.4-5$ & Control Rod Bank & -1.15 & \pm & 0.07 \\
\hline $2.4-6$ & Control Rod Bank & -0.39 & \pm & 0.02 \\
\hline $2.4-7$ & Partial Insertion & -0.073 & \pm & 0.004 \\
\hline $2.4-8$ & Autorod Insertion & -2.82 & \pm & 0.11 \\
\hline $2.4-9$ & Safety/Shutdown Rod 5 & -2.82 & \pm & 0.09 \\
\hline $2.4-10$ & Safety/Shutdown Rod 6 & -2.80 & \pm & 0.16 \\
\hline $2.4-11$ & Safety/Shutdown Rod 7 & -2.72 & \pm & 0.15 \\
\hline $2.4-12$ & Safety/Shutdown Rods 5+6 & -5.95 & \pm & 0.17 \\
\hline $2.4-13$ & Safety/Shutdown Rods 5+7 & -5.73 & \pm & 0.32 \\
\hline $2.4-14$ & Safety/Shutdown Rods 5+8 & -5.75 & \pm & 0.33 \\
\hline $2.4-15$ & Safety/Shutdown Rods 5+6+7 & -9.29 & \pm & 0.21 \\
\hline $2.4-16$ & Safety/Shutdown Rods 5+6+7+8 & -12.67 & \pm & 0.31 \\
\hline
\end{tabular}

Revision: 1 


$$
\begin{gathered}
\text { NEA/NSC/DOC(2006)1 } \\
\text { Gas Cooled (Thermal) Reactor - GCR } \\
\text { PROTEUS-GCR-EXP-004 } \\
\text { CRIT-REAC }
\end{gathered}
$$

\subsection{Benchmark-Model Specifications for Reactivity Coefficient Measurements}

Reactivity coefficient measurements were performed but have not yet been evaluated.

\subsection{Benchmark-Model Specifications for Kinetics Measurements}

Kinetics measurements were performed but have not yet been evaluated.

\subsection{Benchmark-Model Specifications for Reaction-Rate Distribution Measurements}

Reaction-rate distribution measurements were performed but have not yet been evaluated.

\subsection{Benchmark-Model Specifications for Power Distribution Measurements}

Power distribution measurements were not performed.

\subsection{Benchmark-Model Specifications for Isotopic Measurements}

Isotopic measurements were not performed.

\subsection{Benchmark-Model Specifications for Other Miscellaneous Types of Measurements}

Other miscellaneous types of measurements were not performed. 
NEA/NSC/DOC(2006)1

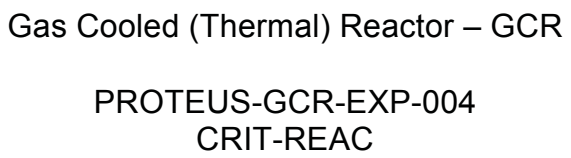

\subsection{RESULTS OF SAMPLE CALCULATIONS}

\subsection{Results of Calculations of the Critical or Subcritical Configurations}

The benchmark models described in Section 3.1 were used with MCNP5 (see Appendix A.1 for sample input deck for Case 1) and ENDF/B-VII.0 neutron cross section data. Random particles are not easily modeled in MCNP, therefore all 9394 TRISO particles were modeled within a cubic lattice with sides $0.1758 \mathrm{~cm}$ in length. All TRISO particles are completely contained within the fueled region of the fuel pebbles (see Figure 4.1-1); this was verified by visually inspecting each layer in a visual editor. The effect of random particle placement was determined to be essentially negligible relative to a regular array of particles in a fuel pebble (see Section 2.1.9.4 of PROTEUS-GCR-EXP-001). ${ }^{\mathrm{a}}$

Monte Carlo calculations were performed with 1,650 generations with 100,000 neutrons per generation. The $\mathrm{k}_{\text {eff }}$ estimates are based on 150 skipped generations and a total of 150,000,000 neutron histories each. Calculated eigenvalues are shown in Table 4.1-1. All calculated eigenvalues are greater than the benchmark value but within $1 \%$ and within the $2 \sigma$ uncertainty. Models developed by Difilippo using MCNP4C with ENDF/B-VI (DLC-189) neutron cross sections did not include Cases 1 and 2 (Cores 9 and 10). ${ }^{\mathrm{b}}$ As noted in PROTEUS-GCR-EXP-001, the models by Difilippo include water content within the graphite reflectors. Evaluation of the water content indicates that the small quantity has a negligible impact on the neutron scattering and only provides additional negative reactivity $(\sim 100 \mathrm{pcm})$ to the system. However, the addition of water absorption seems to be incorrect as the analysis of the equivalent boron content in the graphite reflectors should have already included absorption from water contained within the graphite blocks. Another difference exists for the density of the polyethylene rods; Difilippo reports polyethylene with a mass density of $\sim 0.77 \mathrm{~g} / \mathrm{cm}^{3}$, which is suspiciously low, when the density calculated for the benchmark models is $\sim 0.94 \mathrm{~g} / \mathrm{cm}^{3}$. This latter difference only impacts those models containing polyethylene rods.

Monte Carlo calculations with ENDF/B-VII0.0 of $\mathrm{k}_{\text {eff }}$ for graphite-moderated reactors and assemblies typically compute greater than the benchmark values, as seen for the High Temperature Engineering Test Reactor (HTTR-GCR-RESR-001, -002, and -003), the HTR-10 Pebble-Bed Reactor (HTR10-GCR-RESR-001), and the other HTR-PROTEUS configurations (PROTEUS-GCR-EXP-001, -002, and -003). Computations of the ASTRA critical facility with the MCU-REA1 code agree well with the benchmark $\mathrm{k}_{\text {eff }}$ (ASTRA-GCR-EXP-001) but calculate high when using MCNP. ${ }^{c}$ The MCU computer program was developed to include a special feature to evaluate systems with double-heterogeneity, such as TRISO particles in a HTGR. ${ }^{\mathrm{d}}$ The computational bias using MCNP is on the order of 1-2 \% greater than the benchmark values. The HTTR configurations are closer to $2 \%$ and it has been previously discussed that the bias is possibly due to uncertainties in the impurity content of the graphite blocks ${ }^{\text {e,f }}$ and a need to increase the thermal neutron capture cross section of carbon in both JENDL-3.3 and ENDF/B-VII.0 nuclear data libraries. ${ }^{\mathrm{g}}$

\footnotetext{
${ }^{a}$ Colak, U. and Seker, V., "Monte Carlo Criticality Calculations for a Pebble Bed Reactor with MCNP," Nucl. Sci. Eng., 149, 131-137 (2005).

${ }^{\mathrm{b}}$ Difilippo, F. C., "Monte Carlo Calculations of Pebble Bed Benchmark Configurations of the PROTEUS Facility," Nucl. Sci. Eng., 143, 240-253 (2003).

' Z. Zibi and F. Albornoz, "Validating the MCNP Modelling of the ASTRA Critical Facility," Proc. HTR 2010, Prague, Czech Republic, October 18-20, 2010.

${ }^{\mathrm{d}}$ N. N. Ponomarev-Stepnoi, et al., "Using the MCU Computer Program to Analyze the Results of Critical Experiments with HTGR Fuel Pellets on ASTRA Testing Stand," Atomic Energy, 97, pp. 669-677 (2004).

"K. Yamashita, et al., "Startup Core Physics Tests of High Temperature Engineering Test Reactor (HTTR), (I)," J. At. Energy Soc. Jpn., 42, pp. 30-42 (2000) [in Japanese].

${ }^{\mathrm{f}}$ N. Fujimoto, et al., "Startup Core Physics Tests of High Temperature Engineering Test Reactor (HTTR) (II)," J. At. Energy Soc. Jpn., 42, pp. 458-464 (2000) [in Japanese].

${ }^{g}$ S. Shimakawa, M. Goto, S. Nakagawa, and Y. Tachibana, "Impact of Capture Cross-Section of Carbon on Nuclear Design for HTGRs,” Proc. HTR 2010, Prague, Czech Republic, October 18-20, 2010.
}

Revision: 1

Date: March 31, 2014

Page 193 of 375 
NEA/NSC/DOC(2006)1

Gas Cooled (Thermal) Reactor - GCR

PROTEUS-GCR-EXP-004

CRIT-REAC

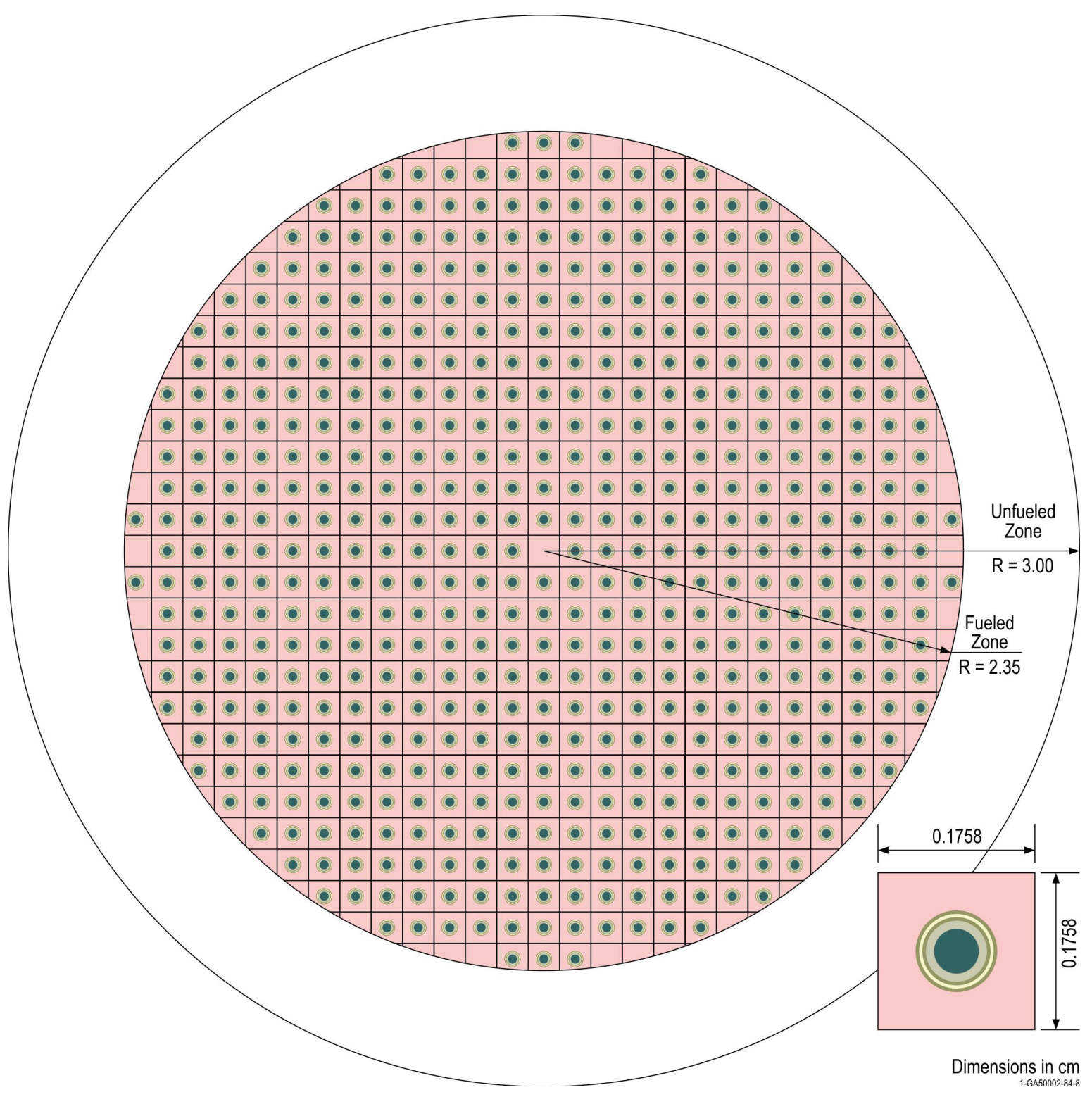

Figure 4.1-1. Regular TRISO Lattice Used in MCNP Calculations of the Benchmark Models.

Revision: 1 
NEA/NSC/DOC(2006)1

\section{Gas Cooled (Thermal) Reactor - GCR \\ PROTEUS-GCR-EXP-004 \\ CRIT-REAC}

Table 4.1-1. Comparison of Benchmark Eigenvalues (MCNP5).

\begin{tabular}{|c|c|c|c|c|c|c|c|c|c|c|}
\hline \multirow{2}{*}{ Case } & \multirow{2}{*}{$\begin{array}{c}\text { Core } \\
\text { (state) }\end{array}$} & \multirow{2}{*}{$\begin{array}{l}\text { Neutron Cross } \\
\text { Section Library }\end{array}$} & \multicolumn{3}{|c|}{ Calculated (MCNP5) } & \multicolumn{3}{|c|}{ Benchmark } & \multirow{2}{*}{$\frac{C-E}{E}(\%)$} & \multirow{2}{*}{$\begin{array}{c}\text { Difference } \\
\text { (pcm) }\end{array}$} \\
\hline & & & $\mathbf{k}_{\mathrm{eff}}$ & \pm & $\sigma$ & $\mathbf{k}_{\text {eff }}$ & \pm & $\sigma$ & & \\
\hline 1 & $9(\# 1)$ & & 1.00581 & \pm & 0.00007 & 1.0029 & \pm & 0.0036 & 0.38 & 377 \\
\hline 2 & 10 & & 1.00667 & \pm & 0.00006 & 1.0020 & \pm & 0.0037 & 0.54 & 543 \\
\hline
\end{tabular}

Additional sample calculations were obtained using the sample input decks provided in Appendix A for the benchmark model provided in Section 3.1 and updating them with ENDF/B-VII.1 neutron cross section data; ${ }^{a}$ the revised input decks were run using MCNP6.1. ${ }^{\mathrm{b}}$ Results are provided in Table 4.1-2. The calculated eigenvalues are now lower than the benchmark values. The result for Case 1 is greater than $1 \%$ from the benchmark value and within a $4 \sigma$ uncertainty. Case 2 results are within the $3 \sigma$ uncertainty. Note that the observed difference between the results shown in Tables 4.1-1 and 4.1-2 is mostly due to the change in the absorption cross section for carbon adopted in ENDF/B-VII.1 from the JENDL-4.0 nuclear data library. The delayed neutron data for ${ }^{235} \mathrm{U}$ in ENDF/B-VII.1 was reverted back to their ENDF/B-VI.8 values instead of the original Keepin data. ${ }^{a}$

Table 4.1-2. Comparison of Benchmark Eigenvalues (MCNP6).

\begin{tabular}{|c|c|c|c|c|c|c|c|c|c|c|}
\hline \multirow{2}{*}{ Case } & \multirow{2}{*}{$\begin{array}{c}\text { Core } \\
\text { (state) }\end{array}$} & \multirow{2}{*}{$\begin{array}{l}\text { Neutron Cross } \\
\text { Section Library }\end{array}$} & \multicolumn{3}{|c|}{ Calculated (MCNP6) } & \multicolumn{3}{|c|}{ Benchmark } & \multirow{2}{*}{$\frac{C-E}{E}(\%)$} & \multirow{2}{*}{$\begin{array}{c}\text { Difference } \\
(\mathbf{p c m})\end{array}$} \\
\hline & & & $\mathbf{k}_{\text {eff }}$ & \pm & $\sigma$ & $\mathbf{k}_{\text {eff }}$ & \pm & $\sigma$ & & \\
\hline 1 & $9(\# 1)$ & & 0.99098 & 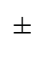 & 0.00 & 1.0029 & \pm & 0.0036 & -1.19 & -1192 \\
\hline 2 & 10 & & 0.99486 & \pm & 0.00006 & 1.0020 & \pm & 0.0037 & -0.71 & -714 \\
\hline
\end{tabular}

\subsection{Results of Buckling and Extrapolation Length Calculations}

Buckling and extrapolation length measurements were performed but have not yet been evaluated.

\subsection{Results of Spectral-Characteristics Calculations}

Spectral characteristics measurements were performed but have not yet been evaluated.

\footnotetext{
${ }^{a}$ M. B. Chadwick, et al., "ENDF/B-VII.1: Nuclear Data for Science and Technology: Cross Sections, Covariances, Fission Product Yields and Decay Data," Nucl. Data Sheets, 112: 2887-2996 (2011).

b J. T. Goorley, et al., "Initial MCNP6 Release Overview - MCNP6 version 1.0," LA-UR-13-22934, Los Alamos National Laboratory (2013).
}

Revision: 1

Date: March 31, 2014 
NEA/NSC/DOC(2006)1

\section{Gas Cooled (Thermal) Reactor - GCR \\ PROTEUS-GCR-EXP-004 \\ CRIT-REAC}

\subsection{Results of Reactivity-Effects Calculations}

The benchmark models described in Section 3.4 were used with MCNP5-1.60 (see Appendix A.1 and A.4 for input decks) and ENDF/B-VII.0 neutron cross section data. For additional details regarding how the TRISO particles were modeled, see Section 4.1. Monte Carlo calculations were performed with 1,650 generations with 100,000 neutrons per generation. The $k_{\text {eff }}$ estimates are based on 150 skipped generations and a total of $150,000,000$ neutron histories each.

The difference between various configurations, as described in Section 3.4.2, were simulated to calculate reactivity worths $(\Delta \mathrm{k} / \mathrm{k})$. These worths were then converted into units of $\rho(\$)$ using a $\beta_{\text {eff }}$ value of $0.00693 \pm 0.00035(5 \%, 1 \sigma)$ for Core 9 and $0.00685 \pm 0.00034(5 \%, 1 \sigma)$ for Core 10 . The Monte Carlo statistical uncertainty is approximately $\$ 0.01$. The uncertainty in the calculated values provided in this section also include the uncertainty in $\beta_{\text {eff; }}$, therefore, calculations using additional neutron cross section libraries were not performed.

Results for the 32 cases are provided in Table 4.4-1 for Core 9 and Table 4.4-2 for Core 10 (16 apiece). There is generally good agreement between calculated and benchmark worths. Most calculations are within $1 \sigma$ to $2 \sigma$ of the benchmark values. At the time of this evaluation the statistical uncertainty in MCNP calculations of the HTR-PROTEUS benchmark models is $\sim 1 \phi$; it is not practical to further reduce this uncertainty with currently available computing resources.

The worth of a control rod is calculated using the following equation:

$\rho(\$)=\frac{k_{\text {inserted }}-k_{\text {withdrawn }}}{k_{\text {inserted }} \times k_{\text {withdrawn }}} \times \frac{1}{\beta_{\text {eff }}}$.

Measurements scaled from another core configuration were evaluated but deemed not acceptable as benchmark data; further information for modeling these data is provided in Appendix F. 
NEA/NSC/DOC(2006)1

\section{Gas Cooled (Thermal) Reactor - GCR \\ PROTEUS-GCR-EXP-004 \\ CRIT-REAC}

Table 4.4-1. Sample Calculations for Reactivity Effects Measurements (Core 9).

\begin{tabular}{|c|c|c|c|c|c|c|c|c|c|c|}
\hline \multirow[t]{2}{*}{ Case } & \multirow[t]{2}{*}{ Measured Parameter } & \multicolumn{3}{|c|}{$\begin{array}{l}\text { Benchmark } \\
\text { Worth }\end{array}$} & \multicolumn{3}{|c|}{$\begin{array}{l}\text { Calculated } \\
\text { Worth }\end{array}$} & \multirow{2}{*}{$\frac{C-E}{E}(\%)$} & \multirow{2}{*}{ \pm} & \multirow{2}{*}{$\sigma^{(\mathbf{a})}$} \\
\hline & & $\rho(\$)$ & \pm & $\boldsymbol{\sigma}$ & $\rho(\$)$ & \pm & $\underline{\sigma}$ & & & \\
\hline $1.4-1$ & Control Rod 1 & -0.41 & \pm & 0.02 & -0.38 & \pm & 0.02 & -7 & \pm & 7 \\
\hline $1.4-2$ & Control Rod 2 & -0.41 & \pm & 0.02 & -0.37 & \pm & 0.02 & -10 & \pm & 7 \\
\hline $1.4-3$ & Control Rod 3 & -0.41 & \pm & 0.02 & -0.38 & \pm & 0.02 & -7 & \pm & 7 \\
\hline $1.4-4$ & Control Rod 4 & -0.41 & \pm & 0.02 & -0.38 & \pm & 0.02 & -7 & \pm & 7 \\
\hline $1.4-5$ & $\begin{array}{c}\text { Control Rod Bank } \\
\text { Full Insertion }\end{array}$ & -1.58 & \pm & 0.09 & -1.55 & \pm & 0.08 & -2 & \pm & 8 \\
\hline $1.4-6$ & $\begin{array}{c}\text { Control Rod Bank } \\
\text { Partial Insertion }\end{array}$ & -0.73 & \pm & 0.04 & -0.70 & \pm & 0.04 & -4 & \pm & 8 \\
\hline $1.4-7$ & Autorod Insertion & -0.10 & \pm & 0.01 & -0.12 & \pm & 0.02 & 20 & \pm & 23 \\
\hline $1.4-8$ & Safety/Shutdown Rod 5 & -3.74 & \pm & 0.17 & -3.78 & \pm & 0.19 & 1 & \pm & 7 \\
\hline $1.4-9$ & Safety/Shutdown Rod 6 & -3.82 & \pm & 0.10 & -3.82 & \pm & 0.19 & 0 & \pm & 6 \\
\hline $1.4-10$ & Safety/Shutdown Rod 7 & -3.70 & \pm & 0.30 & -3.82 & \pm & 0.19 & 3 & \pm & 10 \\
\hline $1.4-11$ & Safety/Shutdown Rod 8 & -3.60 & \pm & 0.29 & -3.70 & \pm & 0.19 & 3 & \pm & 10 \\
\hline $1.4-12$ & Safety/Shutdown Rods $5+6$ & -8.02 & \pm & 0.20 & -8.03 & \pm & 0.40 & 0 & \pm & 6 \\
\hline $1.4-13$ & Safety/Shutdown Rods 5+7 & -7.44 & \pm & 0.60 & -7.76 & \pm & 0.39 & 4 & \pm & 10 \\
\hline $1.4-14$ & Safety/Shutdown Rods 5+8 & -7.40 & \pm & 0.59 & -7.69 & \pm & 0.38 & 4 & \pm & 10 \\
\hline $1.4-15$ & $\begin{array}{l}\text { Safety/Shutdown Rods } \\
5+6+7\end{array}$ & -12.11 & \pm & 0.28 & -12.30 & \pm & 0.61 & 2 & \pm & 6 \\
\hline $1.4-16$ & $\begin{array}{l}\text { Safety/Shutdown Rods } \\
5+6+7+8\end{array}$ & -16.52 & \pm & 0.42 & -16.98 & \pm & 0.85 & 3 & \pm & 6 \\
\hline
\end{tabular}

(a) The uncertainty in $\frac{C-E}{E}(\%)$ is calculated by propagating the uncertainties in both the calculated and benchmark experiment eigenvalues using the following equation: $\sigma=100 \% \times \sqrt{\left(\frac{\sigma_{C}}{E}\right)^{2}+\left(\frac{\sigma_{E} C}{E^{2}}\right)^{2}}$. 
NEA/NSC/DOC(2006)1

Gas Cooled (Thermal) Reactor - GCR

PROTEUS-GCR-EXP-004

CRIT-REAC

Table 4.4-2. Sample Calculations for Reactivity Effects Measurements (Core 10).

\begin{tabular}{|c|c|c|c|c|c|c|c|c|c|c|}
\hline \multirow{2}{*}{ Case } & \multirow[t]{2}{*}{ Measured Parameter } & \multicolumn{3}{|c|}{$\begin{array}{l}\text { Benchmark } \\
\text { Worth }\end{array}$} & \multicolumn{3}{|c|}{$\begin{array}{l}\text { Calculated } \\
\text { Worth }\end{array}$} & \multirow{2}{*}{$\frac{C-E}{E}(\%)$} & \multirow{2}{*}{ \pm} & \multirow{2}{*}{$\sigma^{(\mathbf{a})}$} \\
\hline & & $\rho(\$)$ & \pm & $\sigma$ & $\rho(\$)$ & \pm & $\sigma$ & & & \\
\hline $2.4-1$ & Control Rod 1 & -0.30 & \pm & 0.02 & -0.29 & \pm & 0.02 & -3 & \pm & 9 \\
\hline $2.4-2$ & Control Rod 2 & -0.29 & \pm & 0.02 & -0.28 & \pm & 0.02 & -3 & \pm & 10 \\
\hline $2.4-3$ & Control Rod 3 & -0.29 & \pm & 0.02 & -0.25 & \pm & 0.02 & -14 & \pm & 9 \\
\hline $2.4-4$ & Control Rod 4 & -0.30 & \pm & 0.02 & -0.28 & \pm & 0.02 & -7 & \pm & 9 \\
\hline $2.4-5$ & $\begin{array}{l}\text { Control Rod Bank } \\
\text { Full Insertion }\end{array}$ & -1.15 & \pm & 0.07 & -1.11 & \pm & 0.06 & -3 & \pm & 8 \\
\hline $2.4-6$ & $\begin{array}{l}\text { Control Rod Bank } \\
\text { Partial Insertion } \\
\end{array}$ & -0.39 & \pm & 0.02 & -0.37 & \pm & 0.02 & -5 & \pm & 7 \\
\hline $2.4-7$ & Autorod Insertion & -0.073 & \pm & 0.004 & -0.08 & \pm & 0.01 & 10 & \pm & 15 \\
\hline $2.4-8$ & Safety/Shutdown Rod 5 & -2.82 & \pm & 0.11 & -2.73 & \pm & 0.14 & -3 & \pm & 6 \\
\hline $2.4-9$ & Safety/Shutdown Rod 6 & -2.82 & \pm & 0.09 & -2.75 & \pm & 0.14 & -2 & \pm & 6 \\
\hline $2.4-10$ & Safety/Shutdown Rod 7 & -2.80 & \pm & 0.16 & -2.73 & \pm & 0.14 & -2 & \pm & 7 \\
\hline $2.4-11$ & Safety/Shutdown Rod 8 & -2.72 & \pm & 0.15 & -2.66 & \pm & 0.13 & -2 & \pm & 7 \\
\hline $2.4-12$ & $\begin{array}{l}\text { Safety/Shutdown Rods } \\
5+6\end{array}$ & -5.95 & \pm & 0.17 & -5.70 & \pm & 0.29 & -4 & \pm & 6 \\
\hline $2.4-13$ & $\begin{array}{l}\text { Safety/Shutdown Rods } \\
5+7\end{array}$ & -5.73 & \pm & 0.32 & -5.54 & \pm & 0.28 & -3 & \pm & 7 \\
\hline $2.4-14$ & $\begin{array}{l}\text { Safety/Shutdown Rods } \\
5+8\end{array}$ & -5.75 & \pm & 0.33 & -5.49 & \pm & 0.27 & -5 & \pm & 7 \\
\hline $2.4-15$ & $\begin{array}{c}\text { Safety/Shutdown Rods } \\
5+6+7\end{array}$ & -9.29 & \pm & 0.21 & -8.65 & \pm & 0.43 & -7 & \pm & 5 \\
\hline $2.4-16$ & $\begin{array}{c}\text { Safety/Shutdown Rods } \\
5+6+7+8\end{array}$ & -12.67 & \pm & 0.31 & -11.81 & \pm & 0.59 & -7 & \pm & 5 \\
\hline
\end{tabular}

(a) The uncertainty in $\frac{C-E}{E}(\%)$ is calculated by propagating the uncertainties in both the calculated and benchmark experiment eigenvalues using the following equation: $\sigma=100 \% \times \sqrt{\left(\frac{\sigma_{C}}{E}\right)^{2}+\left(\frac{\sigma_{E} C}{E^{2}}\right)^{2}}$. 


$$
\begin{gathered}
\text { NEA/NSC/DOC(2006)1 } \\
\text { Gas Cooled (Thermal) Reactor - GCR } \\
\text { PROTEUS-GCR-EXP-004 } \\
\text { CRIT-REAC }
\end{gathered}
$$

\subsection{Results of Reactivity Coefficient Calculations}

Reactivity coefficient measurements were performed but have not yet been evaluated.

\subsection{Results of Kinetics Parameter Calculations}

Kinetics measurements were performed but have not yet been evaluated.

\subsection{Results of Reaction-Rate Distribution Calculations}

Reaction-rate distribution measurements were performed but have not yet been evaluated.

\subsection{Results of Power Distribution Calculations}

Power distribution measurements were not performed.

\subsection{Results of Isotopic Calculations}

Isotopic measurements were not performed.

\subsection{Results of Calculations for Other Miscellaneous Types of Measurements}

Other miscellaneous types of measurements were not performed. 
NEA/NSC/DOC(2006)1

\author{
Gas Cooled (Thermal) Reactor - GCR \\ PROTEUS-GCR-EXP-004 \\ CRIT-REAC
}

\title{
5.0 REFERENCES
}

1. Williams, T., "LEU-HTR PROTEUS: Configuration Descriptions and Critical Balances for the Cores of the HTR-PROTEUS Experimental Programme," TM-41-95-18, v. 1.00, Paul Sherrer Institut, Villigen, November 25, 1996.

2. Mathews, D. and Williams, T., "LEU-HTR PROTEUS System Component Description," TM-41-9343, v. 2.0, Paul Scherrer Institut, Villigen, November 25, 1996.

3. Williams, T., Rosselet, M., and Scherer, W. (editors), "Critical Experiments and Reactor Physics Calculations for Low-Enriched High Temperature Gas Cooled Reactors," IAEA-TECDOC-1249, International Atomic Energy Agency, Vienna (2001).

4. Wallerbos, E. J. M., "Reactivity Effects in a Pebble-Bed Type Nuclear Reactor: An Experimental and Calculational Study," Ph.D. Dissertation, Delft University of Technology, Delft, Netherlands (1998).

5. Köberl, O. and Seiler, R., "Detailed Analysis of Pebble-Bed HTR PROTEUS Experiments with the Monte Carlo Code TRIPOLI4," Proc. $2^{\text {nd }}$ International Conference on High Temperature Reactor Technology, Beijing, China, September 22-24, 2004.

6. Chawla, R., Joneja, O. P., Rosselet, M., and Williams, T., "Definition and Analysis of an Experimental Benchmark on Shutdown Rod Worths in LEU-HTR Configurations," Nucl. Tech., 139, 50-60 (2002).

7. Rosselet, M., Chawla, R., and Williams, T., "Epithermal Inverse Kinetic Measurements and Their Interpretation Using a Two-Group Point-Kinetic Model," Nucl. Sci. Eng., 135, 33-47 (2000).

8. Rosselet, M., Williams, T., and Chawla, R., "Subcriticality Measurements Using an Epithermal Pulsed-Neutron-Source Technique in Pebble-Bed HTR Configurations," Ann. Nucl. Energy, 25, 285294 (1998).

9. Rosselet, M., Chawla, R., Joneja, O. P., Williams, T., "Measurement and Analysis of Shutdown Rod Worths in LEU-HTR Configurations," Proc. The $10^{\text {th }}$ International Conference on Emerging Nuclear Energy Systems (ICENES 2000), Petten, The Netherlands, September 25-28, 2000.

10. Rosselet, M., "Reactivity Measurements and their Interpretation in Systems with Large Spatial Effects," Ph.D. Dissertation, École Polytechnique Fédérale de Lausanne, Lausanne, Switzerland (1999). 
NEA/NSC/DOC(2006)1

Gas Cooled (Thermal) Reactor - GCR

PROTEUS-GCR-EXP-004

CRIT-REAC

\section{APPENDIX A: COMPUTER CODES, CROSS SECTIONS, AND TYPICAL INPUT LISTINGS}

\section{A.1 Critical/Subcritical Configurations}

\section{A.1.1 Name(s) of code system(s) used.}

Monte Carlo n-Particle, version 5.1.60 (MCNP5).

\section{A.1.2 Bibliographic references for the codes used.}

X-5 Monte Carlo Team, "MCNP - a General Monte Carlo n-Particle Transport Code, version 5," LA-UR-03-1987, Los Alamos National Laboratory (2003).

\section{A.1.3 Origin of cross-section data.}

The Evaluated Neutron Data File library, ENDF/B-VII.0 a was utilized in the benchmark model analysis.

\section{A.1.4 Spectral calculations and data reduction methods used.}

Not applicable.

\section{A.1.5 Number of energy groups or if continuous-energy cross sections are used in the different phases of calculation.}

Continuous-energy cross sections.

\section{A.1.6 Component calculations.}

- Type of cell calculation - Reactor core, reflectors, and moderator

- Geometry - TRISO particles in graphite pebbles

- Theory used - Not applicable

- Method used - Monte Carlo

- Calculation characteristics

- MCNP5 - histories/cycles/cycles skipped $=100,000 / 1,650 / 150$

continuous-energy cross sections

\section{A.1.7 Other assumptions and characteristics.}

Not applicable.

\footnotetext{
${ }^{a}$ M. B. Chadwick, et al., "ENDF/B-VII.0: Next Generation Evaluated Nuclear Data Library for Nuclear Science and Technology," Nucl. Data Sheets, 107: 2931-3060 (2006).

Revision: 1

Date: March 31, 2014 
NEA/NSC/DOC(2006)1

Gas Cooled (Thermal) Reactor - GCR

PROTEUS-GCR-EXP-004

CRIT-REAC

\section{A.1.8 Typical input listings for each code system type.}

The input deck provided below is for core configuration 9 (Case 1). The following portions of the code need reconfigured for core configuration 10 (Case 2):

- Autorod position,

- Withdrawable control rod positions, and

- Core cavity filled with correct pebble configuration (including polyethylene rods).

MCNP5 Input Deck for Case 1 (Core 9) [can be modified for Case 2 (Core 10)]:

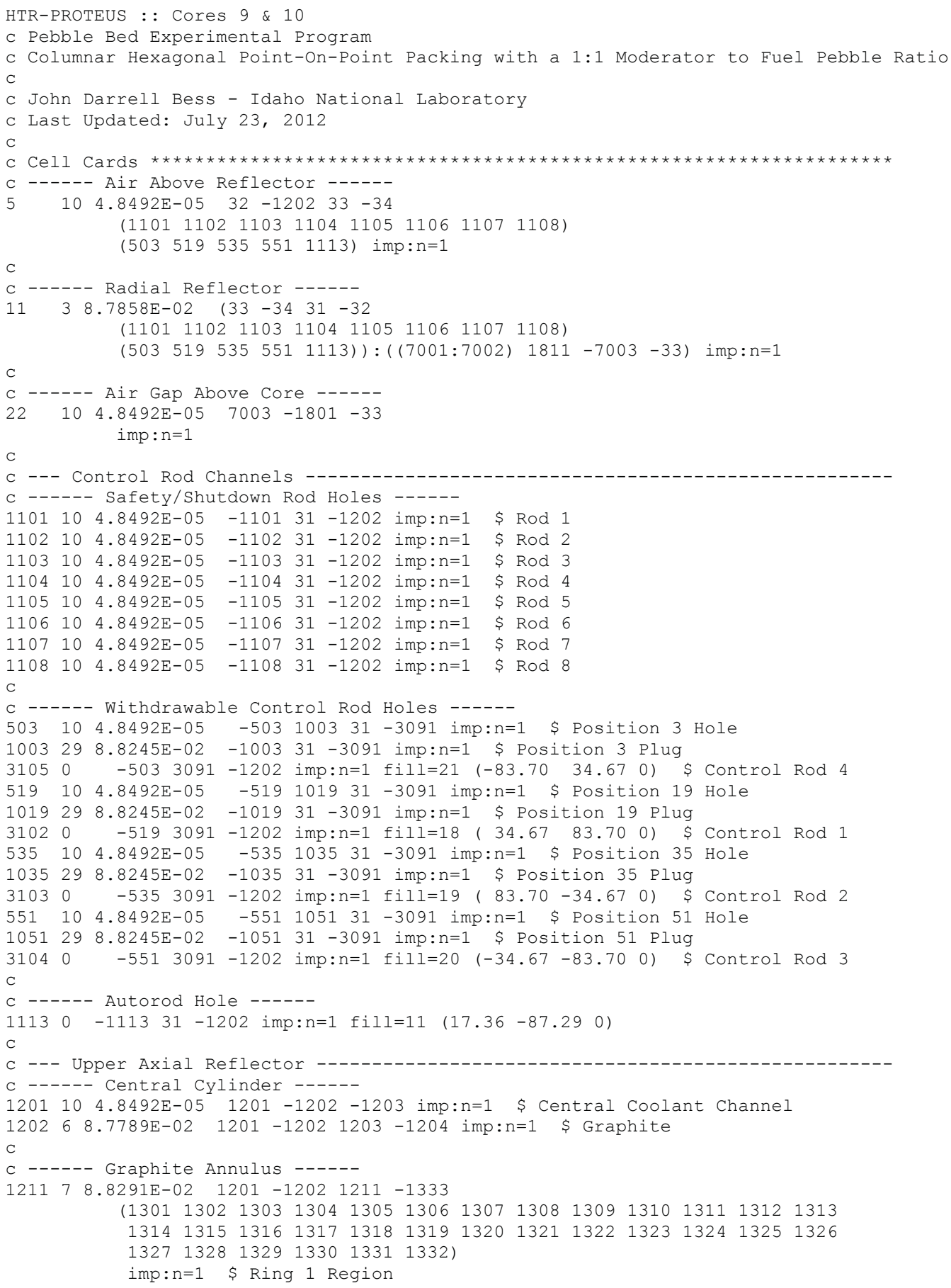

Revision: 1 
NEA/NSC/DOC(2006)1

\section{Gas Cooled (Thermal) Reactor - GCR \\ PROTEUS-GCR-EXP-004 CRIT-REAC}

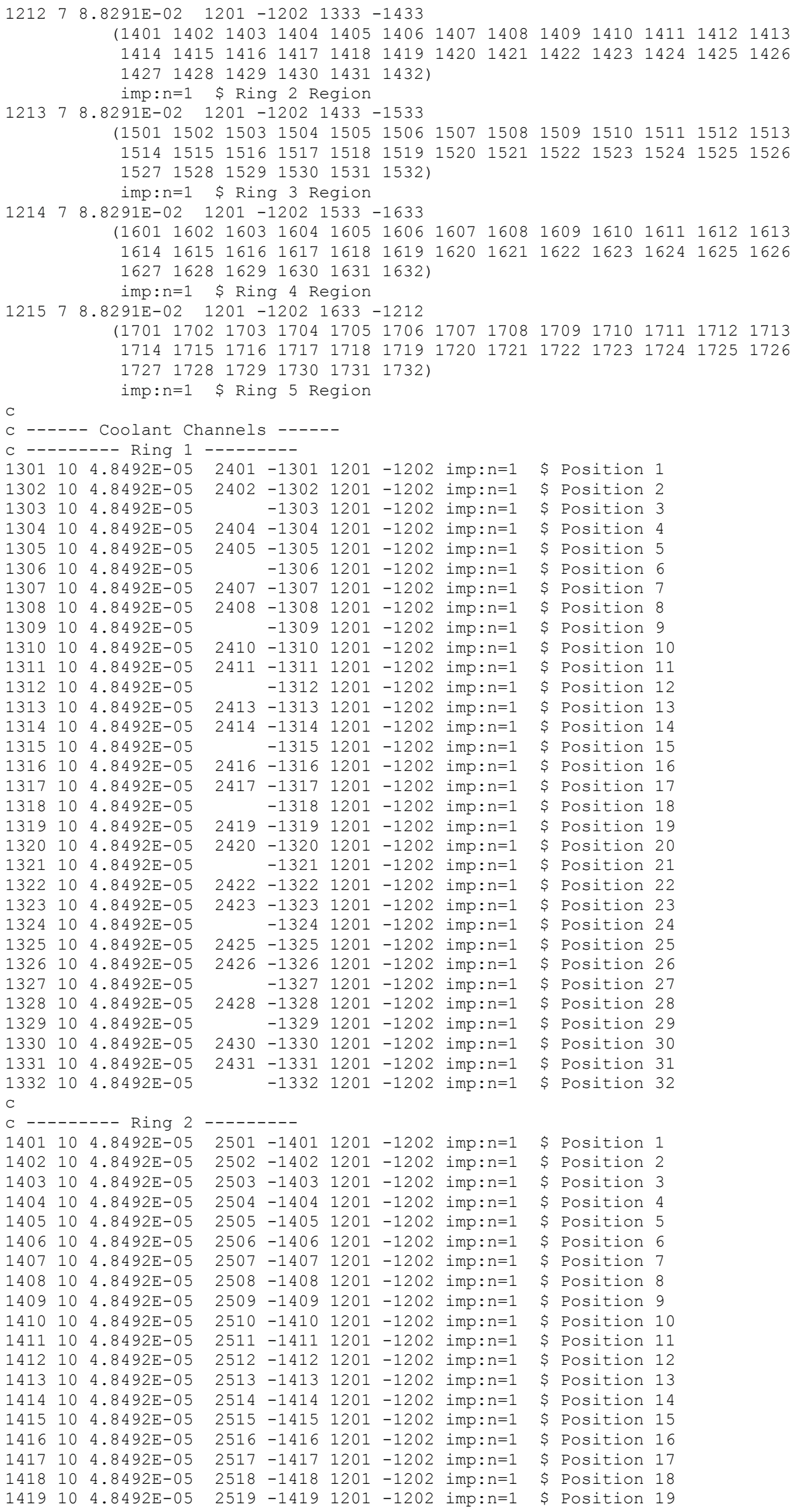

Revision: 1 
NEA/NSC/DOC(2006)1

Gas Cooled (Thermal) Reactor - GCR

PROTEUS-GCR-EXP-004 CRIT-REAC

\begin{tabular}{|c|c|c|c|c|c|c|c|c|c|c|}
\hline 1420 & 10 & $E-05$ & 20 & -1420 & 1 & -1202 & imp: $n=1$ & $\$$ & on & 20 \\
\hline & 10 & $4.8492 \mathrm{E}-05$ & 2521 & -1421 & 201 & -1202 & imp:n=1 & $\$$ & sition & 1 \\
\hline 2 & 10 & $4.8492 E-05$ & 2522 & -1422 & 1201 & -1202 & imp: $n=1$ & $\$$ & Position & 22 \\
\hline 12 & 10 & $4.8492 E-05$ & 2523 & -1423 & 1201 & -1202 & imp: $n=1$ & $\$$ & Position & 23 \\
\hline & 10 & $4.8492 \mathrm{E}-05$ & 2524 & -1424 & 201 & -1202 & imp:n=1 & $\$$ & sition & \\
\hline & 10 & $4.8492 E-05$ & 2525 & -1425 & 201 & -1202 & imp:n=1 & $\$$ & osition & \\
\hline & 10 & $4.8492 \mathrm{E}-05$ & 526 & -1426 & 201 & -1202 & imp: $n=1$ & $\$$ & osition & \\
\hline & 10 & $4.8492 E-05$ & 2527 & -1427 & 201 & -1202 & imp: $n=1$ & $\$$ & osition & 7 \\
\hline & 10 & $4.8492 \mathrm{E}-05$ & 528 & -1428 & 201 & -1202 & imp: $n=1$ & $\$$ & osition & \\
\hline & 10 & $4.8492 \mathrm{E}-05$ & 29 & -1429 & & -1202 & imp: $n=1$ & $\$$ & osition & \\
\hline & 10 & $4.8492 \mathrm{E}-05$ & 30 & -1430 & 201 & -1202 & imp:n=1 & $\$$ & osition & \\
\hline & 10 & $4.8492 \mathrm{E}-05$ & 31 & -1431 & 201 & -1202 & imp: $n=1$ & $\$$ & osition & \\
\hline & 10 & $4.8492 E-05$ & 32 & -1432 & 01 & -1202 & imp:n=1 & $\$$ & & \\
\hline & & & & & & & & & & \\
\hline & & & & 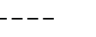 & & & & & & \\
\hline & 0 & $4.8492 E-05$ & & -1501 & 1 & 02 & imp: $n=1$ & $\$$ & ion & 1 \\
\hline & 0 & $4.8492 E-05$ & & -1502 & & -1202 & imp: $n=1$ & $\$$ & ion & \\
\hline & 10 & $4.8492 \mathrm{E}-05$ & & -1503 & 201 & -1202 & imp:n=1 & $\$$ & Position & \\
\hline & 10 & $4.8492 E-05$ & & -1504 & 01 & -1202 & imp: $n=1$ & $\$$ & Posi & \\
\hline & 10 & $4.8492 \mathrm{E}-05$ & & -1505 & 201 & -1202 & imp: $n=1$ & $\$$ & ion & \\
\hline & 10 & $4.8492 \mathrm{E}$ & & -1506 & 201 & -1202 & imp: $n=1$ & $\$$ & ion & \\
\hline & 10 & $4.8492 \mathrm{E}-05$ & 2607 & -1507 & 01 & -1202 & $i m p: n=1$ & $\$$ & Position & 7 \\
\hline & 10 & $4.8492 E-05$ & & -1508 & 01 & -1202 & imp: $n=1$ & $\$$ & Position & \\
\hline & 10 & $4.8492 E-05$ & 2609 & -1509 & 01 & -1202 & imp:n=1 & $\$$ & ion & \\
\hline & 10 & $4.8492 \mathrm{E}-05$ & 2610 & -1510 & 201 & -1202 & imp: $n=1$ & $\$$ & Position & 0 \\
\hline & 10 & $4.8492 E-05$ & & -1511 & 201 & -1202 & imp: $n=1$ & $\$$ & Position & 1 \\
\hline & 10 & $4.8492 \mathrm{E}-05$ & 2612 & -1512 & 1 & -1202 & imp: $n=1$ & $\$$ & Position & 2 \\
\hline & 10 & $4.8492 \mathrm{E}-05$ & & -1513 & & -12 & imp: $n=1$ & $\$$ & Position & \\
\hline & 10 & $4.8492 \mathrm{E}-05$ & & -1514 & 01 & -1202 & imp: $n=1$ & $\$$ & Position & \\
\hline & 10 & $4.8492 E-05$ & & -1515 & 201 & -1202 & imp: $n=1$ & $\$$ & Position & \\
\hline & 10 & $4.8492 \mathrm{E}-05$ & & -1516 & 201 & -12 & imp:n=1 & $\$$ & ion & \\
\hline & 10 & $4.8492 \mathrm{E}-05$ & & -1517 & 201 & -1202 & imp: $n=1$ & $\$$ & Position & 17 \\
\hline & 10 & $4.8492 E-05$ & & -1518 & & -1202 & imp:n=1 & $\$$ & Position & \\
\hline & 10 & $4.8492 E-05$ & & -1519 & 01 & -1202 & imp: $n=1$ & $\$$ & Position & \\
\hline & 10 & $492 E-05$ & & -1520 & & -12 & imp:n=1 & $\$$ & Pos & \\
\hline & 10 & $4.8492 \mathrm{E}-05$ & & -1521 & 1201 & -1202 & $i m p: n=1$ & $\$$ & Position & 21 \\
\hline & 10 & $4.8492 E-05$ & & -1522 & 1201 & -1202 & imp: $n=1$ & $\$$ & Pos & \\
\hline & 10 & $4.8492 \mathrm{E}-05$ & & -1523 & 1201 & -12 & imp: $n=1$ & $\$$ & ion & 25 \\
\hline & 10 & $4.8492 \mathrm{E}-05$ & & -1524 & 1201 & -1202 & imp: $n=1$ & $\$$ & ion & 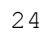 \\
\hline & 10 & $4.8492 \mathrm{E}-05$ & 2625 & -1525 & 1201 & -1202 & imp: $n=1$ & $\$$ & Position & 5 \\
\hline & 10 & $4.8492 E-05$ & & -1526 & 1 & -1202 & imp: $n=1$ & $\$$ & Posi & \\
\hline & 10 & $4.8492 \mathrm{E}-05$ & 27 & -1527 & 1201 & -12 & imp: $n=1$ & $\$$ & ion & 27 \\
\hline & 10 & $4.8492 \mathrm{E}-05$ & & -1528 & 1201 & -1202 & imp: $n=1$ & $\$$ & Position & 28 \\
\hline & 10 & $4.8492 E-05$ & & -1529 & 01 & -1202 & imp:n=1 & $\$$ & Position & 9 \\
\hline & 10 & $4.8492 E-05$ & & -1530 & 1 & -1202 & imp: $n=1$ & $\$$ & Position & 0 \\
\hline & 10 & $4.8492 E-05$ & & -1531 & & -1 & imp:n=1 & $\$$ & Position & - \\
\hline & 10 & $4.8492 \mathrm{E}-05$ & 32 & -1532 & 12 & -12 & imp:n=1 & $\$$ & Position & 32 \\
\hline & & & & & & & & & & \\
\hline & & --- & & 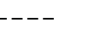 & & & & & & \\
\hline & 10 & $4.8492 E-05$ & & -1601 & & -1202 & imp: $n=1$ & $\$$ & on & 1 \\
\hline & 10 & $4.8492 \mathrm{E}-05$ & & -1602 & 1 & -1202 & imp: $n=1$ & $\$$ & ion & 2 \\
\hline & 10 & $4.8492 \mathrm{E}-05$ & 03 & -1603 & 1201 & -1202 & imp: $n=1$ & $\$$ & Position & 3 \\
\hline & 10 & $4.8492 \mathrm{E}-05$ & 2704 & -1604 & 1201 & -1 & imp:n=1 & $\$$ & ion & 4 \\
\hline & 10 & $4.8492 \mathrm{E}-05$ & & -1605 & 1201 & -1202 & imp: $n=1$ & $\$$ & Position & 5 \\
\hline & 10 & $4.8492 \mathrm{E}-05$ & 6 & -1606 & 1201 & -1202 & imp:n=1 & $\$$ & Position & 6 \\
\hline & 10 & $4.8492 E-05$ & 2707 & -1607 & 1201 & -1202 & imp:n=1 & $\$$ & Position & 7 \\
\hline & 10 & $4.8492 E-05$ & & -1608 & & -1202 & imp: $n=1$ & $\$$ & Position & 8 \\
\hline & 10 & $4.8492 E-05$ & 9 & -1609 & 1. & -1202 & imp: $n=1$ & $\$$ & Position & 9 \\
\hline & 10 & $4.8492 E-05$ & 2710 & -1610 & 1201 & -1202 & imp: $n=1$ & $\$$ & Position & 10 \\
\hline & 10 & $4.8492 \mathrm{E}-05$ & 2711 & -1611 & 1201 & -1202 & imp: $n=1$ & $\$$ & Position & 11 \\
\hline & 10 & $4.8492 \mathrm{E}-05$ & & -1612 & & -1202 & $i m p: n=1$ & $\$$ & Position & 12 \\
\hline & 10 & $4.8492 \mathrm{E}-05$ & 2713 & -1613 & 1201 & -1202 & imp: $n=1$ & $\$$ & Position & 13 \\
\hline & 10 & $4.8492 \mathrm{E}-05$ & 2714 & -1614 & 1201 & -12 & $i m p: n=1$ & $\$$ & Position & 14 \\
\hline & 10 & $4.8492 \mathrm{E}-05$ & 2715 & -1615 & 1201 & -1 & imp:n=1 & $\$$ & Position & \\
\hline & 10 & $4.8492 \mathrm{E}-05$ & & -1616 & 1201 & -1202 & imp: $n=1$ & $\$$ & Position & 16 \\
\hline & 10 & $4.8492 E-05$ & 2717 & -1617 & 1201 & -1202 & imp: $n=1$ & $\$$ & Position & 1 \\
\hline & 10 & $4.8492 \mathrm{E}-05$ & 2718 & -1618 & 1201 & -12 & imp: $n=1$ & $\$$ & Position & 18 \\
\hline & 10 & $4.8492 \mathrm{E}-05$ & & -1619 & 1201 & -12 & imp: $n=1$ & $\$$ & Position & 19 \\
\hline & 10 & $4.8492 \mathrm{E}-05$ & 2720 & -1620 & 1201 & -1202 & imp: $n=1$ & $\$$ & Position & 20 \\
\hline & 10 & $4.8492 \mathrm{E}-05$ & 27 & -1621 & 1201 & -1202 & imp: $n=1$ & $\$$ & Position & 21 \\
\hline & 10 & $4.8492 \mathrm{E}-05$ & 2722 & -1622 & 12 & -12 & imp: $n=1$ & $\$$ & Position & 22 \\
\hline & 10 & $4.8492 \mathrm{E}-05$ & 2723 & -1623 & 1201 & -1202 & imp: $n=1$ & $\$$ & Position & 23 \\
\hline & 10 & $4.8492 E-05$ & 2724 & -1624 & 1201 & -1202 & imp: $n=1$ & $\$$ & Position & 24 \\
\hline & 10 & $4.8492 \mathrm{E}-05$ & 2725 & -1625 & 1201 & -1202 & $i m p: n=1$ & $\$$ & Position & \\
\hline & 10 & $4.8492 E-05$ & 2726 & -1626 & 120 & -1202 & imp: $n=1$ & $\$$ & Position & \\
\hline & 10 & $4.8492 \mathrm{E}-05$ & 2727 & -1627 & 1201 & -1202 & $i m p: n=1$ & $\$$ & Position & 27 \\
\hline
\end{tabular}

Revision: 1 
NEA/NSC/DOC(2006)1

Gas Cooled (Thermal) Reactor - GCR

PROTEUS-GCR-EXP-004 CRIT-REAC

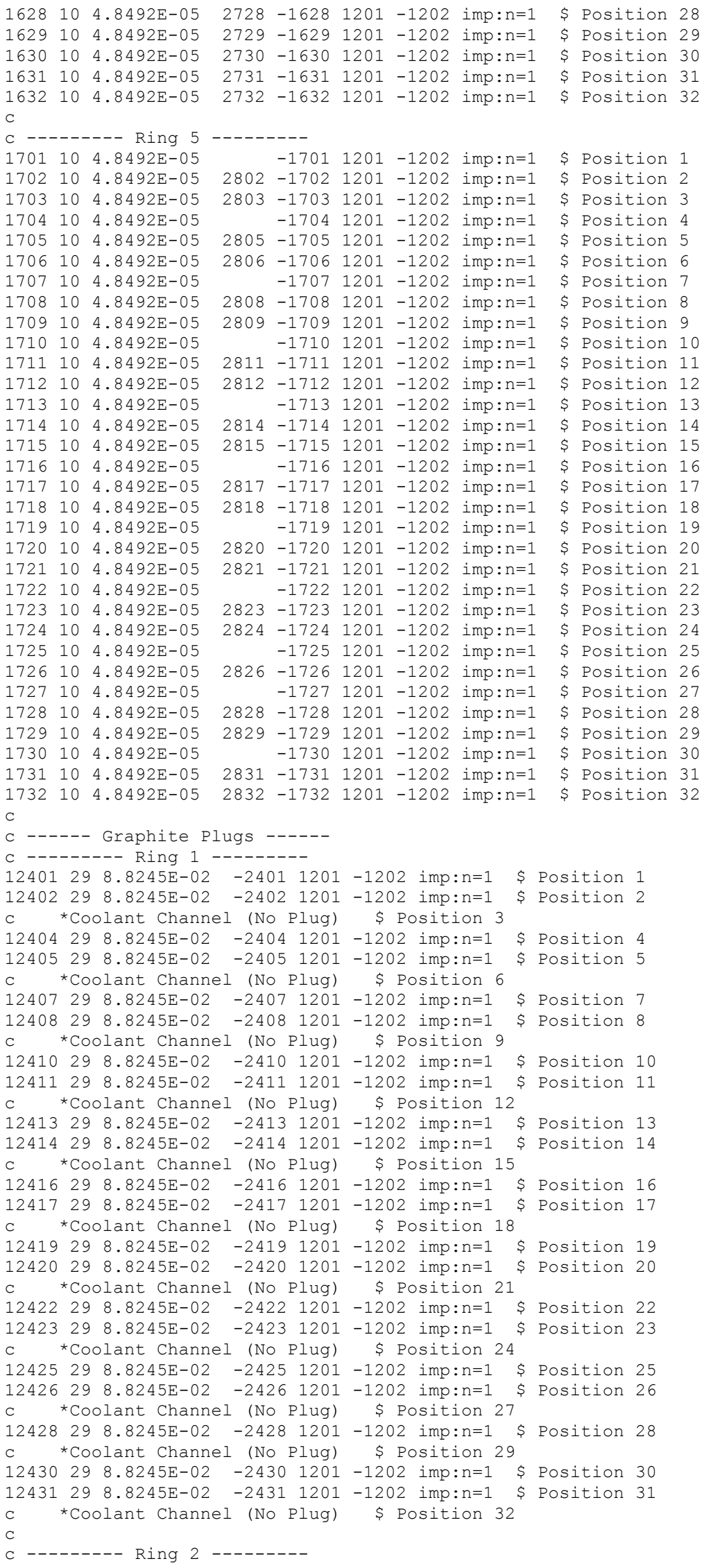

Revision: 1 
NEA/NSC/DOC(2006)1

Gas Cooled (Thermal) Reactor - GCR

PROTEUS-GCR-EXP-004 CRIT-REAC

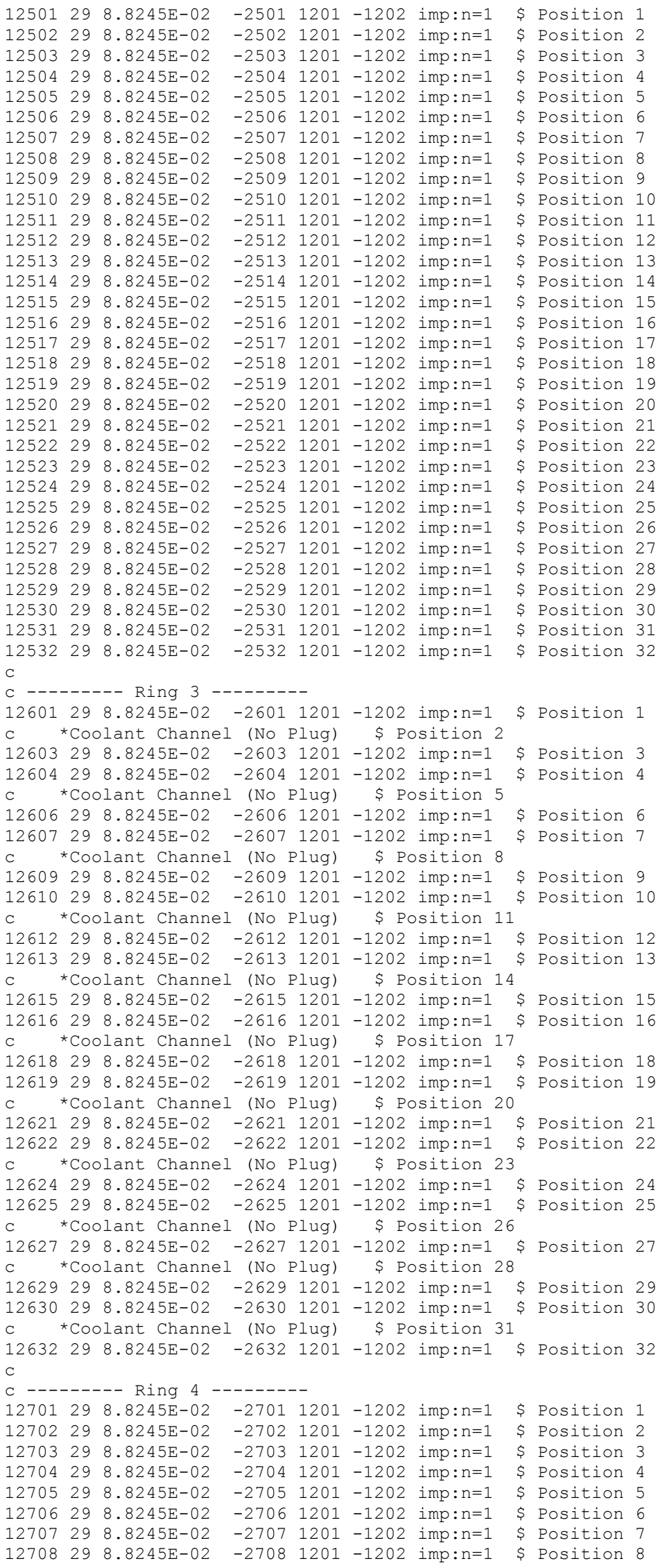

Revision: 1

Date: March 31, 2014

Page 206 of 375 
NEA/NSC/DOC(2006)1

\section{Gas Cooled (Thermal) Reactor - GCR}

\section{PROTEUS-GCR-EXP-004 CRIT-REAC}

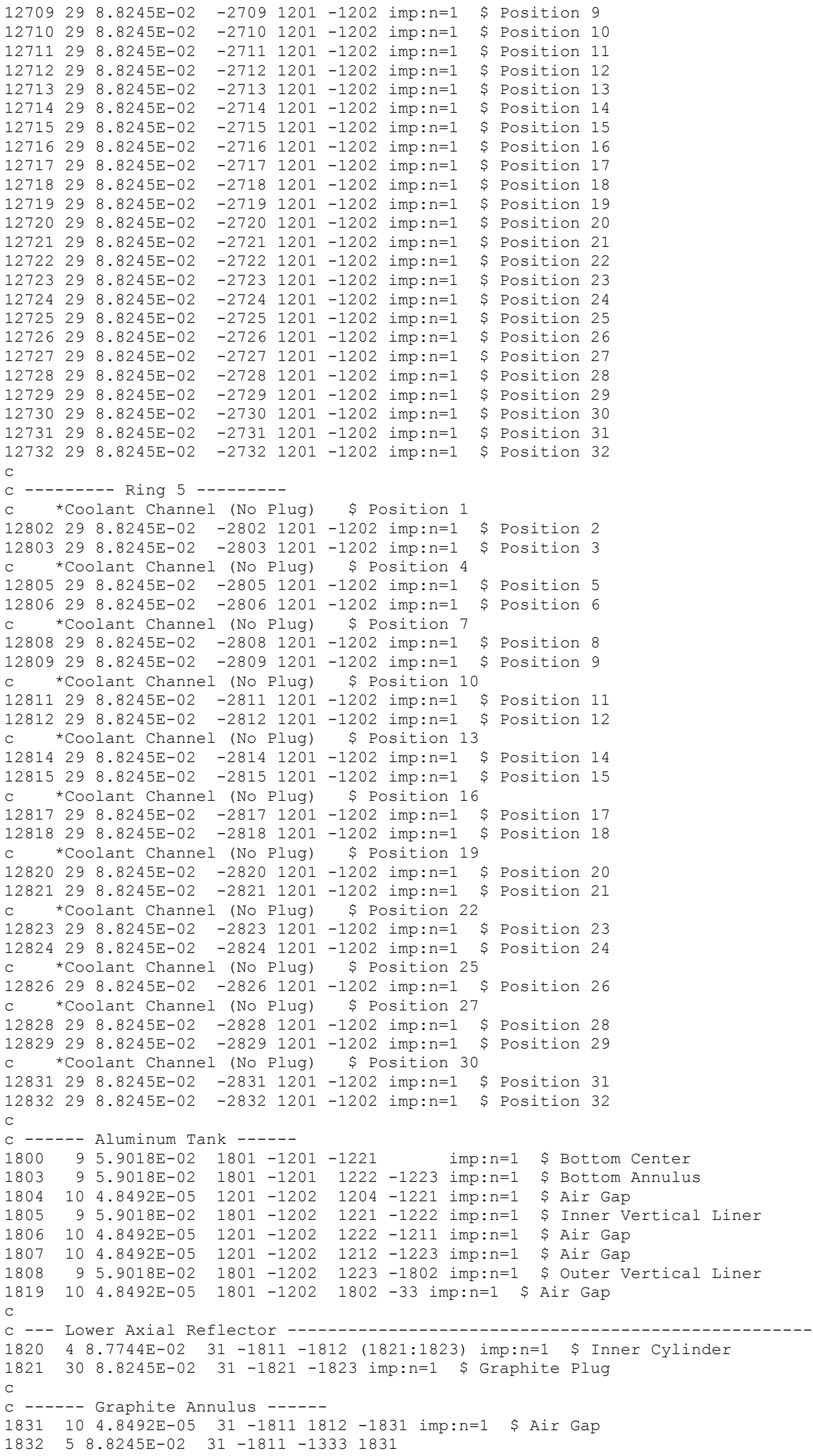

Revision: 1 
NEA/NSC/DOC(2006)1

\section{Gas Cooled (Thermal) Reactor - GCR \\ PROTEUS-GCR-EXP-004 CRIT-REAC}

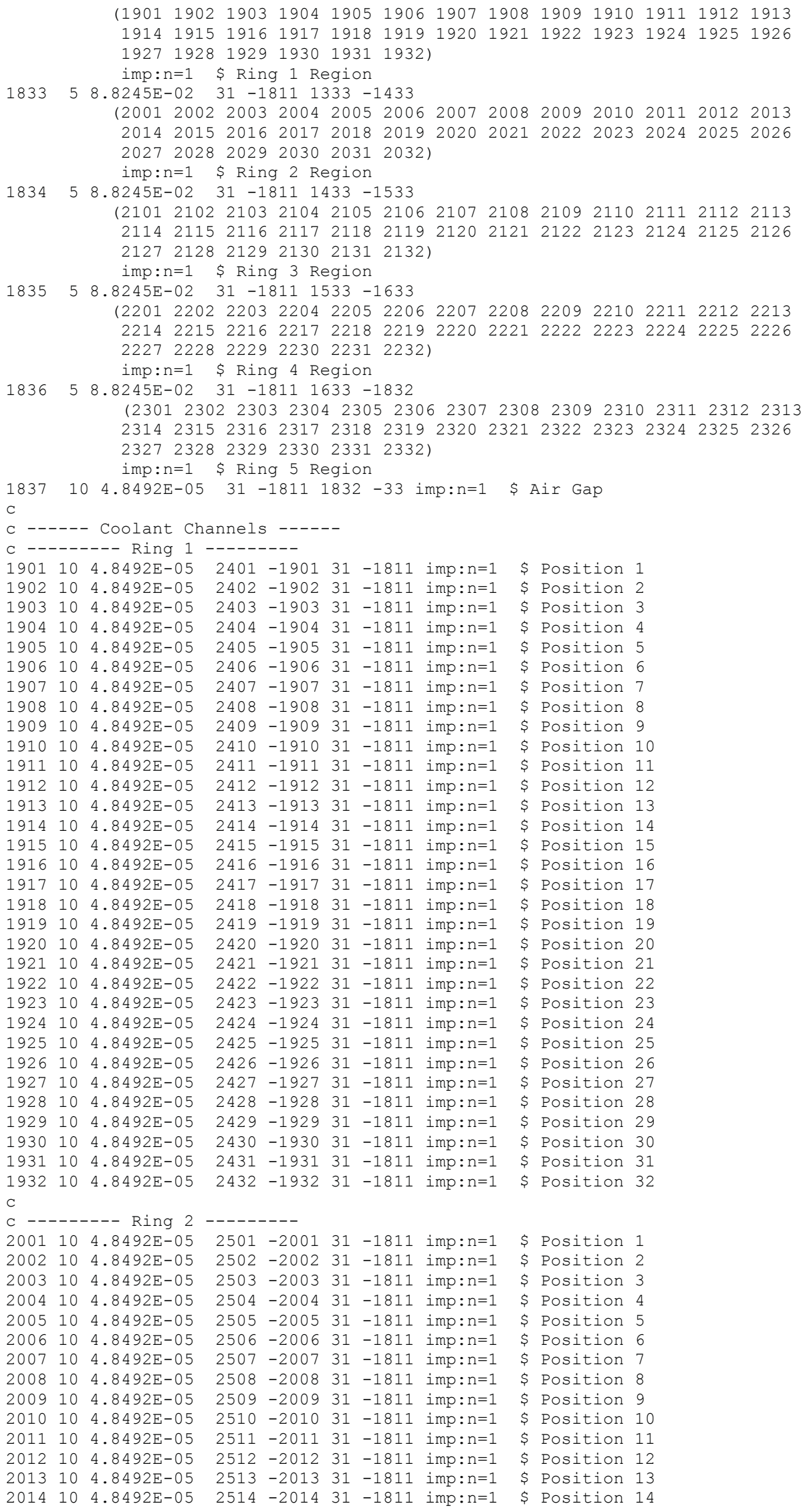

Revision: 1 
NEA/NSC/DOC(2006)1

Gas Cooled (Thermal) Reactor - GCR

PROTEUS-GCR-EXP-004 CRIT-REAC

\begin{tabular}{|c|c|c|c|c|c|c|c|c|c|c|}
\hline 15 & 0 & 5 & 15 & -2015 & 31 & -1811 & $i m p: n=1$ & $\$$ & & \\
\hline & 10 & $492 E-05$ & 16 & -2016 & 31 & -1811 & imp:n=1 & $\$$ & & \\
\hline 1 & 10 & $4.8492 E-05$ & 2517 & -2017 & 31 & -1811 & imp: $n=1$ & $\$$ & osition & \\
\hline$\perp$ & 10 & $4.8492 E-05$ & 518 & -2018 & 31 & -1811 & imp: $n=1$ & $\$$ & osition & \\
\hline & 0 & $4.8492 \mathrm{E}-05$ & 519 & -2019 & 31 & -1811 & $i m p: n=1$ & $\$$ & osition & \\
\hline & 10 & $4.8492 E-05$ & 20 & -2020 & 31 & -1811 & imp:n=1 & $\$$ & osition & \\
\hline 2 & 0 & $4.8492 \mathrm{E}-05$ & 21 & -2021 & 31 & -1811 & $i m p: n=1$ & $\$$ & osition & \\
\hline & 0 & $4.8492 E-05$ & 22 & -2022 & 31 & -1811 & imp: $n=1$ & $\$$ & osition & \\
\hline & 0 & $4.8492 E-05$ & 23 & -2023 & 31 & -1811 & $i m p: n=1$ & $\$$ & & \\
\hline & 10 & $4.8492 \mathrm{E}-05$ & & -2024 & 1 & -1811 & imp: $n=1$ & $\$$ & ositi & \\
\hline & 0 & $4.8492 \mathrm{E}-05$ & 25 & -2025 & 1 & -1811 & imp:n=1 & $\$$ & osition & \\
\hline & 0 & $4.8492 \mathrm{E}-05$ & 26 & -2026 & 31 & -1811 & $i m p: n=1$ & $\$$ & ositio & \\
\hline & 0 & $4.8492 \mathrm{E}-05$ & 27 & -2027 & 1 & -1811 & imp:n=1 & $\$$ & osition & \\
\hline & 0 & $4.8492 \mathrm{E}-05$ & 28 & -2028 & 31 & -1811 & $i m p: n=1$ & $\$$ & osition & \\
\hline & 10 & $4.8492 E-05$ & 29 & -2029 & 31 & -1811 & imp: $n=1$ & $\$$ & osition & \\
\hline & 10 & $4.8492 \mathrm{E}-05$ & 30 & -2030 & 31 & -1811 & imp: $n=1$ & $\$$ & osition & \\
\hline & 10 & $4.8492 E-05$ & & -2031 & 1 & -1811 & $i m p: n=1$ & $\$$ & ition & \\
\hline & 10 & 4.8 & 2 & -2032 & 31 & -18 & imp: $n=1$ & $\$$ & Position & \\
\hline \\
\hline \multicolumn{11}{|c|}{--------- Ring $3--------$} \\
\hline & 10 & $4.8492 \mathrm{E}-05$ & 2601 & -2101 & 31 & -1811 & imp $: n=1$ & $\$$ & osition & 1 \\
\hline & 0 & $92 E-05$ & 02 & -2102 & 31 & -1811 & $i m p: n=1$ & $\$$ & Position & \\
\hline & 0 & $4.8492 \mathrm{E}-05$ & 603 & -2103 & 31 & -1811 & $i m p: n=1$ & $\$$ & Position & \\
\hline & 10 & $4.8492 E-05$ & 04 & -2104 & 31 & -1811 & imp:n=1 & $\$$ & ition & \\
\hline & 10 & $4.8492 E-05$ & 05 & -2105 & 31 & -1811 & imp: $n=1$ & $\$$ & Position & \\
\hline & 10 & $4.8492 E-05$ & 06 & -2106 & 31 & -1811 & imp: $n=1$ & $\$$ & ition & \\
\hline & 10 & $4.8492 \mathrm{E}-05$ & 7 & -2107 & 31 & -1811 & imp: $n=1$ & $\$$ & tion & \\
\hline & 10 & 4.84921 & 08 & -2108 & 31 & -1811 & imp:n=1 & $\$$ & tion & \\
\hline & 10 & $92 E-05$ & 09 & -2109 & 31 & -1811 & imp:n=1 & $\$$ & ition & \\
\hline & 10 & $4.8492 E-05$ & 610 & -2110 & 31 & -1811 & imp: $n=1$ & $\$$ & Eion & \\
\hline & 10 & $4.8492 \mathrm{E}-05$ & 26 & -2111 & 31 & -18 & imp:n=1 & $\$$ & ion & \\
\hline & 10 & $4.8492 \mathrm{E}-05$ & 2612 & -2112 & 31 & -1811 & $i m p: n=1$ & $\$$ & tion & \\
\hline & 10 & $92 E-05$ & 13 & -2113 & 31 & -1811 & imp:n=1 & $\$$ & Eion & \\
\hline & 10 & $4.8492 E-05$ & 2614 & -2114 & 31 & -1811 & $i m p: n=1$ & $\$$ & Pos & a \\
\hline & 10 & $4.8492 \mathrm{E}-05$ & & -2115 & 31 & -1811 & imp:n=1 & $\$$ & Pos & \\
\hline & 10 & $492 E-05$ & 2 & -2116 & 31 & -18 & imp: $n=1$ & $\$$ & Position & \\
\hline & 10 & $4.8492 E-05$ & 17 & -2117 & 31 & -1811 & $i m p: n=1$ & $\$$ & & \\
\hline & 10 & $92 E-05$ & 26 & -21 & 31 & -18 & imp:n=1 & $\$$ & Po & \\
\hline & 10 & $92 E-05$ & 26 & -2119 & 31 & -18 & $i m p: n=1$ & $\$$ & Po: & \\
\hline & 10 & $4.8492 \mathrm{E}-05$ & 20 & -2120 & 31 & -18 & imp:n=1 & $\$$ & Pos & 0 \\
\hline & 10 & $4.8492 E-05$ & 2621 & -2121 & 31 & -1811 & $i m p: n=1$ & $\$$ & Po: & 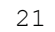 \\
\hline & 10 & $32 E-05$ & 2622 & -2122 & 31 & -1 & imp:n=1 & $\$$ & Pos & 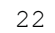 \\
\hline & 10 & 4.8 & 2623 & -2123 & 31 & -18 & $i m p: n=1$ & $\$$ & Pos & 23 \\
\hline & 10 & $4.8492 E-05$ & 2624 & -2124 & 31 & -1811 & imp: $n=1$ & $\$$ & Position & 24 \\
\hline & 10 & $4.8492 \mathrm{E}-05$ & 2625 & -2125 & 31 & -18 & $i m p: n=1$ & $\$$ & tion & $2 J$ \\
\hline & 10 & -05 & 2626 & -2126 & 31 & -1 & imp:n=1 & $\$$ & Pos & 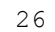 \\
\hline & 10 & $32 E-05$ & 2627 & -2127 & 31 & -18 & imp: $n=1$ & $\$$ & Position & 27 \\
\hline & 10 & $4.8492 E-05$ & 2628 & -2128 & 31 & -1811 & imp: $n=1$ & $\$$ & tion & 8 \\
\hline & 10 & $4.8492 E-05$ & 2629 & -2129 & 31 & $-1 \varepsilon$ & imp: $n=1$ & $\$$ & Po & 29 \\
\hline & 10 & $2 E-05$ & 2630 & -2130 & 31 & -1811 & imp:n=1 & $\$$ & Pos & 30 \\
\hline & 10 & $92 E-05$ & 31 & -2131 & 31 & -18 & imp:n=1 & $\$$ & Pos & 31 \\
\hline 2 & 10 & $4.8492 \mathrm{E}-05$ & 2632 & -2132 & 31 & -18 & imp:n=1 & $\$$ & Eion & 32 \\
\hline \\
\hline & & & & & & & & & & \\
\hline & 10 & 4.8 & & -2201 & 31 & -1811 & imp:n=1 & $\$$ & & 1 \\
\hline & 10 & $4.8492 \mathrm{E}-05$ & 2702 & -2202 & 31 & -1811 & $i m p: n=1$ & $\$$ & Position & \\
\hline & 10 & $4.8492 E-05$ & 2703 & -2203 & 31 & -1811 & imp: $n=1$ & $\$$ & Position & 3 \\
\hline & 10 & $4.8492 E-05$ & 2704 & -2204 & 31 & -1811 & $i m p: n=1$ & $\$$ & Position & 4 \\
\hline & 10 & $4.8492 E-05$ & 2705 & -2205 & 31 & -1811 & imp:n=1 & $\$$ & Pos & \\
\hline & 10 & $4.8492 \mathrm{E}-05$ & 2706 & -2206 & 31 & -1811 & imp:n=1 & $\$$ & Pos & 0 \\
\hline & 10 & $4.8492 \mathrm{E}-05$ & & -2207 & 31 & -1811 & $i m p: n=1$ & $\$$ & Pos & 7 \\
\hline & 10 & $4.8492 \mathrm{E}-05$ & 2708 & -2208 & 31 & -1811 & imp:n=1 & $\$$ & Position & 8 \\
\hline & 10 & $4.8492 \mathrm{E}-05$ & 2709 & -2209 & 31 & -18 & $i m p: n=1$ & $\$$ & ion & 9 \\
\hline & 10 & $4.8492 \mathrm{E}-05$ & 2710 & -2210 & 31 & -1 & imp: $n=1$ & $\$$ & Pos & 10 \\
\hline & 10 & $4.8492 \mathrm{E}-05$ & 2711 & -2211 & 31 & -1811 & $i m p: n=1$ & $\$$ & Position & 11 \\
\hline & 10 & $4.8492 E-05$ & 2712 & -2212 & 31 & -1811 & imp: $n=1$ & $\$$ & Position & 2 \\
\hline & 10 & $4.8492 \mathrm{E}-05$ & 2713 & -2213 & 31 & -1 & imp: $n=1$ & $\$$ & Pos & 13 \\
\hline & 10 & $4.8492 \mathrm{E}-05$ & 2714 & -2214 & 31 & -1811 & $i m p: n=1$ & $\$$ & Position & 14 \\
\hline & 10 & $4.8492 \mathrm{E}-05$ & 2715 & -2215 & 31 & -1811 & imp:n=1 & $\$$ & Position & 15 \\
\hline & 10 & $4.8492 \mathrm{E}-05$ & 2716 & -2216 & 31 & -1811 & imp:n=1 & $\$$ & Positi & 1 \\
\hline & 10 & $4.8492 \mathrm{E}-05$ & 2717 & -2217 & 31 & -1811 & imp:n=1 & $\$$ & Position & 17 \\
\hline & 10 & $4.8492 \mathrm{E}-05$ & 2718 & -2218 & 31 & -1811 & $i m p: n=1$ & $\$$ & Position & 18 \\
\hline & 10 & $4.8492 E-05$ & 2719 & -2219 & 31 & -1811 & imp: $n=1$ & $\$$ & Positio & 19 \\
\hline & 10 & $4.8492 \mathrm{E}-05$ & 2720 & -2220 & 31 & -1811 & $i m p: n=1$ & $\$$ & Positic & 20 \\
\hline & 10 & $4.8492 E-05$ & 2721 & -2221 & 31 & -1811 & $i m p: n=1$ & $\$$ & Positic & 21 \\
\hline & 10 & $4.8492 \mathrm{E}-05$ & 2722 & -2222 & 31 & -1811 & $i m p: n=1$ & $\$$ & Position & 22 \\
\hline
\end{tabular}

Revision: 1 
NEA/NSC/DOC(2006)1

\section{Gas Cooled (Thermal) Reactor - GCR}

\section{PROTEUS-GCR-EXP-004} CRIT-REAC

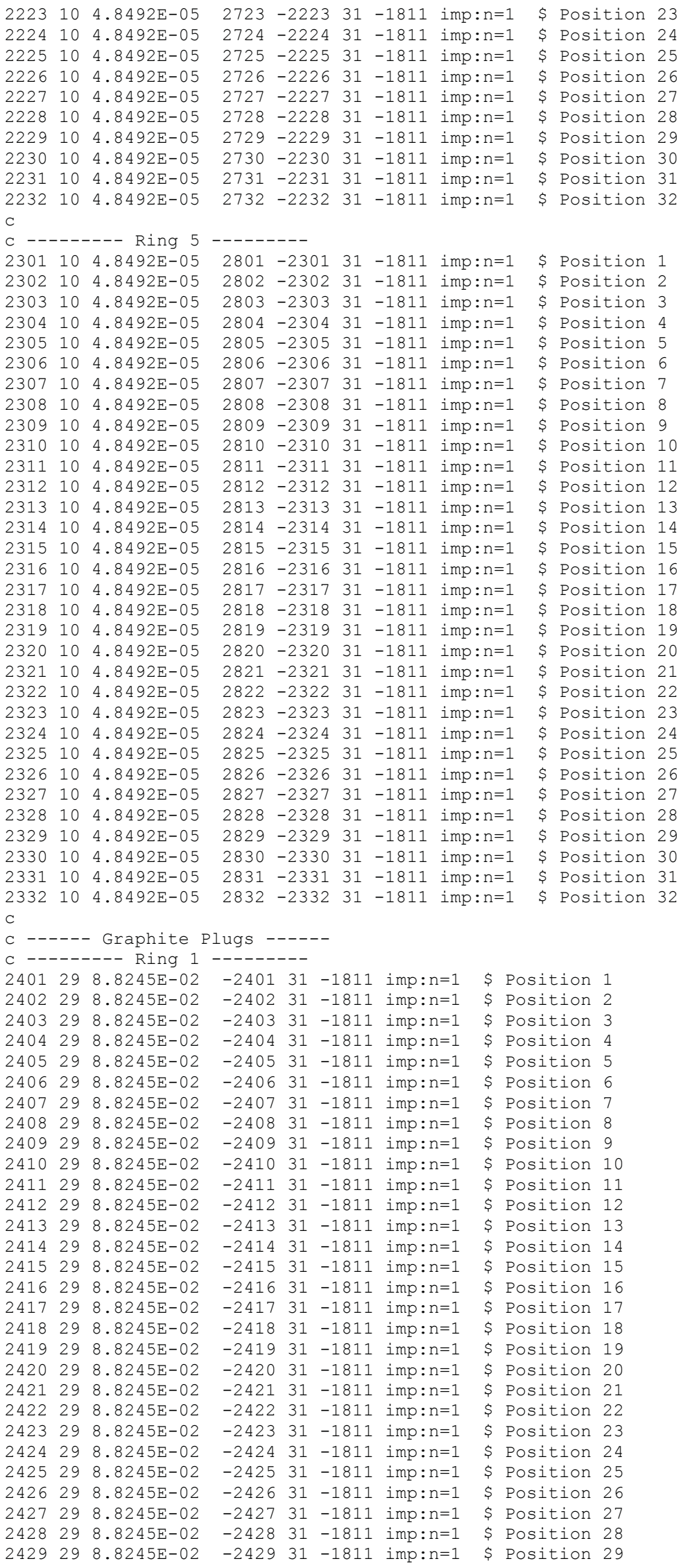

Revision: 1 
NEA/NSC/DOC(2006)1

Gas Cooled (Thermal) Reactor - GCR

PROTEUS-GCR-EXP-004

CRIT-REAC

\begin{tabular}{|c|c|c|c|c|c|c|c|c|c|}
\hline 2430 & 29 & 2 & -2430 & 1 & -1811 & imp: $n=1$ & $\$$ & & \\
\hline & 29 & $8.8245 \mathrm{E}-02$ & -2431 & 31 & -1811 & imp: $n=1$ & $\$$ & ion & \\
\hline 32 & 29 & $8.8245 E-02$ & -2432 & 31 & -1811 & $i m p: n=1$ & $\$$ & Position & 32 \\
\hline & & & & & & & & & \\
\hline & & & & & & & & & \\
\hline & 9 & $8.8245 E-02$ & -2501 & 1 & -1811 & imp: $n=1$ & $\$$ & Position & 1 \\
\hline & 29 & $8.8245 \mathrm{E}-02$ & -2502 & 31 & -1811 & imp: $n=1$ & $\$$ & osition & \\
\hline & 29 & $8.8245 E-02$ & -2503 & 31 & -1811 & imp: $n=1$ & $\$$ & Position & \\
\hline & 29 & $8.8245 E-02$ & -2504 & 31 & -1811 & imp: $n=1$ & $\$$ & osition & \\
\hline & 29 & $8.8245 E-02$ & -2505 & 31 & -1811 & imp: $n=1$ & $\$$ & osition & \\
\hline & 29 & $8.8245 E-02$ & -2506 & 31 & -1811 & imp: $n=1$ & $\$$ & Position & \\
\hline 7 & 29 & $8.8245 E-02$ & -2507 & 31 & -1811 & imp: $n=1$ & $\$$ & Position & \\
\hline & 29 & $8.8245 E-02$ & -2508 & 31 & -1811 & imp: $n=1$ & $\$$ & Position & \\
\hline & 29 & $8.8245 E-02$ & -2509 & 31 & -1811 & imp: $n=1$ & $\$$ & osition & \\
\hline & 29 & $8.8245 E-02$ & -2510 & 31 & -1811 & imp: $n=1$ & $\$$ & Position & \\
\hline & 29 & $8.8245 E-02$ & -2511 & 31 & -1811 & imp: $n=1$ & $\$$ & Position & \\
\hline & 29 & $8.8245 E-02$ & -2512 & 31 & -1811 & imp: $n=1$ & $\$$ & Position & \\
\hline & 29 & $8.8245 \mathrm{E}-02$ & -2513 & 31 & -1811 & imp: $n=1$ & $\$$ & Position & \\
\hline & 29 & $8.8245 E-02$ & -2514 & 31 & -1811 & imp: $n=1$ & $\$$ & ion & \\
\hline & 29 & $8.8245 E-02$ & -2515 & 31 & -1811 & imp: $n=1$ & $\$$ & ion & \\
\hline & 29 & $8.8245 E-02$ & -2516 & 31 & -1811 & imp: $n=1$ & $\$$ & ion & \\
\hline & 29 & $8.8245 E-02$ & -2517 & 31 & -1811 & imp: $n=1$ & $\$$ & Position & 7 \\
\hline & 29 & $8.8245 E-02$ & -2518 & 31 & -1811 & imp: $n=1$ & $\$$ & ion & \\
\hline & 29 & $8.8245 E-02$ & -2519 & & -1811 & imp: $n=1$ & $\$$ & ion & 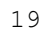 \\
\hline & 29 & $8.8245 \mathrm{E}-02$ & -2520 & 31 & -1811 & $i m p: n=1$ & $\$$ & ion & \\
\hline & 29 & $8.8245 E-02$ & -2521 & 31 & -1811 & imp: $n=1$ & $\$$ & Position & \\
\hline & 29 & $8.8245 E-02$ & -2522 & 31 & -1811 & imp: $n=1$ & $\$$ & Position & \\
\hline & 29 & $8.8245 \mathrm{E}-02$ & -2523 & 31 & -1811 & imp: $n=1$ & $\$$ & Eion & \\
\hline & 29 & $8.8245 E-02$ & -2524 & 31 & -1811 & imp: $n=1$ & $\$$ & ition & \\
\hline & 29 & $8.8245 E-02$ & -2525 & 31 & -1811 & imp: $n=1$ & $\$$ & Position & \\
\hline & 29 & $8.8245 E-02$ & -2526 & 31 & -1811 & imp: $n=1$ & $\$$ & ion & \\
\hline & 29 & $8.8245 E-02$ & -2527 & 31 & -1811 & imp: $n=1$ & $\$$ & tion & 7 \\
\hline & 29 & $8.8245 E-02$ & -2528 & 31 & -1811 & imp: $n=1$ & $\$$ & Position & 8 \\
\hline & 29 & $8.8245 E-02$ & -2529 & 31 & -1811 & imp: $n=1$ & $\$$ & ition & \\
\hline & 29 & $8.8245 E-02$ & -2530 & 31 & -1811 & imp: $n=1$ & $\$$ & Po: & 30 \\
\hline & 29 & $8.8245 E-02$ & -2531 & 31 & -1811 & imp: $n=1$ & $\$$ & tion & 1 \\
\hline & 29 & $8.8245 E-02$ & -2532 & 31 & -1811 & imp: $n=1$ & $\$$ & Po: & 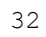 \\
\hline & & & & & & & & & \\
\hline & & $---P$ & -- & & & & & & \\
\hline & 29 & $8.8245 E-02$ & -2601 & 31 & -1811 & imp: $n=1$ & $\$$ & tion & 1 \\
\hline & 29 & $8.8245 E-02$ & -2602 & 31 & -1811 & imp: $n=1$ & $\$$ & Pos & 2 \\
\hline & 29 & $8.8245 E-02$ & -2603 & 31 & -1811 & imp: $n=1$ & $\$$ & Po & 3 \\
\hline & 29 & $8.8245 \mathrm{E}-02$ & -2604 & 31 & -1811 & imp: $n=1$ & $\$$ & tion & 4 \\
\hline & 29 & $8.8245 E-02$ & -2605 & 31 & -1811 & imp: $n=1$ & $\$$ & Position & 5 \\
\hline & 29 & $8.8245 \mathrm{E}-02$ & -2606 & 31 & -1811 & $i m p: n=1$ & $\$$ & tion & 6 \\
\hline & 29 & $8.8245 E-02$ & -2607 & 31 & -1811 & imp: $n=1$ & $\$$ & Pos & 1 \\
\hline & 29 & $8.8245 E-02$ & -2608 & 31 & -1811 & imp: $n=1$ & $\$$ & ition & 8 \\
\hline & 29 & $8.8245 E-02$ & -2609 & 31 & -1811 & imp: $n=1$ & $\$$ & ition & 9 \\
\hline & 29 & $8.8245 E-02$ & -2610 & 31 & -1811 & imp: $n=1$ & $\$$ & $\mathrm{Po}$ & 10 \\
\hline & 29 & $8.8245 \mathrm{E}-02$ & -2611 & 31 & -1811 & imp: $n=1$ & $\$$ & ion & 11 \\
\hline & 29 & $8.8245 E-02$ & -2612 & 31 & -1811 & imp: $n=1$ & $\$$ & Position & 12 \\
\hline & 29 & $8.8245 E-02$ & -2613 & 31 & -1811 & imp: $n=1$ & $\$$ & tion & 13 \\
\hline & 29 & $8.8245 \mathrm{E}-02$ & -2614 & 31 & -1811 & imp: $n=1$ & $\$$ & Po & 14 \\
\hline & 29 & $8.8245 E-02$ & -2615 & 31 & -1811 & imp: $n=1$ & $\$$ & ion & 15 \\
\hline & 29 & $8.8245 \mathrm{E}-02$ & -2616 & 31 & -1811 & imp: $n=1$ & $\$$ & tion & 16 \\
\hline & 29 & $8.8245 E-02$ & -2617 & 31 & -1811 & imp $: n=1$ & $\$$ & tion & 17 \\
\hline & 29 & $8.8245 E-02$ & -2618 & 31 & -1811 & imp: $n=1$ & $\$$ & $\mathrm{Po}$ & 18 \\
\hline & 29 & $8.8245 E-02$ & -2619 & 31 & -1811 & imp: $n=1$ & $\$$ & tion & 19 \\
\hline & 29 & $8.8245 E-02$ & -2620 & 31 & -1811 & imp: $n=1$ & $\$$ & tion & 20 \\
\hline & 29 & $8.8245 E-02$ & -2621 & 31 & -1811 & imp: $n=1$ & $\$$ & Po & 21 \\
\hline & 29 & $8.8245 E-02$ & -2622 & 31 & -1811 & imp: $n=1$ & $\$$ & Po & 22 \\
\hline & 29 & $8.8245 E-02$ & -2623 & 31 & -1811 & imp: $n=1$ & $\$$ & tion & 23 \\
\hline & 29 & $8.8245 E-02$ & -2624 & 31 & -1811 & imp: $n=1$ & $\$$ & Po & 24 \\
\hline & 29 & $8.8245 E-02$ & -2625 & 31 & -1811 & imp: $n=1$ & $\$$ & ion & 25 \\
\hline & 29 & $8.8245 E-02$ & -2626 & 31 & -1811 & imp: $n=1$ & $\$$ & ion & 26 \\
\hline & 29 & $8.8245 E-02$ & -2627 & 31 & -1811 & imp: $n=1$ & $\$$ & tion & 27 \\
\hline & 29 & $8.8245 E-02$ & -2628 & 31 & -1811 & imp: $n=1$ & $\$$ & $\mathrm{Po}$ & 28 \\
\hline & 29 & $8.8245 E-02$ & -2629 & 31 & -1811 & imp: $n=1$ & $\$$ & ion & 29 \\
\hline & 29 & $8.8245 E-02$ & -2630 & 31 & -1811 & imp: $n=1$ & $\$$ & Position & 30 \\
\hline & 29 & $8.8245 E-02$ & -2631 & 31 & -1811 & imp: $n=1$ & $\$$ & Pos & 31 \\
\hline & 29 & $8.8245 E-02$ & -2632 & 31 & -1811 & imp: $n=1$ & $\$$ & Position & 32 \\
\hline & & & & & & & & & \\
\hline & & --- & & & & & & & \\
\hline & 29 & $8.8245 E-02$ & -2 & & -18 & imp: $n=1$ & $\$$ & & \\
\hline & 29 & $8.8245 E-02$ & -2702 & 31 & -1811 & imp: $n=1$ & $\$$ & $\mathrm{Po}$ & 2 \\
\hline & 29 & $8.8245 E-02$ & -2703 & 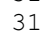 & -1811 & $i m p: n=1$ & $\$$ & ion & 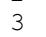 \\
\hline
\end{tabular}

Revision: 1

Date: March 31, 2014

Page 211 of 375 
NEA/NSC/DOC(2006)1

\section{Gas Cooled (Thermal) Reactor - GCR}

\section{PROTEUS-GCR-EXP-004 CRIT-REAC}

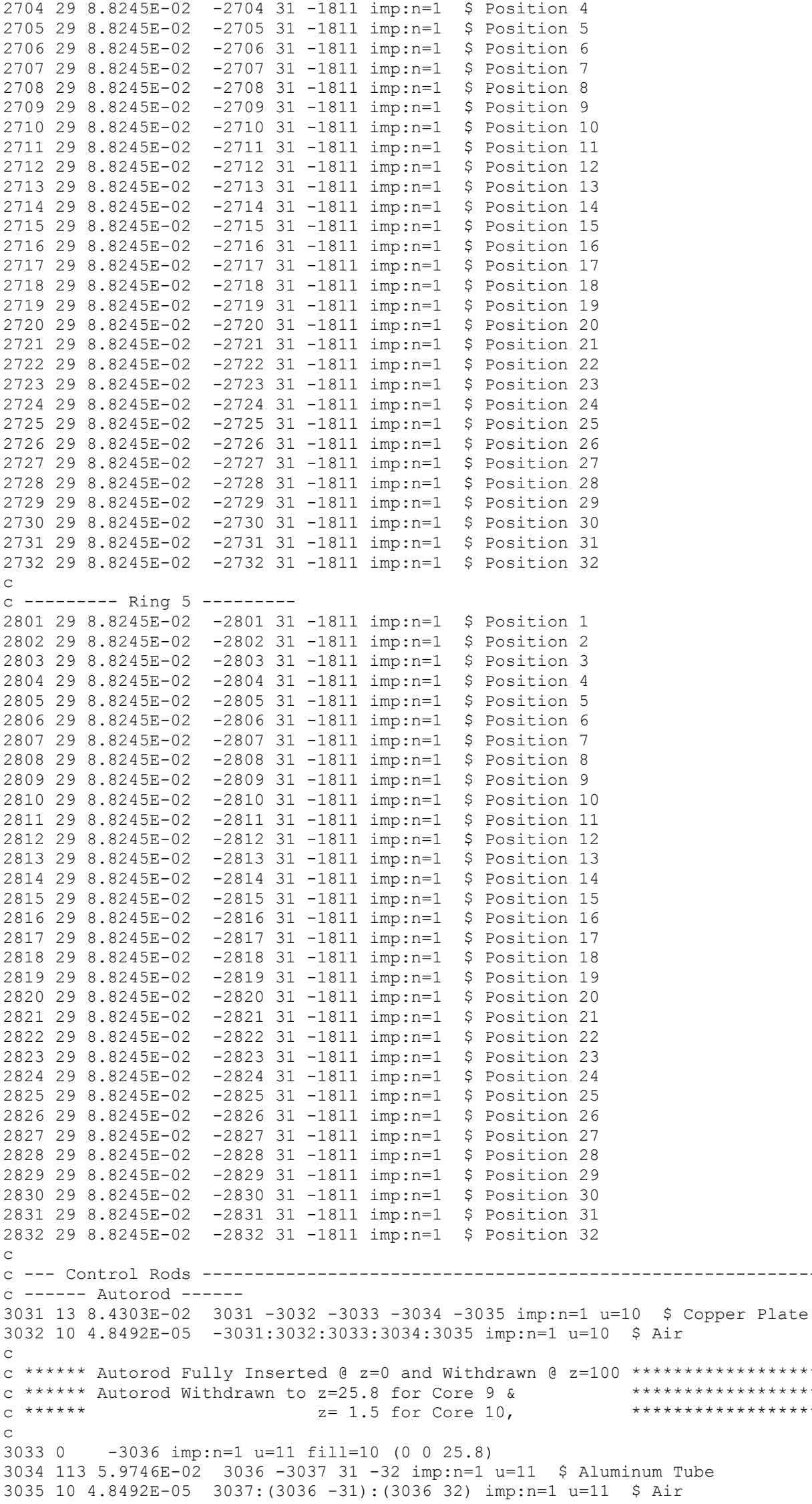

$-270431-1811$ imp: $n=1 \quad \$$ Position 4 $-270531-1811$ imp:n=1 \$ Position 5 -2706 $31-1811$ imp:n=1 \$Position 6 $-270731-1811$ imp:n=1 \$ Position 7 -2708 31 -1811 imp:n=1 \$Position 8 $-270931-1811$ imp:n=1 \$ Position 9 $-271031-1811$ imp: $n=1$ \$ Position 10 Position 12 $-271331-1811$ imp:n=1 \$Position 13 -2714 $31-1811$ imp: $n=1$ \$Position 14 -2715 $31-1811$ imp: $n=1 \quad$ \$ Position 15 -2716 $31-1811$ imp: $n=1$ \$ Position 16 $-271731-1811$ imp:n=1 \$Position 17 $-271831-1811$ imp: $n=1$ \$ Position 18 $-2720 \quad 31-1811$ imp: $n=1 \quad$ \$ Position 20 -2721 $31-1811$ imp:n=1 \$Position 21 -2722 $31-1811$ imp: $n=1$ \$ Position 22 $-272331-1811$ imp: $n=1$ \$ Position 23 -2724 $31-1811$ imp:n=1 \$Position 24 25 $31-1811$ imp: $n=1$ \$ Position 25 $\begin{array}{lllll}-2726 & 31 & -1811 & \text { imp: } n=1 & \text { \$ Position } 26 \\ -2727 & 31 & -1811 & \text { imp: } n=1 & \text { \$ Position } 27\end{array}$ $-272831-1811$ imp:n=1 \$Position 28 -2729 $31-1811$ imp:n=1 \$ Position 29 -2730 $31-1811$ imp: $n=1$ \$ Position 30 -2731 $31-1811$ imp: $n=1$ \$ Position 31 $-273231-1811$ imp:n=1 \$ Position 32

-2801 $31-1811$ imp:n=1 \$Position 1 $-280231-1811$ imp:n=1 \$Position 2 $-280331-1811$ imp: $n=1$ \$Position 3 $-280431-1811$ imp:n=1 \$Position 4 $-280531-1811$ imp:n=1 \$Position 5 $-280631-1811$ imp: $n=1$ \$Position 6 -2808 $31-181$ imp: $n=1 \quad$ \$ Position 7 $-280931-1811$ imp: $n=1$ \$ Position 9 -2810 $31-1811$ imp: $n=1$ \$ Position 10 $-281131-1811$ imp:n=1 \$ Position 11 -2812 $31-1811$ imp:n=1 \$Position 12 $-281331-1811$ imp:n=1 \$Position 13 $-281431-1811$ imp: $n=1 \quad$ \$ Position 14

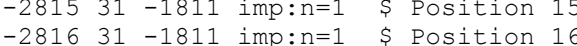
$-281731-1811$ imp: $n=1 \quad$ \$ Position 17 -2818 $31-1811$ imp: $n=1$ \$ Position 18 -2819 $31-1811$ imp:n=1 \$ Position 19 $-282031-1811$ imp:n=1 \$ Position 20 2821 31 -1811 imp:n=1 \$ Position 21 $\begin{array}{llll}-2823 & 31 & -1811 \text { imp: } n=1 & \text { \$ Position } 23\end{array}$ $-282431-1811$ imp: $n=1$ \$ Position 24 $-282531-1811$ imp: $n=1$ \$ Position 25 -2826 $31-1811$ imp:n=1 \$Position 26 $-282731-1811$ imp:n=1 \$ Position 27 $-282831-1811$ imp:n=1 \$ Position 28 $-283031-1811$ imp:n=1 \$Position 29 -2831 $31-1811$ imp: $n=1$ \$ Position 31

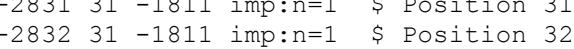

Revision: 1 
NEA/NSC/DOC(2006)1

\section{Gas Cooled (Thermal) Reactor - GCR \\ PROTEUS-GCR-EXP-004 CRIT-REAC}

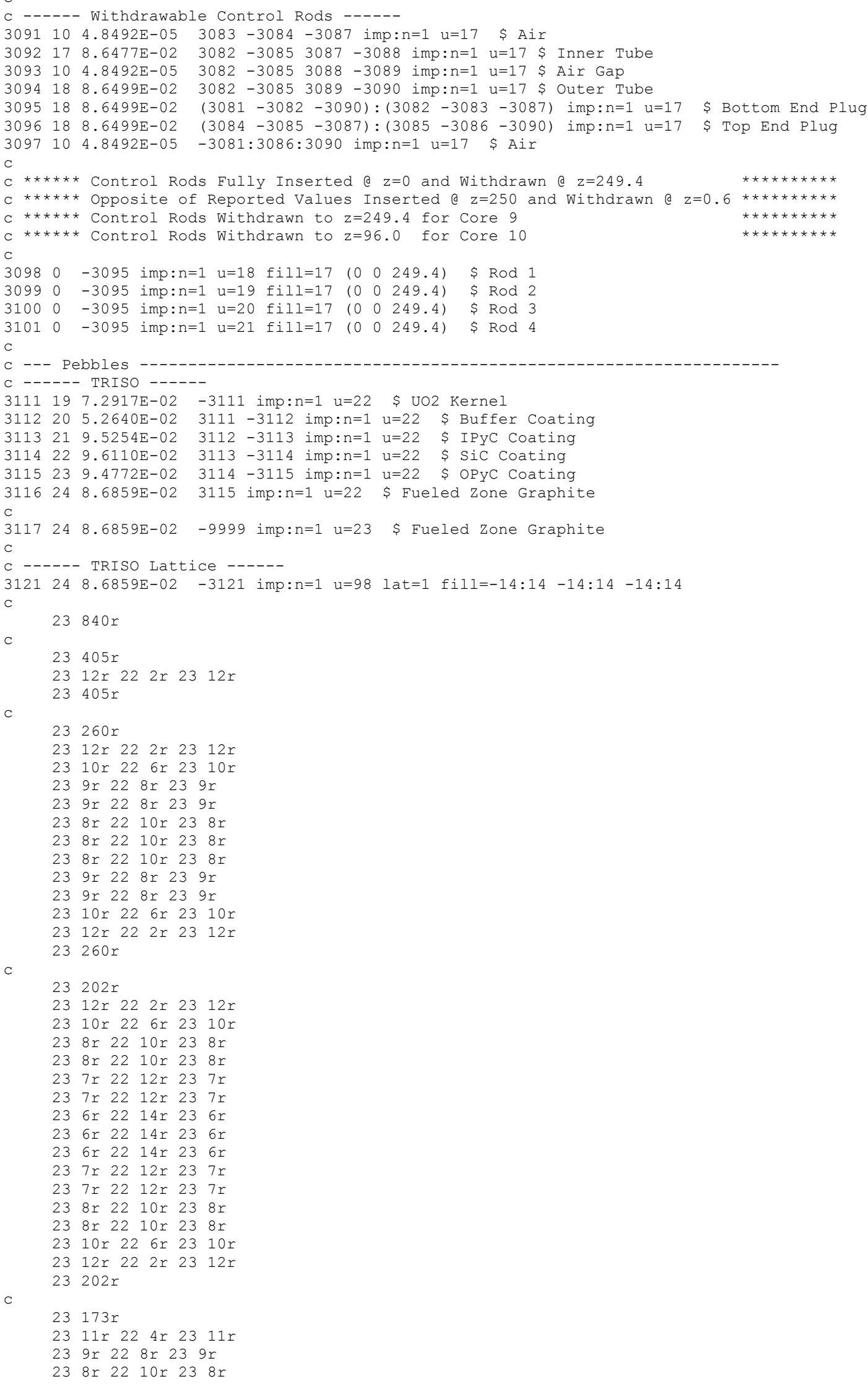

Revision: 1 
NEA/NSC/DOC(2006)1

Gas Cooled (Thermal) Reactor - GCR

\section{PROTEUS-GCR-EXP-004}

CRIT-REAC

37 7r 22 12r $237 r$

$236 r 2214 r 236 r$

$236 r 2214 r 236 r$

$235 r 2216 r 235 r$

$235 r 2216 r 235 r$

$235 r 2216 r 235 r$

$235 r 2216 r 235 r$

$235 r 2216 r 235 r$

$236 r 2214 r 236 r$

$236 r 2214 r 236 r$

$237 r 22$ 12r $237 r$

$238 r 22$ 10r 23 8r

$239 r 22$ 8r 23 9r

23 11r 22 4r 2311 r

$23173 r$

C

$23144 r$

$2310 r 22$ 6r $2310 r$

$238 r 2210 r 238 r$

$237 r 2212 r 237 r$

$236 r 2214 r 236 r$

$235 r 2216 r 235 r$

$235 r 2216 r 235 r$

$234 r 2218 r 234 r$

$234 r 2218 r 234 r$

$234 r 22$ 18r 23 4r

$234 r 22$ 18r $234 r$

$234 r 22$ 18r $234 r$

$234 r 22$ 18r 23 4r

$234 r 22$ 18r $234 r$

$235 r 2216 r 235 r$

$235 r 2216 r 235 r$

$236 r 22$ 14r $236 r$

$237 r 22$ 12r $237 r$

$238 r 2210 r 238 r$

$2310 r 22$ 6r $2310 r$

$23144 r$

C

$23115 r$

2311 r 22 4r $2311 r$

$238 r 2210 r 238 r$

$237 r 2212 r 237 r$

$236 r 2214 r 236 r$

$235 r 2216 r 235 r$

$234 r 22$ 18r $234 r$

$234 r 2218 r 234 r$

$234 r 2218 r 234 r$

$233 r 22$ 20r 23 3r

$233 r 22$ 20r $233 r$

$233 r 22$ 20r 23 3r

$233 r 22$ 20r 23 3r

$233 r 22$ 20r $233 r$

$234 r 22$ 18r 23 4r

$234 r 22$ 18r 23 4r

$234 r 2218 r 234 r$

$235 r 2216 r 235 r$

$236 r 2214 r 236 r$

$237 r 2212 r 237 r$

$238 r 22$ 10r 23 8r

2311 r 22 4r $2311 r$

$23115 r$

C

$\begin{array}{llllll}23 & 86 r & & & \\ 23 & 12 r & 22 & 2 r & 23 & 12 r \\ 23 & 9 r & 22 & 8 r & 23 & 9 r \\ 23 & 7 r & 22 & 12 r & 23 & 7 r \\ 23 & 6 r & 22 & 14 r & 23 & 6 r \\ 23 & 5 r & 22 & 16 r & 23 & 5 r \\ 23 & 4 r & 22 & 18 r & 23 & 4 r \\ 23 & 4 r & 22 & 18 r & 23 & 4 r \\ 23 & 3 r & 22 & 20 r & 23 & 3 r \\ 23 & 3 r & 22 & 20 r & 23 & 3 r \\ 23 & 3 r & 22 & 20 r & 23 & 3 r \\ 23 & 2 r & 22 & 22 r & 23 & 2 r \\ 23 & 2 r & 22 & 22 r & 23 & 2 r \\ 23 & 2 r & 22 & 22 r & 23 & 2 r\end{array}$

Revision: 1

Date: March 31, 2014

Page 214 of 375 
NEA/NSC/DOC(2006)1

Gas Cooled (Thermal) Reactor - GCR

\section{PROTEUS-GCR-EXP-004}

CRIT-REAC

$233 r 22$ 20r $233 r$

$233 r 2220 r 233 r$

$233 r 22$ 20r $233 r$

$234 r 22$ 18r 23 4r

$234 r 22$ 18r 23 4r

$235 r 2216 r 235 r$

$236 r 2214 r 236 r$

$237 r 22$ 12r $237 r$

23 9r 22 8r 23 9r

2312 r $22 \quad 2$ r $23 \quad 12$ r

$2386 r$

$2386 r$

$2310 r 22$ 6r $2310 r$

$238 r 2210 r 238 r$

$236 r 2214 r 236 r$

$235 r 2216 r 23 \quad 5 r$

$234 r 22$ 18r 23 4r

$234 r 22$ 18r $23 \quad 4 r$

$233 r 22$ 20r 23 3r

$233 r 22$ 20r 23 3r

$\begin{array}{lllllllllll}23 & 2 r & 22 & 22 r & 23 & 2 r\end{array}$

$232 r 22$ 22r 23 2r

$232 r 22$ 22r $232 r$

$232 r 22 \quad 22 r 232 r$

$232 r 22 \quad 22 r 232 r$

$232 r 22 \quad 22 r 23 \quad 2 r$

$232 r 22 \quad 22 r 232 r$

$233 r 22 \quad 20 r 233 r$

$233 r 22$ 20r $233 r$

$234 r 2218 r 23$ 4r

$234 r 22 \quad 18 r 23 \quad 4 r$

$235 r 2216 r 235 r$

23 6r 22 14r 23 6r

$238 r 22$ 10r 23 8r

$2310 r 22$ 6r $2310 r$

$2386 r$

c

$\begin{array}{llllll}23 & 57 r & & & & \\ 23 & 12 r & 22 & 2 r & 23 & 12 r \\ 23 & 8 r & 22 & 10 r & 23 & 8 r\end{array}$

$237 r 22$ 12r 23 7r

$235 r 2216 r 235 r$

$234 r 22$ 18r $23 \quad 4 r$

$234 r 2218 r 23 \quad 4 r$

$233 r 22$ 20r $233 r$

$232 r 22 \quad 22 r 232 r$

$232 r 22 \quad 22 r 23 \quad r$

$232 r 22 \quad 22 r 23 \quad 2 r$

$232 r 22 \quad 22 r 23 \quad r$

23 1r 22 24r $231 r$

$\begin{array}{llllll}23 & 1 r & 22 & 24 r & 23 & 1 r\end{array}$

$231 r 22 \quad 24 r 231 r$

$232 r 22 \quad 22 r 232 r$

$232 r 22 \quad 22 r 232 r$

$232 r 22 \quad 22 r 23 \quad r$

$232 r 22$ 22r $232 r$

$233 r 22$ 20r $233 r$

23 4r 22 18r 23 4r

$234 r 22 \quad 18 r 23 \quad 4 r$

$235 r 2216 r 23 \quad 5 r$

$237 r 2212 r 237 r$

23 8r 22 10r $238 r$

2312 r 22 2r $2312 r$

C

$2357 r$

$$
\begin{array}{lllllll}
23 & 57 r & & & \\
23 & 10 r & 22 & 6 r & 23 & 10 r \\
23 & 8 r & 22 & 10 r & 23 & 8 r \\
23 & 6 r & 22 & 14 r & 23 & 6 r \\
23 & 5 r & 22 & 16 r & 23 & 5 r \\
23 & 4 r & 22 & 18 r & 23 & 4 r \\
23 & 3 r & 22 & 20 r & 23 & 3 r \\
23 & 3 r & 22 & 20 r & 23 & 3 r \\
23 & 2 r & 22 & 22 r & 23 & 2 r \\
23 & 2 r & 22 & 22 r & 23 & 2 r
\end{array}
$$

Revision: 1

Date: March 31, 2014

Page 215 of 375 
NEA/NSC/DOC(2006)1

Gas Cooled (Thermal) Reactor - GCR

\section{PROTEUS-GCR-EXP-004}

CRIT-REAC

$231 r 2224 r 231 r$

$231 r 22 \quad 24 r 231 r$

$231 r 22 \quad 24 r 231 r$

23 1r 22 24r 23 1r

23 1r 22 24r 23 1r

$231 r 22 \quad 24 r 231 r$

$231 r 22 \quad 24 r 231 r$

$232 r 22 \quad 22 r 232 r$

$232 r 22$ 22r 23 2r

$233 r 22$ 20r 23 3r

$233 r 22$ 20r $233 r$

$234 r 22 \quad 18 r 23 \quad 4 r$

$235 r 2216 r 235 r$

$236 r 22 \quad 14 r 23 \quad 6 r$

$238 r 22$ 10r 23 8r

$2310 r 22$ 6r 23 10r

C

$2357 r$

$2357 r$

$239 r 228 r 239 r$

$237 r 2212 r 237 r$

$236 r 2214 r 236 r$

$234 r 22$ 18r 23 4r

$234 r 22$ 18r 23 4r

$233 r 22$ 20r $233 r$

$232 r 22 \quad 22 r 23 \quad r$

$232 r 22 \quad 22 r 23 \quad 2 r$

$231 r 22 \quad 24 r 231 r$

$231 r 22 \quad 24 r 231 r$

$231 r 22$ 24r $231 r$

$231 r 22 \quad 24 r 231 r$

$231 r 22 \quad 24 r 231 r$

$231 r 22 \quad 24 r 231 r$

$231 r 22 \quad 24 r 23$ 1r

23 1r 22 24r 231 r

23 1r 22 24r $231 r$

$232 r 22$ 22r 23 2r

$232 r 22$ 22r $232 r$

$233 r 22$ 20r $233 r$

$234 r 22$ 18r 23 4r

$234 r 2218 r 23 \quad 4 r$

$236 r 22 \quad 14 r 23 \quad 6 r$

$237 r 22$ 12r 23 7r

23 9r 22 8r 23 9r

C

$2357 r$

$2357 r$

23 9r 22 8r 23 9r

$237 r 2212 r 237 r$

$235 r 2216 r 235 r$

$234 r 2218 r 23$ 4r

$233 r 22$ 20r $23 \quad 3 r$

$233 r 22$ 20r 23 3r

$232 r 22 \quad 22 r 232 r$

$232 r 22 \quad 22 r 232 r$

$231 r 22 \quad 24 r 231 r$

$231 r 22$ 24r $231 r$

23 1r 22 24r 231 r

$231 r 22 \quad 24 r 23$ 1r

$231 r 22 \quad 24 r 231 r$

23 1r 22 24r 23 1r

$231 r 22 \quad 24 r 23$ 1r

$231 r 22$ 24r $231 r$

$231 r 22 \quad 24 r 23$ 1r

$232 r 22 \quad 22 r 232 r$

$232 r 22 \quad 22 r 23 \quad 2 r$

$233 r 22$ 20r 23 3r

$233 r 22$ 20r $233 r$

$234 r 2218 r 23 \quad 4 r$

$235 r 2216 r 23 \quad 5 r$

$237 r 22$ 12r $237 r$

23 9r 22 8r 23 9r

$2357 r$

C

$2357 r$

238 r 22 10r 238 r

Revision: 1

Date: March 31, 2014

Page 216 of 375 
NEA/NSC/DOC(2006)1

Gas Cooled (Thermal) Reactor - GCR

PROTEUS-GCR-EXP-004 CRIT-REAC

$236 r 2214 r 236 r$

$235 r 2216 r 235 r$

$234 r 2218 r 234 r$

$233 r 22$ 20r 23 3r

$232 r 22$ 22r $232 r$

$232 r 22$ 22r $232 r$

23 1r 22 24r 23 1r

23 1r 22 24r 23 1r

$231 r 2224 r 231 r$

23 1r 22 24r 23 1r

$231 r 22 \quad 24 r 231 r$

$232226 r 23$

23 1r 22 24r 23 1r

$231 r 22$ 24r $231 r$

$231 r 2224 r 231 r$

23 1r 22 24r $231 r$

$231 r 22$ 24r $231 r$

$232 r 22$ 22r 23 2r

$232 r 22$ 22r $232 r$

$233 r 22$ 20r $233 r$

$234 r 22$ 18r 23 4r

$235 r 2216 r 235 r$

$236 r 22$ 14r $236 r$

$238 r 22$ 10r $238 r$

C

$2357 r$

$2328 r$

$\begin{array}{lllllll}23 & 12 r & 22 & 23 & 22 & 23 & 12 r\end{array}$

$238 r 22$ 10r $238 r$

$236 r 2214 r 236 r$

$235 r 2216 r 235 r$

$234 r 22$ 18r 23 4r

$233 r 22$ 20r $233 r$

$232 r 22$ 22r $232 r$

$232 r 22$ 22r $232 r$

$231 r 22$ 24r $231 r$

23 1r 22 24r 23 1r

23 1r 22 24r 23 1r

23 Ir 22 24r $231 r$

$231 r 22$ 24r $231 r$

$231 r 2211$ r 2322 11r 23 1r

23 1r 22 24r 23 1r

$231 r 22$ 24r $231 r$

23 1r 22 24r 23 1r

$231 r 2224 r 231 r$

23 1r 22 24r 23 1r

$232 r 22 \quad 22 r 23 \quad 2 r$

$232 r 22$ 22r $232 r$

$233 r 22$ 20r $233 r$

$234 r 22$ 18r 23 4r

$235 r 2216 r 235 r$

$236 r 2214 r 236 r$

$238 r 2210 r 238 r$

$\begin{array}{lllllll}23 & 12 r & 22 & 23 & 22 & 23 & 12 r\end{array}$

C

$2328 r$

$2357 r$

$238 r 2210 r 238 r$

$236 r 22$ 14r 23 6r

$235 r 2216 r 235 r$

$234 r 2218 r 234 r$

$233 r 22$ 20r $233 r$

$232 r 22 \quad 22 r 232 r$

$232 r 22$ 22r 23 2r

$231 r 2224 r 231 r$

$231 r 22 \quad 24 r 231 r$

23 1r 22 24r $231 r$

$231 r 2224 r 231 r$

$231 r 2224 r 231 r$

$232226 r 23$

23 1r 22 24r 23 1r

23 Ir 22 24r $231 r$

23 1r 22 24r 23 1r

$231 r 22$ 24r $231 r$

$231 r 22$ 24r $231 r$

$232 r 22$ 22r $232 r$

Revision: 1

Date: March 31, 2014

Page 217 of 375 
NEA/NSC/DOC(2006)1

Gas Cooled (Thermal) Reactor - GCR

PROTEUS-GCR-EXP-004

CRIT-REAC

C

$232 r 22$ 22r $232 r$

$233 r 22$ 20r $233 r$

$234 r 2218 r 234 r$

$235 r 2216 r 235 r$

$236 r 2214 r 236 r$

$238 r 2210 r 238 r$

$2357 r$

$2357 r$

$239 r 22$ 8r 23 9r

$237 r 2212 r 237 r$

$235 r 2216 r 235 r$

$234 r 22$ 18r $234 r$

$233 r 22$ 20r $233 r$

$233 r 22$ 20r 23 3r

$232 r 22$ 22r $232 r$

$232 r 22$ 22r $232 r$

$231 r 22$ 24r $231 r$

23 1r 22 24r 23 1r

$231 r 2224 r 231 r$

$231 r 22$ 24r $231 r$

23 1r 22 24r 23 1r

23 1r 22 24r $231 r$

23 1r 22 24r $231 r$

$231 r 22$ 24r $231 r$

$231 r 22$ 24r $231 r$

$232 r 22$ 22r $232 r$

$232 r 22$ 22r $232 r$

$233 r 22$ 20r $233 r$

$233 r 22$ 20r $233 r$

$234 r 22$ 18r $234 r$

$235 r 2216 r 235 r$

$237 r 22$ 12r $237 r$

$239 r 22$ 8r 23 9r

$2357 r$

C

$2357 r$

$239 r 22$ 8r $239 r$

$237 r 2212 r 237 r$

$236 r 2214 r 236 r$

$234 r 2218 r 234 r$

$234 r 2218 r 234 r$

$233 r 22$ 20r $233 r$

$232 r 22 \quad 22 r 232 r$

$232 r 22$ 22r $232 r$

23 1r 22 24r 23 1r

$231 r 22$ 24r $231 r$

$231 r 22$ 24r $231 r$

$231 r 22$ 24r $231 r$

23 Ir 22 24r $231 r$

23 1r 22 24r 23 1r

$231 r 22$ 24r 23 1r

$231 r 22$ 24r 23 1r

$231 r 22$ 24r $231 r$

23 2r 22 22r 23 2r

$232 r 22$ 22r $232 r$

$233 r 22$ 20r $233 r$

$234 r 22$ 18r 23 4r

$234 r 22$ 18r 23 4r

$236 r 2214 r 236 r$

$237 r 2212 r 237 r$

23 9r 22 8r 23 9r

C

$2357 r$

$2357 r$

$2310 r 226 r 2310 r$

$238 r 22$ 10r $238 r$

$236 r 2214 r 236 r$

$235 r 2216 r 235 r$

$234 r 22$ 18r 23 4r

$233 r 22$ 20r 23 3r

$233 r 22$ 20r 23 3r

$232 r 22$ 22r 23 2r

$232 r 22$ 22r $232 r$

$231 r 22$ 24r $231 r$

23 1r 22 24r $231 r$

Revision: 1

Date: March 31, 2014

Page 218 of 375 
NEA/NSC/DOC(2006)1

Gas Cooled (Thermal) Reactor - GCR

PROTEUS-GCR-EXP-004

CRIT-REAC

$231 r 2224 r 231 r$

$231 r 22$ 24r $231 r$

$231 r 22 \quad 24 r 231 r$

$231 r 22$ 24r $231 r$

23 1r 22 24r 23 1r

$232 r 22$ 22r $232 r$

$232 r 22$ 22r $232 r$

$233 r 22$ 20r 23 3r

$233 r 22$ 20r $233 r$

$234 r 22$ 18r 23 4r

$235 r 2216 r 235 r$

$236 r 2214 r 236 r$

$238 r 22$ 10r $238 r$

$2310 r 22$ 6r $2310 r$

C

$2357 r$

$2357 r$

$2312 r 22$ 2r $2312 r$

$238 r 2210 r 238 r$

$237 r 2212 r 237 r$

$235 r 2216 r 235 r$

$234 r 2218 r 234 r$

$234 r 22$ 18r 23 4r

$233 r 22$ 20r $233 r$

$232 r 22$ 22r $232 r$

$232 r 22$ 22r $232 r$

$232 r 22$ 22r $232 r$

$232 r 2222 r 232 r$

23 1r 22 24r 23 1r

$231 r 2224 r 231 r$

$231 r 2224 r 231 r$

$232 r 22$ 22r $232 r$

$232 r 22 \quad 22 r 23 \quad r$

$232 r 22$ 22r $232 r$

$232 r 22$ 22r $232 r$

$233 r 22$ 20r $233 r$

$234 r 22$ 18r 23 4r

$234 r 2218 r 234 r$

$235 r 2216 r 235 r$

$237 r 22$ 12r $237 r$

$238 r 22$ 10r $238 r$

$2312 r 22$ 2r $2312 r$

C

$2357 r$

$2386 r$

$2310 r 22$ 6r $2310 r$

$238 r 22$ 10r 23 8r

$236 r 22$ 14r $236 r$

$235 r 2216 r 235 r$

$234 r 22$ 18r 23 4r

$234 r 22$ 18r 23 4r

$233 r 22$ 20r $233 r$

$233 r 22$ 20r 23 3r

$232 r 22$ 22r $232 r$

$232 r 22 \quad 22 r 23 \quad 2 r$

$232 r 22$ 22r $232 r$

$232 r 22$ 22r $232 r$

$232 r 22$ 22r $232 r$

$232 r 22$ 22r $232 r$

$232 r 22$ 22r $232 r$

$233 r 22$ 20r 23 3r

$233 r 22$ 20r 23 3r

$234 r 22$ 18r 23 4r

$234 r 22$ 18r 23 4r

$235 r 2216 r 235 r$

$236 r 22$ 14r $236 r$

$238 r 22$ 10r $238 r$

$2310 r 22$ 6r $2310 r$

$2386 r$

C
$2386 r$
$2312 r 22 \quad 2 r 2312 r$
23 9r 22 8r 23 9r
$237 r 2212 r 237 r$
$236 r 22$ 14r $236 r$
$235 r 2216 r 235 r$

Revision: 1

Date: March 31, 2014

Page 219 of 375 
NEA/NSC/DOC(2006)1

Gas Cooled (Thermal) Reactor - GCR

\section{PROTEUS-GCR-EXP-004}

CRIT-REAC

$234 r 2218 r 23 \quad 4 r$

$234 r 22$ 18r $234 r$

$233 r 2220 r 233 r$

$233 r 22$ 20r 23 3r

$233 r 2220 r 233 r$

$232 r 22$ 22r $232 r$

$232 r 22$ 22r $232 r$

$232 r 22$ 22r $232 r$

$233 r 22$ 20r $233 r$

$233 r 22$ 20r 23 3r

$233 r 22$ 20r $233 r$

$234 r 22$ 18r 23 4r

$234 r 2218 r 234 r$

$235 r 2216 r 235 r$

$236 r 2214 r 236 r$

$237 r 22$ 12r $237 r$

23 9r 22 8r 23 9r

$2312 r 22 \quad 2 r 2312 r$

$2386 r$

C

$23115 r$

$2311 r 224 r 2311 r$

$238 r 22$ 10r $238 r$

$237 r 22$ 12r $237 r$

$236 r 2214 r 236 r$

$235 r 2216 r 235 r$

$234 r 22$ 18r $234 r$

$234 r 22$ 18r 23 4r

$234 r 22$ 18r 23 4r

$233 r 22$ 20r $233 r$

$233 r 22$ 20r 23 3r

$233 r 22$ 20r $233 r$

$233 r 22$ 20r $233 r$

$233 r 22$ 20r 23 3r

$234 r 2218 r 234 r$

$234 r 22$ 18r $234 r$

$234 r 22$ 18r 23 4r

$235 r 2216 r 235 r$

$236 r 2214 r 236 r$

$237 r 22$ 12r $237 r$

$238 r 2210 r 238 r$

23 11r 22 4r $2311 r$

$23115 r$

C

$23144 r$

$2310 r 22$ 6r $2310 r$

$238 r 2210 r 238 r$

$237 r 2212 r 237 r$

$236 r 2214 r 236 r$

$235 r 2216 r 235 r$

$235 r 22$ 16r $235 r$

$234 r 2218 r 234 r$

$234 r 22$ 18r 23 4r

$234 r 2218 r 234 r$

$234 r 2218 r 234 r$

234 r 22 18r 23 4r

$234 r 22$ 18r $234 r$

$234 r 2218 r 234 r$

$235 r 22$ 16r 23 5r

$235 r 2216 r 235 r$

$236 r 2214 r 236 r$

$237 r 22$ 12r $237 r$

$238 r 2210 r 238 r$

$2310 r 22$ 6r $2310 r$

$23144 r$

C

$$
\begin{array}{llllll}
23 & 173 r & & & \\
23 & 11 r & 22 & 4 r & 23 & 11 r \\
23 & 9 r & 22 & 8 r & 23 & 9 r \\
23 & 8 r & 22 & 10 r & 23 & 8 r \\
23 & 7 r & 22 & 12 r & 23 & 7 r \\
23 & 6 r & 22 & 14 r & 23 & 6 r \\
23 & 6 r & 22 & 14 r & 23 & 6 r \\
23 & 5 r & 22 & 16 r & 23 & 5 r \\
23 & 5 r & 22 & 16 r & 23 & 5 r \\
23 & 5 r & 22 & 16 r & 23 & 5 r
\end{array}
$$

Revision: 1

Date: March 31, 2014

Page 220 of 375 
NEA/NSC/DOC(2006)1

Gas Cooled (Thermal) Reactor - GCR

PROTEUS-GCR-EXP-004

CRIT-REAC

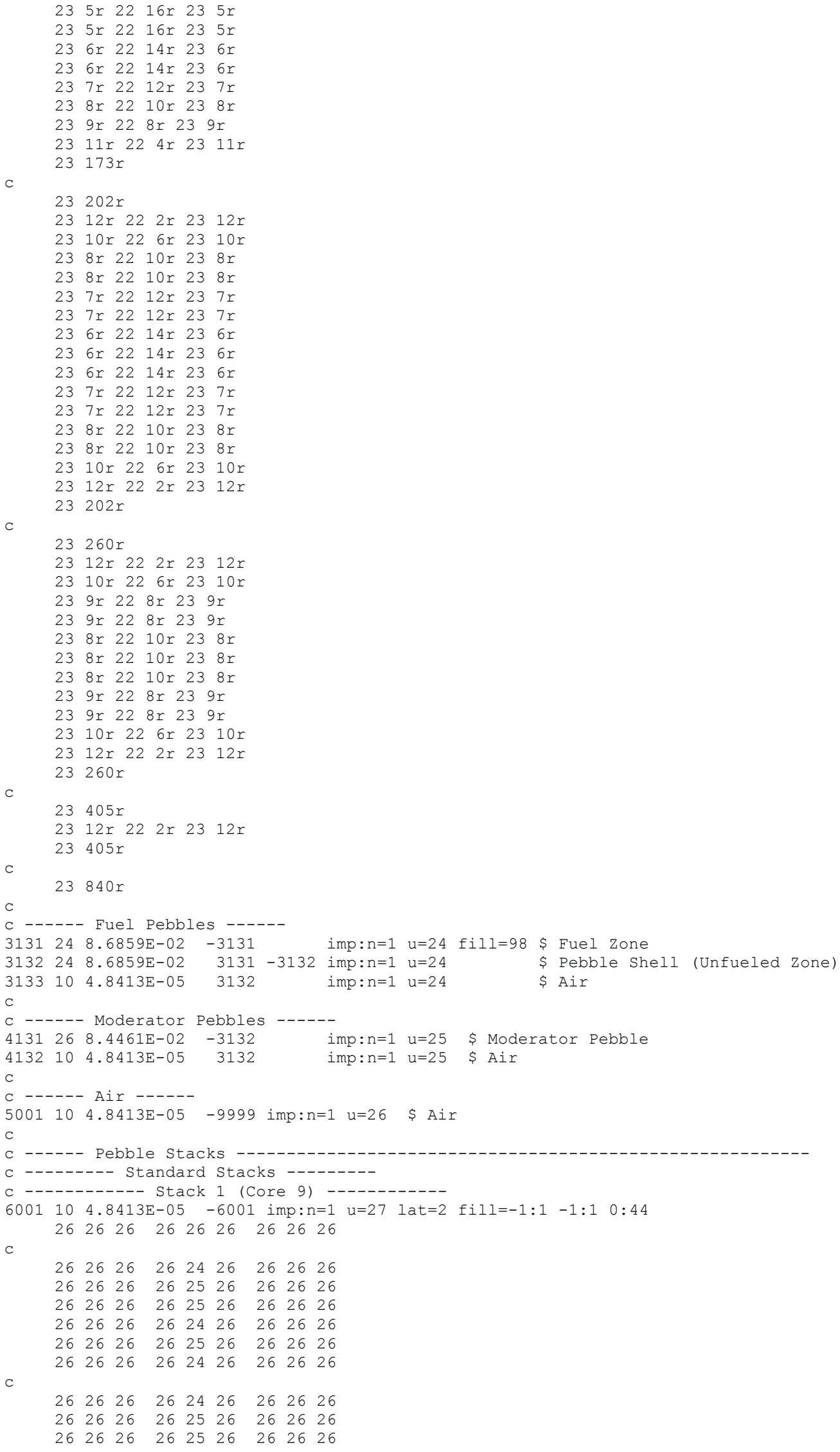

Revision: 1

Date: March 31, 2014

Page 221 of 375 
NEA/NSC/DOC(2006)1

Gas Cooled (Thermal) Reactor - GCR

PROTEUS-GCR-EXP-004

CRIT-REAC

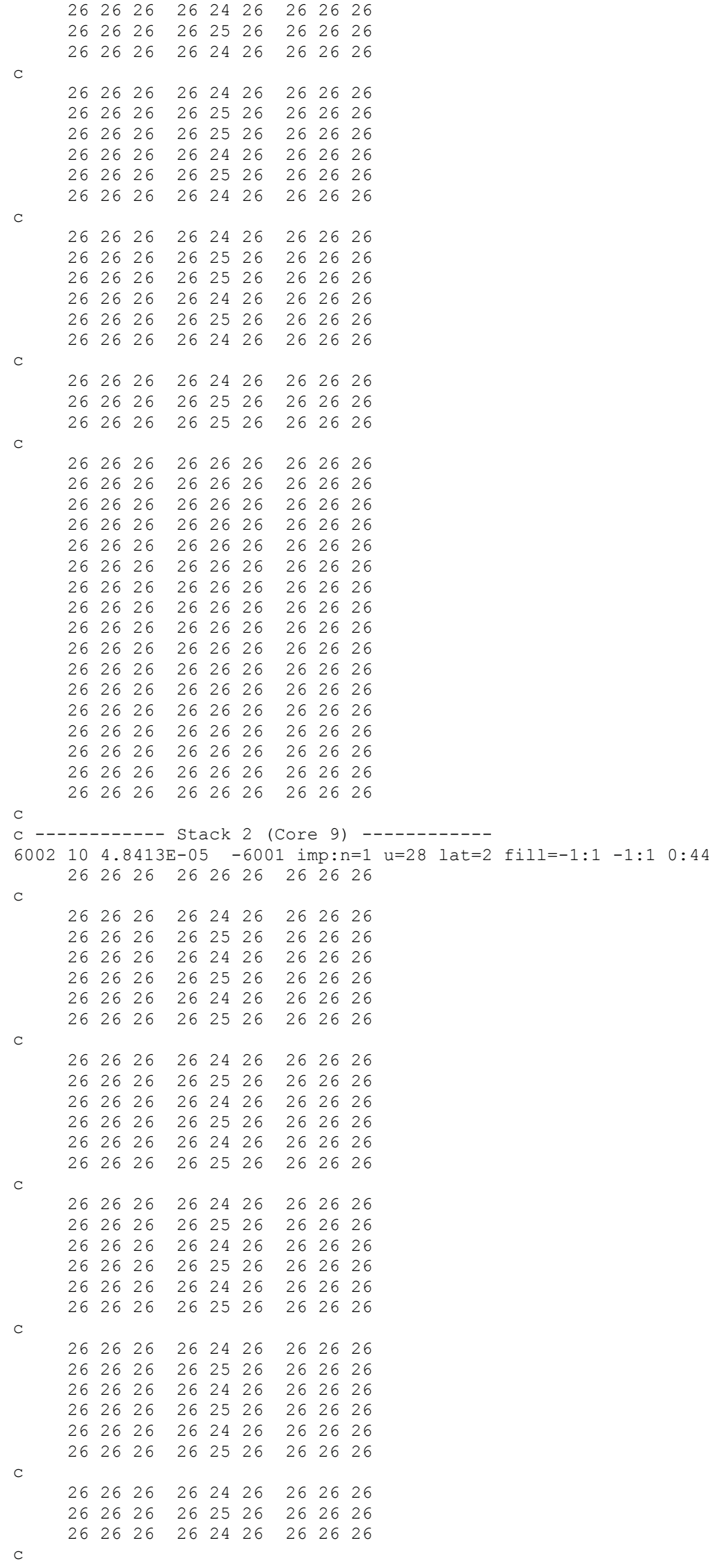

Revision: 1

Date: March 31, 2014

Page 222 of 375 
NEA/NSC/DOC(2006)1

Gas Cooled (Thermal) Reactor - GCR

PROTEUS-GCR-EXP-004

CRIT-REAC

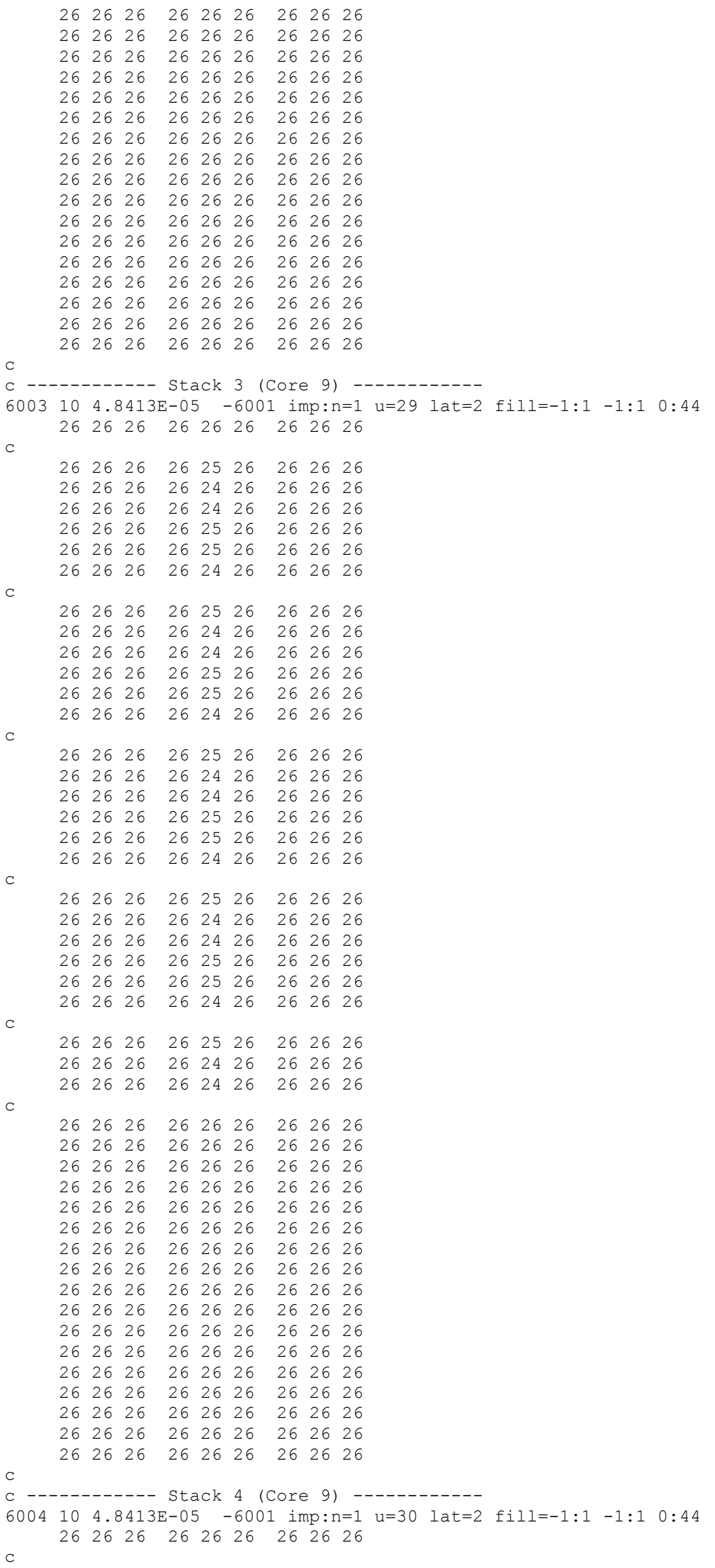

Revision: 1

Date: March 31, 2014

Page 223 of 375 
NEA/NSC/DOC(2006)1

Gas Cooled (Thermal) Reactor - GCR

PROTEUS-GCR-EXP-004

CRIT-REAC

$\begin{array}{lllllllll}26 & 26 & 26 & 26 & 25 & 26 & 26 & 26 & 26 \\ 26 & 26 & 26 & 26 & 24 & 26 & 26 & 26 & 26 \\ 26 & 26 & 26 & 26 & 25 & 26 & 26 & 26 & 26 \\ 26 & 26 & 26 & 26 & 24 & 26 & 26 & 26 & 26 \\ 26 & 26 & 26 & 26 & 24 & 26 & 26 & 26 & 26 \\ 26 & 26 & 26 & 26 & 25 & 26 & 26 & 26 & 26\end{array}$

$\begin{array}{lllllllll}26 & 26 & 26 & 26 & 25 & 26 & 26 & 26 & 26\end{array}$

$\begin{array}{lllllllll}26 & 26 & 26 & 26 & 24 & 26 & 26 & 26 & 26\end{array}$

$\begin{array}{lllllllll}26 & 26 & 26 & 26 & 25 & 26 & 26 & 26 & 26\end{array}$

$\begin{array}{lllllllll}26 & 26 & 26 & 26 & 24 & 26 & 26 & 26 & 26\end{array}$

$\begin{array}{lllllllll}26 & 26 & 26 & 26 & 24 & 26 & 26 & 26 & 26\end{array}$

C

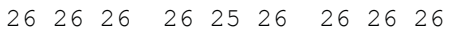

$\begin{array}{lllllllll}26 & 26 & 26 & 26 & 25 & 26 & 26 & 26 & 26\end{array}$

$\begin{array}{lllllllll}26 & 26 & 26 & 26 & 24 & 26 & 26 & 26 & 26\end{array}$

$\begin{array}{lllllllll}26 & 26 & 26 & 26 & 25 & 26 & 26 & 26 & 26\end{array}$

$\begin{array}{lllllllll}26 & 26 & 26 & 26 & 24 & 26 & 26 & 26 & 26\end{array}$

$\begin{array}{lllllllll}26 & 26 & 26 & 26 & 24 & 26 & 26 & 26 & 26\end{array}$

$\begin{array}{lllllllll}26 & 26 & 26 & 26 & 25 & 26 & 26 & 26 & 26\end{array}$

C

$\begin{array}{lllllllll}26 & 26 & 26 & 26 & 25 & 26 & 26 & 26 & 26\end{array}$

$\begin{array}{lllllllll}26 & 26 & 26 & 26 & 24 & 26 & 26 & 26 & 26\end{array}$

$\begin{array}{lllllllll}26 & 26 & 26 & 26 & 25 & 26 & 26 & 26 & 26\end{array}$

$\begin{array}{lllllllll}26 & 26 & 26 & 26 & 24 & 26 & 26 & 26 & 26\end{array}$

$\begin{array}{lllllllll}26 & 26 & 26 & 26 & 24 & 26 & 26 & 26 & 26\end{array}$

C

$\begin{array}{lllllllll}26 & 26 & 26 & 26 & 25 & 26 & 26 & 26 & 26\end{array}$

$\begin{array}{lllllllll}26 & 26 & 26 & 26 & 25 & 26 & 26 & 26 & 26\end{array}$

$\begin{array}{lllllllll}26 & 26 & 26 & 26 & 24 & 26 & 26 & 26 & 26\end{array}$

$\begin{array}{lllllllll}26 & 26 & 26 & 26 & 25 & 26 & 26 & 26 & 26\end{array}$

C

$\begin{array}{lllllllll}26 & 26 & 26 & 26 & 26 & 26 & 26 & 26 & 26\end{array}$

$\begin{array}{lllllllll}26 & 26 & 26 & 26 & 26 & 26 & 26 & 26 & 26\end{array}$

$\begin{array}{lllllllll}26 & 26 & 26 & 26 & 26 & 26 & 26 & 26 & 26\end{array}$

$\begin{array}{lllllllll}26 & 26 & 26 & 26 & 26 & 26 & 26 & 26 & 26\end{array}$

$\begin{array}{lllllllll}26 & 26 & 26 & 26 & 26 & 26 & 26 & 26 & 26\end{array}$

$\begin{array}{lllllllll}26 & 26 & 26 & 26 & 26 & 26 & 26 & 26 & 26\end{array}$

$\begin{array}{lllllllll}26 & 26 & 26 & 26 & 26 & 26 & 26 & 26 & 26\end{array}$

$\begin{array}{lllllllll}26 & 26 & 26 & 26 & 26 & 26 & 26 & 26 & 26\end{array}$

$\begin{array}{lllllllll}26 & 26 & 26 & 26 & 26 & 26 & 26 & 26 & 26\end{array}$

$\begin{array}{lllllllll}26 & 26 & 26 & 26 & 26 & 26 & 26 & 26 & 26\end{array}$

$\begin{array}{lllllllll}26 & 26 & 26 & 26 & 26 & 26 & 26 & 26 & 26\end{array}$

$\begin{array}{lllllllll}26 & 26 & 26 & 26 & 26 & 26 & 26 & 26 & 26\end{array}$

$\begin{array}{lllllllll}26 & 26 & 26 & 26 & 26 & 26 & 26 & 26 & 26\end{array}$

$\begin{array}{lllllllll}26 & 26 & 26 & 26 & 26 & 26 & 26 & 26 & 26\end{array}$

$\begin{array}{lllllllll}26 & 26 & 26 & 26 & 26 & 26 & 26 & 26 & 26\end{array}$

$\begin{array}{lllllllll}26 & 26 & 26 & 26 & 26 & 26 & 26 & 26 & 26\end{array}$

$\begin{array}{lllllllll}26 & 26 & 26 & 26 & 26 & 26 & 26 & 26 & 26\end{array}$

(Core 10) -----------

$6005104.8413 \mathrm{E}-05 \quad-6001$ imp:n=1 $u=31$ lat=2 fill=-1:1 -1:1 0:44 $\begin{array}{lllllllll}26 & 26 & 26 & 26 & 26 & 26 & 26 & 26 & 26\end{array}$

C

$\begin{array}{lllllllll}26 & 26 & 26 & 26 & 24 & 26 & 26 & 26 & 26\end{array}$

$\begin{array}{lllllllll}26 & 26 & 26 & 26 & 25 & 26 & 26 & 26 & 26\end{array}$

$\begin{array}{lllllllll}26 & 26 & 26 & 26 & 25 & 26 & 26 & 26 & 26\end{array}$

$\begin{array}{lllllllll}26 & 26 & 26 & 26 & 24 & 26 & 26 & 26 & 26\end{array}$

$\begin{array}{lllllllll}26 & 26 & 26 & 26 & 25 & 26 & 26 & 26 & 26\end{array}$

C

$\begin{array}{lllllllll}26 & 26 & 26 & 26 & 24 & 26 & 26 & 26 & 26\end{array}$

$\begin{array}{lllllllll}26 & 26 & 26 & 26 & 24 & 26 & 26 & 26 & 26\end{array}$

$\begin{array}{lllllllll}26 & 26 & 26 & 26 & 25 & 26 & 26 & 26 & 26\end{array}$

$\begin{array}{lllllllll}26 & 26 & 26 & 26 & 25 & 26 & 26 & 26 & 26\end{array}$

$\begin{array}{lllllllll}26 & 26 & 26 & 26 & 24 & 26 & 26 & 26 & 26\end{array}$

$\begin{array}{lllllllll}26 & 26 & 26 & 26 & 25 & 26 & 26 & 26 & 26\end{array}$

$\begin{array}{lllllllll}26 & 26 & 26 & 26 & 24 & 26 & 26 & 26 & 26\end{array}$

C

$\begin{array}{lllllllll}26 & 26 & 26 & 26 & 24 & 26 & 26 & 26 & 26\end{array}$

$\begin{array}{lllllllll}26 & 26 & 26 & 26 & 25 & 26 & 26 & 26 & 26\end{array}$

$\begin{array}{lllllllll}26 & 26 & 26 & 26 & 25 & 26 & 26 & 26 & 26\end{array}$

$\begin{array}{lllllllll}26 & 26 & 26 & 26 & 24 & 26 & 26 & 26 & 26\end{array}$

$\begin{array}{lllllllll}26 & 26 & 26 & 26 & 25 & 26 & 26 & 26 & 26\end{array}$

$\begin{array}{lllllllll}26 & 26 & 26 & 26 & 24 & 26 & 26 & 26 & 26\end{array}$

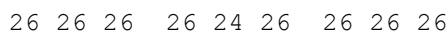

Revision: 1

Date: March 31, 2014

Page 224 of 375 
NEA/NSC/DOC(2006)1

Gas Cooled (Thermal) Reactor - GCR

PROTEUS-GCR-EXP-004

CRIT-REAC

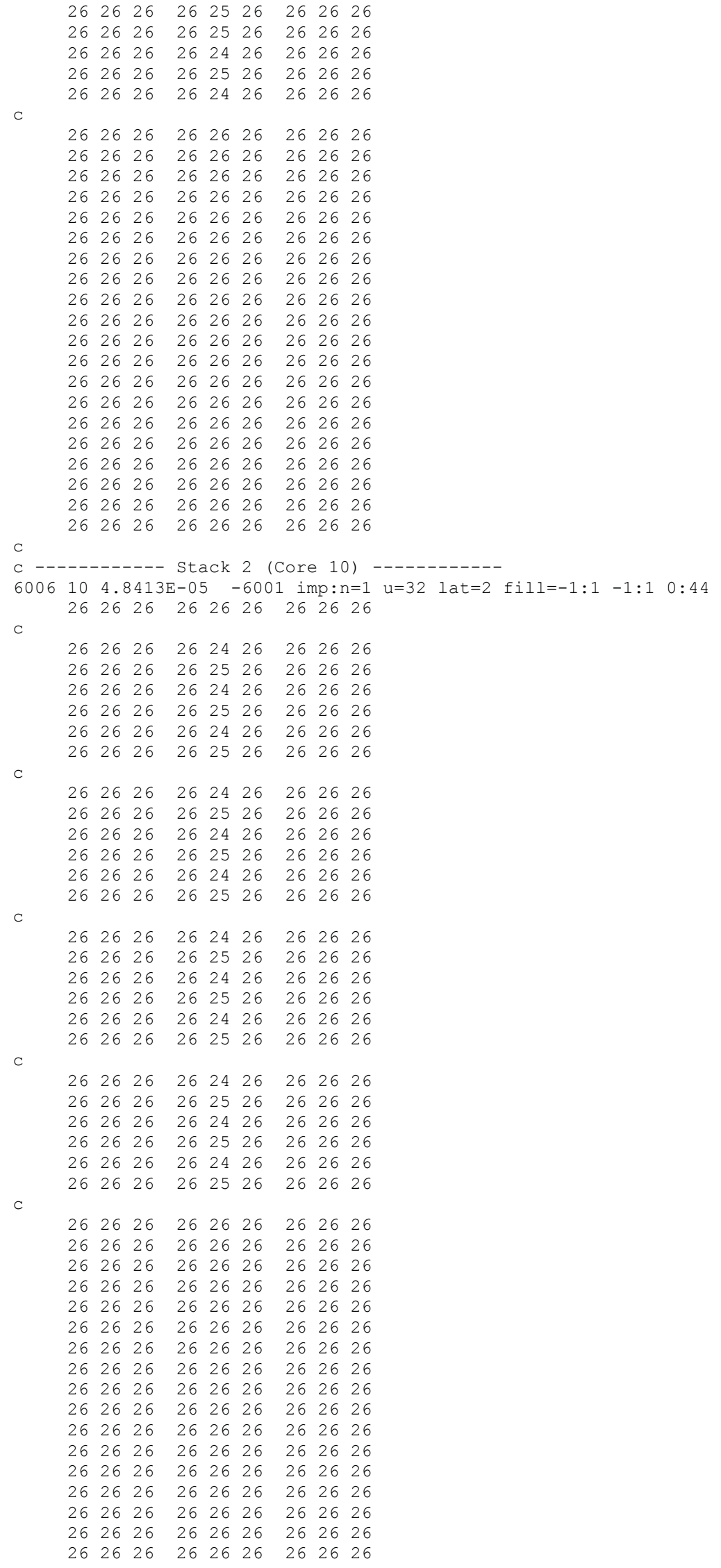

Revision: 1

Date: March 31, 2014

Page 225 of 375 
NEA/NSC/DOC(2006)1

Gas Cooled (Thermal) Reactor - GCR

PROTEUS-GCR-EXP-004

CRIT-REAC

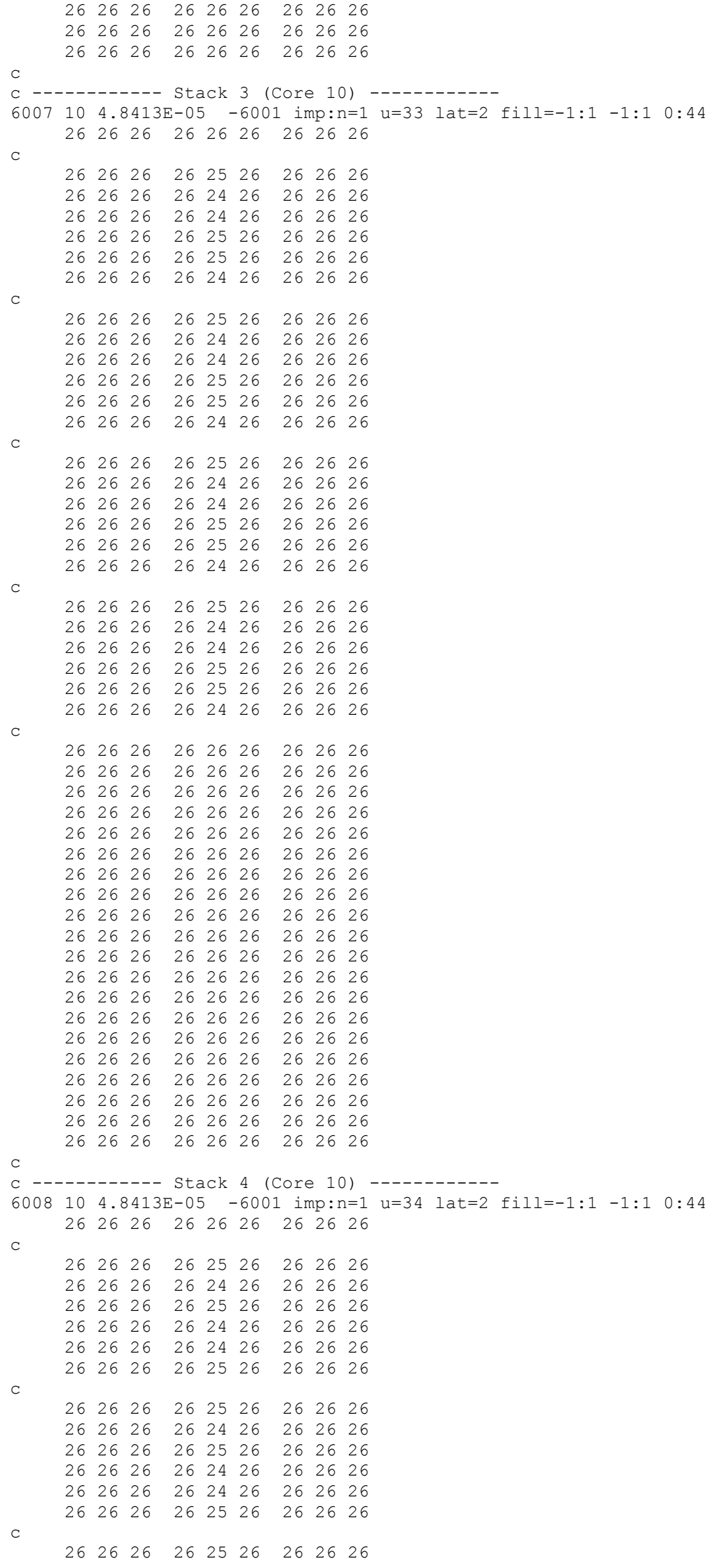

Revision: 1 
NEA/NSC/DOC(2006)1

\section{Gas Cooled (Thermal) Reactor - GCR \\ PROTEUS-GCR-EXP-004 CRIT-REAC}

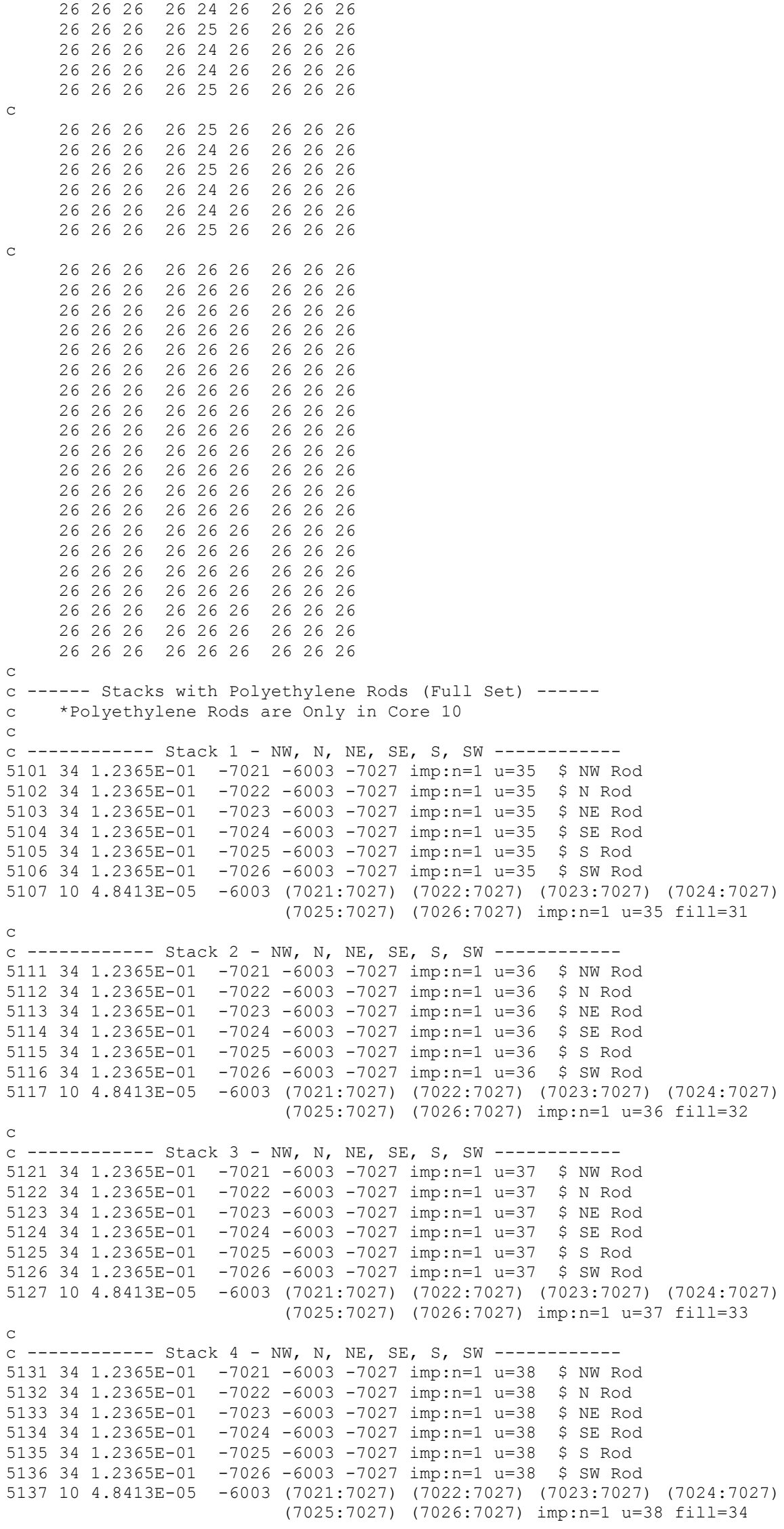

Revision: 1 
NEA/NSC/DOC(2006)1

\section{Gas Cooled (Thermal) Reactor - GCR \\ PROTEUS-GCR-EXP-004 CRIT-REAC}

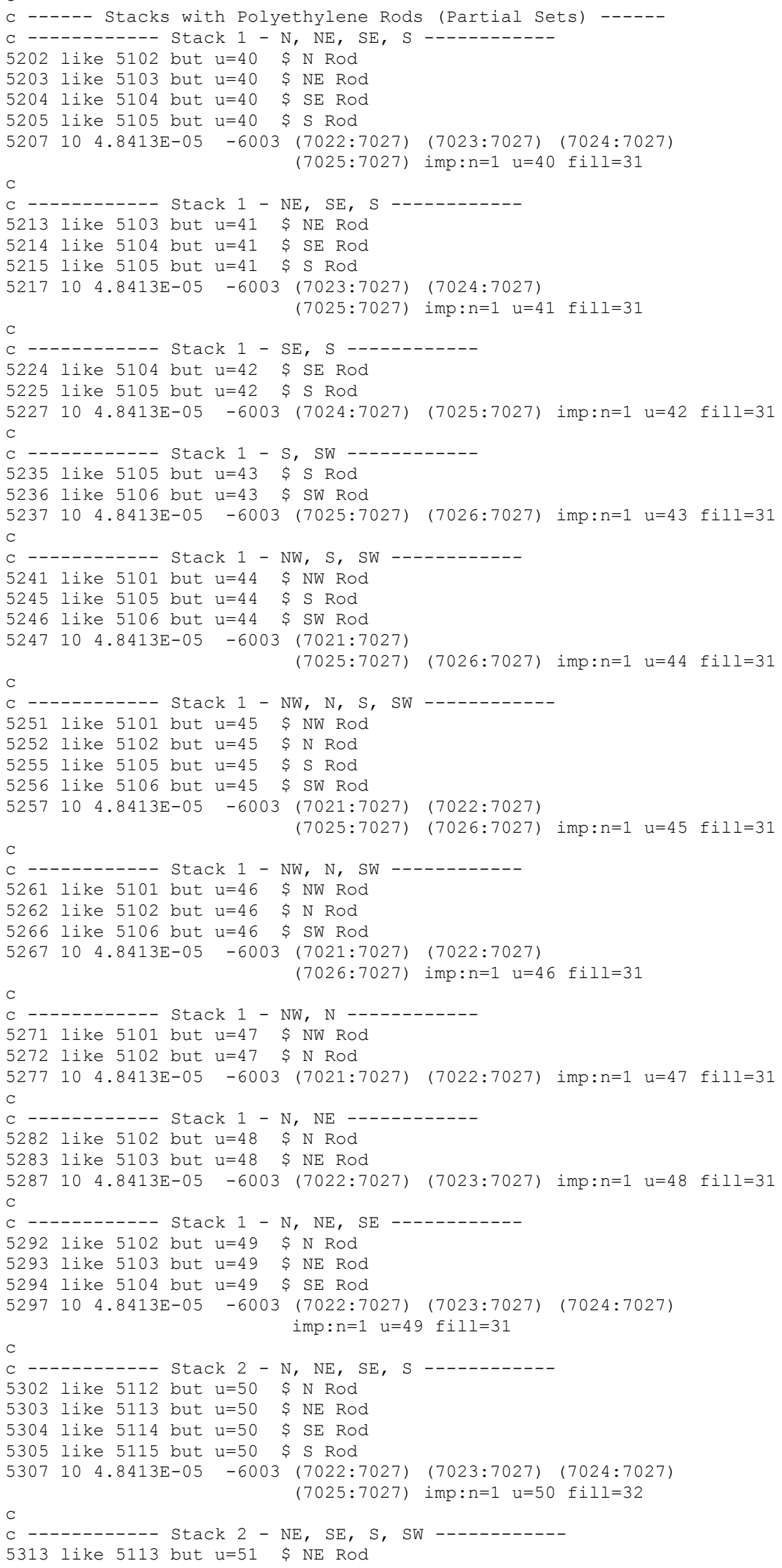

Revision: 1 
NEA/NSC/DOC(2006)1

\section{Gas Cooled (Thermal) Reactor - GCR \\ PROTEUS-GCR-EXP-004 CRIT-REAC}

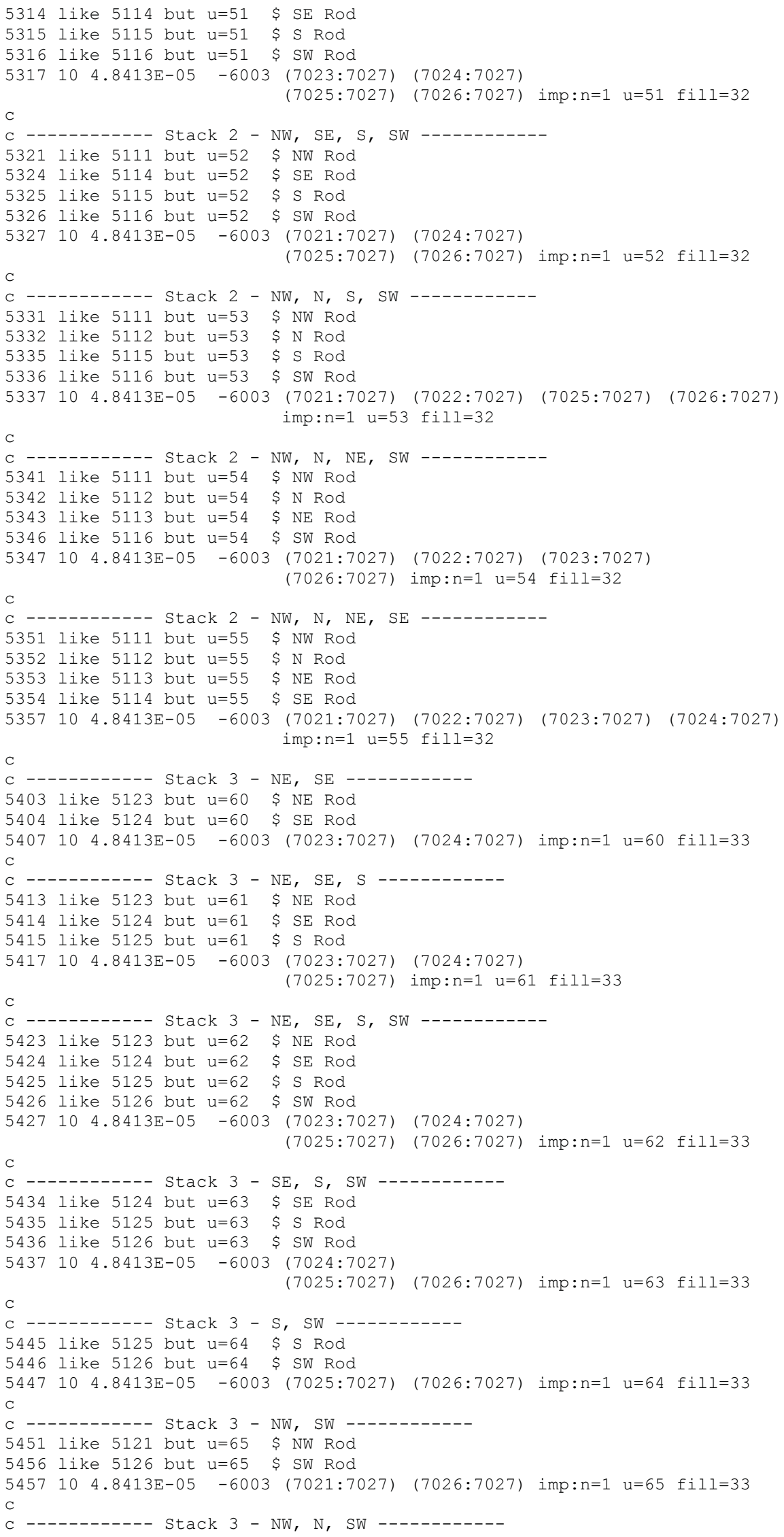

Revision: 1 
NEA/NSC/DOC(2006)1

\section{Gas Cooled (Thermal) Reactor - GCR \\ PROTEUS-GCR-EXP-004 CRIT-REAC}

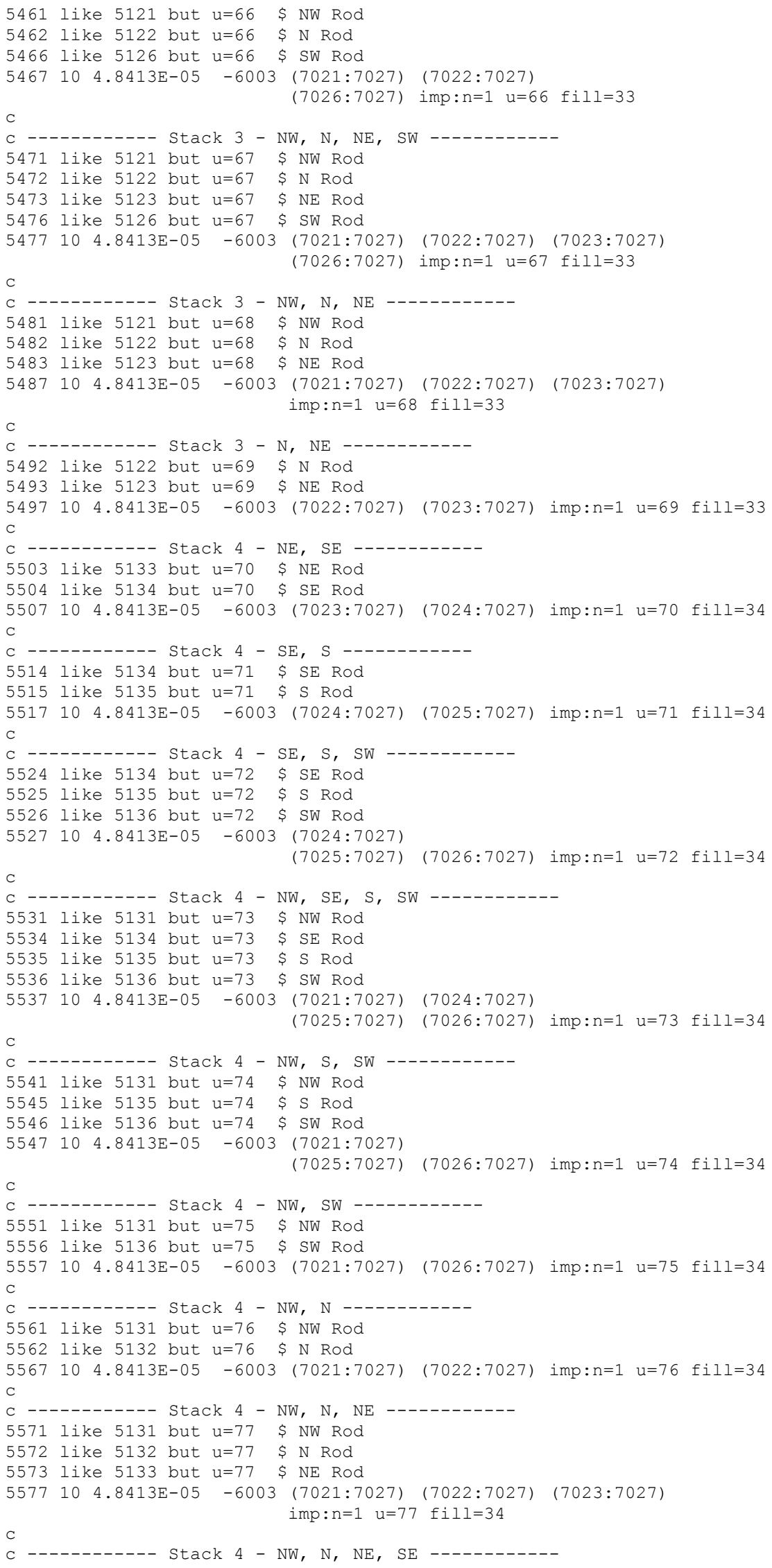

Revision: 1 
NEA/NSC/DOC(2006)1

\section{Gas Cooled (Thermal) Reactor - GCR \\ PROTEUS-GCR-EXP-004 CRIT-REAC}

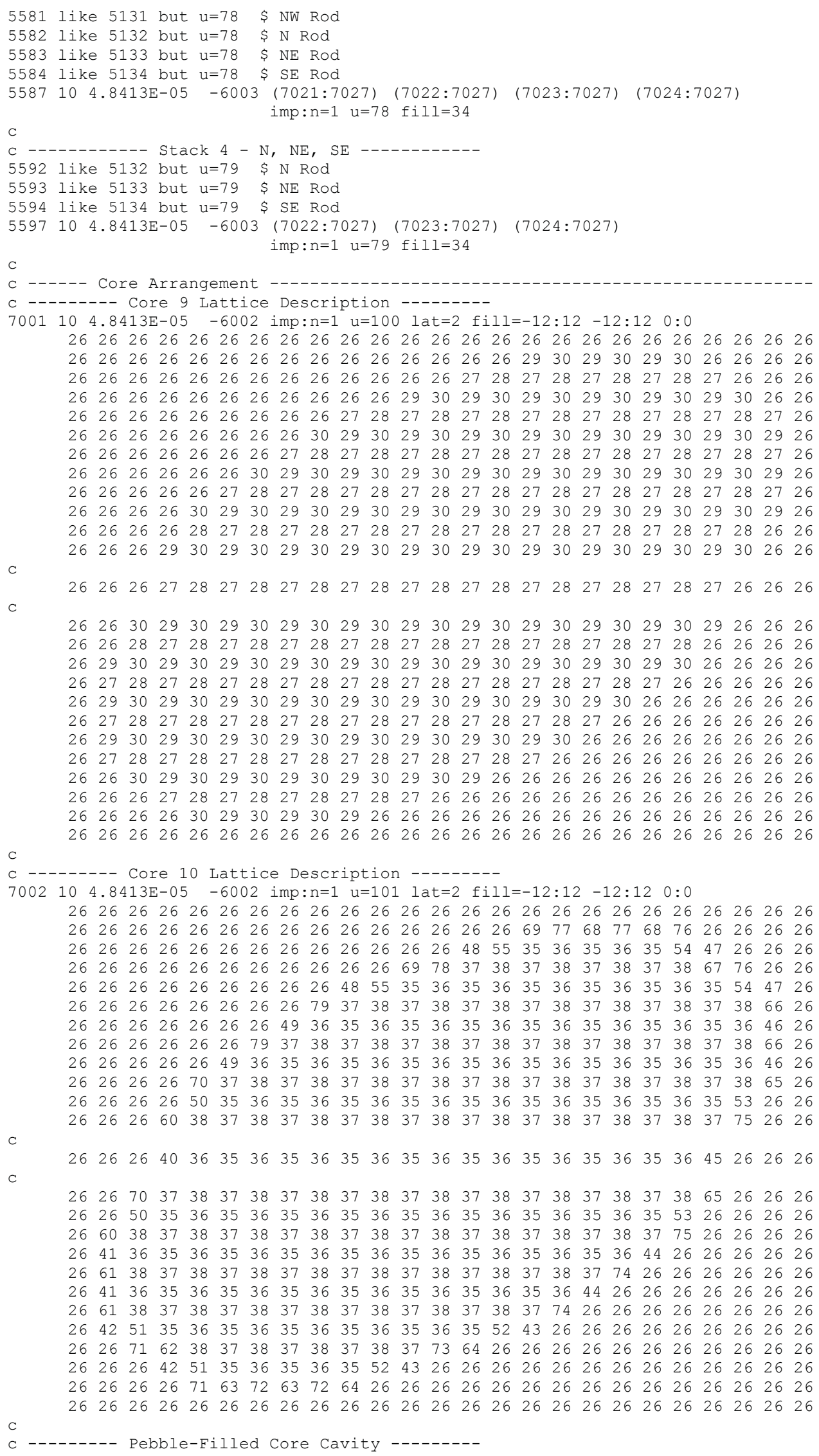

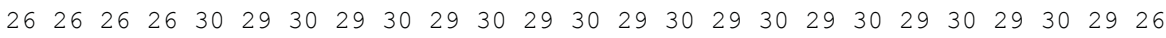
$\begin{array}{lllllllllllllllllllllllll}26 & 26 & 26 & 26 & 28 & 27 & 28 & 27 & 28 & 27 & 28 & 27 & 28 & 27 & 28 & 27 & 28 & 27 & 28 & 27 & 28 & 27 & 28 & 26 & 26\end{array}$

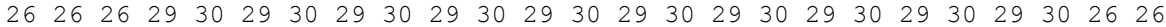
$\begin{array}{lllllllllllllllllllllllll}26 & 29 & 30 & 29 & 30 & 29 & 30 & 29 & 30 & 29 & 30 & 29 & 30 & 29 & 30 & 29 & 30 & 29 & 30 & 29 & 30 & 26 & 26 & 26 & 26\end{array}$ $\begin{array}{llllllllllllllllllllllllllll}26 & 27 & 28 & 27 & 28 & 27 & 28 & 27 & 28 & 27 & 28 & 27 & 28 & 27 & 28 & 27 & 28 & 27 & 28 & 27 & 26 & 26 & 26 & 26 & 26\end{array}$ $\begin{array}{lllllllllllllllllllllllll}26 & 29 & 30 & 29 & 30 & 29 & 30 & 29 & 30 & 29 & 30 & 29 & 30 & 29 & 30 & 29 & 30 & 29 & 30 & 26 & 26 & 26 & 26 & 26 & 26\end{array}$ $\begin{array}{lllllllllllllllllllllllll}26 & 27 & 28 & 27 & 28 & 27 & 28 & 27 & 28 & 27 & 28 & 27 & 28 & 27 & 28 & 27 & 28 & 27 & 26 & 26 & 26 & 26 & 26 & 26 & 26\end{array}$ $\begin{array}{lllllllllllllllllllllllll}26 & 29 & 30 & 29 & 30 & 29 & 30 & 29 & 30 & 29 & 30 & 29 & 30 & 29 & 30 & 29 & 30 & 26 & 26 & 26 & 26 & 26 & 26 & 26 & 26\end{array}$ $\begin{array}{lllllllllllllllllllllllll}26 & 27 & 28 & 27 & 28 & 27 & 28 & 27 & 28 & 27 & 28 & 27 & 28 & 27 & 28 & 27 & 26 & 26 & 26 & 26 & 26 & 26 & 26 & 26 & 26\end{array}$ $\begin{array}{lllllllllllllllllllllllll}26 & 26 & 30 & 29 & 30 & 29 & 30 & 29 & 30 & 29 & 30 & 29 & 30 & 29 & 26 & 26 & 26 & 26 & 26 & 26 & 26 & 26 & 26 & 26 & 26\end{array}$ $\begin{array}{llllllllllllllllllllllllll}26 & 26 & 26 & 27 & 28 & 27 & 28 & 27 & 28 & 27 & 28 & 27 & 26 & 26 & 26 & 26 & 26 & 26 & 26 & 26 & 26 & 26 & 26 & 26 & 26\end{array}$ $\begin{array}{lllllllllllllllllllllllll}26 & 26 & 26 & 26 & 30 & 29 & 30 & 29 & 30 & 29 & 26 & 26 & 26 & 26 & 26 & 26 & 26 & 26 & 26 & 26 & 26 & 26 & 26 & 26 & 26\end{array}$

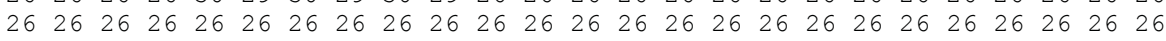
$\begin{array}{llllllllllllllllllllllllll}26 & 26 & 26 & 26 & 26 & 26 & 26 & 26 & 26 & 26 & 26 & 26 & 26 & 26 & 26 & 69 & 77 & 68 & 77 & 68 & 76 & 26 & 26 & 26 & 26\end{array}$

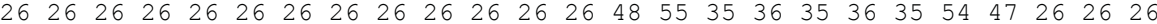
$\begin{array}{lllllllllllllllllllllllll}26 & 26 & 26 & 26 & 26 & 26 & 26 & 26 & 26 & 26 & 26 & 69 & 78 & 37 & 38 & 37 & 38 & 37 & 38 & 37 & 38 & 67 & 76 & 26 & 26\end{array}$

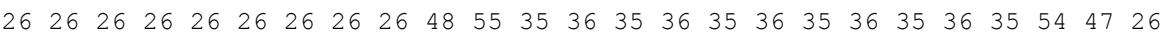
$\begin{array}{lllllllllllllllllllllllll}26 & 26 & 26 & 26 & 26 & 26 & 26 & 26 & 79 & 37 & 38 & 37 & 38 & 37 & 38 & 37 & 38 & 37 & 38 & 37 & 38 & 37 & 38 & 66 & 26\end{array}$ $\begin{array}{lllllllllllllllllllllllll}26 & 26 & 26 & 26 & 26 & 26 & 26 & 49 & 36 & 35 & 36 & 35 & 36 & 35 & 36 & 35 & 36 & 35 & 36 & 35 & 36 & 35 & 36 & 46 & 26\end{array}$ $\begin{array}{llllllllllllllllllllllllllll}26 & 26 & 26 & 26 & 26 & 26 & 79 & 37 & 38 & 37 & 38 & 37 & 38 & 37 & 38 & 37 & 38 & 37 & 38 & 37 & 38 & 37 & 38 & 66 & 26\end{array}$ $\begin{array}{lllllllllllllllllllllllll}26 & 26 & 26 & 26 & 26 & 49 & 36 & 35 & 36 & 35 & 36 & 35 & 36 & 35 & 36 & 35 & 36 & 35 & 36 & 35 & 36 & 35 & 36 & 46 & 26\end{array}$ $\begin{array}{lllllllllllllllllllllllll}26 & 26 & 26 & 26 & 70 & 37 & 38 & 37 & 38 & 37 & 38 & 37 & 38 & 37 & 38 & 37 & 38 & 37 & 38 & 37 & 38 & 37 & 38 & 65 & 26\end{array}$ $\begin{array}{lllllllllllllllllllllllll}26 & 26 & 26 & 26 & 50 & 35 & 36 & 35 & 36 & 35 & 36 & 35 & 36 & 35 & 36 & 35 & 36 & 35 & 36 & 35 & 36 & 35 & 53 & 26 & 26\end{array}$ $\begin{array}{llllllllllllllllllllllllll}26 & 26 & 26 & 60 & 38 & 37 & 38 & 37 & 38 & 37 & 38 & 37 & 38 & 37 & 38 & 37 & 38 & 37 & 38 & 37 & 38 & 37 & 75 & 26 & 26\end{array}$

$\mathrm{C}$ $\begin{array}{lllllllllllllllllllllllll}26 & 26 & 26 & 40 & 36 & 35 & 36 & 35 & 36 & 35 & 36 & 35 & 36 & 35 & 36 & 35 & 36 & 35 & 36 & 35 & 36 & 45 & 26 & 26 & 26\end{array}$

$\mathrm{C}$

$\begin{array}{lllllllllllllllllllllllll}26 & 26 & 70 & 37 & 38 & 37 & 38 & 37 & 38 & 37 & 38 & 37 & 38 & 37 & 38 & 37 & 38 & 37 & 38 & 37 & 38 & 65 & 26 & 26 & 26\end{array}$

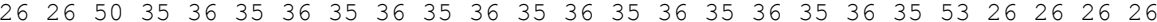
$\begin{array}{lllllllllllllllllllllllll}26 & 60 & 38 & 37 & 38 & 37 & 38 & 37 & 38 & 37 & 38 & 37 & 38 & 37 & 38 & 37 & 38 & 37 & 38 & 37 & 75 & 26 & 26 & 26 & 26\end{array}$ $\begin{array}{lllllllllllllllllllllllllllllll}26 & 41 & 36 & 35 & 36 & 35 & 36 & 35 & 36 & 35 & 36 & 35 & 36 & 35 & 36 & 35 & 36 & 35 & 36 & 44 & 26 & 26 & 26 & 26 & 26\end{array}$ $\begin{array}{lllllllllllllllllllllllll}26 & 61 & 38 & 37 & 38 & 37 & 38 & 37 & 38 & 37 & 38 & 37 & 38 & 37 & 38 & 37 & 38 & 37 & 74 & 26 & 26 & 26 & 26 & 26 & 26\end{array}$ $\begin{array}{lllllllllllllllllllllllllll}26 & 41 & 36 & 35 & 36 & 35 & 36 & 35 & 36 & 35 & 36 & 35 & 36 & 35 & 36 & 35 & 36 & 44 & 26 & 26 & 26 & 26 & 26 & 26 & 26\end{array}$ $\begin{array}{lllllllllllllllllllllllll}26 & 61 & 38 & 37 & 38 & 37 & 38 & 37 & 38 & 37 & 38 & 37 & 38 & 37 & 38 & 37 & 74 & 26 & 26 & 26 & 26 & 26 & 26 & 26 & 26\end{array}$ $\begin{array}{lllllllllllllllllllllllll}26 & 42 & 51 & 35 & 36 & 35 & 36 & 35 & 36 & 35 & 36 & 35 & 36 & 35 & 52 & 43 & 26 & 26 & 26 & 26 & 26 & 26 & 26 & 26 & 26\end{array}$ $\begin{array}{lllllllllllllllllllllllllll}26 & 26 & 71 & 62 & 38 & 37 & 38 & 37 & 38 & 37 & 38 & 37 & 73 & 64 & 26 & 26 & 26 & 26 & 26 & 26 & 26 & 26 & 26 & 26 & 26\end{array}$ $\begin{array}{lllllllllllllllllllllllllll}26 & 26 & 26 & 42 & 51 & 35 & 36 & 35 & 36 & 35 & 52 & 43 & 26 & 26 & 26 & 26 & 26 & 26 & 26 & 26 & 26 & 26 & 26 & 26 & 26\end{array}$

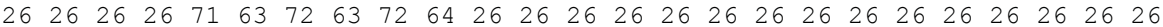

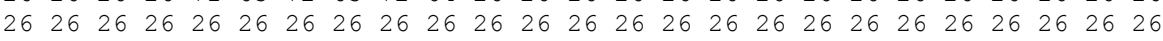

C -------- Pebble-Filled Core Cavity -.-------

Revision: 1 
NEA/NSC/DOC(2006)1

Gas Cooled (Thermal) Reactor - GCR

PROTEUS-GCR-EXP-004

CRIT-REAC

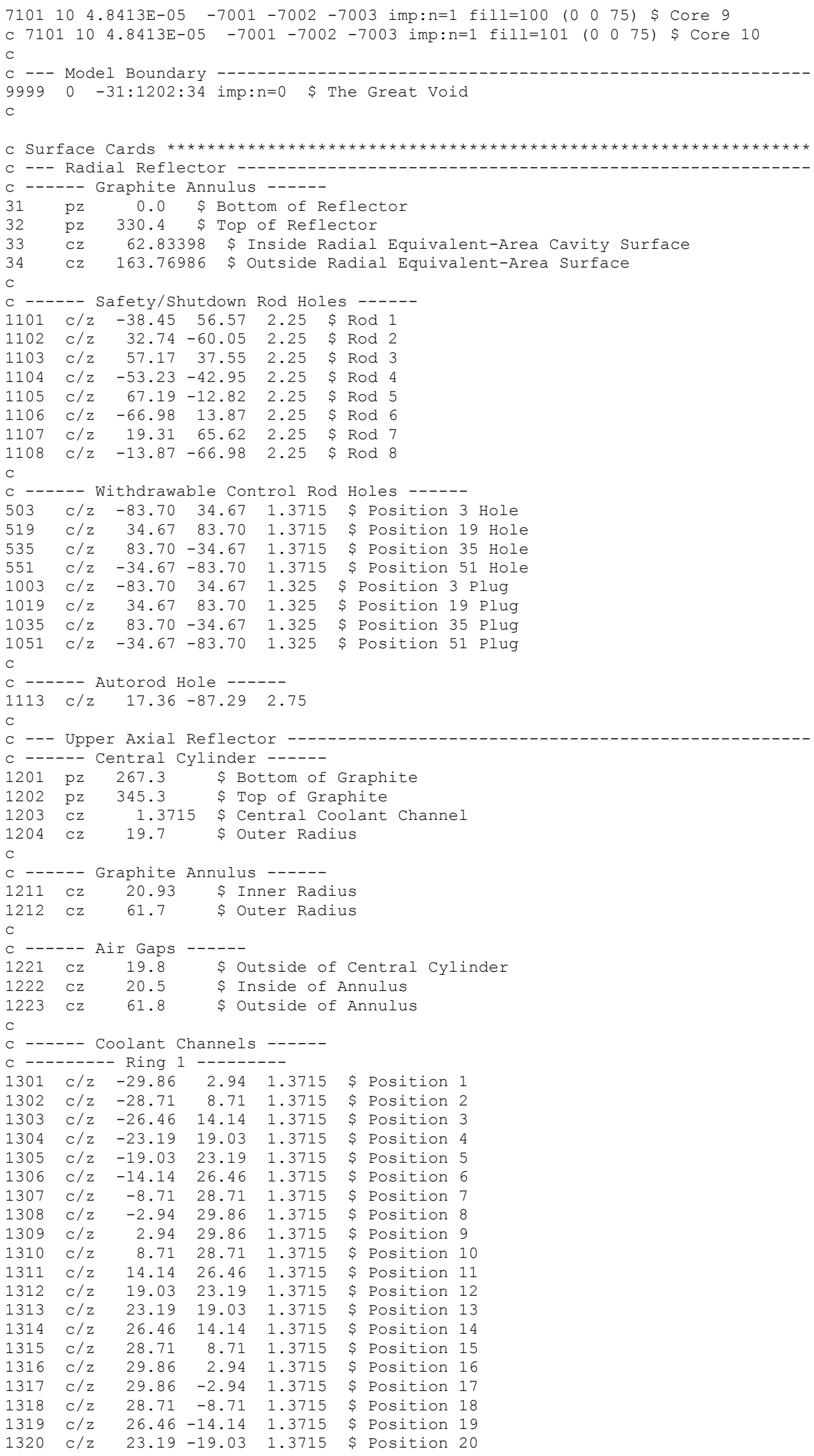

Revision: 1 
NEA/NSC/DOC(2006)1

Gas Cooled (Thermal) Reactor - GCR

PROTEUS-GCR-EXP-004 CRIT-REAC

\begin{tabular}{|c|c|c|c|c|c|c|}
\hline 1321 & $\mathrm{C} / \mathrm{z}$ & 19.03 & -23.19 & 1.3715 & \$ Position 2 & 21 \\
\hline 1322 & $\mathrm{C} / \mathrm{z}$ & 14.14 & -26.46 & 1.3715 & \$ Position 2 & 22 \\
\hline 1323 & $\mathrm{C} / \mathrm{z}$ & 8.71 & -28.71 & 1.3715 & \$ Position 2 & 23 \\
\hline 1324 & $\mathrm{C} / \mathrm{z}$ & 2.94 & -29.86 & 1.3715 & \$ Position 2 & 24 \\
\hline 1325 & $\mathrm{C} / \mathrm{z}$ & -2.94 & -29.86 & 1.3715 & \$ Position 2 & 25 \\
\hline 1326 & $\mathrm{C} / \mathrm{z}$ & -8.71 & -28.71 & 1.3715 & \$ Position 2 & 26 \\
\hline 1327 & $\mathrm{C} / \mathrm{z}$ & -14.14 & -26.46 & 1.3715 & \$ Position 2 & 27 \\
\hline 1328 & $\mathrm{C} / \mathrm{z}$ & -19.03 & -23.19 & 1.3715 & \$ Position 2 & 28 \\
\hline 1329 & $\mathrm{C} / \mathrm{z}$ & -23.19 & -19.03 & 1.3715 & \$ Position 2 & 29 \\
\hline 1330 & $\mathrm{C} / \mathrm{z}$ & -26.46 & -14.14 & 1.3715 & $\$$ Position 3 & 30 \\
\hline 1331 & $\mathrm{C} / \mathrm{z}$ & -28.71 & -8.71 & 1.3715 & \$ Position 3 & 31 \\
\hline $\begin{array}{l}1332 \\
\mathrm{C}\end{array}$ & $\mathrm{C} / \mathrm{z}$ & -29.86 & -2.94 & 1.3715 & \$ Position 3 & 32 \\
\hline $\begin{array}{l}1333 \\
C\end{array}$ & $\mathrm{Cz}$ & 32.75 & \$ Ring & Divider & \multicolumn{2}{|l|}{ for Modeling } \\
\hline-- & ---- & Ring 2 & $2-----$ & --- & & \\
\hline 1401 & $\mathrm{C} / \mathrm{z}$ & -34.82 & 6.93 & 1.3715 & \$ Position 1 & 1 \\
\hline 1402 & $\mathrm{C} / \mathrm{z}$ & -32.80 & 13.59 & 1.3715 & \$ Position 2 & 2 \\
\hline 1403 & $\mathrm{C} / \mathrm{z}$ & -29.52 & 19.72 & 1.3715 & $\$$ Position 3 & 3 \\
\hline 1404 & $\mathrm{C} / \mathrm{z}$ & -25.10 & 25.10 & 1.3715 & \$ Position 4 & 4 \\
\hline 1405 & $\mathrm{C} / \mathrm{z}$ & -19.72 & 29.52 & 1.3715 & \$ Position 5 & 5 \\
\hline 1406 & $\mathrm{C} / \mathrm{z}$ & -13.59 & 32.80 & 1.3715 & \$ Position 6 & 6 \\
\hline 1407 & $\mathrm{C} / \mathrm{z}$ & -6.93 & 34.82 & 1.3715 & \$ Position 7 & 7 \\
\hline 1408 & $\mathrm{C} / \mathrm{z}$ & 0.00 & 35.50 & 1.3715 & \$ Position 8 & 8 \\
\hline 1409 & $\mathrm{C} / \mathrm{z}$ & 6.93 & 34.82 & 1.3715 & \$ Position 9 & 9 \\
\hline 1410 & $\mathrm{C} / \mathrm{z}$ & 13.59 & 32.80 & 1.3715 & \$ Position 1 & 10 \\
\hline 1411 & $\mathrm{C} / \mathrm{z}$ & 19.72 & 29.52 & 1.3715 & $\$$ Position 1 & 11 \\
\hline 1412 & $\mathrm{C} / \mathrm{z}$ & 25.10 & 25.10 & 1.3715 & \$ Position 1 & 12 \\
\hline 1413 & $\mathrm{C} / \mathrm{z}$ & 29.52 & 19.72 & 1.3715 & $\$$ Position 1 & 13 \\
\hline 1414 & $\mathrm{C} / \mathrm{z}$ & 32.80 & 13.59 & 1.3715 & \$ Position 1 & 14 \\
\hline 1415 & $\mathrm{C} / \mathrm{z}$ & 34.82 & 6.93 & 1.3715 & \$ Position 1 & 15 \\
\hline 1416 & $\mathrm{C} / \mathrm{z}$ & 35.50 & 0.00 & 1.3715 & \$ Position 1 & 16 \\
\hline 1417 & $\mathrm{C} / \mathrm{z}$ & 34.82 & -6.93 & 1.3715 & $\$$ Position 1 & 17 \\
\hline 1418 & $\mathrm{C} / \mathrm{z}$ & 32.80 & -13.59 & 1.3715 & \$ Position 1 & 18 \\
\hline 1419 & $\mathrm{C} / \mathrm{z}$ & 29.52 & -19.72 & 1.3715 & \$ Position 1 & 19 \\
\hline 1420 & $c / z$ & 25.10 & -25.10 & 1.3715 & \$ Position 2 & 20 \\
\hline 1421 & $\mathrm{C} / \mathrm{z}$ & 19.72 & -29.52 & 1.3715 & \$ Position 2 & 21 \\
\hline 1422 & $\mathrm{C} / \mathrm{z}$ & 13.59 & -32.80 & 1.3715 & \$ Position 2 & 22 \\
\hline 1423 & $\mathrm{C} / \mathrm{z}$ & 6.93 & -34.82 & 1.3715 & \$ Position 2 & 23 \\
\hline 1424 & $\mathrm{C} / \mathrm{z}$ & 0.00 & -35.50 & 1.3715 & \$ Position 2 & 24 \\
\hline 1425 & $\mathrm{C} / \mathrm{z}$ & -6.93 & -34.82 & 1.3715 & \$ Position 2 & 25 \\
\hline 1426 & $\mathrm{C} / \mathrm{z}$ & -13.59 & -32.80 & 1.3715 & \$ Position 2 & 26 \\
\hline 1427 & $\mathrm{C} / \mathrm{z}$ & -19.72 & -29.52 & 1.3715 & \$ Position 2 & 27 \\
\hline 1428 & $\mathrm{C} / \mathrm{z}$ & -25.10 & -25.10 & 1.3715 & \$ Position 2 & 28 \\
\hline 1429 & $\mathrm{C} / \mathrm{z}$ & -29.52 & -19.72 & 1.3715 & \$ Position 2 & 29 \\
\hline 1430 & $\mathrm{C} / \mathrm{z}$ & -32.80 & -13.59 & 1.3715 & $\$$ Position 3 & 30 \\
\hline 1431 & $\mathrm{C} / \mathrm{z}$ & -34.82 & -6.93 & 1.3715 & $\$$ Position 3 & 31 \\
\hline 1432 & $\mathrm{C} / \mathrm{z}$ & -35.50 & 0.00 & 1.3715 & \$ Position 3 & 32 \\
\hline C & & & & & & \\
\hline 1433 & $\mathrm{Cz}$ & 38.25 & \$ Ring & Divider & for Modeling & g Simplification \\
\hline C & & & & & & \\
\hline C -- - & ---- & - Ring 3 & $3-----$ & --- & & \\
\hline 1501 & $\mathrm{C} / \mathrm{z}$ & -39.23 & 11.90 & 1.3715 & \$ Position 1 & 1 \\
\hline 1502 & $\mathrm{C} / \mathrm{z}$ & -36.16 & 19.33 & 1.3715 & $\$$ Position 2 & 2 \\
\hline 1503 & $\mathrm{C} / \mathrm{z}$ & -31.69 & 26.01 & 1.3715 & \$ Position 3 & 3 \\
\hline 1504 & $\mathrm{C} / \mathrm{z}$ & -26.01 & 31.69 & 1.3715 & \$ Position 4 & 4 \\
\hline 1505 & $\mathrm{C} / \mathrm{z}$ & -19.33 & 36.16 & 1.3715 & \$ Position 5 & 5 \\
\hline 1506 & $\mathrm{C} / \mathrm{z}$ & -11.90 & 39.23 & 1.3715 & \$ Position 6 & 6 \\
\hline 1507 & $\mathrm{C} / \mathrm{z}$ & -4.02 & 40.80 & 1.3715 & \$ Position 7 & 7 \\
\hline 1508 & $\mathrm{C} / \mathrm{z}$ & 4.02 & 40.80 & 1.3715 & \$ Position 8 & 8 \\
\hline 1509 & $\mathrm{C} / \mathrm{z}$ & 11.90 & 39.23 & 1.3715 & \$ Position 9 & 9 \\
\hline 1510 & $\mathrm{C} / \mathrm{z}$ & 19.33 & 36.16 & 1.3715 & \$ Position 1 & 10 \\
\hline 1511 & $\mathrm{C} / \mathrm{z}$ & 26.01 & 31.69 & 1.3715 & \$ Position 1 & 11 \\
\hline 1512 & $\mathrm{C} / \mathrm{z}$ & 31.69 & 26.01 & 1.3715 & \$ Position 1 & 12 \\
\hline 1513 & $\mathrm{C} / \mathrm{z}$ & 36.16 & 19.33 & 1.3715 & \$ Position 1 & 13 \\
\hline 1514 & $\mathrm{C} / \mathrm{z}$ & 39.23 & 11.90 & 1.3715 & $\$$ Position 1 & 14 \\
\hline 1515 & $\mathrm{C} / \mathrm{z}$ & 40.80 & 4.02 & 1.3715 & $\$$ Position 1 & 15 \\
\hline 1516 & $\mathrm{C} / \mathrm{z}$ & 40.80 & -4.02 & 1.3715 & \$ Position 1 & 16 \\
\hline 1517 & $\mathrm{c} / \mathrm{z}$ & 39.23 & -11.90 & 1.3715 & \$ Position 1 & 17 \\
\hline 1518 & $\mathrm{C} / \mathrm{z}$ & 36.16 & -19.33 & 1.3715 & \$ Position 1 & 18 \\
\hline 1519 & $\mathrm{C} / \mathrm{z}$ & 31.69 & -26.01 & 1.3715 & \$ Position 1 & 19 \\
\hline 1520 & $\mathrm{C} / \mathrm{z}$ & 26.01 & -31.69 & 1.3715 & \$ Position 2 & 20 \\
\hline 1521 & $\mathrm{C} / \mathrm{z}$ & 19.33 & -36.16 & 1.3715 & \$ Position 2 & 21 \\
\hline 1522 & $\mathrm{C} / \mathrm{z}$ & 11.90 & -39.23 & 1.3715 & \$ Position 2 & 22 \\
\hline 1523 & $\mathrm{C} / \mathrm{z}$ & 4.02 & -40.80 & 1.3715 & \$ Position 2 & 23 \\
\hline 1524 & $\mathrm{C} / \mathrm{z}$ & -4.02 & -40.80 & 1.3715 & \$ Position 2 & 24 \\
\hline
\end{tabular}

Revision: 1 
NEA/NSC/DOC(2006)1

Gas Cooled (Thermal) Reactor - GCR

PROTEUS-GCR-EXP-004

CRIT-REAC

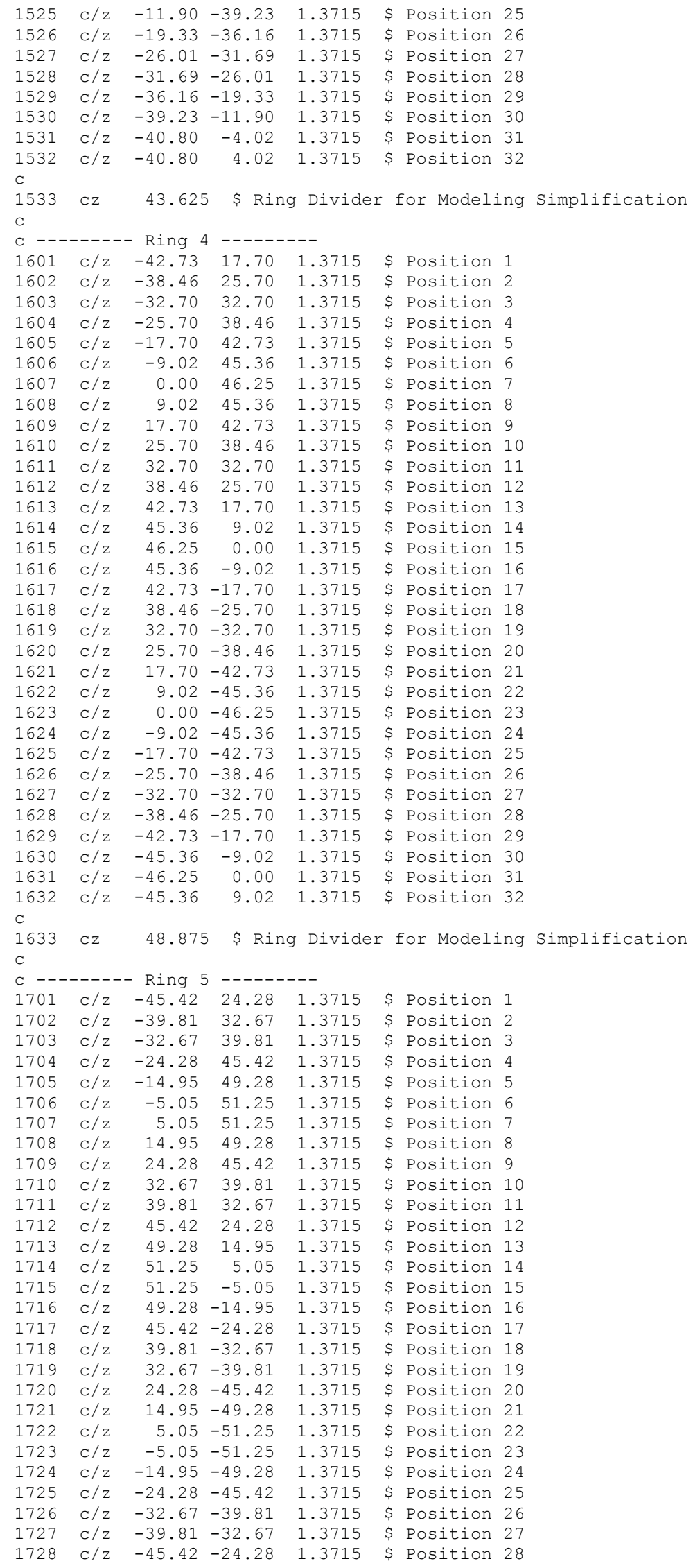

Revision: 1 
NEA/NSC/DOC(2006)1

\section{Gas Cooled (Thermal) Reactor - GCR \\ PROTEUS-GCR-EXP-004 CRIT-REAC}

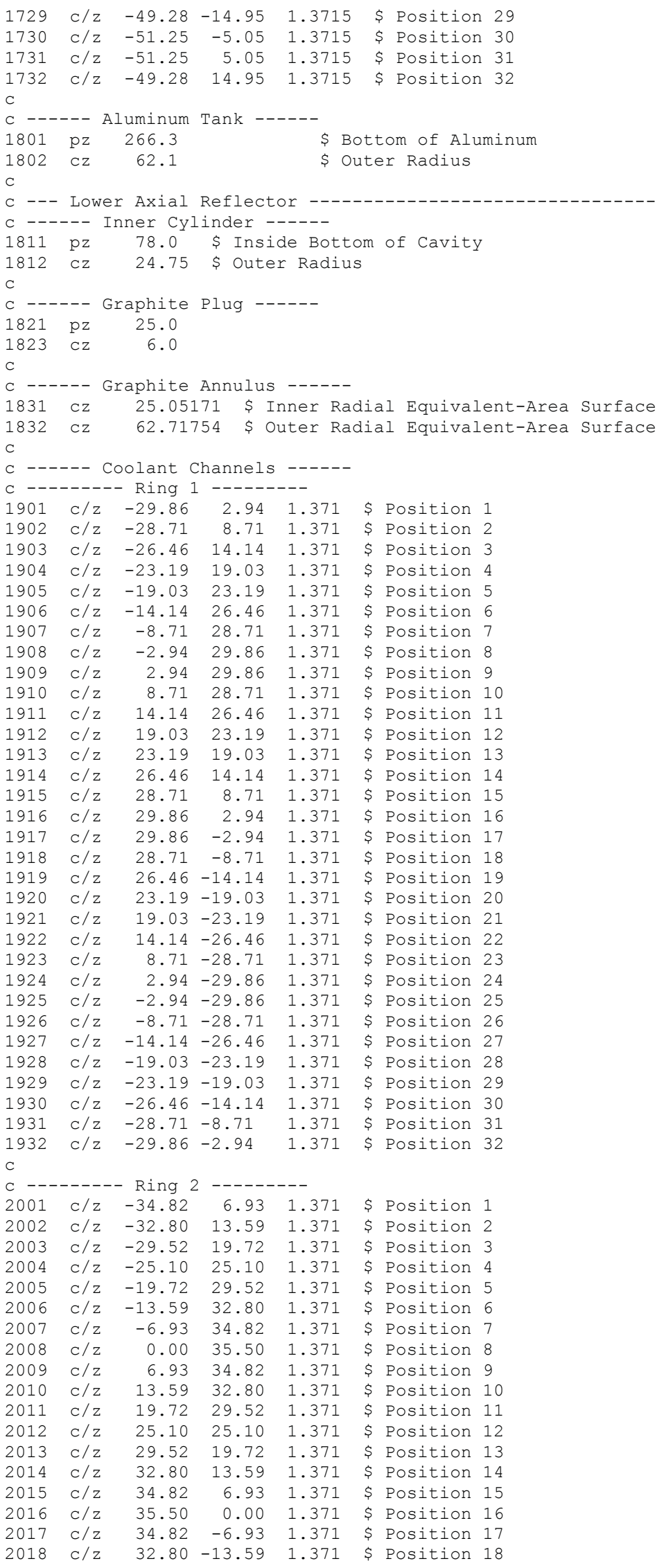

Revision: 1 
NEA/NSC/DOC(2006)1

Gas Cooled (Thermal) Reactor - GCR

PROTEUS-GCR-EXP-004

CRIT-REAC

\begin{tabular}{|c|c|c|c|c|c|c|c|}
\hline 19 & $C / z$ & 29.52 & -19.72 & 1.371 & $\$$ & sition & 19 \\
\hline 20 & $c / z$ & 25.10 & -25.10 & 1.371 & \$ & Position & 2 \\
\hline & $C / z$ & 19.72 & -29.52 & 1.371 & \$ & Position & \\
\hline & $C / z$ & 3.59 & -32.80 & .371 & & Position & \\
\hline & $c / z$ & 6.93 & -34.82 & L. 371 & & Position & \\
\hline & $C / z$ & 0.00 & -35.50 & .371 & & Position & \\
\hline & $C / z$ & -6.93 & -34.82 & L. 371 & & & \\
\hline & $\mathrm{C} / \mathrm{z}$ & -13.59 & -32.80 & .371 & & & \\
\hline & $c / z$ & 19.72 & -29 . & 1.371 & 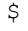 & Po & \\
\hline & $C / z$ & -25.10 & -25.10 & 1.371 & s & & \\
\hline & $C / z$ & -29.52 & -19.72 & .371 & 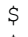 & & \\
\hline & $C / z$ & -32.80 & -13.59 & .371 & $\$$ & & \\
\hline & $C / z$ & -34.82 & -6.93 & .371 & \$ & & \\
\hline & $c / z$ & & 0.00 & .371 & \$ & & \\
\hline \\
\hline & & & & & & & \\
\hline & $C / z$ & -39 & 11 & 1.371 & $\$$ & $n$ & 1 \\
\hline & $C / z$ & -36.16 & 19. & 1.371 & 5 & O & \\
\hline & $C / z$ & -31.69 & 26. & 1.371 & $\$$ & & \\
\hline & $C / z$ & -26.01 & 31. & 1.371 & \$ & Po & 4 \\
\hline & $C / z$ & -19.33 & 36. & 1.371 & $\$$ & $\mathrm{Po}$ & \\
\hline & $c / z$ & -11.90 & 39. & 1.371 & $\$$ & & \\
\hline & $C / z$ & 2 & 40 & 1. & $\$$ & & \\
\hline & $c / z$ & 02 & 40.80 & 1.371 & \$ & EO & \\
\hline & $C / z$ & 11.90 & 39. & 1.371 & $\$$ & $\mathrm{n}$ & \\
\hline & $C / z$ & 3 & 36. & 1.371 & \$ & & \\
\hline & $c / z$ & 26.01 & 31. & 1.371 & $\$$ & & \\
\hline & $C / z$ & 31.69 & 26.01 & 1.371 & $\$$ & & \\
\hline & $c / z$ & 6 & 19 & 1.371 & $\$$ & & \\
\hline & $C / z$ & 3 & 11. & 71 & $\$$ & & \\
\hline & $c / z$ & 40.80 & 4.02 & 1.371 & $\$$ & $n$ & \\
\hline & $C / z$ & 0.80 & -4 & 1.371 & $\$$ & & \\
\hline & $C / z$ & .23 & -11. & & $\$$ & & \\
\hline & $C / z$ & 6 & -1 & 1. & $\$$ & & \\
\hline & $c / z$ & 31.69 & -26.01 & 1.371 & $\$$ & Po: & \\
\hline & $C / z$ & .01 & -31 & 1.371 & \$ & $\mathrm{O}$ & \\
\hline & $C / z$ & 3 & -36 & 1.371 & $\$$ & & \\
\hline & $c / z$ & 11.90 & -39. & 1.371 & \$ & O & \\
\hline & $c / z$ & 4.02 & -40 . & 1 . & S & $n$ & 23 \\
\hline & $c / z$ & -4.02 & -40 . & 1 . & $\$$ & $n$ & \\
\hline & $c / z$ & -11.90 & -39 . & 1 . & $\$$ & & \\
\hline & $c / z$ & -19 & -36 & 1.371 & $\$$ & $\mathrm{Po}$ & 26 \\
\hline & $c / z$ & -26.01 & -31.69 & 1.371 & S & & 27 \\
\hline & $C / z$ & -31.69 & -26 . & 1.371 & $\$$ & & \\
\hline & $c / z$ & -36.16 & -19. & 71 & \$ & & \\
\hline & $C / z$ & -39.23 & -11.90 & 1.371 & S & $\mathrm{PO}$ & 30 \\
\hline & $C / z$ & -40.80 & -4.02 & 1.371 & $\$$ & $n$ & 3 \\
\hline & $C / z$ & -40.80 & 4.02 & 1.371 & $\$$ & 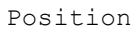 & \\
\hline \\
\hline & & & & . & & & \\
\hline 1 & $c / z$ & -42.73 & 17.70 & 1.3 & $\$$ & $\mathrm{Po}$ & 1 \\
\hline & $c / z$ & -38.46 & 25 & 1 . & $\$$ & & 2 \\
\hline 3 & $c / z$ & -32.70 & 32.70 & 1.371 & \$ & Po & 3 \\
\hline 4 & $C / z$ & -25.70 & 38. & 1.371 & \$ & $\mathrm{Po}$ & 4 \\
\hline 05 & $c / z$ & -17 . & 42.73 & 1.371 & $\$$ & & 5 \\
\hline & $c / z$ & -9.02 & 45. & 1. & $\$$ & $n$ & 6 \\
\hline 07 & $c / z$ & 0.00 & 46.25 & 1. & $\$$ & $\mathrm{Po}$ & 7 \\
\hline 208 & $c / z$ & 9 & 45.36 & 1. & $\$$ & $n$ & 8 \\
\hline & $c / z$ & 0 & 42 & & $\$$ & & 9 \\
\hline & $c / z$ & 25.70 & 38.46 & 1.371 & \$ & Po: & 10 \\
\hline & $c / z$ & 32.70 & 32.70 & 1.371 & $\$$ & Po & 11 \\
\hline & $C / z$ & 38. & 25.70 & 1.371 & $\$$ & & \\
\hline & $c / z$ & 42.73 & 17.70 & 1. & \$ & Pos & 1 \\
\hline 14 & $\mathrm{C} / \mathrm{z}$ & 45.36 & 9.02 & 71 & $\$$ & Po: & 14 \\
\hline 2215 & $C / z$ & 46.25 & 0.00 & 1.371 & $\$$ & Pos: & 15 \\
\hline 2216 & $C / z$ & 45.36 & -9.02 & 1.371 & $\$$ & Pos & 1 \\
\hline 2217 & $c / z$ & 42.73 & -17.70 & 1.371 & $\$$ & Pos: & 1 \\
\hline & $c / z$ & 38.46 & -25.70 & 1.371 & $\$$ & Posi & $1 \varepsilon$ \\
\hline 2219 & $c / z$ & & -32.70 & 1.371 & \$ & Posi & \pm \\
\hline 2220 & $C / z$ & 25.70 & -38.46 & 1.371 & $\$$ & Posi & 20 \\
\hline 2221 & $c / z$ & 17.70 & -42.73 & 1.371 & $\$$ & Posi & 21 \\
\hline $22<2$ & $c / z$ & 9.02 & -45.36 & 1.371 & \$ & Posi & 2 \\
\hline 2223 & $c / z$ & 0.00 & -46.25 & 1.371 & $\$$ & Posi & 2 \\
\hline 2224 & $c / z$ & -9.02 & -45.36 & 1.371 & \$ & Posi & 2 \\
\hline & $c / z$ & & -42.73 & 1.371 & S & Pos & 2 \\
\hline & $c / z$ & 570 & -38.46 & 1.371 & $\$$ & Pos & \\
\hline
\end{tabular}

Revision: 1 
NEA/NSC/DOC(2006)1

Gas Cooled (Thermal) Reactor - GCR

PROTEUS-GCR-EXP-004

CRIT-REAC

\begin{tabular}{|c|c|c|c|c|c|c|c|}
\hline 227 & $\mathrm{C} / \mathrm{z}$ & -32.70 & -32.70 & 1.371 & $\$$ & Position & 27 \\
\hline 28 & $\mathrm{C} / \mathrm{z}$ & -38.46 & -25.70 & 1.371 & & Position & \\
\hline 29 & $\mathrm{C} / \mathrm{z}$ & -42.73 & -17.70 & 1.371 & & Position & \\
\hline & $\mathrm{C} / \mathrm{z}$ & -45.36 & -9.02 & 1.371 & & Position & \\
\hline & $\mathrm{C} / \mathrm{z}$ & -46.25 & 0.00 & 1.371 & & Position & \\
\hline & $\mathrm{C} / \mathrm{z}$ & -45.36 & 9.02 & 1.371 & & Pngition & \\
\hline \\
\hline & & (2019 & & & & & \\
\hline & $\mathrm{C} / \mathrm{z}$ & -45.42 & 24.28 & 1.371 & $\$$ & ion & 1 \\
\hline & $\mathrm{C} / \mathrm{z}$ & -39.81 & 32.67 & 1.371 & \$ & ion & \\
\hline & $\mathrm{C} / \mathrm{z}$ & -32.67 & 39.81 & 1.371 & $\$$ & & \\
\hline & $\mathrm{C} / \mathrm{z}$ & -24.28 & 45.42 & 1371 & & & \\
\hline & $\mathrm{C} / \mathrm{z}$ & -14.95 & 49.28 & 1.371 & \$ & & \\
\hline & $\mathrm{C} / \mathrm{z}$ & -5.05 & 51.25 & 1.371 & \$ & & \\
\hline & $\mathrm{C} / \mathrm{z}$ & 5.05 & 51.25 & 1.371 & $\$$ & & \\
\hline & $\mathrm{C} / \mathrm{z}$ & 14.95 & 49.28 & 1.371 & $\$$ & & \\
\hline & $\mathrm{C} / \mathrm{z}$ & 24.28 & 45. & 1.371 & \$ & & \\
\hline & $\mathrm{C} / \mathrm{z}$ & 32.67 & 39.81 & 1.371 & $\$$ & & \\
\hline & $\mathrm{C} / \mathrm{z}$ & 39.81 & 32 . & 1.371 & $\$$ & & \\
\hline & $\mathrm{C} / \mathrm{z}$ & 45.42 & 24. & 1.371 & $\$$ & & \\
\hline & $\mathrm{C} / \mathrm{z}$ & 49.28 & 14. & 1.371 & $\$$ & & \\
\hline & $\mathrm{C} / \mathrm{z}$ & 51.25 & 5. & 1.371 & \$ & $\mathrm{n}$ & \\
\hline & $\mathrm{C} / \mathrm{z}$ & 51.25 & -5 . & 1.371 & S & & \\
\hline & $\mathrm{C} / \mathrm{z}$ & 49.28 & -14 & 1.371 & $\$$ & & \\
\hline & $\mathrm{C} / \mathrm{z}$ & 45.42 & -24 & 1.371 & \$ & & \\
\hline & $\mathrm{C} / \mathrm{z}$ & 39.81 & -32 . & 1.371 & $\$$ & & 8 \\
\hline & $\mathrm{C} / \mathrm{z}$ & 32.67 & -39 . & 1.371 & $\$$ & & \\
\hline & $\mathrm{C} / \mathrm{z}$ & 24.28 & -45 . & 1.371 & $\$$ & $\mathrm{Po}$ & \\
\hline & $\mathrm{C} / \mathrm{z}$ & 14.95 & -49 . & 1.371 & $\$$ & & 1 \\
\hline & $C / z$ & 5 & -51 & 1.371 & $\$$ & & 2 \\
\hline & $\mathrm{C} / \mathrm{z}$ & -5.05 & -51 & 1.371 & $\$$ & & \\
\hline & $c / z$ & -14.95 & -49 . & 1.371 & $\$$ & & \\
\hline & $\mathrm{C} / \mathrm{z}$ & -24.28 & -45.42 & 1.371 & $\$$ & & $23+2$ \\
\hline & $\mathrm{C} / \mathrm{z}$ & -32.67 & -39 & 1.371 & $\$$ & & \\
\hline & $\mathrm{C} / \mathrm{z}$ & -39.81 & -32 . & 1.371 & $\$$ & & 7 \\
\hline & $\mathrm{C} / \mathrm{z}$ & -45.42 & -24.28 & 1.371 & $\$$ & & 8 \\
\hline & $C / z$ & -49.28 & -14 & 1 . & $\$$ & & 2 \\
\hline & $\mathrm{C} / \mathrm{z}$ & -51.25 & -5 & 1.371 & $\$$ & & 30 \\
\hline & $c / z$ & -51.25 & 5 . & 1.371 & $\$$ & & 31 \\
\hline & $\mathrm{C} / \mathrm{z}$ & -49.28 & 14.95 & 1.3 & $\$$ & & 3 \\
\hline \\
\hline & & & > & & & & \\
\hline \multicolumn{8}{|c|}{--------- Ring $1--------$} \\
\hline & $\mathrm{C} / \mathrm{z}$ & -29.86 & 2.94 & 1.325 & $\$$ & & 1 \\
\hline & $\mathrm{C} / \mathrm{z}$ & -28.71 & 8.71 & 1.325 & $\$$ & & 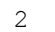 \\
\hline & $\mathrm{c} / \mathrm{z}$ & -26.46 & 14.14 & 1. & $\$$ & $\mathrm{Po}$ & 3 \\
\hline & $\mathrm{C} / \mathrm{z}$ & -23.19 & 19.03 & 1.325 & $\$$ & & 4 \\
\hline & $\mathrm{C} / \mathrm{z}$ & -19.03 & 23. & 1.325 & $\$$ & & 3 \\
\hline & $\mathrm{C} / \mathrm{z}$ & -14.14 & 26.46 & 1.325 & $\$$ & & 6 \\
\hline & $\mathrm{C} / \mathrm{z}$ & -8.71 & 28.71 & 1.325 & $\$$ & & 7 \\
\hline & $\mathrm{C} / \mathrm{z}$ & -2.94 & 29.86 & 1.325 & $\$$ & & 8 \\
\hline & $\mathrm{C} / \mathrm{z}$ & 4 & 29.86 & 1.325 & $\$$ & & 9 \\
\hline & $\mathrm{C} / \mathrm{z}$ & 1 & 28. & 1 & $\$$ & & 10 \\
\hline & $\mathrm{C} / \mathrm{z}$ & 4 & 26.46 & 1.325 & $\$$ & & \\
\hline & $\mathrm{C} / \mathrm{z}$ & 3 & 23.19 & 1.325 & $\$$ & & 12 \\
\hline & $\mathrm{C} / \mathrm{z}$ & 23 & 19.03 & 1.325 & $\$$ & & 13 \\
\hline & $c / z$ & 6.46 & 14.14 & 1.325 & $\$$ & Po & 14 \\
\hline & $\mathrm{C} / \mathrm{z}$ & 3.71 & 8. & 1.325 & $\$$ & & 15 \\
\hline & $c / z$ & 6 & 2 . & 1.325 & $\$$ & & 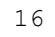 \\
\hline & $\mathrm{C} / \mathrm{z}$ & 6 & -2.94 & 1.325 & $\$$ & & 17 \\
\hline & $c / z$ & 8.71 & -8.71 & 1.325 & \$ & & \\
\hline & $C / z$ & 26.46 & -14.14 & & $\$$ & & $\perp$ \\
\hline & $\mathrm{C} / \mathrm{z}$ & & -19.03 & 1.325 & $\$$ & & 20 \\
\hline & $\mathrm{C} / \mathrm{z}$ & 3 & -23.19 & 1.325 & $\$$ & Po & 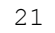 \\
\hline & $c / z$ & 14.14 & -26.46 & 1.325 & $\$$ & Po: & 22 \\
\hline & $C / z$ & 8 . & -28.71 & 1.325 & $\$$ & Po: & 23 \\
\hline & $\mathrm{C} / \mathrm{z}$ & 2.94 & -29.86 & 1.325 & $\$$ & Posi & 24 \\
\hline & $\mathrm{C} / \mathrm{z}$ & -2.94 & -29.86 & 1.325 & $\$$ & Posit & 2 \\
\hline & $c / z$ & -8.71 & -28.71 & 1.325 & $\$$ & POS & 26 \\
\hline & $\mathrm{C} / \mathrm{z}$ & -14.14 & -26.46 & 1.325 & $\$$ & Posi & $2^{7}$ \\
\hline & $c / z$ & -19.03 & -23.19 & 1.325 & $\$$ & Posi & 28 \\
\hline & $c / z$ & -23.19 & -19.03 & 1.325 & 3 & Positi & 2 \\
\hline & $C / z$ & -26.46 & -14.14 & 1.325 & \$ & Posi & 30 \\
\hline & $\mathrm{C} / \mathrm{z}$ & -2 & -8.71 & 1.325 & $\$$ & Pos & 3 \\
\hline 32 & $C / z$ & -29.86 & -2.94 & 1.325 & $\$$ & Posit & 32 \\
\hline
\end{tabular}

C

Revision: 1 
NEA/NSC/DOC(2006)1

Gas Cooled (Thermal) Reactor - GCR

PROTEUS-GCR-EXP-004

CRIT-REAC

\begin{tabular}{|c|c|c|c|c|c|c|c|}
\hline 501 & $\mathrm{C} / \mathrm{z}$ & -34.82 & 6.93 & 1.325 & $\$$ & Position & 1 \\
\hline 02 & $\mathrm{C} / \mathrm{z}$ & -32.80 & 13.59 & 1.325 & $\$$ & Position & 2 \\
\hline & $\mathrm{C} / \mathrm{z}$ & -29.52 & 19.72 & 1.325 & $\$$ & Position & 3 \\
\hline & $\mathrm{C} / \mathrm{z}$ & -25.10 & 25.10 & 1.325 & $\$$ & Position & 4 \\
\hline & $\mathrm{C} / \mathrm{z}$ & -19.72 & 29.52 & 1.325 & $\$$ & Position & \\
\hline & $\mathrm{C} / \mathrm{z}$ & -13.59 & 32.80 & .3 & $\$$ & Pos: & \\
\hline & $\mathrm{C} / \mathrm{z}$ & -6.93 & 34.82 & .3 & $\$$ & Pos: & 7 \\
\hline & $\mathrm{C} / \mathrm{z}$ & 0.00 & 35.50 & .32 & $\$$ & Position & 8 \\
\hline & $\mathrm{C} / \mathrm{z}$ & 6.93 & 34.82 & .32 & $\$$ & Position & \\
\hline & $\mathrm{C} / \mathrm{z}$ & 13.59 & 32.80 & .3 & $\$$ & Position & 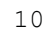 \\
\hline & $\mathrm{C} / \mathrm{z}$ & 19.72 & 29.52 & .3 & $\$$ & Position & 11 \\
\hline & $\mathrm{C} / \mathrm{z}$ & 25.10 & 25.10 & .3 & $\$$ & Posi & 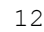 \\
\hline & $\mathrm{c} / \mathrm{z}$ & 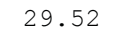 & 19.72 & .3 & $\$$ & Posi & \\
\hline & $\mathrm{C} / \mathrm{z}$ & 30 & 13.59 & 3 & $\$$ & Posi & 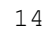 \\
\hline & $\mathrm{C} / \mathrm{z}$ & 34.82 & 6.93 & .3 & $\$$ & Position & \\
\hline & $\mathrm{C} / \mathrm{z}$ & 35.50 & 0.00 & 3 & $\$$ & Position & 1 \\
\hline & $\mathrm{c} / \mathrm{z}$ & 32 & -6.93 & .3 & $\$$ & Posi & \\
\hline & $\mathrm{C} / \mathrm{z}$ & 32.80 & -13.59 & .3 & $\$$ & Posi & 18 \\
\hline & $\mathrm{C} / \mathrm{z}$ & 29.52 & -19.72 & .3 & $\$$ & Posit & \\
\hline & $\mathrm{C} / \mathrm{z}$ & 25.10 & -25.10 & .3 & $\$$ & Posi & 20 \\
\hline & $\mathrm{C} / \mathrm{z}$ & 19.72 & -29.52 & .3 & $\$$ & Posi & 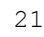 \\
\hline & $\mathrm{C} / \mathrm{z}$ & 13.59 & -32.80 & .3 & $\$$ & Posi & 22 \\
\hline & $\mathrm{C} / \mathrm{z}$ & 6.93 & -34.82 & .3 & $\$$ & Posi & 25 \\
\hline & $\mathrm{C} / \mathrm{z}$ & 0.00 & -35.50 &. & $\$$ & Pos & 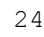 \\
\hline & $\mathrm{C} / \mathrm{z}$ & -6.93 & -34.82 & 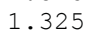 & $\$$ & Pos & \\
\hline & $\mathrm{C} / \mathrm{z}$ & -13.59 & -32.80 & L. & $\$$ & Posi & \\
\hline 27 & $\mathrm{C} / \mathrm{z}$ & -19.72 & -29.52 & .3 & $\$$ & Position & 21 \\
\hline & $\mathrm{C} / \mathrm{z}$ & -25.10 & -25 . & $\therefore$ & \$ & Posi & 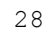 \\
\hline & $\mathrm{C} / \mathrm{z}$ & -29 & -19. & c. & $\$$ & Posi & 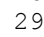 \\
\hline & $\mathrm{C} / \mathrm{z}$ & -32.80 & -13.59 & 1.3 & $\$$ & Position & 30 \\
\hline & $\mathrm{C} / \mathrm{z}$ & -34.82 & -6.93 & 1 . & \$ & Posi & 31 \\
\hline & $\mathrm{C} / \mathrm{z}$ & -35.50 & 0.00 & 1.3 & $\$$ & Pos: & 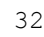 \\
\hline \\
\hline & & ng & & & & & \\
\hline & $\mathrm{C} / \mathrm{z}$ & -39.23 & 11.90 & 1.3 & $\$$ & Pos & 1 \\
\hline & $\mathrm{C} / \mathrm{z}$ & -36 & 19.33 & 1.3 & $\$$ & Po: & 2 \\
\hline & $\mathrm{C} / \mathrm{z}$ & -31.69 & 26.01 & 1.325 & $\$$ & Posi & 3 \\
\hline & $\mathrm{C} / \mathrm{z}$ & -26.01 & 31.69 & 1.3 & $\$$ & Posi & 4 \\
\hline & $\mathrm{C} / \mathrm{z}$ & -19.33 & 36.16 & 1. & $\$$ & Pos & 5 \\
\hline 6 & $\mathrm{C} / \mathrm{z}$ & -11 & 39.23 & 1.3 & $\$$ & Posi & 6 \\
\hline 07 & $\mathrm{C} / \mathrm{z}$ & -4.02 & 40.80 & 1.3 & $\$$ & Posi & 7 \\
\hline 08 & $\mathrm{c} / \mathrm{z}$ & 4.02 & 40.80 & 1.3 & $\$$ & Posi & 8 \\
\hline 09 & $\mathrm{c} / \mathrm{z}$ & 11.90 & 39.23 & 1.3 & $\$$ & Pos & 9 \\
\hline & $\mathrm{C} / \mathrm{z}$ & 19.33 & 36 . & 1. & $\$$ & $\mathrm{PC}$ & 10 \\
\hline 1 & $\mathrm{C} / \mathrm{z}$ & 26.01 & 31.69 & 1.3 & $\$$ & Pos: & 11 \\
\hline & $\mathrm{c} / \mathrm{z}$ & .69 & 26.01 & 1. & $\$$ & Pos & 12 \\
\hline 13 & $\mathrm{C} / \mathrm{z}$ & 6 & 19.33 & 1. & $\$$ & Pos & 13 \\
\hline 4 & $\mathrm{C} / \mathrm{z}$ & 39.23 & 11.90 & 1.3 & $\$$ & Posi & 14 \\
\hline & $\mathrm{c} / \mathrm{z}$ & 40.80 & 4.02 & 1.3 & $\$$ & Posi & 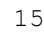 \\
\hline 6 & $\mathrm{C} / \mathrm{z}$ & .80 & -4.02 & 1.3 & $\$$ & Posi & 16 \\
\hline & $\mathrm{c} / \mathrm{z}$ & .23 & -11.90 & 1. & $\$$ & Pos & 17 \\
\hline & $\mathrm{C} / \mathrm{z}$ & 36.16 & -19.33 & 1.3 & $\$$ & Posi & 18 \\
\hline 19 & $\mathrm{C} / \mathrm{z}$ & .69 & -26.01 & 1.3 & $\$$ & Pos & 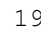 \\
\hline 0 & $\mathrm{C} / \mathrm{z}$ & 01 & -31.69 & 1. & $\$$ & Pos & 20 \\
\hline 1 & $\mathrm{c} / \mathrm{z}$ & 19.33 & -36.16 & 1.3 & $\$$ & Posi & 21 \\
\hline 522 & $c / z$ & 11.90 & -39.23 & 1.32 & $\$$ & Posi & 22 \\
\hline 523 & $\mathrm{C} / \mathrm{z}$ & .02 & -40.80 & 1.325 & $\$$ & Posi & 23 \\
\hline & $c / z$ & -4.02 & -40.80 & 1.3 & $\$$ & Posi & 24 \\
\hline 525 & $\mathrm{C} / \mathrm{z}$ & -11.90 & -39.23 & 1.32 & $\$$ & Posi & 25 \\
\hline 626 & $\mathrm{c} / \mathrm{z}$ & -19.33 & -36.16 & 1.325 & $\$$ & Posi & 26 \\
\hline 627 & $\mathrm{C} / \mathrm{z}$ & -26.01 & -31.69 & 1.325 & $\$$ & Posi & 27 \\
\hline 528 & $\mathrm{C} / \mathrm{z}$ & -31.69 & -26.01 & 1.32 & $\$$ & Posit & 28 \\
\hline 529 & $\mathrm{C} / \mathrm{z}$ & -36.16 & -19.33 & 1.3 & $\$$ & Posi & 29 \\
\hline 630 & $\mathrm{c} / \mathrm{z}$ & -39.23 & -11.90 & 1.3 & $\$$ & Posi & 30 \\
\hline 631 & $\mathrm{C} / \mathrm{z}$ & -40.80 & -4.02 & 1.3 & $\$$ & Posi & 31 \\
\hline 632 & $c / z$ & -40.80 & 4.02 & 1.325 & $\$$ & Posit & 32 \\
\hline \\
\hline & & 1 & & & & & \\
\hline 01 & $\mathrm{C} / \mathrm{z}$ & -42.73 & 17.70 & 1.3 & $\$$ & Position & 1 \\
\hline 702 & $\mathrm{C} / \mathrm{z}$ & -38.46 & 25.70 & 1.325 & $\$$ & Posit & 2 \\
\hline 2703 & $c / z$ & -32.70 & 32.70 & 1.325 & $\$$ & Posit & 3 \\
\hline 2704 & $\mathrm{C} / \mathrm{z}$ & -25.70 & 38.46 & 1.325 & $\$$ & Posit & 4 \\
\hline & $\mathrm{C} / \mathrm{z}$ & -17.70 & 42.73 & 1.325 & $\$$ & Position & 5 \\
\hline & $\mathrm{c} / \mathrm{z}$ & -9.02 & 45.36 & 1.325 & $\$$ & Posit & 6 \\
\hline & $\mathrm{C} / \mathrm{z}$ & 0.00 & 46.25 & 1.325 & $\$$ & Position & \\
\hline
\end{tabular}

Revision: 1 
NEA/NSC/DOC(2006)1

Gas Cooled (Thermal) Reactor - GCR

PROTEUS-GCR-EXP-004

CRIT-REAC

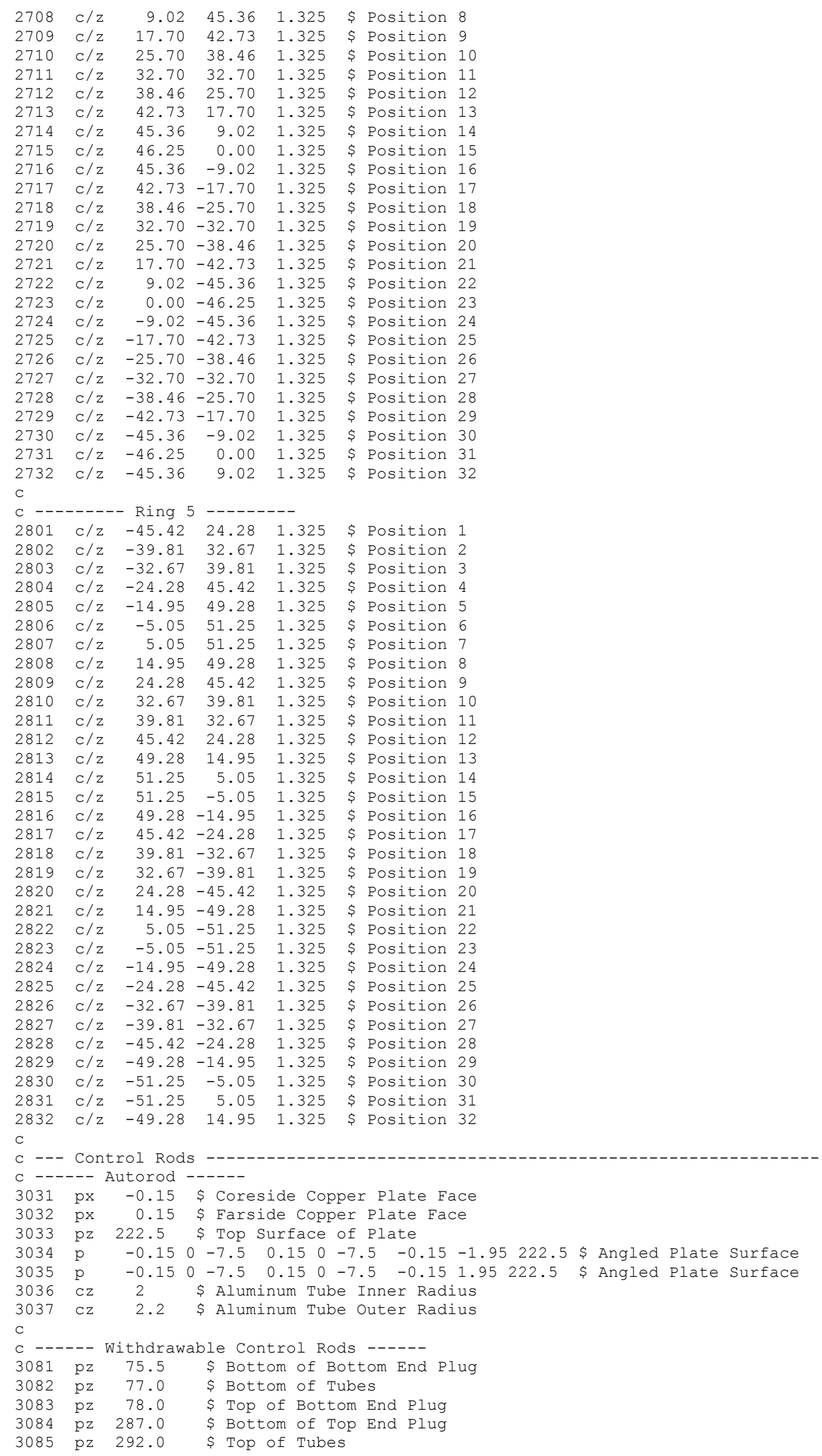

Revision: 1 
NEA/NSC/DOC(2006)1

\section{Gas Cooled (Thermal) Reactor - GCR \\ PROTEUS-GCR-EXP-004 \\ CRIT-REAC}

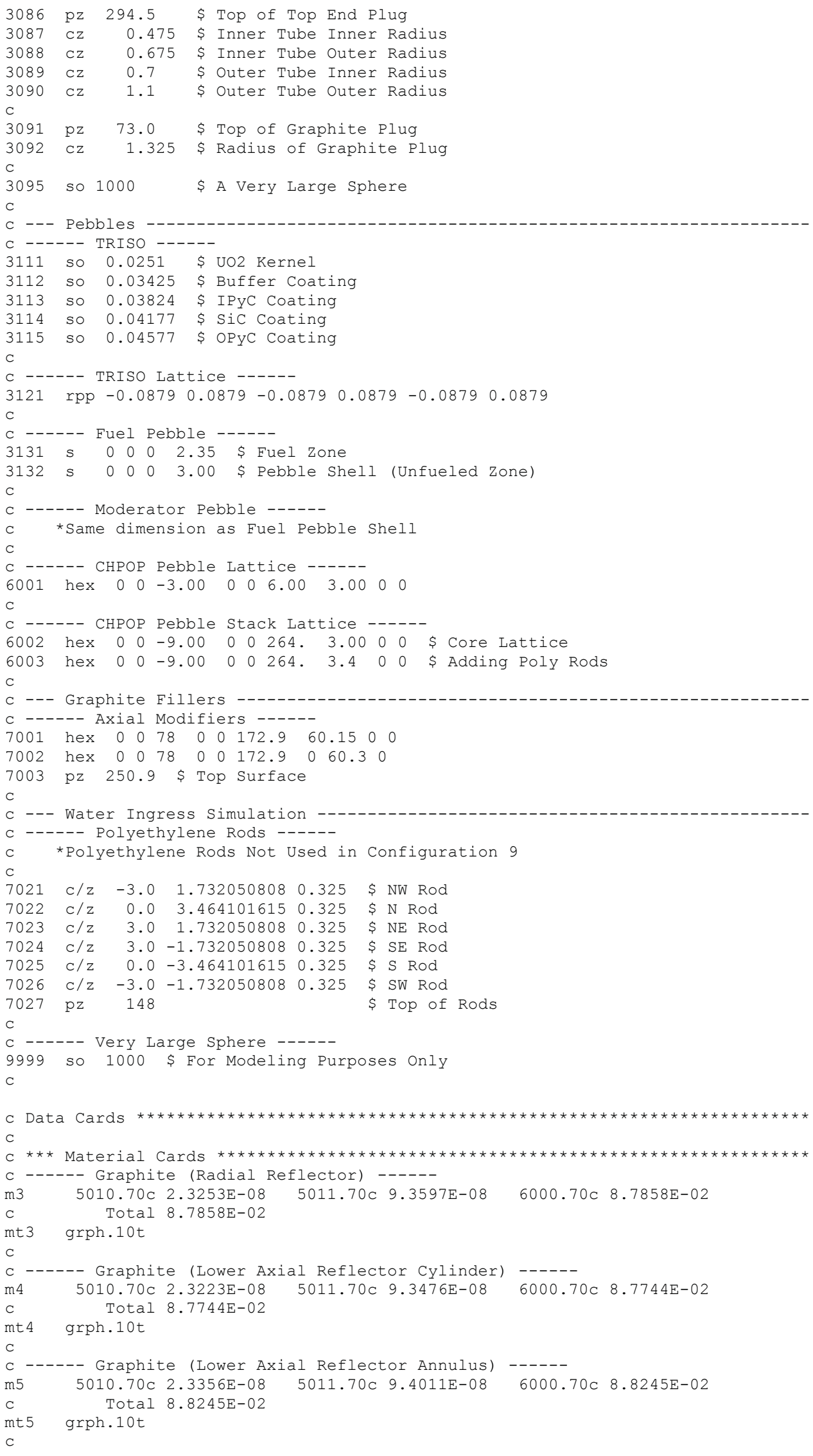

Revision: 1 
NEA/NSC/DOC(2006)1

\section{Gas Cooled (Thermal) Reactor - GCR \\ PROTEUS-GCR-EXP-004 CRIT-REAC}

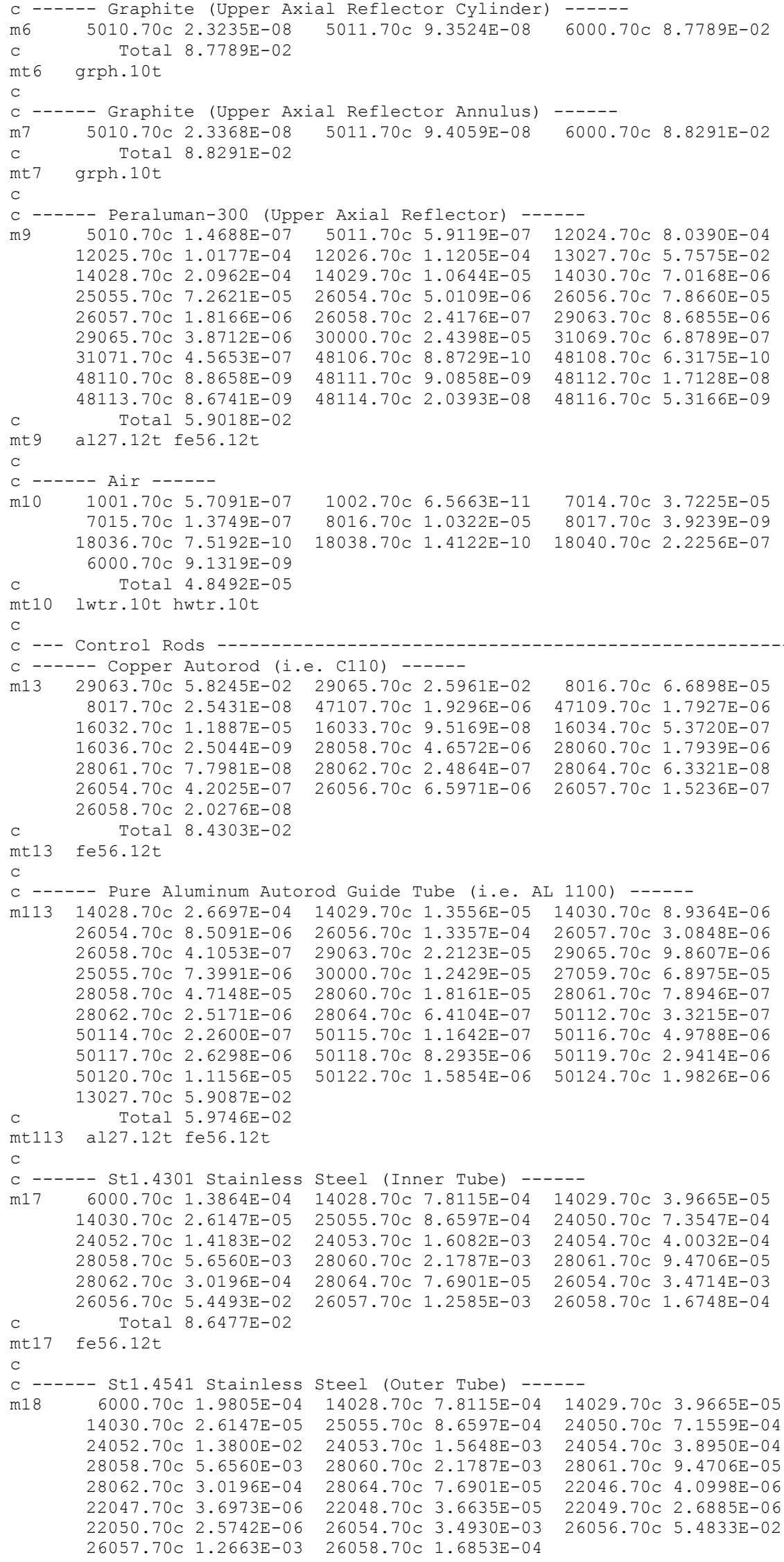

Revision: 1 
NEA/NSC/DOC(2006)1

\section{Gas Cooled (Thermal) Reactor - GCR \\ PROTEUS-GCR-EXP-004 CRIT-REAC}

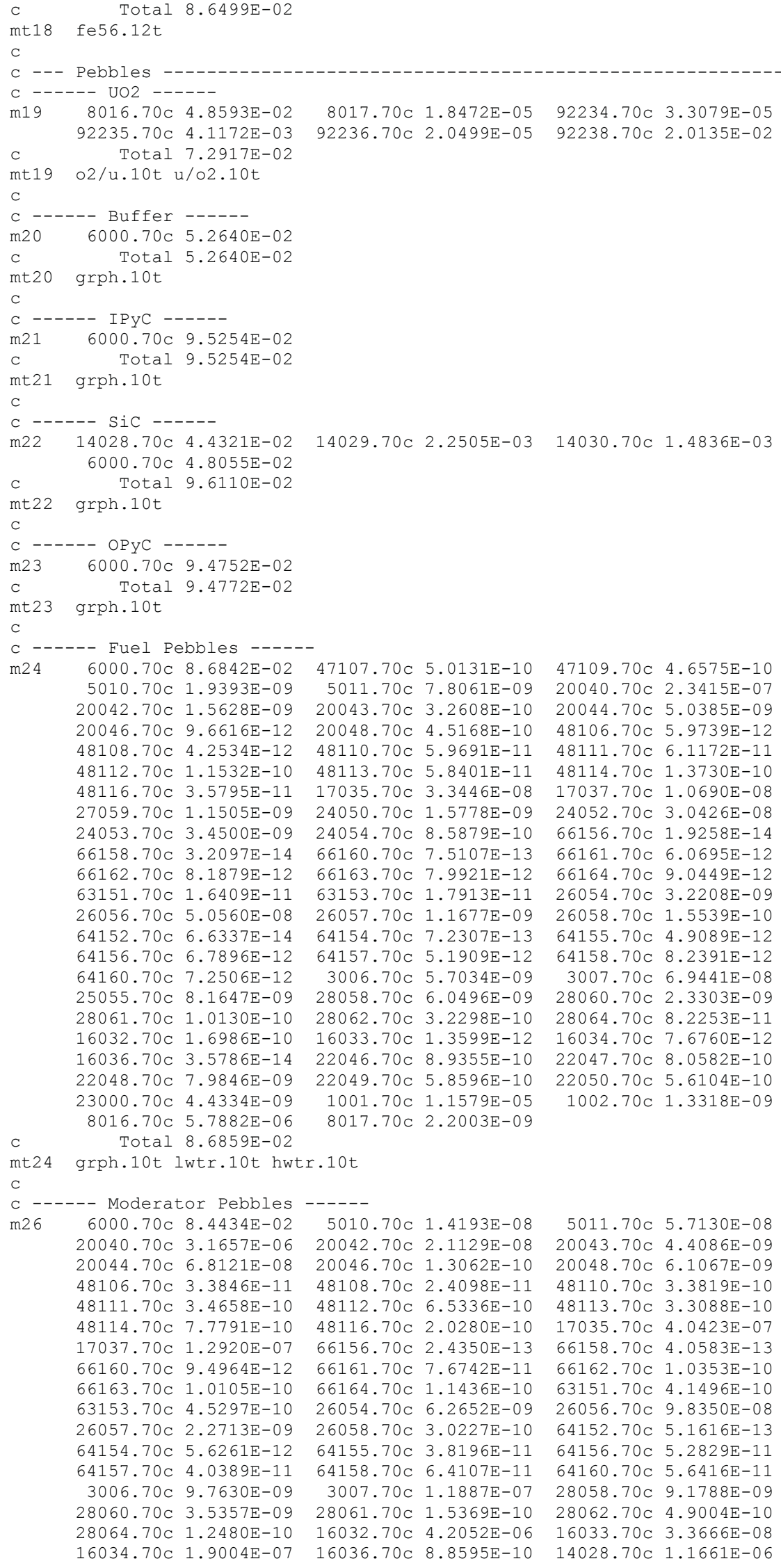

Revision: 1 
NEA/NSC/DOC(2006)1

\section{Gas Cooled (Thermal) Reactor - GCR \\ PROTEUS-GCR-EXP-004 CRIT-REAC}

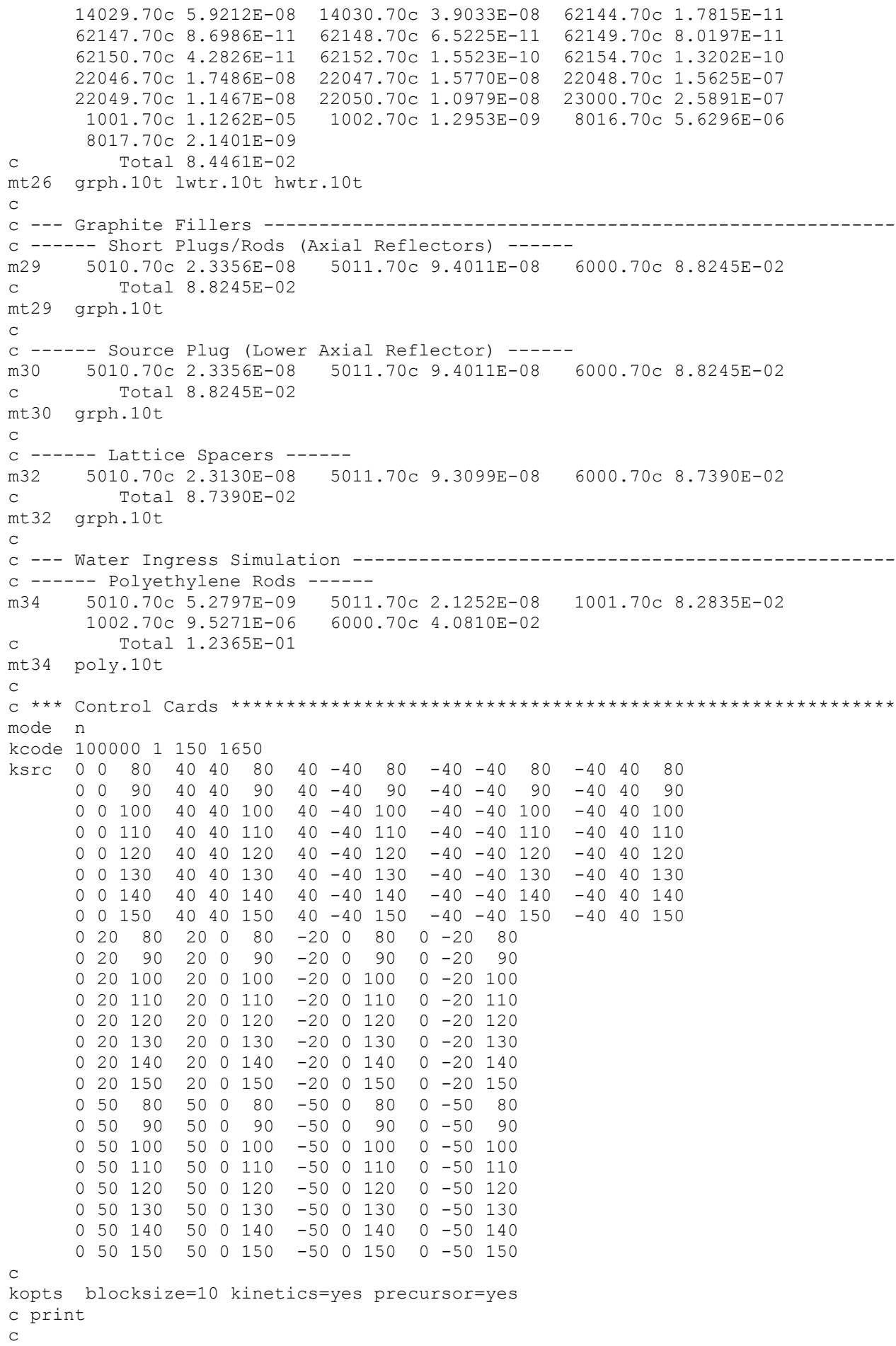

Revision: 1 
NEA/NSC/DOC(2006)1

Gas Cooled (Thermal) Reactor - GCR

PROTEUS-GCR-EXP-004

CRIT-REAC

\section{A.2 Buckling and Extrapolation Length Configurations}

Buckling and extrapolation length measurements were performed but have not yet been evaluated.

\section{A.3 Spectral-Characteristics Configurations}

Spectral characteristics measurements were performed but have not yet been evaluated. 
NEA/NSC/DOC(2006)1

\section{Gas Cooled (Thermal) Reactor - GCR \\ PROTEUS-GCR-EXP-004 \\ CRIT-REAC}

\section{A.4 Reactivity-Effects Configurations}

The input decks for analysis of most reactivity effects measurements are those of the critical configurations (Appendix A.1 described in Section 3.1) with adjustments discussed in Section 3.4.2.

A sample model that includes the safety/shutdown rods for Core 9 is provided below.

\section{MCNP5 Input Deck for Core 9 with Safety/Shutdown Rods:}

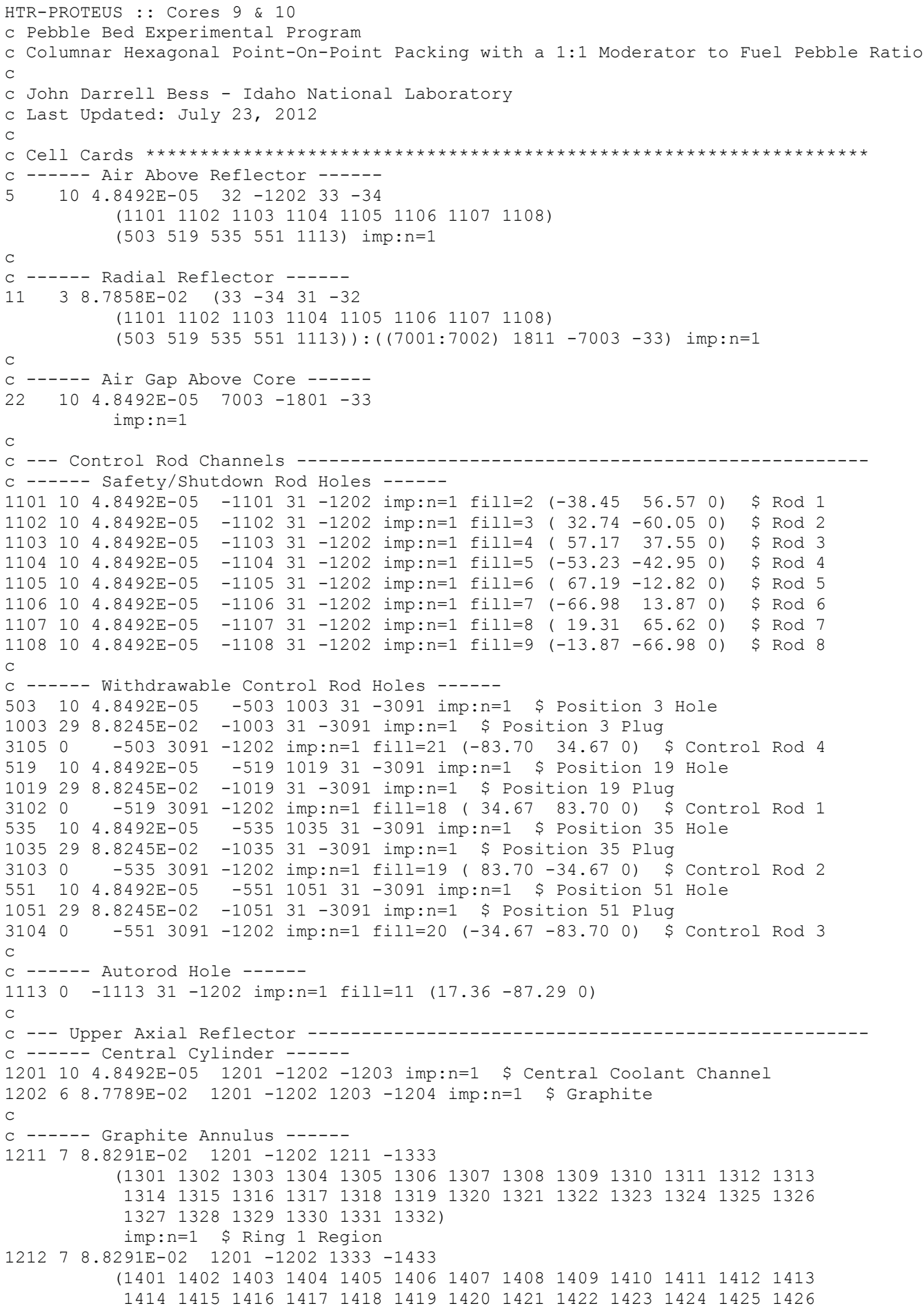

Revision: 1 
NEA/NSC/DOC(2006)1

\section{Gas Cooled (Thermal) Reactor - GCR \\ PROTEUS-GCR-EXP-004 CRIT-REAC}

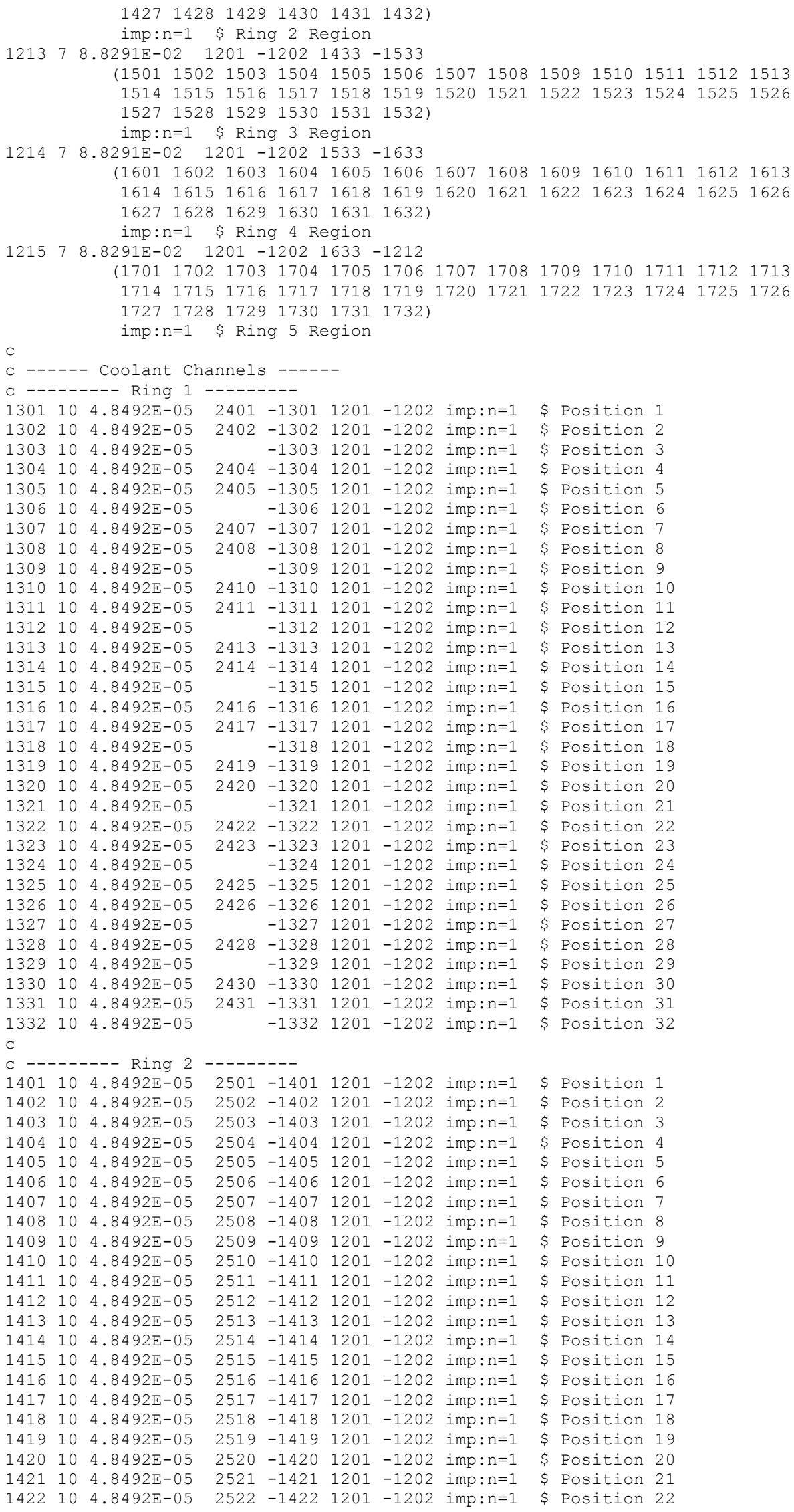

Revision: 1 
NEA/NSC/DOC(2006)1

Gas Cooled (Thermal) Reactor - GCR

PROTEUS-GCR-EXP-004 CRIT-REAC

\begin{tabular}{|c|c|c|c|c|c|c|c|c|c|c|}
\hline 1423 & 0 & $E-05$ & 23 & -1423 & & -1202 & imp: $n=1$ & $\$$ & on & \\
\hline & 10 & $4.8492 \mathrm{E}-05$ & 2524 & -1424 & 201 & -1202 & imp: $n=1$ & $\$$ & sition & 4 \\
\hline 25 & 10 & $4.8492 E-05$ & 2525 & -1425 & 1201 & -1202 & imp: $n=1$ & $\$$ & Position & 25 \\
\hline 2 & 10 & $4.8492 \mathrm{E}-05$ & 2526 & -1426 & 1201 & -1202 & imp: $n=1$ & $\$$ & Position & 26 \\
\hline 27 & 10 & $4.8492 \mathrm{E}-05$ & 2527 & -1427 & 201 & -1202 & imp: $n=1$ & $\$$ & osition & 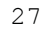 \\
\hline & 10 & $4.8492 \mathrm{E}-05$ & 2528 & -1428 & 201 & -1202 & imp: $n=1$ & $\$$ & osition & \\
\hline 2 & 10 & $4.8492 \mathrm{E}-05$ & 2529 & -1429 & 201 & -1202 & imp: $n=1$ & $\$$ & Position & \\
\hline & 10 & $4.8492 E-05$ & 2530 & -1430 & 201 & -1202 & imp: $n=1$ & $\$$ & osition & \\
\hline & 10 & $4.8492 \mathrm{E}-05$ & 31 & -1431 & 201 & -1202 & imp: $n=1$ & $\$$ & & \\
\hline & 10 & $4.8492 \mathrm{E}-05$ & 32 & -1432 & 01 & -1202 & imp: $n=1$ & $\$$ & & \\
\hline \\
\hline \\
\hline & 0 & 4.849 & \multirow[t]{2}{*}{2601} & -1501 & 01 & -1202 & imp: $n=1$ & $\$$ & on & 1 \\
\hline & 0 & $4.8492 \mathrm{E}-05$ & & -1502 & 201 & -1202 & imp: $n=1$ & $\$$ & osition & \\
\hline & 10 & $4.8492 \mathrm{E}-05$ & & -1503 & 201 & -1202 & imp: $n=1$ & $\$$ & Position & \\
\hline & 10 & $4.8492 \mathrm{E}-05$ & \multirow[t]{2}{*}{2604} & -1504 & 201 & -1202 & imp: $n=1$ & $\$$ & Position & \\
\hline & 10 & $4.8492 E-05$ & & -1505 & & -1202 & imp: $n=1$ & $\$$ & Position & \\
\hline & 10 & $4.8492 \mathrm{E}-05$ & & -1506 & 201 & -1202 & imp: $n=1$ & $\$$ & Position & \\
\hline & 10 & $4.8492 \mathrm{E}-05$ & \multirow[t]{2}{*}{2607} & -1507 & & -1202 & imp: $n=1$ & $\$$ & ion & \\
\hline & 10 & $4.8492 \mathrm{E}-05$ & & -1508 & 201 & -1202 & imp: $n=1$ & $\$$ & ion & \\
\hline & 10 & $4.8492 \mathrm{E}-05$ & & -1509 & 201 & -1202 & imp: $n=1$ & $\$$ & ion & \\
\hline & 10 & $4.8492 \mathrm{E}-05$ & \multirow[t]{2}{*}{2610} & -1510 & 01 & -1202 & imp: $n=1$ & $\$$ & Position & \\
\hline & 10 & $4.8492 E-05$ & & -1511 & 01 & -1202 & imp: $n=1$ & $\$$ & Position & $\perp$ \\
\hline & 10 & $4.8492 \mathrm{E}-05$ & 2612 & -1512 & 01 & -1202 & imp: $n=1$ & $\$$ & ion & \\
\hline & 10 & $4.8492 \mathrm{E}-05$ & 2613 & -1513 & 201 & -1202 & imp: $n=1$ & $\$$ & Position & \\
\hline & 10 & $4.8492 E-05$ & & -1514 & 201 & -1202 & imp: $n=1$ & $\$$ & Position & 4 \\
\hline & 10 & $4.8492 E-05$ & 15 & -1515 & 1 & -1202 & imp: $n=1$ & $\$$ & Position & \\
\hline & 10 & $4.8492 \mathrm{E}-05$ & & -1516 & & -1202 & imp: $n=1$ & $\$$ & Position & \\
\hline & 10 & $4.8492 \mathrm{E}-05$ & & -1517 & 01 & -1202 & imp: $n=1$ & $\$$ & Position & \\
\hline & 10 & $4.8492 E-05$ & & -1518 & 01 & -1202 & imp: $n=1$ & $\$$ & Position & \\
\hline & 10 & $4.8492 \mathrm{E}-05$ & & -1519 & 201 & -1202 & imp: $n=1$ & $\$$ & Position & \\
\hline & 10 & $4.8492 \mathrm{E}-05$ & & -1520 & 201 & -1202 & imp: $n=1$ & $\$$ & Position & 0 \\
\hline & 10 & $4.8492 E-05$ & & -1521 & & -1202 & imp: $n=1$ & $\$$ & Position & \\
\hline & 10 & $4.8492 \mathrm{E}-05$ & & -1522 & 01 & -1202 & imp: $n=1$ & $\$$ & Position & \\
\hline & 10 & $4.8492 \mathrm{E}-05$ & & -1523 & 1 & -12 & imp: $n=1$ & $\$$ & ion & 23 \\
\hline & 10 & $4.8492 \mathrm{E}-05$ & & -1524 & 1201 & -1202 & imp: $n=1$ & $\$$ & Position & 24 \\
\hline & 10 & $4.8492 \mathrm{E}-05$ & & -1525 & 1201 & -1202 & imp: $n=1$ & $\$$ & Pos & \\
\hline & 10 & $4.8492 \mathrm{E}-05$ & & -1526 & 1201 & -12 & imp: $n=1$ & $\$$ & ion & \\
\hline & 10 & $4.8492 E-05$ & 2627 & -1527 & 1201 & -1202 & imp: $n=1$ & $\$$ & ion & 27 \\
\hline & 10 & $4.8492 \mathrm{E}-05$ & & -1528 & 1201 & -1202 & imp: $n=1$ & $\$$ & Position & 28 \\
\hline & 10 & $4.8492 E-05$ & & -1529 & & -1202 & imp: $n=1$ & $\$$ & Posi & \\
\hline & 10 & $4.8492 \mathrm{E}-05$ & 2630 & -1530 & 1 & -12 & imp: $n=1$ & $\$$ & ion & 30 \\
\hline & 10 & $4.8492 \mathrm{E}-05$ & & -1531 & 12 & -1202 & $i m p: n=1$ & $\$$ & Position & 31 \\
\hline & 10 & $4.8492 \mathrm{E}-05$ & 32 & -1532 & & -1202 & imp: $n=1$ & $\$$ & Position & 32 \\
\hline & & & & & & & & & & \\
\hline & & --- & & - & & & & & & \\
\hline & 10 & 4.849 & & -1601 & & -1202 & $i m p: n=1$ & $\$$ & Lon & 1 \\
\hline & 10 & $4.8492 \mathrm{E}-05$ & 02 & -1602 & 1201 & -1202 & imp: $n=1$ & $\$$ & Posit & 2 \\
\hline & 10 & $4.8492 E-05$ & 2703 & -1603 & 1201 & -1202 & $i m p: n=1$ & $\$$ & ition & 3 \\
\hline & 10 & $4.8492 \mathrm{E}-05$ & 2704 & -1604 & 1201 & -1202 & $i m p: n=1$ & $\$$ & Position & 4 \\
\hline & 10 & $4.8492 \mathrm{E}-05$ & & -1605 & 01 & -1202 & imp: $n=1$ & $\$$ & Position & 5 \\
\hline & 10 & $4.8492 \mathrm{E}-05$ & 2706 & -1606 & 1201 & -1202 & $i m p: n=1$ & $\$$ & Position & 6 \\
\hline & 10 & $4.8492 \mathrm{E}-05$ & 2707 & -1607 & 1201 & -12 & imp: $n=1$ & $\$$ & ion & 7 \\
\hline & 10 & $4.8492 \mathrm{E}-05$ & 2708 & -1608 & 1201 & -1202 & $i m p: n=1$ & $\$$ & Position & 8 \\
\hline & 10 & $4.8492 \mathrm{E}-05$ & 09 & -1609 & 1201 & -1202 & imp: $n=1$ & $\$$ & Position & 9 \\
\hline & 10 & $4.8492 \mathrm{E}-05$ & 2710 & -1610 & 1201 & -1202 & $i m p: n=1$ & $\$$ & Position & 10 \\
\hline & 10 & $4.8492 E-05$ & & -1611 & & -1202 & $i m p: n=1$ & $\$$ & Position & 11 \\
\hline & 10 & $4.8492 \mathrm{E}-05$ & 27 & -1612 & 1. & -1202 & imp: $n=1$ & $\$$ & Position & 12 \\
\hline & 10 & $4.8492 \mathrm{E}-05$ & 2713 & -1613 & 1201 & -1202 & imp: $n=1$ & $\$$ & Position & 13 \\
\hline & 10 & $4.8492 \mathrm{E}-05$ & 2714 & -1614 & 1201 & -1202 & imp: $n=1$ & $\$$ & Position & 14 \\
\hline & 10 & $4.8492 \mathrm{E}-05$ & & -1615 & & -1202 & $i m p: n=1$ & $\$$ & Position & 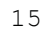 \\
\hline & 10 & $4.8492 \mathrm{E}-05$ & 2716 & -1616 & 1201 & -1202 & imp: $n=1$ & $\$$ & Position & 16 \\
\hline & 10 & $4.8492 \mathrm{E}-05$ & 2717 & -1617 & 1201 & -1202 & $i m p: n=1$ & $\$$ & Position & 17 \\
\hline & 10 & $4.8492 \mathrm{E}-05$ & 2718 & -1618 & 1201 & -1 & imp: $n=1$ & $\$$ & Position & \\
\hline & 10 & $4.8492 \mathrm{E}-05$ & & -1619 & 1201 & -1202 & $i m p: n=1$ & $\$$ & Position & 19 \\
\hline & 10 & $4.8492 E-05$ & 2720 & -1620 & 1201 & -1202 & imp: $n=1$ & $\$$ & Position & 2 \\
\hline & 10 & $4.8492 \mathrm{E}-05$ & 2721 & -1621 & 1201 & -12 & $i m p: n=1$ & $\$$ & Posi & 21 \\
\hline & 10 & $4.8492 \mathrm{E}-05$ & & -1622 & 1201 & -12 & imp: $n=1$ & $\$$ & Position & 22 \\
\hline & 10 & $4.8492 \mathrm{E}-05$ & 2723 & -1623 & 1201 & -1202 & imp: $n=1$ & $\$$ & Position & 23 \\
\hline & 10 & $4.8492 \mathrm{E}-05$ & 2724 & -1624 & 1201 & -1202 & $i m p: n=1$ & $\$$ & Position & \\
\hline & 10 & $4.8492 \mathrm{E}-05$ & 2725 & -1625 & 12 & -1202 & imp: $n=1$ & $\$$ & Position & 25 \\
\hline & 10 & $4.8492 E-05$ & 2726 & -1626 & 1201 & -1202 & $i m p: n=1$ & $\$$ & Position & 26 \\
\hline & 10 & $4.8492 E-05$ & 2727 & -1627 & 1201 & -1202 & imp: $n=1$ & $\$$ & Position & 27 \\
\hline & 10 & $4.8492 \mathrm{E}-05$ & 2728 & -1628 & 1201 & -1202 & $i m p: n=1$ & $\$$ & Position & 28 \\
\hline & 10 & $4.8492 E-05$ & 2729 & -1629 & 1201 & -1202 & $i m p: n=1$ & $\$$ & Position & 29 \\
\hline & 10 & $4.8492 \mathrm{E}-05$ & 2730 & -1630 & 1201 & -1202 & $i m p: n=1$ & $\$$ & Position & 30 \\
\hline
\end{tabular}

Revision: 1 
NEA/NSC/DOC(2006)1

Gas Cooled (Thermal) Reactor - GCR

PROTEUS-GCR-EXP-004 CRIT-REAC

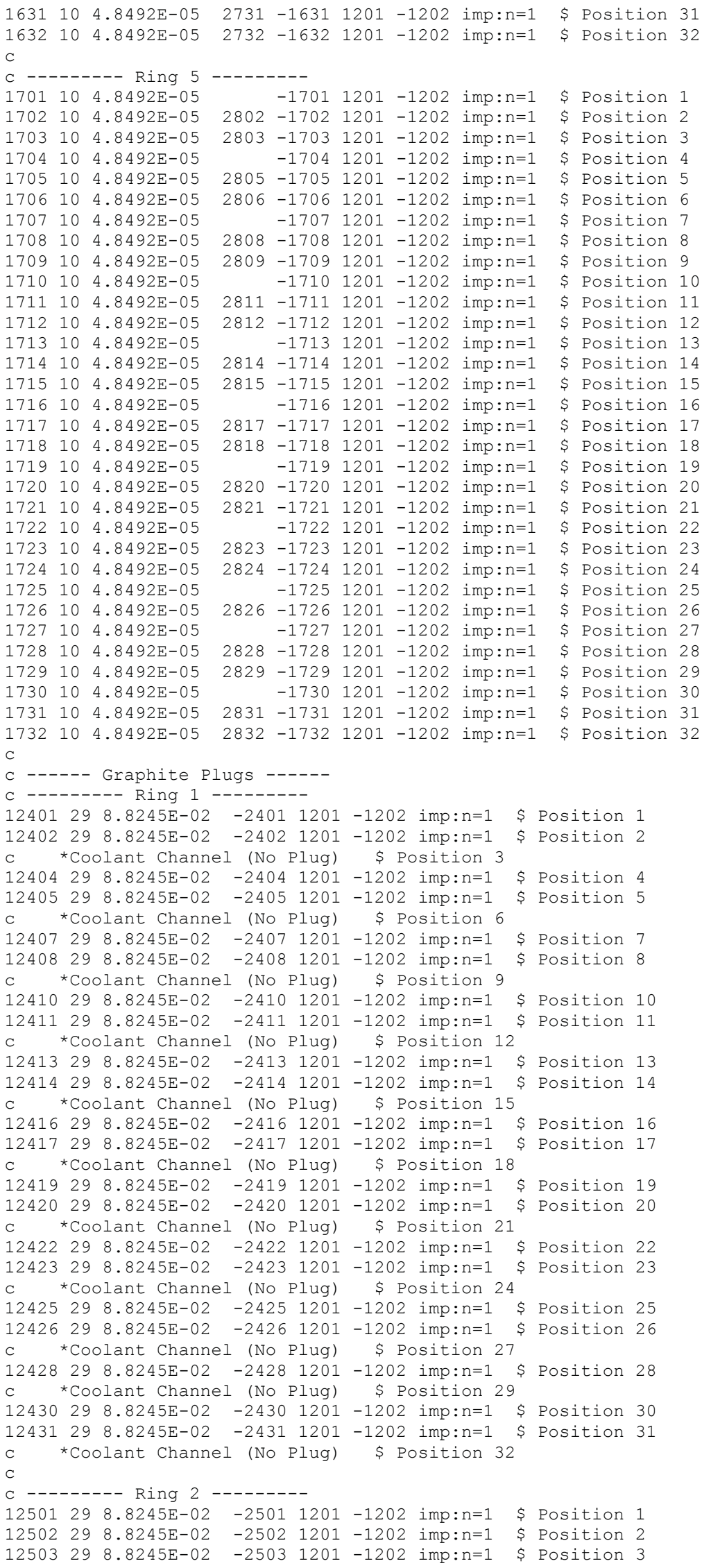

Revision: 1 
NEA/NSC/DOC(2006)1

Gas Cooled (Thermal) Reactor - GCR

PROTEUS-GCR-EXP-004 CRIT-REAC

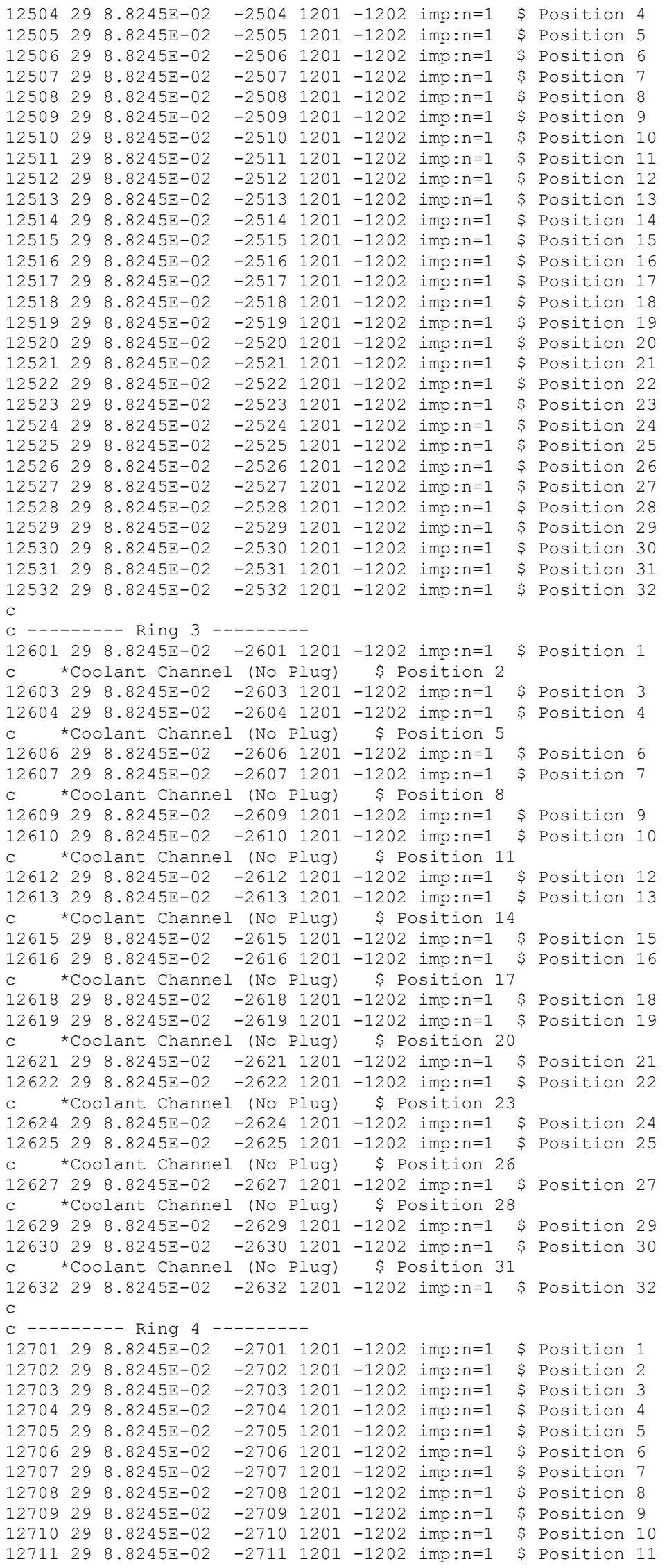

Revision: 1

Date: March 31, 2014

Page 249 of 375 
NEA/NSC/DOC(2006)1

\section{Gas Cooled (Thermal) Reactor - GCR \\ PROTEUS-GCR-EXP-004 CRIT-REAC}

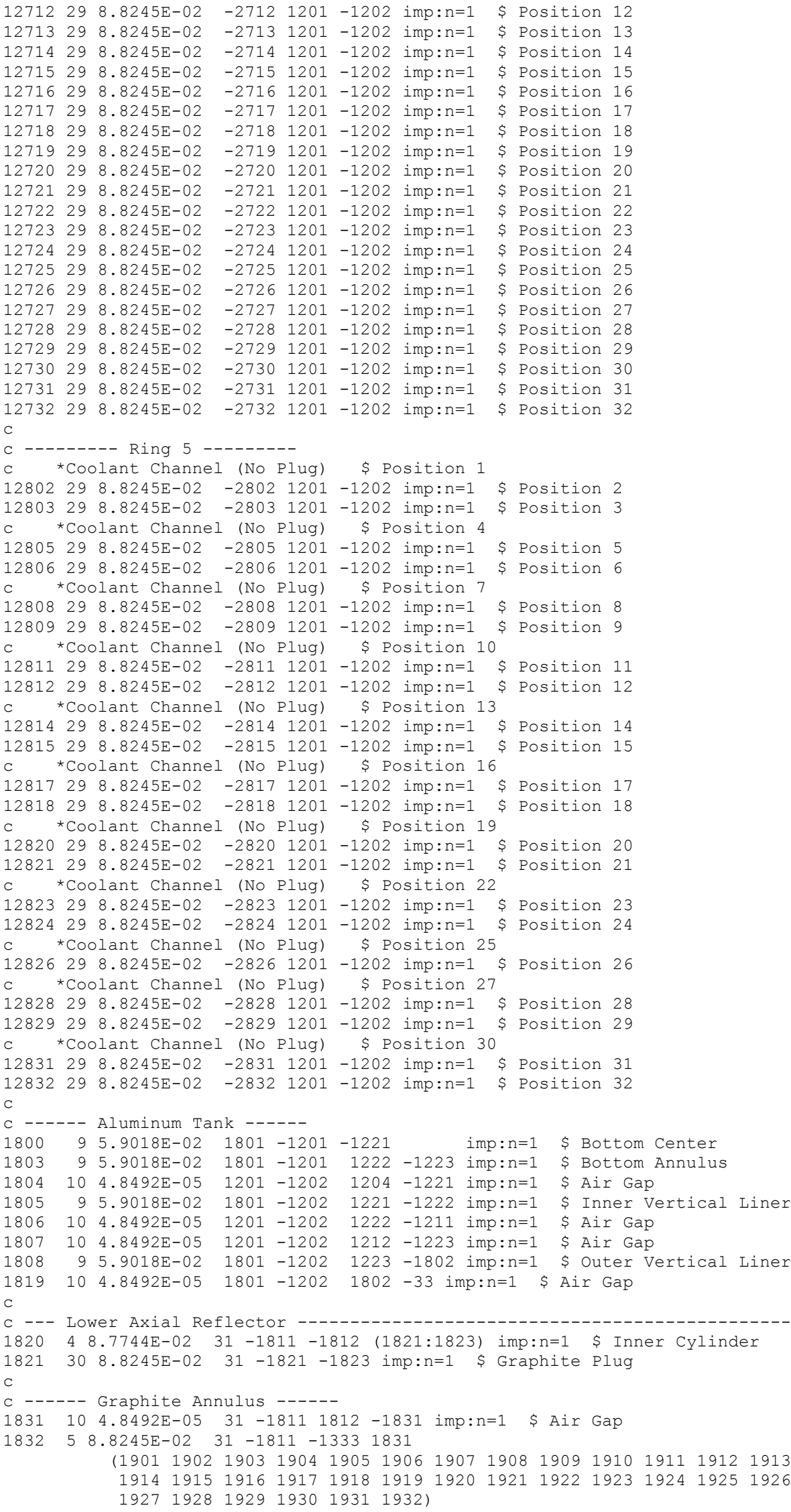

Revision: 1 
NEA/NSC/DOC(2006)1

\section{Gas Cooled (Thermal) Reactor - GCR \\ PROTEUS-GCR-EXP-004 CRIT-REAC}

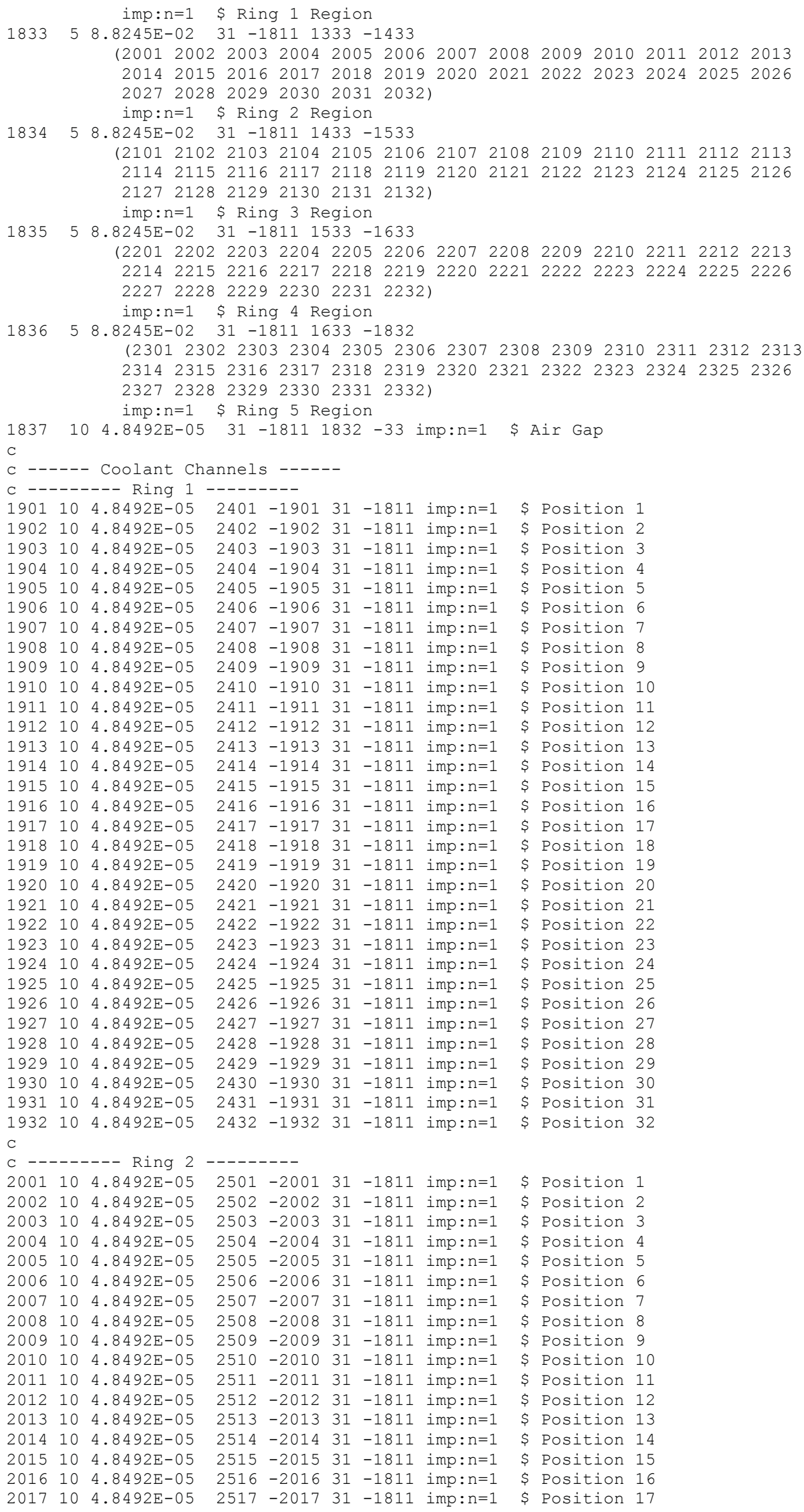

Revision: 1 
NEA/NSC/DOC(2006)1

Gas Cooled (Thermal) Reactor - GCR

PROTEUS-GCR-EXP-004 CRIT-REAC

\begin{tabular}{|c|c|c|c|c|c|c|c|c|c|c|}
\hline 018 & 10 & ( & 18 & -2018 & 1 & -1811 & imp: $n=1$ & & & \\
\hline & 10 & $492 E-05$ & 19 & -2019 & 31 & -1811 & imp:n=1 & $T_{1}$ & & \\
\hline 020 & 10 & $4.8492 E-05$ & 2520 & -2020 & 31 & -1811 & imp: $n=1$ & $\$$ & osition & \\
\hline & 10 & $4.8492 E-05$ & 521 & -2021 & 31 & -1811 & imp: $n=1$ & $\$$ & osition & \\
\hline & 0 & $4.8492 \mathrm{E}-05$ & 522 & -2022 & 31 & -1811 & $i m p: n=1$ & $\$$ & osition & \\
\hline & 10 & $4.8492 E-05$ & 23 & -2023 & 31 & -1811 & imp:n=1 & $\$$ & osition & \\
\hline & 0 & $4.8492 \mathrm{E}-05$ & 524 & -2024 & 31 & -1811 & $i m p: n=1$ & $\$$ & Position & \\
\hline & 0 & $4.8492 E-05$ & 25 & -2025 & 31 & -1811 & imp: $n=1$ & $\$$ & ositio & \\
\hline & 0 & $4.8492 E-05$ & 26 & -2026 & 31 & -1811 & imp: $n=1$ & $\$$ & $n$ & \\
\hline & 0 & $4.8492 \mathrm{E}-05$ & 27 & -2027 & 1 & -1811 & imp: $n=1$ & $\$$ & osition & \\
\hline & 0 & $4.8492 \mathrm{E}-05$ & 28 & -2028 & 1 & -1811 & imp:n=1 & $\$$ & osition & \\
\hline & 0 & $4.8492 \mathrm{E}-05$ & 29 & -2029 & 31 & -1811 & $i m p: n=1$ & $\$$ & ositic & \\
\hline & 10 & $4.8492 \mathrm{E}-05$ & & -2030 & 1 & -1811 & imp:n=1 & $\$$ & osition & \\
\hline & 0 & $92 E-05$ & 1 & -2031 & 31 & -1811 & imp: $n=1$ & $\$$ & osition & \\
\hline & 0 & $92 E-05$ & 2 & -2032 & 31 & -1811 & imp:n=1 & $\$$ & osition & \\
\hline \\
\hline & & 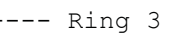 & & 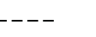 & & & & & & \\
\hline & & 4.8492 & & -2101 & 31 & -1811 & imp: $n=1$ & $\$$ & Position & 1 \\
\hline & 0 & $92 E-05$ & & -2102 & 31 & -1811 & $i m p: n=1$ & $\$$ & $n$ & \\
\hline & 0 & $4.8492 \mathrm{E}-05$ & 3 & -2103 & 1 & -1811 & imp:n=1 & $\$$ & ion & \\
\hline & 10 & 4.8 & & -21 & 31 & -18 & imp: $n=1$ & $\$$ & cion & \\
\hline & 10 & $4.8492 \mathrm{E}-05$ & 5 & -2105 & 31 & -1811 & imp: $n=1$ & $\$$ & Position & \\
\hline & 10 & $4.8492 E-05$ & 06 & -2106 & 31 & -1811 & imp: $n=1$ & $\$$ & Position & \\
\hline & 10 & 4.84921 & 7 & -2107 & 31 & -1811 & imp:n=1 & $\$$ & ition & \\
\hline & 10 & $4.8492 E-05$ & 08 & -2108 & 31 & -1811 & imp: $n=1$ & $\$$ & Position & \\
\hline & 10 & $4.8492 E-05$ & 09 & -2109 & 31 & -1811 & imp: $n=1$ & $\$$ & ition & \\
\hline & 10 & $4.8492 \mathrm{E}-05$ & 0 & -2110 & 31 & -1811 & imp: $n=1$ & $\$$ & tion & \\
\hline & 10 & 4.84921 & & -2111 & 31 & -1811 & imp:n=1 & $\$$ & tion & \\
\hline & 10 & $4.8492 E-05$ & & -2112 & 31 & -1811 & imp:n=1 & $\$$ & Position & \\
\hline & 10 & $4.8492 E-05$ & 13 & -2113 & 31 & -1811 & imp: $n=1$ & $\$$ & Po: & \\
\hline & 10 & $4.8492 \mathrm{E}-05$ & 4 & -2114 & 31 & -18 & imp:n=1 & $\$$ & Pos & \\
\hline & 10 & $4.8492 \mathrm{E}-05$ & 5 & -2115 & 31 & -1811 & $i m p: n=1$ & $\$$ & tion & \\
\hline & 10 & $92 E-05$ & & -2116 & 31 & -1811 & imp:n=1 & $\$$ & ition & \\
\hline & 10 & $4.8492 E-05$ & 17 & -2117 & 31 & -1811 & $i m p: n=1$ & $\$$ & Pos & \\
\hline & 10 & $4.8492 \mathrm{E}-05$ & & -2118 & 31 & -1811 & imp:n=1 & $\$$ & Pos & \\
\hline & 10 & $92 E-05$ & 9 & -2119 & 31 & -18 & imp: $n=1$ & $\$$ & Position & \\
\hline & 10 & $4.8492 E-05$ & 20 & -2120 & 31 & -1811 & $i m p: n=1$ & $\$$ & ion & 0 \\
\hline & 10 & $92 E-05$ & 2 & -2121 & 31 & -18 & imp:n=1 & $\$$ & Pos & \\
\hline & 10 & $4.8492 \mathrm{E}$ & 2 & -2122 & 31 & -1811 & $i m p: n=1$ & $\$$ & Pos & \\
\hline & 10 & $4.8492 E-05$ & 23 & -2123 & 31 & -1811 & imp:n=1 & $\$$ & Eion & \\
\hline & 10 & $4.8492 E-05$ & 24 & -2124 & 31 & -1811 & imp: $n=1$ & $\$$ & Pos & 4 \\
\hline & 10 & -05 & 2 & -21 & 31 & -1 & imp:n=1 & $\$$ & Pos & \\
\hline & 10 & 4.8 & 2626 & -2126 & 31 & -18 & $i m p: n=1$ & $\$$ & ion & 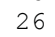 \\
\hline & 10 & $4.8492 E-05$ & 2627 & -2127 & 31 & -1811 & imp: $n=1$ & $\$$ & Position & 7 \\
\hline & 10 & $4.8492 \mathrm{E}-05$ & 26 & -2128 & 31 & -18 & $i m p: n=1$ & $\$$ & tion & 0 \\
\hline & 10 & $4.8492 \mathrm{E}-05$ & & -2129 & 31 & -18 & imp:n=1 & $\$$ & Pos & \\
\hline & 10 & $2 E-05$ & 26 & -2130 & 31 & -18 & $i m p: n=1$ & $\$$ & Position & 30 \\
\hline & 10 & $4.8492 E-05$ & 26 & -2131 & 31 & -1811 & imp: $n=1$ & $\$$ & Position & 1 \\
\hline & 10 & $4.8492 E-05$ & & -21 & 31 & $-1 \varepsilon$ & $i m p: n=1$ & $\$$ & POS & 3 \\
\hline \\
\hline & & & & & & & & & & \\
\hline & 10 & $2 E-05$ & 2701 & -2201 & 31 & -1 & imp:n=1 & $?$ & & 1 \\
\hline & 10 & $4.8492 \mathrm{E}-05$ & 2 & -2202 & 31 & -1 & imp: $n=1$ & $\$$ & Pos & 2 \\
\hline & 10 & $4.8492 \mathrm{E}-05$ & 2703 & -2203 & 31 & -1811 & imp:n=1 & $\$$ & Position & 3 \\
\hline & 10 & $4.8492 \mathrm{E}-05$ & 2704 & -2204 & 31 & -1811 & imp:n=1 & $\$$ & Position & 4 \\
\hline & 10 & $4.8492 E-05$ & 2705 & -2205 & 31 & -1811 & imp: $n=1$ & $\$$ & Position & 5 \\
\hline & 10 & $4.8492 E-05$ & & -2206 & 31 & -1811 & imp: $n=1$ & $\$$ & Position & \\
\hline & 10 & $4.8492 E-05$ & 27 & -2207 & 31 & -1811 & $i m p: n=1$ & $\$$ & Position & 7 \\
\hline & 10 & $4.8492 E-05$ & 2708 & -2208 & 31 & -1811 & imp:n=1 & $\$$ & Pos & $\varepsilon$ \\
\hline & 10 & $4.8492 \mathrm{E}-05$ & 2709 & -2209 & 31 & -18 & imp:n=1 & $\$$ & Pos & 9 \\
\hline & 10 & $4.8492 \mathrm{E}-05$ & & -2210 & 31 & -18 & $i m p: n=1$ & $\$$ & Pos & 10 \\
\hline & 10 & $4.8492 \mathrm{E}-05$ & 2711 & -2211 & 31 & -1811 & imp:n=1 & $\$$ & Position & 1 \\
\hline & 10 & $4.8492 \mathrm{E}-05$ & 2712 & -2212 & 31 & -18 & $i m p: n=1$ & $\$$ & ion & 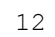 \\
\hline & 10 & $4.8492 \mathrm{E}-05$ & 27 & -2213 & 31 & -1 & imp: $n=1$ & $\$$ & Pos & 13 \\
\hline & 10 & $4.8492 \mathrm{E}-05$ & 27 & -2214 & 31 & -1811 & $i m p: n=1$ & $\$$ & Position & 14 \\
\hline & 10 & $4.8492 E-05$ & 2715 & -2215 & 31 & -1811 & imp: $n=1$ & $\$$ & Position & - \\
\hline & 10 & $4.8492 E-05$ & 2716 & -2216 & 31 & -1 & $i m p: n=1$ & $\$$ & Pos & 16 \\
\hline & 10 & $4.8492 \mathrm{E}-05$ & 2717 & -2217 & 31 & -18 & $i m p: n=1$ & $\$$ & Position & 17 \\
\hline & 10 & $4.8492 \mathrm{E}-05$ & 2718 & -2218 & 31 & -1811 & imp:n=1 & $\$$ & Position & 18 \\
\hline & 10 & $4.8492 \mathrm{E}-05$ & 2719 & -2219 & 31 & & imp:n=1 & $\$$ & Positi & (1) \\
\hline & 10 & $4.8492 \mathrm{E}-05$ & 2720 & -2220 & 31 & -1811 & imp:n=1 & $\$$ & Position & 20 \\
\hline & 10 & $4.8492 \mathrm{E}-05$ & 2721 & -2221 & 31 & -1811 & $i m p: n=1$ & $\$$ & Position & 21 \\
\hline & 10 & $4.8492 E-05$ & 2722 & -2222 & 31 & -1811 & imp: $n=1$ & $\$$ & Positio & 22 \\
\hline & 10 & $4.8492 \mathrm{E}-05$ & 2723 & -2223 & 31 & -1811 & $i m p: n=1$ & $\$$ & Position & 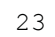 \\
\hline & 10 & $4.8492 E-05$ & 2724 & -2224 & 31 & $-1 \varepsilon$ & $i m p: n=1$ & $\$$ & Position & 24 \\
\hline & 10 & $4.8492 \mathrm{E}-05$ & 2725 & -2225 & 31 & -1811 & $i m p: n=1$ & $\$$ & Position & 25 \\
\hline
\end{tabular}

Revision: 1 
NEA/NSC/DOC(2006)1

\section{Gas Cooled (Thermal) Reactor - GCR}

\section{PROTEUS-GCR-EXP-004} CRIT-REAC

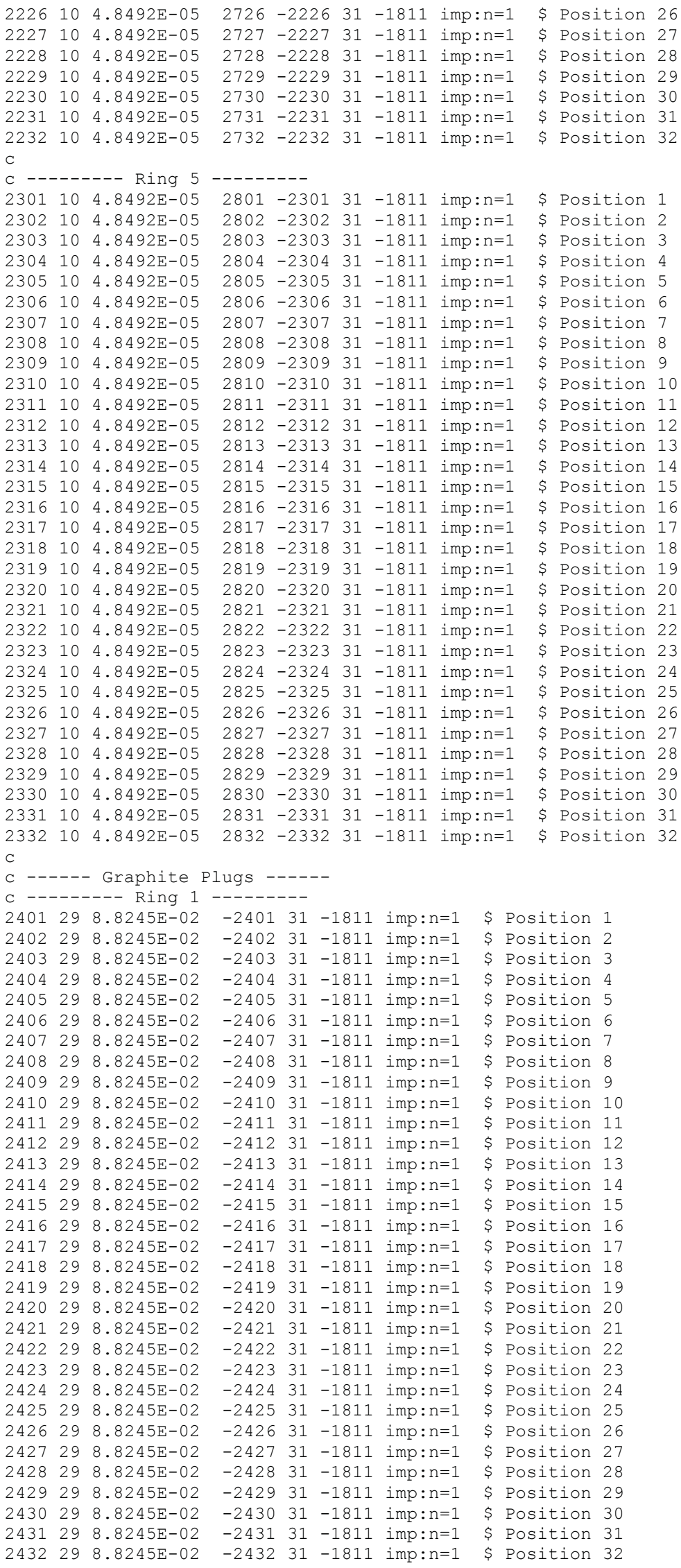

Revision: 1 
NEA/NSC/DOC(2006)1

Gas Cooled (Thermal) Reactor - GCR

PROTEUS-GCR-EXP-004 CRIT-REAC

\begin{tabular}{|c|c|c|c|c|c|c|c|c|c|}
\hline 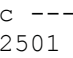 & & 2 & - & 3 & & & & & \\
\hline & 29 & & & 31 & & $i m p: n$ & & & \\
\hline & 29 & & & 31 & -1811 & $i m p: n=1$ & & & \\
\hline & 29 & 8. & & 31 & -1811 & $i m p: n=1$ & & ion & \\
\hline & 29 & 8. & & 31 & -1811 & imp: $n=1$ & & on & \\
\hline & 29 & & & 31 & -1 & imp: $n=1$ & & & \\
\hline & 29 & & & 31 & -18 & imp: $n$ & & on & \\
\hline & 29 & 8. & & 31 & -1811 & imp: $n=1$ & & ion & \\
\hline & 29 & & & 31 & -1811 & imp: $n=1$ & & & \\
\hline & 29 & 02 & & 31 & -1 & $i m p: n$ & & 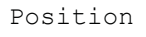 & \\
\hline & 29 & & & 31 & & imp: $n=1$ & & on & \\
\hline & 29 & & & 31 & -1811 & imp: $n=1$ & & & \\
\hline & 29 & $8.8245 E-02$ & & 31 & -1 & $i m p: n$ & & - & \\
\hline & 29 & & & 31 & $-1 \varepsilon$ & imp: $n=1$ & & ion & \\
\hline & 29 & & & 31 & & imp: $n=1$ & 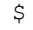 & ion & \\
\hline & 29 & 8. & & 31 & -1 & imp: $n=1$ & & & \\
\hline & 29 & & & 31 & -1 & $: n=1$ & & 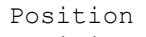 & \\
\hline & 29 & & & 31 & & imp & & Lon & \\
\hline & 29 & & & 31 & & $i m p: n=1$ & & & \\
\hline & 29 & 8.8 & & 31 & -1 & $: n=1$ & & Lon & \\
\hline & 29 & & & 31 & & im & & & \\
\hline & 29 & & & 31 & & imp: $n=1$ & $r$ & ion & \\
\hline & 29 & 8. & & 31 & -1 & $: n=1$ & & & \\
\hline & 29 & & & 31 & -1 & & & 11 & \\
\hline & 29 & & & 31 & & & 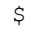 & Lon & \\
\hline & 29 & & & 31 & & $i m p: r$ & $\checkmark$ & ion & \\
\hline & 29 & & & 31 & -1 & & & ion & \\
\hline & 29 & & & 31 & & & & on & \\
\hline & 29 & & & 31 & & in & $\tau$ & Lon & \\
\hline & 29 & & & 31 & & & & & \\
\hline & 29 & & & 31 & -1 & in & & & \\
\hline & 29 & & & 31 & & in & T & & \\
\hline & & & & & & & & & \\
\hline & & & & & & & & & \\
\hline & 29 & 2 & & 31 & & & & & \\
\hline & 29 & & & 31 & & in & s & n & \\
\hline & 29 & & & 31 & & & & n & \\
\hline & 29 & 8 . & -2 & 31 & -18 & in & & 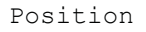 & \\
\hline & 29 & & & 31 & - & & & on & \\
\hline & 29 & & & 31 & - & in & $\$$ & r & \\
\hline & 29 & $45 E-02$ & & 31 & -1 & $i m p: n$ & & on & \\
\hline & 29 & 8. & & 31 & -1 & & & on & \\
\hline & 29 & & & 31 & & imp: $n=1$ & $\$$ & & \\
\hline & 29 & & & 31 & & & & on & \\
\hline & 29 & $E-02$ & & 31 & -1 & & & & \\
\hline & 29 & & & 31 & -1 & & & & \\
\hline & 29 & & & 31 & - & $: n=1$ & \$ & on & \\
\hline & 29 & & & 31 & & imp: $n=1$ & & & \\
\hline & 29 & & & 31 & -1 & im & & on & \\
\hline & 29 & & & 31 & - & im & & & \\
\hline & 29 & & & 31 & - & imp: $n=1$ & ? & on & \\
\hline & 29 & & & 31 & -1 & & & & \\
\hline & 29 & 8. & & 31 & -1 & imp:r & T & 11 & \\
\hline & 29 & 8 . & & 31 & 1 & imp: $n=1$ & S & & \\
\hline & 29 & & & 31 & -1 & imp: $n=1$ & & on & \\
\hline & 29 & & & 31 & & & & 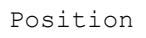 & \\
\hline & 29 & & & 31 & & in & & & \\
\hline & 29 & & & 31 & & imp: $n=1$ & s & & \\
\hline & 29 & & & 31 & -1 & imp: $n=1$ & & ion & \\
\hline & 29 & 8. & & 31 & & imp: $n=1$ & s & & \\
\hline & 29 & 8. & & 31 & - & imp: $n=1$ & \$ & & \\
\hline & 29 & & & 31 & & imp: $n=1$ & & ion & \\
\hline & 29 & 8 . & & 31 & -1 & & T & 1. & \\
\hline & 29 & & & 31 & & $i m p: n=1$ & 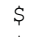 & & \\
\hline & 29 & & & 31 & & imp: $n=1$ & . & on & \\
\hline & 29 & $45 E-02$ & & 31 & -1 & imp: $n=1$ & S & Posi & \\
\hline & & & & & & & & & \\
\hline & & & & & & & & & \\
\hline & 29 & & & 31 & & $i m p: n$ & & & \\
\hline & 29 & $245 \mathrm{~F}$ & & 31 & -1 & $i m p$ & & - & \\
\hline & 29 & $8.8245 E-02$ & & 31 & -1811 & imp: $n=1$ & & & \\
\hline & 29 & $8.8245 E-02$ & & 31 & -1811 & imp: $n=1$ & $\$$ & Position & \\
\hline & 29 & $8.8245 E-02$ & -2705 & 31 & -1811 & $i m p: n=1$ & sta & Position & \\
\hline & 29 & $245 E-02$ & & 31 & -1811 & imp: $n=1$ & $\$$ & Pos: & \\
\hline
\end{tabular}

Revision: 1 
NEA/NSC/DOC(2006)1

\section{Gas Cooled (Thermal) Reactor - GCR}

\section{PROTEUS-GCR-EXP-004 CRIT-REAC}

\begin{tabular}{|c|c|c|c|c|c|c|c|c|c|}
\hline 707 & 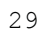 & & & & & imp: $n=1$ & $\$$ & & \\
\hline & 29 & $5 E-02$ & -2708 & 31 & -1811 & imp:n=1 & S & & \\
\hline U & 29 & $8245 E-02$ & -2709 & 31 & -1811 & imp: $n=1$ & $\$$ & ion & \\
\hline & 9 & $.8245 E-02$ & -2710 & 31 & -1811 & imp: $n=1$ & $\$$ & & \\
\hline & 9 & $8245 E-02$ & -2711 & & -1811 & imp:n=1 & & & \\
\hline & 29 & $8245 E-02$ & -2712 & & -1811 & imp: $n=1$ & & & \\
\hline & & $.8245 \mathrm{E}-02$ & -2713 & 31 & -1811 & imp: $n=1$ & & & \\
\hline & & $.8245 E-02$ & -2714 & & -1811 & imp: $n=1$ & & & \\
\hline & & & & & & imp: $n=1$ & & & \\
\hline & & & & & -18 & imp: $n=1$ & & & \\
\hline & & $.8245 \mathrm{E}-02$ & -2717 & & -18 & imp $: n=1$ & & & \\
\hline & & 02 & & & & imp: $n=1$ & & & \\
\hline & & & & & & imp: $n=1$ & & & \\
\hline & & & & & & imp: $n=1$ & & & \\
\hline & & 2 & -27 & & -18 & imp:n=1 & & & \\
\hline & 9 & 2 & & & & imp: $n=1$ & & & \\
\hline & & & & & & imp: $n=1$ & & & \\
\hline & & 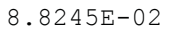 & -27 & & & imp: $n=1$ & & & \\
\hline & 9 & $E-02$ & -27 & & -1 & imp: $n=1$ & & & \\
\hline & & & & & & imp: $n=1$ & & & \\
\hline & & 8 & & & & imp:n=1 & & & \\
\hline & & 2 & -27 & & & imp: $n=1$ & & & \\
\hline & 29 & 8.8 & - & & & $i m p: r$ & 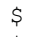 & & \\
\hline & 20 & & & & & imp: $n=1$ & & & \\
\hline & 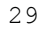 & 8 & & $\perp$ & & imp: $n$ & & & \\
\hline & 29 & 8 & & 0 & & imp & $\$$ & & \\
\hline & & & & & & & & & \\
\hline & & & & & & & & & \\
\hline & & 02 & $-2 \varepsilon$ & & & $n=1$ & $\$$ & & \\
\hline & 9 & 8.8 & -28 & 1 & -1 & imp:n=1 & $\$$ & & \\
\hline & 9 & 8.8 & -28 & & & imp: $n=1$ & $\$$ & & \\
\hline & 29 & 8.8 & -2 & 31 & & imp: $n=1$ & $\$$ & & \\
\hline & 29 & 8.8 & -28 & 31 & -1 & imp: $n=1$ & $\$$ & & \\
\hline & 29 & 8.8 & -28 & 31 & -1 & imp:n=1 & 3 & & \\
\hline & 29 & 8.8 & & 31 & & imp: $n=1$ & $\$$ & & \\
\hline & 29 & 8.8 & -2 & 31 & & imp: $n=1$ & $\$$ & & \\
\hline & 29 & 8.8 & -28 & 1 & -1 & imp:n=1 & $\$$ & & \\
\hline & 29 & 8.8 & -2 & & & imp:n=1 & $\$$ & & \\
\hline & 29 & 8.8 & -2 & 3 & & imp:n=1 & $\$$ & & \\
\hline & 29 & 8.8 & -28 & 3 & -1 & imp: $n=1$ & . & & \\
\hline & 29 & $E-02$ & -28 & & & imp & $\$$ & & \\
\hline & 29 & 8. & -2 & 1 & & imp: & $\$$ & & \\
\hline & 29 & 8.8 & - & 3 & -1 & imp: $n=1$ & $\$$ & & \\
\hline & 29 & 8.8 & -28 & 31 & -1 & imp: $n=1$ & $\$$ & & \\
\hline & 29 & 8. & & & & imp: $n=1$ & $\$$ & & \\
\hline & 29 & 8.8 & & & & imp: $n=1$ & $\$$ & & \\
\hline & 29 & 8.82 & - & 3 & & imp: $n=1$ & $\$$ & & \\
\hline & 29 & $8.8245 \mathrm{E}-$ & -2 & 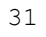 & & imp:n=1 & $\$$ & & \\
\hline & 29 & 8.824 & & & & imp: $n=1$ & $\$$ & & \\
\hline & 29 & 8.82 & -2 & 31 & -1 & imp: $n=1$ & $\$$ & & \\
\hline & 29 & 8.82 & & & & imp: $n=1$ & $\$$ & & \\
\hline & 29 & 8.8 & & & & imp:n=1 & $\$$ & & \\
\hline & 29 & 8.8 & & 31 & -1 & imp: $n=1$ & $\$$ & & \\
\hline & 29 & $8.8245 \mathrm{E}-02$ & -2 & 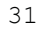 & -18 & imp: $n=1$ & $\$$ & & \\
\hline & 29 & 8.8 & & 3. & & imp: $n=1$ & $\$$ & & \\
\hline & 29 & $8.8245 E-02$ & & & -1 & imp: $n=1$ & $\$$ & & \\
\hline & 29 & $8.8245 E-02$ & -2829 & & -1811 & imp: $n=1$ & $\$$ & Po & \\
\hline & 29 & 8.824 & & & & imp:n=1 & $\$$ & & \\
\hline & 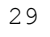 & $8.8245 E-02$ & & & & imp:n=1 & 3 & & \\
\hline & & $8.8245 E-02$ & & & -1 & imp: $n=1$ & $\$$ & & \\
\hline
\end{tabular}

C --- Control Rods C ----- Safety/Shutdown Rods ------

$3001119.1511 \mathrm{E}-02 \quad 3002-3003-3005$ imp:n=1 u=1 \$ Borated Steel Rod

$3002104.8129 \mathrm{E}-053002-3003 \quad 3005-3006$ imp:n=1 u=1 \$ Air

$3003128.6882 \mathrm{E}-02 \quad 3001-3004-3007$ (-3002:3003:3006) imp:n=1 u=1 \$ Steel Tube $3004104.8129 \mathrm{E}-05 \quad-3001: 3004: 3007$ imp:n=1 u=1 \$ Air $\mathrm{C}$

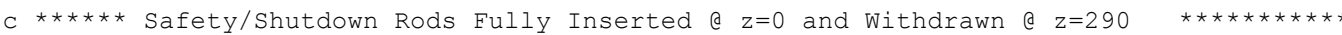
C $\star * * * * *$ Safety/Shutdown Rods were Fully Withdrawn for Cores 1, 1A, 2, $43 * * * * * * * * * * *$ $\mathrm{C}$

Revision: 1 
NEA/NSC/DOC(2006)1

\section{Gas Cooled (Thermal) Reactor - GCR \\ PROTEUS-GCR-EXP-004 CRIT-REAC}

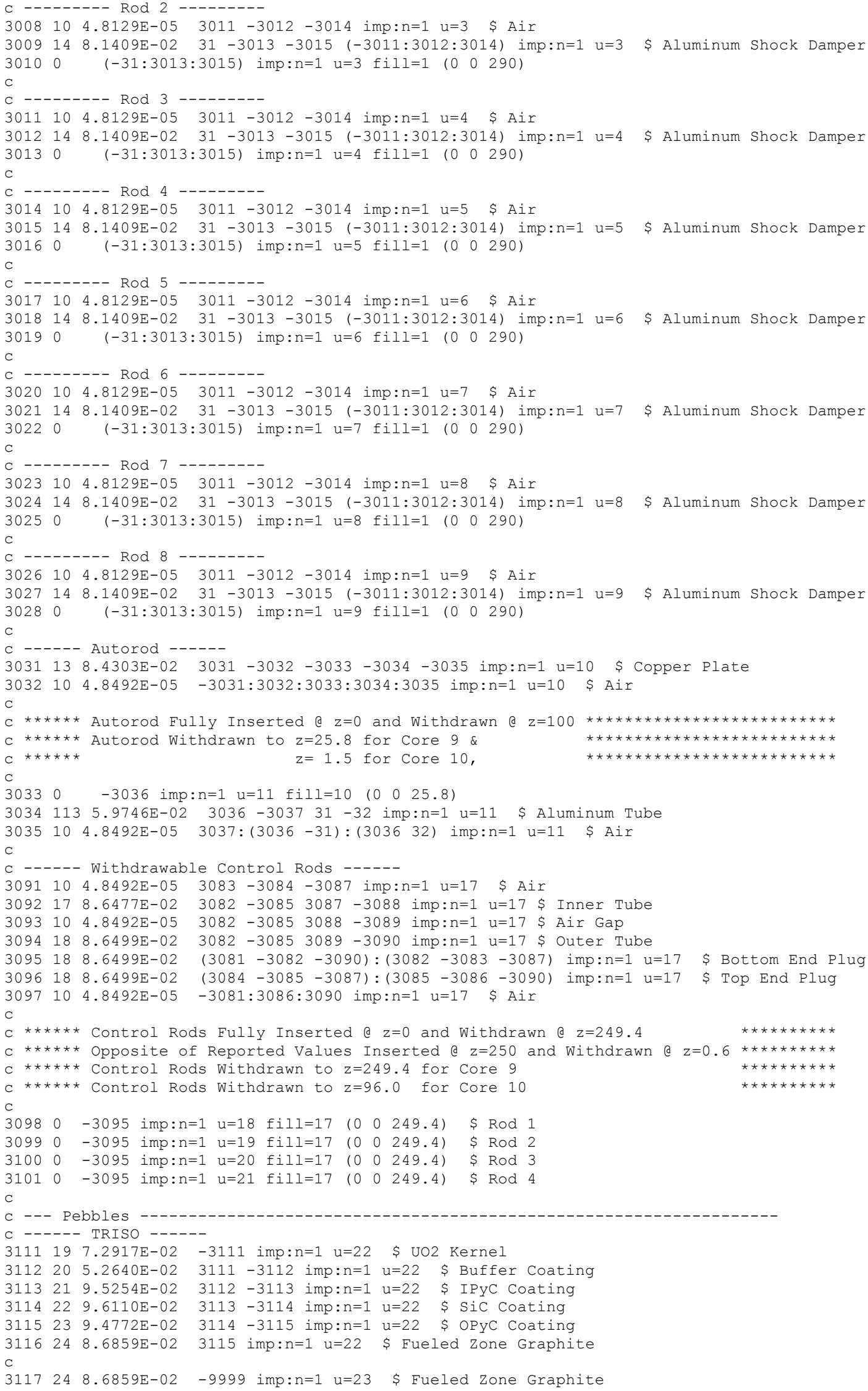

Revision: 1 
NEA/NSC/DOC(2006)1

Gas Cooled (Thermal) Reactor - GCR

PROTEUS-GCR-EXP-004

CRIT-REAC

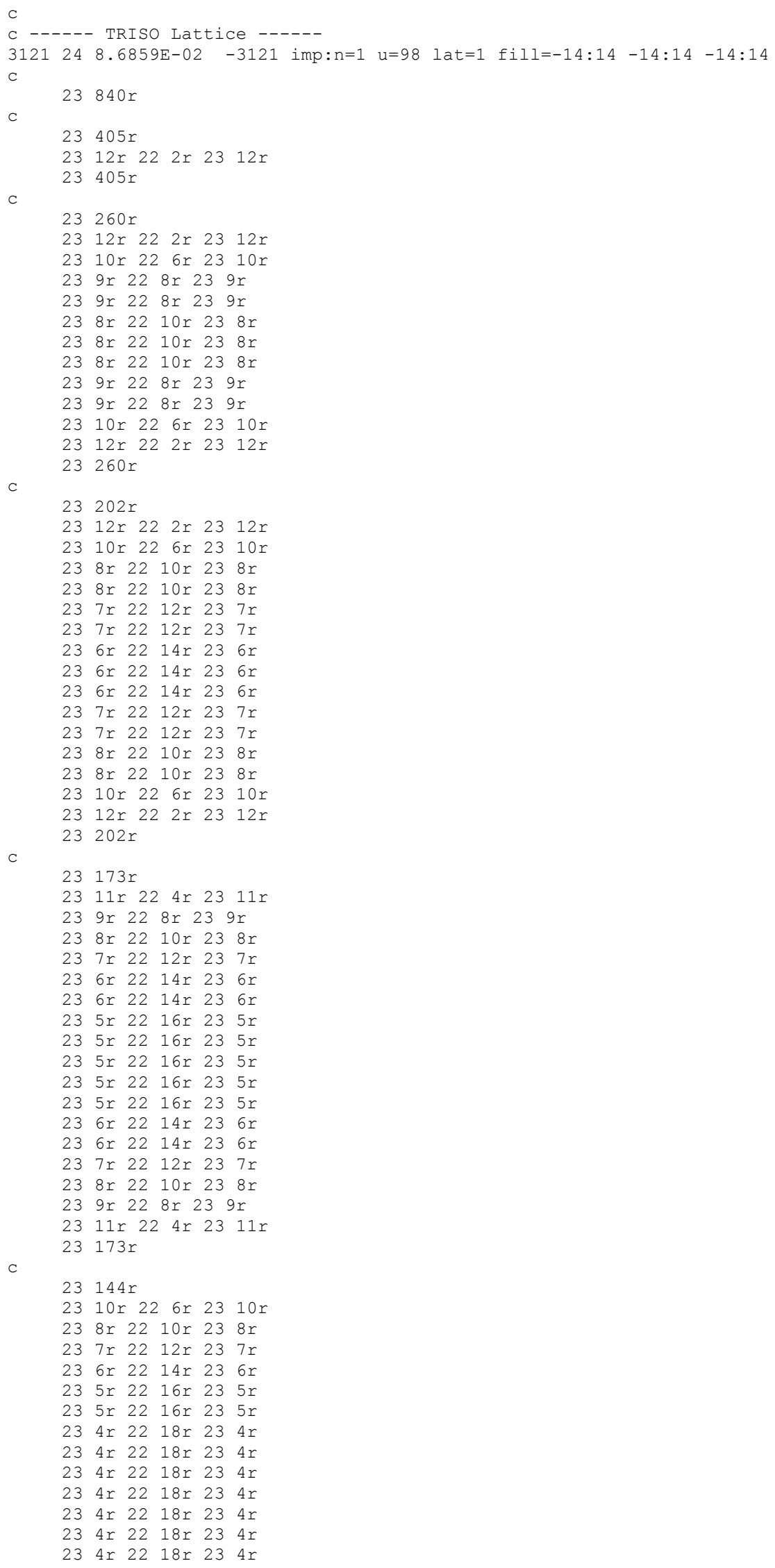

Revision: 1 
NEA/NSC/DOC(2006)1

Gas Cooled (Thermal) Reactor - GCR

\section{PROTEUS-GCR-EXP-004}

CRIT-REAC

$235 r 2216 r 235 r$

$235 r 22 \quad 16 r 23 \quad 5 r$

$236 r 2214 r 236 r$

$237 r 2212 r 237 r$

$238 r 22$ 10r 23 8r

$2310 r 22$ 6r 23 10r

$23144 r$

C

$23115 r$

2311 r 22 4r 2311 r

$238 r 22$ 10r 23 8r

$237 r 2212 r 23$ 7r

$236 r 2214 r 236 r$

$235 r 2216 r 23 \quad 5 r$

$234 r 2218 r 23$ 4r

$234 r 22 \quad 18 r 23 \quad 4 r$

$234 r 22 \quad 18 r 23 \quad 4 r$

$233 r 22$ 20r 23 3r

$233 r 22$ 20r $233 r$

$233 r 22$ 20r $233 r$

$233 r 22$ 20r 23 3r

$233 r 22$ 20r $233 r$

$234 r 22$ 18r 23 4r

$234 r 22$ 18r 23 4r

$234 r 22$ 18r $23 \quad 4 r$

$235 r 22 \quad 16 r 23 \quad 5 r$

$236 r 22 \quad 14 r 23 \quad 6 r$

$237 r 2212 r 237 r$

$238 r 22$ 10r 23 8r

$2311 r 22$ 4r 2311 r

$23115 r$

C

$2386 r$

2312 r 22 2r 2312 r

$239 r 228 r 239 r$

$237 r 22$ 12r $237 r$

$236 r 22 \quad 14 r 23 \quad 6 r$

$235 r 2216 r 235 r$

$234 r 2218 r 23 \quad 4 r$

23 4r 22 18r 23 4r

$233 r 22$ 20r $233 r$

$233 r 22$ 20r 23 3r

$233 r 22 \quad 20 r 233 r$

$232 r 22$ 22r $232 r$

$232 r 22 \quad 22 r 23 \quad r$

$232 r 22$ 22r $232 r$

$233 r 22$ 20r $233 r$

$233 r 22$ 20r 23 3r

$233 r 22$ 20r $233 r$

$234 r 2218 r 23 \quad 4 r$

$234 r 22$ 18r 23 4r

$235 r 22$ 16r $23 \quad 5 r$

$236 r 22 \quad 14 r \quad 23 \quad 6 r$

$237 r 22$ 12r 23 7r

23 9r 22 8r 23 9r

2312 r 22 2r 2312 r

C

$2386 r$

$$
\begin{array}{lllllll}
23 & 86 r & & & & \\
23 & 10 r & 22 & 6 r & 23 & 10 r \\
23 & 8 r & 22 & 10 r & 23 & 8 r \\
23 & 6 r & 22 & 14 r & 23 & 6 r \\
23 & 5 r & 22 & 16 r & 23 & 5 r \\
23 & 4 r & 22 & 18 r & 23 & 4 r \\
23 & 4 r & 22 & 18 r & 23 & 4 r \\
23 & 3 r & 22 & 20 r & 23 & 3 r \\
23 & 3 r & 22 & 20 r & 23 & 3 r \\
23 & 2 r & 22 & 22 r & 23 & 2 r \\
23 & 2 r & 22 & 22 r & 23 & 2 r \\
23 & 2 r & 22 & 22 r & 23 & 2 r \\
23 & 2 r & 22 & 22 r & 23 & 2 r \\
23 & 2 r & 22 & 22 r & 23 & 2 r \\
23 & 2 r & 22 & 22 r & 23 & 2 r \\
23 & 2 r & 22 & 22 r & 23 & 2 r \\
23 & 3 r & 22 & 20 r & 23 & 3 r \\
23 & 3 r & 22 & 20 r & 23 & 3 r
\end{array}
$$

Revision: 1

Date: March 31, 2014

Page 258 of 375 
NEA/NSC/DOC(2006)1

Gas Cooled (Thermal) Reactor - GCR

\section{PROTEUS-GCR-EXP-004}

CRIT-REAC

C

$234 r 2218 r 234 r$

$234 r 2218 r 234 r$

$235 r 2216 r 235 r$

$236 r 2214 r 236 r$

$238 r 2210 r 238 r$

$2310 r 22$ 6r $2310 r$

$2386 r$

$2357 r$

2312 r 22 2r $2312 r$

$238 r 2210 r 238 r$

$237 r 22$ 12r $237 r$

$235 r 2216 r 235 r$

$234 r 22$ 18r 23 4r

$234 r 22$ 18r 23 4r

$233 r 22$ 20r $233 r$

$232 r 22$ 22r $232 r$

$232 r 22$ 22r $232 r$

$232 r 22$ 22r $232 r$

$232 r 22$ 22r $232 r$

23 1r 22 24r 23 1r

23 1r 22 24r 23 1r

23 1r 22 24r 23 1r

$232 r 22$ 22r $232 r$

$232 r 22 \quad 22 \quad 232 r$

$232 r 22$ 22r $232 r$

$232 r 22$ 22r $232 r$

$233 r 22$ 20r $233 r$

$234 r 22$ 18r $234 r$

$234 r 2218 r 234 r$

$235 r 2216 r 235 r$

$237 r 2212 r 237 r$

$238 r 22$ 10r $238 r$

2312 r 22 2r $2312 r$

C$$
2357 r
$$

$2357 r$

$2310 r 22$ 6r $2310 r$

$238 r 2210 r 238 r$

$236 r 2214 r 236 r$

$235 r 2216 r 235 r$

$234 r 22$ 18r 23 4r

$233 r 22$ 20r $233 r$

$233 r 22$ 20r $233 r$

$232 r 22$ 22r $232 r$

$232 r 22$ 22r $232 r$

$231 r 22 \quad 24 r 231 r$

$231 r 22$ 24r $231 r$

23 1r 22 24r $231 r$

23 Ir 22 24r $231 r$

23 1r 22 24r 23 1r

23 1r 22 24r 23 1r

$231 r 22$ 24r 23 1r

$232 r 22$ 22r $232 r$

$232 r 22$ 22r $232 r$

$233 r 22$ 20r 23 3r

$233 r 22$ 20r $233 r$

$234 r 22$ 18r 23 4r

$235 r 2216 r 235 r$

$236 r 2214 r 236 r$

$238 r 2210 r 238 r$

$2310 r 22$ 6r $2310 r$

C

$2357 r$

$2357 r$

$239 r 228 r 239 r$

$237 r 2212 r 237 r$

$236 r 2214 r 236 r$

$234 r 22$ 18r $234 r$

$234 r 2218 r 234 r$

$233 r 22$ 20r 23 3r

$232 r 22$ 22r $232 r$

$232 r 22$ 22r $232 r$

$231 r 2224 r 231 r$

$231 r 22$ 24r $231 r$

$231 r 22$ 24r $231 r$

Revision: 1

Date: March 31, 2014

Page 259 of 375 
NEA/NSC/DOC(2006)1

Gas Cooled (Thermal) Reactor - GCR

PROTEUS-GCR-EXP-004

CRIT-REAC

$231 r 2224 r 231 r$

$231 r 22$ 24r $231 r$

$231 r 2224 r 231 r$

$231 r 2224 r 231 r$

23 1r 22 24r 23 1r

23 Ir 22 24r $231 r$

$232 r 22$ 22r $232 r$

$232 r 22$ 22r $232 r$

$233 r 22$ 20r $233 r$

$234 r 22$ 18r 23 4r

$234 r 22$ 18r $234 r$

$236 r 2214 r 236 r$

$237 r 2212 r 237 r$

23 9r 22 8r 23 9r

C

$2357 r$

$2357 r$

$239 r 22$ 8r $239 r$

$237 r 2212 r 237 r$

$235 r 2216 r 235 r$

$234 r 22$ 18r 23 4r

$233 r 22$ 20r $233 r$

$233 r 22$ 20r 23 3r

$232 r 22$ 22r $232 r$

$232 r 22$ 22r $232 r$

$231 r 2224 r 231 r$

$231 r 22$ 24r $231 r$

$231 r 22$ 24r $231 r$

$231 r 22$ 24r $231 r$

23 1r 22 24r 23 1r

$231 r 2224 r 231 r$

$231 r 22$ 24r $231 r$

23 1r 22 24r 23 1r

23 1r 22 24r $231 r$

$232 r 22$ 22r $232 r$

$232 r 22$ 22r $232 r$

$233 r 22$ 20r 23 3r

$233 r 22$ 20r $233 r$

$234 r 22$ 18r $234 r$

$235 r 2216 r 235 r$

$237 r 22$ 12r $237 r$

23 9r 22 8r 23 9r

$2357 r$

C

$2357 r$

23 8r 22 10r $238 r$

$236 r 2214 r 236 r$

$235 r 2216 r 235 r$

$234 r 2218 r 234 r$

$233 r 22$ 20r $233 r$

$232 r 22$ 22r $232 r$

$232 r 22 \quad 22 \quad 232 r$

23 1r 22 24r $231 r$

23 1r 22 24r 23 1r

23 1r 22 24r 23 1r

$231 r 2224 r 231 r$

$231 r 2224 r 231 r$

$232226 r 23$

23 1r 22 24r 23 1r

$231 r 22$ 24r 23 1r

$231 r 22$ 24r $231 r$

23 1r 22 24r 23 1r

23 1r 22 24r 23 1r

$232 r 22$ 22r $232 r$

$232 r 22$ 22r $232 r$

$233 r 22$ 20r $233 r$

$234 r 2218 r 234 r$

$235 r 2216 r 235 r$

$236 r 22$ 14r $236 r$

$238 r 2210 r 238 r$

C

$2357 r$

$2328 r$

$\begin{array}{lllllll}23 & 12 r & 22 & 23 & 22 & 23 & 12 r\end{array}$

23 8r 22 10r 23 8r

$236 r 2214 r 236 r$

Revision: 1

Date: March 31, 2014

Page 260 of 375 
NEA/NSC/DOC(2006)1

Gas Cooled (Thermal) Reactor - GCR

PROTEUS-GCR-EXP-004

CRIT-REAC

$235 r 2216 r 235 r$

$234 r 22$ 18r $234 r$

$233 r 22$ 20r $233 r$

$232 r 22$ 22r $232 r$

$232 r 22 \quad 22 r 23 \quad 2 r$

23 Ir 22 24r $231 r$

23 1r 22 24r 23 1r

23 1r 22 24r 23 1r

$231 r 22$ 24r $231 r$

23 1r 22 24r 23 1r

23 Ir 2211 r 2322 11r 23 1r

$231 r 22$ 24r $231 r$

23 1r 22 24r 23 1r

$231 r 22 \quad 24 r 231 r$

$231 r 2224 r 231 r$

23 1r 22 24r $231 r$

$232 r 22$ 22r $232 r$

$232 r 22$ 22r 23 2r

$233 r 22$ 20r $233 r$

$234 r 22$ 18r 23 4r

$235 r 22$ 16r $235 r$

$236 r 2214 r 236 r$

$238 r 2210 r 238 r$

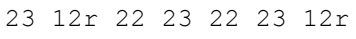

C

$2328 r$

$2357 r$

$238 r 2210 r 238 r$

$236 r 2214 r 236 r$

$235 r 2216 r 235 r$

$234 r 22$ 18r 23 4r

$233 r 22$ 20r $233 r$

$232 r 22$ 22r $232 r$

$232 r 22$ 22r $232 r$

$231 r 22$ 24r 23 1r

23 1r 22 24r 23 1r

$231 r 22$ 24r $231 r$

23 1r 22 24r 23 1r

23 1r 22 24r 23 1r

2322 26r 23

23 1r 22 24r $231 r$

$231 r 22$ 24r 23 1r

$231 r 22$ 24r $231 r$

23 1r 22 24r 23 1r

23 Ir 22 24r $231 r$

$232 r 22$ 22r $232 r$

$232 r 22$ 22r $232 r$

$233 r 22$ 20r $233 r$

$234 r 2218 r 234 r$

$235 r 2216 r 235 r$

$236 r 22$ 14r $236 r$

$238 r 2210 r 238 r$

$2357 r$

C

$\begin{array}{llllll}23 & 57 r & & \\ 23 & 9 r & 22 & 8 r & 23 & 9 r \\ 23 & 7 r & 22 & 12 r & 23 & 7 r \\ 23 & 5 r & 22 & 16 r & 23 & 5 r \\ 23 & 4 r & 22 & 18 r & 23 & 4 r \\ 23 & 3 r & 22 & 20 r & 23 & 3 r \\ 23 & 3 r & 22 & 20 r & 23 & 3 r \\ 23 & 2 r & 22 & 22 r & 23 & 2 r \\ 23 & 2 r & 22 & 22 r & 23 & 2 r \\ 23 & 1 r & 22 & 24 r & 23 & 1 r \\ 23 & 1 r & 22 & 24 r & 23 & 1 r \\ 23 & 1 r & 22 & 24 r & 23 & 1 r \\ 23 & 1 r & 22 & 24 r & 23 & 1 r \\ 23 & 1 r & 22 & 24 r & 23 & 1 r \\ 23 & 1 r & 22 & 24 r & 23 & 1 r \\ 23 & 1 r & 22 & 24 r & 23 & 1 r \\ 23 & 1 r & 22 & 24 r & 23 & 1 r \\ 23 & 1 r & 22 & 24 r & 23 & 1 r \\ 23 & 2 r & 22 & 22 r & 23 & 2 r \\ 23 & 2 r & 22 & 22 r & 23 & 2 r \\ 23 & 3 r & 22 & 20 r & 23 & 3 r \\ 23 & 3 r & 22 & 20 r & 23 & 3 r\end{array}$

Revision: 1

Date: March 31, 2014

Page 261 of 375 
NEA/NSC/DOC(2006)1

Gas Cooled (Thermal) Reactor - GCR

\section{PROTEUS-GCR-EXP-004}

CRIT-REAC

$234 r 22 \quad 18 r 23 \quad 4 r$

$235 r 2216 r 235 r$

$237 r 2212 r 237 r$

23 9r 22 8r 23 9r

$2357 r$

$2357 r$

23 9r 22 8r 23 9r

$237 r 2212 r 237 r$

$236 r 22 \quad 14 r 23$ 6r

$234 r 22$ 18r 23 4r

23 4r 22 18r 23 4r

$233 r 22$ 20r 23 3r

$232 r 22 \quad 22 r 23 \quad r$

$232 r 22 \quad 22 r 23 \quad 2 r$

$231 r 22 \quad 24 r 231 r$

$231 r 22 \quad 24 r 231 r$

$231 r 22 \quad 24 r 23$ 1r

$231 r 22 \quad 24 r 231 r$

$231 r 22 \quad 24 r 231 r$

$231 r 22 \quad 24 r 23$ 1r

$\begin{array}{llllll}23 & 1 r & 22 & 24 r & 23 & 1 r\end{array}$

23 1r 22 24r 23 1r

$231 r 22 \quad 24 r 23 \quad 1 r$

$232 r 22 \quad 22 r 232 r$

$232 r 22$ 22r 23 2r

$233 r 22$ 20r $233 r$

$234 r 22 \quad 18 r 23 \quad 4 r$

$234 r 22 \quad 18 r 23 \quad 4 r$

$236 r 22$ 14r $236 r$

$237 r 2212 r \quad 237 r$

23 9r 22 8r 23 9r

C

$2357 r$

$2357 r$

$2310 r 22$ 6r 23 10r

$238 r 2210 r 238 r$

$236 r 22$ 14r $236 r$

$235 r 2216 r 235 r$

$234 r 2218 r 23 \quad 4 r$

$233 r 22$ 20r $233 r$

$233 r 22$ 20r 23 3r

$232 r 22 \quad 22 r 23 \quad r$

$232 r 22 \quad 22 r 23 \quad r$

$231 r 22 \quad 24 r 231 r$

$231 r 22$ 24r $231 r$

$231 r 22 \quad 24 r 231 r$

$231 r 22 \quad 24 r 231 r$

$231 r 22 \quad 24 r 231 r$

$231 r 22 \quad 24 r 231 r$

$231 r 22 \quad 24 r 23$ 1r

$\begin{array}{llllll}23 & 2 r & 22 & 22 r & 23 & 2 r\end{array}$

$232 r 22 \quad 22 r 23 \quad r$

$233 r 22$ 20r $233 r$

$233 r 22$ 20r $233 r$

$234 r 22$ 18r 23 4r

$235 r 22$ 16r $23 \quad 5 r$

$236 r 22 \quad 14 r 23 \quad 6 r$

$238 r 2210 r 23$ 8r

$2310 r 22$ 6r $2310 r$

$2357 r$

C

$\begin{array}{llllll}23 & 57 r & & & & \\ 23 & 12 r & 22 & 2 r & 23 & 12 r \\ 23 & 8 r & 22 & 10 r & 23 & 8 r \\ 23 & 7 r & 22 & 12 r & 23 & 7 r \\ 23 & 5 r & 22 & 16 r & 23 & 5 r \\ 23 & 4 r & 22 & 18 r & 23 & 4 r \\ 23 & 4 r & 22 & 18 r & 23 & 4 r \\ 23 & 3 r & 22 & 20 r & 23 & 3 r \\ 23 & 2 r & 22 & 22 r & 23 & 2 r \\ 23 & 2 r & 22 & 22 r & 23 & 2 r \\ 23 & 2 r & 22 & 22 r & 23 & 2 r \\ 23 & 2 r & 22 & 22 r & 23 & 2 r \\ 23 & 1 r & 22 & 24 r & 23 & 1 r \\ 23 & 1 r & 22 & 24 r & 23 & 1 r\end{array}$

Revision: 1

Date: March 31, 2014

Page 262 of 375 
NEA/NSC/DOC(2006)1

Gas Cooled (Thermal) Reactor - GCR

PROTEUS-GCR-EXP-004

CRIT-REAC

C

$231 r 22 \quad 24 r 231 r$

$232 r 22 \quad 22 r 232 r$

$\begin{array}{llllll}23 & 2 r & 22 & 22 r & 23 & 2 r\end{array}$

$232 r 22 \quad 22 r 23 \quad 2 r$

$232 r 22 \quad 22 r 23 \quad 2 r$

$233 r 22$ 20r $233 r$

$234 r 2218 r 23 \quad 4 r$

$234 r 22 \quad 18 r \quad 23 \quad 4 r$

$235 r 2216 r 23 \quad 5 r$

$237 r 2212 r 237 r$

$238 r 22$ 10r 23 8r

2312 r 22 2r 2312 r

$2357 r$

$2386 r$

$2310 r 22$ 6r $2310 r$

23 8r 22 10r 23 8r

$236 r 22 \quad 14 r 23 \quad 6 r$

$235 r 2216 r 235 r$

$234 r 22 \quad 18 r 23 \quad 4 r$

$234 r 22$ 18r 23 4r

$233 r 22$ 20r $23 \quad 3 r$

$233 r 22$ 20r 23 3r

$232 r 22$ 22r $232 r$

$232 r 22 \quad 22 r 232 r$

23 2r 22 22r 23 2r

$232 r 22 \quad 22 r 23 \quad 2 r$

$232 r 22 \quad 22 r 23 \quad r$

$232 r 22 \quad 22 r 23 \quad r$

$232 r 22 \quad 22 r 232 r$

$233 r 22$ 20r 23 3r

$233 r 22$ 20r $233 r$

$234 r 22$ 18r $23 \quad 4 r$

$234 r 22$ 18r 23 4r

$235 r 2216 r 235 r$

$236 r 22 \quad 14 r 23 \quad 6 r$

$238 r 22$ 10r 23 8r

$2310 r 22$ 6r $2310 r$

C

$2386 r$

$2312 r 22 \quad 2 r 2312 r$

23 9r 22 8r 23 9r

$237 r 2212$ r $237 r$

$236 r 22$ 14r 23 6r

$235 r 2216 r 235 r$

23 4r 22 18r 23 4r

$234 r 22$ 18r 23 4r

$233 r 22$ 20r $233 r$

$233 r 22$ 20r 23 3r

$233 r 22$ 20r 23 3r

23 2r 22 22r 23 2r

23 2r 22 22r 23 2r

$232 r 22 \quad 22 r 232 r$

$233 r 22$ 20r $233 r$

$233 r 22$ 20r 23 3r

$233 r 22$ 20r $233 r$

$234 r 22$ 18r 23 4r

$234 r 22$ 18r 23 4r

$235 r 2216 r 235 r$

$236 r 2214 r 23$ 6r

$237 r 22$ 12r 23 7r

23 9r 22 8r 23 9r

2312 r 22 2r $2312 r$

$2386 r$

$$
\begin{array}{lllllll}
23 & 115 r & & & \\
23 & 11 r & 22 & 4 r & 23 & 11 r \\
23 & 8 r & 22 & 10 r & 23 & 8 r \\
23 & 7 r & 22 & 12 r & 23 & 7 r \\
23 & 6 r & 22 & 14 r & 23 & 6 r \\
23 & 5 r & 22 & 16 r & 23 & 5 r \\
23 & 4 r & 22 & 18 r & 23 & 4 r \\
23 & 4 r & 22 & 18 r & 23 & 4 r \\
23 & 4 r & 22 & 18 r & 23 & 4 r \\
23 & 3 r & 22 & 20 r & 23 & 3 r
\end{array}
$$

Revision: 1

Date: March 31, 2014
Page 263 of 375 
NEA/NSC/DOC(2006)1

Gas Cooled (Thermal) Reactor - GCR

\section{PROTEUS-GCR-EXP-004}

CRIT-REAC

$233 r 22$ 20r $233 r$

$233 r 2220 r 233 r$

$233 r 22$ 20r $233 r$

$233 r 22$ 20r 23 3r

$234 r 22$ 18r 23 4r

$234 r 22 \quad 18 r 23$ 4r

$234 r 22$ 18r 23 4r

$235 r 2216 r 235 r$

$236 r 2214 r 23 \quad 6 r$

$237 r 2212 r 237 r$

23 8r 22 10r 23 8r

2311 r 22 4r $2311 r$

$23115 r$

$23144 r$

$2310 r 22$ 6r $2310 r$

$238 r 2210 r 238 r$

$\begin{array}{llllll}23 & 7 r & 22 & 12 r & 23 & 7 r\end{array}$

$\begin{array}{llllll}23 & 6 r & 22 & 14 r & 23 & 6 r\end{array}$

$235 r 22 \quad 16 r 23 \quad 5 r$

$235 r 2216 r 235 r$

$234 r 2218 r 234 r$

$234 r 22$ 18r 23 4r

$234 r 22$ 18r 23 4r

$234 r 22$ 18r $234 r$

$234 r 22 \quad 18 r 23 \quad 4 r$

$234 r 22$ 18r $23 \quad 4 r$

$234 r 22$ 18r 23 4r

$235 r 22 \quad 16 r 23 \quad 5 r$

$235 r 2216 r 235 r$

$236 r 22 \quad 14 r 23 \quad 6 r$

$\begin{array}{llllll}23 & 7 r & 22 & 12 r & 23 & 7 r\end{array}$

23 8r 22 10r 23 8r

$2310 r 22$ 6r 23 10r

C

$23144 r$

$23173 r$

2311 r 22 4r 2311 r

23 9r 22 8r 23 9r

$238 r 22$ 10r 23 8r

$\begin{array}{llllll}23 & 7 r & 22 & 12 r & 23 & 7 r\end{array}$

$236 r 22$ 14r 23 6r

$236 r 22 \quad 14 r 236 r$

$235 r 2216 r 235 r$

$235 r 2216 r 235 r$

$235 r 2216 r 235 r$

$235 r 2216 r 235 r$

$235 r 2216 r 235 r$

$236 r 2214 r 236 r$

$236 r 22 \quad 14 r 236 r$

$237 r 22$ 12r 23 7r

$238 r 2210 r 238 r$

23 9r 22 8r 23 9r

2311 r 22 4r 2311 r

$23173 r$

$23202 x$

$2312 r 22$ 2r $2312 r$

23 10r 22 6r 23 10r

23 8r 22 10r 23 8r

$238 r 2210 r 238 r$

$237 r 2212 r 237 r$

$237 r 22$ 12r $237 r$

$236 r 2214 r 236 r$

$236 r 2214 r 23 \quad 6 r$

$236 r 22$ 14r $236 r$

$237 r 2212 r 237 r$

$237 r 2212 r 237 r$

$238 r 2210 r 238 r$

$238 r 22$ 10r 23 8r

$2310 r 22$ 6r 23 10r

$2312 r 22$ 2r $2312 r$

$23202 r$

C

$23260 r$

2312 r 22 2r 2312 r

Revision: 1

Date: March 31, 2014

Page 264 of 375 
NEA/NSC/DOC(2006)1

Gas Cooled (Thermal) Reactor - GCR

\section{PROTEUS-GCR-EXP-004}

CRIT-REAC

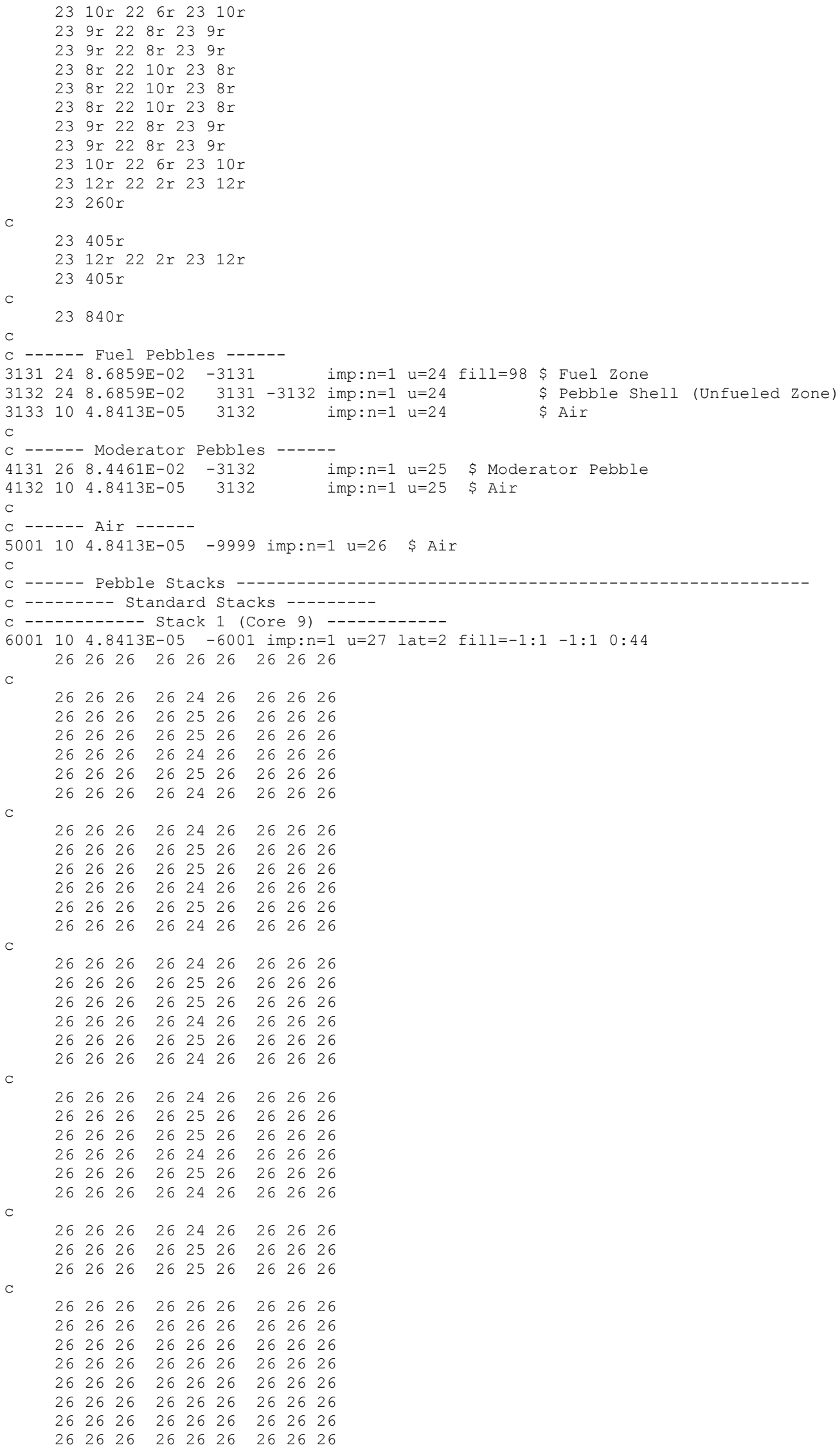

Revision: 1

Date: March 31, 2014

Page 265 of 375 
NEA/NSC/DOC(2006)1

Gas Cooled (Thermal) Reactor - GCR

PROTEUS-GCR-EXP-004

CRIT-REAC

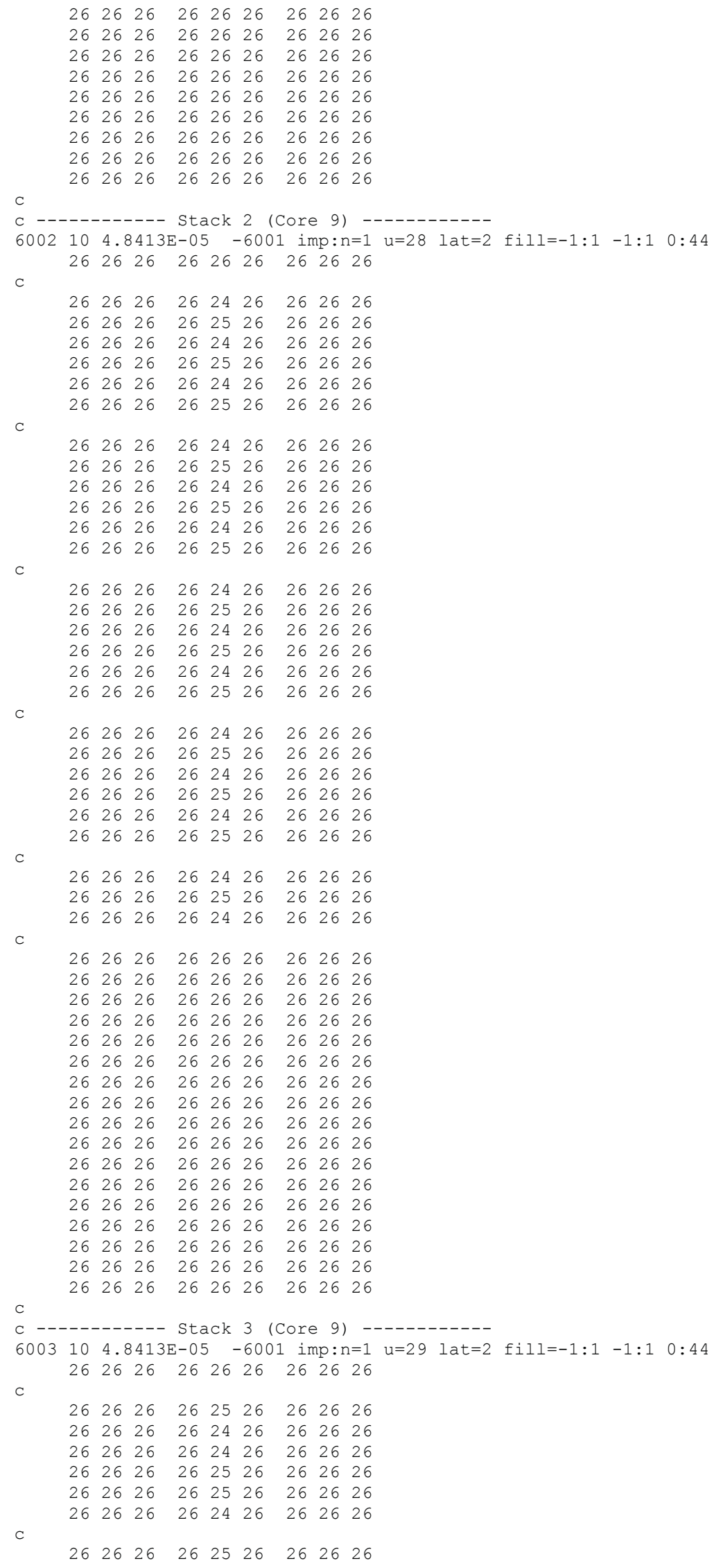

Revision: 1

Date: March 31, 2014

Page 266 of 375 
NEA/NSC/DOC(2006)1

Gas Cooled (Thermal) Reactor - GCR

PROTEUS-GCR-EXP-004

CRIT-REAC

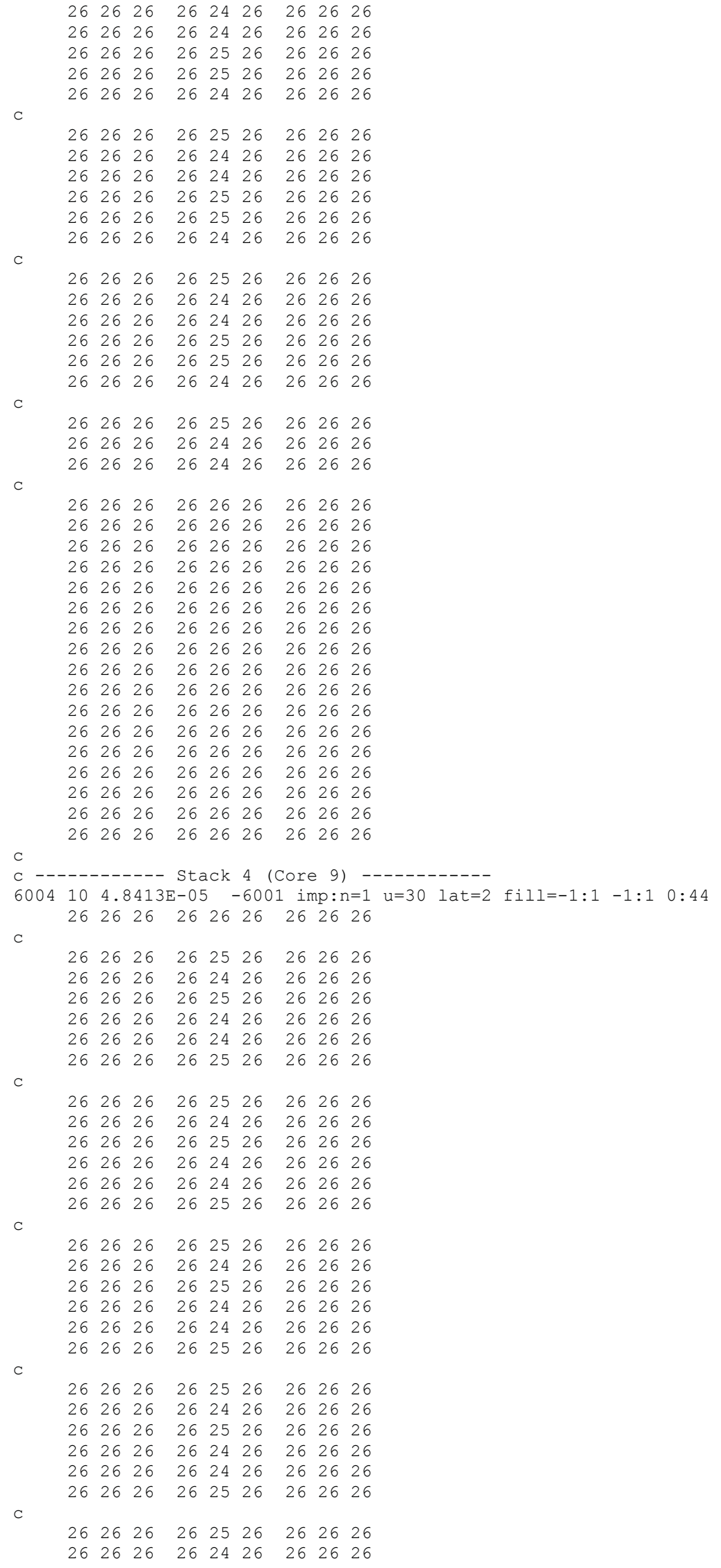

Revision: 1

Date: March 31, 2014

Page 267 of 375 
NEA/NSC/DOC(2006)1

Gas Cooled (Thermal) Reactor - GCR

PROTEUS-GCR-EXP-004

CRIT-REAC

c

$\begin{array}{lllllllll}26 & 26 & 26 & 26 & 25 & 26 & 26 & 26 & 26 \\ 26 & 26 & 26 & 26 & 26 & 26 & 26 & 26 & 26 \\ 26 & 26 & 26 & 26 & 26 & 26 & 26 & 26 & 26 \\ 26 & 26 & 26 & 26 & 26 & 26 & 26 & 26 & 26 \\ 26 & 26 & 26 & 26 & 26 & 26 & 26 & 26 & 26 \\ 26 & 26 & 26 & 26 & 26 & 26 & 26 & 26 & 26 \\ 26 & 26 & 26 & 26 & 26 & 26 & 26 & 26 & 26 \\ 26 & 26 & 26 & 26 & 26 & 26 & 26 & 26 & 26 \\ 26 & 26 & 26 & 26 & 26 & 26 & 26 & 26 & 26 \\ 26 & 26 & 26 & 26 & 26 & 26 & 26 & 26 & 26 \\ 26 & 26 & 26 & 26 & 26 & 26 & 26 & 26 & 26 \\ 26 & 26 & 26 & 26 & 26 & 26 & 26 & 26 & 26 \\ 26 & 26 & 26 & 26 & 26 & 26 & 26 & 26 & 26 \\ 26 & 26 & 26 & 26 & 26 & 26 & 26 & 26 & 26 \\ 26 & 26 & 26 & 26 & 26 & 26 & 26 & 26 & 26 \\ 26 & 26 & 26 & 26 & 26 & 26 & 26 & 26 & 26 \\ 26 & 26 & 26 & 26 & 26 & 26 & 26 & 26 & 26 \\ 26 & 26 & 26 & 26 & 26 & 26 & 26 & 26 & 26\end{array}$

$6005104.8413 \mathrm{E}-05 \quad-6001$ imp:n=1 $u=31$ lat=2 fill=-1:1 -1:1 $0: 44$ $\begin{array}{lllllllll}26 & 26 & 26 & 26 & 26 & 26 & 26 & 26 & 26\end{array}$

$\begin{array}{lllllllll}26 & 26 & 26 & 26 & 24 & 26 & 26 & 26 & 26\end{array}$

$\begin{array}{lllllllll}26 & 26 & 26 & 26 & 25 & 26 & 26 & 26 & 26\end{array}$

$\begin{array}{lllllllll}26 & 26 & 26 & 26 & 25 & 26 & 26 & 26 & 26\end{array}$

$\begin{array}{lllllllll}26 & 26 & 26 & 26 & 24 & 26 & 26 & 26 & 26\end{array}$

$\begin{array}{lllllllll}26 & 26 & 26 & 26 & 25 & 26 & 26 & 26 & 26\end{array}$

C

$262626 \quad 262426 \quad 26 \quad 2626$

$\begin{array}{lllllllll}26 & 26 & 26 & 26 & 24 & 26 & 26 & 26 & 26\end{array}$

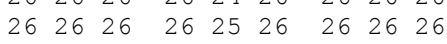

$\begin{array}{lllllllll}26 & 26 & 26 & 26 & 25 & 26 & 26 & 26 & 26\end{array}$

$\begin{array}{lllllllll}26 & 26 & 26 & 26 & 24 & 26 & 26 & 26 & 26\end{array}$

$\begin{array}{lllllllll}26 & 26 & 26 & 26 & 25 & 26 & 26 & 26 & 26\end{array}$

$\begin{array}{lllllllll}26 & 26 & 26 & 26 & 24 & 26 & 26 & 26 & 26\end{array}$

C

$\begin{array}{lllllllll}26 & 26 & 26 & 26 & 24 & 26 & 26 & 26 & 26\end{array}$

$\begin{array}{lllllllll}26 & 26 & 26 & 26 & 25 & 26 & 26 & 26 & 26\end{array}$

$\begin{array}{lllllllll}26 & 26 & 26 & 26 & 25 & 26 & 26 & 26 & 26\end{array}$

$\begin{array}{lllllllll}26 & 26 & 26 & 26 & 24 & 26 & 26 & 26 & 26\end{array}$

$\begin{array}{lllllllll}26 & 26 & 26 & 26 & 25 & 26 & 26 & 26 & 26\end{array}$

C

$\begin{array}{lllllllll}26 & 26 & 26 & 26 & 24 & 26 & 26 & 26 & 26\end{array}$

$\begin{array}{lllllllll}26 & 26 & 26 & 26 & 25 & 26 & 26 & 26 & 26\end{array}$

$\begin{array}{lllllllll}26 & 26 & 26 & 26 & 25 & 26 & 26 & 26 & 26\end{array}$

$\begin{array}{lllllllll}26 & 26 & 26 & 26 & 24 & 26 & 26 & 26 & 26\end{array}$

$\begin{array}{lllllllll}26 & 26 & 26 & 26 & 25 & 26 & 26 & 26 & 26\end{array}$

$\begin{array}{lllllllll}26 & 26 & 26 & 26 & 24 & 26 & 26 & 26 & 26\end{array}$

C

$\begin{array}{lllllllll}26 & 26 & 26 & 26 & 26 & 26 & 26 & 26 & 26\end{array}$

$\begin{array}{lllllllll}26 & 26 & 26 & 26 & 26 & 26 & 26 & 26 & 26\end{array}$

$\begin{array}{lllllllll}26 & 26 & 26 & 26 & 26 & 26 & 26 & 26 & 26\end{array}$

$\begin{array}{lllllllll}26 & 26 & 26 & 26 & 26 & 26 & 26 & 26 & 26\end{array}$

$\begin{array}{lllllllll}26 & 26 & 26 & 26 & 26 & 26 & 26 & 26 & 26\end{array}$

$\begin{array}{lllllllll}26 & 26 & 26 & 26 & 26 & 26 & 26 & 26 & 26\end{array}$

$\begin{array}{lllllllll}26 & 26 & 26 & 26 & 26 & 26 & 26 & 26 & 26\end{array}$

$\begin{array}{lllllllll}26 & 26 & 26 & 26 & 26 & 26 & 26 & 26 & 26\end{array}$

$\begin{array}{lllllllll}26 & 26 & 26 & 26 & 26 & 26 & 26 & 26 & 26\end{array}$

$\begin{array}{lllllllll}26 & 26 & 26 & 26 & 26 & 26 & 26 & 26 & 26\end{array}$

$\begin{array}{lllllllll}26 & 26 & 26 & 26 & 26 & 26 & 26 & 26 & 26\end{array}$

$\begin{array}{lllllllll}26 & 26 & 26 & 26 & 26 & 26 & 26 & 26 & 26\end{array}$

$\begin{array}{lllllllll}26 & 26 & 26 & 26 & 26 & 26 & 26 & 26 & 26\end{array}$

$\begin{array}{lllllllll}26 & 26 & 26 & 26 & 26 & 26 & 26 & 26 & 26\end{array}$

$\begin{array}{lllllllll}26 & 26 & 26 & 26 & 26 & 26 & 26 & 26 & 26\end{array}$

$\begin{array}{lllllllll}26 & 26 & 26 & 26 & 26 & 26 & 26 & 26 & 26\end{array}$

$\begin{array}{lllllllll}26 & 26 & 26 & 26 & 26 & 26 & 26 & 26 & 26\end{array}$

$\begin{array}{lllllllll}26 & 26 & 26 & 26 & 26 & 26 & 26 & 26 & 26\end{array}$

$\begin{array}{lllllllll}26 & 26 & 26 & 26 & 26 & 26 & 26 & 26 & 26\end{array}$

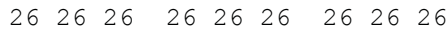

c ------------ Stack 2 (Core 10) ------------

$6006104.8413 \mathrm{E}-05 \quad-6001$ imp: $n=1 \quad u=32$ lat=2 fill=-1:1 -1:1 0:44

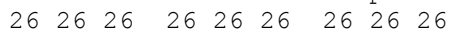

Revision: 1

Date: March 31, 2014

Page 268 of 375 
NEA/NSC/DOC(2006)1

Gas Cooled (Thermal) Reactor - GCR

PROTEUS-GCR-EXP-004

CRIT-REAC

C

C

$\begin{array}{lllllllll}26 & 26 & 26 & 26 & 24 & 26 & 26 & 26 & 26\end{array}$

$\begin{array}{lllllllll}26 & 26 & 26 & 26 & 25 & 26 & 26 & 26 & 26\end{array}$

$\begin{array}{lllllllll}26 & 26 & 26 & 26 & 24 & 26 & 26 & 26 & 26\end{array}$

$\begin{array}{lllllllll}26 & 26 & 26 & 26 & 25 & 26 & 26 & 26 & 26\end{array}$

$\begin{array}{lllllllll}26 & 26 & 26 & 26 & 24 & 26 & 26 & 26 & 26\end{array}$

$\begin{array}{lllllllll}26 & 26 & 26 & 26 & 25 & 26 & 26 & 26 & 26\end{array}$

$\begin{array}{lllllllll}26 & 26 & 26 & 26 & 24 & 26 & 26 & 26 & 26\end{array}$

$\begin{array}{lllllllll}26 & 26 & 26 & 26 & 25 & 26 & 26 & 26 & 26\end{array}$

$\begin{array}{lllllllll}26 & 26 & 26 & 26 & 24 & 26 & 26 & 26 & 26\end{array}$

$\begin{array}{lllllllll}26 & 26 & 26 & 26 & 25 & 26 & 26 & 26 & 26\end{array}$

$\begin{array}{lllllllll}26 & 26 & 26 & 26 & 24 & 26 & 26 & 26 & 26\end{array}$

$\begin{array}{lllllllll}26 & 26 & 26 & 26 & 25 & 26 & 26 & 26 & 26\end{array}$

$\begin{array}{lllllllll}26 & 26 & 26 & 26 & 24 & 26 & 26 & 26 & 26\end{array}$

$\begin{array}{lllllllll}26 & 26 & 26 & 26 & 25 & 26 & 26 & 26 & 26\end{array}$

$\begin{array}{lllllllll}26 & 26 & 26 & 26 & 24 & 26 & 26 & 26 & 26\end{array}$

$\begin{array}{lllllllll}26 & 26 & 26 & 26 & 25 & 26 & 26 & 26 & 26\end{array}$

$\begin{array}{lllllllll}26 & 26 & 26 & 26 & 24 & 26 & 26 & 26 & 26\end{array}$

$\begin{array}{lllllllll}26 & 26 & 26 & 26 & 25 & 26 & 26 & 26 & 26\end{array}$

$\begin{array}{lllllllll}26 & 26 & 26 & 26 & 24 & 26 & 26 & 26 & 26\end{array}$

$\begin{array}{lllllllll}26 & 26 & 26 & 26 & 25 & 26 & 26 & 26 & 26\end{array}$

$\begin{array}{lllllllll}26 & 26 & 26 & 26 & 24 & 26 & 26 & 26 & 26\end{array}$

$\begin{array}{lllllllll}26 & 26 & 26 & 26 & 25 & 26 & 26 & 26 & 26\end{array}$

$\begin{array}{lllllllll}26 & 26 & 26 & 26 & 24 & 26 & 26 & 26 & 26\end{array}$

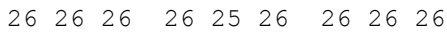

$\begin{array}{lllllllll}26 & 26 & 26 & 26 & 26 & 26 & 26 & 26 & 26\end{array}$

$\begin{array}{lllllllll}26 & 26 & 26 & 26 & 26 & 26 & 26 & 26 & 26\end{array}$

$\begin{array}{lllllllll}26 & 26 & 26 & 26 & 26 & 26 & 26 & 26 & 26\end{array}$

$\begin{array}{lllllllll}26 & 26 & 26 & 26 & 26 & 26 & 26 & 26 & 26\end{array}$

$\begin{array}{lllllllll}26 & 26 & 26 & 26 & 26 & 26 & 26 & 26 & 26\end{array}$

$\begin{array}{lllllllll}26 & 26 & 26 & 26 & 26 & 26 & 26 & 26 & 26\end{array}$

$\begin{array}{lllllllll}26 & 26 & 26 & 26 & 26 & 26 & 26 & 26 & 26\end{array}$

$\begin{array}{lllllllll}26 & 26 & 26 & 26 & 26 & 26 & 26 & 26 & 26\end{array}$

$\begin{array}{lllllllll}26 & 26 & 26 & 26 & 26 & 26 & 26 & 26 & 26\end{array}$

$\begin{array}{lllllllll}26 & 26 & 26 & 26 & 26 & 26 & 26 & 26 & 26\end{array}$

$\begin{array}{lllllllll}26 & 26 & 26 & 26 & 26 & 26 & 26 & 26 & 26\end{array}$

$\begin{array}{lllllllll}26 & 26 & 26 & 26 & 26 & 26 & 26 & 26 & 26\end{array}$

$\begin{array}{lllllllll}26 & 26 & 26 & 26 & 26 & 26 & 26 & 26 & 26\end{array}$

$\begin{array}{lllllllll}26 & 26 & 26 & 26 & 26 & 26 & 26 & 26 & 26\end{array}$

$\begin{array}{lllllllll}26 & 26 & 26 & 26 & 26 & 26 & 26 & 26 & 26\end{array}$

$\begin{array}{lllllllll}26 & 26 & 26 & 26 & 26 & 26 & 26 & 26 & 26\end{array}$

$\begin{array}{lllllllll}26 & 26 & 26 & 26 & 26 & 26 & 26 & 26 & 26\end{array}$

$\begin{array}{lllllllll}26 & 26 & 26 & 26 & 26 & 26 & 26 & 26 & 26\end{array}$

$\begin{array}{lllllllll}26 & 26 & 26 & 26 & 26 & 26 & 26 & 26 & 26\end{array}$

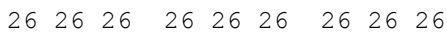

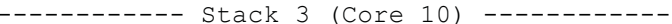

$6007104.8413 \mathrm{E}-05-6001$ imp:n=1 $u=33$ lat=2 fill=-1:1 -1:1 $0: 44$ $\begin{array}{lllllllll}26 & 26 & 26 & 26 & 26 & 26 & 26 & 26 & 26\end{array}$

C

$\begin{array}{lllllllll}26 & 26 & 26 & 26 & 25 & 26 & 26 & 26 & 26\end{array}$

$\begin{array}{lllllllll}26 & 26 & 26 & 26 & 24 & 26 & 26 & 26 & 26\end{array}$

$\begin{array}{lllllllll}26 & 26 & 26 & 26 & 24 & 26 & 26 & 26 & 26\end{array}$

$\begin{array}{lllllllll}26 & 26 & 26 & 26 & 25 & 26 & 26 & 26 & 26\end{array}$

$\begin{array}{lllllllll}26 & 26 & 26 & 26 & 25 & 26 & 26 & 26 & 26\end{array}$

C

$\begin{array}{lllllllll}26 & 26 & 26 & 26 & 24 & 26 & 26 & 26 & 26\end{array}$

$\begin{array}{lllllllll}26 & 26 & 26 & 26 & 25 & 26 & 26 & 26 & 26\end{array}$

$\begin{array}{lllllllll}26 & 26 & 26 & 26 & 24 & 26 & 26 & 26 & 26\end{array}$

$\begin{array}{lllllllll}26 & 26 & 26 & 26 & 24 & 26 & 26 & 26 & 26\end{array}$

$\begin{array}{lllllllll}26 & 26 & 26 & 26 & 25 & 26 & 26 & 26 & 26\end{array}$

$\begin{array}{lllllllll}26 & 26 & 26 & 26 & 25 & 26 & 26 & 26 & 26\end{array}$

$\begin{array}{lllllllll}26 & 26 & 26 & 26 & 24 & 26 & 26 & 26 & 26\end{array}$

C

$\begin{array}{lllllllll}26 & 26 & 26 & 26 & 25 & 26 & 26 & 26 & 26\end{array}$

$\begin{array}{lllllllll}26 & 26 & 26 & 26 & 24 & 26 & 26 & 26 & 26\end{array}$

$\begin{array}{lllllllll}26 & 26 & 26 & 26 & 24 & 26 & 26 & 26 & 26\end{array}$

$\begin{array}{lllllllll}26 & 26 & 26 & 26 & 25 & 26 & 26 & 26 & 26\end{array}$

$\begin{array}{lllllllll}26 & 26 & 26 & 26 & 25 & 26 & 26 & 26 & 26\end{array}$

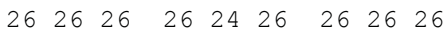

$\begin{array}{lllllllll}26 & 26 & 26 & 26 & 25 & 26 & 26 & 26 & 26\end{array}$

Revision: 1

Date: March 31, 2014

Page 269 of 375 
NEA/NSC/DOC(2006)1

Gas Cooled (Thermal) Reactor - GCR

PROTEUS-GCR-EXP-004

CRIT-REAC

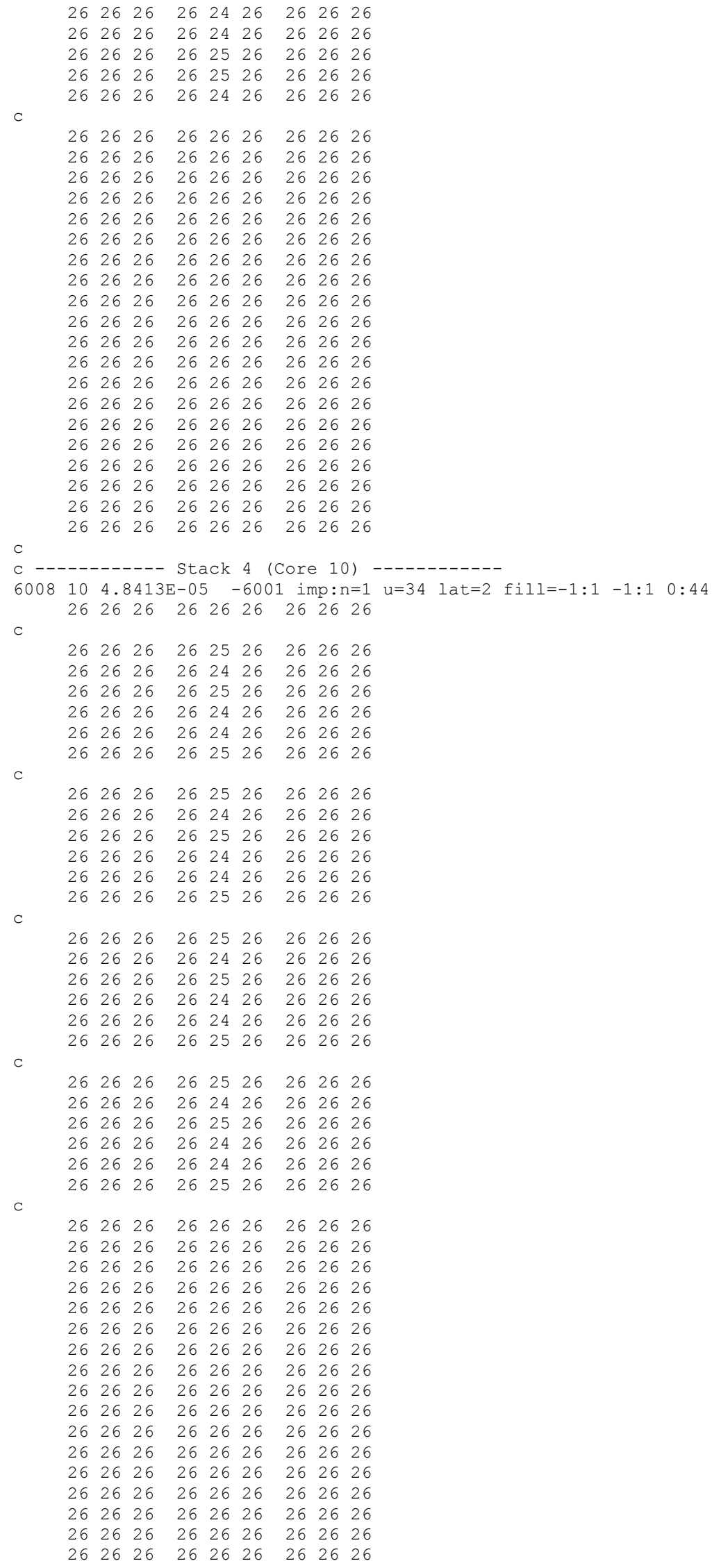

Revision: 1

Date: March 31, 2014

Page 270 of 375 
NEA/NSC/DOC(2006)1

\section{Gas Cooled (Thermal) Reactor - GCR \\ PROTEUS-GCR-EXP-004 CRIT-REAC}

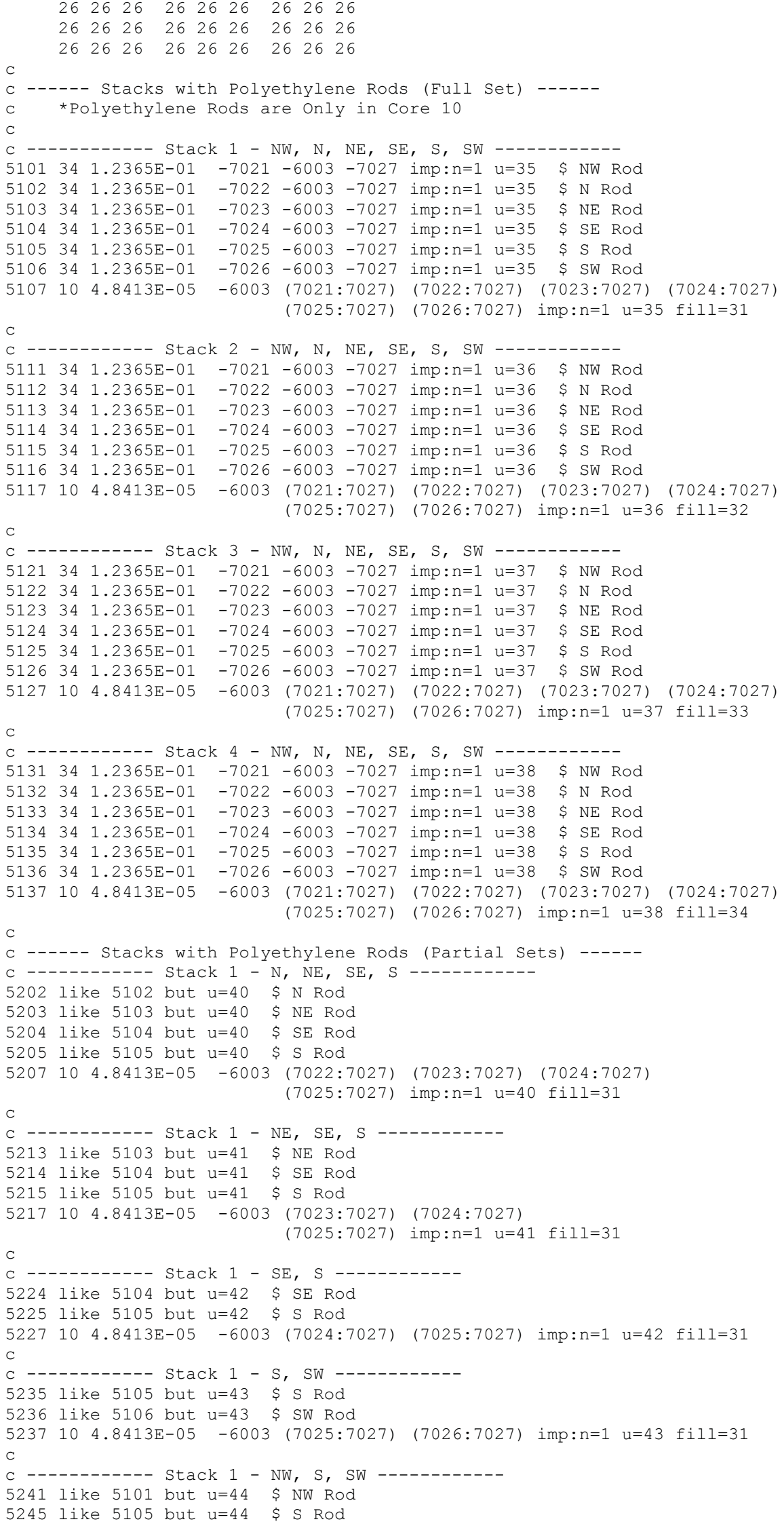

Revision: 1 
NEA/NSC/DOC(2006)1

\section{Gas Cooled (Thermal) Reactor - GCR \\ PROTEUS-GCR-EXP-004 CRIT-REAC}

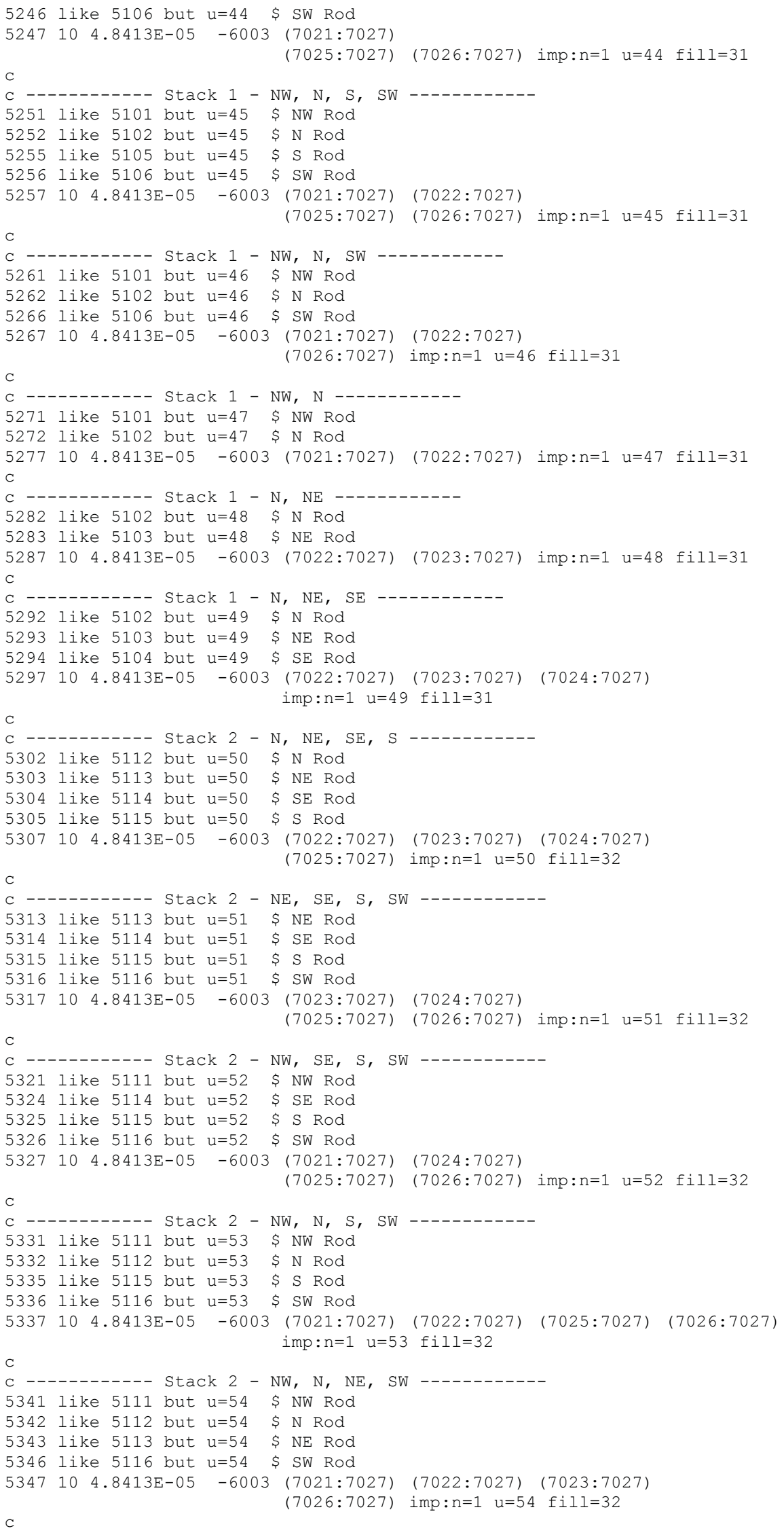

Revision: 1 
NEA/NSC/DOC(2006)1

\section{Gas Cooled (Thermal) Reactor - GCR \\ PROTEUS-GCR-EXP-004 CRIT-REAC}

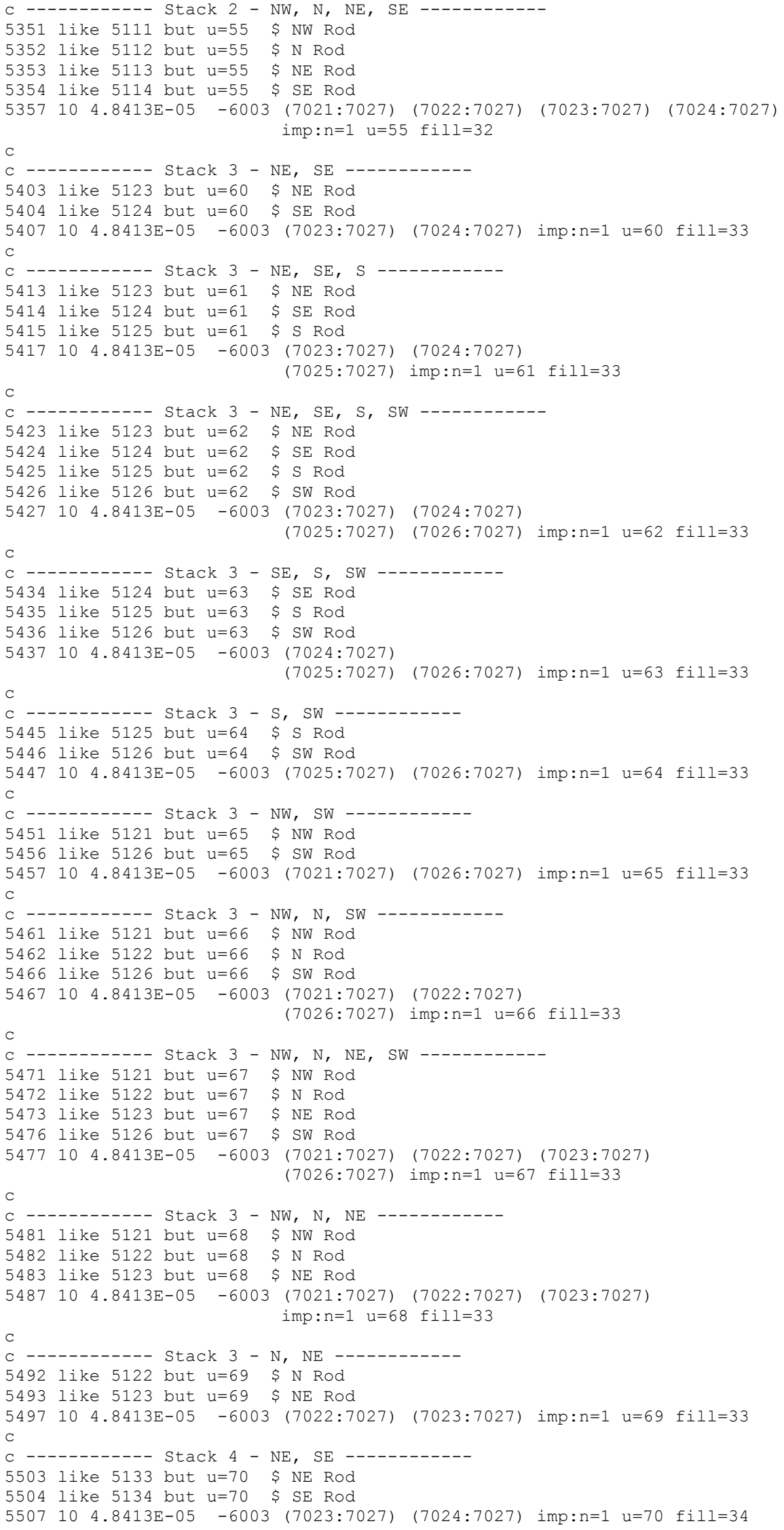

Revision: 1 
NEA/NSC/DOC(2006)1

\section{Gas Cooled (Thermal) Reactor - GCR \\ PROTEUS-GCR-EXP-004 CRIT-REAC}

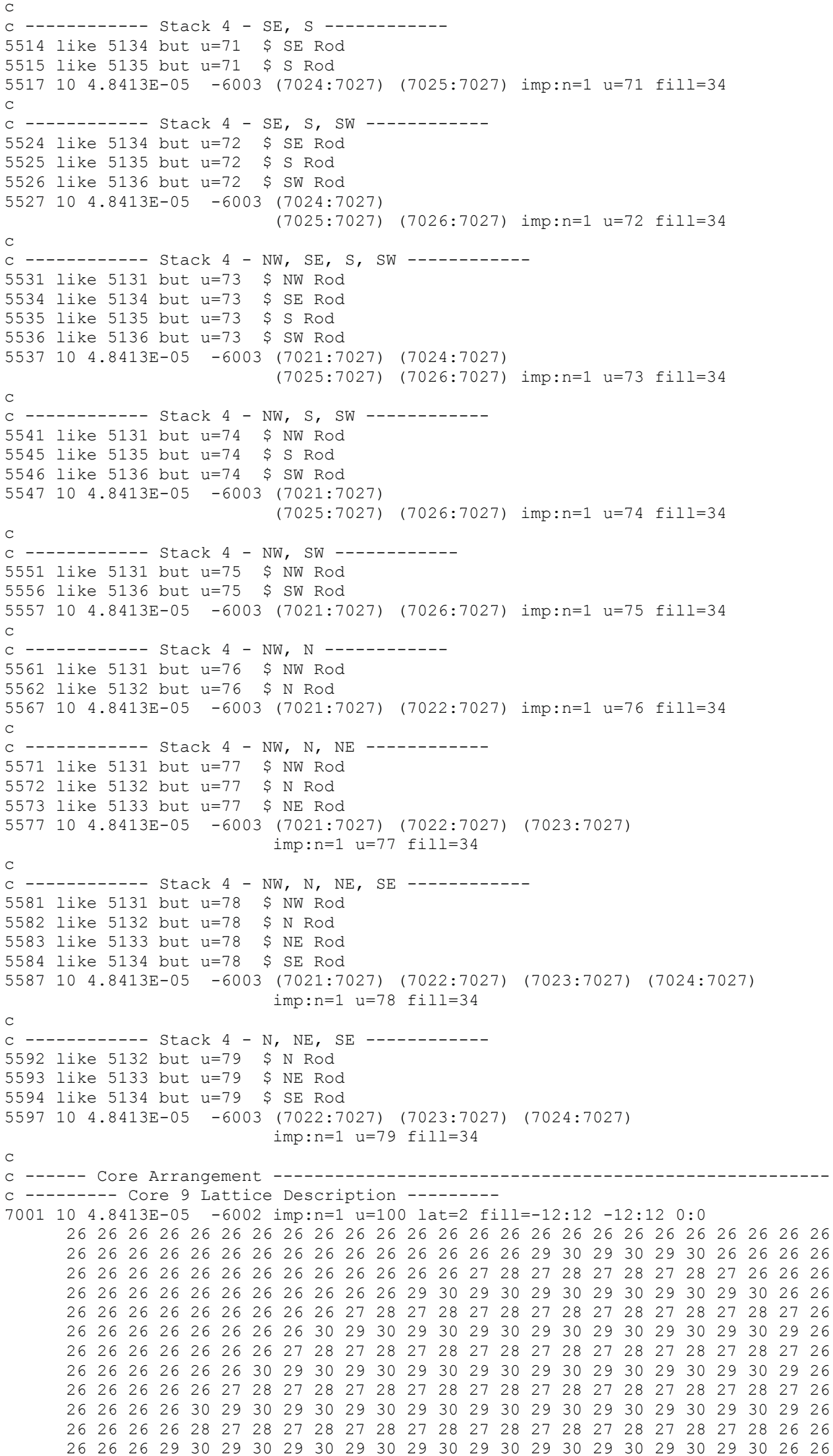

Revision: 1 
NEA/NSC/DOC(2006)1

\section{Gas Cooled (Thermal) Reactor - GCR \\ PROTEUS-GCR-EXP-004 CRIT-REAC} C -------- Core 10 Lattice Description --------

$7002104.8413 E-05 \quad-6002$ imp:n=1 u=101 lat=2 fill=-12:12 -12:12 0:0

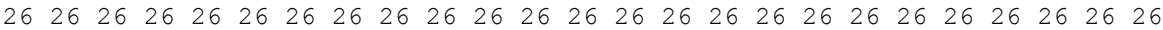

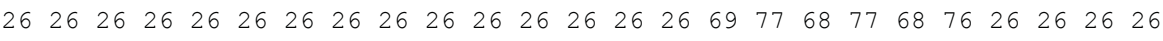

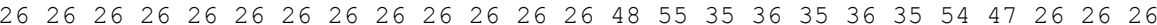
$\begin{array}{lllllllllllllllllllllllll}26 & 26 & 26 & 26 & 26 & 26 & 26 & 26 & 26 & 26 & 26 & 69 & 78 & 37 & 38 & 37 & 38 & 37 & 38 & 37 & 38 & 67 & 76 & 26 & 26\end{array}$

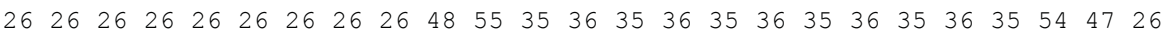
$\begin{array}{lllllllllllllllllllllllll}26 & 26 & 26 & 26 & 26 & 26 & 26 & 26 & 79 & 37 & 38 & 37 & 38 & 37 & 38 & 37 & 38 & 37 & 38 & 37 & 38 & 37 & 38 & 66 & 26\end{array}$

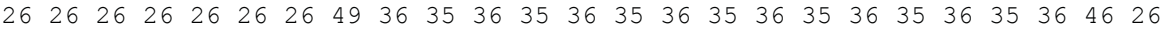
$\begin{array}{lllllllllllllllllllllllll}26 & 26 & 26 & 26 & 26 & 26 & 79 & 37 & 38 & 37 & 38 & 37 & 38 & 37 & 38 & 37 & 38 & 37 & 38 & 37 & 38 & 37 & 38 & 66 & 26\end{array}$ $\begin{array}{lllllllllllllllllllllllll}26 & 26 & 26 & 26 & 26 & 49 & 36 & 35 & 36 & 35 & 36 & 35 & 36 & 35 & 36 & 35 & 36 & 35 & 36 & 35 & 36 & 35 & 36 & 46 & 26\end{array}$ $\begin{array}{lllllllllllllllllllllllll}26 & 26 & 26 & 26 & 70 & 37 & 38 & 37 & 38 & 37 & 38 & 37 & 38 & 37 & 38 & 37 & 38 & 37 & 38 & 37 & 38 & 37 & 38 & 65 & 26\end{array}$ $\begin{array}{lllllllllllllllllllllllll}26 & 26 & 26 & 26 & 50 & 35 & 36 & 35 & 36 & 35 & 36 & 35 & 36 & 35 & 36 & 35 & 36 & 35 & 36 & 35 & 36 & 35 & 53 & 26 & 26\end{array}$ $\begin{array}{lllllllllllllllllllllllll}26 & 26 & 26 & 60 & 38 & 37 & 38 & 37 & 38 & 37 & 38 & 37 & 38 & 37 & 38 & 37 & 38 & 37 & 38 & 37 & 38 & 37 & 75 & 26 & 26\end{array}$

$\mathrm{C}$

$262626 \quad 40 \quad 36 \quad 35 \quad 36 \quad 35 \quad 36 \quad 35 \quad 36 \quad 35 \quad 36 \quad 35 \quad 36 \quad 35 \quad 36 \quad 35 \quad 36 \quad 35 \quad 36 \quad 45 \quad 26 \quad 26 \quad 26$

$\begin{array}{llllllllllllllllllllllllll}26 & 26 & 70 & 37 & 38 & 37 & 38 & 37 & 38 & 37 & 38 & 37 & 38 & 37 & 38 & 37 & 38 & 37 & 38 & 37 & 38 & 65 & 26 & 26 & 26\end{array}$

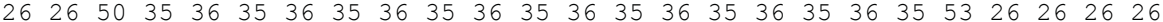
$\begin{array}{lllllllllllllllllllllllll}26 & 60 & 38 & 37 & 38 & 37 & 38 & 37 & 38 & 37 & 38 & 37 & 38 & 37 & 38 & 37 & 38 & 37 & 38 & 37 & 75 & 26 & 26 & 26 & 26\end{array}$ $\begin{array}{llllllllllllllllllllllllll}26 & 41 & 36 & 35 & 36 & 35 & 36 & 35 & 36 & 35 & 36 & 35 & 36 & 35 & 36 & 35 & 36 & 35 & 36 & 44 & 26 & 26 & 26 & 26 & 26\end{array}$ $\begin{array}{lllllllllllllllllllllllll}26 & 61 & 38 & 37 & 38 & 37 & 38 & 37 & 38 & 37 & 38 & 37 & 38 & 37 & 38 & 37 & 38 & 37 & 74 & 26 & 26 & 26 & 26 & 26 & 26\end{array}$ $\begin{array}{lllllllllllllllllllllllll}26 & 41 & 36 & 35 & 36 & 35 & 36 & 35 & 36 & 35 & 36 & 35 & 36 & 35 & 36 & 35 & 36 & 44 & 26 & 26 & 26 & 26 & 26 & 26 & 26\end{array}$ $\begin{array}{lllllllllllllllllllllllll}26 & 61 & 38 & 37 & 38 & 37 & 38 & 37 & 38 & 37 & 38 & 37 & 38 & 37 & 38 & 37 & 74 & 26 & 26 & 26 & 26 & 26 & 26 & 26 & 26\end{array}$ $\begin{array}{lllllllllllllllllllllllll}26 & 42 & 51 & 35 & 36 & 35 & 36 & 35 & 36 & 35 & 36 & 35 & 36 & 35 & 52 & 43 & 26 & 26 & 26 & 26 & 26 & 26 & 26 & 26 & 26\end{array}$ $\begin{array}{lllllllllllllllllllllllll}26 & 26 & 71 & 62 & 38 & 37 & 38 & 37 & 38 & 37 & 38 & 37 & 73 & 64 & 26 & 26 & 26 & 26 & 26 & 26 & 26 & 26 & 26 & 26 & 26\end{array}$ $\begin{array}{lllllllllllllllllllllllll}26 & 26 & 26 & 42 & 51 & 35 & 36 & 35 & 36 & 35 & 52 & 43 & 26 & 26 & 26 & 26 & 26 & 26 & 26 & 26 & 26 & 26 & 26 & 26 & 26\end{array}$ $\begin{array}{lllllllllllllllllllllllll}26 & 26 & 26 & 26 & 71 & 63 & 72 & 63 & 72 & 64 & 26 & 26 & 26 & 26 & 26 & 26 & 26 & 26 & 26 & 26 & 26 & 26 & 26 & 26 & 26\end{array}$

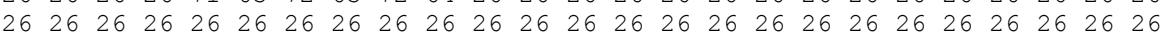
c -------- Pebble-Filled Core Cavity ---------

$7101 \quad 104.8413 \mathrm{E}-05 \quad-7001-7002-7003$ imp: $n=1$ fill=100 (0 0 75) \$ Core 9

C $7101104.8413 \mathrm{E}-05$-7001 -7002 -7003 imp:n=1 fill=101 (0 0 75) $\$$ Core 10

$\mathrm{C}$

C --- Model Boundary

$99990-31: 1202: 34$ imp:n=0 \$ The Great Void

C

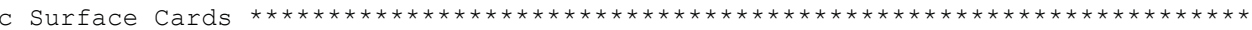

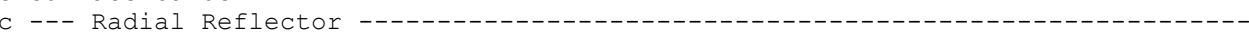

C ------ Graphite Annulus ------

$31 \mathrm{pz} \quad 0.0 \quad$ \$ Bottom of Reflector

$32 \mathrm{pz} \quad 330.4 \quad \$$ Top of Reflector

$33 \mathrm{Cz} \quad 62.83398$ \$ Inside Radial Equivalent-Area Cavity Surface

$34 \mathrm{Cz} \quad 163.76986$ \$ Outside Radial Equivalent-Area Surface

C ------ Safety/Shutdown Rod Holes ------

$1101 \mathrm{C} / \mathrm{z} \quad-38.45 \quad 56.57 \quad 2.25 \quad$ \$ Rod 1

$1102 \mathrm{c} / \mathrm{z} \quad 32.74-60.05 \quad 2.25 \quad \$$ Rod 2

$1103 \mathrm{c} / \mathrm{z} \quad 57.17 \quad 37.55 \quad 2.25 \quad$ \$ Rod 3

$1104 \mathrm{c} / \mathrm{z}-53.23-42.95 \quad 2.25 \quad$ \$ Rod 4

$1105 \mathrm{c} / \mathrm{z} \quad 67.19-12.82 \quad 2.25 \quad \$ \operatorname{Rod} 5$

$\begin{array}{llllll}1106 & \mathrm{c} / \mathrm{z} & -66.98 & 13.87 & 2.25 & \$ \text { Rod } 6\end{array}$

$\begin{array}{llllll}1107 & \mathrm{c} / \mathrm{z} & 19.31 & 65.62 & 2.25 & \$ \mathrm{Rod} 7\end{array}$

$1108 \mathrm{C} / \mathrm{z} \quad-13.87-66.98 \quad 2.25 \quad \$ \operatorname{Rod} 8$ $\mathrm{C}$

c ----- Withdrawable Control Rod Holes ------

$503 \mathrm{c} / \mathrm{z} \quad-83.70 \quad 34.67 \quad 1.3715$ \$ Position 3 Hole

$519 \mathrm{c} / \mathrm{z} \quad 34.67 \quad 83.70 \quad 1.3715 \quad$ \$ Position $19 \mathrm{Hole}$

$535 \mathrm{c} / \mathrm{z} \quad 83.70-34.67 \quad 1.3715$ \$ Position $35 \mathrm{Hole}$

$551 \mathrm{c} / \mathrm{z} \quad-34.67-83.70 \quad 1.3715$ \$ Position 51 Hole

Revision: 1

Date: March 31, 2014

Page 275 of 375 
NEA/NSC/DOC(2006)1

Gas Cooled (Thermal) Reactor - GCR

\section{PROTEUS-GCR-EXP-004} CRIT-REAC

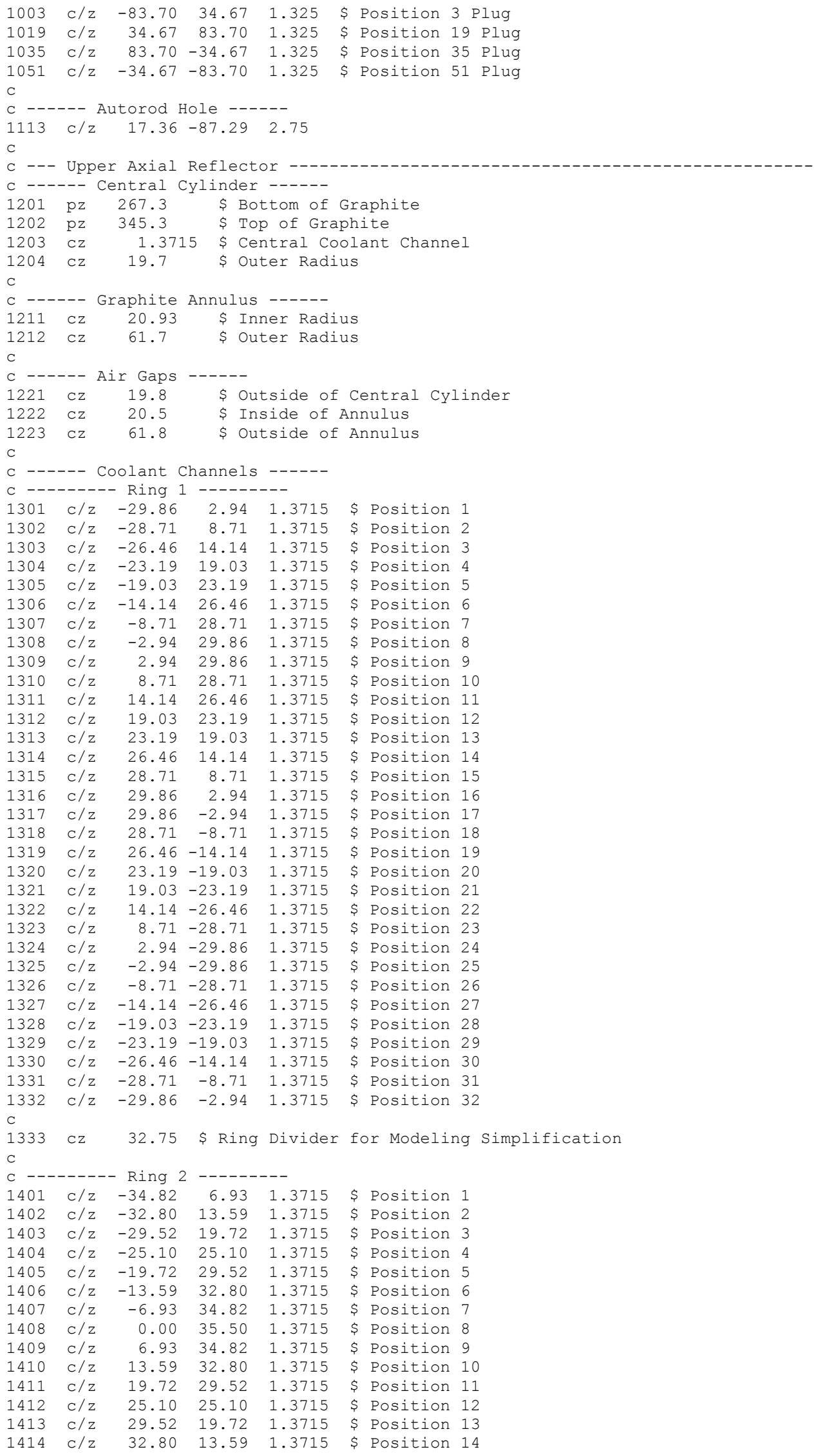

Revision: 1 
NEA/NSC/DOC(2006)1

Gas Cooled (Thermal) Reactor - GCR

PROTEUS-GCR-EXP-004 CRIT-REAC

\begin{tabular}{|c|c|c|c|c|c|c|}
\hline 1415 & $\mathrm{C} / \mathrm{z}$ & 34.82 & 6.93 & 1.3715 & \$ Position 15 & \\
\hline 1416 & $\mathrm{C} / \mathrm{z}$ & 35.50 & 0.00 & 1.3715 & \$ Position 16 & \\
\hline 1417 & $\mathrm{C} / \mathrm{z}$ & 34.82 & -6.93 & 1.3715 & \$ Position 17 & \\
\hline 1418 & $\mathrm{C} / \mathrm{z}$ & 32.80 & -13.59 & 1.3715 & \$ Position 18 & \\
\hline 1419 & $\mathrm{C} / \mathrm{z}$ & 29.52 & -19.72 & 1.3715 & \$ Position 19 & \\
\hline 1420 & $\mathrm{C} / \mathrm{z}$ & 25.10 & -25.10 & 1.3715 & \$ Position 20 & \\
\hline 1421 & $\mathrm{C} / \mathrm{z}$ & 19.72 & -29.52 & 1.3715 & \$ Position 21 & \\
\hline 1422 & $\mathrm{C} / \mathrm{z}$ & 13.59 & -32.80 & 1.3715 & \$ Position 22 & \\
\hline 1423 & $\mathrm{C} / \mathrm{z}$ & 6.93 & -34.82 & 1.3715 & \$ Position 23 & \\
\hline 1424 & $\mathrm{C} / \mathrm{z}$ & 0.00 & -35.50 & 1.3715 & \$ Position 24 & \\
\hline 1425 & $\mathrm{C} / \mathrm{z}$ & -6.93 & -34.82 & 1.3715 & \$ Position 25 & \\
\hline 1426 & $\mathrm{C} / \mathrm{z}$ & -13.59 & -32.80 & 1.3715 & \$ Position 26 & \\
\hline 1427 & $\mathrm{C} / \mathrm{z}$ & -19.72 & -29.52 & 1.3715 & \$ Position 27 & \\
\hline 1428 & $\mathrm{C} / \mathrm{z}$ & -25.10 & -25.10 & 1.3715 & \$ Position 28 & \\
\hline 1429 & $\mathrm{C} / \mathrm{z}$ & -29.52 & -19.72 & 1.3715 & \$ Position 29 & \\
\hline 1430 & $\mathrm{C} / \mathrm{z}$ & -32.80 & -13.59 & 1.3715 & \$ Position 30 & \\
\hline 1431 & $\mathrm{C} / \mathrm{z}$ & -34.82 & -6.93 & 1.3715 & \$ Position 31 & \\
\hline 1432 & $\mathrm{C} / \mathrm{z}$ & -35.50 & 0.00 & 1.3715 & \$ Position 32 & \\
\hline & & & & & & \\
\hline 1433 & $\mathrm{Cz}$ & 38.25 & \$ Ring & Divider & \multirow[t]{2}{*}{ for Modeling } & Simplification \\
\hline \multirow{2}{*}{\multicolumn{7}{|c|}{ C }} \\
\hline & & & & & & \\
\hline 1501 & $\mathrm{C} / \mathrm{z}$ & -39.23 & 11.90 & 1.3715 & \$ Position 1 & \\
\hline 1502 & $\mathrm{C} / \mathrm{z}$ & -36.16 & 19.33 & 1.3715 & \$ Position 2 & \\
\hline 1503 & $\mathrm{C} / \mathrm{z}$ & -31.69 & 26.01 & 1.3715 & \$ Position 3 & \\
\hline 1504 & $\mathrm{C} / \mathrm{z}$ & -26.01 & 31.69 & 1.3715 & \$ Position 4 & \\
\hline 1505 & $\mathrm{C} / \mathrm{z}$ & -19.33 & 36.16 & 1.3715 & \$ Position 5 & \\
\hline 1506 & $\mathrm{C} / \mathrm{z}$ & -11.90 & 39.23 & 1.3715 & \$ Position 6 & \\
\hline 1507 & $\mathrm{C} / \mathrm{z}$ & -4.02 & 40.80 & 1.3715 & \$ Position 7 & \\
\hline 1508 & $\mathrm{C} / \mathrm{z}$ & 4.02 & 40.80 & 1.3715 & $\$$ Position 8 & \\
\hline 1509 & $\mathrm{C} / \mathrm{z}$ & 11.90 & 39.23 & 1.3715 & \$ Position 9 & \\
\hline 1510 & $\mathrm{C} / \mathrm{z}$ & 19.33 & 36.16 & 1.3715 & \$ Position 10 & \\
\hline 1511 & $\mathrm{C} / \mathrm{z}$ & 26.01 & 31.69 & 1.3715 & \$ Position 11 & \\
\hline 1512 & $\mathrm{C} / \mathrm{z}$ & 31.69 & 26.01 & 1.3715 & \$ Position 12 & \\
\hline 1513 & $\mathrm{C} / \mathrm{z}$ & 36.16 & 19.33 & 1.3715 & \$ Position 13 & \\
\hline 1514 & $\mathrm{C} / \mathrm{z}$ & 39.23 & 11.90 & 1.3715 & \$ Position 14 & \\
\hline 1515 & $\mathrm{C} / \mathrm{z}$ & 40.80 & 4.02 & 1.3715 & \$ Position 15 & \\
\hline 1516 & $\mathrm{C} / \mathrm{z}$ & 40.80 & -4.02 & 1.3715 & \$ Position 16 & \\
\hline 1517 & $\mathrm{C} / \mathrm{z}$ & 39.23 & $-11 \cdot 90$ & 1.3715 & \$ Position 17 & \\
\hline 1518 & $\mathrm{C} / \mathrm{z}$ & 36.16 & -19.33 & 1.3715 & \$ Position 18 & \\
\hline 1519 & $\mathrm{C} / \mathrm{z}$ & 31.69 & -26.01 & 1.3715 & \$ Position 19 & \\
\hline 1520 & $\mathrm{C} / \mathrm{z}$ & 26.01 & -31.69 & 1.3715 & \$ Position 20 & \\
\hline 1521 & $\mathrm{C} / \mathrm{z}$ & 19.33 & -36.16 & 1.3715 & \$ Position 21 & \\
\hline 1522 & $\mathrm{C} / \mathrm{z}$ & 11.90 & -39.23 & 1.3715 & \$ Position 22 & \\
\hline 1523 & $\mathrm{C} / \mathrm{z}$ & 4.02 & -40.80 & 1.3715 & \$ Position 23 & \\
\hline 1524 & $\mathrm{C} / \mathrm{z}$ & -4.02 & -40.80 & 1.3715 & \$ Position 24 & \\
\hline 1525 & $\mathrm{C} / \mathrm{z}$ & -11.90 & -39.23 & 1.3715 & \$ Position 25 & \\
\hline 1526 & $\mathrm{C} / \mathrm{z}$ & -19.33 & -36.16 & 1.3715 & \$ Position 26 & \\
\hline 1527 & $\mathrm{C} / \mathrm{z}$ & -26.01 & -31.69 & 1.3715 & \$ Position 27 & \\
\hline 1528 & $\mathrm{C} / \mathrm{z}$ & -31.69 & -26.01 & 1.3715 & \$ Position 28 & \\
\hline 1529 & $\mathrm{C} / \mathrm{z}$ & -36.16 & -19.33 & 1.3715 & \$ Position 29 & \\
\hline 1530 & $\mathrm{C} / \mathrm{z}$ & -39.23 & -11.90 & 1.3715 & \$ Position 30 & \\
\hline 1531 & $\mathrm{C} / \mathrm{z}$ & -40.80 & -4.02 & 1.3715 & \$ Position 31 & \\
\hline 1532 & $\mathrm{C} / \mathrm{z}$ & -40.80 & 4.02 & 1.3715 & \$ Position 32 & \\
\hline \multicolumn{7}{|c|}{ 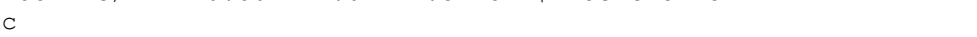 } \\
\hline 1533 & $\mathrm{Cz}$ & 43.625 & \$ Ring & Divider & for Modeling & Simplification \\
\hline \multicolumn{7}{|l|}{ C } \\
\hline C -- & ---- & - Ring 4 & $4-1$ & --- & & \\
\hline 1601 & $\mathrm{C} / \mathrm{z}$ & -42.73 & 17.70 & 1.3715 & \$ Position 1 & \\
\hline 1602 & $\mathrm{C} / \mathrm{z}$ & -38.46 & 25.70 & 1.3715 & $\$$ Position 2 & \\
\hline 1603 & $\mathrm{C} / \mathrm{z}$ & -32.70 & 32.70 & 1.3715 & \$ Position 3 & \\
\hline 1604 & $\mathrm{C} / \mathrm{z}$ & -25.70 & 38.46 & 1.3715 & \$ Position 4 & \\
\hline 1605 & $\mathrm{C} / \mathrm{z}$ & -17.70 & 42.73 & 1.3715 & \$ Position 5 & \\
\hline 1606 & $\mathrm{C} / \mathrm{z}$ & -9.02 & 45.36 & 1.3715 & \$ Position 6 & \\
\hline 1607 & $\mathrm{C} / \mathrm{z}$ & 0.00 & 46.25 & 1.3715 & \$ Position 7 & \\
\hline 1608 & $\mathrm{C} / \mathrm{z}$ & 9.02 & 45.36 & 1.3715 & \$ Position 8 & \\
\hline 1609 & $\mathrm{C} / \mathrm{z}$ & 17.70 & 42.73 & 1.3715 & \$ Position 9 & \\
\hline 1610 & $\mathrm{C} / \mathrm{z}$ & 25.70 & 38.46 & 1.3715 & \$ Position 10 & \\
\hline 1611 & $\mathrm{C} / \mathrm{z}$ & 32.70 & 32.70 & 1.3715 & \$ Position 11 & \\
\hline 1612 & $\mathrm{C} / \mathrm{z}$ & 38.46 & 25.70 & 1.3715 & \$ Position 12 & \\
\hline 1613 & $\mathrm{C} / \mathrm{z}$ & 42.73 & 17.70 & 1.3715 & \$ Position 13 & \\
\hline 1614 & $\mathrm{C} / \mathrm{z}$ & 45.36 & 9.02 & 1.3715 & \$ Position 14 & \\
\hline 1615 & $\mathrm{C} / \mathrm{z}$ & 46.25 & 0.00 & 1.3715 & \$ Position 15 & \\
\hline 1616 & $\mathrm{C} / \mathrm{z}$ & 45.36 & -9.02 & 1.3715 & \$ Position 16 & \\
\hline 1617 & $\mathrm{C} / \mathrm{z}$ & 42.73 & -17.70 & 1.3715 & \$ Position 17 & \\
\hline 1618 & $\mathrm{C} / \mathrm{z}$ & 38.46 & -25.70 & 1.3715 & \$ Position 18 & \\
\hline
\end{tabular}

Revision: 1 
NEA/NSC/DOC(2006)1

Gas Cooled (Thermal) Reactor - GCR

PROTEUS-GCR-EXP-004

CRIT-REAC

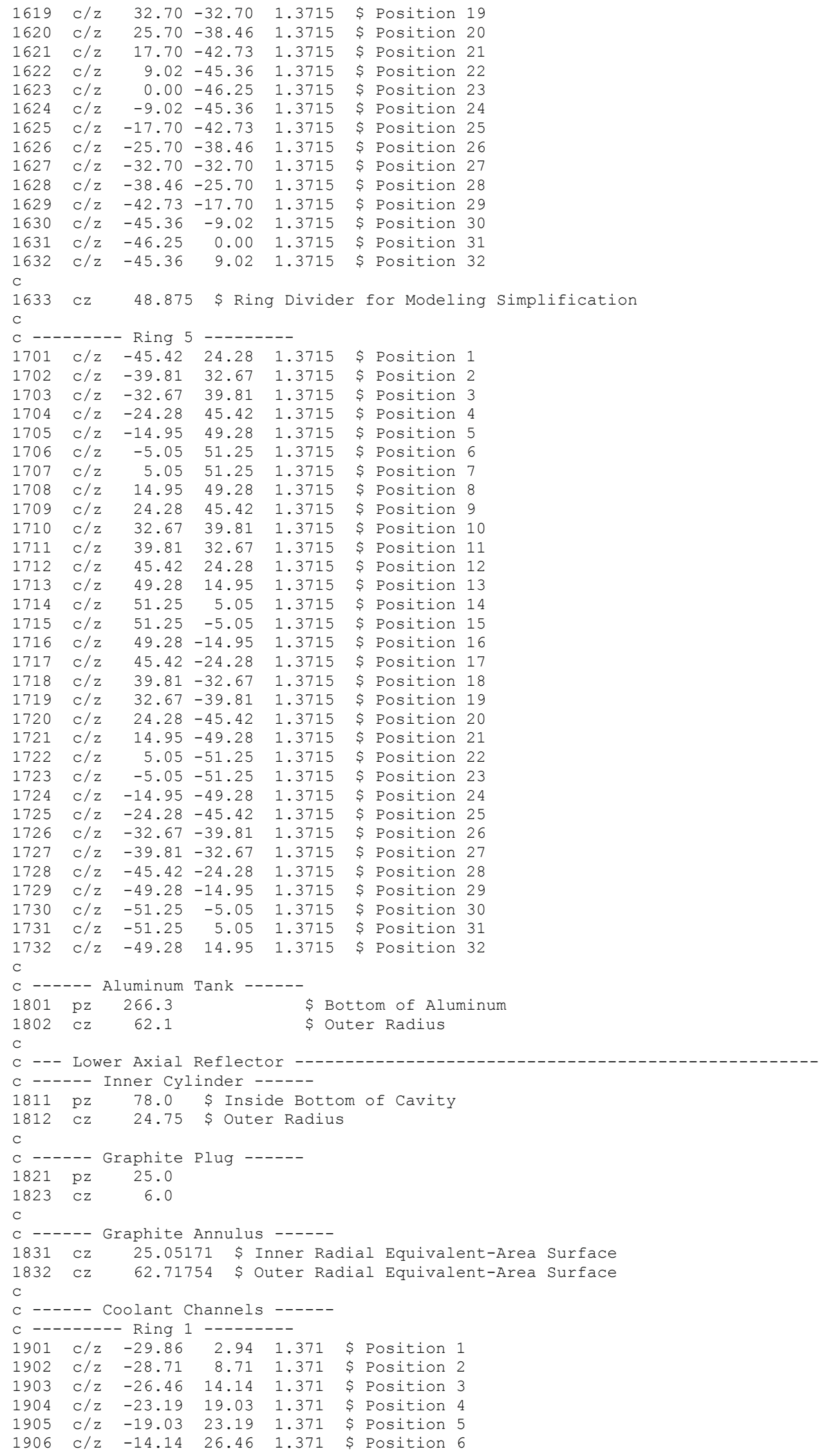

Revision: 1 
NEA/NSC/DOC(2006)1

Gas Cooled (Thermal) Reactor - GCR

PROTEUS-GCR-EXP-004

CRIT-REAC

\begin{tabular}{|c|c|c|c|c|c|c|c|}
\hline 07 & $\mathrm{C} / \mathrm{z}$ & -8.71 & 28.71 & 1.371 & $\$$ & Position & 7 \\
\hline 08 & $c / z$ & -2.94 & 29.86 & 1.371 & \$ & Position & 8 \\
\hline & $\mathrm{C} / \mathrm{z}$ & 2.94 & 29.86 & 1.371 & $\$$ & Position & \\
\hline & $\mathrm{C} / \mathrm{z}$ & 8.71 & 28.71 & .371 & & Posi & \\
\hline & $\mathrm{C} / \mathrm{z}$ & 14.14 & 26.46 & L. 371 & Y & Pos: & \\
\hline & $\mathrm{C} / \mathrm{z}$ & 19.03 & 23.19 & .371 & ; & Pos: & \\
\hline & $c / z$ & 23.19 & 03 & L. 371 & & & \\
\hline & $\mathrm{C} / \mathrm{z}$ & 26.46 & 1 & 371 & & Po & \\
\hline & $c / z$ & 28.71 & 8 . & 1.371 & ? & Po & \\
\hline & $\mathrm{C} / \mathrm{z}$ & 29.86 & 2 . & 1.371 & s & & \\
\hline & $c / z$ & 29.86 & -2 & 1.371 & 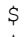 & & \\
\hline & $\mathrm{C} / \mathrm{z}$ & 28.71 & -8 & .371 & $\$$ & Po & \\
\hline & $\mathrm{C} / \mathrm{z}$ & 26.46 & -14. & .371 & \$ & & \\
\hline & $\mathrm{C} / \mathrm{z}$ & 23.19 & -19. & .371 & & & \\
\hline & $C / z$ & 19.03 & -23 . & .371 & ? & Po & \\
\hline & $c / z$ & 14.14 & -26. & .371 & \$ & Po & \\
\hline & $\mathrm{C} / \mathrm{z}$ & 8.71 & -28 & 371 & \$ & & \\
\hline & $C / z$ & 2.94 & -29 . & .371 & 5 & $n$ & \\
\hline & $\mathrm{C} / \mathrm{z}$ & -2.94 & -29 . & .371 & $\$$ & & \\
\hline & $\mathrm{C} / \mathrm{z}$ & -8.71 & -28 & .371 & \$ & Po & \\
\hline & $\mathrm{C} / \mathrm{z}$ & -14.14 & -26 & L. 371 & $\$$ & Po & \\
\hline & $c / z$ & 19.03 & -23 & 371 & $\$$ & $\mathrm{Po}$ & r \\
\hline & $\mathrm{C} / \mathrm{z}$ & -23.19 & -19 & 371 & $\$$ & & \\
\hline & $\mathrm{C} / \mathrm{z}$ & -26.46 & -14.14 & L. 371 & \$ & Po & \\
\hline & $C / z$ & -28.71 & -8.71 & .371 & $\$$ & $n$ & $3 \perp$ \\
\hline & $\mathrm{C} / \mathrm{z}$ & -29.86 & -2.9 & .371 & $\$$ & & \\
\hline \\
\hline & & 92 & & & & & \\
\hline & $c / z$ & -34.82 & 6. & 1.371 & $\$$ & on & 1 \\
\hline & $C / z$ & -32.80 & 13 & 1 . & $\$$ & $n$ & \\
\hline & $c / z$ & -29.52 & 19.72 & 1.371 & $\$$ & Po & 3 \\
\hline & $C / z$ & -25.10 & 25.10 & 1.371 & $\$$ & Po & 4 \\
\hline & $C / z$ & -1 & 29 & 71 & \$ & $\mathrm{pn}$ & \\
\hline & $\mathrm{C} / \mathrm{z}$ & -13 & 32 . & 1. & $\$$ & & 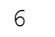 \\
\hline & $C / z$ & -6.93 & 34.82 & 1.371 & $\$$ & Pos & 7 \\
\hline & $c / z$ & .00 & 35. & 1.371 & \$ & Po & \\
\hline & $C / z$ & 6.93 & 34.82 & 1. & $\$$ & & 9 \\
\hline & $c / z$ & 13.59 & 32. & 1 . & $\$$ & $\mathrm{Po}$ & 10 \\
\hline & $c / z$ & .72 & 29. & 1.371 & $\$$ & $\mathrm{Po}$ & - \\
\hline & $c / z$ & 0 & 0 & 1 . & \$ & $\mathrm{Po}$ & -2 \\
\hline & $c / z$ & 2 & 19 & 1 . & $\$$ & & \\
\hline & $C / z$ & 32.80 & 13. & 1 . & $\$$ & $\mathrm{Po}$ & 14 \\
\hline & $\mathrm{C} / \mathrm{z}$ & 34.82 & 6. & 1.371 & $\$$ & & 5 \\
\hline & $C / z$ & 35.50 & 0. & 1.371 & $\$$ & $n$ & \\
\hline & $C / z$ & .82 & -6 . & 1 . & \$ & & \\
\hline & $\mathrm{C} / \mathrm{z}$ & .80 & -13. & 1 . & \$ & & 18 \\
\hline & $c / z$ & 29.52 & -19.72 & 1.371 & $\$$ & Po & 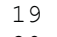 \\
\hline 20 & $\mathrm{C} / \mathrm{z}$ & 25.10 & -25.10 & 1.371 & $\$$ & & 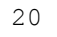 \\
\hline & $c / z$ & 19.72 & -29 . & 1.371 & \$ & Po & \\
\hline 22 & $\mathrm{C} / \mathrm{z}$ & 13.59 & -32.80 & 1.371 & S & Po & 22 \\
\hline 3 & $c / z$ & .93 & -34.82 & 1.371 & $\$$ & $\mathrm{Pc}$ & 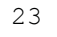 \\
\hline & $C / z$ & 0.00 & -35 & 1. & $\$$ & $\mathrm{Po}$ & 24 \\
\hline & $\mathrm{C} / \mathrm{z}$ & -6.93 & -34.82 & 1.371 & \$ & Po & 25 \\
\hline 26 & $C / z$ & -13.59 & -32 . & 1.371 & $\$$ & & 26 \\
\hline 27 & $C / z$ & -19.72 & -29 . & 1.371 & $\$$ & $\mathrm{Po}$ & -2 \\
\hline & $c / z$ & -25.10 & -25 . & 1 . & \$ & $\mathrm{Po}$ & $2 \zeta$ \\
\hline & $C / z$ & -29.52 & -19.72 & 1 . & $\$$ & & 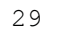 \\
\hline 30 & $c / z$ & -32.80 & -13.59 & 1. & $\$$ & $\mathrm{Po}$ & 30 \\
\hline & $c / z$ & -34.82 & -6.93 & & $\$$ & & 31 \\
\hline 32 & $c / z$ & -35.50 & 0.00 & 1. & $\$$ & Po: & 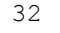 \\
\hline \\
\hline & & 3 & & -_ & & & \\
\hline & $c / z$ & -39.23 & 1 & 1.3 & $\$$ & & \\
\hline & $\mathrm{C} / \mathrm{z}$ & -36.16 & 1 & & $\$$ & Po: & 2 \\
\hline & $c / z$ & -31.69 & 26.01 & & $\$$ & Po: & 3 \\
\hline & $C / z$ & -26.01 & 31.69 & 1.371 & $\$$ & Pos & 4 \\
\hline & $c / z$ & -19.33 & 36.16 & 1.371 & $\$$ & Pos & 5 \\
\hline & $\mathrm{C} / \mathrm{z}$ & -11.90 & 39.23 & 1.371 & $\$$ & Pos & 6 \\
\hline & $c / z$ & -4.02 & 40.80 & 1.371 & $\$$ & Pos & 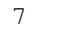 \\
\hline & $c / z$ & 4.02 & 40.80 & 1.371 & $\$$ & Pos: & 8 \\
\hline & $c / z$ & 11.90 & 39.23 & 1.371 & 3 & Pos & 9 \\
\hline & $c / z$ & 19.33 & 36.16 & 1.371 & $\$$ & Pos & \\
\hline & $\mathrm{C} / \mathrm{z}$ & 26.01 & 31.69 & 1.371 & S & Pos & \\
\hline & $c / z$ & 31.69 & 26.01 & 1.371 & \$ & Pos: & \\
\hline & $C / z$ & 36.16 & 19. & & & POS & \\
\hline & $c / z$ & 39.23 & 11.90 & 1.371 & $T$ & Pos & \\
\hline
\end{tabular}

Revision: 1 
NEA/NSC/DOC(2006)1

Gas Cooled (Thermal) Reactor - GCR

PROTEUS-GCR-EXP-004

CRIT-REAC

\begin{tabular}{|c|c|c|c|c|c|c|c|}
\hline 15 & $\mathrm{C} / \mathrm{z}$ & 80 & 02 & 1.371 & $\$$ & ion & \\
\hline 16 & $\mathrm{C} / \mathrm{z}$ & 40.80 & -4.02 & 1.371 & 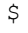 & Position & \\
\hline 17 & $\mathrm{C} / \mathrm{z}$ & 9.23 & -11.90 & 1.371 & $\$$ & Position & \\
\hline & $\mathrm{C} / \mathrm{z}$ & 6.16 & -19.33 & .371 & & Pos & \\
\hline & $\mathrm{C} / \mathrm{z}$ & 31.69 & -26.01 & .371 & & Pos: & \\
\hline & $\mathrm{C} / \mathrm{z}$ & 26.01 & -31.69 & L. 371 & & Pos: & \\
\hline & $\mathrm{C} / \mathrm{z}$ & 9.33 & -36.16 & L. 371 & & & \\
\hline & $\mathrm{C} / \mathrm{z}$ & 1.90 & -39.23 & .371 & & & \\
\hline & $\mathrm{C} / \mathrm{z}$ & 4.02 & -40.80 & 1.371 & & Po & \\
\hline & $\mathrm{C} / \mathrm{z}$ & -4.02 & -40.80 & L. 371 & & & \\
\hline & $\mathrm{C} / \mathrm{z}$ & 11.90 & -39.23 & .371 & & & \\
\hline & $\mathrm{C} / \mathrm{z}$ & 19.33 & -36.16 & .371 & S & & \\
\hline & $\mathrm{C} / \mathrm{z}$ & -26.01 & -31.69 & L. 371 & \$ & & \\
\hline & $\mathrm{C} / \mathrm{z}$ & -31.69 & -26.01 & L. 371 & \$ & & \\
\hline & $\mathrm{C} / \mathrm{z}$ & -36.16 & -19. & L. 371 & & & \\
\hline & $c / z$ & -39.23 & $-11 \cdot 90$ & .371 & \$ & & \\
\hline & $\mathrm{C} / \mathrm{z}$ & -40.80 & -4.02 & .371 & \$ & & \\
\hline & $\mathrm{C} / \mathrm{z}$ & -40.80 & 4.02 & 371 & 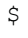 & & \\
\hline \\
\hline & & 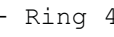 & & & & & \\
\hline & $\mathrm{c} / \mathrm{z}$ & -42.73 & 17.70 & 1.371 & $\$$ & 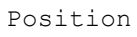 & \\
\hline & $c / z$ & -38.46 & 25. & 1.371 & & 0 & \\
\hline & $\mathrm{C} / \mathrm{z}$ & -32.70 & 32 . & 1.371 & $\$$ & & \\
\hline & $\mathrm{C} / \mathrm{z}$ & -25.70 & 38.46 & 1.371 & \$ & Po & 4 \\
\hline & $\mathrm{C} / \mathrm{z}$ & -17.70 & 42.73 & 1.371 & $\$$ & $\mathrm{n}$ & \\
\hline & $\mathrm{C} / \mathrm{z}$ & -9.02 & 45.36 & 1.371 & $\$$ & & \\
\hline & $\mathrm{C} / \mathrm{z}$ & 0.00 & 46.25 & 1.371 & $\$$ & & \\
\hline 08 & $\mathrm{c} / \mathrm{z}$ & 9.02 & 45.36 & 1.371 & $\$$ & & \\
\hline 9 & $c / z$ & 17.70 & 42.73 & 1.371 & $\$$ & & \\
\hline & $c / z$ & 25.70 & 38.46 & 71 & $\$$ & & \\
\hline & $c / z$ & 32.70 & 32. & 1.371 & \$ & & \\
\hline 12 & $c / z$ & 38.46 & 25 . & 1.371 & $\$$ & & \\
\hline & $\mathrm{C} / \mathrm{z}$ & 42.73 & 17. & 1. & $\$$ & & \\
\hline & $\mathrm{c} / \mathrm{z}$ & 45.36 & 9. & 1 . & $\$$ & & \\
\hline 15 & $c / z$ & 46.25 & 0.00 & 1.371 & $\$$ & & \\
\hline 16 & $c / z$ & 45.36 & -9.02 & 1.371 & \$ & & \\
\hline & $c / z$ & 42.73 & -17.70 & 1.371 & $\$$ & & \\
\hline 18 & $c / z$ & 38.46 & -25 & 371 & \$ & & \\
\hline 19 & $c / z$ & 32.70 & -32.70 & 1. & $\$$ & & \\
\hline 20 & $c / z$ & 25.70 & -38 & 71 & \$ & & \\
\hline 21 & $\mathrm{c} / \mathrm{z}$ & 17.70 & -42 . & 1 . & $\$$ & & \\
\hline 22 & $c / z$ & 9.02 & -45 & 371 & $\$$ & & \\
\hline 23 & $c / z$ & 0.00 & -46.25 & 1.371 & \$ & & \\
\hline 24 & $c / z$ & -9.02 & -45.36 & 1.371 & $\$$ & & \\
\hline 25 & $c / z$ & -17.70 & -42 . & 71 & \$ & & \\
\hline 26 & $c / z$ & -25.70 & -38.46 & 71 & $\$$ & & \\
\hline 227 & $c / z$ & -32.70 & -32.70 & 1.371 & $\$$ & Po & \\
\hline 228 & $\mathrm{C} / \mathrm{z}$ & -38.46 & -25.70 & 1.371 & $\$$ & & \\
\hline 229 & $c / z$ & -42.73 & -17.70 & 71 & \$ & & \\
\hline 230 & $c / z$ & -45.36 & -9.02 & 1.371 & S & & 5 \\
\hline 231 & $\mathrm{C} / \mathrm{z}$ & -46.25 & 0.00 & 1.371 & $\$$ & $\mathrm{Po}$ & 31 \\
\hline 2232 & $c / z$ & -45.36 & 9.02 & 1.3 & $\$$ & & 3 \\
\hline \\
\hline & & & & & & & \\
\hline & $\mathrm{C} / \mathrm{z}$ & -45.42 & 24.28 & 1.371 & $\$$ & & 1 \\
\hline & $c / z$ & -39.81 & 32.67 & 1 . & s & & \\
\hline & $c / z$ & -32.67 & 39.81 & 1 . & \$ & & 3 \\
\hline 4 & $\mathrm{C} / \mathrm{z}$ & -24.28 & 45.42 & 1 . & $\$$ & & 4 \\
\hline & $c / z$ & -14.95 & 49.28 & & $\$$ & & 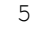 \\
\hline & $c / z$ & -5.05 & 51.25 & 1. & \$ & $\mathrm{Po}$ & 6 \\
\hline & $c / z$ & 5.05 & 51.25 & 1.371 & $\$$ & & 7 \\
\hline & $c / z$ & 14.95 & 49.28 & 1.371 & $\$$ & & 8 \\
\hline & $c / z$ & 24.28 & 45.42 & 1.371 & $\$$ & & 9 \\
\hline & $c / z$ & 32.67 & 39. & & $\$$ & & 10 \\
\hline & $c / z$ & 39.81 & 32.67 & 1.371 & $\$$ & Po & \\
\hline & $c / z$ & 45.42 & 24.28 & 1.371 & $\$$ & Po & $\perp$ \\
\hline & $c / z$ & 49.28 & 14.95 & 1.371 & $\$$ & Pos & 1 \\
\hline & $c / z$ & 51.25 & 5. & 1.371 & $\$$ & Pos & 1 \\
\hline & $c / z$ & 51.25 & -5.05 & 1.371 & \$ & Pos & 15 \\
\hline 2316 & $c / z$ & 49.28 & $-14 \cdot 95$ & 1.371 & \$ & Pos: & 16 \\
\hline & $c / z$ & 45.42 & -24.28 & 1.371 & $\$$ & Posi & 1 \\
\hline & $c / z$ & 39.81 & -32.67 & 1.371 & $\$$ & & \\
\hline & $c / z$ & 32.67 & -39.81 & 1.371 & $\$$ & Pos & -1 \\
\hline & $c / z$ & 24.28 & -45.42 & 1.371 & \$ & Posi & 20 \\
\hline & $c / z$ & 14.95 & -49.28 & 1. & & & \\
\hline & $c / z$ & 5.05 & -51.25 & 1.371 & 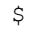 & Pos & \\
\hline
\end{tabular}

Revision: 1 
NEA/NSC/DOC(2006)1

Gas Cooled (Thermal) Reactor - GCR

PROTEUS-GCR-EXP-004

CRIT-REAC

\begin{tabular}{|c|c|c|c|c|c|c|c|}
\hline 23 & $c / z$ & -5.05 & -51.25 & 1.371 & $\$$ & Position & 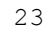 \\
\hline 324 & $c / z$ & -14.95 & -49.28 & 1.371 & S & Position & 2 \\
\hline & $c / z$ & -24.28 & -45.42 & 1.371 & \$ & Position & \\
\hline & $c / z$ & -32.67 & -39.81 & .371 & & Pos: & \\
\hline & $c / z$ & -39.81 & -32.67 & 1.371 & 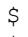 & Pos: & \\
\hline & $c / z$ & -45.42 & -24.28 & .371 & & Posi & \\
\hline & $c / z$ & -49.28 & -14.95 & L. 371 & & & \\
\hline & $c / z$ & -51.25 & -5.05 & .371 & & Pos & \\
\hline & $c / z$ & -51.25 & 5 . & 1.371 & \$ & Pos & \\
\hline & $\mathrm{C} / \mathrm{z}$ & -49.28 & 14.95 & 1.371 & 5 & & \\
\hline \\
\hline \multicolumn{8}{|c|}{------ Graphite Plugs ------ } \\
\hline \multicolumn{8}{|c|}{-------- Ring $1--------$} \\
\hline & $\mathrm{c} / \mathrm{z}$ & -29.86 & 2.94 & 1.325 & $\$$ & Po & 1 \\
\hline & $\mathrm{c} / \mathrm{z}$ & -28.71 & 8.71 & 1.325 & ? & Po & \\
\hline & $\mathrm{c} / \mathrm{z}$ & -26.46 & 14.14 & 1.325 & \$ & Po & \\
\hline & $\mathrm{c} / \mathrm{z}$ & -23.19 & 19.03 & 1.325 & $\$$ & Po & \\
\hline & $\mathrm{c} / \mathrm{z}$ & -19.03 & 23.19 & 1.325 & T & Po & \\
\hline & $c / z$ & -14.14 & 26. & 1.325 & $\$$ & Po & \\
\hline & $c / z$ & -8 . & 28.71 & 1.325 & \$ & Po & \\
\hline & $\mathrm{c} / \mathrm{z}$ & -2.94 & 29.86 & 1.325 & $\$$ & Po: & \\
\hline & $c / z$ & 2.94 & 29 & 1.325 & S & Po & \\
\hline & $c / z$ & 8. & 28 & 1. & $\$$ & & \\
\hline & $c / z$ & 14. & 26. & 1.325 & $p$ & Po: & \\
\hline & $c / z$ & 19 & 23 & 1.325 & $\$$ & $\mathrm{n}$ & \\
\hline & $c / z$ & & 19 & 1 & $\$$ & & \\
\hline & $c / z$ & 26 & 14 & 5 & $\$$ & & \\
\hline & $c / z$ & 28.71 & 8.71 & 1.325 & ? & $\mathrm{n}$ & \\
\hline & $c / z$ & 25 & 2 . & $\cdot$ & $\$$ & & \\
\hline & $c / z$ & & -2 . & 5 & $\$$ & $\mathrm{n}$ & \\
\hline & $c / z$ & 28.71 & -8 & 5 & \$ & $n$ & \\
\hline & $c / z$ & 26.46 & -14 . & 5 & $\$$ & $\mathrm{n}$ & 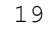 \\
\hline & $c / z$ & & -19 & & P & & \\
\hline & $\mathrm{c} / \mathrm{z}$ & & -23 & 1 . & $\$$ & & \\
\hline & $c / z$ & 14.14 & -26.46 & 1.325 & $\$$ & Po & 2 \\
\hline & $c / z$ & 1 & -28.71 & 1 . & \$ & Po & 3 \\
\hline & $c / z$ & 4 & -29 . & 1 . & $\$$ & & \\
\hline & $c / z$ & -2.94 & -29.86 & 1 . & \$ & $\mathrm{Po}$ & \\
\hline & $c / z$ & -8.71 & -28.71 & 1 . & \$ & $\mathrm{Po}$ & 2 \\
\hline & $c / z$ & -14.14 & -26 & & $\$$ & $\mathrm{Po}$ & 7 \\
\hline & $c / z$ & -19 & -23 & 1 . & $\$$ & & \\
\hline & $c / z$ & -23.19 & -19. & 1. & $\$$ & & \\
\hline & $c / z$ & -26.46 & -14.14 & 1. & S & & 30 \\
\hline & $c / z$ & -28.71 & -8.71 & 1. & $\$$ & $n$ & \\
\hline & $c / z$ & -29 & -2 & 1 . & \$ & & 3 \\
\hline \\
\hline & & 20. & & - & & & \\
\hline & $c / z$ & -34.82 & 6.93 & 1.3 & $\$$ & Po & 1 \\
\hline & $c / z$ & -32.80 & 13 & & $\$$ & Po & 2 \\
\hline & $c / z$ & -29.52 & 19.72 & 1.325 & \$ & Po & 3 \\
\hline & $c / z$ & -25.10 & 25.10 & 1. & $\$$ & $\mathrm{Po}$ & 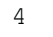 \\
\hline & $c / z$ & -1 & 29 & 1. & \$ & & 5 \\
\hline & $c / z$ & -13.59 & 32.80 & 1.325 & $\$$ & Po & 6 \\
\hline & $c / z$ & -6.93 & 34.82 & 1. & $\$$ & Po & 7 \\
\hline & $c / z$ & 0 & 50 & 1. & \$ & $n$ & 8 \\
\hline & $c / z$ & & 34.82 & 1 . & \$ & Po & 9 \\
\hline & $c / z$ & 13.59 & 32.80 & & $\$$ & & 10 \\
\hline & $c / z$ & 1. & 29 & 1 . & $\$$ & $\mathrm{Po}$ & 11 \\
\hline & $c / z$ & & & & \$ & & 12 \\
\hline & $c / z$ & 29. & 19. & & $\$$ & Po & 1 \\
\hline & $c / z$ & 32.80 & 13.59 & 1. & $\$$ & Po & 14 \\
\hline & $c / z$ & & 6.93 & 1. & $\$$ & & \\
\hline & $c / z$ & 35.50 & 0.00 & & \$ & Pos & 1 \\
\hline & $c / z$ & 34.82 & -6 . & & $\$$ & Po & 17 \\
\hline & $c / z$ & 32.80 & -13.59 & 1. & $\$$ & Pos & 18 \\
\hline & $c / z$ & 29.52 & -19.72 & 1.3 & $\$$ & Pos & 19 \\
\hline & $c / z$ & 25.10 & -25.10 & 1.325 & $\$$ & Pos & 2 \\
\hline & $c / z$ & 19.72 & -29 . & & $\$$ & Pos & 21 \\
\hline & $c / z$ & 13.59 & -32.80 & 1.325 & $\$$ & Pos: & - \\
\hline 252 & $c / z$ & 6.93 & -34.82 & 1.325 & $\$$ & Pos: & 23 \\
\hline & $c / z$ & 0.00 & -35.50 & 1.325 & $\$$ & Posi & 2 \\
\hline & $c / z$ & -6.93 & -34.82 & 1.325 & $\$$ & Pos & 2 \\
\hline & $c / z$ & -13.59 & -32.80 & 1.325 & $\$$ & Pos: & 20 \\
\hline L & $c / z$ & -19.72 & -29.52 & 1.325 & $\$$ & Pos: & 2 \\
\hline & $c / z$ & -25.10 & -25.10 & 1.325 & S & Pos & 2 \\
\hline & $c / z$ & -29.52 & -19.72 & 1.325 & $\$$ & Pos & \\
\hline
\end{tabular}

Revision: 1 
NEA/NSC/DOC(2006)1

Gas Cooled (Thermal) Reactor - GCR

PROTEUS-GCR-EXP-004

CRIT-REAC

\begin{tabular}{|c|c|c|c|c|c|c|c|}
\hline 30 & $\mathrm{C} / \mathrm{z}$ & -32.80 & -13.59 & 1.325 & $\$$ & Position & 30 \\
\hline 37 & $\mathrm{C} / \mathrm{z}$ & -34.82 & -6.93 & 1.325 & $?$ & Position & 31 \\
\hline & $C / z$ & -35.50 & 0.00 & 1.325 & \$ & Position & \\
\hline \\
\hline \\
\hline & $c / z$ & -39.23 & 11.90 & 1.325 & $\$$ & Position & 1 \\
\hline & $\mathrm{C} / \mathrm{z}$ & -36.16 & 19.33 & & $\$$ & & \\
\hline & $\mathrm{C} / \mathrm{z}$ & -31.69 & 26.01 & 1.32 & $\$$ & Posi & \\
\hline & $\mathrm{C} / \mathrm{z}$ & -26.01 & 31.69 & 1.3 & $\$$ & Pos: & \\
\hline & $\mathrm{C} / \mathrm{z}$ & -19.33 & 36.16 & 1.325 & $\$$ & Pos: & \\
\hline & $\mathrm{C} / \mathrm{z}$ & -11.90 & 39.23 & 1.32 & $\$$ & Po: & \\
\hline & $\mathrm{C} / \mathrm{z}$ & -4.02 & 40.80 & 1.32 & 3 & Pos & \\
\hline & $\mathrm{C} / \mathrm{z}$ & 4.02 & 40.80 & 1.325 & $\$$ & Posi & \\
\hline & $\mathrm{C} / \mathrm{z}$ & 11.90 & 39.23 & L. 325 & $\$$ & Posit & \\
\hline & $C / z$ & 19 & 36. & 1.32 & $\$$ & Po & 10 \\
\hline & $\mathrm{C} / \mathrm{z}$ & 26.01 & 31.69 & .32 & $\$$ & Po & \\
\hline & $\mathrm{C} / \mathrm{z}$ & 31.69 & 26.01 & 1.325 & $\$$ & Po & \\
\hline & $\mathrm{C} / \mathrm{z}$ & 36.16 & 19.33 & .3 & \$ & Po: & \\
\hline & $\mathrm{C} / \mathrm{z}$ & 39.23 & 11.90 & & \$ & Po & \\
\hline & $\mathrm{C} / \mathrm{z}$ & 40.80 & 4. & . & \$ & Posi & \\
\hline & $\mathrm{C} / \mathrm{z}$ & 40.80 & -4 . & .32 & $\$$ & Posi & \\
\hline & $\mathrm{C} / \mathrm{z}$ & 39.23 & -11 & . & \$ & Posi & \\
\hline & $\mathrm{C} / \mathrm{z}$ & 36.16 & -19 & & $\$$ & Posi & \\
\hline & $\mathrm{C} / \mathrm{z}$ & 31.69 & -26.01 & 1.32 & \$ & Posit & \\
\hline & $\mathrm{C} / \mathrm{z}$ & 26.01 & -31 & .3 & $\$$ & Posit & \\
\hline & $\mathrm{C} / \mathrm{z}$ & 19.33 & -36 & 1 & $\$$ & Posi & 1 \\
\hline & $c / z$ & 11.90 & -39.23 & & $\$$ & Posi & \\
\hline & $\mathrm{C} / \mathrm{z}$ & 4.02 & -40.80 & L. & $\$$ & osi & \\
\hline & $\mathrm{C} / \mathrm{z}$ & -4.02 & -40 . & & $\$$ & $\mathrm{n}$ & 4 \\
\hline & $\mathrm{C} / \mathrm{z}$ & $-11 \cdot 90$ & -39 . & & $\$$ & Pos & 5 \\
\hline & $c / z$ & -19.33 & -36.16 & 1. & $\$$ & Posi & \\
\hline & $c / z$ & -26.01 & -31.69 & 1.325 & $\$$ & Posit & 21 \\
\hline & $C / z$ & -31.69 & -26 & 1. & \$ & & $\xi$ \\
\hline & $c / z$ & -36.16 & -19. & 1. & $\$$ & Po & \\
\hline & $C / z$ & -39.23 & $-11 \cdot 90$ & 1.325 & $\$$ & Posi & 3 \\
\hline & $\mathrm{C} / \mathrm{z}$ & -40.80 & -4.02 & & $\$$ & Posi & $\perp$ \\
\hline & $C / z$ & -40.80 & 4.02 & 1. & $\$$ & Pos: & \\
\hline \\
\hline & $-x_{-1}$ & ing & & & & & \\
\hline & $C / z$ & -42.73 & 17.70 & 1.32 & $\$$ & Po & 1 \\
\hline & $c / z$ & -38.46 & 25. & 1. & $\$$ & Po & - \\
\hline & $c / z$ & -32.70 & 32.70 & 1. & \$ & Posi & 3 \\
\hline & $\mathrm{C} / \mathrm{z}$ & -25.70 & 38.46 & 1.325 & $\$$ & Posi & 4 \\
\hline & $\mathrm{C} / \mathrm{z}$ & -17.70 & 42.73 & 1.325 & $\$$ & Po & 5 \\
\hline & $c / z$ & -9.02 & 45 & 1 . & $\$$ & Pos & 6 \\
\hline & $\mathrm{C} / \mathrm{z}$ & 0.00 & 46. & 1 . & $\$$ & Pos & 7 \\
\hline & $c / z$ & 9.02 & 45.36 & 1.3 & $\$$ & Posi & 8 \\
\hline & $\mathrm{C} / \mathrm{z}$ & 17.70 & 42.73 & 1.325 & $\$$ & Pos & 9 \\
\hline & $c / z$ & 25.70 & 38. & 1 . & $\$$ & Posi & 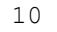 \\
\hline & $\mathrm{C} / \mathrm{z}$ & 32.70 & 32 . & 1.3 & $\$$ & Posi & 1 \\
\hline & $c / z$ & 38.46 & 25. & 1.32 & $\$$ & Po: & \\
\hline & $\mathrm{C} / \mathrm{z}$ & 42.73 & 17. & 1. & $\$$ & Po: & 13 \\
\hline & $\mathrm{C} / \mathrm{z}$ & 45.36 & 9.02 & 1.325 & \$ & Posi & 1 \\
\hline & $\mathrm{C} / \mathrm{z}$ & 46.25 & 0 . & 1. & $\$$ & Po: & 15 \\
\hline & $c / z$ & 45.36 & -9.02 & 1. & $\$$ & Pos & 16 \\
\hline & $c / z$ & 42.73 & -17 . & 1 . & \$ & Pos & 17 \\
\hline & $C / z$ & 38.46 & -25.70 & 1. & $\$$ & Pos & 1 \\
\hline & $c / z$ & 32.70 & -32 & 1. & $\$$ & Po: & 19 \\
\hline & $c / z$ & 25.70 & -38 & 1 . & $\$$ & Po: & 20 \\
\hline & $c / z$ & 17.70 & -42.73 & 1. & $\$$ & Posi & 21 \\
\hline & $c / z$ & 9.02 & -45.36 & 1.325 & $\$$ & Pos & 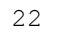 \\
\hline & $C / z$ & 0.00 & -46 . & 1.325 & $\$$ & Posi & 23 \\
\hline & $c / z$ & -9.02 & -45.36 & 1.325 & \$ & Posi & 2 \\
\hline & $\mathrm{C} / \mathrm{z}$ & -17.70 & -42.73 & & $\$$ & Posi & 2 \\
\hline & $\mathrm{C} / \mathrm{z}$ & -25.70 & -38.46 & & $\$$ & Posi & 2 \\
\hline & $\mathrm{C} / \mathrm{z}$ & -32.70 & -32.70 & 1.325 & $\$$ & Posi & 2 \\
\hline & $c / z$ & -38.46 & -25.70 & 1.325 & $\$$ & Posit & 2 \\
\hline & $\mathrm{C} / \mathrm{z}$ & -42.73 & -17.70 & & $\$$ & Posi & \\
\hline & $c / z$ & -45.36 & -9.02 & 1.325 & $\$$ & Posi & 30 \\
\hline & $\mathrm{C} / \mathrm{z}$ & -46.25 & 0.00 & 1.325 & $\$$ & Posit & 3 \\
\hline & $c / z$ & -45.36 & 9.02 & 1.325 & $\$$ & Posit & \\
\hline \\
\hline & & Bing 5 & & & & & \\
\hline & $C / z$ & .42 & 24.28 & 1.3 & & Pos & 1 \\
\hline & $\mathrm{C} / \mathrm{z}$ & & 32 . & & & Pos & \\
\hline & $c / z$ & -32.67 & 39.81 & 1.325 & & Pos & \\
\hline
\end{tabular}

Revision: 1 
NEA/NSC/DOC(2006)1

Gas Cooled (Thermal) Reactor - GCR

PROTEUS-GCR-EXP-004

CRIT-REAC

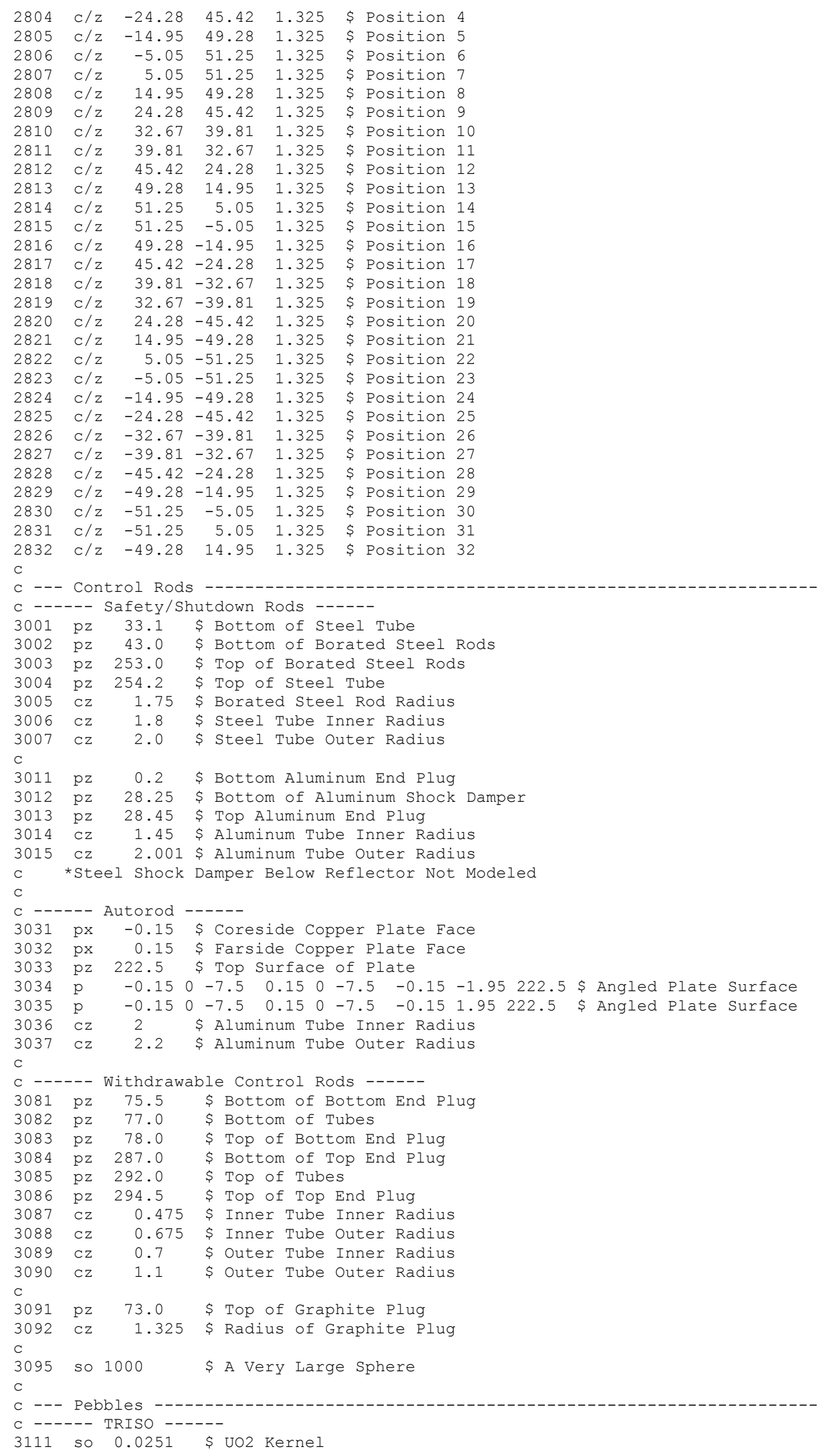

Revision: 1 
NEA/NSC/DOC(2006)1

\section{Gas Cooled (Thermal) Reactor - GCR \\ PROTEUS-GCR-EXP-004 \\ CRIT-REAC}

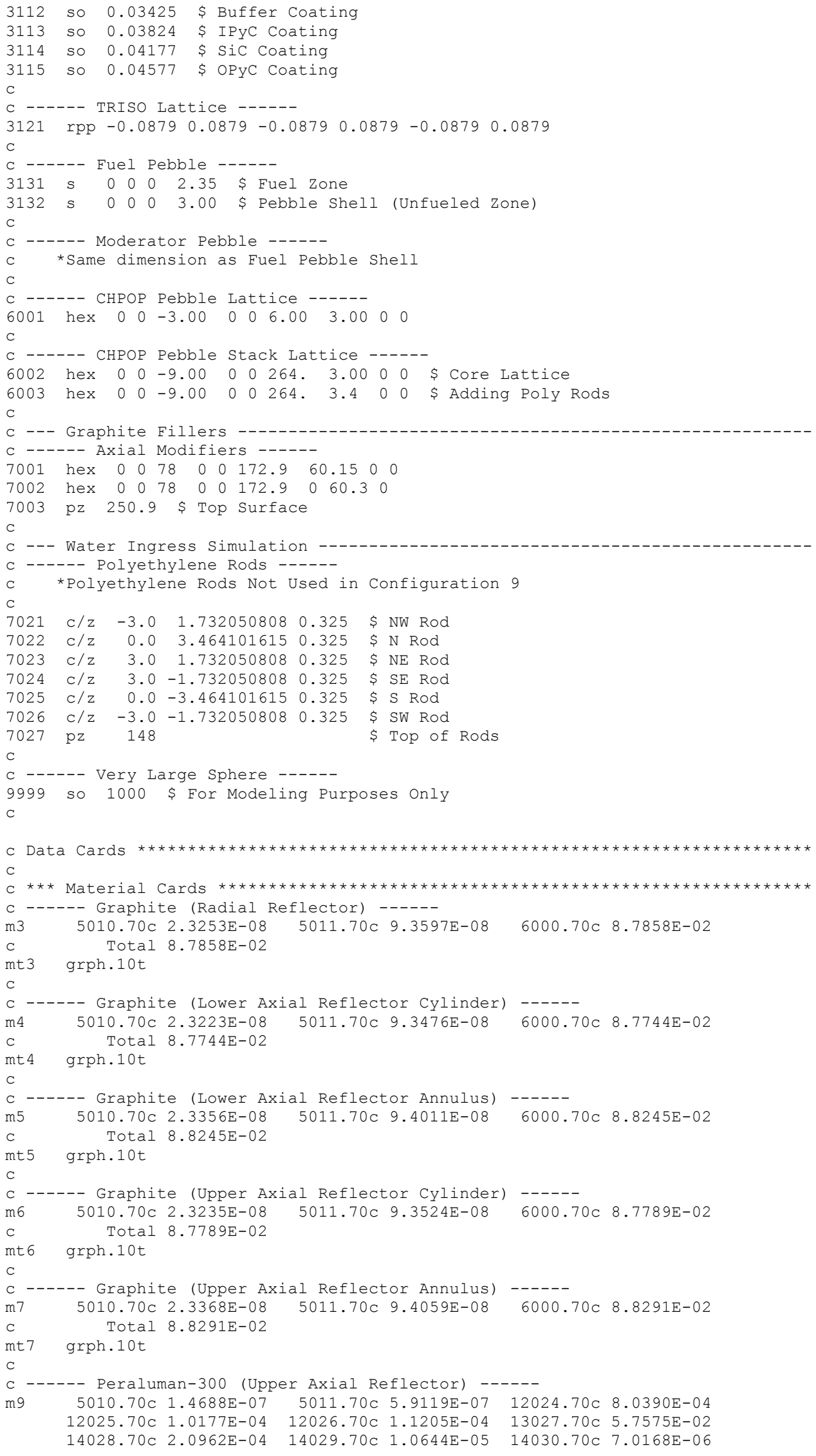

Revision: 1 
NEA/NSC/DOC(2006)1

\section{Gas Cooled (Thermal) Reactor - GCR \\ PROTEUS-GCR-EXP-004 CRIT-REAC}

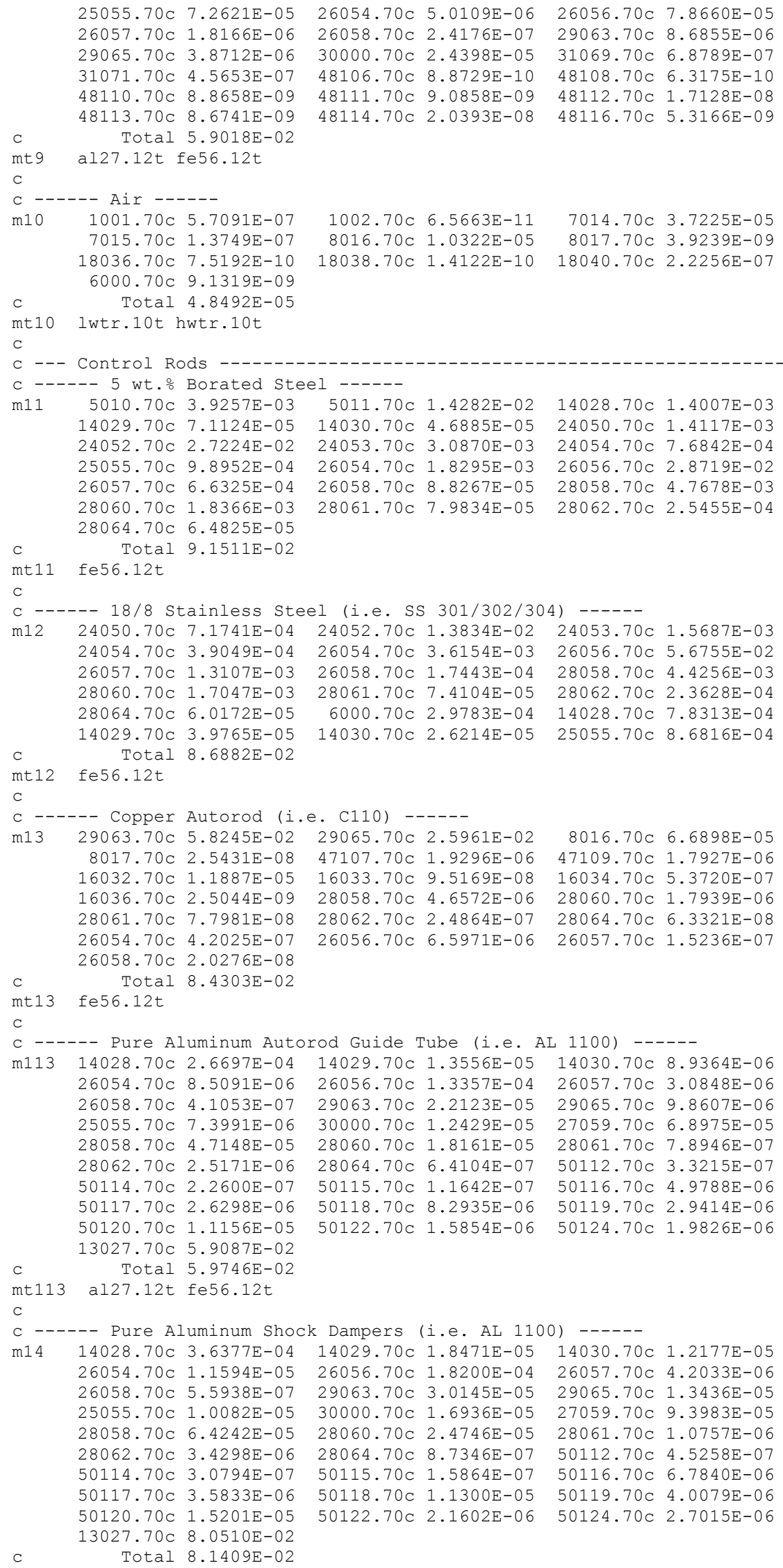

$26054.70 \mathrm{C} 5.0109 \mathrm{E}-06$ $26058.70 \mathrm{C} 2.4176 \mathrm{E}-07$ $30000.70 \mathrm{C} 2.4398 \mathrm{E}-05$ $48106.70 \mathrm{C} 8.8729 \mathrm{E}-10$ $48111.70 \mathrm{C} 9.0858 \mathrm{E}-09$ $48114.70 \mathrm{C} \quad 2.0393 \mathrm{E}-08 \quad 48116.70 \mathrm{C} \quad 5.3166 \mathrm{E}-09$

Revision: 1 
NEA/NSC/DOC(2006)1

\section{Gas Cooled (Thermal) Reactor - GCR \\ PROTEUS-GCR-EXP-004 CRIT-REAC}

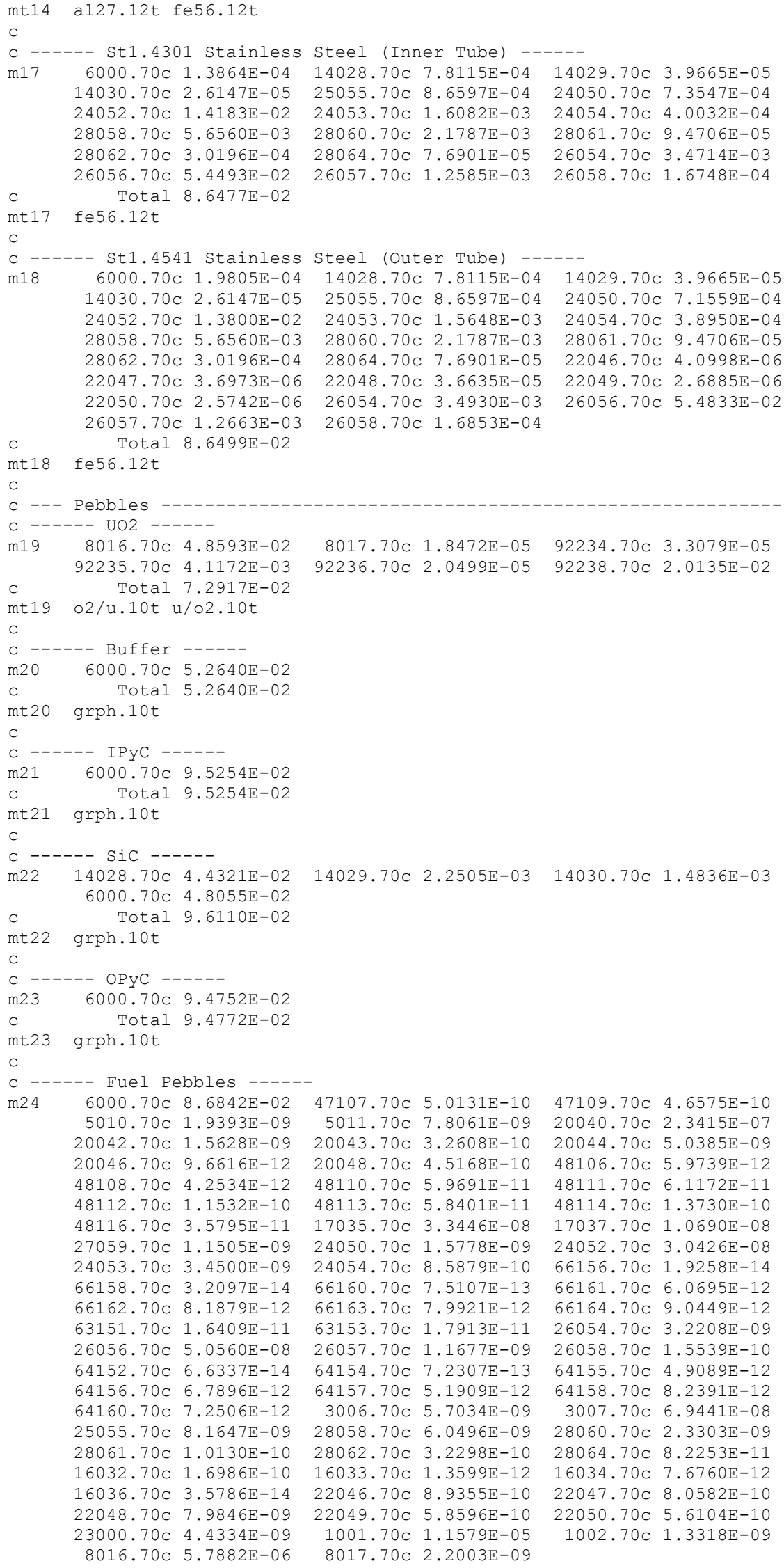

$47109.70 \mathrm{C} \quad 4.6575 \mathrm{E}-10$ $20040.70 \mathrm{C} 2.3415 \mathrm{E}-07$ $20044.70 \mathrm{C} 5.0385 \mathrm{E}-09$ $48106.70 \mathrm{C} \quad 5.9739 \mathrm{E}-12$ $48111.70 \mathrm{C} \quad 6.1172 \mathrm{E}-11$ $48114.70 \mathrm{C} 1.3730 \mathrm{E}-10$ $17037.70 \mathrm{C} 1.0690 \mathrm{E}-08$ $24052.70 \mathrm{C} 3.0426 \mathrm{E}-08$ $66156.70 \mathrm{C} \quad 1.9258 \mathrm{E}-14$ $66161.70 \mathrm{C} \quad 6.0695 \mathrm{E}-12$ $66164.70 \mathrm{C} 9.0449 \mathrm{E}-12$ $26054.70 \mathrm{C} 3.2208 \mathrm{E}-09$ $26058.70 \mathrm{C} 1.5539 \mathrm{E}-10$ $64155.70 \mathrm{C} \quad 4.9089 \mathrm{E}-12$ $64158.70 \mathrm{C} 8.2391 \mathrm{E}-12$ $3007.70 \mathrm{C} 6.9441 \mathrm{E}-08$ $28060.70 \mathrm{C} 2.3303 \mathrm{E}-09$ $28064.70 \mathrm{C} 8.2253 \mathrm{E}-11$ $16034.70 \mathrm{C} 7.6760 \mathrm{E}-12$ $22047.70 \mathrm{C} 8.0582 \mathrm{E}-10$ $22050.70 \mathrm{C} 5.6104 \mathrm{E}-10$ $1002.70 \mathrm{C} 1.3318 \mathrm{E}-09$

Revision: 1 
NEA/NSC/DOC(2006)1

\section{Gas Cooled (Thermal) Reactor - GCR \\ PROTEUS-GCR-EXP-004 CRIT-REAC}

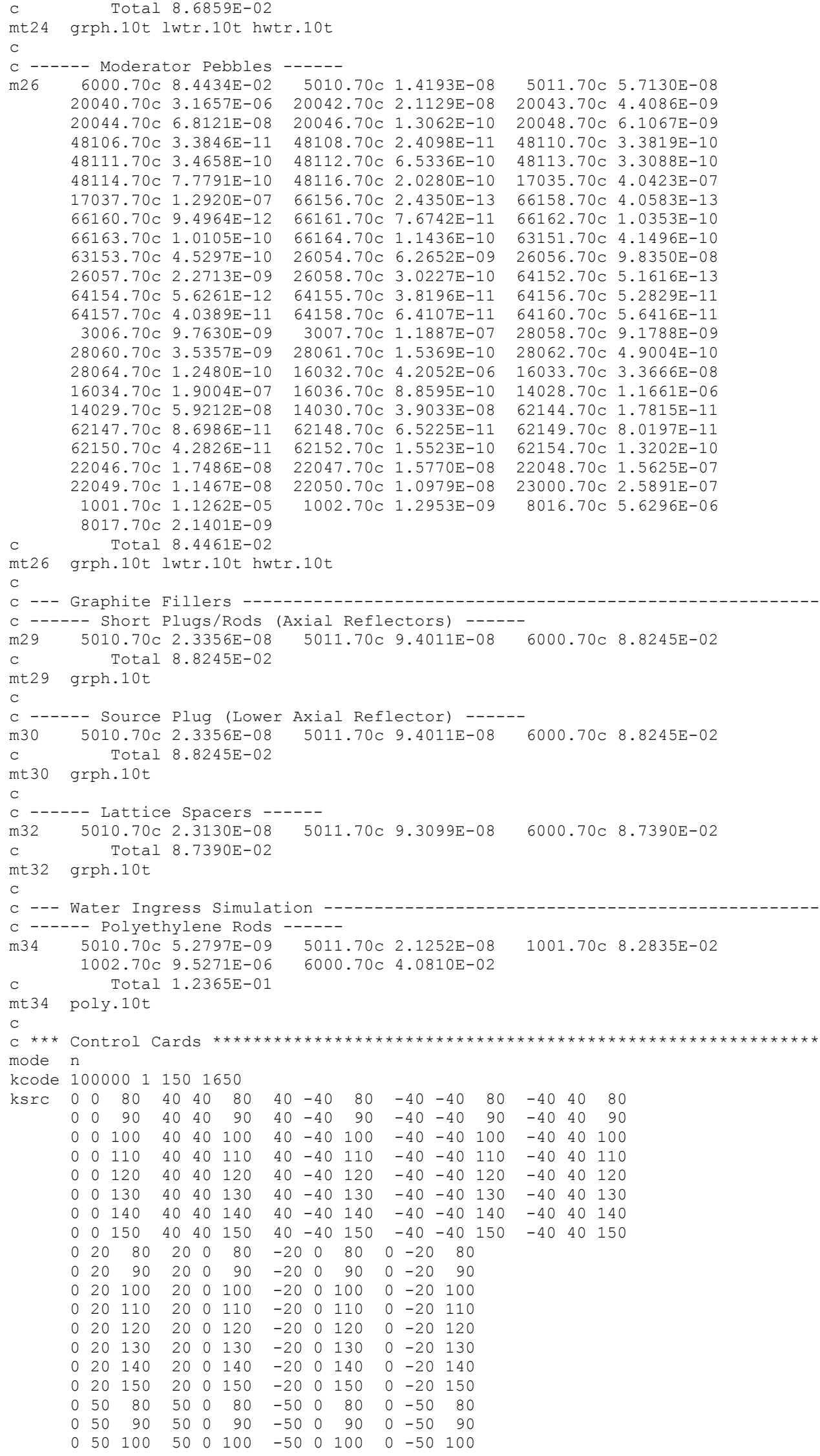

Revision: 1 


\section{NEA/NSC/DOC(2006)1}

\section{Gas Cooled (Thermal) Reactor - GCR}

\section{PROTEUS-GCR-EXP-004}

CRIT-REAC

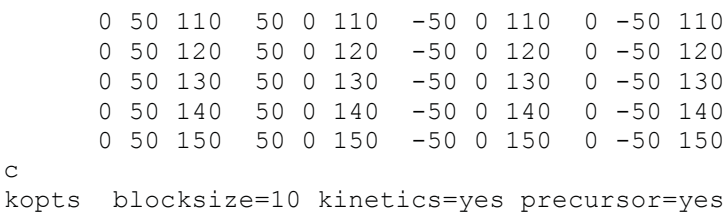

Revision: 1 


\section{NEA/NSC/DOC(2006)1 \\ Gas Cooled (Thermal) Reactor - GCR \\ PROTEUS-GCR-EXP-004 \\ CRIT-REAC}

\section{A.5 Reactivity Coefficient Configurations}

Reactivity coefficient measurements were performed but have not yet been evaluated.

\section{A.6 Kinetics Parameter Configurations}

Kinetics measurements were performed but have not yet been evaluated.

\section{A.7 Reaction-Rate Configurations}

Reaction-rate distribution measurements were performed but have not yet been evaluated.

\section{A.8 Power Distribution Configurations}

Power distribution measurements were not performed.

\section{A.9 Isotopic Configurations}

Isotopic measurements were not performed.

\section{A.10 Configurations of Other Miscellaneous Types of Measurements}

Other miscellaneous types of measurements were not performed. 
NEA/NSC/DOC(2006)1

Gas Cooled (Thermal) Reactor - GCR

PROTEUS-GCR-EXP-004

CRIT-REAC

\section{APPENDIX B: CALCULATED SPECTRAL DATA}

The neutron spectral calculations provided below were obtained from the output files for the input decks used to obtain the results in Section 4.1. Spectral data using the ENDF/B-VII.0 neutron cross section library is provided here for the MCNP5 analysis.

\section{B.1 MCNP-Calculated Spectral Data}

A summary of the computed neutron spectral data using MCNP5 for the benchmark model is provided in Tables B.1-1 and B.1-2 for Cases 1 and 2 (Cores 9 and 10), respectively.

Table B.1-1. Neutron Spectral Data for Benchmark Model for Case 1 (Core 9).

\begin{tabular}{|c|c|}
\hline $\begin{array}{l}\text { Neutron Cross } \\
\text { Section Library }\end{array}$ & ENDF/B-VII.0 \\
\hline $\mathbf{k}_{\text {eff }}$ & 1.00667 \\
\hline$\pm \sigma_{\mathrm{k}}$ & 0.00007 \\
\hline Neutron Leakage $(\%)^{(a)}$ & 16.18 \\
\hline \multirow{2}{*}{$\begin{array}{c}\text { Fission Fraction, } \\
\text { by Energy (\%) }\end{array}$} & 95.02 \\
\hline & 4.65 \\
\hline Fast $(>100 \mathrm{keV})$ & 0.33 \\
\hline $\begin{array}{c}\text { Average Number of } \\
\text { Neutrons Produced } \\
\text { per Fission }\end{array}$ & 2.437 \\
\hline $\begin{array}{c}\text { Energy of Average } \\
\text { Neutron Lethargy } \\
\text { Causing Fission (eV) }\end{array}$ & 0.054995 \\
\hline $\begin{array}{c}\text { Neutron Generation } \\
\text { Time, } \Lambda \text { (msec) }\end{array}$ & 2.09855 \\
\hline $\operatorname{Rossi}^{\alpha} \alpha\left(\mathrm{msec}^{-1}\right)$ & $-3.30178 \mathrm{E}-03$ \\
\hline$\beta_{\text {eff }}$ & 0.00693 \\
\hline
\end{tabular}

(a) The neutron leakage is calculated using the neutron balance tables provided in the MCNP output file. The weight fraction of neutrons lost due to escaping the boundaries of the benchmark model are divided by the total weight fraction of neutron loss. 
Gas Cooled (Thermal) Reactor - GCR

PROTEUS-GCR-EXP-004

CRIT-REAC

Table B.1-2. Neutron Spectral Data for Benchmark Model for Case 2 (Core 10).

\begin{tabular}{|c|c|}
\hline $\begin{array}{c}\text { Neutron Cross } \\
\text { Section Library } \\
\end{array}$ & ENDF/B-VII.0 \\
\hline $\mathbf{k}_{\text {eff }}$ & 1.00743 \\
\hline$\pm \sigma_{k}$ & 0.00006 \\
\hline Neutron Leakage $(\%)^{(a)}$ & 13.66 \\
\hline Thermal $(<0.625 \mathrm{eV})$ & 96.52 \\
\hline $\begin{array}{l}\text { Fission Fraction, } \\
\text { by Eneroy }(\%)\end{array}$ & 3.18 \\
\hline Fast $(>100 \mathrm{keV})$ & 0.30 \\
\hline $\begin{array}{c}\text { Average Number of } \\
\text { Neutrons Produced } \\
\text { per Fission } \\
\end{array}$ & 2.437 \\
\hline $\begin{array}{c}\text { Energy of Average } \\
\text { Neutron Lethargy } \\
\text { Causing Fission (eV) }\end{array}$ & 0.044361 \\
\hline $\begin{array}{c}\text { Neutron Generation } \\
\text { Time, } \Lambda \text { (msec) }\end{array}$ & 1.69449 \\
\hline Rossi- $\alpha\left(\mathrm{msec}^{-1}\right)$ & $-4.04482 \mathrm{E}-03$ \\
\hline$\beta_{\text {eff }}$ & 0.00685 \\
\hline
\end{tabular}

(a) The neutron leakage is calculated using the neutron balance tables provided in the MCNP output file. The weight fraction of neutrons lost due to escaping the boundaries of the benchmark model are divided by the total weight fraction of neutron loss. 
NEA/NSC/DOC(2006)1

Gas Cooled (Thermal) Reactor - GCR

PROTEUS-GCR-EXP-004

CRIT-REAC

\section{APPENDIX C: DETAILED MODELS OF HTR-PROTEUS}

\section{C.1 Detailed MCNP Models of the HTR-PROTEUS (NOT BENCHMARKED)}

Detailed models of HTR-PROTEUS core configurations 9 and 10 were prepared to evaluate biases in the benchmark model. Because the effects of many of the model simplifications produced small or otherwise negligible biases (in regards to criticality) in the benchmark model, development of a detailed benchmark model was unnecessary. An example MCNP5 input deck, using ENDF/B-VII.0 neutron cross section data, is preserved in this appendix for future use. Calculations were performed with 1,650 generations with 100,000 neutrons per generation. The $\mathrm{k}_{\text {eff }}$ estimates (with the first 150 generations skipped) are the result of 150,000,000 neutron histories. Calculated results obtained with this input deck are provided in Tables C.1-1 and C.1-2 for each core, respectively.

The input deck provided below is for core configuration 9. The following portions of the code need reconfigured for core configuration 10 :

- Autorod position,

- Withdrawable control rod positions,

- Core cavity filled with correct pebble configuration (including polyethylene rods), and

- Change air composition and atom density.

Table C.1-1. Neutron Spectral Data for Detailed Model (Core 9).

\begin{tabular}{|c|c|}
\hline $\begin{array}{l}\text { Neutron Cross } \\
\text { Section Library }\end{array}$ & ENDF/B-VII.0 \\
\hline $\mathbf{k}_{\text {eff }}$ & \\
\hline$\pm \sigma_{k}$ & 0.00007 \\
\hline Neutron Leakage $(\%)^{(a)}$ & 1.84 \\
\hline Thermal $(<0.625 \mathrm{eV})$ & 95.00 \\
\hline $\begin{array}{l}\text { Fission Fraction, } \\
\text { by Energy }(\%)\end{array}$ & 4.66 \\
\hline Fast $(>100 \mathrm{keV})$ & 0.33 \\
\hline $\begin{array}{c}\text { Average Number of } \\
\text { Neutrons Produced } \\
\text { per Fission } \\
\end{array}$ & 2.437 \\
\hline $\begin{array}{c}\text { Energy of Average } \\
\text { Neutron Lethargy } \\
\text { Causing Fission (eV) }\end{array}$ & 0.055035 \\
\hline $\begin{array}{c}\text { Neutron Generation } \\
\text { Time, } \Lambda \text { (msec) } \\
\end{array}$ & 2.11558 \\
\hline Rossi- $\alpha\left(\mathrm{msec}^{-1}\right)$ & $-3.29941 \mathrm{E}-03$ \\
\hline$\beta_{\text {eff }}$ & 0.00698 \\
\hline
\end{tabular}

(a) The neutron leakage is calculated using the neutron balance tables provided in the MCNP output file. The weight fraction of neutrons lost due to escaping the boundaries of the benchmark model are divided by the total weight fraction of neutron loss. 
NEA/NSC/DOC(2006)1

Gas Cooled (Thermal) Reactor - GCR

PROTEUS-GCR-EXP-004

CRIT-REAC

Table C.1-2. Neutron Spectral Data for Detailed Model (Core 10).

\begin{tabular}{|c|c|}
\hline $\begin{array}{l}\text { Neutron Cross } \\
\text { Section Library } \\
\end{array}$ & ENDF/B-VII.0 \\
\hline $\mathbf{k}_{\text {eff }}$ & 1.00647 \\
\hline$\pm \sigma_{\mathrm{k}}$ & 0.00006 \\
\hline Neutron Leakage $(\%)^{(a)}$ & 1.43 \\
\hline Thermal $(<0.625 \mathrm{eV})$ & 96.52 \\
\hline \multirow{2}{*}{$\begin{array}{c}\text { Fission Fraction, } \\
\text { by Energy (\%) }\end{array}$} & 3.18 \\
\hline & 0.30 \\
\hline $\begin{array}{c}\text { Average Number of } \\
\text { Neutrons Produced } \\
\text { per Fission }\end{array}$ & 2.437 \\
\hline $\begin{array}{c}\text { Energy of Average } \\
\text { Neutron Lethargy } \\
\text { Causing Fission (eV) }\end{array}$ & 0.044377 \\
\hline $\begin{array}{c}\text { Neutron Generation } \\
\text { Time, } \Lambda \text { (msec) }\end{array}$ & 1.70417 \\
\hline $\operatorname{Rossi}-\alpha\left(\mathrm{msec}^{-1}\right)$ & $-4.05453 \mathrm{E}-03$ \\
\hline$\beta_{\text {eff }}$ & 0.00691 \\
\hline
\end{tabular}

(a) The neutron leakage is calculated using the neutron balance tables provided in the MCNP output file. The weight fraction of neutrons lost due to escaping the boundaries of the benchmark model are divided by the total weight fraction of neutron loss.

Revision: 1 
NEA/NSC/DOC(2006)1

Gas Cooled (Thermal) Reactor - GCR

PROTEUS-GCR-EXP-004

CRIT-REAC

\section{C.2 Input Listing for Detailed Models}

\section{MCNP5 Input Deck for Core 9 (can be modified for Core 10):}

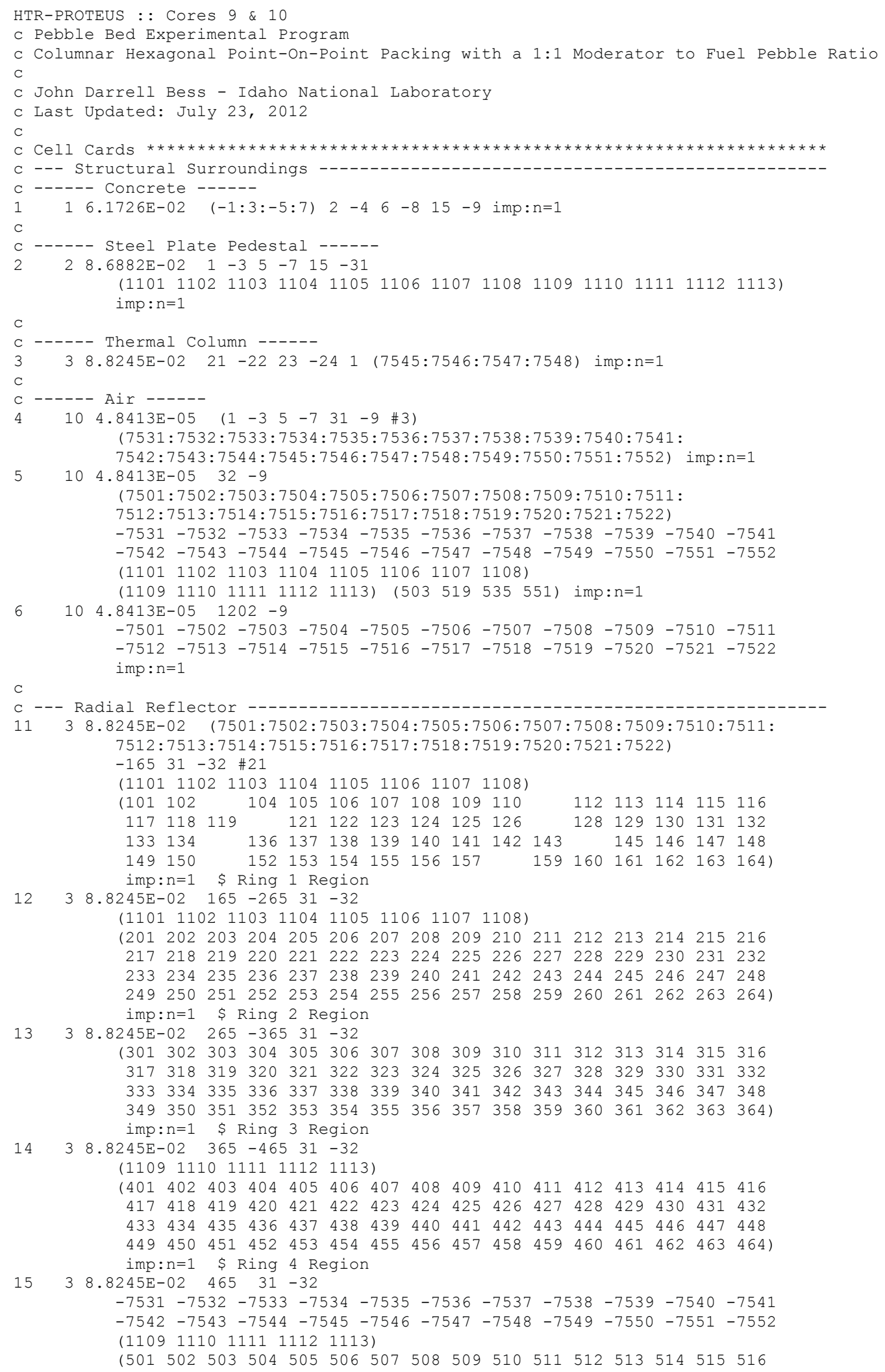

Revision: 1 
NEA/NSC/DOC(2006)1

\section{Gas Cooled (Thermal) Reactor - GCR \\ PROTEUS-GCR-EXP-004 CRIT-REAC}

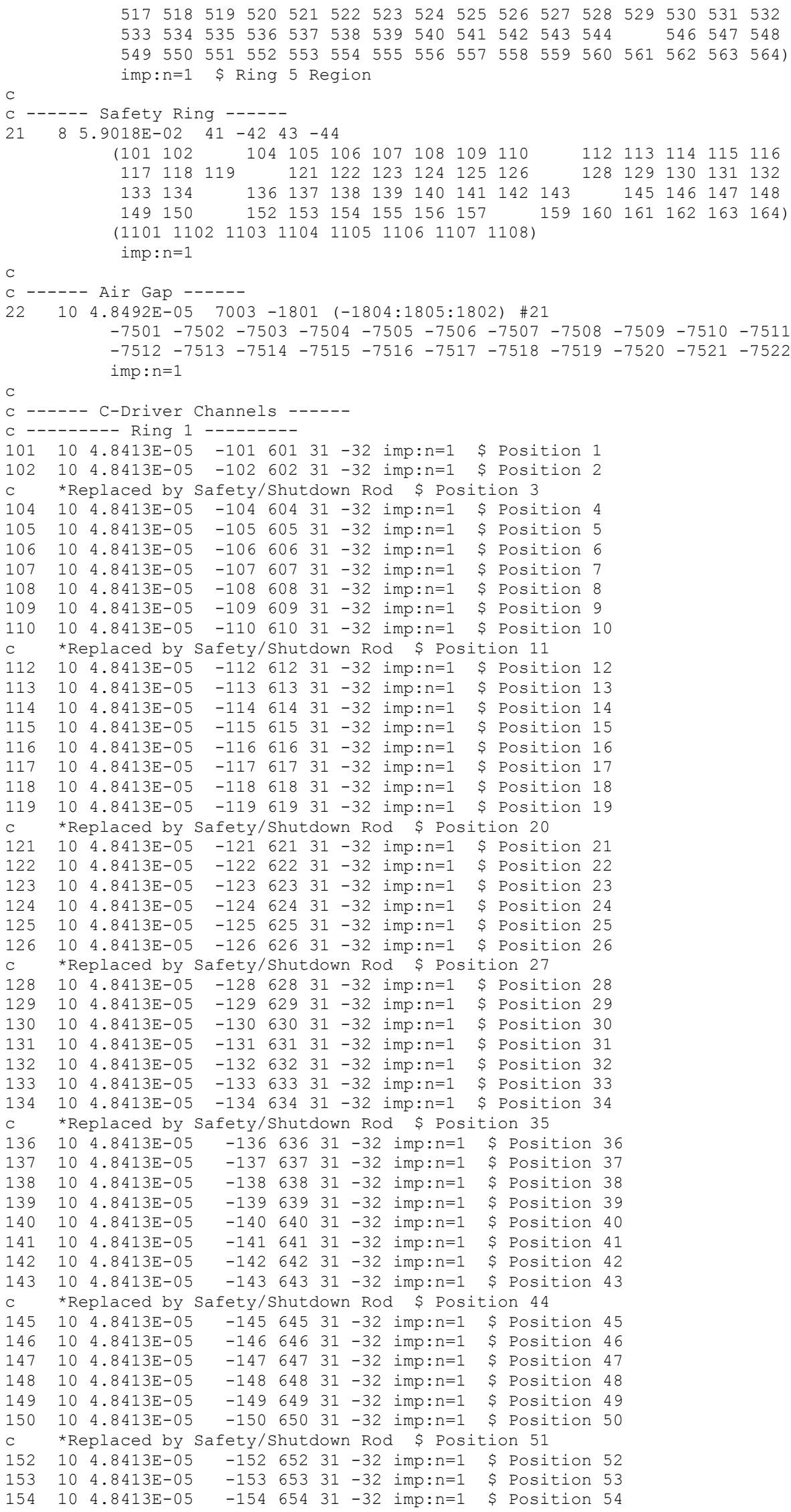

Revision: 1 
NEA/NSC/DOC(2006)1

\section{Gas Cooled (Thermal) Reactor - GCR}

\section{PROTEUS-GCR-EXP-004} CRIT-REAC

\begin{tabular}{|c|c|c|c|c|c|c|c|c|c|c|}
\hline 55 & 0 & $4.8413 E-05$ & -155 & 655 & 31 & -32 & imp $: n=1$ & $\$$ & ion & \\
\hline & 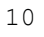 & 4. $8413 E-05$ & -156 & 656 & 31 & -32 & imp: $n=1$ & & sition & \\
\hline 5 & 10 & $4.8413 E-05$ & -157 & 657 & 31 & -32 & imp: $n=1$ & & Position & \\
\hline & \multicolumn{10}{|c|}{ *Replaced by Safety/Shutdown Rod \$ Position 58} \\
\hline & 10 & $4.8413 E-05$ & -159 & 659 & 31 & -32 & imp:n=1 & $\$$ & Position & \\
\hline & 10 & $4.8413 E-05$ & -160 & 60 & 31 & -32 & imp: $n=1$ & $r$ & sition & \\
\hline o & 10 & $4.8413 E-05$ & -161 & 661 & 31 & -32 & imp:n=1 & $\$$ & sition & \\
\hline & 0 & $4.8413 E-05$ & -162 & 662 & 31 & -32 & imp: $: n=1$ & $\$$ & sition & \\
\hline & 0 & $4.8413 E-05$ & -163 & 663 & & -32 & imp: $n=1$ & $\$$ & & \\
\hline & 0 & $4.8413 E-05$ & -164 & 64 & & -32 & imp: $n=1$ & $\$$ & & \\
\hline \\
\hline & & & & & & & & & & \\
\hline & 10 & 4.841 & -201 & & 31 & -32 & imp: $n=1$ & $\$$ & ion & \\
\hline & 10 & $3 E-05$ & -202 & 02 & 31 & -32 & imp: $n=1$ & $\$$ & & \\
\hline & 0 & $4.8413 E-05$ & -203 & 03 & 31 & -32 & imp: $n=1$ & $\$$ & tion & \\
\hline 4 & 10 & $4.8413 E-05$ & -204 & 704 & 31 & -32 & imp: $n=1$ & $\$$ & ition & \\
\hline & 0 & 4.84 & -205 & & & & imp: $n=1$ & $\$$ & & \\
\hline ) & 10 & $3 E-05$ & -206 & 706 & 31 & -32 & imp: $n=1$ & $\$$ & ion & \\
\hline & 10 & 4.84 & -207 & 07 & 31 & -32 & imp: $n=1$ & $\$$ & & \\
\hline & 0 & 4.84 & -208 & 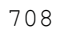 & & -32 & imp: $: n=1$ & $\$$ & & \\
\hline & 0 & 4.84 & -209 & 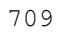 & & -32 & imp: $n=1$ & $\$$ & & \\
\hline & 0 & $3 E-05$ & -210 & 710 & 31 & -32 & imp: $n=1$ & $\$$ & ion & \\
\hline & 0 & $4.8413 \mathrm{E}-$ & -211 & & 31 & -32 & imp: $n=1$ & $\$$ & ion & \\
\hline & 10 & 4.84 & -212 & & & & imp: $n=1$ & $\$$ & & \\
\hline & 10 & 4.84 & -213 & & 31 & -32 & $i m p: n=1$ & $\$$ & ion & \\
\hline & 10 & 4.84 & -214 & & 31 & -32 & imp: $n=1$ & $\$$ & & \\
\hline & 10 & 4.84 & -215 & & 1 & -32 & imp: $n=1$ & $\$$ & & \\
\hline & 10 & 4.84 & -216 & & & -32 & imp: $n=1$ & $\$$ & & \\
\hline & 10 & 4.84 & -217 & & 31 & -32 & imp: $n=1$ & $\$$ & & \\
\hline & 10 & 4.84 & -218 & & 31 & -32 & imp:n=1 & $\$$ & & \\
\hline & 10 & 4.84 & -219 & 7 & $\perp$ & -32 & imp: $n=1$ & $\$$ & & \\
\hline & 10 & 4.84 & -220 & 720 & 31 & -32 & imp:n=1 & $\$$ & & \\
\hline & 10 & 4.84 & -221 & & 31 & -32 & imp: $: n=1$ & $\$$ & & \\
\hline $22-2+3-1-1$ & 10 & $4.8413 E-05$ & -222 & 722 & 31 & -32 & imp: $n=1$ & $\$$ & on & \\
\hline & 10 & 4.84131 & -223 & & & -32 & imp: $n=1$ & $\$$ & & \\
\hline 2 & 10 & 4.84 & -224 & 724 & 31 & -32 & $i m p: n=1$ & $\$$ & & \\
\hline & 10 & 4.84 & -225 & & 31 & -32 & imp:n=1 & $\$$ & & \\
\hline ? & 10 & 4.84 & -226 & & 1 & -32 & imp: $n=1$ & $\$$ & & \\
\hline 27 & 10 & 4.84 & -227 & & 1 & -32 & imp: $n=1$ & $\$$ & & \\
\hline 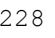 & 10 & $3 E-05$ & -228 & & 31 & -32 & imp: $n=1$ & $\$$ & $\mathrm{Po}$ & \\
\hline 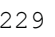 & 10 & $3 E-05$ & -229 & 729 & 1 & -32 & imp: $n=1$ & $\$$ & $n$ & 9 \\
\hline & 10 & 4.84 & -230 & & & -32 & imp: $n=1$ & $\$$ & & 0 \\
\hline & 10 & 4.84 & -231 & 7 & 1 & -32 & $i m p: n=1$ & $\$$ & $n$ & 1 \\
\hline 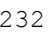 & 10 & $4.8413 E-05$ & -232 & 73 & 31 & -32 & imp: $n=1$ & $\$$ & ion & \\
\hline 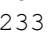 & 10 & $4.8413 E-05$ & -233 & & & -32 & $i m p: n=1$ & $\$$ & & \\
\hline & 10 & 4.8 & -23 & & & -32 & imp: $n=1$ & $\$$ & & 4 \\
\hline 2 & 10 & 4.84 & -2 & & 31 & -32 & imp: $n=1$ & $\$$ & ion & ( \\
\hline & 10 & $4.8413 E-05$ & -236 & & 31 & -32 & imp:n=1 & $\$$ & ion & \\
\hline 3 & 10 & $4.8413 E-0$ & -237 & & & -32 & imp: $n=1$ & $\$$ & & \\
\hline 38 & 10 & 4.84 & -238 & & 31 & -32 & imp:n=1 & $\$$ & on & 8 \\
\hline & 10 & $4.8413 E-05$ & -239 & 9 & 31 & -32 & imp: $n=1$ & $\$$ & on & \\
\hline 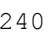 & 10 & $4.8413 E-05$ & -240 & 740 & & -32 & imp:n=1 & $\$$ & ion & 0 \\
\hline & 10 & $4.8413 \mathrm{E}$ & -241 & & & -32 & imp:n=1 & $\$$ & on & \\
\hline & 10 & 4.84 & -242 & & 31 & -32 & imp:n=1 & $\$$ & on & \\
\hline & 10 & $4.8413 E-05$ & -243 & 743 & 31 & -32 & imp: $n=1$ & $\$$ & ion & \\
\hline & 10 & $4.8413 E-0$ & -244 & 744 & 3 & -32 & imp: $n=1$ & $\$$ & ion & \\
\hline 2 & 10 & $4.8413 E-05$ & -245 & & & -32 & imp: $n=1$ & $\$$ & ion & \\
\hline & 10 & $4.8413 E-05$ & -246 & 746 & 31 & -32 & imp: $n=1$ & $\$$ & ion & \\
\hline & 10 & $4.8413 E-0$ & -247 & 747 & & -32 & imp: $n=1$ & $\$$ & ion & \\
\hline 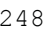 & 10 & $4.8413 E-05$ & -248 & 748 & & -32 & imp: $n=1$ & $\$$ & ion & 0 \\
\hline & 10 & $4.8413 E-05$ & -249 & 749 & 31 & -32 & imp:n=1 & $\$$ & ion & 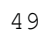 \\
\hline & 10 & $4.8413 E-05$ & -250 & 750 & 31 & -32 & imp: $n=1$ & $\$$ & ion & 50 \\
\hline & 10 & $4.8413 \mathrm{E}-0$ & -251 & 751 & & -32 & $i m p: n=1$ & $\$$ & ion & \\
\hline & 10 & $4.8413 E-0$ & -252 & 752 & & -32 & imp: $n=1$ & $\$$ & ion & \\
\hline 253 & 10 & $4.8413 E-05$ & -253 & 753 & 31 & -32 & imp:n=1 & $\$$ & ion & 53 \\
\hline & 10 & $4.8413 E-05$ & -254 & 754 & 3 & -32 & imp: $n=1$ & $\$$ & ion & \\
\hline & 10 & $4.8413 E-05$ & -255 & 755 & 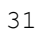 & -32 & imp: $n=1$ & $\$$ & ion & \\
\hline & 10 & $4.8413 E-05$ & -256 & & 3 & -32 & imp:n=1 & $\$$ & ion & \\
\hline 2 & 10 & $4.8413 E-05$ & -257 & 757 & 31 & -32 & imp: $n=1$ & $\$$ & Position & 7 \\
\hline & 10 & $4.8413 E-05$ & -258 & 758 & 3. & -32 & imp:n=1 & $\$$ & ion & \\
\hline & 10 & $4.8413 E-05$ & -259 & 759 & & -32 & imp: $n=1$ & $\$$ & tion & \\
\hline 60 & 10 & $4.8413 E-05$ & -260 & 760 & 31 & -32 & imp: $n=1$ & $\$$ & Position & 50 \\
\hline 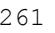 & 10 & $4.8413 E-05$ & -261 & 761 & 31 & -32 & imp: $n=1$ & $\$$ & Position & 61 \\
\hline & 10 & $4.8413 E-05$ & -262 & 762 & 31 & -32 & imp: $n=1$ & $\$$ & Position & \\
\hline 26 & 10 & $4.8413 E-05$ & -263 & 763 & 31 & -32 & imp: $n=1$ & $\$$ & Position & 63 \\
\hline 264 & 10 & $4.8413 E-05$ & -264 & 764 & 31 & -32 & imp: $n=1$ & $\$$ & Position & 64 \\
\hline
\end{tabular}

Revision: 1 
NEA/NSC/DOC(2006)1

\section{Gas Cooled (Thermal) Reactor - GCR}

\section{PROTEUS-GCR-EXP-004 CRIT-REAC}

\begin{tabular}{|c|c|c|c|c|c|c|c|c|c|c|}
\hline 01 & 10 & $4.8413 E-05$ & -301 & 801 & 31 & -32 & $i m p: n=1$ & $\$$ & Position & 1 \\
\hline 02 & 10 & $4.8413 E-05$ & -302 & 802 & 31 & -32 & imp: $n=1$ & $\$$ & osition & 2 \\
\hline 03 & 10 & $4.8413 E-05$ & -303 & 803 & 31 & -32 & imp: $n=1$ & $\$$ & Position & 3 \\
\hline 04 & 10 & $4.8413 E-05$ & -304 & 804 & 31 & -32 & $i m p: n=1$ & $\$$ & Position & 4 \\
\hline 5 & 10 & $4.8413 E-05$ & -305 & 805 & 31 & -32 & imp: $n=1$ & $\$$ & Position & 5 \\
\hline 6 & 10 & $4.8413 E-05$ & -306 & 806 & 31 & -32 & $i m p: n=1$ & $\$$ & Posi & \\
\hline 07 & 10 & $4.8413 E-05$ & -307 & 807 & 31 & -32 & $i m p: n=1$ & $\$$ & Position & \\
\hline 8 & 10 & $4.8413 E-05$ & -308 & 808 & 31 & -32 & $i m p: n=1$ & $\$$ & Position & 8 \\
\hline 99 & 10 & $4.8413 E-05$ & -309 & 809 & 31 & -32 & imp: $n=1$ & $\$$ & Position & \\
\hline 10 & 10 & $4.8413 E-05$ & -310 & 810 & 31 & -32 & imp: $n=1$ & $\$$ & Position & \\
\hline 1 & 10 & $4.8413 E-05$ & -311 & 811 & 31 & -32 & $i m p: n=1$ & $\$$ & Position & 1 \\
\hline 2 & 10 & $4.8413 E-05$ & -312 & 812 & 31 & -32 & imp: $n=1$ & $\$$ & Position & 2 \\
\hline 13 & 10 & $4.8413 E-05$ & -313 & 813 & 31 & -32 & imp: $n=1$ & $\$$ & Position & 3 \\
\hline 4 & 10 & $4.8413 E-05$ & -314 & 814 & 31 & -32 & $i m p: n=1$ & $\$$ & Position & \\
\hline 5 & 10 & $113 E-05$ & -315 & 815 & 31 & -32 & $i m p: n=1$ & $\$$ & Position & \\
\hline 16 & 10 & $4.8413 E-05$ & -316 & 816 & 31 & -32 & $i m p: n=1$ & $\$$ & Position & 6 \\
\hline 17 & 10 & $4.8413 E-05$ & -317 & 817 & 31 & -32 & $i m p: n=1$ & $\$$ & Posi & \\
\hline 8 & 10 & $3 E-05$ & -318 & 818 & 31 & -32 & imp: $n=1$ & $\$$ & Posi & \\
\hline 9 & 10 & $113 E-05$ & -319 & 819 & 31 & -32 & $i m p: n=1$ & $\$$ & Position & \\
\hline 20 & 10 & $4.8413 \mathrm{E}-05$ & -320 & 820 & 31 & -32 & imp: $n=1$ & $\$$ & Position & \\
\hline 21 & 10 & $4.8413 E-05$ & -321 & 821 & 31 & -32 & $i m p: n=1$ & \$ & ion & \\
\hline 22 & 10 & $4.8413 E-05$ & -322 & 822 & 31 & -32 & imp: $n=1$ & $\$$ & Position & 22 \\
\hline 23 & 10 & $4.8413 E-05$ & -323 & 823 & 31 & -32 & $i m p: n=1$ & $\$$ & Position & \\
\hline 24 & 10 & $4.8413 E-05$ & -324 & 824 & 31 & -32 & $i m p: n=1$ & $\$$ & on & 24 \\
\hline 25 & 10 & $13 E-05$ & -325 & 825 & 31 & -32 & imp: $n=1$ & 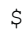 & on & \\
\hline 26 & 10 & $13 E-05$ & -326 & 826 & 31 & -32 & $i m p: n=1$ & $\$$ & Posi & 6 \\
\hline 27 & 10 & $4.8413 E-05$ & -327 & 827 & 31 & -32 & imp: $n=1$ & $\$$ & Posi & 7 \\
\hline 28 & 10 & $413 E-05$ & -328 & 828 & 31 & -32 & imp: $n=$ & S & on & \\
\hline 29 & 10 & 4. & -329 & 829 & 31 & -32 & imp: $n=1$ & $\$$ & Pos & 9 \\
\hline 30 & 10 & $413 E-05$ & -330 & 830 & 31 & -32 & imp: $n=1$ & $\$$ & Position & 0 \\
\hline 31 & 10 & $4.8413 E-05$ & -331 & 831 & 31 & -32 & imp: $n=1$ & $\$$ & Position & 1 \\
\hline 32 & 10 & $4.8413 E-05$ & -332 & 832 & 31 & -32 & $i m p: n$ & S & $\mathrm{Pc}$ & 2 \\
\hline 33 & 10 & $413 E-05$ & -333 & 833 & 31 & -32 & imp: $n=1$ & $\$$ & Posi & 33 \\
\hline 34 & 10 & $4.8413 E-05$ & -334 & 834 & 31 & -32 & imp: $n=1$ & $\$$ & Position & 4 \\
\hline 35 & 10 & $413 E-05$ & -335 & 835 & 31 & -32 & $i m p: n=1$ & $\$$ & $\mathrm{Po}$ & \\
\hline 36 & 10 & 4. & -336 & 836 & 31 & -32 & imp: $n=1$ & $\$$ & Pos & 36 \\
\hline 37 & 10 & $413 E-05$ & -337 & 837 & 31 & -32 & $i m p: n=1$ & $\$$ & Position & 7 \\
\hline 38 & 10 & $4.8413 E-05$ & -338 & 838 & 31 & -32 & imp: $n=1$ & $\$$ & Posi & 8 \\
\hline 39 & 10 & $4.8413 E-05$ & -339 & 839 & 31 & -32 & imp:n=1 & S & Pos & 39 \\
\hline 340 & 10 & $13 E-05$ & -340 & 840 & 31 & -32 & imp: $n=1$ & $\$$ & Posi & 40 \\
\hline 41 & 10 & $4.8413 E-05$ & -341 & 841 & 31 & -32 & $i m p: n=1$ & $\$$ & Position & 41 \\
\hline 342 & 10 & $4.8413 E-05$ & -342 & 842 & 31 & -32 & $i m p: n=1$ & $\$$ & Posi & 42 \\
\hline 343 & 10 & $4.8413 E-05$ & -343 & 843 & 31 & -32 & imp: $n=1$ & $\$$ & Posi & 43 \\
\hline 344 & 10 & $4.8413 E-05$ & -344 & 844 & 31 & -32 & $i m p: n=1$ & $\$$ & Pos & 44 \\
\hline 345 & 10 & $4.8413 E-05$ & -345 & 845 & 31 & -32 & $i m p: n=1$ & $\$$ & Posi & 45 \\
\hline 46 & 10 & $4.8413 E-05$ & -346 & 846 & 31 & -32 & $i m p: n=1$ & $\$$ & Pos & 46 \\
\hline 347 & 10 & $413 E-05$ & -347 & 847 & 31 & -32 & imp: $n=1$ & $\$$ & Posi & 47 \\
\hline 348 & 10 & $4.8413 E-05$ & -348 & 848 & 31 & -32 & imp: $n=1$ & \$ & Position & 48 \\
\hline 349 & 10 & $4.8413 E-05$ & -349 & 849 & 31 & -32 & imp: $n=1$ & $\$$ & Position & 49 \\
\hline 350 & 10 & $4.8413 E-05$ & -350 & 850 & 31 & -32 & imp: $n=1$ & $\$$ & Posi & 50 \\
\hline 351 & 10 & $4.8413 E-05$ & -351 & 851 & 31 & -32 & $i m p: n=1$ & $\$$ & Position & 51 \\
\hline 352 & 10 & $4.8413 E-05$ & -352 & 852 & 31 & -32 & $i m p: n=1$ & $\$$ & Posi & 52 \\
\hline 353 & 10 & $4.8413 E-05$ & -353 & 853 & 31 & -32 & imp: $n=1$ & $\$$ & Position & 53 \\
\hline 354 & 10 & $4.8413 E-05$ & -354 & 854 & 31 & -32 & imp: $n=1$ & $\$$ & Posi & 54 \\
\hline 355 & 10 & $4.8413 E-05$ & -355 & 855 & 31 & -32 & $i m p: n=1$ & $\$$ & Position & 5 \\
\hline 356 & 10 & $4.8413 E-05$ & -356 & 856 & 31 & -32 & $i m p: n=1$ & $\$$ & Position & 56 \\
\hline 357 & 10 & $4.8413 E-05$ & -357 & 857 & 31 & -32 & imp: $n=1$ & $\$$ & Position & 57 \\
\hline 358 & 10 & $4.8413 E-05$ & -358 & 858 & 31 & -32 & imp: $n=1$ & $\$$ & Position & 58 \\
\hline 359 & 10 & $4.8413 E-05$ & -359 & 859 & 31 & -32 & $i m p: n=1$ & $\$$ & Position & 59 \\
\hline 360 & 10 & $4.8413 E-05$ & -360 & 860 & 31 & -32 & imp: $n=1$ & $\$$ & Position & 60 \\
\hline 361 & 10 & $4.8413 E-05$ & -361 & 861 & 31 & -32 & imp: $n=1$ & $\$$ & Posi & 61 \\
\hline 362 & 10 & $4.8413 E-05$ & -362 & 862 & 31 & -32 & $i m p: n=1$ & $\$$ & Position & 62 \\
\hline 363 & 10 & $4.8413 E-05$ & -363 & 863 & 31 & -32 & imp: $n=1$ & $\$$ & Position & 63 \\
\hline 364 & 10 & $4.8413 E-05$ & -364 & 864 & 31 & -32 & $i m p: n=1$ & \$ & Posi & \\
\hline \\
\hline & & & & & & & & & & \\
\hline 401 & 10 & $4.8413 E-05$ & -401 & 901 & 31 & -32 & $1 \mathrm{mp}: \mathrm{n}=1$ & 3 & & 1 \\
\hline 402 & 10 & $4.8413 E-05$ & -402 & 902 & 31 & -32 & imp: $n=1$ & $\$$ & Position & . \\
\hline 403 & 10 & $4.8413 E-05$ & -403 & 903 & 31 & -32 & imp: $n=1$ & $\$$ & Position & 3 \\
\hline 404 & 10 & $4.8413 E-05$ & -404 & 904 & 31 & -32 & imp: $n=1$ & $\$$ & Position & 4 \\
\hline 405 & 10 & $4.8413 E-05$ & -405 & 905 & 31 & -32 & imp: $n=1$ & $\$$ & Position & \\
\hline 406 & 10 & $4.8413 E-05$ & -406 & 906 & 31 & -32 & imp: $n=1$ & $\$$ & Position & 6 \\
\hline 407 & 10 & $4.8413 E-05$ & -407 & 907 & 31 & -32 & $i m p: n=1$ & $\$$ & Position & 7 \\
\hline 408 & 10 & $4.8413 E-05$ & -408 & 908 & 31 & -32 & $i m p: n=1$ & $\$$ & Position & 8 \\
\hline
\end{tabular}

Revision: 1 
NEA/NSC/DOC(2006)1

\section{Gas Cooled (Thermal) Reactor - GCR}

\section{PROTEUS-GCR-EXP-004 CRIT-REAC}

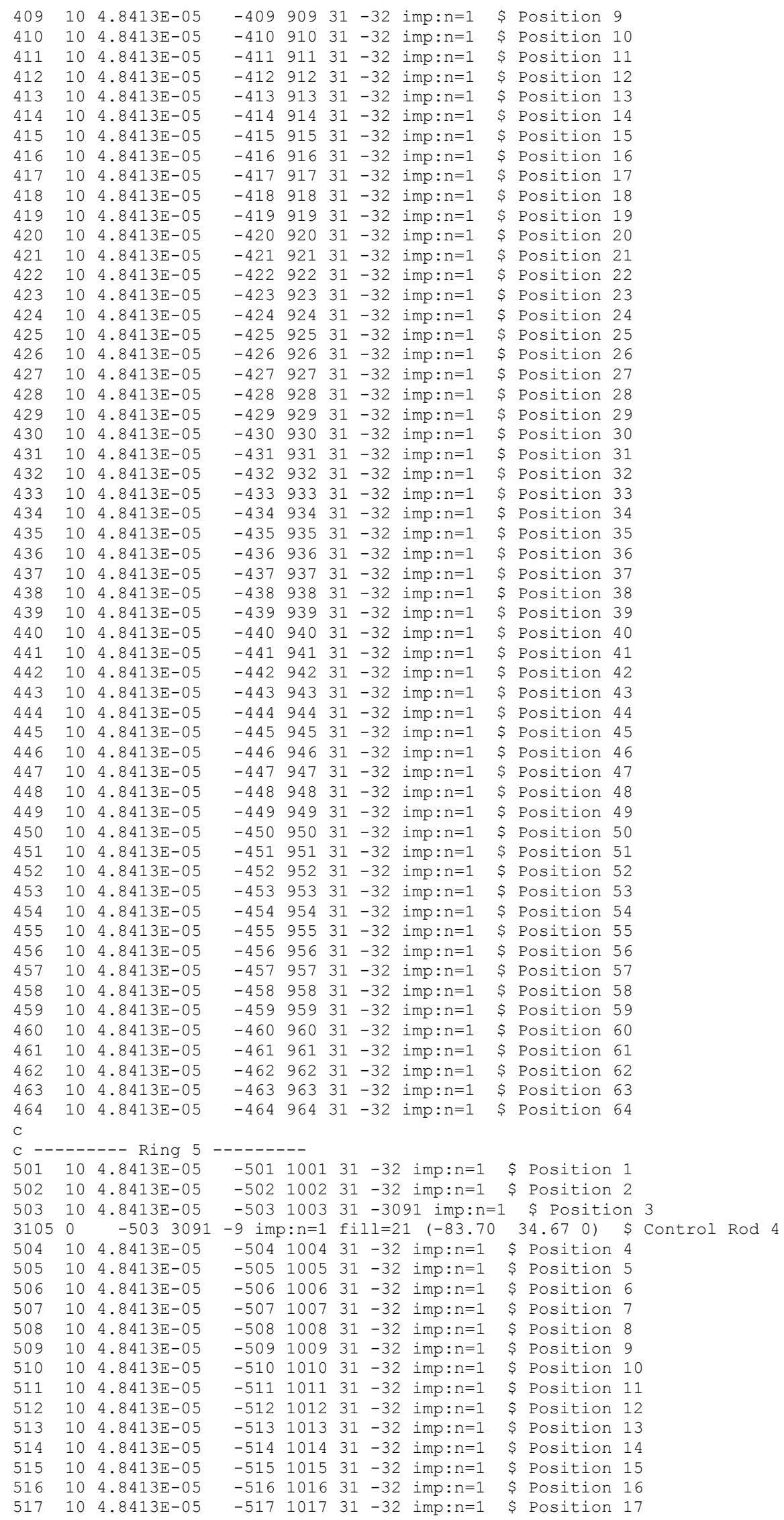

Revision: 1 
NEA/NSC/DOC(2006)1

\section{Gas Cooled (Thermal) Reactor - GCR}

\section{PROTEUS-GCR-EXP-004 CRIT-REAC}

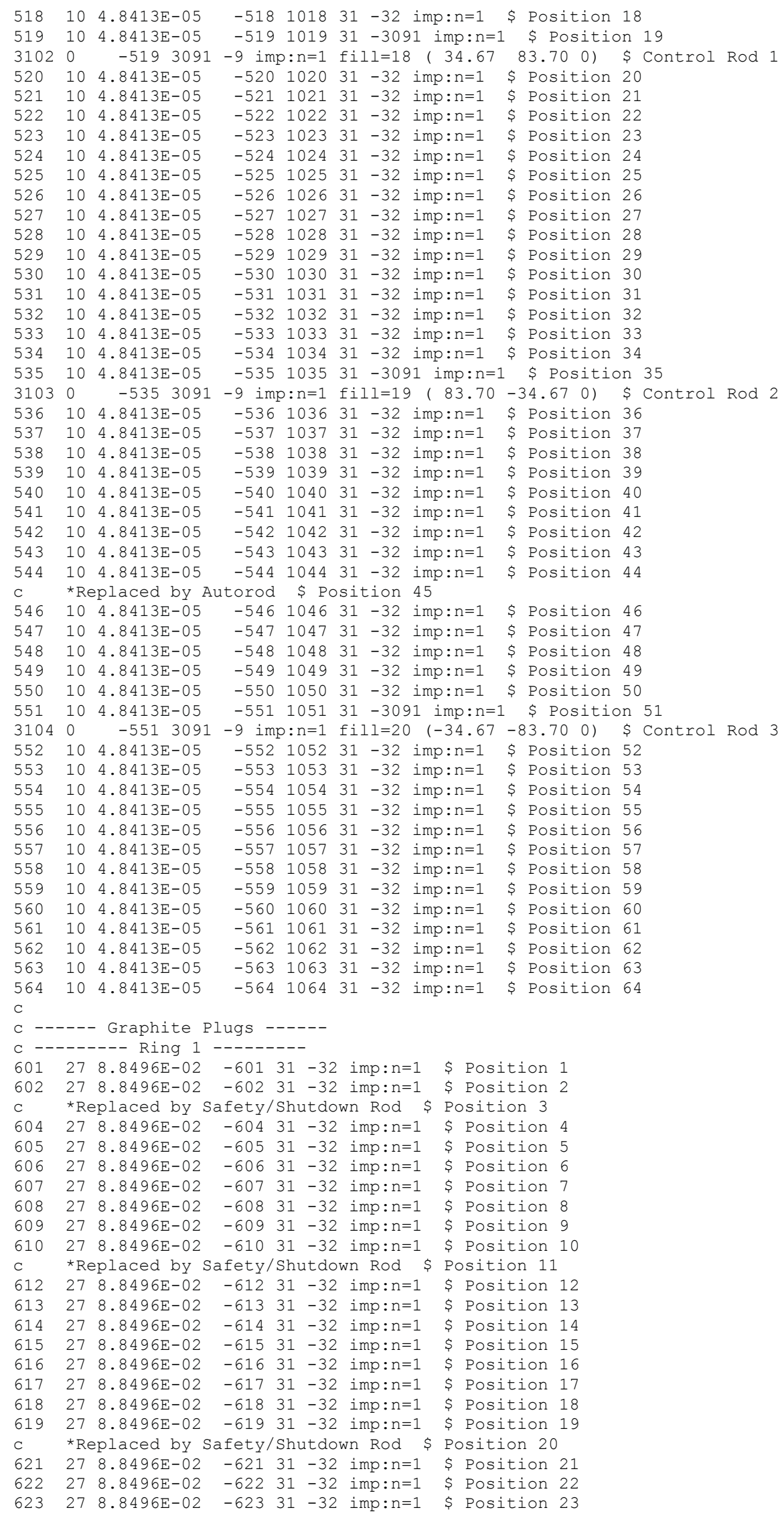

Revision: 1 
NEA/NSC/DOC(2006)1

\section{Gas Cooled (Thermal) Reactor - GCR}

\section{PROTEUS-GCR-EXP-004 CRIT-REAC}

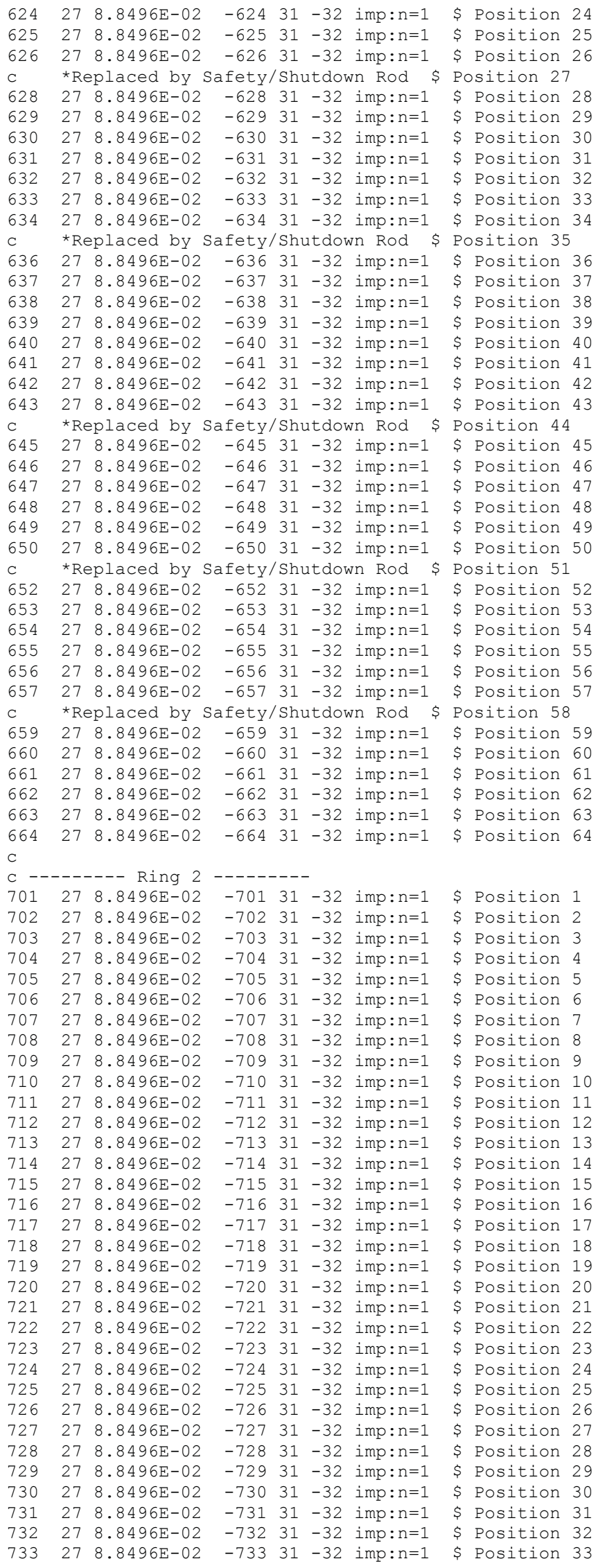

Revision: 1 
NEA/NSC/DOC(2006)1

Gas Cooled (Thermal) Reactor - GCR

PROTEUS-GCR-EXP-004 CRIT-REAC

\begin{tabular}{|c|c|c|c|c|c|c|c|c|c|}
\hline 734 & 27 & $3496 \mathrm{E}-02$ & -734 & 31 & -32 & imp: $n=1$ & $\$$ & sition & 34 \\
\hline 35 & 27 & $8.8496 \mathrm{E}-02$ & -735 & 31 & -32 & imp: $n=1$ & & ion & \\
\hline 36 & 27 & $8.8496 \mathrm{E}-02$ & -736 & 31 & -32 & imp: $n=1$ & $\$$ & sition & 36 \\
\hline 37 & 27 & $8.8496 \mathrm{E}-02$ & -737 & 31 & -32 & imp: $n=1$ & $\$$ & osition & \\
\hline 38 & 27 & $8.8496 \mathrm{E}-02$ & -738 & 31 & -32 & imp: $n=1$ & S & sition & \\
\hline 39 & 27 & $8.8496 \mathrm{E}-02$ & -739 & 31 & -32 & imp: $n=1$ & S & ion & \\
\hline 10 & 27 & $8.8496 \mathrm{E}-02$ & -740 & 31 & -32 & imp: $n=1$ & $\$$ & sition & \\
\hline 41 & 27 & $8.8496 \mathrm{E}-02$ & -741 & 31 & -32 & imp: $n=1$ & $\$$ & ion & \\
\hline 42 & 27 & $8.8496 \mathrm{E}-02$ & -742 & 31 & -32 & imp: $n=1$ & $\$$ & & \\
\hline & 27 & $8.8496 \mathrm{E}-02$ & -743 & 31 & -32 & imp: $n=1$ & $\$$ & & \\
\hline \pm & 27 & $8.8496 \mathrm{E}-02$ & -744 & 31 & -32 & imp: $n=1$ & $\$$ & $\mathrm{n}$ & \\
\hline 4 & 27 & $8.8496 \mathrm{E}-02$ & -745 & 31 & -32 & imp: $n=1$ & $\$$ & & \\
\hline 1 & 27 & $8.8496 \mathrm{E}-02$ & -746 & 31 & -32 & imp: $n=1$ & $\$$ & & \\
\hline 7 & 27 & $8.8496 \mathrm{E}-02$ & -747 & 31 & -32 & imp: $n=1$ & $\$$ & ion & \\
\hline 4 & 27 & $8.8496 \mathrm{E}-02$ & -748 & 31 & -32 & imp: $n=1$ & $\$$ & & \\
\hline 74 & 27 & $8.8496 \mathrm{E}-02$ & -749 & 31 & -32 & imp: $n=1$ & $\$$ & & \\
\hline 75 & 27 & $8.8496 \mathrm{E}-02$ & -750 & 31 & -32 & imp: $n=1$ & $\$$ & & \\
\hline 5 & 27 & $8.8496 \mathrm{E}-02$ & -751 & 31 & -32 & imp: $n=1$ & $\$$ & & \\
\hline 5 & 27 & $8.8496 \mathrm{E}-02$ & -752 & 31 & -32 & imp: $n=1$ & $\$$ & & \\
\hline 75 & 27 & $8.8496 \mathrm{E}-02$ & -753 & 31 & -32 & imp: $n=1$ & $\$$ & & \\
\hline 75 & 27 & 8.84 & -754 & 31 & -32 & imp: $n=1$ & $\$$ & & \\
\hline 5 & 27 & $8.8496 \mathrm{E}-02$ & -755 & 31 & -32 & imp: $n=1$ & $\$$ & & \\
\hline 56 & 27 & $8.8496 \mathrm{E}-02$ & -756 & 31 & -32 & imp: $n=1$ & $\$$ & & \\
\hline 75 & 27 & $8.8496 \mathrm{E}-02$ & -757 & 31 & -32 & imp: $n=1$ & $\$$ & & \\
\hline 75 & 27 & $8.8496 \mathrm{E}-02$ & -758 & 31 & -32 & imp: $n=1$ & $\$$ & & \\
\hline 1 & 27 & $8.8496 \mathrm{E}-02$ & -759 & 31 & -32 & imp: $n=1$ & $\$$ & & \\
\hline 76 & 27 & $8.8496 \mathrm{E}-02$ & -760 & 31 & -32 & imp: $n=1$ & $\$$ & & \\
\hline 76 & 27 & $8.8496 \mathrm{E}-02$ & -761 & 31 & -32 & imp: $n=1$ & $\$$ & & \\
\hline 76 & 27 & $8.8496 \mathrm{E}-02$ & -762 & 31 & -32 & imp: $n=1$ & $\$$ & & \\
\hline 76 & 27 & $8.8496 \mathrm{E}-02$ & -763 & 31 & -32 & imp: $n=1$ & $\$$ & & \\
\hline 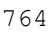 & 27 & $8.8496 \mathrm{E}-02$ & -764 & 31 & -32 & imp: $n=1$ & $\$$ & & \\
\hline \\
\hline & & & & & & & & & \\
\hline 01 & 27 & $8.8496 \mathrm{E}-02$ & -801 & 31 & -32 & imp: $n=1$ & $\$$ & & \\
\hline 802 & 27 & $8.8496 \mathrm{E}-02$ & -802 & 31 & -32 & imp: $n=1$ & $\$$ & & \\
\hline 803 & 27 & $8.8496 \mathrm{E}-02$ & -803 & 31 & -32 & imp: $n=1$ & $\$$ & $\mathrm{n}$ & 3 \\
\hline 804 & 27 & $8.8496 \mathrm{E}-02$ & -804 & 31 & -32 & imp: $n=1$ & $\$$ & n & \\
\hline 80 & 27 & $8.8496 \mathrm{E}-02$ & -805 & 31 & -32 & imp: $n=1$ & $\$$ & & \\
\hline 06 & 27 & $8.8496 \mathrm{E}-02$ & -806 & 31 & -32 & imp: $n=1$ & $\$$ & & \\
\hline 807 & 27 & $8.8496 \mathrm{E}-02$ & -807 & 31 & -32 & imp: $n=1$ & $\$$ & & \\
\hline 808 & 27 & $8.8496 \mathrm{E}-02$ & -808 & 31 & -32 & imp: $n=1$ & $\$$ & & \\
\hline 80 & 27 & $8.8496 \mathrm{E}-02$ & -809 & 31 & -32 & imp: $n=1$ & $\$$ & & \\
\hline 810 & 27 & $8.8496 \mathrm{E}-02$ & -810 & 31 & -32 & imp: $n=1$ & $\$$ & & \\
\hline 811 & 27 & $8.8496 \mathrm{E}-02$ & -811 & 31 & -32 & imp: $n=1$ & $\$$ & & \\
\hline 812 & 27 & $8.8496 \mathrm{E}-02$ & -812 & 31 & -32 & imp: $n=1$ & $\$$ & & \\
\hline 813 & 27 & $8.8496 \mathrm{E}-02$ & -813 & 31 & -32 & imp: $n=1$ & $\$$ & & \\
\hline 814 & 27 & $8.8496 \mathrm{E}-02$ & -814 & 31 & -32 & imp: $n=1$ & $\$$ & & \\
\hline 815 & 27 & $8.8496 \mathrm{E}-02$ & -815 & 31 & -32 & imp: $n=1$ & $\$$ & & \\
\hline 81 & 27 & $8.8496 \mathrm{E}-02$ & -816 & 31 & -32 & imp: $n=1$ & $\$$ & & \\
\hline 81 & 27 & $8.8496 \mathrm{E}-02$ & -817 & 31 & -32 & imp: $n=1$ & $\$$ & & \\
\hline 818 & 27 & $8.8496 \mathrm{E}-02$ & -818 & 31 & -32 & imp: $n=1$ & $\$$ & & \\
\hline 819 & 27 & $8.8496 \mathrm{E}-02$ & -819 & 31 & -32 & imp: $n=1$ & $\$$ & n & \\
\hline 820 & 27 & $8.8496 \mathrm{E}-02$ & -820 & 31 & -32 & imp: $n=1$ & $\$$ & & \\
\hline 821 & 27 & $8.8496 \mathrm{E}-02$ & -821 & 31 & -32 & imp: $n=1$ & $\$$ & & \\
\hline 822 & 27 & $8.8496 \mathrm{E}-02$ & -822 & 31 & -32 & imp: $n=1$ & $\$$ & - & \\
\hline 823 & 27 & $8.8496 \mathrm{E}-02$ & -823 & 31 & -32 & imp: $n=1$ & $\$$ & & \\
\hline 824 & 27 & $8.8496 \mathrm{E}-02$ & -824 & 31 & -32 & imp: $n=1$ & $\$$ & & \\
\hline 825 & 27 & $8.8496 \mathrm{E}-02$ & -825 & 31 & -32 & imp: $n=1$ & $\$$ & & \\
\hline 826 & 27 & $8.8496 \mathrm{E}-02$ & -826 & 31 & -32 & imp: $n=1$ & $\$$ & & \\
\hline 827 & 27 & $8.8496 \mathrm{E}-02$ & -827 & 31 & -32 & imp: $n=1$ & $\$$ & & \\
\hline 828 & 27 & $8.8496 \mathrm{E}-02$ & -828 & 31 & -32 & imp: $n=1$ & $\$$ & & \\
\hline 829 & 27 & $8.8496 \mathrm{E}-02$ & -829 & 31 & -32 & imp: $n=1$ & $\$$ & $\mathrm{P}$ & \\
\hline 830 & 27 & $8.8496 \mathrm{E}-02$ & -830 & 31 & -32 & imp: $n=1$ & $\$$ & & \\
\hline 831 & 27 & $8.8496 \mathrm{E}-02$ & -831 & 31 & -32 & imp: $n=1$ & $\$$ & & \\
\hline 832 & 27 & $8.8496 \mathrm{E}-02$ & -832 & 31 & -32 & imp: $n=1$ & $\$$ & & \\
\hline 833 & 27 & $8.8496 \mathrm{E}-02$ & -833 & 31 & -32 & imp: $n=1$ & $\$$ & & \\
\hline 834 & 27 & $8.8496 \mathrm{E}-02$ & -834 & 31 & -32 & imp: $n=1$ & $\$$ & & \\
\hline 835 & 27 & $8.8496 \mathrm{E}-02$ & -835 & 31 & -32 & imp: $n=1$ & $\$$ & & \\
\hline 836 & 27 & $8.8496 \mathrm{E}-02$ & -836 & 31 & -32 & imp: $n=1$ & $\$$ & Po & \\
\hline 837 & 27 & $8.8496 \mathrm{E}-02$ & -837 & 31 & -32 & imp: $n=1$ & $\$$ & & \\
\hline 838 & 27 & $8.8496 \mathrm{E}-02$ & -838 & 31 & -32 & imp: $n=1$ & $\$$ & & \\
\hline 839 & 27 & $8.8496 \mathrm{E}-02$ & -839 & 31 & -32 & imp: $n=1$ & $\$$ & & \\
\hline 840 & 27 & $8.8496 \mathrm{E}-02$ & -840 & 31 & -32 & imp: $n=1$ & $\$$ & Pos & \\
\hline 841 & 27 & $8.8496 \mathrm{E}-02$ & -841 & 31 & -32 & imp: $n=1$ & $\$$ & & \\
\hline 842 & 27 & $8.8496 \mathrm{E}-02$ & -842 & 31 & -32 & imp: $n=1$ & $\$$ & & \\
\hline 843 & 27 & $8.8496 \mathrm{E}-02$ & -843 & 31 & -32 & imp: $n=1$ & $\$$ & Pos & \\
\hline
\end{tabular}

Revision: 1 
NEA/NSC/DOC(2006)1

Gas Cooled (Thermal) Reactor - GCR

PROTEUS-GCR-EXP-004 CRIT-REAC

\begin{tabular}{|c|c|c|c|c|c|c|c|c|c|}
\hline 44 & r & $5 E-02$ & 34 & 1 & -32 & imp: $n=1$ & & & \\
\hline & 7 & $496 \mathrm{E}-02$ & 845 & 31 & -32 & imp: $n=1$ & & & \\
\hline & 27 & $496 \mathrm{E}-02$ & 346 & 31 & -32 & imp: $n=1$ & & sition & \\
\hline & 27 & $.8496 \mathrm{E}-02$ & -847 & 31 & -32 & imp: $n=1$ & & & \\
\hline & 7 & $.8496 \mathrm{E}-02$ & -848 & 31 & -32 & imp: $n=1$ & & & \\
\hline & 7 & $.8496 \mathrm{E}-02$ & -849 & 1 & -32 & imp: $n=1$ & & & \\
\hline & 7 & $8496 \mathrm{E}-02$ & 50 & 31 & -32 & imp: $n=1$ & & n & \\
\hline & & $496 E-02$ & -851 & 31 & -32 & imp: $n=1$ & & & \\
\hline & 7 & $.8496 \mathrm{E}-02$ & -852 & 1 & -32 & imp: $n=1$ & & & \\
\hline & 7 & 8106 & & 1 & -32 & imp: $n=1$ & & & \\
\hline & $\pi$ & 2 & 54 & 1 & -32 & imp: $n=1$ & & & \\
\hline & 7 & -02 & 355 & 1 & -32 & imp: $n=1$ & & & \\
\hline & 7 & & & 1 & & imp: $n=1$ & & & \\
\hline & 7 & & & 1 & & imp: $n=1$ & & & \\
\hline & 7 & & & 1 & & imp: $n=1$ & & & \\
\hline & 27 & 2 & & 1 & & imp: $n=1$ & & & \\
\hline & & & & 1 & & imp: $n=1$ & & & \\
\hline & 7 & & & 1 & -32 & imp: $n=1$ & & & \\
\hline & 7 & & & $\perp$ & & imp: $n=1$ & & & \\
\hline & 7 & & & 1 & & $m p: n$ & & & \\
\hline & 7 & & & & & $m p: n$ & $\$$ & & \\
\hline \\
\hline & & & & & & & & & \\
\hline & 27 & & & 1 & 32 & imp: $n=1$ & $\$$ & 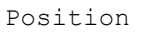 & \\
\hline & 27 & & & 31 & -32 & imp: $n=1$ & $\$$ & $n$ & \\
\hline & 27 & 8.8 & & 31 & -32 & imp: $n=1$ & $\$$ & & \\
\hline & 27 & 8.8 & 4 & 1 & -32 & $i m p: n$ & $\$$ & th & \\
\hline & 27 & 8. & & 1 & -32 & imp: $n=1$ & $\$$ & & \\
\hline & 27 & 8 . & & 31 & -32 & imp: $n=1$ & $\$$ & & \\
\hline & 27 & 8.8 & & 1 & -32 & imp:r & $\$$ & & \\
\hline & 27 & 8. & - & 31 & -32 & imp: & $\gamma$ & & \\
\hline & 27 & 8.8 & & 31 & -32 & imp: & $\$$ & & \\
\hline & 27 & 8 & & 31 & -32 & imp & $\$$ & & \\
\hline & 27 & 8.8 & & 31 & -32 & imp:l & $\$$ & 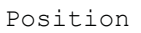 & \\
\hline & 27 & 8.8 & & 31 & -32 & imp: 1 & 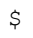 & & \\
\hline & 27 & 8. & & 31 & -32 & imp: 1 & $\$$ & Po & \\
\hline & 27 & 8. & & 1 & -32 & imp: $n=1$ & $\$$ & & \\
\hline & 27 & 8. & & 31 & -32 & imp: & ? & & \\
\hline & 27 & 8. & & 31 & -32 & imp: & $\$$ & & \\
\hline & 27 & 8. & & 31 & -32 & $i m p: r$ & $\$$ & $P_{0}$ & \\
\hline & 27 & 8. & & 1 & - & imp & & & \\
\hline & 27 & & & 31 & & imp & T & & \\
\hline & 27 & 8. & & 31 & -32 & imp: $n$ & $\$$ & & \\
\hline & 27 & 8. & & 31 & 2 & imp: $n=1$ & 4 & & \\
\hline & 27 & 8. & & 31 & & imp: $n$ & $r$ & & \\
\hline & 27 & 8 . & & 31 & & imp: $n$ & $\$$ & & \\
\hline & 27 & 8. & & 31 & -32 & imp: $n=1$ & $\$$ & & \\
\hline & 27 & 8.8 & & 31 & -32 & imp: $n=1$ & $\$$ & & \\
\hline & 27 & 8. & & 31 & -32 & imp: & $\$$ & & \\
\hline & 27 & 8. & & 31 & -32 & imp: $n=1$ & $\$$ & & \\
\hline & 27 & 8.8 & & 31 & -32 & imp: $n=1$ & $\$$ & & \\
\hline & 27 & 8.8 & & 31 & -32 & imp: $n=1$ & $\$$ & 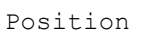 & \\
\hline & 27 & 8. & & 31 & & imp: & $\$$ & - & \\
\hline & 27 & 8. & & 31 & -32 & imp: $n=1$ & $T$ & & \\
\hline & 27 & 8.8 & & 31 & -32 & imp: $n=1$ & $\$$ & & \\
\hline & 27 & 8.8 & & 31 & -32 & imp: $n=1$ & $\$$ & Po & \\
\hline & 27 & 8. & & 31 & -32 & imp: $n=1$ & $\$$ & & \\
\hline & 27 & 8.8 & & 31 & -32 & imp: $n=1$ & $\$$ & & \\
\hline & 27 & 8.8 & & 31 & -32 & imp & $\$$ & & \\
\hline & 27 & 8. & & 31 & -32 & imp: $n=1$ & $\$$ & & \\
\hline & 27 & 8. & & 31 & -32 & imp:n=1 & $\$$ & & \\
\hline & 27 & 8.8 & & 31 & -32 & imp: $n=1$ & $\$$ & $\mathrm{Po}$ & \\
\hline & 27 & 8.8 & & 31 & & $i m p: r$ & $r$ & & \\
\hline & 27 & 8.8 & & 31 & & imp: $n=1$ & $r$ & & \\
\hline & 27 & 8.8 & & 31 & -32 & imp: $n=1$ & $\$$ & & \\
\hline & 27 & $96 \mathrm{E}-$ & & 31 & -32 & imp: $n=1$ & $\$$ & 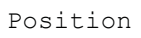 & \\
\hline & 27 & 8.8 & & 31 & & imp: $n$ & $\$$ & & \\
\hline & 27 & 8.8 & & 31 & & imp: $n=1$ & $T$ & $\mathrm{Po}$ & \\
\hline & 27 & 8.8 & & 31 & -32 & imp: $n=1$ & $\$$ & Pos: & \\
\hline & 27 & 8.8 & & 31 & -32 & imp & $\$$ & & \\
\hline & 27 & 8.8 & & 31 & & imp: $n=1$ & $\$$ & & \\
\hline & 27 & 8.8 & & 31 & -32 & imp: $n=1$ & 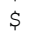 & Posi & \\
\hline & 27 & $8.8496 \mathrm{E}-02$ & & 31 & -32 & imp: $n=1$ & $\$$ & Positio & \\
\hline & 27 & 8.8 & & 31 & & imp: $n=1$ & s & & \\
\hline & 27 & 8.8 & & I & & imp: $n=1$ & & & \\
\hline & 27 & $8.8496 E-02$ & & 31 & & imp: $n=1$ & $\$$ & Pos & \\
\hline
\end{tabular}

Revision: 1 
NEA/NSC/DOC(2006)1

Gas Cooled (Thermal) Reactor - GCR

\section{PROTEUS-GCR-EXP-004 CRIT-REAC}

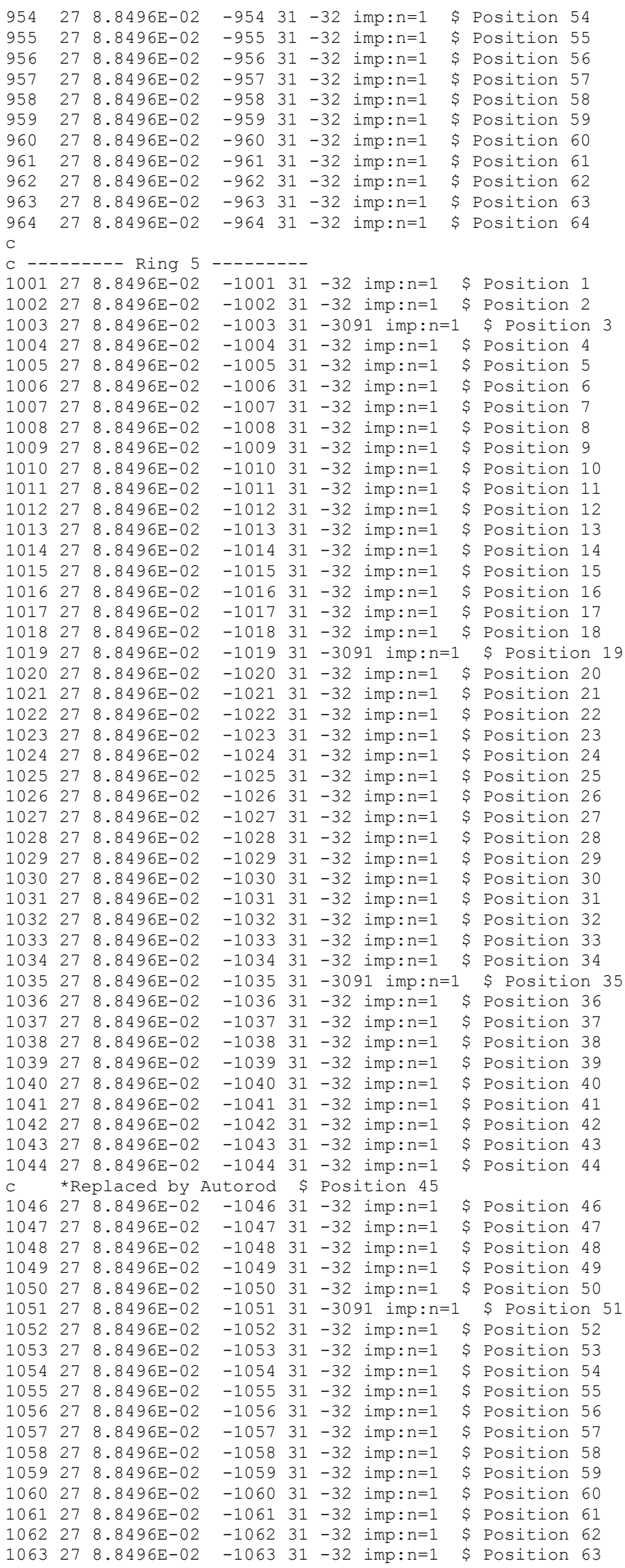

Revision: 1 
NEA/NSC/DOC(2006)1

\section{Gas Cooled (Thermal) Reactor - GCR \\ PROTEUS-GCR-EXP-004 CRIT-REAC}

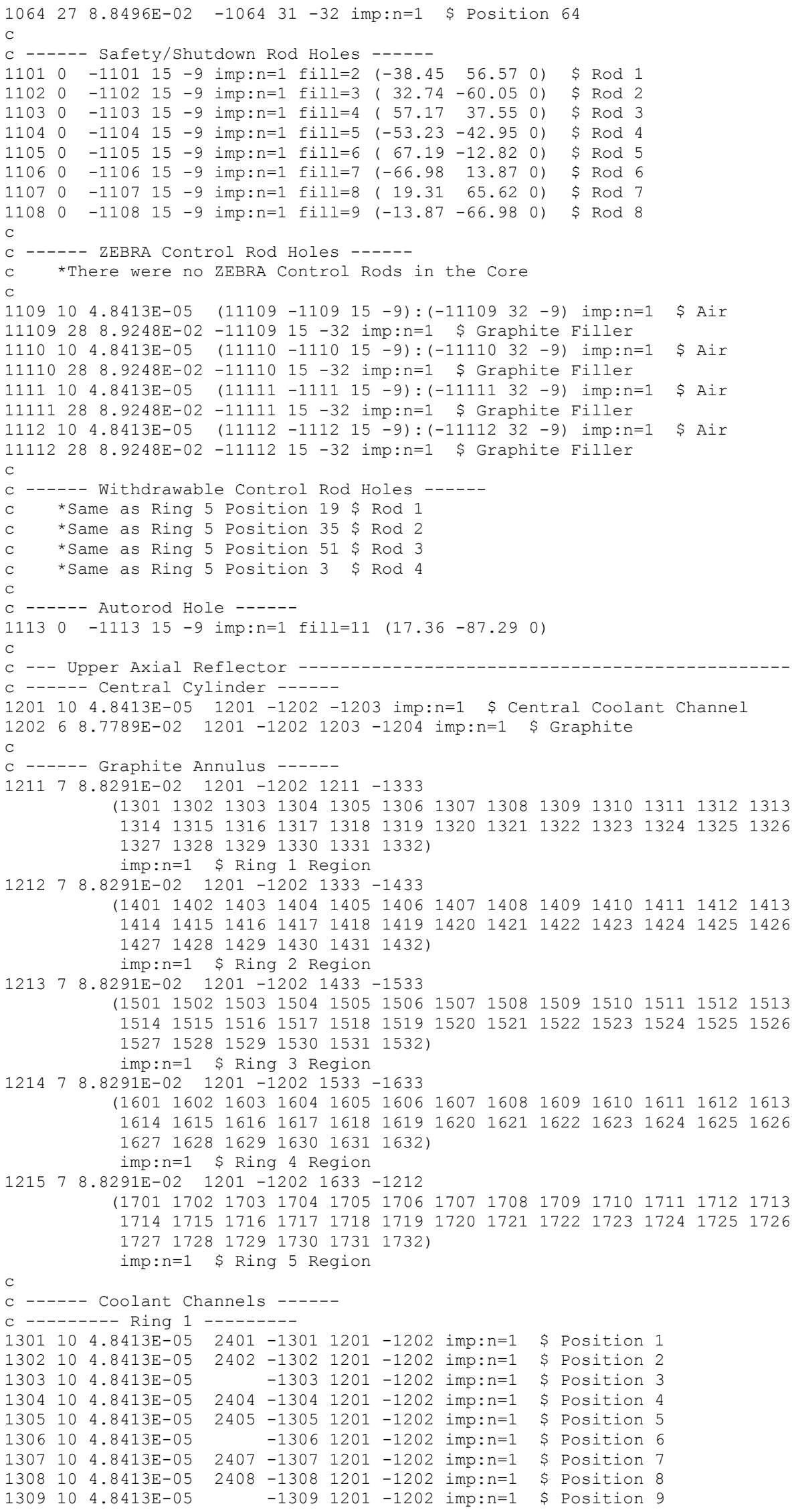

Revision: 1 
NEA/NSC/DOC(2006)1

Gas Cooled (Thermal) Reactor - GCR

PROTEUS-GCR-EXP-004 CRIT-REAC

\begin{tabular}{|c|c|c|c|c|c|c|c|c|c|c|}
\hline 10 & 10 & $E-05$ & 10 & -1310 & 1 & 02 & imp:n=1 & 7 & & \\
\hline & 10 & $4.8413 \mathrm{E}-05$ & 2411 & -1311 & 201 & -1202 & imp: $n=1$ & 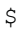 & Eion & 1 \\
\hline 312 & 10 & $4.8413 E-05$ & & -1312 & 1201 & -1202 & imp: $n=1$ & $\$$ & Position & 12 \\
\hline 31 & 10 & $4.8413 E-05$ & 2413 & -1313 & 1201 & -1202 & imp: $n=1$ & $\$$ & Position & 13 \\
\hline & 0 & $4.8413 E-05$ & 2414 & -1314 & 1201 & -1202 & $i m p: n=1$ & $\$$ & tion & \\
\hline & 10 & $4.8413 E-05$ & & -1315 & 201 & -1202 & imp: $n=1$ & $\$$ & ion & \\
\hline & LO & $4.8413 E-05$ & 2416 & -1316 & 201 & -1202 & imp:n=1 & $\$$ & sition & \\
\hline & 0 & $4.8413 E-05$ & 2417 & -1317 & 201 & -1202 & imp: $n=1$ & $\$$ & sition & \\
\hline & 10 & $4.8413 E-05$ & & -1318 & 201 & -1202 & imp: $n=1$ & $\$$ & & \\
\hline & 0 & $4.8413 E-05$ & 19 & -1319 & 01 & -1202 & imp: $n=1$ & $\$$ & & \\
\hline & 0 & $4.8413 E-05$ & 2420 & -1320 & 201 & -1202 & imp:n=1 & $\$$ & $\mathrm{n}$ & \\
\hline & 10 & $4.8413 E-05$ & & -1321 & 201 & -1202 & imp:n=1 & $\$$ & & \\
\hline & 10 & $4.8413 E-05$ & 22 & -1322 & & -1202 & imp: $n=1$ & $\$$ & & \\
\hline & 10 & $4.8413 E-05$ & 2423 & -1323 & 201 & -1202 & $i m p: n=1$ & $\$$ & ion & \\
\hline & 10 & $4.8413 E-05$ & & -1324 & 201 & -1202 & imp: $n=1$ & $\$$ & & \\
\hline & 10 & $4.8413 E-05$ & 2425 & -1325 & 201 & -1202 & $i m p: n=1$ & $\$$ & $\mathrm{n}$ & \\
\hline & 10 & $4.8413 E-05$ & 2426 & -1326 & & -1202 & imp: $n=1$ & $\$$ & & \\
\hline & 10 & $4.8413 \mathrm{E}-05$ & & -1327 & 1 & -1202 & imp: $n=1$ & $\$$ & Lon & 7 \\
\hline & 10 & $4.8413 E-05$ & & -1328 & 201 & -1202 & imp: $n=1$ & $\$$ & & \\
\hline & 10 & $4.8413 E-05$ & & -1329 & 01 & -1202 & imp: $n=1$ & $\$$ & $\mathrm{n}$ & \\
\hline & 10 & $4.8413 E-05$ & & -1330 & & -1202 & imp: $n=1$ & $\$$ & & \\
\hline & 10 & $4.8413 E-05$ & 2431 & -1331 & 1 & -1202 & $i m p: n=1$ & $\$$ & & \\
\hline & 10 & $4.8413 E-05$ & & -1332 & & -1202 & imp: $n=1$ & $\$$ & & \\
\hline & & & & & & & & & & \\
\hline & & & & & & & & & & \\
\hline & 0 & 4.84 & & -1401 & & -1202 & imp: $n=1$ & $\$$ & $n$ & 1 \\
\hline & 10 & $4.8413 E-05$ & 2502 & -1402 & 1201 & -1202 & imp: $n=1$ & $\$$ & on & \\
\hline & 10 & $4.8413 E-05$ & 2503 & -1403 & 1201 & -1202 & imp: $n=1$ & $\$$ & on & \\
\hline & 10 & $4.8413 E-05$ & 2504 & -1404 & 201 & -1202 & imp: $n=1$ & $\$$ & & \\
\hline & 10 & $4.8413 E-05$ & 2505 & -1405 & 201 & -1202 & imp: $n=1$ & $\$$ & $n$ & \\
\hline & 10 & $4.8413 E-05$ & 2506 & -1406 & 201 & -1202 & $i m p: n=1$ & $\$$ & n & \\
\hline & 10 & $4.8413 E-05$ & 2507 & -1407 & 1201 & -1202 & imp: $n=1$ & $\$$ & & 7 \\
\hline & 10 & $4.8413 E-05$ & 2508 & -1408 & 1201 & -1202 & $i m p: n=1$ & $\$$ & Po & \\
\hline & 10 & $4.8413 E-05$ & 2509 & -1409 & 1201 & -1202 & imp: $n=1$ & $\$$ & $\mathrm{pn}$ & 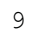 \\
\hline & 10 & $4.8413 E-05$ & 2510 & -1410 & 201 & -1202 & imp: $n=1$ & $\$$ & & 10 \\
\hline & 10 & $4.8413 \mathrm{E}-05$ & 2511 & -1411 & 1201 & -1202 & imp: $n=1$ & $\$$ & & \\
\hline & 10 & $4.8413 E-05$ & 2512 & -1412 & 1201 & -1202 & imp: $n=1$ & $\$$ & & \\
\hline & 10 & $4.8413 E-05$ & 2513 & -14 & 1201 & -1202 & $i m p: n=1$ & $\$$ & & \\
\hline & 10 & $4.8413 E-05$ & 2514 & -14 & 1201 & -1202 & imp: $n=1$ & $\$$ & & \\
\hline & 10 & $4.8413 E-05$ & 2515 & -1415 & 1201 & -1202 & imp: $n=1$ & $\$$ & Po & \\
\hline & 10 & $4.8413 E-05$ & 2516 & -1416 & 201 & -1202 & imp: $n=1$ & $\$$ & $\mathrm{n}$ & \\
\hline & 10 & $4.8413 E-05$ & 2517 & -1 & 1201 & -1 & imp: $n=1$ & $\$$ & & \\
\hline & 10 & $4.8413 \mathrm{E}-05$ & 2518 & -14 & 1201 & -1202 & imp: $n=1$ & $\$$ & & 8 \\
\hline & 10 & $4.8413 E-05$ & 2519 & -1419 & 1201 & -1202 & imp: $n=1$ & $\$$ & & \\
\hline & 10 & $4.8413 E-05$ & 2520 & -1420 & 1201 & -1202 & imp: $n=1$ & $\$$ & & \\
\hline & 10 & $4.8413 E-05$ & 2521 & -1421 & 1201 & -12 & imp: $n=1$ & $\$$ & & \\
\hline & 10 & $4.8413 E-05$ & 2522 & -1422 & 1201 & -1202 & imp: $n=1$ & $\$$ & on & 2 \\
\hline & 10 & $4.8413 E-05$ & 2523 & -1423 & 1201 & -1202 & imp: $n=1$ & $\$$ & & \\
\hline & 10 & $4.8413 E-05$ & 2524 & -1424 & 1201 & -1202 & $i m p: n=1$ & $\$$ & & \\
\hline & 10 & $4.8413 E-05$ & 2525 & -1425 & 1201 & -1202 & imp: $n=1$ & $\$$ & & \\
\hline & 10 & $4.8413 E-05$ & 2526 & -1426 & 1201 & -1202 & $i m p: n=1$ & $\$$ & Po & \\
\hline & 10 & $4.8413 E-05$ & 2527 & -1427 & 1201 & -1202 & imp: $n=1$ & $\$$ & $\mathrm{n}$ & 7 \\
\hline & 10 & $4.8413 E-05$ & 2528 & -1428 & 1201 & -1202 & imp: $n=1$ & $\$$ & $\mathrm{pn}$ & \\
\hline & 10 & $4.8413 E-05$ & 2529 & -1429 & 1201 & -12 & imp: $n=1$ & $\$$ & $\mathrm{Po}$ & 9 \\
\hline & 10 & $4.8413 E-05$ & 2530 & -1430 & 1201 & -1202 & imp: $n=1$ & $\$$ & Po & \\
\hline & 10 & $4.8413 E-05$ & 2531 & -1431 & 1201 & -1202 & imp: $n=1$ & $\$$ & & 1 \\
\hline & 10 & $4.8413 E-05$ & 2532 & -1432 & 1201 & -1202 & imp: $n=1$ & $\$$ & & 32 \\
\hline & & & & & & & & & & \\
\hline & & & & & & & & & & \\
\hline & 10 & $4.8413 E-05$ & 2601 & -1501 & 1201 & -12 & $i m p: n=1$ & $\$$ & & 1 \\
\hline & 10 & $4.8413 \mathrm{E}-05$ & & -1502 & 1201 & -1202 & $i m p: n=1$ & $\$$ & $\mathrm{Po}$ & 2 \\
\hline & 10 & $4.8413 E-05$ & & -1503 & 1201 & -1202 & imp: $n=1$ & $\$$ & $\mathrm{Po}$ & 3 \\
\hline & 10 & $4.8413 E-05$ & 2604 & -1504 & 1201 & -1202 & $i m p: n=1$ & $\$$ & & 4 \\
\hline & 10 & $4.8413 E-05$ & & -1505 & 1201 & -1202 & imp: $n=1$ & $\$$ & & 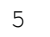 \\
\hline & 10 & $4.8413 E-05$ & 2606 & -1506 & 1201 & -1202 & $i m p: n=1$ & $\$$ & Po & 6 \\
\hline م & 10 & $4.8413 E-05$ & 2607 & -1507 & 1201 & -1202 & imp: $n=1$ & $\$$ & Po & 7 \\
\hline & 10 & $4.8413 E-05$ & & -1508 & 1201 & -1202 & imp: $n=1$ & $\$$ & & 8 \\
\hline & 10 & $4.8413 E-05$ & 2609 & -1509 & 1201 & -1202 & $i m p: n=1$ & $\$$ & Pos & 9 \\
\hline & 10 & $4.8413 E-05$ & 2610 & -1510 & 1201 & -1202 & imp: $n=1$ & $\$$ & Pos & 10 \\
\hline & 10 & $4.8413 E-05$ & & -1511 & 1201 & -1202 & imp: $n=1$ & $\$$ & & 11 \\
\hline & 10 & $4.8413 E-05$ & 2612 & -1512 & 1201 & -1202 & $i m p: n=1$ & $\$$ & Po & \\
\hline & 10 & $4.8413 E-05$ & 2613 & -1513 & 1201 & -1202 & $i m p: n=1$ & $\$$ & Pos & 13 \\
\hline & 10 & $4.8413 E-05$ & & -1514 & 1201 & -1202 & imp: $n=1$ & $\$$ & Posit & 14 \\
\hline & 10 & $4.8413 E-05$ & 2615 & -1515 & 1201 & -1202 & $i m p: n=1$ & $\$$ & & \\
\hline & 10 & $4.8413 E-05$ & 2616 & -1516 & 1201 & -1202 & $i m p: n=1$ & $\$$ & Pos & 16 \\
\hline 517 & 10 & $4.8413 E-05$ & & -1517 & 1201 & -1202 & imp: $n=1$ & $\$$ & Posi & 17 \\
\hline
\end{tabular}

Revision: 1 
NEA/NSC/DOC(2006)1

Gas Cooled (Thermal) Reactor - GCR

PROTEUS-GCR-EXP-004 CRIT-REAC

\begin{tabular}{|c|c|c|c|c|c|c|c|c|c|c|}
\hline 518 & 10 & $E-05$ & 18 & -1518 & 01 & -1202 & imp: $n=1$ & s & Lon & 18 \\
\hline & 10 & $4.8413 E-05$ & 2619 & -1519 & 201 & -1202 & imp: $n=1$ & S & sition & 19 \\
\hline 520 & 10 & $4.8413 E-05$ & & -1520 & 1201 & -1202 & imp: $n=1$ & $\$$ & Position & 20 \\
\hline 52 & 10 & $4.8413 E-05$ & 2621 & -1521 & 1201 & -1202 & imp: $n=1$ & $\$$ & Position & 21 \\
\hline & 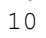 & $4.8413 E-05$ & 2622 & -1522 & 1201 & -1202 & imp: $n=1$ & $\$$ & Position & \\
\hline & LO & $4.8413 E-05$ & & -1523 & 201 & -1202 & imp: $n=1$ & $\$$ & Position & 23 \\
\hline 2 & 0 & $4.8413 E-05$ & 2624 & -1524 & 201 & -1202 & imp: $n=1$ & $\$$ & Position & 4 \\
\hline & t & $4.8413 E-05$ & 2625 & -1525 & 201 & -1202 & imp: $n=1$ & $\$$ & Position & \\
\hline & 10 & $4.8413 E-05$ & & -1526 & 201 & -1202 & imp: $n=1$ & $\$$ & Position & \\
\hline & 0 & $4.8413 E-05$ & 2627 & -1527 & 201 & -1202 & imp: $n=1$ & $\$$ & Position & \\
\hline & 0 & $4.8413 E-05$ & & -1528 & 201 & -1202 & imp: $n=1$ & $\$$ & Position & \\
\hline & 10 & $4.8413 E-05$ & 529 & -1529 & 201 & -1202 & imp: $n=1$ & $\$$ & Position & \\
\hline & 10 & $4.8413 E-05$ & 630 & -1530 & & -1202 & imp: $n=1$ & $\$$ & Position & \\
\hline & Sto & $4.8413 E-05$ & & -1531 & 201 & -1202 & imp: $n=1$ & $\$$ & Position & \\
\hline & 10 & $4.8413 E-05$ & 32 & -1532 & 201 & -1202 & imp: $n=1$ & $\$$ & Position & \\
\hline \\
\hline \\
\hline & & $3 E-05$ & & -1601 & 201 & -1202 & imp: $n=1$ & $\$$ & Position & 1 \\
\hline & 0 & $4.8413 E-05$ & 2702 & -1602 & 201 & -1202 & imp: $n=1$ & $\$$ & tion & \\
\hline & 0 & $4.8413 E-05$ & 03 & -1603 & 201 & -1202 & imp: $n=1$ & $\$$ & ion & \\
\hline & 10 & $4.8413 E-05$ & 2704 & -1604 & 201 & -1202 & imp: $n=1$ & $\$$ & ion & \\
\hline & 10 & $4.8413 E-05$ & 2705 & -1605 & 201 & -1202 & imp: $n=1$ & $\$$ & Position & \\
\hline & 10 & $4.8413 E-05$ & 2706 & -1606 & 201 & -1202 & imp: $n=1$ & $\$$ & Position & 0 \\
\hline & 10 & $4.8413 E-05$ & 2707 & -1607 & 201 & -1202 & imp: $n=1$ & $\$$ & Position & \\
\hline & 10 & $4.8413 E-05$ & 2708 & -1608 & 201 & -1202 & imp: $n=1$ & $\$$ & Position & 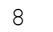 \\
\hline & 10 & $4.8413 E-05$ & 2709 & -1609 & 201 & -1202 & imp: $n=1$ & $\$$ & Position & $y$ \\
\hline & 10 & $4.8413 E-05$ & 2710 & -1610 & 201 & -12 & imp: $n=1$ & $\$$ & Pos & 10 \\
\hline & 10 & $4.8413 E-05$ & 2711 & -1611 & & -12 & imp: $n=1$ & $\$$ & ion & \\
\hline & 10 & $4.8413 E-05$ & 2712 & -1612 & 201 & -1202 & imp: $n=1$ & $\$$ & Position & \\
\hline & 10 & $4.8413 E-05$ & 2713 & -1613 & 201 & -1202 & imp: $n=1$ & $\$$ & Position & 13 \\
\hline & 10 & $4.8413 E-05$ & 2714 & -1614 & 1 & -12 & imp: $n=1$ & $\$$ & ion & 4 \\
\hline & 10 & $4.8413 E-05$ & 2715 & -1615 & 1201 & -12 & imp: $n=1$ & $\$$ & Position & \\
\hline & 10 & $4.8413 E-05$ & 2716 & -1616 & 1201 & -1202 & imp: $n=1$ & $\$$ & Position & \\
\hline & 10 & $4.8413 E-05$ & 2717 & -1617 & 1201 & -1202 & imp: $n=1$ & $\$$ & Position & 17 \\
\hline & 10 & $4.8413 E-05$ & 2718 & -1618 & 1 & -12 & imp: $n=1$ & $\$$ & ion & \\
\hline & 10 & $4.8413 E-05$ & 2719 & -1619 & 1201 & -1202 & imp: $n=1$ & $\$$ & Position & 9 \\
\hline & 10 & $4.8413 E-05$ & 2720 & -1620 & 1201 & -1202 & imp: $n=1$ & $\$$ & ion & 20 \\
\hline & 10 & $4.8413 E-05$ & 2721 & -1621 & 1 & -1 & imp:n=1 & $\$$ & ion & 21 \\
\hline & 10 & $4.8413 E-05$ & 2722 & -1622 & 1201 & -12 & imp: $n=1$ & $\$$ & ion & \\
\hline & 10 & $4.8413 E-05$ & 2723 & -1623 & 1201 & -1202 & imp: $n=1$ & $\$$ & Position & 3 \\
\hline & 10 & $4.8413 E-05$ & 2724 & -1624 & 01 & -1202 & $i m p: n=1$ & $\$$ & Position & 24 \\
\hline & 10 & $4.8413 E-05$ & 2725 & -1625 & 1 & -1 & imp: $n=1$ & $\$$ & Po: & \\
\hline & 10 & $4.8413 E-05$ & 2726 & -1626 & 12 & -12 & imp: $n=1$ & $\$$ & Position & 26 \\
\hline & 10 & $4.8413 E-05$ & 2727 & -1627 & 1201 & -1202 & imp: $n=1$ & $\$$ & Position & 2 \\
\hline & 10 & $4.8413 E-05$ & 2728 & -1628 & 1201 & -12 & imp: $n=1$ & $\$$ & Position & $\angle 0$ \\
\hline & 10 & $4.8413 E-05$ & 2729 & -1629 & 1201 & -12 & imp: $n=1$ & $\$$ & Position & \\
\hline & 10 & $4.8413 E-05$ & 2730 & -1630 & 1201 & -12 & imp: $n=1$ & $\$$ & Position & 30 \\
\hline & 10 & $4.8413 E-05$ & 2731 & -1631 & 1 & -1202 & imp: $n=1$ & $\$$ & Position & 31 \\
\hline & 10 & $4.8413 E-05$ & 2732 & -1632 & & -12 & imp: $n=1$ & $\$$ & Po & 32 \\
\hline \multicolumn{11}{|c|}{1} \\
\hline & & & & & & & & & & \\
\hline & 10 & $4.8413 E-05$ & & -1701 & 1201 & -1202 & imp: $n=1$ & $\$$ & n & $\perp$ \\
\hline & 10 & $4.8413 E-05$ & 2802 & -1702 & 1201 & -12 & imp: $n=1$ & $\$$ & ion & 2 \\
\hline & 10 & $4.8413 E-05$ & 2803 & -1703 & 1201 & -1202 & $i m p: n=1$ & $\$$ & Position & 3 \\
\hline & 10 & $4.8413 E-05$ & & -1704 & 1201 & -1202 & imp: $n=1$ & $\$$ & Position & 4 \\
\hline & 10 & $4.8413 E-05$ & 2805 & -1705 & 1201 & -1202 & imp: $n=1$ & $\$$ & Position & 5 \\
\hline & 10 & $4.8413 E-05$ & 2806 & -1706 & & -1202 & $i m p: n=1$ & $\$$ & Position & 6 \\
\hline & 10 & $4.8413 E-05$ & & -1707 & 12 & -1202 & imp: $n=1$ & $\$$ & Position & 7 \\
\hline & 10 & $4.8413 E-05$ & 2808 & -1708 & 1201 & -1202 & imp: $n=1$ & $\$$ & Position & 8 \\
\hline & 10 & $4.8413 E-05$ & 2809 & -1709 & 1201 & -1202 & imp: $n=1$ & $\$$ & Position & 9 \\
\hline & 10 & $4.8413 E-05$ & & -1710 & & -1202 & imp: $n=1$ & $\$$ & Position & 10 \\
\hline & 10 & $4.8413 E-05$ & 2811 & -1711 & 1201 & -1202 & imp: $n=1$ & $\$$ & Position & 11 \\
\hline & 10 & $4.8413 E-05$ & 2812 & -1712 & 1201 & -1202 & $i m p: n=1$ & $\$$ & Position & 12 \\
\hline & 10 & $4.8413 E-05$ & & -1713 & 1201 & -12 & imp: $n=1$ & $\$$ & Position & 13 \\
\hline & 10 & $4.8413 E-05$ & 2814 & -1714 & 1201 & -1202 & $i m p: n=1$ & $\$$ & Position & 14 \\
\hline & 10 & $4.8413 E-05$ & 2815 & -1715 & 1201 & -1202 & imp: $n=1$ & $\$$ & Position & 15 \\
\hline & 10 & $4.8413 E-05$ & & -1716 & 1201 & -12 & imp: $n=1$ & $\$$ & Position & 16 \\
\hline & 10 & $4.8413 E-05$ & 2817 & -1717 & 1201 & -1202 & imp: $n=1$ & $\$$ & Position & 17 \\
\hline & 10 & $4.8413 E-05$ & 2818 & -1718 & 1201 & -1202 & imp: $n=1$ & $\$$ & Position & 18 \\
\hline & 10 & $4.8413 E-05$ & & -1719 & 1201 & -12 & imp: $n=1$ & $\$$ & Positi & 19 \\
\hline & 10 & $4.8413 E-05$ & 2820 & -1720 & 1201 & -1202 & imp: $n=1$ & $\$$ & Position & 20 \\
\hline & 10 & $4.8413 E-05$ & 2821 & -1721 & 1201 & -1202 & $i m p: n=1$ & $\$$ & Position & 21 \\
\hline & 10 & $4.8413 E-05$ & & -1722 & 1201 & -1202 & imp: $n=1$ & $\$$ & Position & 22 \\
\hline & 10 & $4.8413 E-05$ & 2823 & -1723 & 1201 & -1202 & imp: $n=1$ & $\$$ & Positi & 23 \\
\hline & 10 & $4.8413 E-05$ & 2824 & -1724 & 1201 & -1202 & $i m p: n=1$ & $\$$ & Position & 24 \\
\hline & 10 & $4.8413 E-05$ & & -1725 & 1201 & -1202 & imp: $n=1$ & $\$$ & Position & 25 \\
\hline
\end{tabular}

Revision: 1 
NEA/NSC/DOC(2006)1

Gas Cooled (Thermal) Reactor - GCR

\section{PROTEUS-GCR-EXP-004} CRIT-REAC

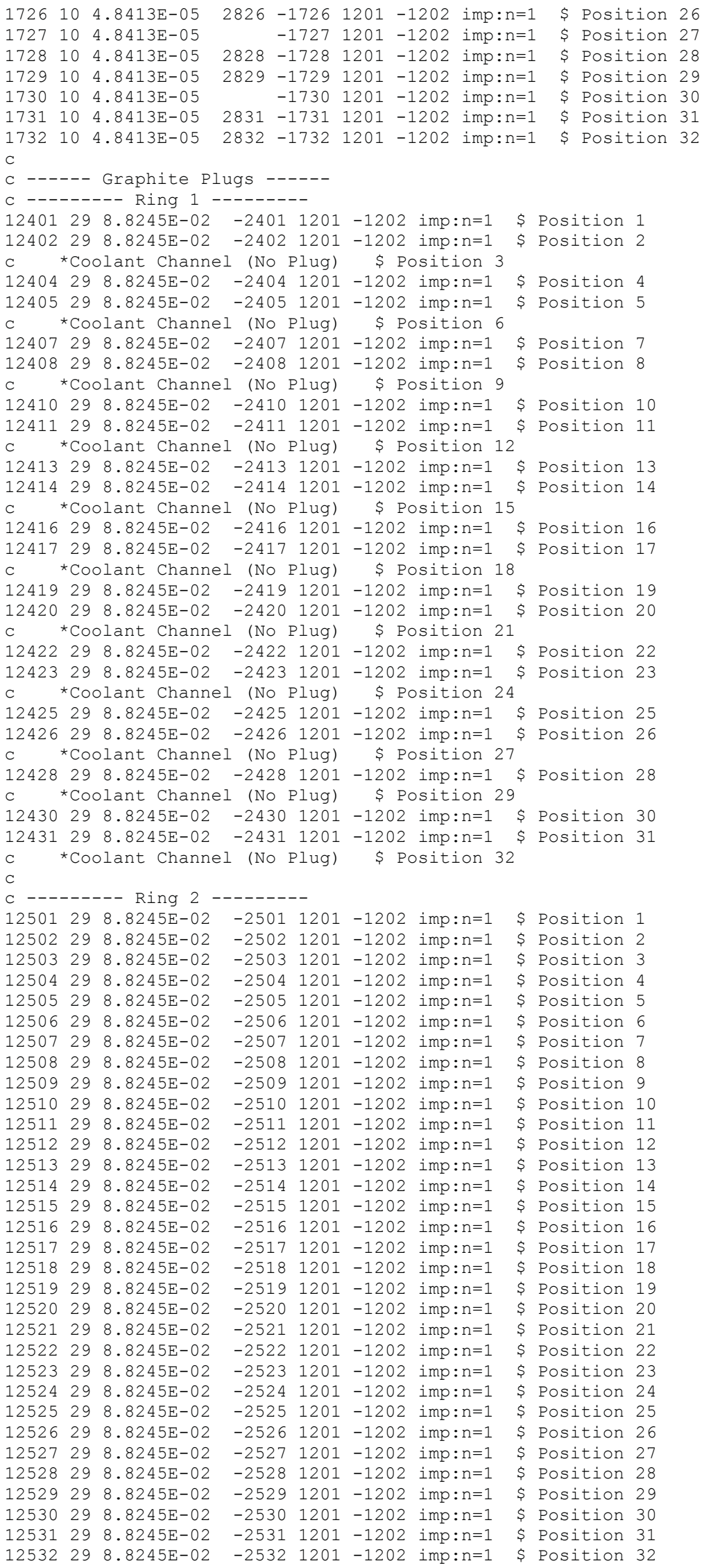

Revision: 1 
NEA/NSC/DOC(2006)1

Gas Cooled (Thermal) Reactor - GCR

PROTEUS-GCR-EXP-004 CRIT-REAC

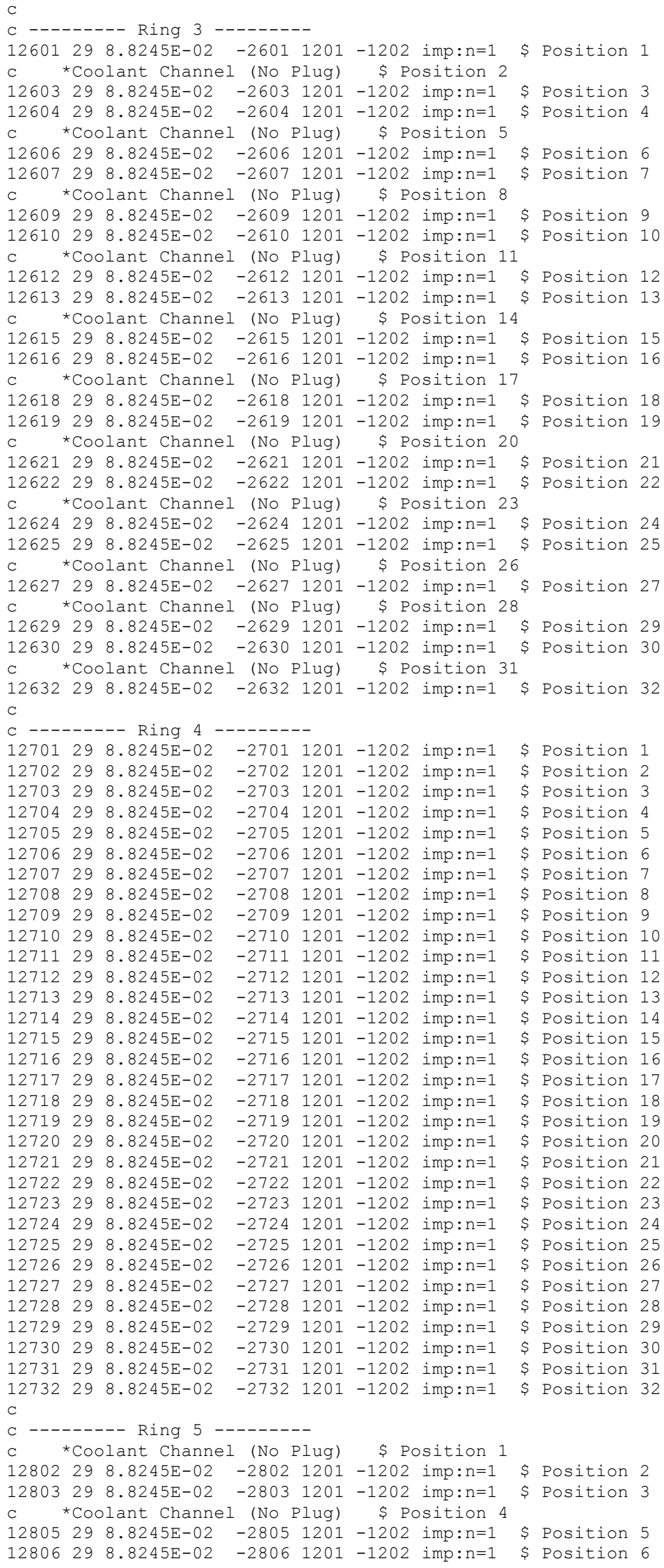

Revision: 1 
NEA/NSC/DOC(2006)1

\section{Gas Cooled (Thermal) Reactor - GCR \\ PROTEUS-GCR-EXP-004 CRIT-REAC}

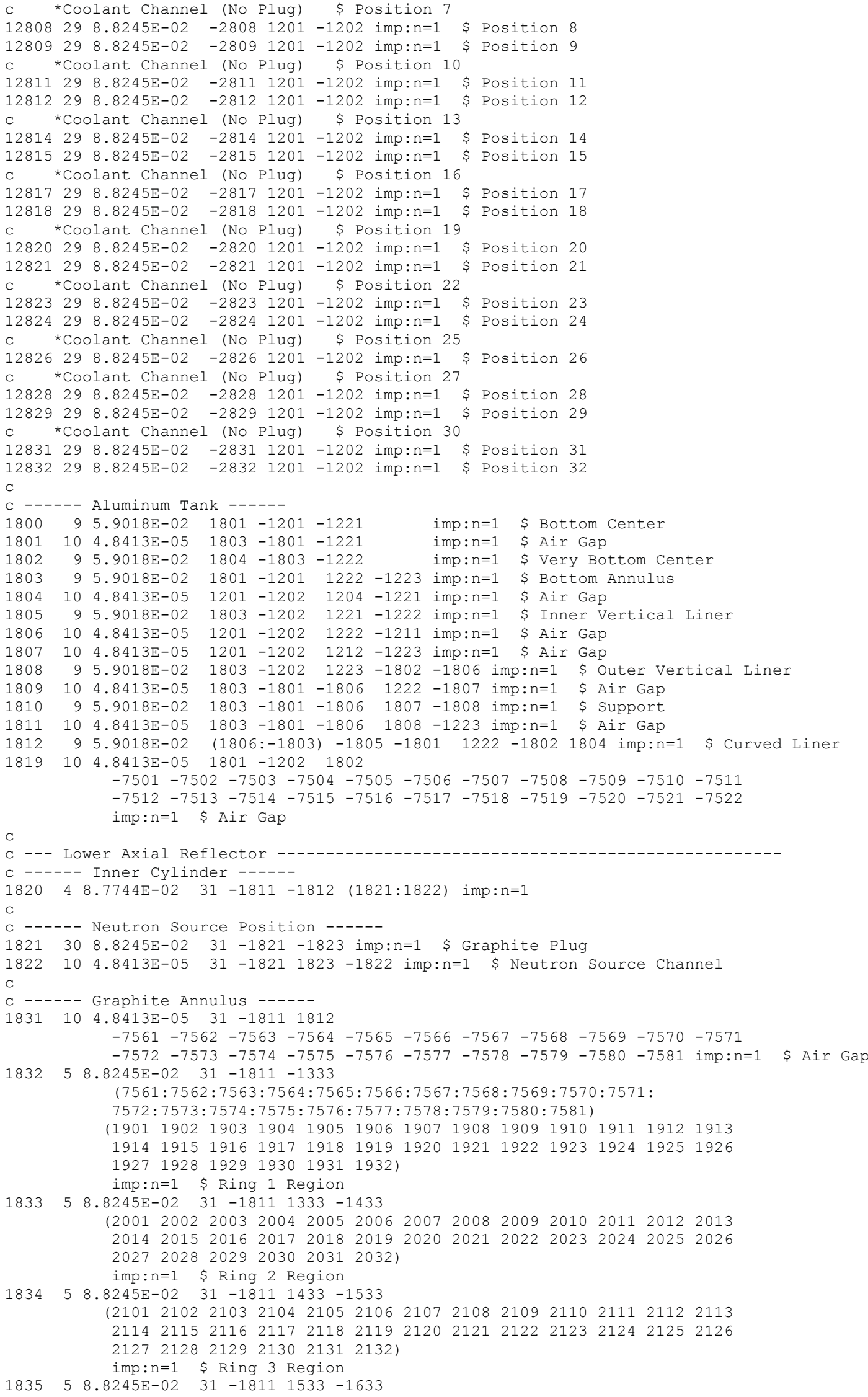

Revision: 1 
NEA/NSC/DOC(2006)1

\section{Gas Cooled (Thermal) Reactor - GCR \\ PROTEUS-GCR-EXP-004 CRIT-REAC}

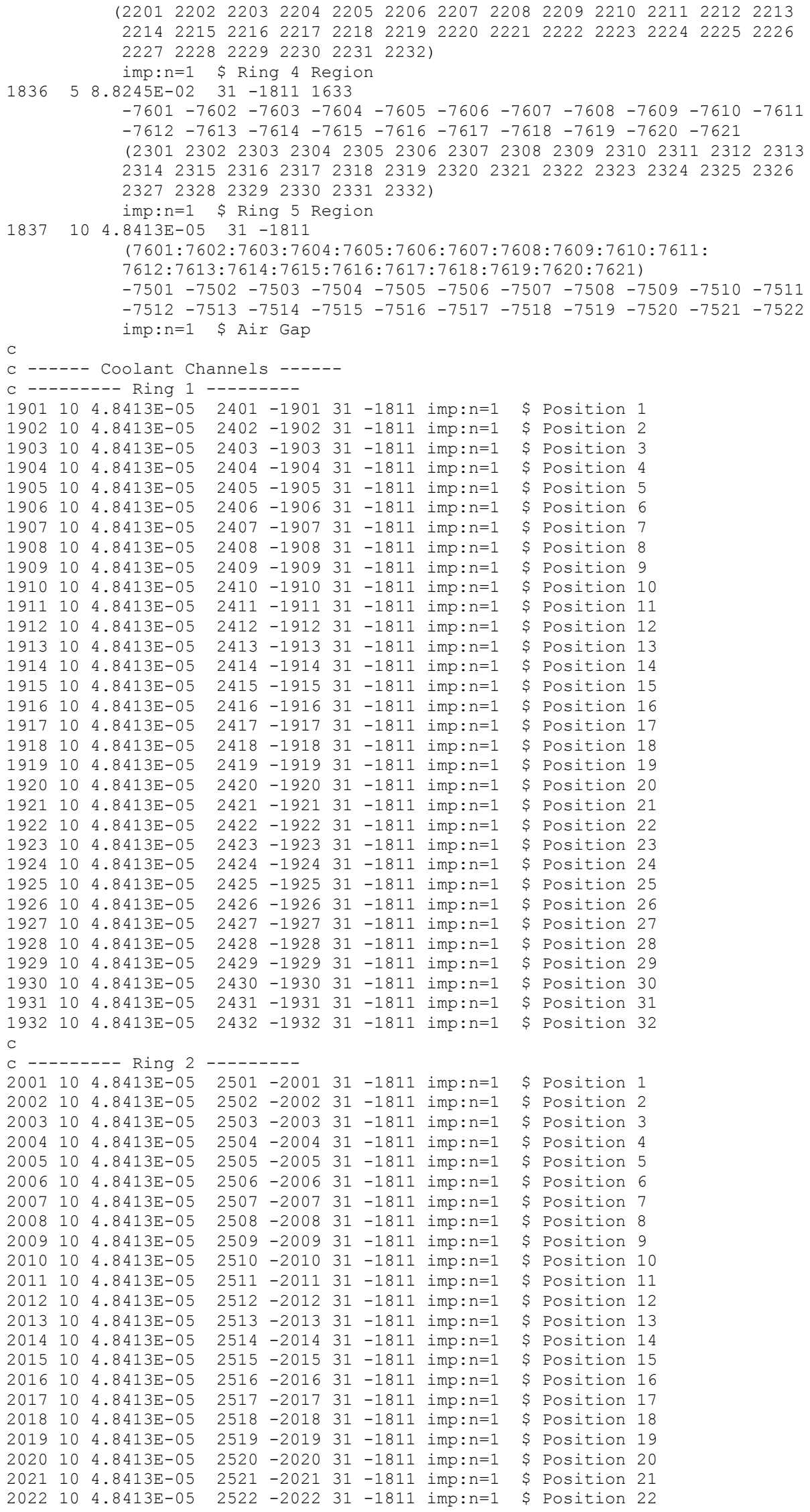

Revision: 1 
NEA/NSC/DOC(2006)1

\section{Gas Cooled (Thermal) Reactor - GCR}

\section{PROTEUS-GCR-EXP-004} CRIT-REAC

\begin{tabular}{|c|c|c|c|c|c|c|c|c|c|c|}
\hline 23 & 10 & U & 23 & -2023 & 1 & 1811 & $i m p: n=1$ & & & \\
\hline & 10 & $413 E-05$ & 2524 & -2024 & 31 & -1811 & imp:n=1 & $T_{T}$ & sition & 24 \\
\hline 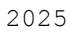 & 10 & $4.8413 E-05$ & 2525 & -2025 & 31 & -1811 & imp:n=1 & $\$$ & Position & 25 \\
\hline & 10 & $4.8413 E-05$ & 2526 & -2026 & 31 & -1811 & imp: $n=1$ & $\$$ & Position & \\
\hline & 0 & $4.8413 E-05$ & 2527 & -2027 & 31 & -1811 & $i m p: n=1$ & $\$$ & osition & \\
\hline & 0 & $4.8413 E-05$ & 528 & -2028 & 31 & -1811 & imp:n=1 & $\$$ & osition & \\
\hline 2 & 10 & $4.8413 E-05$ & 529 & -2029 & 31 & -1811 & $i m p: n=1$ & $\$$ & Position & \\
\hline & 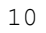 & $4.8413 E-05$ & 30 & -2030 & 31 & -1811 & imp: $n=1$ & $\$$ & ositio & \\
\hline & 0 & $4.8413 \mathrm{E}-05$ & 31 & -2031 & 31 & -1811 & imp:n=1 & $\$$ & 年 & \\
\hline & 10 & $4.8413 \mathrm{E}-05$ & 32 & -2032 & 81 & -1811 & imp: $n=1$ & $\$$ & it. & \\
\hline \\
\hline \\
\hline & & 4.84 & & -2101 & 1 & -1811 & imp:n=1 & $\$$ & osition & 1 \\
\hline & & $4.8413 E-05$ & 02 & -2102 & 31 & -1811 & $i m p: n=1$ & $\$$ & osition & \\
\hline & 0 & $4.8413 E-05$ & 3 & -2103 & 31 & -1811 & imp: $n=1$ & $\$$ & Position & \\
\hline & 10 & $4.8413 E-05$ & & -2104 & 31 & -1811 & imp: $n=1$ & $\$$ & Position & \\
\hline & 10 & $4.8413 E-05$ & & -2105 & 1 & -1811 & $i m p: n=1$ & $\$$ & osition & \\
\hline & 0 & $4.8413 \mathrm{E}-05$ & 506 & -2106 & 31 & -1811 & imp:n=1 & $\$$ & Position & \\
\hline & 0 & $4.8413 E-05$ & 07 & -2107 & 31 & -1811 & $i m p: n=1$ & $\$$ & Position & \\
\hline & 10 & $4.8413 E-05$ & 8 & -2108 & 1 & -1811 & imp:n=1 & $\$$ & ion & \\
\hline & 10 & $4.8413 \mathrm{E}-$ & 9 & -2109 & 31 & -1811 & imp:n=1 & $\$$ & tion & \\
\hline & 10 & $4.8413 E-05$ & 510 & -2110 & 31 & -1811 & imp:n=1 & $\$$ & Position & 10 \\
\hline & 10 & $4.8413 E-05$ & $\perp \perp$ & -2111 & 31 & -1811 & imp: $n=1$ & $\$$ & Position & \\
\hline & 10 & $4.8413 E-05$ & 12 & -2112 & 1 & -1811 & imp:n=1 & $\$$ & osition & \\
\hline & 10 & $4.8413 E-05$ & & -2113 & 31 & -1811 & $i m p: n=1$ & $\$$ & Position & \\
\hline & 10 & $4.8413 E-05$ & & -2114 & 31 & -1811 & imp:n=1 & $\$$ & Position & \\
\hline & 10 & $4.8413 E-05$ & 5 & -2115 & 31 & -1811 & imp:n=1 & $\$$ & tion & \\
\hline & 10 & $4.8413 E-05$ & & -2116 & 1 & -1811 & imp:n=1 & $\$$ & tion & \\
\hline & 10 & $4.8413 E-05$ & & -2117 & 31 & -1811 & imp:n=1 & $\$$ & Position & \\
\hline & 10 & $4.8413 E-05$ & 18 & -2118 & 31 & -1811 & imp: $n=1$ & $\$$ & Po: & \\
\hline & 10 & $4.8413 E-05$ & 2619 & -21 & 31 & -18 & imp: $n=1$ & $\$$ & Pos & \\
\hline & 10 & $4.8413 E-05$ & 2620 & -2120 & 31 & -1811 & imp:n=1 & $\$$ & Position & \\
\hline & 10 & $4.8413 E-05$ & 21 & -2121 & 31 & -1811 & imp:n=1 & $\$$ & Position & \\
\hline & 10 & $4.8413 E-05$ & 22 & -2122 & 31 & -1811 & $i m p: n=1$ & $\$$ & Eion & \\
\hline & 10 & $4.8413 E-05$ & 23 & -2123 & 31 & -1 & imp:n=1 & $\$$ & Pos & \\
\hline & 10 & $4.8413 E-05$ & 2624 & -2124 & 31 & -18 & imp: $n=1$ & $\$$ & Position & \\
\hline & 10 & $4.8413 E-05$ & 25 & -2125 & 31 & -1811 & $i m p: n=1$ & $\$$ & Pos & \\
\hline & 10 & $4.8413 E-05$ & 2626 & -21 & 31 & $-1 \varepsilon$ & $i m p: n=1$ & $\$$ & Pos & \\
\hline & 10 & $4.8413 E-05$ & 2627 & -2127 & 31 & -18 & $i m p: n=1$ & $\$$ & Pos & \\
\hline & 10 & $4.8413 E-05$ & 28 & -2128 & 31 & -18 & imp:n=1 & $\$$ & Position & \\
\hline & 10 & $4.8413 E-05$ & 29 & -2129 & 31 & -18 & $i m p: n=1$ & $\$$ & Pos & \\
\hline & 10 & $3 E-05$ & 26 & -21 & 31 & -1 & imp:n=1 & $\$$ & Pos & \\
\hline & 10 & $13 E-05$ & 2631 & -21 & 31 & -18 & $i m p: n=1$ & $\$$ & ion & \\
\hline & 10 & $4.8413 E-05$ & & -2132 & 31 & -18 & imp: $n=1$ & $\$$ & Pos & \\
\hline \\
\hline & & -- & & - & & & & & & \\
\hline & 10 & $4.8413 E-05$ & & -2201 & 31 & -1811 & imp: $n=1$ & $\$$ & Pos & 1 \\
\hline & 10 & $4.8413 E-05$ & 02 & -2202 & 31 & -1811 & imp: $: n=1$ & $\$$ & tion & \\
\hline & 10 & $4.8413 E-05$ & 2703 & -2203 & 31 & -18 & imp: $n=1$ & $\$$ & Pos & \\
\hline & 10 & $4.8413 E-05$ & 2704 & -2204 & 31 & -1811 & imp:n=1 & $\$$ & ion & \\
\hline & 10 & $4.8413 E-05$ & 27 & -2205 & 31 & -1811 & imp:n=1 & $\$$ & Position & \\
\hline & 10 & $4.8413 E-05$ & 2706 & -2206 & 31 & -1811 & imp:n=1 & $\$$ & Eion & \\
\hline & 10 & $4.8413 E-05$ & 2707 & -2207 & 31 & -18 & imp:n=1 & $\$$ & Pos & f \\
\hline & 10 & $4.8413 E-05$ & 2708 & -2208 & 31 & -18 & imp: $n=1$ & $\$$ & Position & 8 \\
\hline & 10 & $4.8413 E-05$ & 2709 & -2209 & 31 & -1811 & imp:n=1 & $\$$ & Position & 9 \\
\hline & 10 & $4.8413 E-05$ & 2710 & -2210 & 31 & -1811 & $i m p: n=1$ & $\$$ & tion & 10 \\
\hline & 10 & $4.8413 E-05$ & & -2211 & 31 & -18 & imp: $n=1$ & $\$$ & Pos & 1 \\
\hline & 10 & $4.8413 E-05$ & 2712 & -2212 & 31 & -1811 & $i m p: n=1$ & $\$$ & Position & 2 \\
\hline & 10 & $4.8413 E-05$ & 2713 & -2213 & 31 & -1811 & imp:n=1 & $\$$ & Pos & 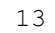 \\
\hline & 10 & $4.8413 E-05$ & 2714 & -2214 & 31 & -18 & imp:n=1 & $\$$ & Pos & 4 \\
\hline & 10 & $4.8413 E-05$ & & -2215 & 31 & -18 & $i m p: n=1$ & $\$$ & Pos & 15 \\
\hline & 10 & $4.8413 E-05$ & 2716 & -2216 & 31 & -1811 & imp:n=1 & $\$$ & Position & 6 \\
\hline & 10 & $4.8413 E-05$ & 27 & -2217 & 31 & -18 & $i m p: n=1$ & $\$$ & ion & 7 \\
\hline & 10 & $4.8413 E-05$ & 27 & -2218 & 31 & -1 & imp: $n=1$ & $\$$ & Position & 年 \\
\hline & 10 & $4.8413 E-05$ & & -2219 & 31 & -1811 & $i m p: n=1$ & $\$$ & Position & 19 \\
\hline & 10 & $4.8413 E-05$ & 2720 & -2220 & 31 & -1811 & imp: $n=1$ & $\$$ & Position & 20 \\
\hline & 10 & $4.8413 E-05$ & 272 & -2221 & 31 & -1 & imp: $n=1$ & $\$$ & Pos & 21 \\
\hline & 10 & $4.8413 E-05$ & 2722 & -2222 & 31 & -18 & $i m p: n=1$ & $\$$ & Position & 22 \\
\hline & 10 & $4.8413 E-05$ & 2723 & -2223 & 31 & -1811 & imp:n=1 & $\$$ & Position & 23 \\
\hline & 10 & $4.8413 E-05$ & 2724 & -2224 & 31 & -1811 & imp:n=1 & $\$$ & Positi & 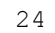 \\
\hline & 10 & $4.8413 E-05$ & 2725 & -2225 & 31 & $-1 \varepsilon$ & imp:n=1 & $\$$ & Position & 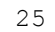 \\
\hline & 10 & $4.8413 E-05$ & 2726 & -2226 & 31 & -1811 & $i m p: n=1$ & $\$$ & Position & 26 \\
\hline & 10 & $4.8413 E-05$ & 2727 & -2227 & 31 & -1811 & imp: $n=1$ & $\$$ & Positio & 27 \\
\hline & 10 & $4.8413 E-05$ & 2728 & -2228 & 31 & -1811 & $i m p: n=1$ & $\$$ & Positic & . \\
\hline & 10 & $4.8413 E-05$ & 2729 & -2229 & 31 & -1811 & $i m p: n=1$ & $\$$ & Positic & 29 \\
\hline & 10 & $4.8413 E-05$ & 2730 & -2230 & 31 & -1811 & $i m p: n=1$ & $\$$ & Position & 30 \\
\hline
\end{tabular}

Revision: 1 
NEA/NSC/DOC(2006)1

\section{Gas Cooled (Thermal) Reactor - GCR}

\section{PROTEUS-GCR-EXP-004} CRIT-REAC

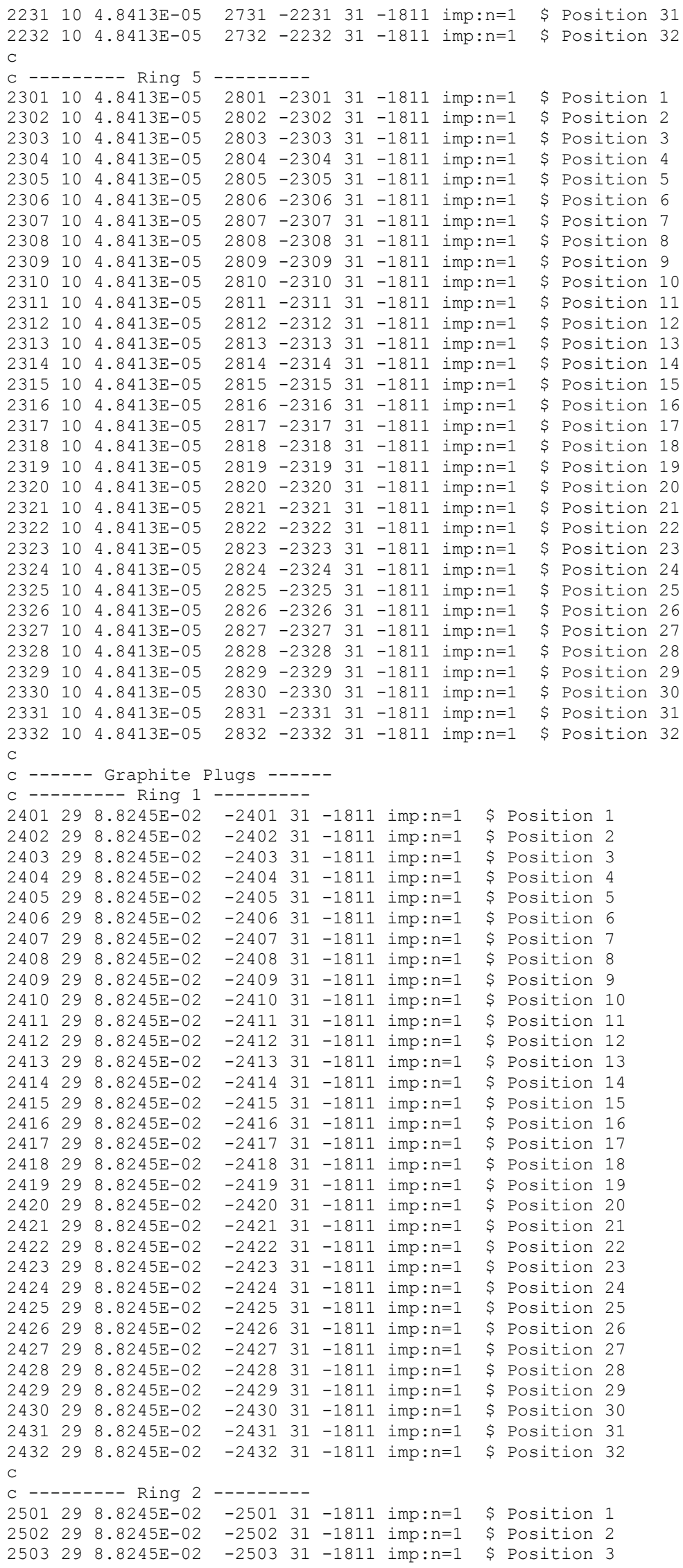

Revision: 1 
NEA/NSC/DOC(2006)1

Gas Cooled (Thermal) Reactor - GCR

PROTEUS-GCR-EXP-004

CRIT-REAC

\begin{tabular}{|c|c|c|c|c|c|c|c|c|c|}
\hline 504 & 9 & & & & & $m p: n=1$ & & & \\
\hline & 9 & & & 31 & -1811 & imp: $n=1$ & & & \\
\hline & 9 & $5 E-02$ & -2506 & 31 & -1811 & imp: $n=1$ & $T$ & ion & \\
\hline & 9 & $8245 E-02$ & -2507 & 31 & -1811 & imp: $n=1$ & & & \\
\hline & 9 & $.8245 E-02$ & -2508 & 31 & -1811 & imp: $n=1$ & & & \\
\hline & & $5 E-02$ & -2509 & & -1811 & imp: $n=1$ & & & \\
\hline & & $8245 E-02$ & & 1 & -18 & imp:n=1 & \$ & & \\
\hline & & $245 E-02$ & -2511 & 1 & -1811 & imp: $n=1$ & & & \\
\hline & & $.8245 \mathrm{E}-02$ & -2512 & & -1811 & imp: $n=1$ & & & \\
\hline & & 82015 & 25 & & -18 & imp: $n=1$ & & & \\
\hline & & .824 & & 1 & -1811 & imp: $n=1$ & & & \\
\hline & & $8.8245 E-02$ & 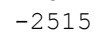 & & -1811 & imp: $n=1$ & $?$ & & \\
\hline & & 8.8 & & & & imp: $n=1$ & & & \\
\hline & & & & & & imp: $n=1$ & T & & \\
\hline & & 8.8 & & 1 & -18 & imp: $n=1$ & $\$$ & & \\
\hline & & $8.8245 E-02$ & & & -18 & imp: $n=1$ & $\$$ & & \\
\hline & & & & & & imp: $n=1$ & T & & \\
\hline & & 8.8 & , & 31 & -1 & imp: $n=1$ & $\$$ & & \\
\hline & & 8.8 & & & $-1 \varepsilon$ & imp: $n=1$ & . & & \\
\hline & & 8.8 & & & 1 & imp: $n$ & & & \\
\hline & & & & & & imp: $n$ & $\$$ & & \\
\hline & & 8.8 & & 31 & -1 & $i m p: n=1$ & $\$$ & & \\
\hline & & 8.8 & & & -18 & imp: $n$ & $\$$ & $\mathrm{n}$ & \\
\hline & & 8.8 & & & & imp: $n$ & & & \\
\hline & & 8.8 & & 1 & -1 & imp: $n=1$ & $\$$ & & \\
\hline & & 8.8 & & 1 & -18 & imp: $n=1$ & $\$$ & & \\
\hline & & 8.8 & & & -18 & imp: 1 & $\$$ & & \\
\hline & & 8. & & & & imp: $n=1$ & $\$$ & $n$ & \\
\hline & & 8.8 & & 31 & -18 r & imp: $n=1$ & $\$$ & & \\
\hline & & & & & & & & & \\
\hline & & --- & & & & & & & \\
\hline & & & & 1 & 1 & imp: $n=1$ & $\$$ & Po & \\
\hline & & 8.8 & & 31 & 1 & imp: $n=1$ & $\$$ & $\mathrm{Po}$ & \\
\hline & 9 & 8.8 & & 31 & -18 & imp: $n=1$ & $\$$ & $\mathrm{Po}$ & \\
\hline & 20 & 8.8 & & & -1 & imp: $n=1$ & $T$ & $\mathrm{Po}$ & \\
\hline & a & 8.8 & & 31 & -1 & imp: $n=1$ & $\$$ & Po & \\
\hline & a & 8.8 & & 31 & -18 & imp: $n=1$ & $\$$ & $\mathrm{Po}$ & \\
\hline & 29 & 8.8 & & & 1 & imp: & $\$$ & $\mathrm{Po}$ & \\
\hline & 29 & 8.8 & & & -1 & imp: & $\$$ & & \\
\hline & 29 & 8.8 & & 31 & -1 & imp: $n=1$ & $\$$ & $\mathrm{Po}$ & \\
\hline & 29 & 8.8 & & & -18 & imp: $n=1$ & $\$$ & & \\
\hline & 9 & 8.8 & & & 1 & imp: $n=1$ & 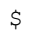 & $\mathrm{Po}$ & \\
\hline & 9 & 8. & & & -1 & imp: $n=1$ & $\$$ & & \\
\hline & 29 & 8.8 & & 31 & -18 & imp: $n=1$ & $\$$ & & \\
\hline & 29 & 8.8 & -2 & & -18 & imp: $n=1$ & $\$$ & & \\
\hline & 29 & 8.8 & & & -1 & imp: $n=1$ & $\$$ & & \\
\hline & a & 8.8 & & 31 & -1 & imp: $n=1$ & $\$$ & & \\
\hline & 20 & 8.8 & -2 & 31 & -18 & imp: $n=1$ & $\$$ & & \\
\hline & 29 & 8.8 & -2 & & -18 & imp: & $\$$ & & \\
\hline & 9 & 8.8 & & & -1 & imp: $n=1$ & $\$$ & & \\
\hline & م & 8.8 & & 31 & -18 & imp: $n=1$ & $\$$ & $\mathrm{Po}$ & \\
\hline & 29 & $8.8245 E-02$ & -2 & & -1811 & imp: $n=1$ & $\$$ & $\mathrm{Po}$ & \\
\hline & 29 & 8.8 & & & -1 & imp: $n=1$ & $\$$ & $\mathrm{Po}$ & \\
\hline & 20 & 8.8 & & & -1 & imp: $n=1$ & $\$$ & & \\
\hline & 29 & 8.8 & -2 & 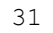 & -18 & imp: $n=1$ & $\$$ & & \\
\hline & 29 & $8.8245 E-02$ & -2625 & & -1811 & imp: $n=1$ & $\$$ & $\mathrm{Po}$ & \\
\hline & 29 & 8.8245 & & & -18 & imp: $n=1$ & $\$$ & & \\
\hline & 29 & $8.8245 E-02$ & & 31 & -18 & imp: $n=1$ & $\$$ & & \\
\hline & 29 & 8.8245 & -2 & & -1811 & imp: $n=1$ & $\$$ & & \\
\hline & 29 & 8.8 & -2 & & -18 & imp: $n=1$ & $\$$ & & \\
\hline & 29 & 8.8 & & & -18 & imp: $n=1$ & $\$$ & & \\
\hline & 29 & $8.8245 \mathrm{f}$ & -2 & 3 & -1811 & imp: $n=1$ & $\$$ & $\mathrm{Po}$ & \\
\hline & 29 & $8.8245 E-02$ & -2 & 3 & -1811 & imp: $n=1$ & $\$$ & Pos & \\
\hline & & & & & & & & & \\
\hline & & & & & & & & & \\
\hline & & 8.8 & & & & & 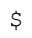 & & \\
\hline & 29 & 8.824 & & & -1 & imp: $n=1$ & $\$$ & & \\
\hline & 29 & 8.8245 & & & -1811 & imp: $n=1$ & $\$$ & Pos & \\
\hline & 29 & $8.8245 E-02$ & & 3 & -1811 & imp: $n=1$ & $\$$ & Positi & \\
\hline & 29 & $8.8245 E-02$ & & 3 & -1811 & imp: $n=1$ & $\$$ & & \\
\hline & 29 & $8.8245 E-02$ & & & -1811 & imp: $n=1$ & $\$$ & Pos & \\
\hline & 29 & $8.8245 E-02$ & & 31 & -1811 & imp: $n=1$ & $\$$ & Posi & \\
\hline & 29 & $8.8245 E-02$ & & 31 & -1811 & imp: $n=1$ & $\$$ & Position & \\
\hline & 29 & $8.8245 E-02$ & & . & -1811 & imp: $n=1$ & 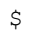 & Posi & \\
\hline & 29 & $8.8245 \mathrm{E}-$ & & & -1811 & imp: $n=1$ & S & & \\
\hline & & $8.8245 E-02$ & & 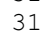 & -1811 & imp: $n=1$ & $\$$ & Pos & \\
\hline
\end{tabular}

Revision: 1 
NEA/NSC/DOC(2006)1

\section{Gas Cooled (Thermal) Reactor - GCR}

\section{PROTEUS-GCR-EXP-004 CRIT-REAC}

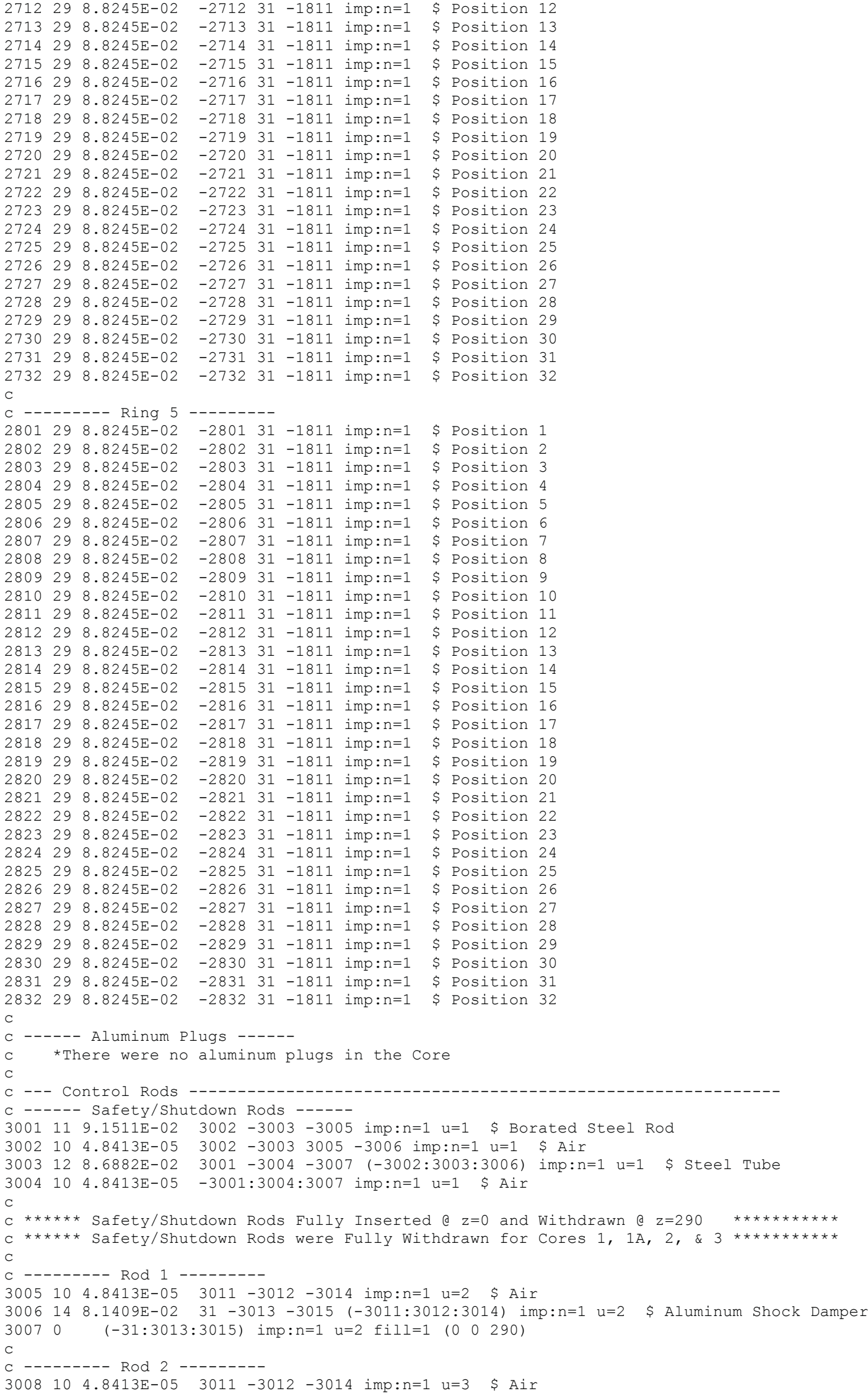

Revision: 1 
NEA/NSC/DOC(2006)1

\section{Gas Cooled (Thermal) Reactor - GCR \\ PROTEUS-GCR-EXP-004 CRIT-REAC}

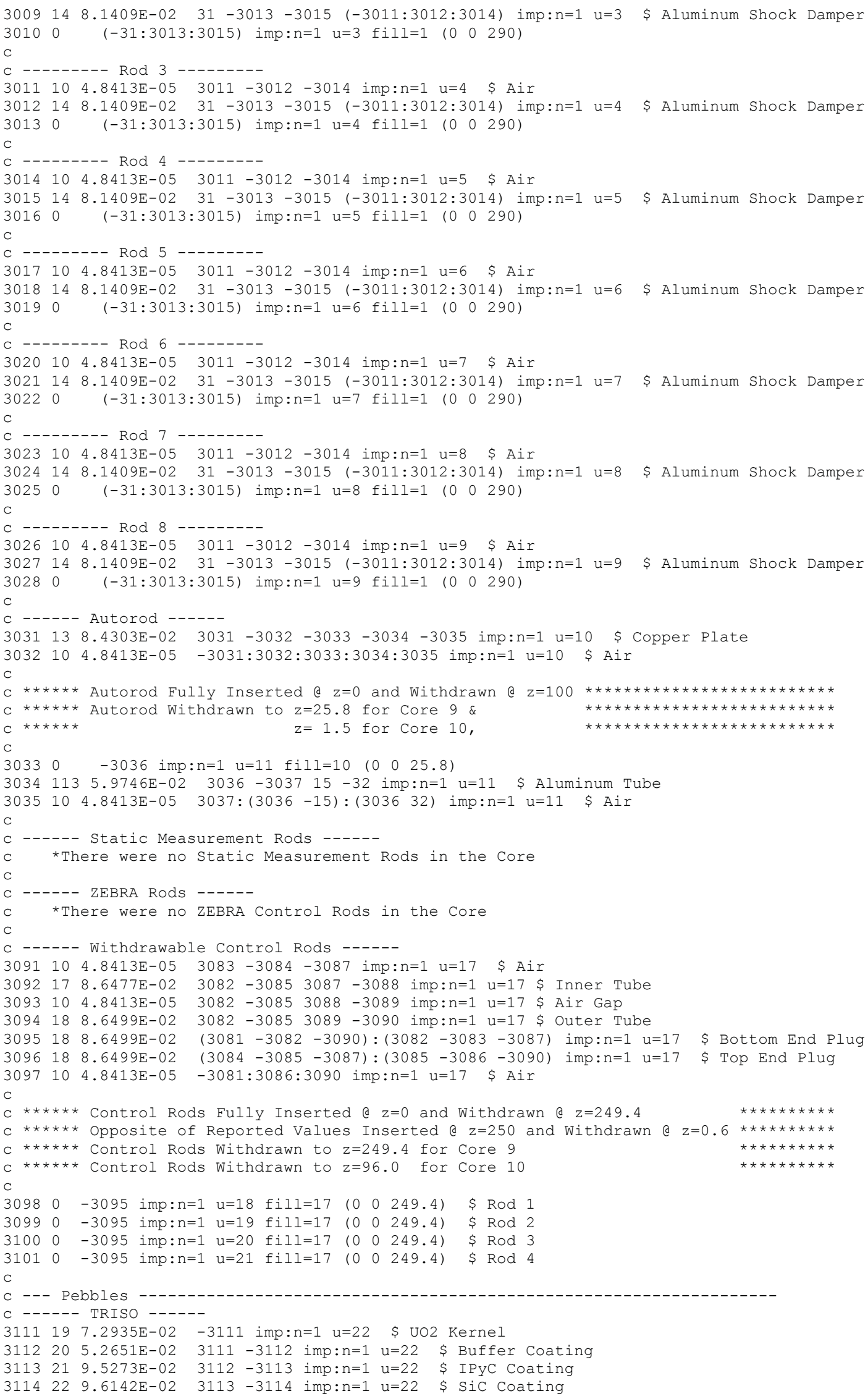

Revision: 1 
NEA/NSC/DOC(2006)1

\section{Gas Cooled (Thermal) Reactor - GCR}

\section{PROTEUS-GCR-EXP-004 CRIT-REAC}

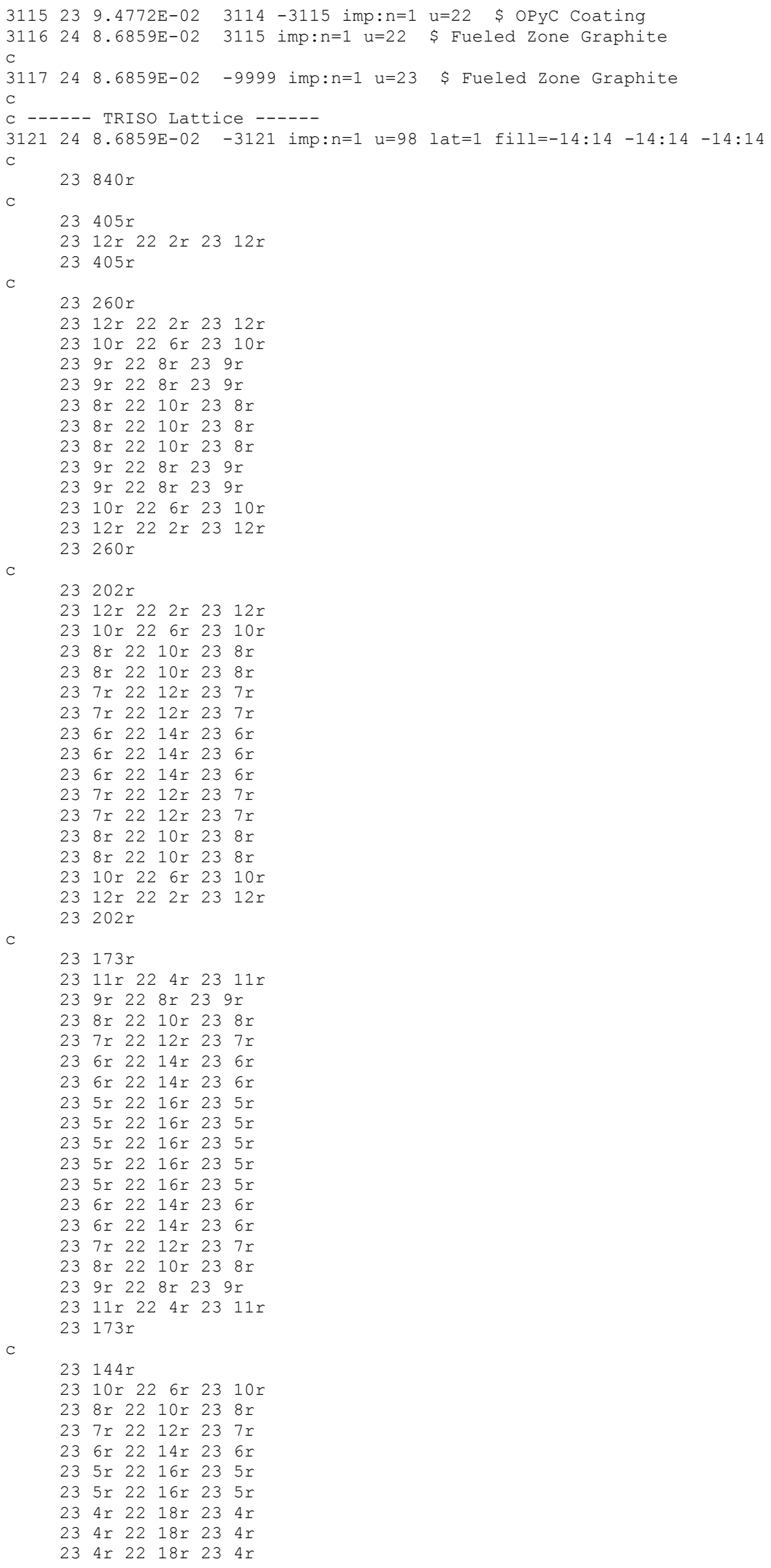

Revision: 1 
NEA/NSC/DOC(2006)1

Gas Cooled (Thermal) Reactor - GCR

PROTEUS-GCR-EXP-004

CRIT-REAC

$234 r 2218 r 234 r$

$234 r 22$ 18r 23 4r

$234 r 2218 r 234 r$

$234 r 22$ 18r 23 4r

$235 r 2216 r 235 r$

$235 r 2216 r 235 r$

$236 r 2214 r 236 r$

$237 r 22$ 12r $237 r$

$238 r 2210 r 238 r$

23 10r 22 6r $2310 r$

$23144 r$

C

$23115 r$

$2311 r 22$ 4r $2311 r$

$238 r 22$ 10r 23 8r

$237 r 2212 r 237 r$

$236 r 2214 r 236 r$

$235 r 2216 r 235 r$

$234 r 2218 r 234 r$

$234 r 22$ 18r 23 4r

$234 r 22$ 18r 23 4r

$233 r 22$ 20r $233 r$

$233 r 22$ 20r $233 r$

$233 r 22$ 20r $233 r$

$233 r 22$ 20r $233 r$

$233 r 22$ 20r $233 r$

$234 r 22$ 18r $234 r$

$234 r 22$ 18r 23 4r

$234 r 22$ 18r 23 4r

$235 r 2216 r 235 r$

$236 r 2214 r 236 r$

$237 r 2212 r 237 r$

$238 r 2210 r 238 r$

23 11r 22 4r $2311 r$

$23115 r$

C

$2386 r$

2312 r 22 2r 2312 r

23 9r 22 8r 23 9r

$237 r 2212 r 237 r$

$236 r 2214 r 236 r$

$235 r 2216 r 235 r$

$234 r 2218 r 234 r$

$234 r 2218 r 234 r$

$233 r 22$ 20r 23 3r

$233 r 22$ 20r 23 3r

$233 r 22$ 20r $233 r$

$232 r 22$ 22r $232 r$

$232 r 22$ 22r $232 r$

$232 r 22$ 22r $232 r$

$233 r 22$ 20r 23 3r

$233 r 22$ 20r $233 r$

$233 r 22$ 20r 23 3r

$234 r 22$ 18r 23 4r

$234 r 2218 r 234 r$

$235 r 2216 r 235 r$

$236 r 2214 r 236 r$

$237 r 2212 r 237 r$

23 9r 22 8r 23 9r

2312 r 22 2r $2312 r$

$2386 r$

C

$\begin{array}{llllll}23 & 86 r & & \\ 23 & 10 r & 22 & 6 r & 23 & 10 r \\ 23 & 8 r & 22 & 10 r & 23 & 8 r \\ 23 & 6 r & 22 & 14 r & 23 & 6 r \\ 23 & 5 r & 22 & 16 r & 23 & 5 r \\ 23 & 4 r & 22 & 18 r & 23 & 4 r \\ 23 & 4 r & 22 & 18 r & 23 & 4 r \\ 23 & 3 r & 22 & 20 r & 23 & 3 r \\ 23 & 3 r & 22 & 20 r & 23 & 3 r \\ 23 & 2 r & 22 & 22 r & 23 & 2 r \\ 23 & 2 r & 22 & 22 r & 23 & 2 r \\ 23 & 2 r & 22 & 22 r & 23 & 2 r \\ 23 & 2 r & 22 & 22 r & 23 & 2 r \\ 23 & 2 r & 22 & 22 r & 23 & 2 r\end{array}$

Revision: 1

Date: March 31, 2014

Page 317 of 375 
NEA/NSC/DOC(2006)1

Gas Cooled (Thermal) Reactor - GCR

\section{PROTEUS-GCR-EXP-004}

CRIT-REAC

$232 r 2222 r 232 r$

$232 r 22$ 22r $232 r$

$233 r 22$ 20r $233 r$

$233 r 22$ 20r 23 3r

$234 r 2218 r 234 r$

$234 r 22$ 18r $234 r$

$235 r 2216 r 235 r$

$236 r 22$ 14r $236 r$

$238 r 22$ 10r $238 r$

$2310 r 226 r 2310 r$

$2386 r$

$2357 r$

$2312 r 22 \quad 2 r 23 \quad 12 r$

$238 r 22$ 10r 23 8r

$237 r 22$ 12r $237 r$

$235 r 2216 r 235 r$

$234 r 22$ 18r 23 4r

$234 r 22$ 18r 23 4r

$233 r 22$ 20r $233 r$

$232 r 22$ 22r $232 r$

$232 r 22 \quad 22 r 23 \quad 2 r$

$232 r 22$ 22r $232 r$

$232 r 22$ 22r $232 r$

$231 r 22$ 24r $231 r$

$231 r 2224 r 231 r$

$231 r 22$ 24r $231 r$

$232 r 22$ 22r $232 r$

$232 r 22$ 22r 23 2r

$232 r 22$ 22r $232 r$

$232 r 22$ 22r $232 r$

$233 r 22$ 20r $233 r$

$234 r 2218 r 234 r$

$234 r 22$ 18r 23 4r

$235 r 2216 r 235 r$

$237 r 22 \quad 12 r 23 \quad 7 r$

$238 r 2210 r 238 r$

$2312 r 22 \quad 2 r 2312 r$

C

$2357 r$

$2357 r$

$2310 r 22$ 6r $2310 r$

$238 r 2210 r 238 r$

$236 r 22 \quad 14 r 23 \quad 6 r$

$235 r 2216 r 235 r$

$234 r 2218 r 234 r$

$233 r 22$ 20r $233 r$

$233 r 22$ 20r 23 3r

$232 r 22$ 22r $232 r$

$232 r 22$ 22r $232 r$

23 1r 22 24r 23 1r

$231 r 2224 r 231 r$

$231 r 22$ 24r $231 r$

23 1r 22 24r 23 1r

23 1r 22 24r 23 1r

$231 r 2224$ r $231 r$

$231 r 2224 r 231 r$

$232 r 22$ 22r $232 r$

$232 r 22$ 22r $232 r$

$233 r 22$ 20r $233 r$

$233 r 22$ 20r $233 r$

$234 r 22$ 18r 23 4r

$235 r 2216 r 235 r$

$236 r 22$ 14r $236 r$

$238 r 2210 r 238 r$

$2310 r 22$ 6r $2310 r$

$2357 r$

C

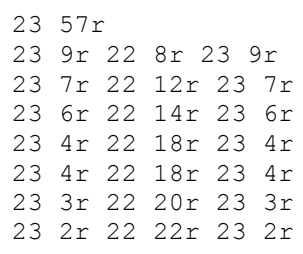

Revision: 1

Date: March 31, 2014

Page 318 of 375 
NEA/NSC/DOC(2006)1

Gas Cooled (Thermal) Reactor - GCR

\section{PROTEUS-GCR-EXP-004}

CRIT-REAC

$232 r 2222 r 232 r$

$231 r 2224 r 231 r$

$231 r 22$ 24r $231 r$

$231 r 22$ 24r $231 r$

$231 r 22$ 24r $231 r$

23 1r 22 24r 23 1r

$231 r 2224 r 231 r$

23 1r 22 24r 23 1r

$231 r 22$ 24r $231 r$

$231 r 22$ 24r 23 1r

$232 r 22$ 22r $232 r$

23 2r 22 22r 23 2r

$233 r 22$ 20r $233 r$

$234 r 2218 r 23$ 4r

$234 r 2218 r 234 r$

$236 r 2214 r 236 r$

$237 r 2212 r 237 r$

$239 r 22$ 8r $239 r$

$2357 r$

$2357 r$

23 9r 22 8r 23 9r

$237 r 2212 r 237 r$

$235 r 2216 r 235 r$

$234 r 22$ 18r $23 \quad 4 r$

$233 r 22$ 20r 23 3r

$233 r 22$ 20r $233 r$

$232 r 22 \quad 22 r 232 r$

$232 r 22 \quad 22 r 232 r$

$231 r 22 \quad 24 r 231 r$

$231 r 22 \quad 24 r 231 r$

$231 r 22 \quad 24 r 231 r$

23 1r 22 24r 23 1r

23 1r 22 24r 23 1r

23 1r 22 24r 231 r

23 1r 22 24r 23 1r

$231 r 22 \quad 24 r 231 r$

$231 r 22$ 24r $231 r$

$232 r 22 \quad 22 r 23 \quad r$

$232 r 22 \quad 22 r 23 \quad r$

$233 r 22$ 20r $233 r$

$233 r 22$ 20r 23 3r

$234 r 22$ 18r 23 4r

$235 r 2216 r 235 r$

$237 r 22$ 12r 23 7r

23 9r 22 8r 23 9r

$2357 r$

$2357 r$

$238 r 2210 r 238 r$

$236 r 2214 r 236 r$

$235 r 2216 r 23 \quad 5 r$

$234 r 22$ 18r 23 4r

$233 r 22$ 20r $233 r$

$232 r 22 \quad 22 \quad 23 \quad 2 r$

$232 r 22 \quad 22 r 232 r$

$231 r 22$ 24r $231 r$

$231 r 22 \quad 24 r 231 r$

23 1r 22 24r 23 1r

$231 r 22 \quad 24 r 231 r$

$231 r 22 \quad 24 r 231 r$

$\begin{array}{llll}23 & 22 & 26 r & 23\end{array}$

$231 r 22 \quad 24 r 231 r$

$231 r 22 \quad 24 r 23$ 1r

$231 r 22 \quad 24 r 231 r$

23 1r 22 24r 23 1r

$231 r 22 \quad 24 r 231 r$

$232 r 22 \quad 22 r 232 r$

$232 r 22 \quad 22 r 232 r$

$233 r 22$ 20r 23 3r

$234 r 22 \quad 18 r 23 \quad 4 r$

$235 r 2216 r 23 \quad 5 r$

$236 r 22$ 14r 23 6r

$238 r 2210 r 238 r$

$2357 r$

C

Revision: 1

Date: March 31, 2014

Page 319 of 375 
NEA/NSC/DOC(2006)1

Gas Cooled (Thermal) Reactor - GCR

PROTEUS-GCR-EXP-004

CRIT-REAC

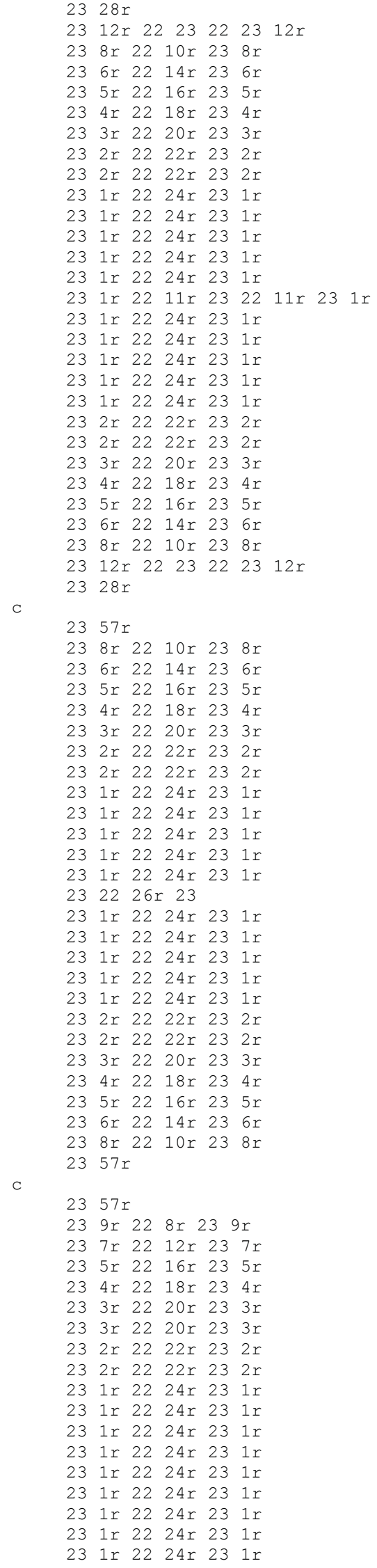

Revision: 1

Date: March 31, 2014

Page 320 of 375 
NEA/NSC/DOC(2006)1

Gas Cooled (Thermal) Reactor - GCR

\section{PROTEUS-GCR-EXP-004}

CRIT-REAC

$232 r 22 \quad 22 r 23 \quad 2 r$

$232 r 22 \quad 22 r 232 r$

$233 r 22$ 20r $233 r$

$233 r 22$ 20r 23 3r

$234 r 22$ 18r 23 4r

$235 r 2216 r 23 \quad 5 r$

$237 r 2212 r 237 r$

23 9r 22 8r 23 9r

$2357 r$

$2357 r$

23 9r 22 8r 23 9r

$\begin{array}{llllll}23 & 7 r & 22 & 12 r & 23 & 7 r\end{array}$

$236 r 22 \quad 14 r 23 \quad 6 r$

$234 r 22$ 18r 23 4r

$234 r 22$ 18r $234 r$

$233 r 22$ 20r $233 r$

$232 r 22 \quad 22 r 23 \quad 2 r$

$232 r 22 \quad 22 r 232 r$

$231 r 22 \quad 24 r 231 r$

$231 r 22 \quad 24 r 23$ 1r

$\begin{array}{llllll}23 & 1 r & 22 & 24 r & 23 & 1 r\end{array}$

$231 r 22 \quad 24 r 23$ 1r

$231 r 22$ 24r $231 r$

$231 r 22$ 24r $231 r$

$231 r 22 \quad 4 r 231 r$

$231 r 22 \quad 24 r 231 r$

$231 r 22 \quad 24 r 231 r$

$232 r 22 \quad 22 r 232 r$

$232 r 22 \quad 22 r 232 r$

$233 r 22$ 20r $233 r$

$234 r 2218 r 23 \quad 4 r$

$234 r 22$ 18r 23 4r

$236 r 22 \quad 14 r 23 \quad 6 r$

$237 r 22$ 12r 237 r

23 9r 22 8r 23 9r

$2357 r$

c

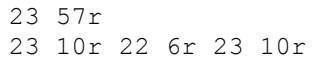

23 8r 22 10r 23 8r

$236 r 2214 r 236 r$

$235 r 2216 r 235 r$

$234 r 22$ 18r $23 \quad 4 r$

$233 r 22$ 20r 23 3r

$233 r 22$ 20r $233 r$

$232 r 22 \quad 22 r 232 r$

$232 r 22 \quad 22 r 232 r$

$231 r 22 \quad 24 r 231 r$

$231 r 22 \quad 24 r 231 r$

$231 r 22 \quad 24 r 231 r$

$\begin{array}{llllll}23 & 1 r & 22 & 24 r & 23 & 1 r\end{array}$

$231 r 22 \quad 24 r 23$ 1r

$231 r 22 \quad 24 r 231 r$

$231 r 22 \quad 24 r 231 r$

$232 r 22$ 22r 23 2r

$232 r 22$ 22r $232 r$

$233 r 22 \quad 20 r 233 r$

$233 r 22$ 20r 23 3r

$234 r 22 \quad 18 r 23 \quad 4 r$

$235 r 2216 r 23 \quad 5 r$

$236 r 22$ 14r 23 6r

$238 r 22$ 10r $238 r$

23 10r 22 6r 23 10r

C

$2357 r$

$$
\begin{array}{lllllll}
23 & 57 r & & & & \\
23 & 12 r & 22 & 2 r & 23 & 12 r \\
23 & 8 r & 22 & 10 r & 23 & 8 r \\
23 & 7 r & 22 & 12 r & 23 & 7 r \\
23 & 5 r & 22 & 16 r & 23 & 5 r \\
23 & 4 r & 22 & 18 r & 23 & 4 r \\
23 & 4 r & 22 & 18 r & 23 & 4 r \\
23 & 3 r & 22 & 20 r & 23 & 3 r \\
23 & 2 r & 22 & 22 r & 23 & 2 r \\
23 & 2 r & 22 & 22 r & 23 & 2 r
\end{array}
$$

Revision: 1

Date: March 31, 2014

Page 321 of 375 
NEA/NSC/DOC(2006)1

Gas Cooled (Thermal) Reactor - GCR

\section{PROTEUS-GCR-EXP-004}

CRIT-REAC

$232 r 22$ 22r $232 r$

$232 r 22$ 22r $232 r$

$231 r 22$ 24r $231 r$

$231 r 22$ 24r $231 r$

23 1r 22 24r 23 1r

$232 r 22$ 22r $232 r$

$232 r 22$ 22r $232 r$

$232 r 22$ 22r $232 r$

$232 r 22$ 22r $232 r$

$233 r 22$ 20r 23 3r

$234 r 2218 r 234 r$

$234 r 22$ 18r 23 4r

$235 r 22$ 16r $235 r$

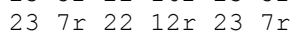

$238 r 2210 r 238 r$

2312 r 22 2r 2312 r

$2357 r$

C

$2386 r$

$2310 r 22$ 6r $2310 r$

$238 r 2210 r 238 r$

$236 r 22$ 14r $236 r$

$235 r 2216 r 235 r$

$234 r 22$ 18r $234 r$

$234 r 2218 r 234 r$

$233 r 22$ 20r 23 3r

$233 r 22$ 20r $233 r$

$232 r 22$ 22r $232 r$

$232 r 22$ 22r $232 r$

$232 r 22 \quad 22 r 232 r$

$232 r 22$ 22r $232 r$

$232 r 22$ 22r $232 r$

$232 r 22 \quad 22 r 232 r$

$232 r 22$ 22r $232 r$

$233 r 22$ 20r $233 r$

$233 r 22$ 20r $233 r$

$234 r 2218 r 234 r$

$234 r 2218 r 234 r$

$235 r 2216 r 235 r$

$236 r 22$ 14r $236 r$

$238 r 2210 r 238 r$

$2310 r 22$ 6r $2310 r$

C

$2386 r$

$2386 r$

$2312 r 22 \quad 2 r 2312 r$

$239 r 22$ 8r 23 9r

$237 r 22$ 12r $237 r$

$236 r 2214 r 236 r$

$235 r 2216 r 235 r$

$234 r 22$ 18r 23 4r

23 4r 22 18r 23 4r

$233 r 22$ 20r 23 3r

$233 r 22$ 20r $233 r$

$233 r 22$ 20r $233 r$

$232 r 22$ 22r $232 r$

$232 r 22 \quad 22 \quad 232 r$

$232 r 22$ 22r $232 r$

$233 r 22$ 20r 23 3r

$233 r 22$ 20r $233 r$

$233 r 22$ 20r 23 3r

$234 r 22$ 18r 23 4r

$234 r 22$ 18r $234 r$

$235 r 2216 r 235 r$

$236 r 2214 r 236 r$

$237 r 2212 r 237 r$

23 9r 22 8r 23 9r

$2312 r 22$ 2r $2312 r$

$2386 r$

C

$$
\begin{array}{llllll}
23 & 115 r & & & \\
23 & 11 r & 22 & 4 r & 23 & 11 r \\
23 & 8 r & 22 & 10 r & 23 & 8 r \\
23 & 7 r & 22 & 12 r & 23 & 7 r \\
23 & 6 r & 22 & 14 r & 23 & 6 r \\
23 & 5 r 22 & 16 r & 23 & 5 r
\end{array}
$$

Revision: 1

Date: March 31, 2014

Page 322 of 375 
NEA/NSC/DOC(2006)1

Gas Cooled (Thermal) Reactor - GCR

\section{PROTEUS-GCR-EXP-004}

CRIT-REAC

$234 r 2218 r 234 r$

$234 r 2218 r 234 r$

$234 r 2218 r 234 r$

$233 r 22$ 20r 23 3r

$233 r 2220 r 233 r$

$233 r 22$ 20r $233 r$

$233 r 22$ 20r $233 r$

$233 r 22$ 20r 23 3r

$234 r 22$ 18r $234 r$

$234 r 22$ 18r 23 4r

$234 r 2218 r 234 r$

$235 r 2216 r 235 r$

$236 r 2214 r 236 r$

$237 r 22$ 12r $237 r$

$238 r 22$ 10r $238 r$

$2311 r 22$ 4r $2311 r$

$23115 r$

$23144 r$

$2310 r 22$ 6r $2310 r$

$238 r 2210 r 238 r$

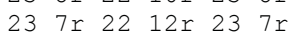

$236 r 2214 r 236 r$

$235 r 2216 r 235 r$

$235 r 2216 r 235 r$

$234 r 22$ 18r 23 4r

$234 r 22$ 18r $234 r$

$234 r 22$ 18r 23 4r

$234 r 22$ 18r $234 r$

$234 r 2218 r 234 r$

$234 r 22$ 18r 23 4r

$234 r 22$ 18r 23 4r

$235 r 2216 r 235 r$

$235 r 2216 r 235 r$

$236 r 2214 r 236 r$

$237 r 2212 r 237 r$

$238 r 2210 r 238 r$

$2310 r 226 r 2310 r$

$23144 r$

$23173 r$

2311 r 22 4r $2311 r$

$239 r 22$ 8r 23 9r

$238 r 2210 r 238 r$

$237 r 2212 r 237 r$

$236 r 2214 r 236 r$

$236 r 2214 r 236 r$

$235 r 2216 r 235 r$

$235 r 2216 r 235 r$

$235 r 2216 r 235 r$

$235 r 2216 r 235 r$

$235 r 2216 r 235 r$

$236 r 22$ 14r 23 6r

$236 r 2214 r 236 r$

$237 r 2212 r 237 r$

$238 r 22$ 10r $238 r$

$239 r 22$ 8r 23 9r

$2311 r 22$ 4r $2311 r$

$23173 r$

C

$\begin{array}{llllll}23 & 202 r & & \\ 23 & 12 r & 22 & 2 r & 23 & 12 r \\ 23 & 10 r & 22 & 6 r & 23 & 10 r \\ 23 & 8 r & 22 & 10 r & 23 & 8 r \\ 23 & 8 r & 22 & 10 r & 23 & 8 r \\ 23 & 7 r & 22 & 12 r & 23 & 7 r \\ 23 & 7 r & 22 & 12 r & 23 & 7 r \\ 23 & 6 r & 22 & 14 r & 23 & 6 r \\ 23 & 6 r & 22 & 14 r & 23 & 6 r \\ 23 & 6 r & 22 & 14 r & 23 & 6 r \\ 23 & 7 r & 22 & 12 r & 23 & 7 r \\ 23 & 7 r & 22 & 12 r & 23 & 7 r \\ 23 & 8 r & 22 & 10 r & 23 & 8 r \\ 23 & 8 r & 22 & 10 r & 23 & 8 r \\ 23 & 10 r & 22 & 6 r & 23 & 10 r \\ 23 & 12 r & 22 & 2 r & 23 & 12 r\end{array}$

Revision: 1

Date: March 31, 2014

Page 323 of 375 
NEA/NSC/DOC(2006)1

Gas Cooled (Thermal) Reactor - GCR

\section{PROTEUS-GCR-EXP-004}

CRIT-REAC

C

$23202 r$

$23260 r$

$2312 r 22 \quad 2 r 23 \quad 12 r$

$2310 r 22$ 6r $2310 r$

$239 r 22$ 8r 23 9r

23 9r 22 8r 23 9r

$238 r 2210 r 238 r$

$238 r 22$ 10r $238 r$

$238 r 22$ 10r 23 8r

$239 r 228 r 239 r$

$239 r 22$ 8r 23 9r

$2310 r 22$ 6r $2310 r$

$2312 r 22 \quad 2 r 2312 r$

$23260 r$

C

$23405 r$

2312 r 22 2r $2312 r$

$23405 r$

C

$23840 r$

C

Revision: 1 
NEA/NSC/DOC(2006)1

Gas Cooled (Thermal) Reactor - GCR

PROTEUS-GCR-EXP-004

CRIT-REAC

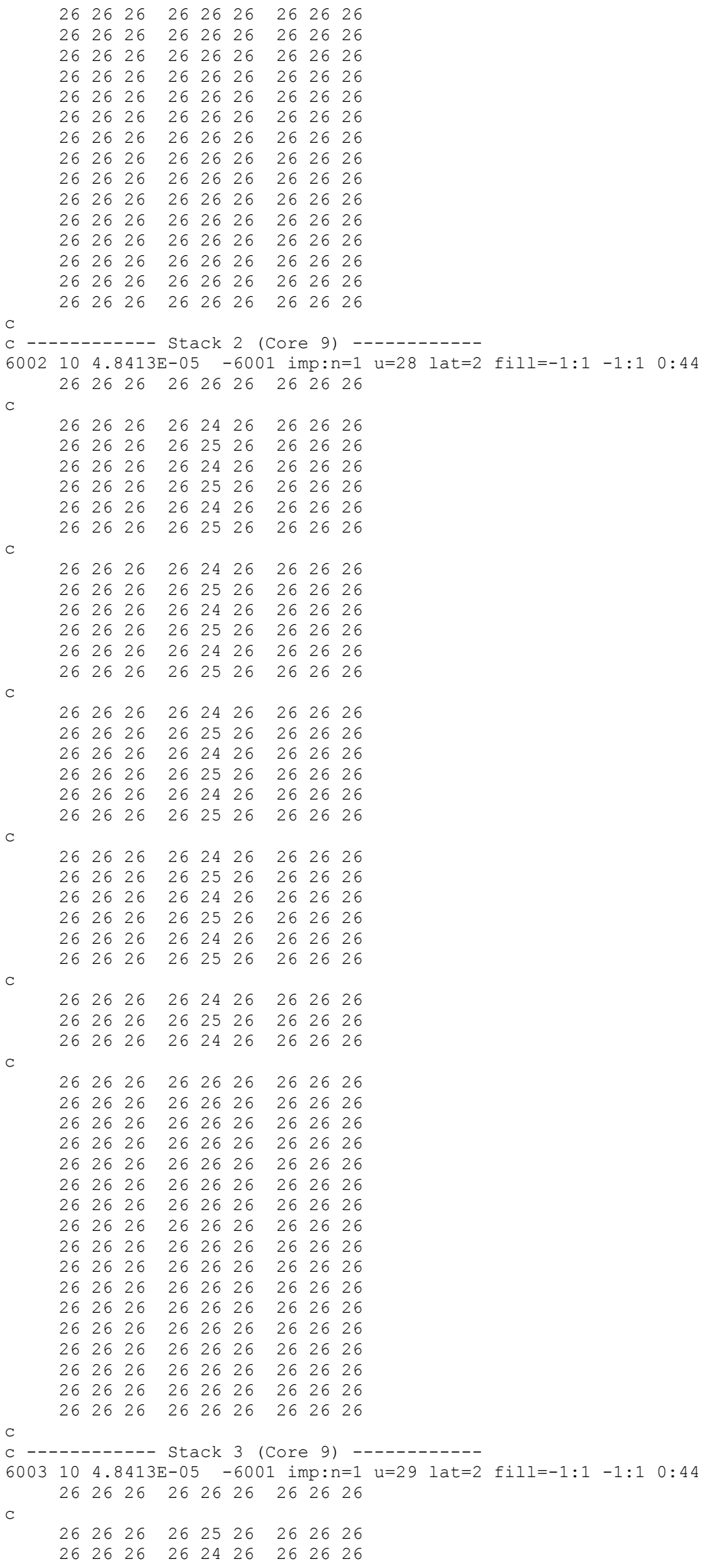

Revision: 1

Date: March 31, 2014

Page 325 of 375 
NEA/NSC/DOC(2006)1

Gas Cooled (Thermal) Reactor - GCR

PROTEUS-GCR-EXP-004

CRIT-REAC

C

$\begin{array}{lllllllll}26 & 26 & 26 & 26 & 24 & 26 & 26 & 26 & 26\end{array}$

$\begin{array}{lllllllll}26 & 26 & 26 & 26 & 25 & 26 & 26 & 26 & 26\end{array}$

$\begin{array}{lllllllll}26 & 26 & 26 & 26 & 25 & 26 & 26 & 26 & 26\end{array}$

$\begin{array}{lllllllll}26 & 26 & 26 & 26 & 24 & 26 & 26 & 26 & 26\end{array}$

$\begin{array}{lllllllll}26 & 26 & 26 & 26 & 25 & 26 & 26 & 26 & 26\end{array}$

$\begin{array}{lllllllll}26 & 26 & 26 & 26 & 24 & 26 & 26 & 26 & 26\end{array}$

$\begin{array}{lllllllll}26 & 26 & 26 & 26 & 24 & 26 & 26 & 26 & 26\end{array}$

$\begin{array}{lllllllll}26 & 26 & 26 & 26 & 25 & 26 & 26 & 26 & 26\end{array}$

$\begin{array}{lllllllll}26 & 26 & 26 & 26 & 25 & 26 & 26 & 26 & 26\end{array}$

$\begin{array}{lllllllll}26 & 26 & 26 & 26 & 24 & 26 & 26 & 26 & 26\end{array}$

$\begin{array}{lllllllll}26 & 26 & 26 & 26 & 25 & 26 & 26 & 26 & 26\end{array}$

$\begin{array}{lllllllll}26 & 26 & 26 & 26 & 24 & 26 & 26 & 26 & 26\end{array}$

$\begin{array}{lllllllll}26 & 26 & 26 & 26 & 24 & 26 & 26 & 26 & 26\end{array}$

$\begin{array}{lllllllll}26 & 26 & 26 & 26 & 25 & 26 & 26 & 26 & 26\end{array}$

$\begin{array}{lllllllll}26 & 26 & 26 & 26 & 25 & 26 & 26 & 26 & 26\end{array}$

$\begin{array}{lllllllll}26 & 26 & 26 & 26 & 24 & 26 & 26 & 26 & 26\end{array}$

C

$\begin{array}{lllllllll}26 & 26 & 26 & 26 & 25 & 26 & 26 & 26 & 26\end{array}$

$\begin{array}{lllllllll}26 & 26 & 26 & 26 & 24 & 26 & 26 & 26 & 26\end{array}$

$\begin{array}{lllllllll}26 & 26 & 26 & 26 & 24 & 26 & 26 & 26 & 26\end{array}$

$\begin{array}{lllllllll}26 & 26 & 26 & 26 & 25 & 26 & 26 & 26 & 26\end{array}$

$\begin{array}{lllllllll}26 & 26 & 26 & 26 & 25 & 26 & 26 & 26 & 26\end{array}$

C

$\begin{array}{lllllllll}26 & 26 & 26 & 26 & 25 & 26 & 26 & 26 & 26\end{array}$

$\begin{array}{lllllllll}26 & 26 & 26 & 26 & 24 & 26 & 26 & 26 & 26\end{array}$

$\begin{array}{lllllllll}26 & 26 & 26 & 26 & 24 & 26 & 26 & 26 & 26\end{array}$

C

$\begin{array}{lllllllll}26 & 26 & 26 & 26 & 26 & 26 & 26 & 26 & 26\end{array}$

$\begin{array}{lllllllll}26 & 26 & 26 & 26 & 26 & 26 & 26 & 26 & 26\end{array}$

$\begin{array}{lllllllll}26 & 26 & 26 & 26 & 26 & 26 & 26 & 26 & 26\end{array}$

$\begin{array}{lllllllll}26 & 26 & 26 & 26 & 26 & 26 & 26 & 26 & 26\end{array}$

$\begin{array}{lllllllll}26 & 26 & 26 & 26 & 26 & 26 & 26 & 26 & 26\end{array}$

$\begin{array}{lllllllll}26 & 26 & 26 & 26 & 26 & 26 & 26 & 26 & 26\end{array}$

$\begin{array}{lllllllll}26 & 26 & 26 & 26 & 26 & 26 & 26 & 26 & 26\end{array}$

$\begin{array}{lllllllll}26 & 26 & 26 & 26 & 26 & 26 & 26 & 26 & 26\end{array}$

$\begin{array}{lllllllll}26 & 26 & 26 & 26 & 26 & 26 & 26 & 26 & 26\end{array}$

$\begin{array}{lllllllll}26 & 26 & 26 & 26 & 26 & 26 & 26 & 26 & 26\end{array}$

$\begin{array}{lllllllll}26 & 26 & 26 & 26 & 26 & 26 & 26 & 26 & 26\end{array}$

$\begin{array}{lllllllll}26 & 26 & 26 & 26 & 26 & 26 & 26 & 26 & 26\end{array}$

$\begin{array}{lllllllll}26 & 26 & 26 & 26 & 26 & 26 & 26 & 26 & 26\end{array}$

$\begin{array}{lllllllll}26 & 26 & 26 & 26 & 26 & 26 & 26 & 26 & 26\end{array}$

$\begin{array}{lllllllll}26 & 26 & 26 & 26 & 26 & 26 & 26 & 26 & 26\end{array}$

$\begin{array}{lllllllll}26 & 26 & 26 & 26 & 26 & 26 & 26 & 26 & 26\end{array}$

C

$\begin{array}{lllllllll}26 & 26 & 26 & 26 & 26 & 26 & 26 & 26 & 26\end{array}$

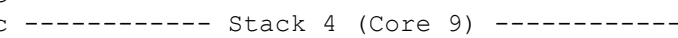

$6004104.8413 \mathrm{E}-05 \quad-6001$ imp:n=1 $u=30$ lat=2 fill=-1:1 -1:1 0:44

$\begin{array}{lllllllll}26 & 26 & 26 & 26 & 26 & 26 & 26 & 26 & 26\end{array}$

C

$\begin{array}{lllllllll}26 & 26 & 26 & 26 & 25 & 26 & 26 & 26 & 26\end{array}$

$\begin{array}{lllllllll}26 & 26 & 26 & 26 & 24 & 26 & 26 & 26 & 26\end{array}$

$\begin{array}{lllllllll}26 & 26 & 26 & 26 & 25 & 26 & 26 & 26 & 26\end{array}$

$\begin{array}{lllllllll}26 & 26 & 26 & 26 & 24 & 26 & 26 & 26 & 26\end{array}$

$\begin{array}{lllllllll}26 & 26 & 26 & 26 & 24 & 26 & 26 & 26 & 26\end{array}$

$\begin{array}{lllllllll}26 & 26 & 26 & 26 & 25 & 26 & 26 & 26 & 26\end{array}$

C

$\begin{array}{lllllllll}26 & 26 & 26 & 26 & 25 & 26 & 26 & 26 & 26\end{array}$

$\begin{array}{lllllllll}26 & 26 & 26 & 26 & 24 & 26 & 26 & 26 & 26\end{array}$

$\begin{array}{lllllllll}26 & 26 & 26 & 26 & 25 & 26 & 26 & 26 & 26\end{array}$

$\begin{array}{lllllllll}26 & 26 & 26 & 26 & 24 & 26 & 26 & 26 & 26\end{array}$

$\begin{array}{lllllllll}26 & 26 & 26 & 26 & 24 & 26 & 26 & 26 & 26\end{array}$

C

$\begin{array}{lllllllll}26 & 26 & 26 & 26 & 25 & 26 & 26 & 26 & 26\end{array}$

$\begin{array}{lllllllll}26 & 26 & 26 & 26 & 25 & 26 & 26 & 26 & 26\end{array}$

$\begin{array}{lllllllll}26 & 26 & 26 & 26 & 24 & 26 & 26 & 26 & 26\end{array}$

$\begin{array}{lllllllll}26 & 26 & 26 & 26 & 25 & 26 & 26 & 26 & 26\end{array}$

$\begin{array}{lllllllll}26 & 26 & 26 & 26 & 24 & 26 & 26 & 26 & 26\end{array}$

$\begin{array}{lllllllll}26 & 26 & 26 & 26 & 24 & 26 & 26 & 26 & 26\end{array}$

$\begin{array}{lllllllll}26 & 26 & 26 & 26 & 25 & 26 & 26 & 26 & 26\end{array}$

C

$\begin{array}{lllllllll}26 & 26 & 26 & 26 & 25 & 26 & 26 & 26 & 26\end{array}$

$\begin{array}{lllllllll}26 & 26 & 26 & 26 & 24 & 26 & 26 & 26 & 26\end{array}$

$\begin{array}{lllllllll}26 & 26 & 26 & 26 & 25 & 26 & 26 & 26 & 26\end{array}$

Revision: 1

Date: March 31, 2014

Page 326 of 375 
NEA/NSC/DOC(2006)1

Gas Cooled (Thermal) Reactor - GCR

PROTEUS-GCR-EXP-004

CRIT-REAC

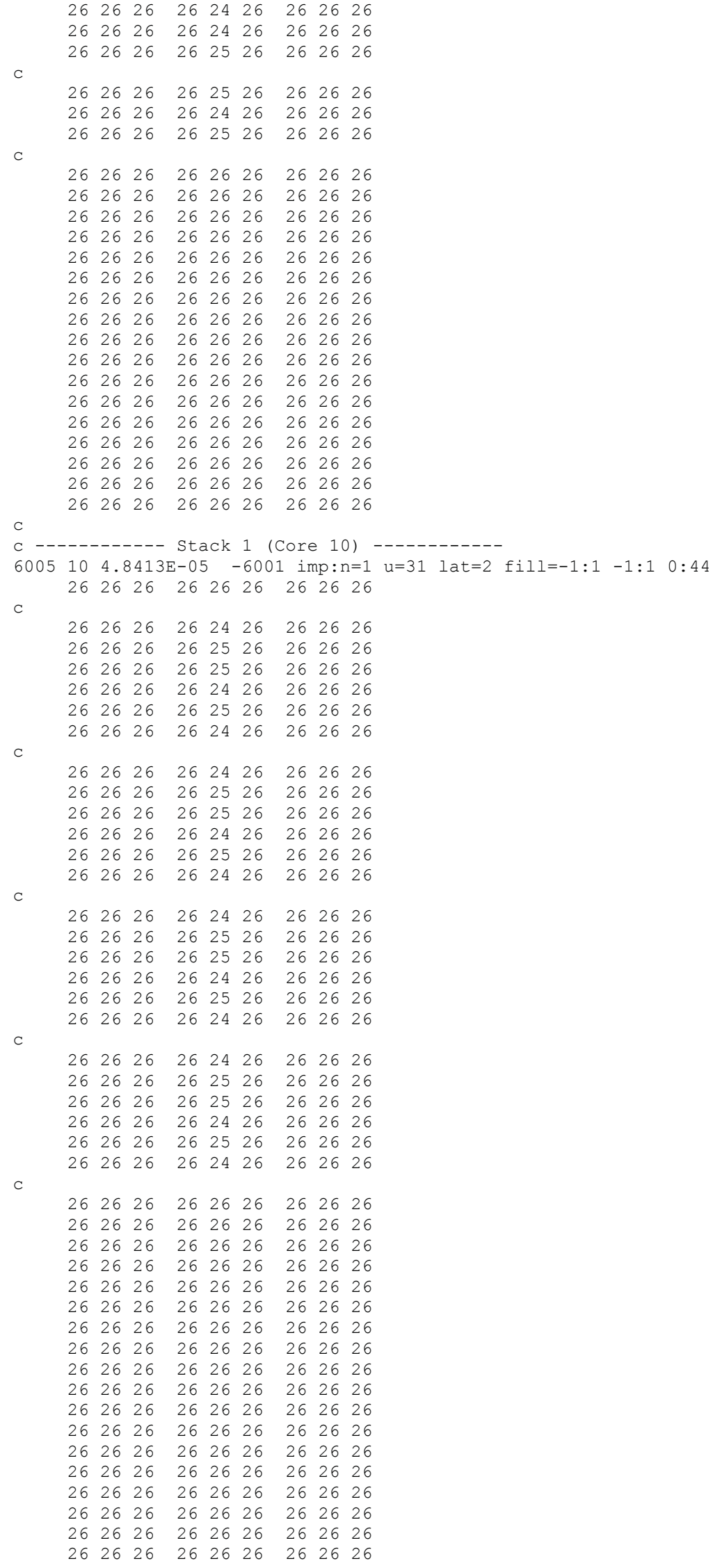

Revision: 1

Date: March 31, 2014

Page 327 of 375 
NEA/NSC/DOC(2006)1

Gas Cooled (Thermal) Reactor - GCR

PROTEUS-GCR-EXP-004

CRIT-REAC

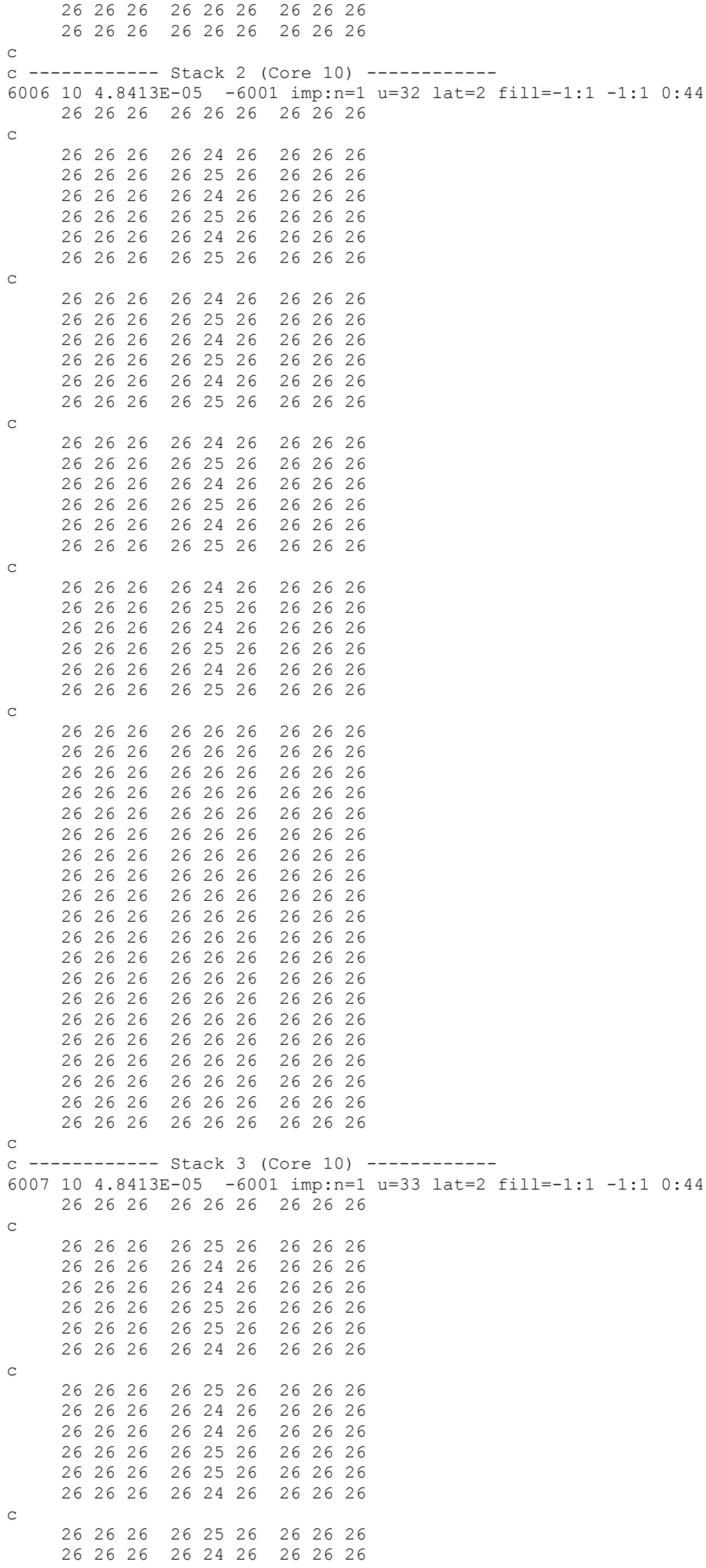

Revision: 1 
NEA/NSC/DOC(2006)1

Gas Cooled (Thermal) Reactor - GCR

PROTEUS-GCR-EXP-004

CRIT-REAC

C

C

$\begin{array}{lllllllll}26 & 26 & 26 & 26 & 24 & 26 & 26 & 26 & 26 \\ 26 & 26 & 26 & 26 & 25 & 26 & 26 & 26 & 26 \\ 26 & 26 & 26 & 26 & 25 & 26 & 26 & 26 & 26 \\ 26 & 26 & 26 & 26 & 24 & 26 & 26 & 26 & 26 \\ 26 & 26 & 26 & 26 & 25 & 26 & 26 & 26 & 26 \\ 26 & 26 & 26 & 26 & 24 & 26 & 26 & 26 & 26 \\ 26 & 26 & 26 & 26 & 24 & 26 & 26 & 26 & 26 \\ 26 & 26 & 26 & 26 & 25 & 26 & 26 & 26 & 26 \\ 26 & 26 & 26 & 26 & 25 & 26 & 26 & 26 & 26\end{array}$

$\begin{array}{lllllllll}26 & 26 & 26 & 26 & 26 & 26 & 26 & 26 & 26\end{array}$

$\begin{array}{lllllllll}26 & 26 & 26 & 26 & 26 & 26 & 26 & 26 & 26\end{array}$

$\begin{array}{lllllllll}26 & 26 & 26 & 26 & 26 & 26 & 26 & 26 & 26\end{array}$

$\begin{array}{lllllllll}26 & 26 & 26 & 26 & 26 & 26 & 26 & 26 & 26\end{array}$

$\begin{array}{lllllllll}26 & 26 & 26 & 26 & 26 & 26 & 26 & 26 & 26\end{array}$

$\begin{array}{lllllllll}26 & 26 & 26 & 26 & 26 & 26 & 26 & 26 & 26\end{array}$

$\begin{array}{lllllllll}26 & 26 & 26 & 26 & 26 & 26 & 26 & 26 & 26\end{array}$

$\begin{array}{lllllllll}26 & 26 & 26 & 26 & 26 & 26 & 26 & 26 & 26\end{array}$

$\begin{array}{lllllllll}26 & 26 & 26 & 26 & 26 & 26 & 26 & 26 & 26\end{array}$

$\begin{array}{lllllllll}26 & 26 & 26 & 26 & 26 & 26 & 26 & 26 & 26\end{array}$

$\begin{array}{lllllllll}26 & 26 & 26 & 26 & 26 & 26 & 26 & 26 & 26\end{array}$

$\begin{array}{lllllllll}26 & 26 & 26 & 26 & 26 & 26 & 26 & 26 & 26\end{array}$

$\begin{array}{lllllllll}26 & 26 & 26 & 26 & 26 & 26 & 26 & 26 & 26\end{array}$

$\begin{array}{lllllllll}26 & 26 & 26 & 26 & 26 & 26 & 26 & 26 & 26\end{array}$

$\begin{array}{lllllllll}26 & 26 & 26 & 26 & 26 & 26 & 26 & 26 & 26\end{array}$

$\begin{array}{lllllllll}26 & 26 & 26 & 26 & 26 & 26 & 26 & 26 & 26\end{array}$

$\begin{array}{lllllllll}26 & 26 & 26 & 26 & 26 & 26 & 26 & 26 & 26\end{array}$

$\begin{array}{lllllllll}26 & 26 & 26 & 26 & 26 & 26 & 26 & 26 & 26\end{array}$

$\begin{array}{lllllllll}26 & 26 & 26 & 26 & 26 & 26 & 26 & 26 & 26\end{array}$

$\begin{array}{lllllllll}26 & 26 & 26 & 26 & 26 & 26 & 26 & 26 & 26\end{array}$

$\mathrm{C}$

-------- Stack 4 (Core 10)

$6008104.8413 \mathrm{E}-05 \quad-6001$ imp:n=1 u=34 lat=2 fill=-1:1 -1:1 0:44

$262626262626 \quad 262626$

$\begin{array}{lllllllll}26 & 26 & 26 & 26 & 25 & 26 & 26 & 26 & 26\end{array}$

$\begin{array}{lllllllll}26 & 26 & 26 & 26 & 24 & 26 & 26 & 26 & 26\end{array}$

$\begin{array}{lllllllll}26 & 26 & 26 & 26 & 25 & 26 & 26 & 26 & 26\end{array}$

$\begin{array}{lllllllll}26 & 26 & 26 & 26 & 24 & 26 & 26 & 26 & 26\end{array}$

$\begin{array}{lllllllll}26 & 26 & 26 & 26 & 24 & 26 & 26 & 26 & 26\end{array}$

C

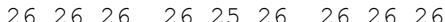

$\begin{array}{lllllllll}26 & 26 & 26 & 26 & 25 & 26 & 26 & 26 & 26\end{array}$

$\begin{array}{lllllllll}26 & 26 & 26 & 26 & 24 & 26 & 26 & 26 & 26\end{array}$

$\begin{array}{lllllllll}26 & 26 & 26 & 26 & 25 & 26 & 26 & 26 & 26\end{array}$

$\begin{array}{lllllllll}26 & 26 & 26 & 26 & 24 & 26 & 26 & 26 & 26\end{array}$

$\begin{array}{lllllllll}26 & 26 & 26 & 26 & 24 & 26 & 26 & 26 & 26\end{array}$

$\begin{array}{lllllllll}26 & 26 & 26 & 26 & 25 & 26 & 26 & 26 & 26\end{array}$

C

$\begin{array}{lllllllll}26 & 26 & 26 & 26 & 25 & 26 & 26 & 26 & 26\end{array}$

$\begin{array}{lllllllll}26 & 26 & 26 & 26 & 24 & 26 & 26 & 26 & 26\end{array}$

$\begin{array}{lllllllll}26 & 26 & 26 & 26 & 25 & 26 & 26 & 26 & 26\end{array}$

$\begin{array}{lllllllll}26 & 26 & 26 & 26 & 24 & 26 & 26 & 26 & 26\end{array}$

$\begin{array}{lllllllll}26 & 26 & 26 & 26 & 24 & 26 & 26 & 26 & 26\end{array}$

C

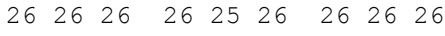

$\begin{array}{lllllllll}26 & 26 & 26 & 26 & 25 & 26 & 26 & 26 & 26\end{array}$

$\begin{array}{lllllllll}26 & 26 & 26 & 26 & 24 & 26 & 26 & 26 & 26\end{array}$

$\begin{array}{lllllllll}26 & 26 & 26 & 26 & 25 & 26 & 26 & 26 & 26\end{array}$

$\begin{array}{lllllllll}26 & 26 & 26 & 26 & 24 & 26 & 26 & 26 & 26\end{array}$

$\begin{array}{lllllllll}26 & 26 & 26 & 26 & 24 & 26 & 26 & 26 & 26\end{array}$

$\begin{array}{lllllllll}26 & 26 & 26 & 26 & 25 & 26 & 26 & 26 & 26\end{array}$

C

$\begin{array}{lllllllll}26 & 26 & 26 & 26 & 26 & 26 & 26 & 26 & 26\end{array}$

$\begin{array}{lllllllll}26 & 26 & 26 & 26 & 26 & 26 & 26 & 26 & 26\end{array}$

$\begin{array}{lllllllll}26 & 26 & 26 & 26 & 26 & 26 & 26 & 26 & 26\end{array}$

$\begin{array}{lllllllll}26 & 26 & 26 & 26 & 26 & 26 & 26 & 26 & 26\end{array}$

$\begin{array}{lllllllll}26 & 26 & 26 & 26 & 26 & 26 & 26 & 26 & 26\end{array}$

$\begin{array}{lllllllll}26 & 26 & 26 & 26 & 26 & 26 & 26 & 26 & 26\end{array}$

$\begin{array}{lllllllll}26 & 26 & 26 & 26 & 26 & 26 & 26 & 26 & 26\end{array}$

$\begin{array}{lllllllll}26 & 26 & 26 & 26 & 26 & 26 & 26 & 26 & 26\end{array}$

$\begin{array}{lllllllll}26 & 26 & 26 & 26 & 26 & 26 & 26 & 26 & 26\end{array}$

$\begin{array}{lllllllll}26 & 26 & 26 & 26 & 26 & 26 & 26 & 26 & 26\end{array}$

$\begin{array}{lllllllll}26 & 26 & 26 & 26 & 26 & 26 & 26 & 26 & 26\end{array}$

Revision: 1

Date: March 31, 2014

Page 329 of 375 
NEA/NSC/DOC(2006)1

\section{Gas Cooled (Thermal) Reactor - GCR \\ PROTEUS-GCR-EXP-004 CRIT-REAC}

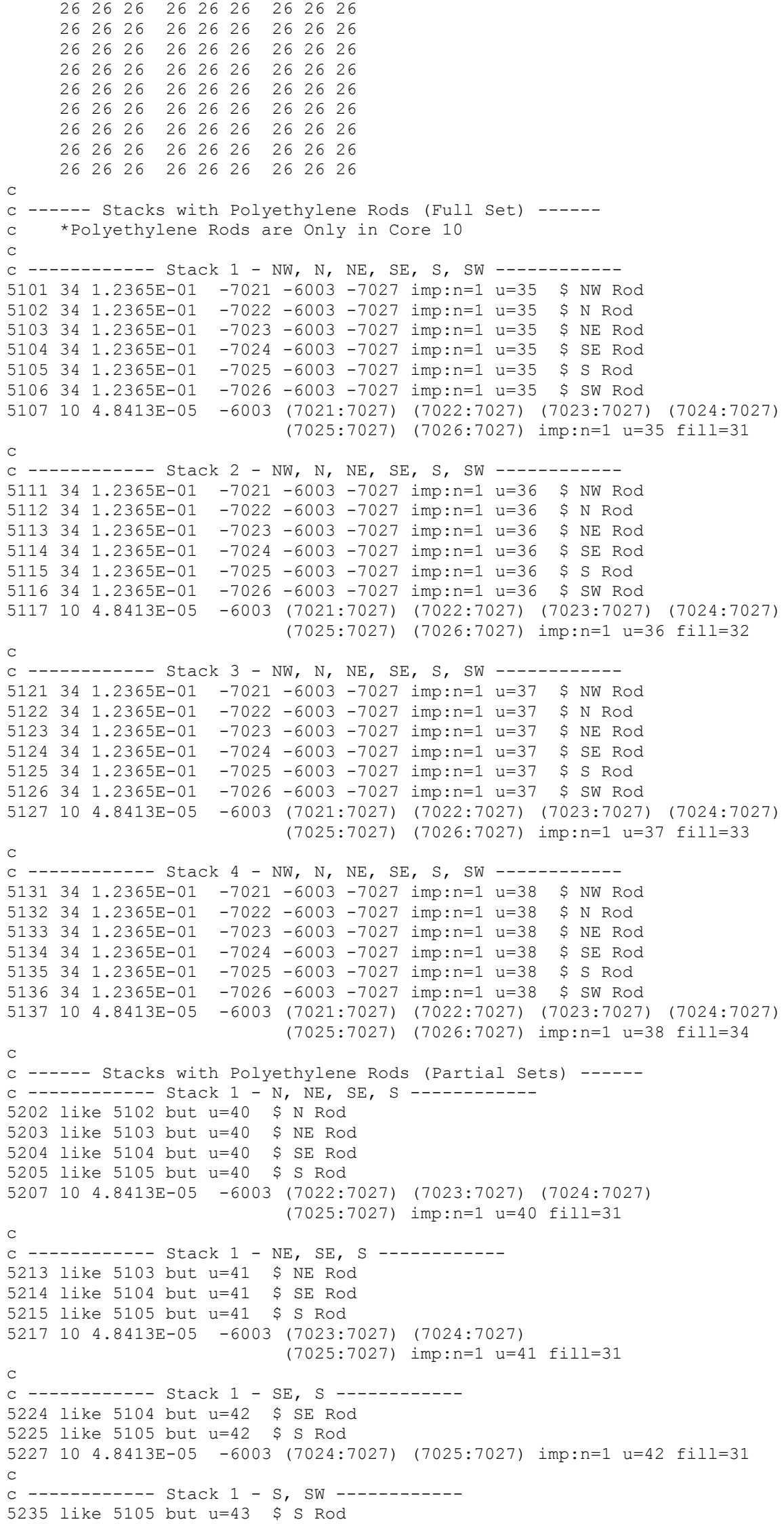

Revision: 1 
NEA/NSC/DOC(2006)1

\section{Gas Cooled (Thermal) Reactor - GCR \\ PROTEUS-GCR-EXP-004 \\ CRIT-REAC}

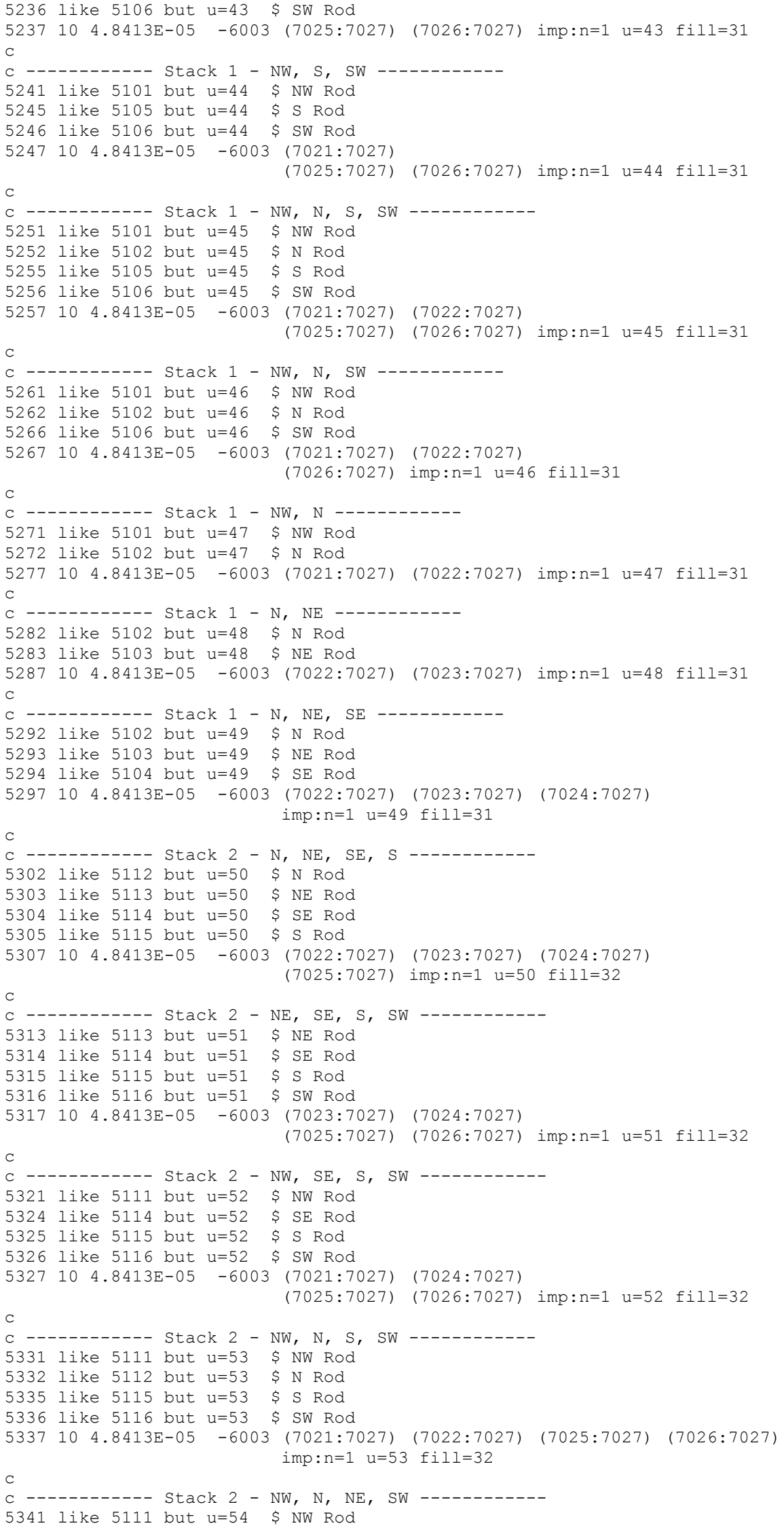

Revision: 1 
NEA/NSC/DOC(2006)1

\section{Gas Cooled (Thermal) Reactor - GCR \\ PROTEUS-GCR-EXP-004 CRIT-REAC}

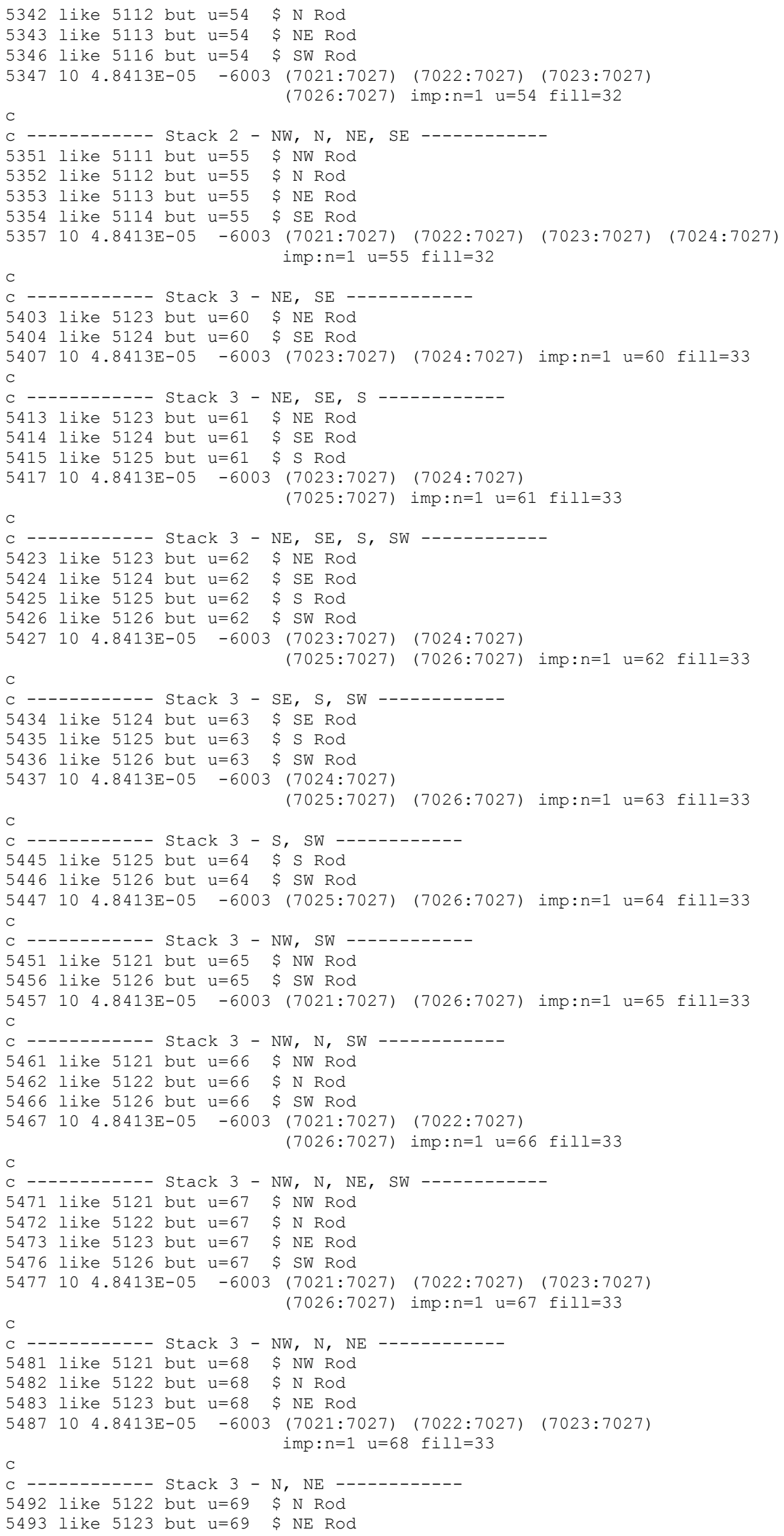

Revision: 1 
NEA/NSC/DOC(2006)1

\section{Gas Cooled (Thermal) Reactor - GCR \\ PROTEUS-GCR-EXP-004 \\ CRIT-REAC}

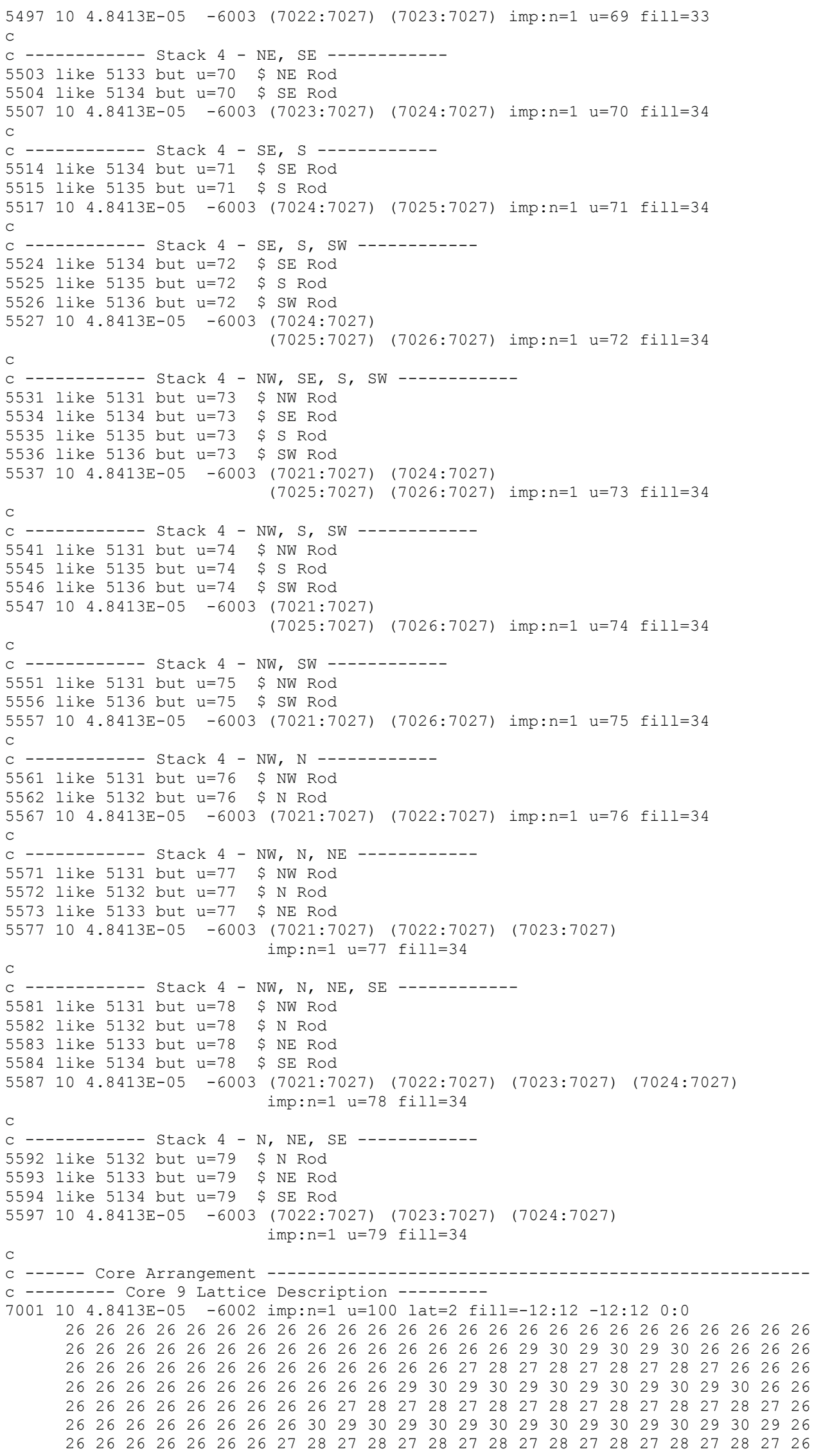

Revision: 1 
NEA/NSC/DOC(2006)1

\section{Gas Cooled (Thermal) Reactor - GCR \\ PROTEUS-GCR-EXP-004 CRIT-REAC}

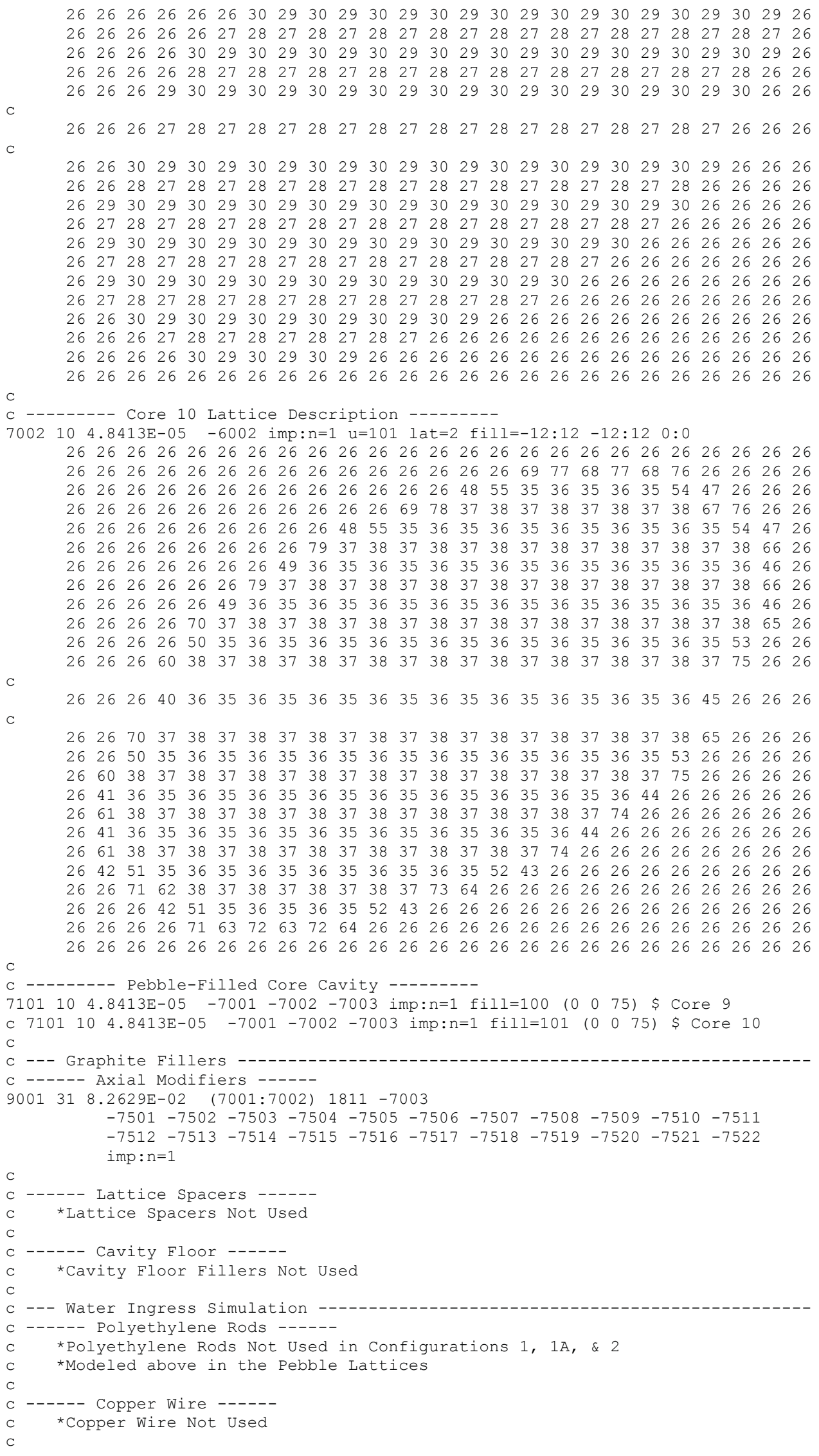

Revision: 1

Date: March 31, 2014

Page 334 of 375 
NEA/NSC/DOC(2006)1

\section{Gas Cooled (Thermal) Reactor - GCR \\ PROTEUS-GCR-EXP-004 CRIT-REAC}

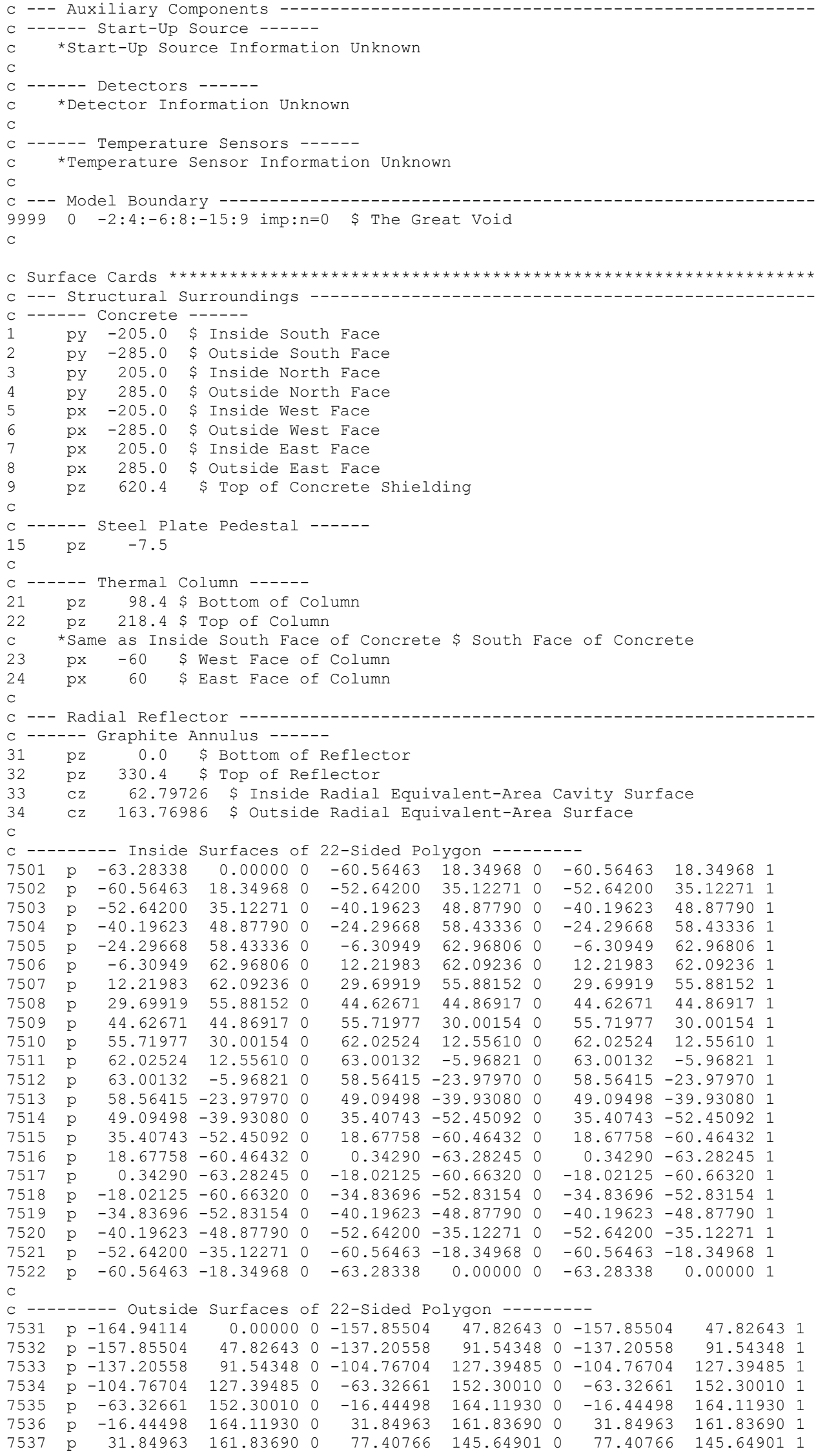

Revision: 1 
NEA/NSC/DOC(2006)1

\section{Gas Cooled (Thermal) Reactor - GCR}

\section{PROTEUS-GCR-EXP-004 CRIT-REAC}

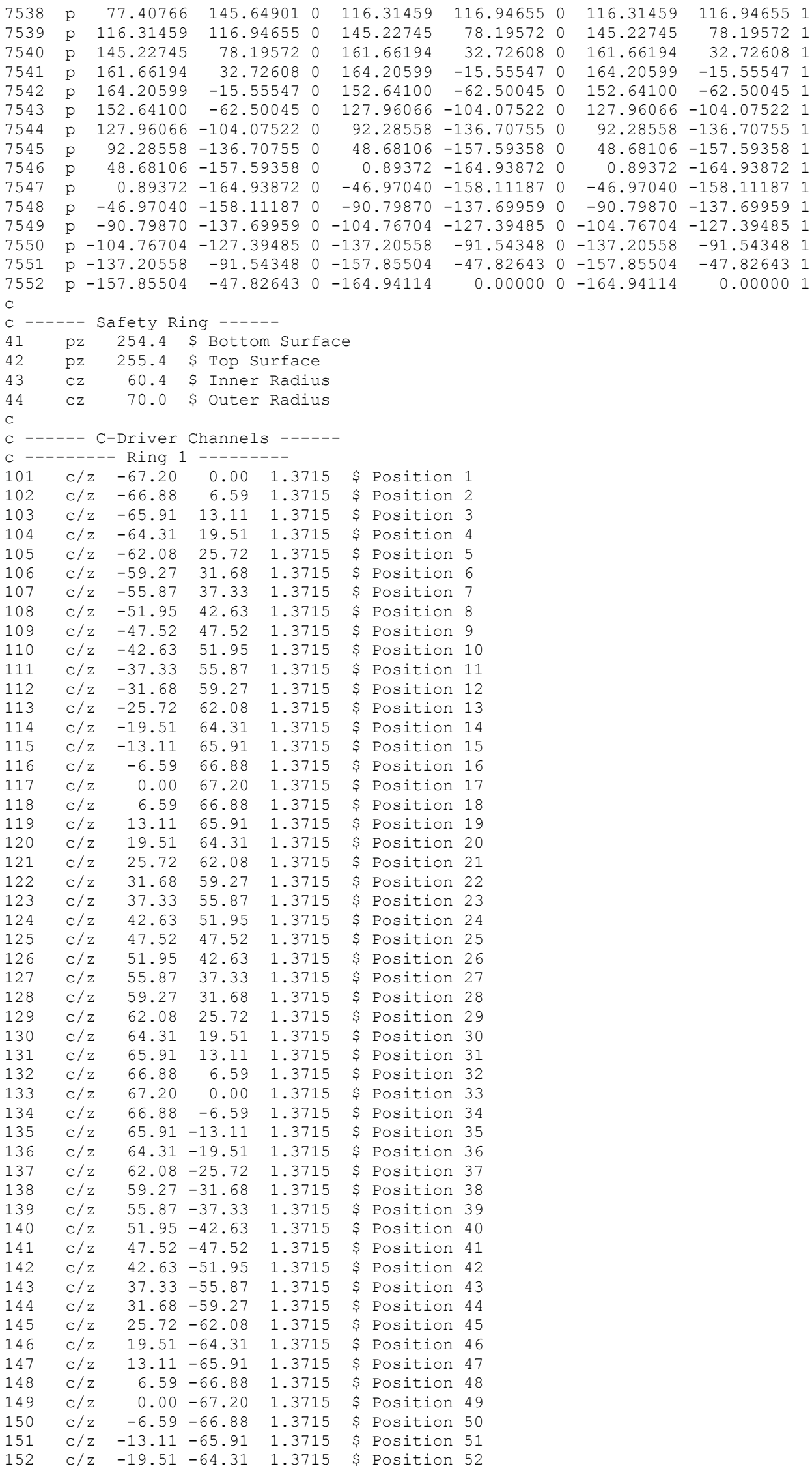

Revision: 1 
NEA/NSC/DOC(2006)1

Gas Cooled (Thermal) Reactor - GCR

PROTEUS-GCR-EXP-004 CRIT-REAC

\begin{tabular}{|c|c|c|c|c|c|c|}
\hline 153 & $\mathrm{c} / \mathrm{z}$ & -25.72 & -62.08 & 1.3715 & \$ Position 53 & \\
\hline 154 & $c / z$ & -31.68 & -59.27 & 1.3715 & \$ Position 54 & \\
\hline 155 & $\mathrm{c} / \mathrm{z}$ & -37.33 & -55.87 & 1.3715 & \$ Position 55 & \\
\hline 156 & $\mathrm{c} / \mathrm{z}$ & -42.63 & -51.95 & 1.3715 & \$ Position 56 & \\
\hline 157 & $\mathrm{c} / \mathrm{z}$ & -47.52 & -47.52 & 1.3715 & \$ Position 57 & \\
\hline 158 & $\mathrm{c} / \mathrm{z}$ & -51.95 & -42.63 & 1.3715 & \$ Position 58 & \\
\hline 159 & $c / z$ & -55.87 & -37.33 & 1.3715 & \$ Position 59 & \\
\hline 160 & $c / z$ & -59.27 & -31.68 & 1.3715 & \$ Position 60 & \\
\hline 161 & $\mathrm{c} / \mathrm{z}$ & -62.08 & -25.72 & 1.3715 & \$ Position 61 & \\
\hline 162 & $\mathrm{c} / \mathrm{z}$ & -64.31 & -19.51 & 1.3715 & \$ Position 62 & \\
\hline 163 & $\mathrm{c} / \mathrm{z}$ & -65.91 & -13.11 & 1.3715 & \$ Position 63 & \\
\hline 164 & $c / z$ & -66.88 & -6.59 & 1.3715 & \$ Position 64 & \\
\hline & & & & & & \\
\hline 165 & $\mathrm{Cz}$ & 70.125 & \$ Ring & g Divider & for Modeling & Simplification \\
\hline & & & & & & \\
\hline & --- & Ring 2 & ----- & --- & & \\
\hline 201 & $\mathrm{c} / \mathrm{z}$ & -72.96 & 3.58 & 1.3715 & \$Position 1 & \\
\hline 202 & $c / z$ & -72.26 & 10.72 & 1.3715 & \$ Position 2 & \\
\hline 203 & $\mathrm{c} / \mathrm{z}$ & -70.86 & 17.75 & 1.3715 & \$ Position 3 & \\
\hline 204 & $\mathrm{c} / \mathrm{z}$ & -68.78 & 24.61 & 1.3715 & \$ Position 4 & \\
\hline 205 & $\mathrm{c} / \mathrm{z}$ & -66.04 & 31.23 & 1.3715 & \$ Position 5 & \\
\hline 206 & $c / z$ & -62.66 & 37.56 & 1.3715 & \$ Position 6 & \\
\hline 207 & $\mathrm{c} / \mathrm{z}$ & -58.67 & 43.52 & 1.3715 & \$ Position 7 & \\
\hline 208 & $\mathrm{c} / \mathrm{z}$ & -54.13 & 49.06 & 1.3715 & \$ Position 8 & \\
\hline 209 & $\mathrm{c} / \mathrm{z}$ & -49.06 & 54.13 & 1.3715 & \$ Position 9 & \\
\hline 210 & $\mathrm{c} / \mathrm{z}$ & -43.52 & 58.67 & 1.3715 & \$ Position 10 & \\
\hline 211 & $\mathrm{c} / \mathrm{z}$ & -37.56 & 62.66 & 1.3715 & \$ Position 11 & \\
\hline 212 & $\mathrm{c} / \mathrm{z}$ & -31.23 & 66.04 & 1.3715 & \$ Position 12 & \\
\hline 213 & $c / z$ & -24.61 & 68.78 & 1.3715 & \$ Position 13 & \\
\hline 214 & $\mathrm{c} / \mathrm{z}$ & -17.75 & 70.86 & 1.3715 & \$ Position 14 & \\
\hline 215 & $\mathrm{c} / \mathrm{z}$ & -10.72 & 72.26 & 1.3715 & \$ Position 15 & \\
\hline 216 & $\mathrm{c} / \mathrm{z}$ & -3.58 & 72.96 & 1.3715 & \$ Position 16 & \\
\hline 217 & $\mathrm{c} / \mathrm{z}$ & 3.58 & 72.96 & 1.3715 & \$ Position 17 & \\
\hline 218 & $\mathrm{c} / \mathrm{z}$ & 10.72 & 72.26 & 1.3715 & \$ Position 18 & \\
\hline 219 & $\mathrm{c} / \mathrm{z}$ & 17.75 & 70.86 & 1.3715 & \$ Position 19 & \\
\hline 220 & $\mathrm{c} / \mathrm{z}$ & 24.61 & 68.78 & 1.3715 & \$ Position 20 & \\
\hline 221 & $\mathrm{C} / \mathrm{z}$ & 31.23 & 66.04 & 1.3715 & \$ Position 21 & \\
\hline 222 & $\mathrm{c} / \mathrm{z}$ & 37.56 & 62.66 & 1.3715 & \$ Position 22 & \\
\hline 223 & $\mathrm{c} / \mathrm{z}$ & 43.52 & 58.67 & 1.3715 & \$ Position 23 & \\
\hline 224 & $c / z$ & 49.06 & 54.13 & 1.3715 & \$ Position 24 & \\
\hline 225 & $\mathrm{c} / \mathrm{z}$ & 54.13 & 49.06 & 1.3715 & \$ Position 25 & \\
\hline 226 & $\mathrm{c} / \mathrm{z}$ & 58.67 & 43.52 & 1.3715 & \$ Position 26 & \\
\hline 227 & $\mathrm{C} / \mathrm{z}$ & 62.66 & 37.56 & 1.3715 & \$ Position 27 & \\
\hline 228 & $\mathrm{c} / \mathrm{z}$ & 66.04 & 31.23 & 1.3715 & \$ Position 28 & \\
\hline 229 & $\mathrm{c} / \mathrm{z}$ & 68.78 & 24.61 & 1.3715 & \$ Position 29 & \\
\hline 230 & $\mathrm{c} / \mathrm{z}$ & 70.86 & 17.75 & 1.3715 & \$ Position 30 & \\
\hline 231 & $\mathrm{C} / \mathrm{z}$ & 72.26 & 10.72 & 1.3715 & \$ Position 31 & \\
\hline 232 & $\mathrm{c} / \mathrm{z}$ & 72.96 & 3.58 & 1.3715 & \$ Position 32 & \\
\hline 233 & $\mathrm{c} / \mathrm{z}$ & 72.96 & -3.58 & 1.3715 & \$ Position 33 & \\
\hline 234 & $\mathrm{c} / \mathrm{z}$ & 72.26 & -10.72 & 1.3715 & \$ Position 34 & \\
\hline 235 & $\mathrm{c} / \mathrm{z}$ & 70.86 & -17.75 & 1.3715 & \$ Position 35 & \\
\hline 236 & $\mathrm{c} / \mathrm{z}$ & 68.78 & -24.61 & 1.3715 & \$ Position 36 & \\
\hline 237 & $\mathrm{C} / \mathrm{z}$ & 66.04 & -31.23 & 1.3715 & \$ Position 37 & \\
\hline 238 & $\mathrm{c} / \mathrm{z}$ & 62.66 & -37.56 & 1.3715 & \$ Position 38 & \\
\hline 239 & $\mathrm{c} / \mathrm{z}$ & 58.67 & -43.52 & 1.3715 & \$ Position 39 & \\
\hline 240 & $\mathrm{c} / \mathrm{z}$ & 54.13 & -49.06 & 1.3715 & \$ Position 40 & \\
\hline 241 & $\mathrm{c} / \mathrm{z}$ & 49.06 & -54.13 & 1.3715 & \$ Position 41 & \\
\hline 242 & $\mathrm{C} / \mathrm{z}$ & 43.52 & -58.67 & 1.3715 & \$ Position 42 & \\
\hline 243 & $\mathrm{c} / \mathrm{z}$ & 37.56 & -62.66 & 1.3715 & \$ Position 43 & \\
\hline 244 & $\mathrm{c} / \mathrm{z}$ & 31.23 & -66.04 & 1.3715 & \$ Position 44 & \\
\hline 245 & $\mathrm{c} / \mathrm{z}$ & 24.61 & -68.78 & 1.3715 & \$ Position 45 & \\
\hline 246 & $\mathrm{c} / \mathrm{z}$ & 17.75 & -70.86 & 1.3715 & \$ Position 46 & \\
\hline 247 & $\mathrm{c} / \mathrm{z}$ & 10.72 & -72.26 & 1.3715 & \$ Position 47 & \\
\hline 248 & $\mathrm{c} / \mathrm{z}$ & 3.58 & -72.96 & 1.3715 & \$ Position 48 & \\
\hline 249 & $\mathrm{c} / \mathrm{z}$ & -3.58 & -72.96 & 1.3715 & \$ Position 49 & \\
\hline 250 & $\mathrm{c} / \mathrm{z}$ & -10.72 & -72.26 & 1.3715 & \$ Position 50 & \\
\hline 251 & $\mathrm{c} / \mathrm{z}$ & -17.75 & -70.86 & 1.3715 & \$ Position 51 & \\
\hline 252 & $\mathrm{c} / \mathrm{z}$ & -24.61 & -68.78 & 1.3715 & \$ Position 52 & \\
\hline 253 & $\mathrm{c} / \mathrm{z}$ & -31.23 & -66.04 & 1.3715 & \$ Position 53 & \\
\hline 254 & $\mathrm{C} / \mathrm{z}$ & -37.56 & -62.66 & 1.3715 & \$ Position 54 & \\
\hline 255 & $\mathrm{c} / \mathrm{z}$ & -43.52 & -58.67 & 1.3715 & \$ Position 55 & \\
\hline 256 & $\mathrm{c} / \mathrm{z}$ & -49.06 & -54.13 & 1.3715 & \$ Position 56 & \\
\hline 257 & $\mathrm{c} / \mathrm{z}$ & -54.13 & -49.06 & 1.3715 & \$ Position 57 & \\
\hline 258 & $\mathrm{C} / \mathrm{z}$ & -58.67 & -43.52 & 1.3715 & \$ Position 58 & \\
\hline 259 & $\mathrm{c} / \mathrm{z}$ & -62.66 & -37.56 & 1.3715 & \$ Position 59 & \\
\hline 260 & $\mathrm{c} / \mathrm{z}$ & -66.04 & -31.23 & 1.3715 & \$ Position 60 & \\
\hline
\end{tabular}

Revision: 1 
NEA/NSC/DOC(2006)1

Gas Cooled (Thermal) Reactor - GCR

PROTEUS-GCR-EXP-004

CRIT-REAC

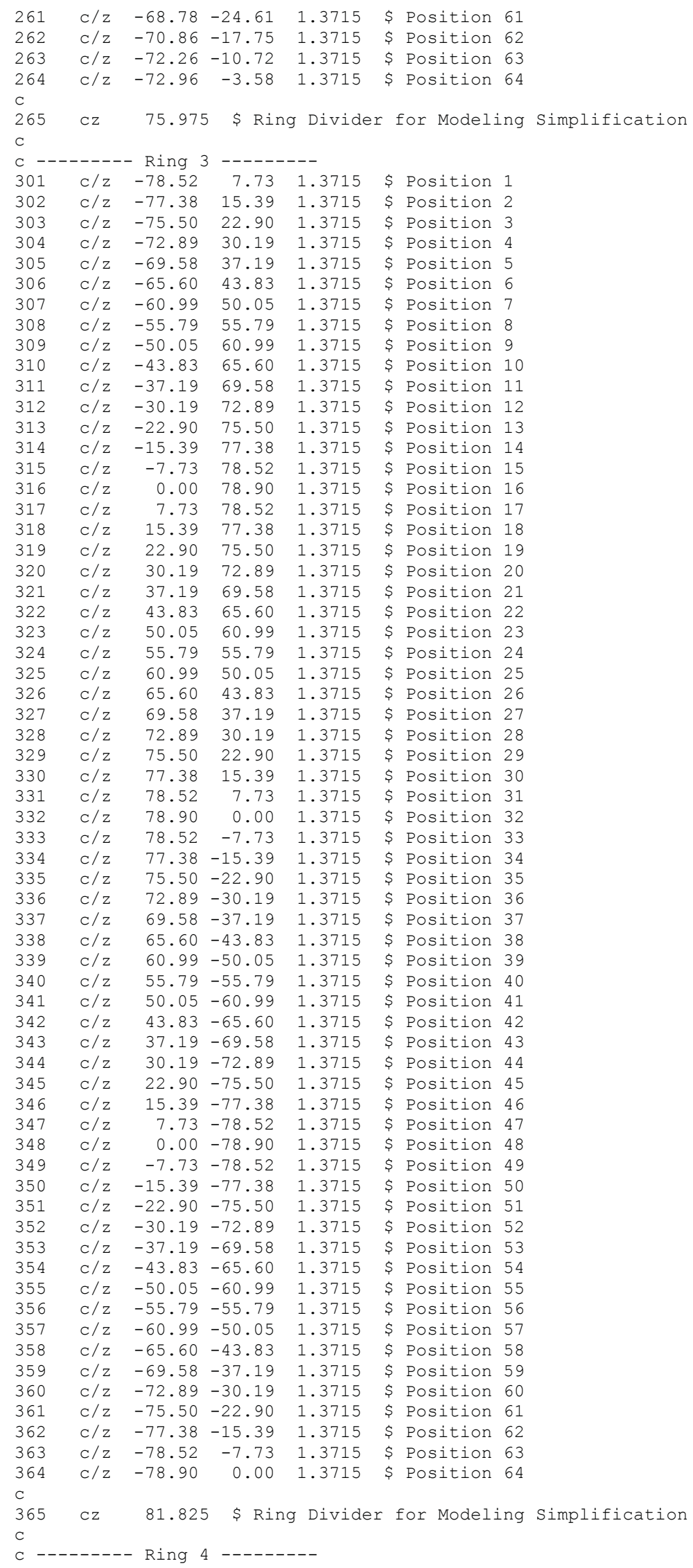

Revision: 1 
NEA/NSC/DOC(2006)1

Gas Cooled (Thermal) Reactor - GCR

PROTEUS-GCR-EXP-004 CRIT-REAC

\begin{tabular}{|c|c|c|c|c|c|c|}
\hline 401 & $\mathrm{C} / \mathrm{z}$ & -83.83 & 12.44 & 1.3715 & \$ Position 1 & \\
\hline 402 & $\mathrm{C} / \mathrm{z}$ & -82.21 & 20.59 & 1.3715 & \$ Position 2 & \\
\hline 403 & $\mathrm{C} / \mathrm{z}$ & -79.80 & 28.55 & 1.3715 & \$ Position 3 & \\
\hline 404 & $\mathrm{C} / \mathrm{z}$ & -76.61 & 36.24 & 1.3715 & \$ Position 4 & \\
\hline 405 & $\mathrm{C} / \mathrm{z}$ & -72.69 & 43.57 & 1.3715 & \$ Position 5 & \\
\hline 406 & $\mathrm{C} / \mathrm{z}$ & -68.07 & 50.49 & 1.3715 & \$ Position 6 & \\
\hline 407 & $c / z$ & -62.80 & 56.91 & 1.3715 & \$ Position 7 & \\
\hline 408 & $\mathrm{C} / \mathrm{z}$ & -56.91 & 62.80 & 1.3715 & \$ Position 8 & \\
\hline 409 & $\mathrm{C} / \mathrm{z}$ & -50.49 & 68.07 & 1.3715 & \$ Position 9 & \\
\hline 410 & $\mathrm{C} / \mathrm{z}$ & -43.57 & 72.69 & 1.3715 & \$ Position 10 & \\
\hline 411 & $\mathrm{C} / \mathrm{z}$ & -36.24 & 76.61 & 1.3715 & \$ Position 11 & \\
\hline 412 & $\mathrm{C} / \mathrm{z}$ & -28.55 & 79.80 & 1.3715 & \$ Position 12 & \\
\hline 413 & $\mathrm{C} / \mathrm{z}$ & -20.59 & 82.21 & 1.3715 & \$ Position 13 & \\
\hline 414 & $\mathrm{C} / \mathrm{z}$ & -12.44 & 83.83 & 1.3715 & \$ Position 14 & \\
\hline 415 & $\mathrm{C} / \mathrm{z}$ & -4.16 & 84.65 & 1.3715 & \$ Position 15 & \\
\hline 416 & $\mathrm{C} / \mathrm{z}$ & 4.16 & 84.65 & 1.3715 & \$ Position 16 & \\
\hline 417 & $\mathrm{C} / \mathrm{z}$ & 12.44 & 83.83 & 1.3715 & \$ Position 17 & \\
\hline 418 & $\mathrm{C} / \mathrm{z}$ & 20.59 & 82.21 & 1.3715 & \$ Position 18 & \\
\hline 419 & $\mathrm{C} / \mathrm{z}$ & 28.55 & 79.80 & 1.3715 & \$ Position 19 & \\
\hline 420 & $\mathrm{C} / \mathrm{z}$ & 36.24 & 76.61 & 1.3715 & \$ Position 20 & \\
\hline 421 & $\mathrm{C} / \mathrm{z}$ & 43.57 & 72.69 & 1.3715 & \$ Position 21 & \\
\hline 422 & $\mathrm{C} / \mathrm{z}$ & 50.49 & 68.07 & 1.3715 & \$ Position 22 & \\
\hline 423 & $\mathrm{C} / \mathrm{z}$ & 56.91 & 62.80 & 1.3715 & \$ Position 23 & \\
\hline 424 & $\mathrm{C} / \mathrm{z}$ & 62.80 & 56.91 & 1.3715 & \$ Position 24 & \\
\hline 425 & $\mathrm{C} / \mathrm{z}$ & 68.07 & 50.49 & 1.3715 & \$ Position 25 & \\
\hline 426 & $\mathrm{C} / \mathrm{z}$ & 72.69 & 43.57 & 1.3715 & \$ Position 26 & \\
\hline 427 & $\mathrm{C} / \mathrm{z}$ & 76.61 & 36.24 & 1.3715 & \$ Position 27 & \\
\hline 428 & $\mathrm{C} / \mathrm{z}$ & 79.80 & 28.55 & 1.3715 & \$ Position 28 & \\
\hline 429 & $\mathrm{C} / \mathrm{z}$ & 82.21 & 20.59 & 1.3715 & \$ Position 29 & \\
\hline 430 & $\mathrm{C} / \mathrm{z}$ & 83.83 & 12.44 & 1.3715 & $\$$ Position 30 & \\
\hline 431 & $\mathrm{C} / \mathrm{z}$ & 84.65 & 4.16 & 1.3715 & \$ Position 31 & \\
\hline 432 & $\mathrm{C} / \mathrm{z}$ & 84.65 & -4.16 & 1.3715 & \$ Position 32 & \\
\hline 433 & $\mathrm{C} / \mathrm{z}$ & 83.83 & -12.44 & 1.3715 & \$ Position 33 & \\
\hline 434 & $\mathrm{C} / \mathrm{z}$ & 82.21 & -20.59 & 1.3715 & \$ Position 34 & \\
\hline 435 & $\mathrm{C} / \mathrm{z}$ & 79.80 & -28.55 & 1.3715 & \$ Position 35 & \\
\hline 436 & $\mathrm{C} / \mathrm{z}$ & 76.61 & -36.24 & 1.3715 & \$ Position 36 & \\
\hline 437 & $\mathrm{C} / \mathrm{z}$ & 72.69 & -43.57 & 1.3715 & \$ Position 37 & \\
\hline 438 & $\mathrm{C} / \mathrm{z}$ & 68.07 & -50.49 & 1.3715 & \$ Position 38 & \\
\hline 439 & $\mathrm{C} / \mathrm{z}$ & 62.80 & -56.91 & 1.3715 & \$ Position 39 & \\
\hline 440 & $\mathrm{C} / \mathrm{z}$ & 56.91 & -62.80 & 1.3715 & \$ Position 40 & \\
\hline 441 & $\mathrm{C} / \mathrm{z}$ & 50.49 & -68.07 & 1.3715 & \$ Position 41 & \\
\hline 442 & $\mathrm{C} / \mathrm{z}$ & 43.57 & -72.69 & 1.3715 & \$ Position 42 & \\
\hline 443 & $\mathrm{C} / \mathrm{z}$ & 36.24 & -76.61 & 1.3715 & \$ Position 43 & \\
\hline 444 & $\mathrm{C} / \mathrm{z}$ & 28.55 & -79.80 & 1.3715 & \$ Position 44 & \\
\hline 445 & $\mathrm{C} / \mathrm{z}$ & 20.59 & -82.21 & 1.3715 & \$ Position 45 & \\
\hline 446 & $\mathrm{C} / \mathrm{z}$ & 12.44 & -83.83 & 1.3715 & \$ Position 46 & \\
\hline 447 & $\mathrm{C} / \mathrm{z}$ & 4.16 & -84.65 & 1.3715 & \$ Position 47 & \\
\hline 448 & $\mathrm{C} / \mathrm{z}$ & -4.16 & -84.65 & 1.3715 & $\$$ Position 48 & \\
\hline 449 & $\mathrm{C} / \mathrm{z}$ & -12.44 & -83.83 & 1.3715 & \$ Position 49 & \\
\hline 450 & $\mathrm{C} / \mathrm{z}$ & -20.59 & -82.21 & 1.3715 & \$ Position 50 & \\
\hline 451 & $\mathrm{C} / \mathrm{z}$ & -28.55 & -79.80 & 1.3715 & \$ Position 51 & \\
\hline 452 & $\mathrm{C} / \mathrm{z}$ & -36.24 & -76.61 & 1.3715 & \$ Position 52 & \\
\hline 453 & $\mathrm{C} / \mathrm{z}$ & -43.57 & -72.69 & 1.3715 & \$ Position 53 & \\
\hline 454 & $\mathrm{C} / \mathrm{z}$ & -50.49 & -68.07 & 1.3715 & \$ Position 54 & \\
\hline 455 & $\mathrm{C} / \mathrm{z}$ & -56.91 & -62.80 & 1.3715 & \$ Position 55 & \\
\hline 456 & $\mathrm{C} / \mathrm{z}$ & -62.80 & -56.91 & 1.3715 & \$ Position 56 & \\
\hline 457 & $\mathrm{C} / \mathrm{z}$ & -68.07 & -50.49 & 1.3715 & \$ Position 57 & \\
\hline 458 & $\mathrm{C} / \mathrm{z}$ & -72.69 & -43.57 & 1.3715 & \$ Position 58 & \\
\hline 459 & $\mathrm{C} / \mathrm{z}$ & -76.61 & -36.24 & 1.3715 & \$ Position 59 & \\
\hline 460 & $\mathrm{C} / \mathrm{z}$ & -79.80 & -28.55 & 1.3715 & \$ Position 60 & \\
\hline 461 & $\mathrm{C} / \mathrm{z}$ & -82.21 & -20.59 & 1.3715 & \$ Position 61 & \\
\hline 462 & $\mathrm{C} / \mathrm{z}$ & -83.83 & -12.44 & 1.3715 & \$ Position 62 & \\
\hline 463 & $\mathrm{C} / \mathrm{z}$ & -84.65 & -4.16 & 1.3715 & \$ Position 63 & \\
\hline 464 & $\mathrm{C} / \mathrm{z}$ & -84.65 & 4.16 & 1.3715 & \$ Position 64 & \\
\hline C & & & & & & \\
\hline 465 & $\mathrm{Cz}$ & 87.675 & \$ Ring & \multirow[t]{2}{*}{ Divider } & \multirow[t]{2}{*}{ for Modeling } & \multirow[t]{2}{*}{ Simplification } \\
\hline C & & & & & & \\
\hline & ---- & - Ring 5 & ---- & -- & & \\
\hline 501 & $\mathrm{c} / \mathrm{z}$ & -88.86 & 17.68 & 1.3715 & \$ Position 1 & \\
\hline 502 & $\mathrm{C} / \mathrm{z}$ & -86.70 & 26.30 & 1.3715 & \$ Position 2 & \\
\hline 503 & $\mathrm{C} / \mathrm{z}$ & -83.70 & 34.67 & 1.3715 & \$ Position 3 & \\
\hline 504 & $\mathrm{C} / \mathrm{z}$ & -79.90 & 42.71 & 1.3715 & \$ Position 4 & \\
\hline 505 & $\mathrm{C} / \mathrm{z}$ & -75.33 & 50.33 & 1.3715 & \$ Position 5 & \\
\hline 506 & $\mathrm{C} / \mathrm{z}$ & -70.03 & 57.48 & 1.3715 & \$ Position 6 & \\
\hline 507 & $\mathrm{C} / \mathrm{z}$ & -64.06 & 64.06 & 1.3715 & \$ Position 7 & \\
\hline 508 & $\mathrm{C} / \mathrm{z}$ & -57.48 & 70.03 & 1.3715 & \$ Position 8 & \\
\hline
\end{tabular}

Revision: 1 
NEA/NSC/DOC(2006)1

Gas Cooled (Thermal) Reactor - GCR

PROTEUS-GCR-EXP-004

CRIT-REAC

\begin{tabular}{|c|c|c|c|c|c|c|c|}
\hline 509 & $c / z$ & -50.33 & 75.33 & 1.3715 & \$ & Position & 9 \\
\hline 0 & $c / z$ & -42.71 & 79.90 & 1.3715 & $\$$ & Position & \\
\hline & $c / z$ & -34.67 & 83.70 & 1.3715 & s & Position & \\
\hline & $c / z$ & -26.30 & 86.70 & 1.3715 & S & $\mathrm{n}$ & \\
\hline & $c / z$ & -17.68 & 88.86 & 1.3715 & \$ & Pos & \\
\hline & $c / z$ & -8.88 & 90.16 & 1.3715 & S & Po: & \\
\hline & $c / z$ & 0.00 & 0.60 & 1.3715 & & & \\
\hline & $c / z$ & 8.88 & & 1.3715 & \$ & & \\
\hline & $c / z$ & 17.68 & 88.86 & 1.3715 & $\$$ & $\mathrm{Po}$ & \\
\hline & $\mathrm{C} / \mathrm{z}$ & 26.30 & 86.70 & 1.3715 & & & \\
\hline & $c / z$ & 34.67 & 83.70 & 1.3715 & s & & \\
\hline & $c / z$ & 42.71 & & 1.37 & \$ & & \\
\hline & $c / z$ & 50.33 & & 1.3715 & 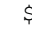 & & \\
\hline & $c / z$ & 57.48 & 70.03 & 1.3715 & $\$$ & & \\
\hline & $c / z$ & 64.06 & 6 & 1.3715 & \$ & & \\
\hline 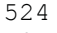 & $c / z$ & 70.03 & 57.48 & $1.3^{7}$ & S & & \\
\hline & $c / z$ & 75.33 & 3 & 1.3715 & S & & \\
\hline & $c / z$ & 79.90 & 42. & 1.3715 & s & & \\
\hline & $c / z$ & 83.70 & 34. & 1.37 & \$ & & \\
\hline D & $c / z$ & 86.70 & 26. & 1.37 & S & & \\
\hline 29 & $c / z$ & 88.86 & 17.68 & 1.3715 & \$ & $\mathrm{Po}$ & \\
\hline D & $c / z$ & 90.16 & 8 . & 1.37 & $\$$ & & \\
\hline & $c / z$ & 90.60 & 0 . & 1.3 & \$ & & \\
\hline & $c / z$ & 90.16 & -8.88 & 1.3715 & S & $\mathrm{Pc}$ & \\
\hline & $c / z$ & 88.86 & -17 . & 1.3715 & S & & \\
\hline & $c / z$ & 86.70 & -26 & 1.3 & $\$$ & & \\
\hline & $c / z$ & 83.70 & -34 & 1.3 & \$ & & \\
\hline & $\mathrm{C} / \mathrm{z}$ & 79.90 & -42.71 & 1.3715 & S & & \\
\hline & $c / z$ & 75.33 & -50 & 1.37 & $\$$ & & \\
\hline & $c / z$ & 70 & -57 & 1.3 & $\$$ & & \\
\hline & $c / z$ & 64.06 & -64.06 & 1.3715 & \$ & & \\
\hline 4 & $c / z$ & 57.48 & -70 . & 1.3715 & $\$$ & & \\
\hline & $c / z$ & 50.33 & -7 & 1.37 & \$ & & \\
\hline & $\mathrm{c} / \mathrm{z}$ & 42.71 & -75 & 1.3 & \$ & & \\
\hline & $c / z$ & 34.67 & -83.70 & 1.3715 & \$ & $\mathrm{Pc}$ & \\
\hline & $c / z$ & 26.30 & -86 & 1.37 & $\$$ & & \\
\hline & $c / z$ & 17.68 & -88 & 1.3 & $\$$ & & \\
\hline & $c / z$ & 8.88 & -90 & 1.3715 & \$ & & \\
\hline 7 & $c / z$ & 0.00 & -90.60 & 1.3715 & $\$$ & & \\
\hline 4 & $c / z$ & -8.88 & -90 . & 1.37 & $\$$ & & \\
\hline 4 & $c / z$ & -17.68 & -88 & 1.3 & \$ & & \\
\hline & $c / z$ & -26.30 & -86.70 & 1.3715 & $\$$ & & \\
\hline 5 & $c / z$ & -34.67 & -83.70 & 1.3715 & $\$$ & & \\
\hline 52 & $c / z$ & -42.71 & -79. & 1.37 & $\$$ & & \\
\hline & $c / z$ & -50.33 & -75. & 1.37 & S & & \\
\hline & $\mathrm{C} / \mathrm{z}$ & -57.48 & -70.03 & 1.3715 & \$ & & \\
\hline & $c / z$ & -64.06 & -64.06 & 1.3715 & $\$$ & & \\
\hline 5 & $c / z$ & -70.03 & -57.48 & 1.37 & $\$$ & & \\
\hline 57 & $c / z$ & -75.33 & -50.33 & 1.3715 & 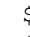 & & \\
\hline 5 & $c / z$ & -79.90 & -42 . & 1.3715 & $\$$ & & \\
\hline$\pi$ & $c / z$ & -83.70 & -34 & 1.3715 & $\$$ & & \\
\hline 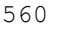 & $c / z$ & -86.70 & -26.30 & 1.37 & 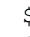 & & \\
\hline$\sigma$ & $c / z$ & -88.86 & -17.68 & 1.3715 & 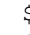 & & \\
\hline 56 & $c / z$ & -90.16 & -8.88 & 1.37 & s & & \\
\hline 56 & $c / z$ & -90.60 & 0.00 & 1.3715 & $\$$ & & \\
\hline 56 & $c / z$ & -90.16 & 8.88 & 1.3 & $\$$ & & \\
\hline \\
\hline \multicolumn{8}{|c|}{ c ------ Graphite Plugs ------ } \\
\hline \multicolumn{8}{|c|}{------ Ring 1 --------- } \\
\hline 601 & $\mathrm{c} / \mathrm{z}$ & -67.20 & 0.00 & 1.32 & s & Po & \pm \\
\hline 602 & $\mathrm{c} / \mathrm{z}$ & -66.88 & 6.59 & 1.325 & $\$$ & Pos & 2 \\
\hline 60 & $c / z$ & -65.91 & 13.11 & 1.325 & $\$$ & Pos & 3 \\
\hline & $\mathrm{C} / \mathrm{z}$ & -64.31 & 19.51 & 1.325 & $\$$ & Pos: & 4 \\
\hline 60 & $\mathrm{c} / \mathrm{z}$ & -62.08 & 25.72 & 1.325 & $\$$ & Pos & 5 \\
\hline 60 & $c / z$ & -59.27 & 31.68 & 1.325 & $\$$ & Pos & 6 \\
\hline $60^{\circ}$ & $c / z$ & -55.87 & 37.33 & 1.325 & $\$$ & Pos & 7 \\
\hline 608 & $c / z$ & -51.95 & 42.63 & 1.325 & $\$$ & Pos: & 8 \\
\hline & $c / z$ & -47.52 & 47.52 & 1.325 & $\$$ & Pos & 9 \\
\hline 61 & $c / z$ & -42.63 & 51.95 & 1.325 & $\$$ & Pos & 10 \\
\hline 611 & $c / z$ & -37.33 & 55.87 & 1.325 & $\$$ & Pos & 11 \\
\hline 61 & $c / z$ & -31.68 & 59.27 & 1.325 & $\$$ & Pos & 12 \\
\hline 61 & $c / z$ & -25.72 & 62.08 & 1.325 & $\$$ & Pos & 13 \\
\hline 61 & $c / z$ & -19.51 & 64.31 & 1.325 & $\$$ & Pos & 14 \\
\hline 61 & $c / z$ & -13.11 & 65.91 & 1.325 & $\$$ & Pos: & 15 \\
\hline & $c / z$ & -6.59 & 66.88 & 1.3 & $\$$ & Pos & \\
\hline & $\mathrm{C} / \mathrm{z}$ & 0.00 & 67.20 & 1.325 & $r$ & Pos & \\
\hline
\end{tabular}

Revision: 1 
NEA/NSC/DOC(2006)1

Gas Cooled (Thermal) Reactor - GCR

PROTEUS-GCR-EXP-004

CRIT-REAC

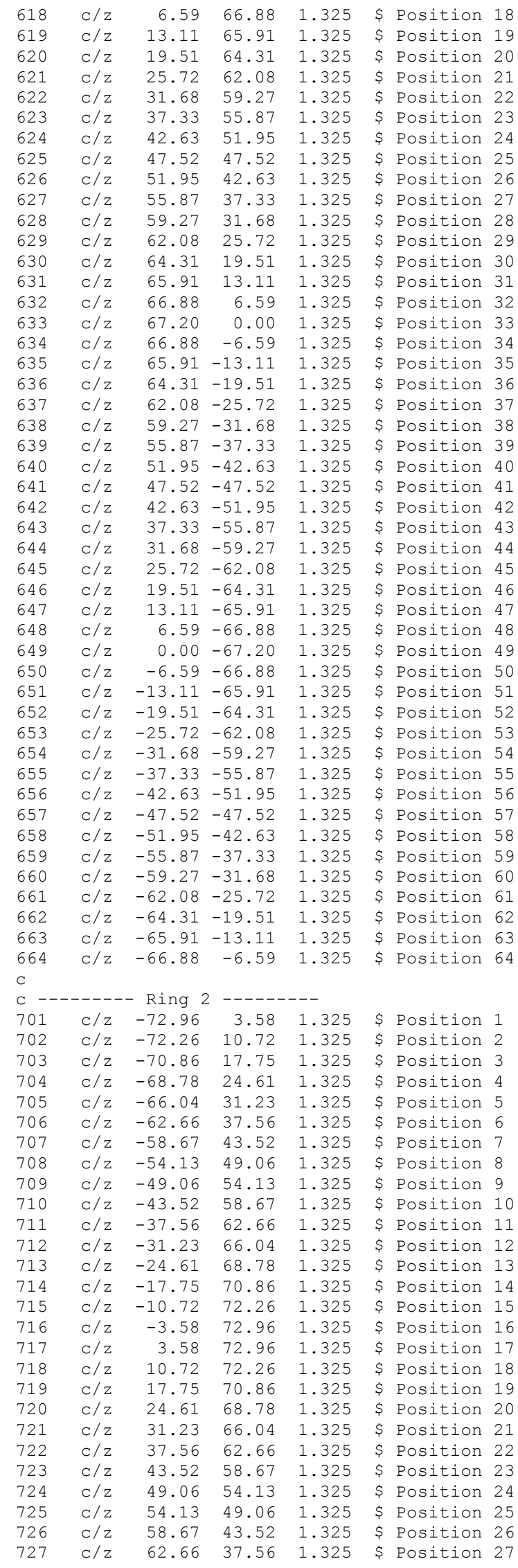

Revision: 1 
NEA/NSC/DOC(2006)1

Gas Cooled (Thermal) Reactor - GCR

PROTEUS-GCR-EXP-004

CRIT-REAC

\begin{tabular}{|c|c|c|c|c|c|c|c|}
\hline 728 & $\mathrm{c} / \mathrm{z}$ & 66.04 & 31.23 & 1.325 & \$ & sition & 28 \\
\hline 700 & $c / z$ & 68.78 & 24.61 & 1.325 & & sition & \\
\hline 30 & $\mathrm{c} / \mathrm{z}$ & 70.86 & 17.75 & 1.325 & 3 & Position & 30 \\
\hline 31 & $c / z$ & 72.26 & 10.72 & 1.325 & $\$$ & osition & \\
\hline & $\mathrm{c} / \mathrm{z}$ & 72.96 & 3.58 & 1.325 & $\$$ & osition & \\
\hline & $\mathrm{c} / \mathrm{z}$ & 72.96 & -3.58 & 1.325 & $\$$ & jon & \\
\hline & $c / z$ & 72.26 & -10.72 & 1.325 & \$ & ositio & \\
\hline & $c / z$ & 70.86 & -17.75 & 1.325 & $\$$ & - & \\
\hline & $\mathrm{c} / \mathrm{z}$ & 68.78 & -24.61 & 1.325 & \$ & & \\
\hline & $\mathrm{c} / \mathrm{z}$ & 66.04 & -31.23 & 1.325 & $\$$ & & \\
\hline & $\mathrm{c} / \mathrm{z}$ & 62.66 & -37.56 & 1.325 & $\$$ & 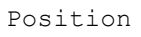 & \\
\hline & $\mathrm{c} / \mathrm{z}$ & 58.67 & -43.52 & 1.325 & $\$$ & & \\
\hline & $\mathrm{C} / \mathrm{z}$ & 54.13 & -49.06 & 1.325 & \$ & & \\
\hline & $\mathrm{C} / \mathrm{z}$ & 49.06 & -54.13 & 1.32 & \$ & 4 & \\
\hline & $c / z$ & 43.52 & -58.67 & 1.325 & $\$$ & \pm & \\
\hline & $\mathrm{c} / \mathrm{z}$ & 37.56 & -62.66 & 1.325 & $\$$ & & \\
\hline & $\mathrm{c} / \mathrm{z}$ & 31.23 & -66.04 & 1.325 & $\$$ & & \\
\hline & $c / z$ & 24.61 & -68.78 & 1.325 & $\$$ & & \\
\hline & $\mathrm{c} / \mathrm{z}$ & 17.75 & -70.86 & 1.325 & $\$$ & & \\
\hline 4 & $c / z$ & 10.72 & -72.26 & 1.3 & $\$$ & & \\
\hline & $\mathrm{c} / \mathrm{z}$ & 3.58 & -72.96 & 1.3 & $\$$ & & \\
\hline & $c / z$ & -3.58 & -72.96 & 1.3 & $\$$ & & \\
\hline 50 & $\mathrm{c} / \mathrm{z}$ & -10.72 & -72.26 & 1.3 & $\$$ & & \\
\hline 75 & $\mathrm{C} / \mathrm{z}$ & -17.75 & -70.86 & 1.3 & \$ & & \\
\hline 75 & $c / z$ & -24.61 & -68.78 & 1.3 & $\$$ & & \\
\hline 5 & $c / z$ & -31.23 & -66.04 & 1.3 & $\$$ & & \\
\hline 5 & $c / z$ & -37.56 & -62.66 & 1.3 & $\$$ & & \\
\hline 75 & $\mathrm{c} / \mathrm{z}$ & -43.52 & -58.67 & 1.3 & $\$$ & & \\
\hline & $c / z$ & -49.06 & -54.13 & 1.3 & $\$$ & & \\
\hline 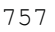 & $\mathrm{C} / \mathrm{z}$ & -54.13 & -49.06 & 1.3 & $\$$ & & \\
\hline 75 & $c / z$ & -58.67 & -43.52 & 1.3 & $\$$ & & \\
\hline 75 & $\mathrm{c} / \mathrm{z}$ & -62.66 & -37.56 & 1.3 & $\$$ & & \\
\hline D & $c / z$ & -66.04 & -31.23 & 1. & $\$$ & 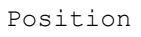 & \\
\hline 761 & $\mathrm{c} / \mathrm{z}$ & -68.78 & -24.61 & 1.325 & $\$$ & n & \\
\hline 76 & $c / z$ & -70.86 & -17.75 & 1.3 & $\$$ & & \\
\hline 76 & $\mathrm{c} / \mathrm{z}$ & -72.26 & -10.72 & 1.3 & $\$$ & & \\
\hline 76 & $c / z$ & -72.96 & -3.58 & 1 . & $\$$ & & \\
\hline \\
\hline & & & P & -- & & & \\
\hline 1 & $c / z$ & -78.52 & 7.73 & 1.3 & $\$$ & & 1 \\
\hline & $\mathrm{C} / \mathrm{z}$ & -77.38 & 15.39 & 1.325 & $\$$ & $\mathrm{n}$ & \\
\hline t. & $c / z$ & -75.50 & 22.90 & 1. & $\$$ & & \\
\hline 4 & $\mathrm{C} / \mathrm{z}$ & -72.89 & 30.19 & 1. & $\$$ & & \\
\hline 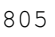 & $\mathrm{c} / \mathrm{z}$ & -69.58 & 37.19 & 1.325 & $\$$ & 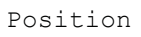 & \\
\hline 806 & $c / z$ & -65.60 & 43.83 & 1.32 & $\$$ & & \\
\hline & $\mathrm{c} / \mathrm{z}$ & -60.99 & 50. & 1.3 & $\$$ & & \\
\hline 8 & $\mathrm{C} / \mathrm{z}$ & -55.79 & 55.79 & 1.3 & $\$$ & & 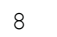 \\
\hline 809 & $\mathrm{C} / \mathrm{z}$ & -50.05 & 60.99 & 1.3 & $\$$ & & 9 \\
\hline 810 & $c / z$ & -43.83 & 65.60 & 1.3 & $\$$ & & c \\
\hline & $\mathrm{c} / \mathrm{z}$ & -37.19 & 69.58 & 1. & $\$$ & & \\
\hline & $\mathrm{c} / \mathrm{z}$ & -30.19 & 72.89 & 1.3 & $\$$ & on & \\
\hline & $\mathrm{c} / \mathrm{z}$ & -22.90 & 75.50 & 1.325 & $\$$ & $n$ & \\
\hline 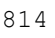 & $\mathrm{c} / \mathrm{z}$ & -15.39 & 77. & 1. & $\$$ & & \\
\hline & $\mathrm{c} / \mathrm{z}$ & -7.73 & 78.52 & 1. & $\$$ & & \\
\hline & $\mathrm{c} / \mathrm{z}$ & 0.00 & 78.90 & 1. & $\$$ & 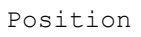 & \\
\hline 817 & $\mathrm{c} / \mathrm{z}$ & 7.73 & 78.52 & 1.32 & $\$$ & & \\
\hline 818 & $\mathrm{c} / \mathrm{z}$ & 15.39 & 77 & & $\$$ & & \\
\hline & $\mathrm{c} / \mathrm{z}$ & 22.90 & 75.50 & 1. & $\$$ & & \\
\hline 820 & $c / z$ & 30.19 & 72.89 & 1.3 & $\$$ & & \\
\hline 821 & $c / z$ & 37.19 & 69.58 & 1. & $\$$ & & \\
\hline 822 & $\mathrm{c} / \mathrm{z}$ & 43.83 & 65. & 1. & $\$$ & & \\
\hline 823 & $\mathrm{c} / \mathrm{z}$ & 50.05 & 60.99 & 1.325 & $\$$ & $\mathrm{P}^{2}$ & \\
\hline 824 & $\mathrm{c} / \mathrm{z}$ & 55.79 & 55. & 1 . & $\$$ & & \\
\hline & $\mathrm{c} / \mathrm{z}$ & 60.99 & 50. & 1.3 & $\$$ & & \\
\hline & $\mathrm{c} / \mathrm{z}$ & 65.60 & 43.83 & 1. & $\$$ & & \\
\hline 827 & $\mathrm{C} / \mathrm{z}$ & 69.58 & 37.19 & 1.325 & $\$$ & & \\
\hline 82 & $\mathrm{c} / \mathrm{z}$ & 72.89 & 30 . & & $\$$ & & \\
\hline 829 & $\mathrm{c} / \mathrm{z}$ & 75.50 & 22.90 & 1. & $\$$ & & \\
\hline 830 & $c / z$ & 77.38 & 15.39 & 1.3 & $\$$ & & \\
\hline 831 & $\mathrm{c} / \mathrm{z}$ & 78.52 & 7. & 1. & $\$$ & & \\
\hline 832 & $\mathrm{c} / \mathrm{z}$ & 78.90 & 0.00 & & $\$$ & & \\
\hline 83 & $c / z$ & 78.52 & -7.73 & 1. & $\$$ & & \\
\hline 83 & $\mathrm{c} / \mathrm{z}$ & 77.38 & -15.39 & 1. & $\$$ & Po & \\
\hline & $\mathrm{c} / \mathrm{z}$ & 75.50 & -22.90 & & $\$$ & & \\
\hline & $\mathrm{c} / \mathrm{z}$ & 72.89 & -30 . & & $\$$ & & \\
\hline 837 & $\mathrm{c} / \mathrm{z}$ & 69.58 & -37.19 & 1. 325 & $\$$ & & \\
\hline
\end{tabular}

Revision: 1 
NEA/NSC/DOC(2006)1

Gas Cooled (Thermal) Reactor - GCR

PROTEUS-GCR-EXP-004

CRIT-REAC

\begin{tabular}{|c|c|c|c|c|c|c|c|}
\hline 38 & $\mathrm{C} / \mathrm{z}$ & 65.60 & -43.83 & 1.325 & $\$$ & Position & 38 \\
\hline & $\mathrm{C} / \mathrm{z}$ & 60.99 & -50.05 & 1.325 & c & Position & \\
\hline & $\mathrm{C} / \mathrm{z}$ & 55.79 & -55.79 & 1.325 & $\$$ & Position & 40 \\
\hline & $\mathrm{C} / \mathrm{z}$ & 50.05 & -60.99 & 1.325 & \$ & Position & \\
\hline & $\mathrm{C} / \mathrm{z}$ & 43.83 & -65.60 & 1.325 & $\$$ & Position & \\
\hline & $\mathrm{C} / \mathrm{z}$ & 37.19 & -69.58 & 1.32 & $\$$ & Position & \\
\hline & $\mathrm{C} / \mathrm{z}$ & 30.19 & -72.89 & 1.325 & $\$$ & osition & \\
\hline & $\mathrm{C} / \mathrm{z}$ & 22.90 & -75.50 & 1.325 & $\$$ & $\mathrm{n}$ & \\
\hline & $\mathrm{C} / \mathrm{z}$ & 15.39 & -77.38 & 1.325 & \$ & & \\
\hline & $\mathrm{C} / \mathrm{z}$ & 7.73 & -78.52 & 1.32 & $\$$ & & \\
\hline & $\mathrm{C} / \mathrm{z}$ & 0.00 & -78.90 & 1.325 & $\$$ & ition & \\
\hline & $\mathrm{C} / \mathrm{z}$ & -7.73 & -78.52 & 1.325 & $\$$ & & \\
\hline & $\mathrm{C} / \mathrm{z}$ & -15.39 & -77.38 & 1.3 & $\$$ & & \\
\hline & $\mathrm{C} / \mathrm{z}$ & 22.90 & -75 & 1.32 & $\$$ & & \\
\hline & $\mathrm{C} / \mathrm{z}$ & 30.19 & -72.89 & 1.32 & $\$$ & ition & \\
\hline & $\mathrm{C} / \mathrm{z}$ & -37.19 & -69.58 & 1.325 & $\$$ & $\mathrm{Po}$ & \\
\hline & $\mathrm{C} / \mathrm{z}$ & -43.83 & -65.60 & 1.32 & $\$$ & & \\
\hline & $\mathrm{C} / \mathrm{z}$ & -50.05 & -60 . & 1.32 & $\$$ & E & \\
\hline & $\mathrm{C} / \mathrm{z}$ & -55.79 & -55.79 & 1.32 & $\$$ & & \\
\hline & $\mathrm{C} / \mathrm{z}$ & -60.99 & -50.05 & 1.325 & $\$$ & on & \\
\hline & $\mathrm{C} / \mathrm{z}$ & -65 & -43 & 1.32 & $\$$ & & \\
\hline & $\mathrm{C} / \mathrm{z}$ & -69.58 & -37 . & 1.3 & $\$$ & Po & \\
\hline & $\mathrm{C} / \mathrm{z}$ & -72.89 & -30.19 & 1.325 & $\$$ & Po & \\
\hline & $\mathrm{C} / \mathrm{z}$ & -75.50 & -22.90 & 1. & $\$$ & & \\
\hline & $\mathrm{C} / \mathrm{z}$ & -77.38 & -15 . & 1.3 & $\$$ & & \\
\hline & $\mathrm{C} / \mathrm{z}$ & -78.52 & -7.73 & 1.3 & $\$$ & & \\
\hline & $\mathrm{C} / \mathrm{z}$ & -78.90 & 0.00 & 1.3 & $\$$ & & \\
\hline \\
\hline & - & indy & & & & & \\
\hline & $\mathrm{C} / \mathrm{z}$ & -83.83 & 12.44 & 1.325 & $\$$ & Po: & 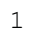 \\
\hline ) & $\mathrm{C} / \mathrm{z}$ & -82.21 & 20. & 1. & $\$$ & Po & \\
\hline & $\mathrm{C} / \mathrm{z}$ & -79.80 & 28. & 1.325 & $\$$ & Po & 3 \\
\hline & $\mathrm{C} / \mathrm{z}$ & -76.61 & 36.24 & 1.325 & $\$$ & Po & 4 \\
\hline & $\mathrm{C} / \mathrm{z}$ & -72.69 & 43.57 & 1.325 & $\$$ & Po & 5 \\
\hline & $\mathrm{C} / \mathrm{z}$ & -68.07 & 50. & 1. & $\$$ & $\mathrm{Po}$ & 6 \\
\hline & $\mathrm{C} / \mathrm{z}$ & -62.80 & 56. & 1.3 & $\$$ & Po & 7 \\
\hline & $\mathrm{C} / \mathrm{z}$ & -56.91 & 62. & 1.3 & $\$$ & & 8 \\
\hline 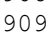 & $\mathrm{C} / \mathrm{z}$ & -50.49 & 68. & 1.3 & $\$$ & & 9 \\
\hline & $\mathrm{C} / \mathrm{z}$ & -43.57 & 72. & 1. & $\$$ & & \\
\hline & $c / z$ & -36.24 & 76 & 1.3 & $\$$ & Po & \\
\hline & $\mathrm{C} / \mathrm{z}$ & -28.55 & 79.80 & 1.325 & $\$$ & & \\
\hline & $\mathrm{C} / \mathrm{z}$ & -20.59 & 82. & 1. & $\$$ & $\mathrm{Po}$ & \\
\hline & $\mathrm{C} / \mathrm{z}$ & -12.44 & 83. & 1.3 & $\$$ & & \\
\hline & $\mathrm{C} / \mathrm{z}$ & -4.16 & 84. & 1.325 & $\$$ & $\mathrm{Po}$ & \\
\hline & $\mathrm{C} / \mathrm{z}$ & 4.16 & 84. & 1.3 & $\$$ & & \\
\hline & $\mathrm{C} / \mathrm{z}$ & 12.44 & 83. & 1.3 & $\$$ & & \\
\hline & $\mathrm{C} / \mathrm{z}$ & 20 & 82. & 1.3 & $\$$ & & \\
\hline & $\mathrm{C} / \mathrm{z}$ & 28.55 & 79.80 & 1.32 & $\$$ & $n$ & \\
\hline & $c / z$ & 36.24 & 76. & 1.3 & $\$$ & & \\
\hline & $\mathrm{C} / \mathrm{z}$ & 43.57 & 72. & 1. & $\$$ & & \\
\hline & $\mathrm{C} / \mathrm{z}$ & .49 & 68. & 1. 325 & $\$$ & $\mathrm{Po}$ & \\
\hline & $\mathrm{C} / \mathrm{z}$ & 6.91 & 62.80 & 1.325 & $\$$ & $\mathrm{Po}$ & 2 \\
\hline D. & $\mathrm{C} / \mathrm{z}$ & 30 & 56. & 1.32 & $\$$ & $\mathrm{Po}$ & \\
\hline & $\mathrm{C} / \mathrm{z}$ & 07 & 50 & 1. & $\$$ & & \\
\hline & $\mathrm{C} / \mathrm{z}$ & 72.69 & 43.57 & 1.325 & $\$$ & $\mathrm{Po}$ & \\
\hline 87 & $\mathrm{C} / \mathrm{z}$ & 5.61 & 36.24 & 1.325 & $\$$ & Po & \\
\hline 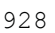 & $\mathrm{C} / \mathrm{z}$ & 9.80 & 28. & 1.325 & $\$$ & & \\
\hline & $\mathrm{C} / \mathrm{z}$ & 82.21 & 20. & 1.325 & $\$$ & & 2 \\
\hline & $\mathrm{C} / \mathrm{z}$ & 3.83 & 12 . & 1.325 & $\$$ & & 3 \\
\hline & $\mathrm{C} / \mathrm{z}$ & 4.65 & 4. & 1.325 & $\$$ & & 3 \\
\hline & $\mathrm{C} / \mathrm{z}$ & 65 & -4.16 & 1.325 & $\$$ & & \\
\hline & $\mathrm{C} / \mathrm{z}$ & 3.83 & -12.44 & 1.325 & $\$$ & Po: & 3 \\
\hline & $\mathrm{C} / \mathrm{z}$ & .21 & -20 . & 1.325 & $\$$ & $\mathrm{Po}$ & 3 \\
\hline & $\mathrm{C} / \mathrm{z}$ & 80 & -28 & 1.325 & $\$$ & Po & 3 \\
\hline & $\mathrm{C} / \mathrm{z}$ & 76.61 & -36.24 & 1.325 & $\$$ & & 3 \\
\hline & $\mathrm{C} / \mathrm{z}$ & 72.69 & -43.57 & 1.325 & $\$$ & Po & 3 \\
\hline & $\mathrm{C} / \mathrm{z}$ & 68.07 & -50 . & 1.325 & $\$$ & & \\
\hline & $\mathrm{C} / \mathrm{z}$ & 62.80 & -56.91 & 1.325 & $\$$ & $\mathrm{Po}$ & 3 \\
\hline & $\mathrm{C} / \mathrm{z}$ & 56.91 & -62.80 & 1.325 & $\$$ & Pos: & 4 \\
\hline & $\mathrm{C} / \mathrm{z}$ & 0.49 & -68.07 & 1.325 & $\$$ & & \\
\hline & $\mathrm{C} / \mathrm{z}$ & 43.57 & -72.69 & 1.325 & $\$$ & Pos & \\
\hline 94 & $\mathrm{C} / \mathrm{z}$ & 36.24 & -76.61 & 1.325 & $\$$ & Posi & 7 \\
\hline 94 & $\mathrm{C} / \mathrm{z}$ & 28.55 & -79.80 & 1.325 & $\$$ & Position & 4 \\
\hline & $\mathrm{C} / \mathrm{z}$ & 20.59 & -82.21 & 1.325 & $\$$ & Posi & \\
\hline & $\mathrm{C} / \mathrm{z}$ & 12.44 & -83.83 & 1.325 & $\$$ & Pos & $I$ \\
\hline 947 & $\mathrm{C} / \mathrm{z}$ & 4.16 & -84.65 & 1.325 & $\$$ & Pos: & 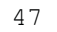 \\
\hline
\end{tabular}

Revision: 1 
NEA/NSC/DOC(2006)1

Gas Cooled (Thermal) Reactor - GCR

PROTEUS-GCR-EXP-004

CRIT-REAC

\begin{tabular}{|c|c|c|c|c|c|c|c|}
\hline 48 & $\mathrm{c} / \mathrm{z}$ & -4.16 & -84.65 & 1.325 & $\$$ & osition & 48 \\
\hline & $\mathrm{C} / \mathrm{z}$ & -12.44 & -83.83 & 1.325 & & Position & \\
\hline & $\mathrm{C} / \mathrm{z}$ & -20.59 & -82.21 & 1.325 & $?$ & Position & 50 \\
\hline & $\mathrm{C} / \mathrm{z}$ & -28.55 & -79.80 & 1.325 & & osition & \\
\hline & $\mathrm{C} / \mathrm{z}$ & -36.24 & -76.61 & 1.325 & & 年- & \\
\hline & $\mathrm{C} / \mathrm{z}$ & -43.57 & -72.69 & 1.3 & & & \\
\hline & $\mathrm{C} / \mathrm{z}$ & -50.49 & -68.07 & . 32 & & S & \\
\hline & $\mathrm{C} / \mathrm{z}$ & -56.91 & -62.80 & 1.3 & s & & \\
\hline & $\mathrm{C} / \mathrm{z}$ & -62.80 & -56.91 & 1.3 & & & \\
\hline & $\mathrm{C} / \mathrm{z}$ & -68.07 & -50.49 & 13 & S & & \\
\hline & $\mathrm{C} / \mathrm{z}$ & -72.69 & -43.57 & 1.32 & $\$$ & n & \\
\hline & $\mathrm{C} / \mathrm{z}$ & -76.61 & -36.24 & 1.32 & $\$$ & & \\
\hline & $\mathrm{C} / \mathrm{z}$ & -79.80 & -28.55 & 1. & S & & \\
\hline & $\mathrm{C} / \mathrm{z}$ & -82.21 & -20 & 1 & \$ & & \\
\hline & $\mathrm{C} / \mathrm{z}$ & -83.83 & -12.44 & 1.3 & $\$$ & & \\
\hline & $\mathrm{C} / \mathrm{z}$ & -84.65 & -4.16 & 1.32 & $\$$ & & \\
\hline & $\mathrm{C} / \mathrm{z}$ & -84.65 & 4.16 & 1.3 & $\$$ & & \\
\hline \\
\hline & & 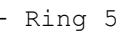 & & & & & \\
\hline & $\mathrm{C} / \mathrm{z}$ & -88 & + & 1.32 & $\$$ & on & \\
\hline & $\mathrm{C} / \mathrm{z}$ & -86 & & 1 . & $\$$ & & \\
\hline & $\mathrm{C} / \mathrm{z}$ & -83.70 & 34 . & 1.3 & $\$$ & $n$ & \\
\hline & $\mathrm{C} / \mathrm{z}$ & -79.90 & 1 & 1 . & $\$$ & & \\
\hline & $\mathrm{C} / \mathrm{z}$ & -75.33 & & 1 . & $\$$ & & \\
\hline & $\mathrm{C} / \mathrm{z}$ & -70 & & 1.3 & $\$$ & & \\
\hline & $\mathrm{C} / \mathrm{z}$ & -64.06 & 64. & 1. & $\$$ & & \\
\hline & $\mathrm{C} / \mathrm{z}$ & -57.48 & 3 & 1 . & S & & \\
\hline & $\mathrm{C} / \mathrm{z}$ & -50.33 & & 1. & $\$$ & & \\
\hline & $\mathrm{C} / \mathrm{z}$ & -42.71 & 0 & 1. & $\$$ & & \\
\hline & $\mathrm{C} / \mathrm{z}$ & -34.67 & 83.70 & 1. & $\$$ & & \\
\hline & $\mathrm{C} / \mathrm{z}$ & -26.30 & & 1. & $\$$ & & \\
\hline & $\mathrm{C} / \mathrm{z}$ & -17.68 & & 1. & $\$$ & & \\
\hline & $\mathrm{C} / \mathrm{z}$ & -8.88 & & 1. & $\$$ & & \\
\hline & $\mathrm{C} / \mathrm{z}$ & .00 & 0 & 1 . & $\$$ & & \\
\hline & $\mathrm{C} / \mathrm{z}$ & .88 & & 1 . & $\$$ & & \\
\hline & $C / z$ & 17.68 & 6 & 1. & $\$$ & & \\
\hline & $\mathrm{C} / \mathrm{z}$ & 26.30 & 0 & 1 . & \$ & & \\
\hline & $c / z$ & 7 & & 1. & $\$$ & & \\
\hline & $\mathrm{C} / \mathrm{z}$ & 42 & & 1 & $\$$ & & \\
\hline & $c / z$ & 33 & 75 & 1 . & $\$$ & & \\
\hline & $\mathrm{C} / \mathrm{z}$ & 57.48 & 70 & 1 . & $\$$ & & \\
\hline & $\mathrm{C} / \mathrm{z}$ & 6 & & 1. & $\$$ & & \\
\hline & $\mathrm{C} / \mathrm{z}$ & 3 & & 1. & $\$$ & & \\
\hline & $\mathrm{C} / \mathrm{z}$ & 75.33 & 50 & 1 . & \$ & & \\
\hline & $\mathrm{C} / \mathrm{z}$ & 0 & & 1 . & $\$$ & & \\
\hline & $\mathrm{C} / \mathrm{z}$ & 0 & & 1. & $\$$ & & \\
\hline & $C / z$ & 0 & 26 & 1 . & $\$$ & & \\
\hline & $\mathrm{C} / \mathrm{z}$ & 88.86 & 17. & 1. & $\$$ & & \\
\hline & $c / z$ & 6 & 8 . & 1. & $\$$ & & \\
\hline & $\mathrm{C} / \mathrm{z}$ & 0 & & & $\$$ & & \\
\hline & $\mathrm{C} / \mathrm{z}$ & 6 & -8 & 1. & $\$$ & & \\
\hline & $\mathrm{C} / \mathrm{z}$ & 88.86 & -17 . & 1. & $\$$ & & \\
\hline & $\mathrm{C} / \mathrm{z}$ & 0 & -26 & 1. & $\$$ & & \\
\hline & $\mathrm{C} / \mathrm{z}$ & 70 & -34 & 1. & $\$$ & & \\
\hline & $\mathrm{C} / \mathrm{z}$ & 79.90 & -42 . & 1. & $\$$ & & \\
\hline & $\mathrm{C} / \mathrm{z}$ & 75.33 & -50.33 & 1 & $\$$ & & \\
\hline & $\mathrm{C} / \mathrm{z}$ & 3 & -57. & 1 & $\$$ & & \\
\hline & $\mathrm{C} / \mathrm{z}$ & 64.06 & -64. & 1. & $\$$ & & \\
\hline & $\mathrm{C} / \mathrm{z}$ & 57.48 & -70 & 1. & $\$$ & & \\
\hline & $\mathrm{C} / \mathrm{z}$ & 50.33 & -75 & & $\$$ & & \\
\hline & $\mathrm{C} / \mathrm{z}$ & 42.71 & -79 & & \$ & & \\
\hline & $\mathrm{C} / \mathrm{z}$ & 34.67 & -83 . & 1.3 & $\$$ & & \\
\hline & $\mathrm{C} / \mathrm{z}$ & 26.30 & -86 & 1 . & $\$$ & & \\
\hline & $\mathrm{C} / \mathrm{z}$ & 17.68 & -88 & 1. & $\$$ & & \\
\hline & $\mathrm{C} / \mathrm{z}$ & 8.88 & -90 . & 1. & $\$$ & & \\
\hline & $\mathrm{C} / \mathrm{z}$ & 0.00 & -90.60 & 1.3 & $\$$ & & \\
\hline & $c / z$ & -8.88 & -90 . & & $\$$ & & \\
\hline & $\mathrm{C} / \mathrm{z}$ & -17.68 & -88 & 1.325 & $\$$ & & \\
\hline & $c / z$ & -26.30 & -86.70 & 1.325 & $\$$ & Pos & \\
\hline & $\mathrm{C} / \mathrm{z}$ & -34.67 & -83 & 1. & $\$$ & & \\
\hline & $\mathrm{C} / \mathrm{z}$ & -42.71 & -79. & 1.325 & $\$$ & & \\
\hline & $\mathrm{C} / \mathrm{z}$ & -50.33 & -75 . & 1.325 & $\$$ & Posi & \\
\hline & $\mathrm{C} / \mathrm{z}$ & -57.48 & -70.03 & 1.325 & $\$$ & Posi & \\
\hline & $\mathrm{C} / \mathrm{z}$ & -64.06 & -64 . & 1.325 & $r$ & & \\
\hline & $\mathrm{C} / \mathrm{z}$ & -70.03 & -57 . & 1.325 & $\$$ & Pos & \\
\hline & $\mathrm{C} / \mathrm{z}$ & -75.33 & -50.33 & 1.325 & $\$$ & Pos & \\
\hline
\end{tabular}

Revision: 1 
NEA/NSC/DOC(2006)1

Gas Cooled (Thermal) Reactor - GCR

PROTEUS-GCR-EXP-004

CRIT-REAC

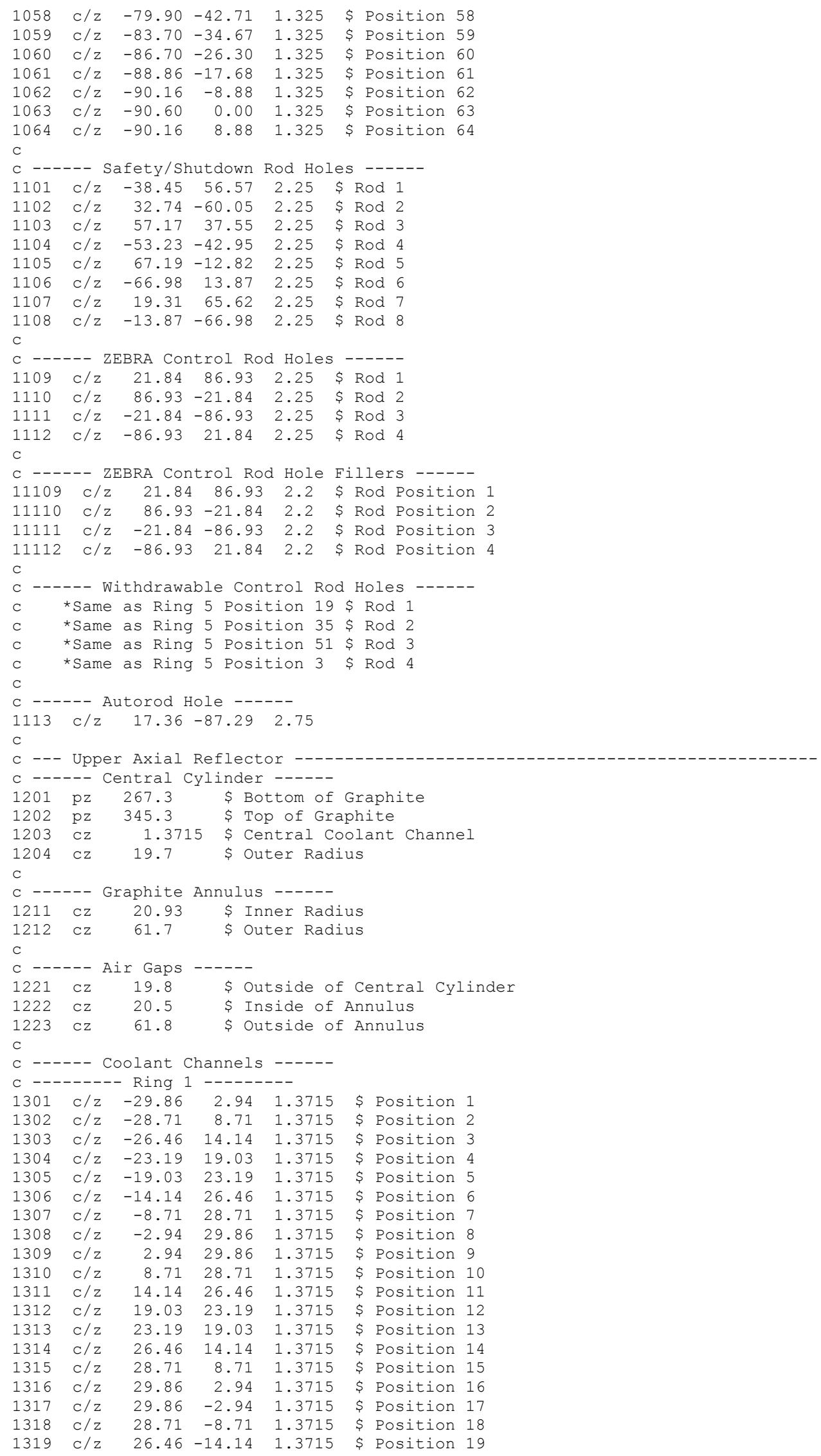

Revision: 1 
NEA/NSC/DOC(2006)1

Gas Cooled (Thermal) Reactor - GCR

PROTEUS-GCR-EXP-004 CRIT-REAC

\begin{tabular}{|c|c|c|c|c|c|c|}
\hline 1320 & $c / z$ & 23.19 & -19.03 & 1.3715 & \$ Position & 20 \\
\hline 1321 & $c / z$ & 19.03 & -23.19 & 1.3715 & \$ Position 2 & 21 \\
\hline 1322 & $\mathrm{c} / \mathrm{z}$ & 14.14 & -26.46 & 1.3715 & \$ Position & 22 \\
\hline 1323 & $c / z$ & 8.71 & -28.71 & 1.3715 & \$ Position & 23 \\
\hline 1324 & $c / z$ & 2.94 & -29.86 & 1.3715 & \$ Position & 24 \\
\hline 1325 & $c / z$ & -2.94 & -29.86 & 1.3715 & \$ Position 2 & 25 \\
\hline 1326 & $\mathrm{c} / \mathrm{z}$ & -8.71 & -28.71 & 1.3715 & \$ Position 2 & 26 \\
\hline 1327 & $\mathrm{c} / \mathrm{z}$ & -14.14 & -26.46 & 1.3715 & \$ Position & 27 \\
\hline 1328 & $c / z$ & -19.03 & -23.19 & 1.3715 & $\$$ Position 2 & 28 \\
\hline 1329 & $\mathrm{c} / \mathrm{z}$ & -23.19 & -19.03 & 1.3715 & \$ Position & 29 \\
\hline 1330 & $c / z$ & -26.46 & -14.14 & 1.3715 & \$ Position & 30 \\
\hline 1331 & $c / z$ & -28.71 & -8.71 & 1.3715 & \$ Position & 31 \\
\hline $\begin{array}{l}1332 \\
\mathrm{C}\end{array}$ & $c / z$ & -29.86 & -2.94 & 1.3715 & $\$$ Position & 32 \\
\hline $\begin{array}{l}1333 \\
c\end{array}$ & $\mathrm{Cz}$ & 32.75 & \$ Ring & Divider & for Modeling & g Simplification \\
\hline C --- & --- & Ring 2 & $2-----$ & --- & & \\
\hline 1401 & $\mathrm{c} / \mathrm{z}$ & -34.82 & 6.93 & 1.3715 & \$ Position 1 & 1 \\
\hline 1402 & $c / z$ & -32.80 & 13.59 & 1.3715 & \$ Position 2 & 2 \\
\hline 1403 & $c / z$ & -29.52 & 19.72 & 1.3715 & \$ Position 3 & 3 \\
\hline 1404 & $\mathrm{c} / \mathrm{z}$ & -25.10 & 25.10 & 1.3715 & \$ Position & 4 \\
\hline 1405 & $c / z$ & -19.72 & 29.52 & 1.3715 & \$ Position & 5 \\
\hline 1406 & $c / z$ & -13.59 & 32.80 & 1.3715 & $\$$ Position & 6 \\
\hline 1407 & $c / z$ & -6.93 & 34.82 & 1.3715 & \$ Position & 7 \\
\hline 1408 & $\mathrm{c} / \mathrm{z}$ & 0.00 & 35.50 & 1.3715 & \$ Position $\varepsilon$ & 8 \\
\hline 1409 & $c / z$ & 6.93 & 34.82 & 1.3715 & \$ Position & 9 \\
\hline 1410 & $c / z$ & 13.59 & 32.80 & 1.3715 & \$ Position 1 & 10 \\
\hline 1411 & $\mathrm{c} / \mathrm{z}$ & 19.72 & 29.52 & 1.3715 & \$ Position & 11 \\
\hline 1412 & $c / z$ & 25.10 & 25.10 & 1.3715 & \$ Position & 12 \\
\hline 1413 & $\mathrm{c} / \mathrm{z}$ & 29.52 & 19.72 & 1.3715 & \$ Position & 13 \\
\hline 1414 & $c / z$ & 32.80 & 13.59 & 1.3715 & \$ Position & 14 \\
\hline 1415 & $c / z$ & 34.82 & 6.93 & 1.3715 & \$ Position & 15 \\
\hline 1416 & $c / z$ & 35.50 & 0.00 & 1.3715 & \$ Position & 16 \\
\hline 1417 & $c / z$ & 34.82 & -6.93 & 1.3715 & \$ Position & 17 \\
\hline 1418 & $c / z$ & 32.80 & -13.59 & 1.3715 & \$ Position & 18 \\
\hline 1419 & $c / z$ & 29.52 & -19.72 & 1.3715 & $\$$ Position & 19 \\
\hline 1420 & $c / z$ & 25.10 & -25.10 & 1.3715 & \$ Position & 20 \\
\hline 1421 & $c / z$ & 19.72 & -29.52 & 1.3715 & \$ Position 2 & 21 \\
\hline 1422 & $c / z$ & 13.59 & -32.80 & 1.3715 & $\$$ Position 2 & 22 \\
\hline 1423 & $c / z$ & 6.93 & -34.82 & 1.3715 & $\$$ Position 2 & 23 \\
\hline 1424 & $c / z$ & 0.00 & -35.50 & 1.3715 & \$ Position 2 & 24 \\
\hline 1425 & $c / z$ & -6.93 & -34.82 & 1.3715 & \$ Position 2 & 25 \\
\hline 1426 & $c / z$ & -13.59 & -32.80 & 1.3715 & $\$$ Position 2 & 26 \\
\hline 1427 & $\mathrm{C} / \mathrm{z}$ & -19.72 & -29.52 & 1.3715 & \$ Position 2 & 27 \\
\hline 1428 & $c / z$ & -25.10 & -25.10 & 1.3715 & \$ Position 2 & 28 \\
\hline 1429 & $\mathrm{c} / \mathrm{z}$ & -29.52 & -19.72 & 1.3715 & \$ Position 2 & 29 \\
\hline 1430 & $c / z$ & -32.80 & -13.59 & 1.3715 & $\$$ Position 3 & 30 \\
\hline 1431 & $\mathrm{c} / \mathrm{z}$ & -34.82 & -6.93 & 1.3715 & \$ Position 3 & 31 \\
\hline $\begin{array}{l}1432 \\
\mathrm{C}\end{array}$ & $c / z$ & -35.50 & 0.00 & 1.3715 & $\$$ Position 3 & 32 \\
\hline $\begin{array}{l}1433 \\
C\end{array}$ & $\mathrm{Cz}$ & 38.25 & \$ Ring & Divider & for Modeling & g Simplification \\
\hline$C---$ & ----- & - Ring 3 & $3-----$ & --- & & \\
\hline 1501 & $\mathrm{C} / \mathrm{z}$ & -39.23 & 11.90 & 1.3715 & \$ Position 1 & 1 \\
\hline 1502 & $c / z$ & -36.16 & 19.33 & 1.3715 & \$ Position 2 & 2 \\
\hline 1503 & $c / z$ & -31.69 & 26.01 & 1.3715 & $\$$ Position 3 & 3 \\
\hline 1504 & $c / z$ & -26.01 & 31.69 & 1.3715 & \$ Position & 4 \\
\hline 1505 & $\mathrm{c} / \mathrm{z}$ & -19.33 & 36.16 & 1.3715 & \$ Position 5 & 5 \\
\hline 1506 & $c / z$ & $-11 \cdot 90$ & 39.23 & 1.3715 & $\$$ Position 6 & 6 \\
\hline 1507 & $c / z$ & -4.02 & 40.80 & 1.3715 & \$ Position 7 & 7 \\
\hline 1508 & $c / z$ & 4.02 & 40.80 & 1.3715 & $\$$ Position & 8 \\
\hline 1509 & $c / z$ & 11.90 & 39.23 & 1.3715 & \$ Position & 9 \\
\hline 1510 & $c / z$ & 19.33 & 36.16 & 1.3715 & \$ Position 1 & 10 \\
\hline 1511 & $c / z$ & 26.01 & 31.69 & 1.3715 & \$ Position 1 & 11 \\
\hline 1512 & $c / z$ & 31.69 & 26.01 & 1.3715 & $\$$ Position 1 & 12 \\
\hline 1513 & $\mathrm{c} / \mathrm{z}$ & 36.16 & 19.33 & 1.3715 & \$ Position 1 & 13 \\
\hline 1514 & $c / z$ & 39.23 & 11.90 & 1.3715 & \$ Position 1 & 14 \\
\hline 1515 & $\mathrm{c} / \mathrm{z}$ & 40.80 & 4.02 & 1.3715 & \$ Position 1 & 15 \\
\hline 1516 & $c / z$ & 40.80 & -4.02 & 1.3715 & \$ Position 1 & 16 \\
\hline 1517 & $c / z$ & 39.23 & $-11 \cdot 90$ & 1.3715 & \$ Position 1 & 17 \\
\hline 1518 & $c / z$ & 36.16 & -19.33 & 1.3715 & \$ Position 1 & 18 \\
\hline 1519 & $c / z$ & 31.69 & -26.01 & 1.3715 & \$ Position 1 & 19 \\
\hline 1520 & $c / z$ & 26.01 & -31.69 & 1.3715 & \$ Position 2 & 20 \\
\hline 1521 & $c / z$ & 19.33 & -36.16 & 1.3715 & \$ Position 2 & 21 \\
\hline 1522 & $c / z$ & 11.90 & -39.23 & 1.3715 & \$ Position 2 & 22 \\
\hline 1523 & $c / z$ & 4.02 & -40.80 & 1.3715 & \$ Position 2 & 23 \\
\hline
\end{tabular}

Revision: 1 
NEA/NSC/DOC(2006)1

Gas Cooled (Thermal) Reactor - GCR

PROTEUS-GCR-EXP-004 CRIT-REAC

\begin{tabular}{|c|c|c|c|c|c|c|}
\hline 1524 & $\mathrm{C} / \mathrm{z}$ & -4.02 & -40.80 & 1.3715 & \$ Position 24 & \\
\hline 1525 & $\mathrm{C} / \mathrm{z}$ & $-11 \cdot 90$ & -39.23 & 1.3715 & \$ Position 25 & \\
\hline 1526 & $\mathrm{C} / \mathrm{z}$ & -19.33 & -36.16 & 1.3715 & \$ Position 26 & \\
\hline 1527 & $\mathrm{C} / \mathrm{z}$ & -26.01 & -31.69 & 1.3715 & \$ Position 27 & \\
\hline 1528 & $\mathrm{C} / \mathrm{z}$ & -31.69 & -26.01 & 1.3715 & \$ Position 28 & \\
\hline 529 & $\mathrm{C} / \mathrm{z}$ & -36.16 & -19.33 & 1.3715 & \$ Position 29 & \\
\hline 530 & $c / z$ & -39.23 & -11.90 & 1.3715 & \$ Position 30 & \\
\hline 31 & $\mathrm{C} / \mathrm{z}$ & -40.80 & -4.02 & 1.3715 & \$ Position 31 & \\
\hline 532 & $\mathrm{C} / \mathrm{z}$ & -40.80 & 4.02 & 1.3715 & \$ Position 32 & \\
\hline & & & & & & \\
\hline & $\mathrm{Cz}$ & 43.625 & \$ Ring & 9 Divider & for Modeling & Simplification \\
\hline & & & & & & \\
\hline & ---- & Ring 4 & $4-----$ & --- & & \\
\hline 601 & $\mathrm{C} / \mathrm{z}$ & -42.73 & 17.70 & 1.3715 & \$ Position 1 & \\
\hline 2 & $\mathrm{C} / \mathrm{z}$ & -38.46 & 25.70 & 1.3715 & \$ Position 2 & \\
\hline 603 & $\mathrm{C} / \mathrm{z}$ & -32.70 & 32.70 & 1.3715 & $\$$ Position 3 & \\
\hline 604 & $\mathrm{C} / \mathrm{z}$ & -25.70 & 38.46 & 1.3715 & \$ Position 4 & \\
\hline 505 & $\mathrm{C} / \mathrm{z}$ & -17.70 & 42.73 & 1.3715 & \$ Position 5 & \\
\hline 506 & $\mathrm{C} / \mathrm{z}$ & -9.02 & 45.36 & 1.3715 & \$ Position 6 & \\
\hline 607 & $\mathrm{C} / \mathrm{z}$ & 0.00 & 46.25 & 1.3715 & \$ Position 7 & \\
\hline 608 & $\mathrm{C} / \mathrm{z}$ & 9.02 & 45.36 & 1.3715 & \$ Position 8 & \\
\hline 509 & $\mathrm{C} / \mathrm{z}$ & 17.70 & 42.73 & 1.3715 & \$ Position 9 & \\
\hline 610 & $\mathrm{C} / \mathrm{z}$ & 25.70 & 38.46 & 1.3715 & \$ Position 10 & \\
\hline 611 & $\mathrm{C} / \mathrm{z}$ & 32.70 & 32.70 & 1.3715 & \$ Position 11 & \\
\hline 1612 & $\mathrm{C} / \mathrm{z}$ & 38.46 & 25.70 & 1.3715 & \$ Position 12 & \\
\hline 1613 & $\mathrm{C} / \mathrm{z}$ & 42.73 & 17.70 & 1.3715 & \$ Position 13 & \\
\hline 1614 & $\mathrm{C} / \mathrm{z}$ & 45.36 & 9.02 & 1.3715 & \$ Position 14 & \\
\hline 1615 & $\mathrm{C} / \mathrm{z}$ & 46.25 & 0.00 & 1.3715 & \$ Position 15 & \\
\hline 1616 & $\mathrm{C} / \mathrm{z}$ & 45.36 & -9.02 & 1.3715 & \$ Position 16 & \\
\hline 1617 & $\mathrm{C} / \mathrm{z}$ & 42.73 & -17.70 & 1.3715 & \$ Position 17 & \\
\hline 1618 & $\mathrm{C} / \mathrm{z}$ & 38.46 & -25.70 & 1.3715 & \$ Position 18 & \\
\hline 1619 & $\mathrm{C} / \mathrm{z}$ & 32.70 & -32.70 & 1.3715 & \$ Position 19 & \\
\hline 1620 & $\mathrm{C} / \mathrm{z}$ & 25.70 & -38.46 & 1.3715 & \$ Position 20 & \\
\hline 1621 & $\mathrm{C} / \mathrm{z}$ & 17.70 & -42.73 & 1.3715 & \$ Position 21 & \\
\hline 1622 & $\mathrm{C} / \mathrm{z}$ & 9.02 & -45.36 & 1.3715 & \$ Position 22 & \\
\hline 1623 & $\mathrm{C} / \mathrm{z}$ & 0.00 & -46.25 & 1.3715 & \$ Position 23 & \\
\hline 1624 & $\mathrm{C} / \mathrm{z}$ & -9.02 & -45.36 & 1.3715 & \$ Position 24 & \\
\hline 1625 & $\mathrm{C} / \mathrm{z}$ & -17.70 & -42.73 & 1.3715 & \$ Position 25 & \\
\hline 1626 & $\mathrm{C} / \mathrm{z}$ & -25.70 & -38.46 & 1.3715 & \$ Position 26 & \\
\hline 1627 & $\mathrm{C} / \mathrm{z}$ & -32.70 & -32.70 & 1.3715 & \$ Position 27 & \\
\hline 1628 & $\mathrm{C} / \mathrm{z}$ & -38.46 & -25.70 & 1.3715 & \$ Position 28 & \\
\hline 1629 & $\mathrm{C} / \mathrm{z}$ & -42.73 & -17.70 & 1.3715 & \$ Position 29 & \\
\hline 1630 & $\mathrm{C} / \mathrm{z}$ & -45.36 & -9.02 & 1.3715 & \$ Position 30 & \\
\hline 1631 & $\mathrm{C} / \mathrm{z}$ & -46.25 & 0.00 & 1.3715 & \$ Position 31 & \\
\hline 1632 & $\mathrm{C} / \mathrm{z}$ & -45.36 & 9.02 & 1.3715 & \$ Position 32 & \\
\hline & & & & & & \\
\hline 1633 & $\mathrm{Cz}$ & 48.875 & \$ Ring & g Divider & for Modeling & Simplification \\
\hline C & & & & & & \\
\hline$C---$ & ---- & - Ring 5 & $5-----$ & --- & & \\
\hline 1701 & $\mathrm{C} / \mathrm{z}$ & -45.42 & 24.28 & 1.3715 & \$ Position 1 & \\
\hline 1702 & $\mathrm{C} / \mathrm{z}$ & -39.81 & 32.67 & 1.3715 & \$ Position 2 & \\
\hline 1703 & $\mathrm{C} / \mathrm{z}$ & -32.67 & 39.81 & 1.3715 & $\$$ Position 3 & \\
\hline 1704 & $\mathrm{C} / \mathrm{z}$ & -24.28 & 45.42 & 1.3715 & \$ Position 4 & \\
\hline 1705 & $\mathrm{C} / \mathrm{z}$ & -14.95 & 49.28 & 1.3715 & \$ Position 5 & \\
\hline 1706 & $\mathrm{C} / \mathrm{z}$ & -5.05 & 51.25 & 1.3715 & \$ Position 6 & \\
\hline 1707 & $\mathrm{C} / \mathrm{z}$ & 5.05 & 51.25 & 1.3715 & \$ Position 7 & \\
\hline 1708 & $\mathrm{C} / \mathrm{z}$ & 14.95 & 49.28 & 1.3715 & \$ Position 8 & \\
\hline 1709 & $\mathrm{C} / \mathrm{z}$ & 24.28 & 45.42 & 1.3715 & \$ Position 9 & \\
\hline 1710 & $\mathrm{C} / \mathrm{z}$ & 32.67 & 39.81 & 1.3715 & \$ Position 10 & \\
\hline 1711 & $\mathrm{C} / \mathrm{z}$ & 39.81 & 32.67 & 1.3715 & \$ Position 11 & \\
\hline 1712 & $\mathrm{C} / \mathrm{z}$ & 45.42 & 24.28 & 1.3715 & \$ Position 12 & \\
\hline 1713 & $\mathrm{C} / \mathrm{z}$ & 49.28 & 14.95 & 1.3715 & \$ Position 13 & \\
\hline 1714 & $\mathrm{C} / \mathrm{z}$ & 51.25 & 5.05 & 1.3715 & \$ Position 14 & \\
\hline 1715 & $\mathrm{C} / \mathrm{z}$ & 51.25 & -5.05 & 1.3715 & \$ Position 15 & \\
\hline 1716 & $\mathrm{C} / \mathrm{z}$ & 49.28 & -14.95 & 1.3715 & \$ Position 16 & \\
\hline 1717 & $\mathrm{C} / \mathrm{z}$ & 45.42 & -24.28 & 1.3715 & \$ Position 17 & \\
\hline 1718 & $\mathrm{C} / \mathrm{z}$ & 39.81 & -32.67 & 1.3715 & \$ Position 18 & \\
\hline 1719 & $\mathrm{C} / \mathrm{z}$ & 32.67 & -39.81 & 1.3715 & \$ Position 19 & \\
\hline 1720 & $\mathrm{c} / \mathrm{z}$ & 24.28 & -45.42 & 1.3715 & \$ Position 20 & \\
\hline 1721 & $\mathrm{C} / \mathrm{z}$ & 14.95 & -49.28 & 1.3715 & \$ Position 21 & \\
\hline 1722 & $\mathrm{C} / \mathrm{z}$ & 5.05 & -51.25 & 1.3715 & \$ Position 22 & \\
\hline 1723 & $\mathrm{C} / \mathrm{z}$ & -5.05 & -51.25 & 1.3715 & \$ Position 23 & \\
\hline 1724 & $\mathrm{C} / \mathrm{z}$ & -14.95 & -49.28 & 1.3715 & \$ Position 24 & \\
\hline 1725 & $\mathrm{C} / \mathrm{z}$ & -24.28 & -45.42 & 1.3715 & \$ Position 25 & \\
\hline 1726 & $\mathrm{C} / \mathrm{z}$ & -32.67 & -39.81 & 1.3715 & \$ Position 26 & \\
\hline 1727 & $\mathrm{C} / \mathrm{z}$ & -39.81 & -32.67 & 1.3715 & \$ Position 27 & \\
\hline
\end{tabular}

Revision: 1 
NEA/NSC/DOC(2006)1

\section{Gas Cooled (Thermal) Reactor - GCR \\ PROTEUS-GCR-EXP-004 CRIT-REAC}

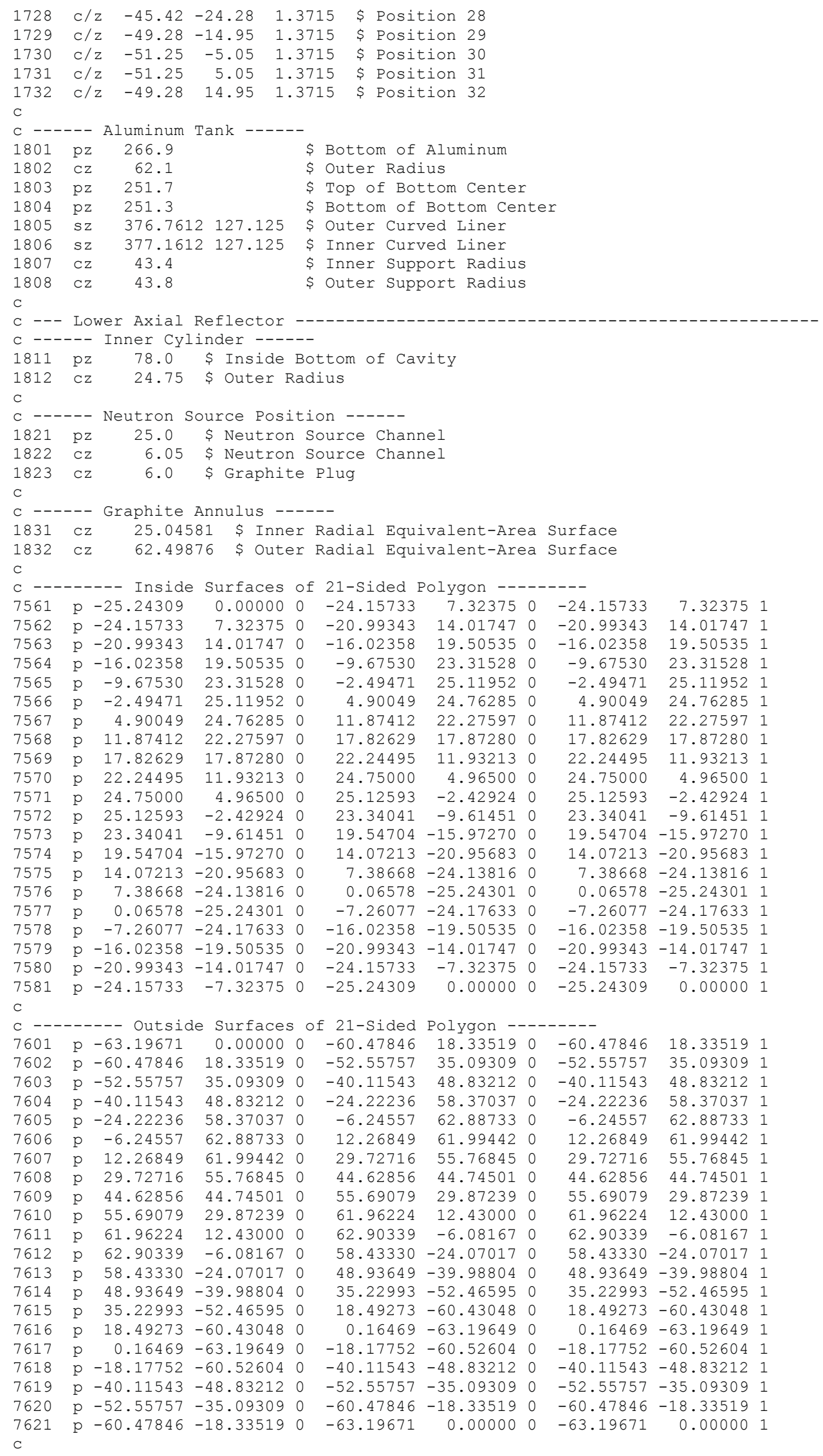

Revision: 1 
NEA/NSC/DOC(2006)1

Gas Cooled (Thermal) Reactor - GCR

PROTEUS-GCR-EXP-004

CRIT-REAC

\begin{tabular}{|c|c|c|c|c|c|c|c|}
\hline 01 & $\mathrm{C} / \mathrm{z}$ & -29.86 & 2.94 & 1.371 & $\$$ & Position & 1 \\
\hline & $\mathrm{C} / \mathrm{z}$ & -28.71 & 8.71 & 1.371 & $\$$ & Position & . \\
\hline 0. & $c / z$ & -26.46 & 14.14 & 1.371 & $\$$ & Position & 3 \\
\hline 04 & $\mathrm{C} / \mathrm{z}$ & -23.19 & 19.03 & 1.371 & $\$$ & Position & 4 \\
\hline & $\mathrm{C} / \mathrm{z}$ & -19.03 & 23.19 & 1.371 & $\$$ & Position & J \\
\hline & $\mathrm{C} / \mathrm{z}$ & -14 & 26.46 & 1.371 & $\$$ & Position & 6 \\
\hline 7 & $\mathrm{c} / \mathrm{z}$ & -8.71 & 28.71 & 1.371 & $\$$ & Position & 7 \\
\hline 8 & $\mathrm{C} / \mathrm{z}$ & -2.94 & 29.86 & 1.371 & $\$$ & Position & 8 \\
\hline 09 & $\mathrm{C} / \mathrm{z}$ & 2.94 & 29.86 & 1.371 & $\$$ & Position & 9 \\
\hline 0 & $c / z$ & 8.71 & 28.71 & 1.371 & $\$$ & tion & 10 \\
\hline 11 & $\mathrm{C} / \mathrm{z}$ & 14.14 & 26.46 & 1.371 & $\$$ & ion & 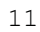 \\
\hline 12 & $\mathrm{c} / \mathrm{z}$ & & 23.19 & 1.371 & $\$$ & Lon & 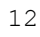 \\
\hline 13 & $\mathrm{C} / \mathrm{z}$ & 9 & 19.03 & 1.371 & $\$$ & ion & 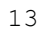 \\
\hline 4 & $\mathrm{c} / \mathrm{z}$ & 26.46 & 14.14 & 1.371 & $\$$ & ion & 14 \\
\hline 15 & $\mathrm{C} / \mathrm{z}$ & 28.71 & 8.71 & 1.371 & $\$$ & ion & 15 \\
\hline 16 & $\mathrm{c} / \mathrm{z}$ & 29.86 & 2.94 & 1.371 & $\$$ & ion & 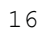 \\
\hline 17 & $\mathrm{C} / \mathrm{z}$ & 29.86 & -2.94 & 1. & $\$$ & ion & 17 \\
\hline 18 & $\mathrm{C} / \mathrm{z}$ & 28.71 & -8 . & 1.371 & $\$$ & ion & 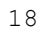 \\
\hline 19 & $\mathrm{C} / \mathrm{z}$ & 26.46 & -14.14 & 1.371 & \$ & ion & \\
\hline 20 & $\mathrm{C} / \mathrm{z}$ & & -19.03 & 1.3 & $\$$ & on & 20 \\
\hline 21 & $c / z$ & 03 & -23.19 & 1. & $\$$ & ion & 21 \\
\hline 22 & $\mathrm{C} / \mathrm{z}$ & 14.14 & -26.46 & 1.371 & $\$$ & ion & 22 \\
\hline 23 & $\mathrm{C} / \mathrm{z}$ & .71 & -28.71 & 1. & $\$$ & on & 20 \\
\hline 24 & $\mathrm{C} / \mathrm{z}$ & 2.94 & -29. & & $\$$ & ion & 24 \\
\hline 25 & $\mathrm{C} / \mathrm{z}$ & -2.94 & -29.86 & 1. & $\$$ & ion & 25 \\
\hline 26 & $\mathrm{C} / \mathrm{z}$ & -8.71 & -28.71 & 1.371 & $\$$ & ion & 26 \\
\hline 27 & $\mathrm{C} / \mathrm{z}$ & -14.14 & -26.46 & & $\$$ & ion & 2 \\
\hline 28 & $\mathrm{C} / \mathrm{z}$ & -19 & -23 & 1. & $\$$ & ion & 20 \\
\hline 29 & $\mathrm{C} / \mathrm{z}$ & -23.19 & -19.03 & 1.371 & $\$$ & ion & 29 \\
\hline 30 & $\mathrm{C} / \mathrm{z}$ & -26.46 & -14.14 & 1. & $\$$ & on & 0 \\
\hline 31 & $\mathrm{C} / \mathrm{z}$ & -28.71 & -8.71 & & $\$$ & on & 31 \\
\hline 932 & $\mathrm{C} / \mathrm{z}$ & -29.86 & -2.94 & 1.371 & $\$$ & ion & 32 \\
\hline \\
\hline \multicolumn{8}{|c|}{ C -------- Ring 2 -------- } \\
\hline 01 & $\mathrm{c} / \mathrm{z}$ & -34.82 & 6.93 & 1.371 & $\$$ & ion & 1 \\
\hline 02 & $\mathrm{C} / \mathrm{z}$ & -32.80 & 13.59 & 1. & $\$$ & ion & 2 \\
\hline 03 & $\mathrm{c} / \mathrm{z}$ & -29.52 & 19.72 & 1.371 & $\$$ & ion & 3 \\
\hline 04 & $\mathrm{C} / \mathrm{z}$ & -25.10 & 25.10 & 1 . & $\$$ & ion & 4 \\
\hline 05 & $\mathrm{c} / \mathrm{z}$ & -19.72 & 29.52 & 1. & $\$$ & ion & 5 \\
\hline 06 & $\mathrm{C} / \mathrm{z}$ & -13.59 & 32.80 & 1.371 & $\$$ & ion & 6 \\
\hline 07 & $\mathrm{C} / \mathrm{z}$ & -6.93 & 34.82 & 1.371 & $\$$ & ion & 7 \\
\hline 08 & $\mathrm{c} / \mathrm{z}$ & 0.00 & 35.50 & 1. & $\$$ & ion & 8 \\
\hline 09 & $\mathrm{C} / \mathrm{z}$ & 6.93 & 34 & 1 . & $\$$ & ion & 9 \\
\hline 10 & $\mathrm{C} / \mathrm{z}$ & 13.59 & 32.80 & 1.371 & $\$$ & Lon & 10 \\
\hline 11 & $\mathrm{C} / \mathrm{z}$ & 72 & 29. & 1. & $\$$ & ion & 11 \\
\hline 12 & $\mathrm{C} / \mathrm{z}$ & 0 & 25 & 1. & $\$$ & on & 12 \\
\hline 13 & $\mathrm{c} / \mathrm{z}$ & 29.52 & 19.72 & 1.371 & $\$$ & ion & 13 \\
\hline 14 & $c / z$ & 32.80 & 13. & 1.371 & $\$$ & ion & 14 \\
\hline 015 & $\mathrm{C} / \mathrm{z}$ & 82 & 6.93 & 1.371 & $\$$ & ion & 15 \\
\hline 01 & $c / z$ & 50 & 0 . & 1. & $\$$ & ion & 16 \\
\hline 17 & $\mathrm{C} / \mathrm{z}$ & .82 & -6.93 & 1.371 & $\$$ & ion & 17 \\
\hline 18 & $c / z$ & .80 & -13 . & 1 . & $\$$ & ion & 18 \\
\hline 19 & $c / z$ & 52 & -19. & 1. & $\$$ & on & 19 \\
\hline 020 & $c / z$ & 10 & -25.10 & 1.371 & $\$$ & ion & 20 \\
\hline 021 & $c / z$ & 19.72 & -29 . & 1.371 & $\$$ & ion & 21 \\
\hline 022 & $\mathrm{C} / \mathrm{z}$ & 13.59 & -32 . & 1.371 & $\$$ & ion & 22 \\
\hline 23 & $c / z$ & 6.93 & -34 . & 1.371 & $\$$ & ion & 23 \\
\hline 024 & $\mathrm{C} / \mathrm{z}$ & 0.00 & -35.50 & 1.371 & $\$$ & ion & 24 \\
\hline 025 & $\mathrm{c} / \mathrm{z}$ & -6.93 & -34.82 & 1.371 & $\$$ & ion & 25 \\
\hline 2026 & $\mathrm{C} / \mathrm{z}$ & -13.59 & -32.80 & 1.371 & $\$$ & ion & 26 \\
\hline 2027 & $\mathrm{C} / \mathrm{z}$ & -19.72 & -29.52 & 1.371 & $\$$ & Eion & 27 \\
\hline 28 & $\mathrm{C} / \mathrm{z}$ & -25.10 & -25 . & 1.371 & $\$$ & ion & 28 \\
\hline 2029 & $\mathrm{C} / \mathrm{z}$ & -29.52 & -19.72 & 1.371 & $\$$ & ion & 29 \\
\hline 2030 & $\mathrm{C} / \mathrm{z}$ & -32.80 & -13.59 & 1.371 & $\$$ & Position & 30 \\
\hline 2031 & $\mathrm{C} / \mathrm{z}$ & -34.82 & -6.93 & 1.371 & $\$$ & Position & 31 \\
\hline 2032 & $\mathrm{C} / \mathrm{z}$ & -35.50 & 0.00 & 1.371 & $\$$ & Position & 32 \\
\hline \multirow{2}{*}{\multicolumn{8}{|c|}{ C }} \\
\hline & & & & & & & \\
\hline & $\mathrm{C} / \mathrm{z}$ & 23 & 11.9 & 1.3 & $\$$ & & 1 \\
\hline 2102 & $\mathrm{c} / \mathrm{z}$ & -36.16 & 19.33 & 1.371 & $\$$ & Position & \\
\hline 2103 & $\mathrm{C} / \mathrm{z}$ & -31.69 & 26.01 & 1.371 & $\$$ & Position & 3 \\
\hline & $\mathrm{C} / \mathrm{z}$ & -26.01 & 31.69 & 1.371 & $\$$ & Position & 4 \\
\hline & $\mathrm{C} / \mathrm{z}$ & -19.33 & 36.16 & 1.371 & $\$$ & Position & \\
\hline 106 & $\mathrm{C} / \mathrm{z}$ & -11.90 & 39.23 & 1.371 & $\$$ & Position & 0 \\
\hline
\end{tabular}

Revision: 1 
NEA/NSC/DOC(2006)1

Gas Cooled (Thermal) Reactor - GCR

PROTEUS-GCR-EXP-004

CRIT-REAC

\begin{tabular}{|c|c|c|c|c|c|c|c|}
\hline 07 & $\mathrm{C} / \mathrm{z}$ & -4.02 & 40.80 & 1.371 & $\$$ & Position & 7 \\
\hline 08 & $\mathrm{C} / \mathrm{z}$ & 4.02 & 40.80 & 1.371 & ? & Position & 8 \\
\hline & $\mathrm{C} / \mathrm{z}$ & 11.90 & 39.23 & 1.371 & \$ & Position & \\
\hline & $c / z$ & 19.33 & 36.16 & .371 & & Pos & \\
\hline & $\mathrm{C} / \mathrm{z}$ & 26.01 & 31.69 & 1.371 & r & Pos: & \\
\hline & $\mathrm{C} / \mathrm{z}$ & 31.69 & 26.01 & 1.371 & & Pos: & \\
\hline & $\mathrm{C} / \mathrm{z}$ & 36.16 & 19.33 & 1.371 & & & \\
\hline & $\mathrm{C} / \mathrm{z}$ & 39.23 & 11.90 & 371 & & & \\
\hline & $c / z$ & 40.80 & 4.02 & 1.371 & & Po & \\
\hline & $\mathrm{C} / \mathrm{z}$ & 40.80 & -4.02 & 1.371 & & & \\
\hline & $c / z$ & 39.23 & $-11 \cdot 90$ & 1.371 & & & \\
\hline & $\mathrm{C} / \mathrm{z}$ & 36.16 & 19. & .371 & S & & \\
\hline & $\mathrm{C} / \mathrm{z}$ & 31.69 & -26. & 1.371 & $\$$ & & \\
\hline & $\mathrm{C} / \mathrm{z}$ & 26.01 & -31 . & 1.371 & & & \\
\hline & $C / z$ & 19.33 & -36 & 1371 & & 2 & \\
\hline & $c / z$ & 11.90 & -39. & .371 & \$ & & \\
\hline & $\mathrm{C} / \mathrm{z}$ & 4.02 & -40 . & .371 & \$ & & \\
\hline & $C / z$ & -4.02 & -40 . & .371 & 5 & $\mathrm{n}$ & 24 \\
\hline & $\mathrm{C} / \mathrm{z}$ & 11.90 & -39. & .371 & $\$$ & & \\
\hline & $\mathrm{C} / \mathrm{z}$ & 19.33 & -36 & .371 & \$ & Po & \\
\hline & $\mathrm{C} / \mathrm{z}$ & -26.01 & -31 & 1.371 & $\$$ & Po & \\
\hline & $c / z$ & -31.69 & -26 & 1.371 & $\$$ & $n$ & c \\
\hline & $\mathrm{C} / \mathrm{z}$ & -36 & -19 & & $\$$ & & \\
\hline & $\mathrm{C} / \mathrm{z}$ & -39.23 & -11. & 1.371 & \$ & Po & \\
\hline & $C / z$ & -40.80 & -4 & .371 & 5 & $n$ & \\
\hline & $\mathrm{C} / \mathrm{z}$ & -40.80 & 4. & 1.371 & $\$$ & & \\
\hline \\
\hline & & 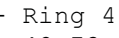 & & & & & \\
\hline & $c / z$ & -42.73 & 17. & 1.371 & $\$$ & on & \\
\hline & $C / z$ & -38.46 & 25 & & $\$$ & & \\
\hline & $c / z$ & -32.70 & 32. & 1.371 & \$ & Po & \\
\hline & $C / z$ & -25.70 & 38. & 1.371 & $\$$ & $\mathrm{Po}$ & 4 \\
\hline & $C / z$ & -17.70 & 42 & 1 . & $\$$ & on & \\
\hline & $\mathrm{C} / \mathrm{z}$ & -9.02 & 45 & 1 . & $\$$ & & 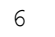 \\
\hline & $C / z$ & 0.00 & 46.25 & 1.371 & $\$$ & Po & 7 \\
\hline & $c / z$ & 9.02 & 45. & 1.371 & $\$$ & n & \\
\hline & $C / z$ & 17.70 & 42. & 1. & $\$$ & & ( \\
\hline & $c / z$ & 25.70 & 38 & 1 . & \$ & & \\
\hline & $c / z$ & 32.70 & 32 . & 1 . & 3 & $\mathrm{Po}$ & - \\
\hline & $c / z$ & 38.46 & 25 & 1 & $\$$ & $\mathrm{n}$ & 2 \\
\hline & $c / z$ & 42.73 & 17. & 1 . & $\$$ & & \\
\hline & $C / z$ & 45.36 & 9. & 1. & $\$$ & & 14 \\
\hline & $c / z$ & 46.25 & 0 . & 1.3 & $\$$ & & 5 \\
\hline & $C / z$ & 45.36 & -9. & 1.371 & $\$$ & & \\
\hline & $C / z$ & 42.73 & -17. & 1 & $\$$ & & \\
\hline & $\mathrm{C} / \mathrm{z}$ & 38.46 & -25 . & 71 & $\$$ & & 18 \\
\hline & $C / z$ & 32.70 & -32.70 & 1.371 & $\$$ & & 19 \\
\hline 20 & $\mathrm{C} / \mathrm{z}$ & 25.70 & -38 & 1.371 & $\$$ & & 20 \\
\hline & $c / z$ & 17.70 & -42 . & & \$ & $\mathrm{Po}$ & 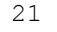 \\
\hline 22 & $C / z$ & 9.02 & -45 . & 1.371 & \$ & & 22 \\
\hline 3 & $c / z$ & 0.00 & -46 & 1.371 & \$ & $\mathrm{Pc}$ & 23 \\
\hline & $c / z$ & -9 & -45 & 1 & $\$$ & & 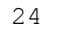 \\
\hline & $\mathrm{C} / \mathrm{z}$ & -17.70 & -42 . & 1.371 & $\$$ & $\mathrm{n}$ & 3 \\
\hline 26 & $c / z$ & -25.70 & -38 & 1.371 & $\$$ & & 26 \\
\hline 227 & $C / z$ & -32.70 & -32 . & 1.371 & $\$$ & & 27 \\
\hline & $c / z$ & -38.46 & -25 . & & \$ & & $2 \zeta$ \\
\hline & $C / z$ & -42.73 & -17 . & & $\$$ & & \\
\hline 30 & $c / z$ & -45.36 & -9 . & 1 & $\$$ & & 30 \\
\hline & $c / z$ & -46 & & & $\$$ & & 31 \\
\hline 2232 & $c / z$ & -45.36 & 9. & 1. & $\$$ & Po & \\
\hline \multicolumn{8}{|l|}{ C } \\
\hline & & Ring 5 & & - & & & \\
\hline & $c / z$ & -45.42 & 24. & & & & \\
\hline & $\mathrm{C} / \mathrm{z}$ & -39.81 & 32 . & & s & Po & \\
\hline & $c / z$ & -32.67 & 39. & 71 & \$ & Po & 3 \\
\hline & $C / z$ & -24.28 & 45. & 1. & $\$$ & Pos & t \\
\hline & $c / z$ & -14.95 & 49. & 1.371 & $\$$ & Pos & 5 \\
\hline & $\mathrm{C} / \mathrm{z}$ & -5.05 & 51. & 1 . & $\$$ & Po: & 6 \\
\hline & $c / z$ & & 51. & 1. & $\$$ & Pos & 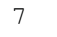 \\
\hline & $c / z$ & 14.95 & 49.28 & 1.371 & $\$$ & Pos & 8 \\
\hline & $c / z$ & 24.28 & 45.42 & 1.371 & \$ & Pos & 0 \\
\hline & $c / z$ & 32.67 & 39. & 1.371 & $\$$ & Pos & \\
\hline & $\mathrm{C} / \mathrm{z}$ & 39.81 & 32.67 & 1.371 & $\$$ & Pos & 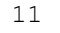 \\
\hline & $c / z$ & 45.42 & 24.28 & 1.371 & $\$$ & Pos & \\
\hline & $C / z$ & 49.28 & 14. & & & Po: & \\
\hline & $c / z$ & 51.25 & 5. & 1.371 & $T$ & Pos & \\
\hline
\end{tabular}

Revision: 1 
NEA/NSC/DOC(2006)1

Gas Cooled (Thermal) Reactor - GCR

PROTEUS-GCR-EXP-004

CRIT-REAC

\begin{tabular}{|c|c|c|c|c|c|c|c|}
\hline 15 & $\mathrm{C} / \mathrm{z}$ & 51.25 & -5.05 & 1.371 & $\$$ & sition & \\
\hline$\perp$ & $\mathrm{C} / \mathrm{z}$ & 49.28 & -14.95 & 1.371 & S & Position & \\
\hline & $\mathrm{C} / \mathrm{z}$ & 5.42 & -24.28 & 1.371 & \$ & Position & \\
\hline & $\mathrm{c} / \mathrm{z}$ & 9.81 & -32.67 & .371 & & Position & \\
\hline & $\mathrm{c} / \mathrm{z}$ & 32.67 & -39.81 & 1.371 & $\tau$ & Position & \\
\hline & $\mathrm{C} / \mathrm{z}$ & 24.28 & -45.42 & 1.371 & & Posit & \\
\hline & $\mathrm{C} / \mathrm{z}$ & 4.95 & -49.28 & 1.371 & & & \\
\hline & $\mathrm{C} / \mathrm{z}$ & 5.05 & -51.25 & .371 & & & \\
\hline & $\mathrm{C} / \mathrm{z}$ & -5.05 & -51.25 & 1.371 & & Po & \\
\hline & $\mathrm{C} / \mathrm{z}$ & 14.95 & -49.28 & 1.371 & & & \\
\hline & $\mathrm{c} / \mathrm{z}$ & 24.28 & -45.42 & 1.371 & & & \\
\hline & $\mathrm{c} / \mathrm{z}$ & 32.67 & -39 . & 1.371 & $\$$ & & \\
\hline & $\mathrm{C} / \mathrm{z}$ & -39.81 & -32 & 1.371 & \$ & & \\
\hline & $\mathrm{c} / \mathrm{z}$ & -45.42 & -24 & 1.371 & \$ & & \\
\hline & $\mathrm{C} / \mathrm{z}$ & -49.28 & -14.95 & 1.371 & $r$ & & \\
\hline & $\mathrm{C} / \mathrm{z}$ & -51.25 & -5 . & 1.371 & \$ & & \\
\hline & $\mathrm{C} / \mathrm{z}$ & -51.25 & 5. & 1.371 & \$ & & \\
\hline & $\mathrm{C} / \mathrm{z}$ & -49.28 & 14.95 & 1.371 & 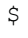 & & \\
\hline \\
\hline & & Pats & tala & & & & \\
\hline \multicolumn{8}{|c|}{--------- Ring 1 --------- } \\
\hline & $\mathrm{c} / \mathrm{z}$ & -29.86 & 2.94 & 1.325 & $\$$ & Pos & \\
\hline & $\mathrm{c} / \mathrm{z}$ & -28.71 & 8 . & 1.325 & $\$$ & & \\
\hline & $\mathrm{c} / \mathrm{z}$ & -26.46 & 14.14 & 1.325 & $\$$ & Po & P \\
\hline & $\mathrm{C} / \mathrm{z}$ & -23.19 & 19.03 & 1.325 & $\$$ & $\mathrm{n}$ & \\
\hline & $\mathrm{C} / \mathrm{z}$ & -19.03 & 23. & 1.325 & \$ & & \\
\hline & $\mathrm{C} / \mathrm{z}$ & -14.14 & 26. & 1.325 & \$ & & \\
\hline & $\mathrm{C} / \mathrm{z}$ & -8.71 & 28.71 & 1.325 & \$ & & \\
\hline & $\mathrm{c} / \mathrm{z}$ & -2.94 & 29.86 & 1.325 & $\$$ & & \\
\hline & $\mathrm{C} / \mathrm{z}$ & 2.94 & 29. & 1.325 & $\$$ & & \\
\hline & $\mathrm{c} / \mathrm{z}$ & 8.71 & 28.71 & 1.325 & $\$$ & $\mathrm{Po}$ & \\
\hline & $c / z$ & 14.14 & 26. & 1.325 & $\$$ & & \\
\hline & $\mathrm{C} / \mathrm{z}$ & 3 & 23 & 1 . & \$ & & \\
\hline & $\mathrm{C} / \mathrm{z}$ & 23.19 & 19 & 1 . & $\$$ & & \\
\hline & $\mathrm{c} / \mathrm{z}$ & 26.46 & 14.14 & 1.325 & $\$$ & Po & \\
\hline & $\mathrm{C} / \mathrm{z}$ & .71 & 8. & 1.325 & 3 & & \\
\hline & $\mathrm{C} / \mathrm{z}$ & .86 & 2 . & 1.325 & $\$$ & & \\
\hline & $\mathrm{c} / \mathrm{z}$ & 9.86 & -2.94 & 1 . & $\$$ & & \\
\hline & $\mathrm{c} / \mathrm{z}$ & 8.71 & -8.71 & 1. & $\$$ & & \\
\hline & $c / z$ & .46 & -14 . & 1 . & \$ & & \\
\hline & $\mathrm{C} / \mathrm{z}$ & 23.19 & -19 . & 1 . & $\$$ & & \\
\hline & $\mathrm{c} / \mathrm{z}$ & .03 & -23 & 1 . & $\$$ & & \\
\hline & $\mathrm{C} / \mathrm{z}$ & 14.14 & -26.46 & 1.325 & \$ & & \\
\hline & $\mathrm{C} / \mathrm{z}$ & 8.71 & -28.71 & 1.325 & $\$$ & & \\
\hline & $\mathrm{C} / \mathrm{z}$ & 2.94 & -29.86 & 1 . & \$ & & \\
\hline & $\mathrm{C} / \mathrm{z}$ & -2.94 & -29.86 & 1.325 & S & & \\
\hline 26 & $\mathrm{C} / \mathrm{z}$ & -8.71 & -28.71 & 1.325 & $\$$ & & \\
\hline & $\mathrm{C} / \mathrm{z}$ & -14.14 & -26.46 & 1.325 & $\$$ & & \\
\hline & $\mathrm{c} / \mathrm{z}$ & -19.03 & -23.19 & 1. & \$ & $\mathrm{Po}$ & \\
\hline 29 & $\mathrm{C} / \mathrm{z}$ & -23.19 & -19.03 & 1.325 & $\$$ & & \\
\hline & $\mathrm{C} / \mathrm{z}$ & -26.46 & -14.14 & 1.325 & $\$$ & Po & 3( \\
\hline & $\mathrm{C} / \mathrm{z}$ & -28.71 & -8 . & & \$ & & 31 \\
\hline & $c / z$ & -29.86 & -2.94 & 1.325 & $\$$ & & 0 \\
\hline \\
\hline & & 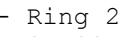 & - & -- & & & \\
\hline & $\mathrm{C} / \mathrm{z}$ & -34.82 & 6. & 1.3 & $\$$ & & 1 \\
\hline & $\mathrm{C} / \mathrm{z}$ & -32.80 & 13. & 1.3 & $\$$ & & 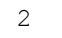 \\
\hline & $\mathrm{c} / \mathrm{z}$ & -29.52 & 19. & 1 . & $\$$ & & 3 \\
\hline & $\mathrm{c} / \mathrm{z}$ & -25 & 25. & & \$ & & 4 \\
\hline & $c / z$ & -19.72 & 29.52 & 1. & $\$$ & Po & 5 \\
\hline & $\mathrm{C} / \mathrm{z}$ & -13.59 & 32.80 & 1.325 & $\$$ & & 6 \\
\hline & $\mathrm{C} / \mathrm{z}$ & -6.93 & 34.82 & 1.325 & $\$$ & & 7 \\
\hline & $C / z$ & 0.00 & 35.50 & 1.325 & $\$$ & & 8 \\
\hline & $\mathrm{C} / \mathrm{z}$ & 6.93 & 34.82 & 1. & $\$$ & & 9 \\
\hline & $\mathrm{C} / \mathrm{z}$ & 13.59 & 32.80 & 1.325 & $\$$ & Po & 10 \\
\hline & $\mathrm{C} / \mathrm{z}$ & 19.72 & 29.52 & 1.325 & $\$$ & Po: & 11 \\
\hline & $\mathrm{c} / \mathrm{z}$ & 25.10 & 25.10 & 1.325 & $\$$ & Pos & 1 \\
\hline & $\mathrm{C} / \mathrm{z}$ & 29.52 & 19.72 & 1.325 & $\$$ & Po: & \\
\hline & $\mathrm{c} / \mathrm{z}$ & 32.80 & 13.59 & 1.325 & \$ & Pos & \\
\hline & $\mathrm{C} / \mathrm{z}$ & 34.82 & 6.93 & 1.325 & $\$$ & Pos & 15 \\
\hline & $\mathrm{c} / \mathrm{z}$ & 35.50 & 0.00 & 1.325 & $\$$ & Posi & 1 \\
\hline & $\mathrm{c} / \mathrm{z}$ & 34.82 & -6.93 & 1.325 & $\$$ & & $\perp$ \\
\hline & $\mathrm{C} / \mathrm{z}$ & 32.80 & -13.59 & 1.325 & $\$$ & Pos & 10 \\
\hline & $c / z$ & 29.52 & -19.72 & 1.325 & \$ & Pos & \\
\hline & $\mathrm{C} / \mathrm{z}$ & 25.10 & -25.10 & 1.325 & $r$ & & \\
\hline & $c / z$ & 19.72 & -29.52 & 1.325 & 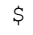 & Pos & \\
\hline
\end{tabular}

Revision: 1 
NEA/NSC/DOC(2006)1

Gas Cooled (Thermal) Reactor - GCR

PROTEUS-GCR-EXP-004

CRIT-REAC

\begin{tabular}{|c|c|c|c|c|c|c|c|}
\hline 22 & $/ z$ & .59 & -32.80 & 1.325 & $\$$ & sition & 22 \\
\hline & $\mathrm{C} / \mathrm{z}$ & 5.93 & -34.82 & 1.325 & & sition & \\
\hline 524 & $\mathrm{C} / \mathrm{z}$ & 0.00 & -35.50 & 1.325 & $\$$ & Position & \\
\hline & $\mathrm{C} / \mathrm{z}$ & -6.93 & -34.82 & 1.325 & $\$$ & Position & \\
\hline & $c / z$ & -13.59 & -32.80 & 1.325 & & & \\
\hline & $\mathrm{C} / \mathrm{z}$ & -19.72 & -29.52 & 1.32 & $\$$ & ositio & \\
\hline & $\mathrm{C} / \mathrm{z}$ & -25.10 & -25.10 & 1.325 & 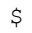 & osition & \\
\hline & $\mathrm{C} / \mathrm{z}$ & -29.52 & -19.72 & 1.325 & $\$$ & Posit & \\
\hline & $\mathrm{C} / \mathrm{z}$ & -32.80 & -13.59 & $3 x^{-1}$ & $\$$ & 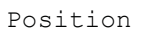 & \\
\hline & $\mathrm{C} / \mathrm{z}$ & -34.82 & -6.93 & 1.32 & $\$$ & os & \\
\hline & $\mathrm{C} / \mathrm{z}$ & -35.50 & 0.00 & 1.325 & $\$$ & 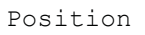 & \\
\hline \\
\hline & & 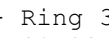 & & & & & \\
\hline & $\mathrm{C} / \mathrm{z}$ & -39.23 & 11.90 & 1.325 & $\$$ & osition & \\
\hline & $\mathrm{c} / \mathrm{z}$ & -36.16 & 9.33 & 1.325 & $\$$ & on & \\
\hline & $\mathrm{C} / \mathrm{z}$ & -31.69 & 1 & 1. & \$ & on & \\
\hline & $\mathrm{C} / \mathrm{z}$ & -26 . & 31. & 1. & $\$$ & on & \\
\hline & $\mathrm{C} / \mathrm{z}$ & -19.33 & 36.16 & 1.3 & $\$$ & tion & \\
\hline & $c / z$ & -11.90 & 39.23 & 1. & $\$$ & on & \\
\hline & $\mathrm{C} / \mathrm{z}$ & -4.02 & & 1. & $\$$ & & \\
\hline & $\mathrm{c} / \mathrm{z}$ & 4. & 40. & 1.32 & $\$$ & O & \\
\hline & $\mathrm{C} / \mathrm{z}$ & 11.90 & 39.23 & 1.32 & $\$$ & & \\
\hline & $\mathrm{C} / \mathrm{z}$ & 19. & 6 & 1 & $\$$ & & \\
\hline & $\mathrm{C} / \mathrm{z}$ & 26. & 31 & 1. & \$ & & \\
\hline & $\mathrm{C} / \mathrm{z}$ & 31.69 & 26. & 1 & $\$$ & on & \\
\hline & $C / z$ & 36.16 & 19.33 & 1.32 & $\$$ & J11 & \\
\hline & $\mathrm{C} / \mathrm{z}$ & 39. & 0 & 1. & $\$$ & & \\
\hline & $\mathrm{C} / \mathrm{z}$ & 40. & 4. & 1.3 & $\$$ & $\mathrm{n}$ & \\
\hline & $\mathrm{C} / \mathrm{z}$ & 40. & -4.02 & 1. & \$ & & \\
\hline & $\mathrm{C} / \mathrm{z}$ & 39.23 & -11.90 & 1.3 & $\$$ & 011 & \\
\hline & $\mathrm{C} / \mathrm{z}$ & 36.16 & -19.33 & 1.3 & $\$$ & & \\
\hline & $\mathrm{C} / \mathrm{z}$ & 31.69 & -26 & 1. & $\$$ & & \\
\hline & $\mathrm{C} / \mathrm{z}$ & 26.01 & -31.69 & 1.3 & $\$$ & $\mathrm{Pc}$ & \\
\hline & $c / z$ & 19. & -36 & 1. & $\$$ & & \\
\hline & $C / z$ & 11.90 & -39 & 1. & $\$$ & & \\
\hline & $\mathrm{C} / \mathrm{z}$ & 2 & -40.80 & 1.3 & $\$$ & on & \\
\hline & $C / z$ & -4.02 & -40.80 & 1. & $\$$ & on & \\
\hline & $\mathrm{C} / \mathrm{z}$ & -11.90 & -39 . & 1. & $\$$ & & \\
\hline & $\mathrm{C} / \mathrm{z}$ & -19. & -36.16 & 1 . & $\$$ & & \\
\hline & $\mathrm{C} / \mathrm{z}$ & -26.01 & -31.69 & 1.32 & $\$$ & $\mathrm{Pc}$ & \\
\hline & $\mathrm{C} / \mathrm{z}$ & -31.69 & -26 & 1. & $\$$ & & \\
\hline & $\mathrm{C} / \mathrm{z}$ & -36.16 & -19 & 1. & $\$$ & & \\
\hline & $\mathrm{C} / \mathrm{z}$ & -39. & -11.90 & 1. & $\$$ & $\mathrm{Pc}$ & \\
\hline & $C / z$ & -40.80 & -4.02 & 1.3 & $\$$ & $\mathrm{P}$ & \\
\hline & $\mathrm{C} / \mathrm{z}$ & -40.80 & 4.02 & 1.3 & $\$$ & $P$ & \\
\hline \multicolumn{8}{|l|}{ C } \\
\hline & & & & & & & \\
\hline & $\mathrm{C} / \mathrm{z}$ & -42.73 & 17.70 & 1.325 & $\$$ & Po & \\
\hline & $C / z$ & -38.46 & 25.70 & 1.3 & $\$$ & Po & \\
\hline & $\mathrm{C} / \mathrm{z}$ & -32.70 & 32. & 1 . & $\$$ & $\mathrm{Po}$ & \\
\hline & $C / z$ & -25.70 & 38.46 & 1.3 & $\$$ & $\mathrm{Po}$ & \\
\hline & $C / z$ & -17 . & 42 & 1. & $\$$ & $\mathrm{Po}$ & \\
\hline & $\mathrm{C} / \mathrm{z}$ & -9.02 & 36 & 1. & $\$$ & & \\
\hline & $\mathrm{C} / \mathrm{z}$ & & 46. & 1 . & $\$$ & $\mathrm{Po}$ & \\
\hline & $c / z$ & 9.02 & 45.36 & 1.3 & $\$$ & $\mathrm{Pc}$ & \\
\hline & $\mathrm{c} / \mathrm{z}$ & 17. & 42 & 1. & $\$$ & & \\
\hline & $c / z$ & 25. & 38.46 & 1 . & $\$$ & & \\
\hline & $\mathrm{C} / \mathrm{z}$ & 32.70 & 32.70 & 1.3 & $\$$ & & \\
\hline & $c / z$ & 38. & 25. & 1. & $\$$ & & \\
\hline & $\mathrm{C} / \mathrm{z}$ & 42. & 17. & & $\$$ & & \\
\hline & $\mathrm{c} / \mathrm{z}$ & 45. & 9.02 & 1. & $\$$ & & \\
\hline & $C / z$ & 46.25 & 0 . & 1.3 & $\$$ & & \\
\hline & $\mathrm{C} / \mathrm{z}$ & 45. & -9 . & 1. & $\$$ & Po & \\
\hline & $\mathrm{C} / \mathrm{z}$ & 42. & -17 & 1. & $\$$ & & \\
\hline & $\mathrm{C} / \mathrm{z}$ & 38. & -25.70 & 1.3 & $\$$ & Po & \\
\hline & $C / z$ & 32. & -32.70 & 1. & $\$$ & Po & \\
\hline & $\mathrm{C} / \mathrm{z}$ & 25. & -38.46 & & $\$$ & & \\
\hline & $C / z$ & 17.70 & -42.73 & 1. & $\$$ & Po: & \\
\hline & $c / z$ & 9. & -45 . & & $\$$ & & \\
\hline & $C / z$ & 0 . & -46 & 1. & $\$$ & Po & \\
\hline & $C / z$ & -9 . & -45 . & 1. & $\$$ & Po: & \\
\hline & $\mathrm{C} / \mathrm{z}$ & -17.70 & -42.73 & 1.3 & $\$$ & Pos & \\
\hline & $\mathrm{C} / \mathrm{z}$ & -25 . & -38 & & $\$$ & & \\
\hline & $\mathrm{C} / \mathrm{z}$ & -32.70 & -32 & 1. & $\$$ & Po: & \\
\hline & $C / z$ & -38.46 & -25.70 & 1.3 & $\$$ & Po: & \\
\hline 272 & $\mathrm{C} / \mathrm{z}$ & -42.73 & -17.70 & 1.3 & $\$$ & Pos & \\
\hline
\end{tabular}

Revision: 1 
NEA/NSC/DOC(2006)1

Gas Cooled (Thermal) Reactor - GCR

PROTEUS-GCR-EXP-004

CRIT-REAC

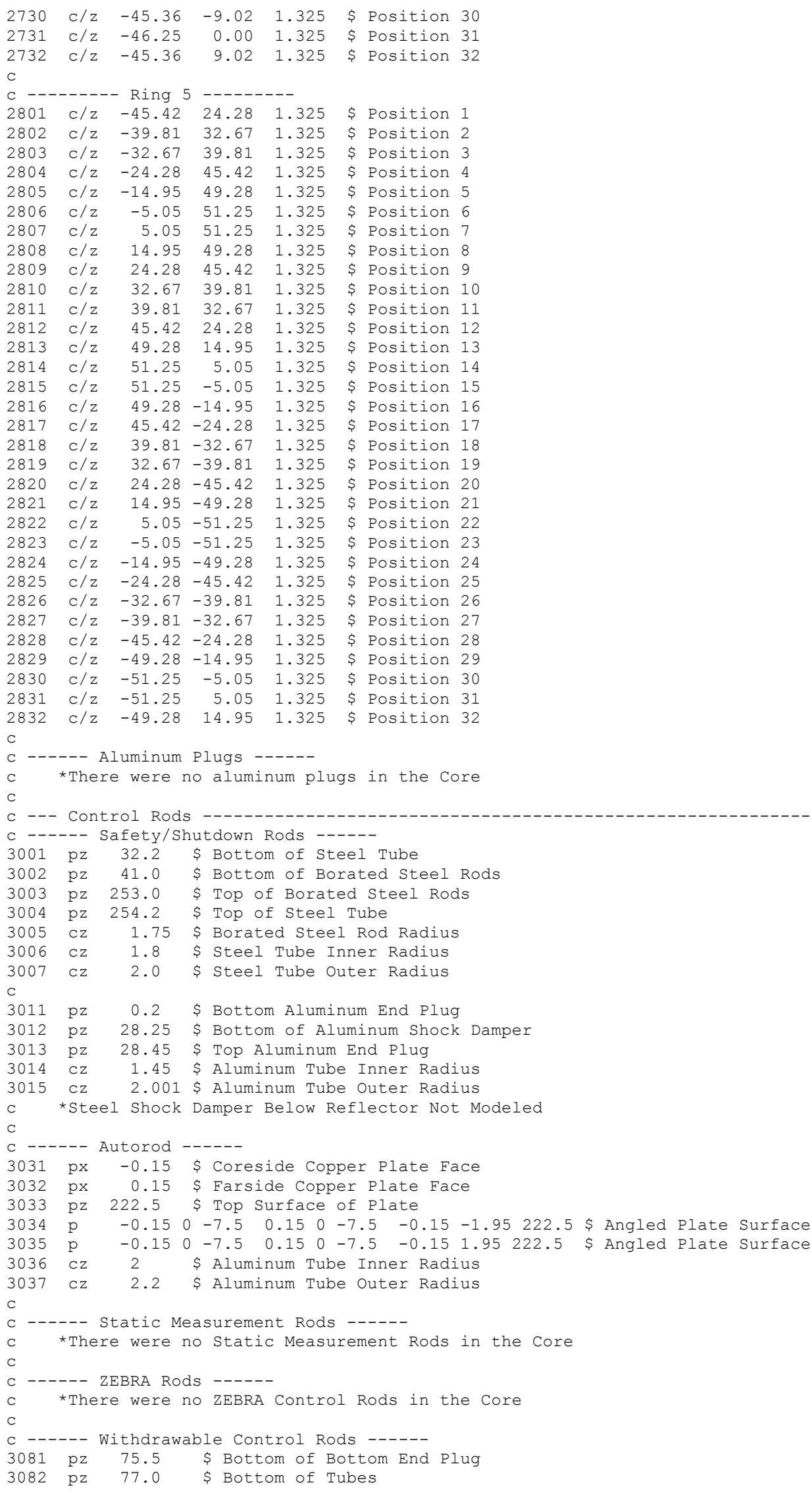

Revision: 1 
NEA/NSC/DOC(2006)1

\section{Gas Cooled (Thermal) Reactor - GCR \\ PROTEUS-GCR-EXP-004 \\ CRIT-REAC}

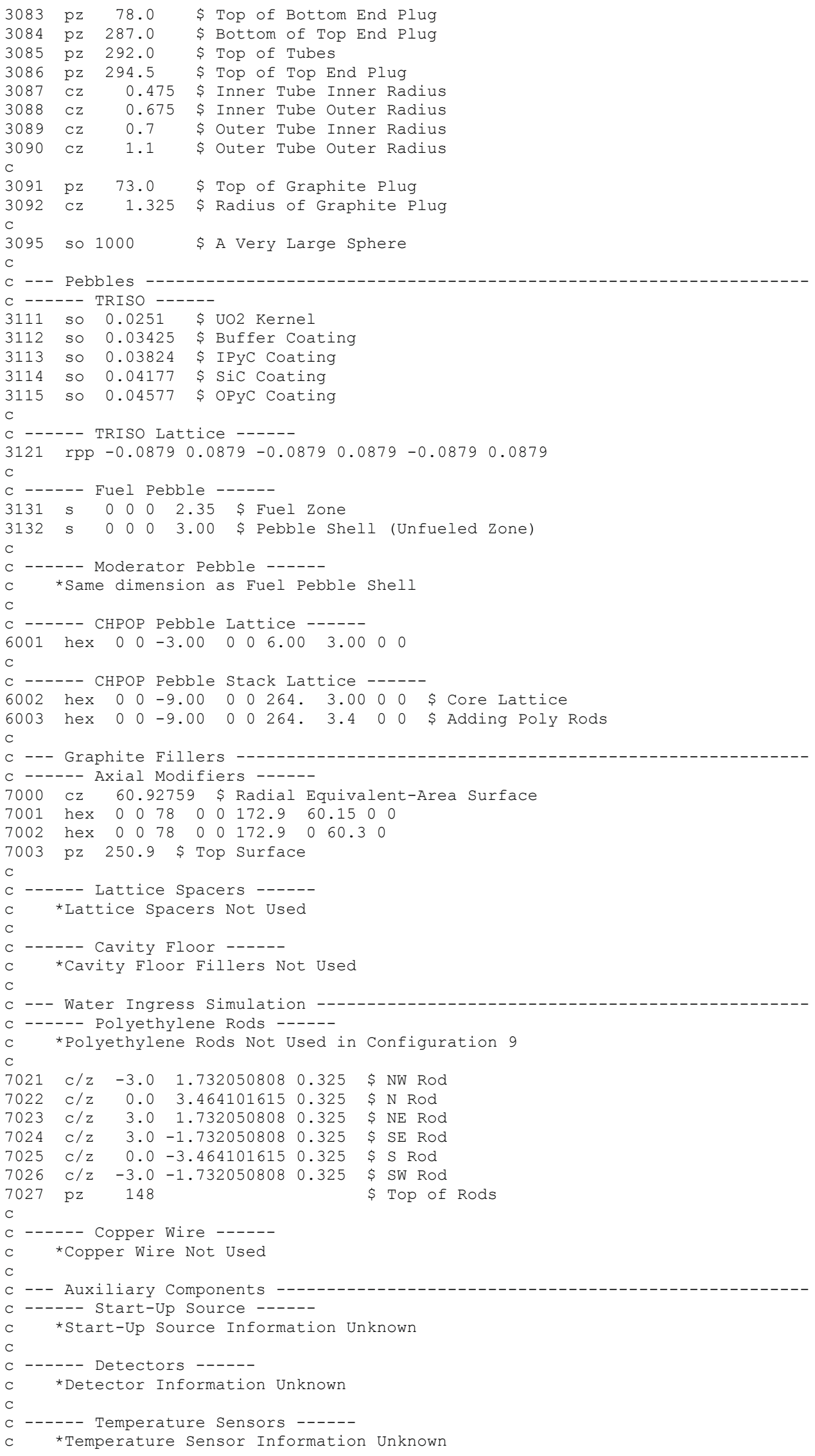

Revision: 1 
NEA/NSC/DOC(2006)1

\section{Gas Cooled (Thermal) Reactor - GCR \\ PROTEUS-GCR-EXP-004 CRIT-REAC}

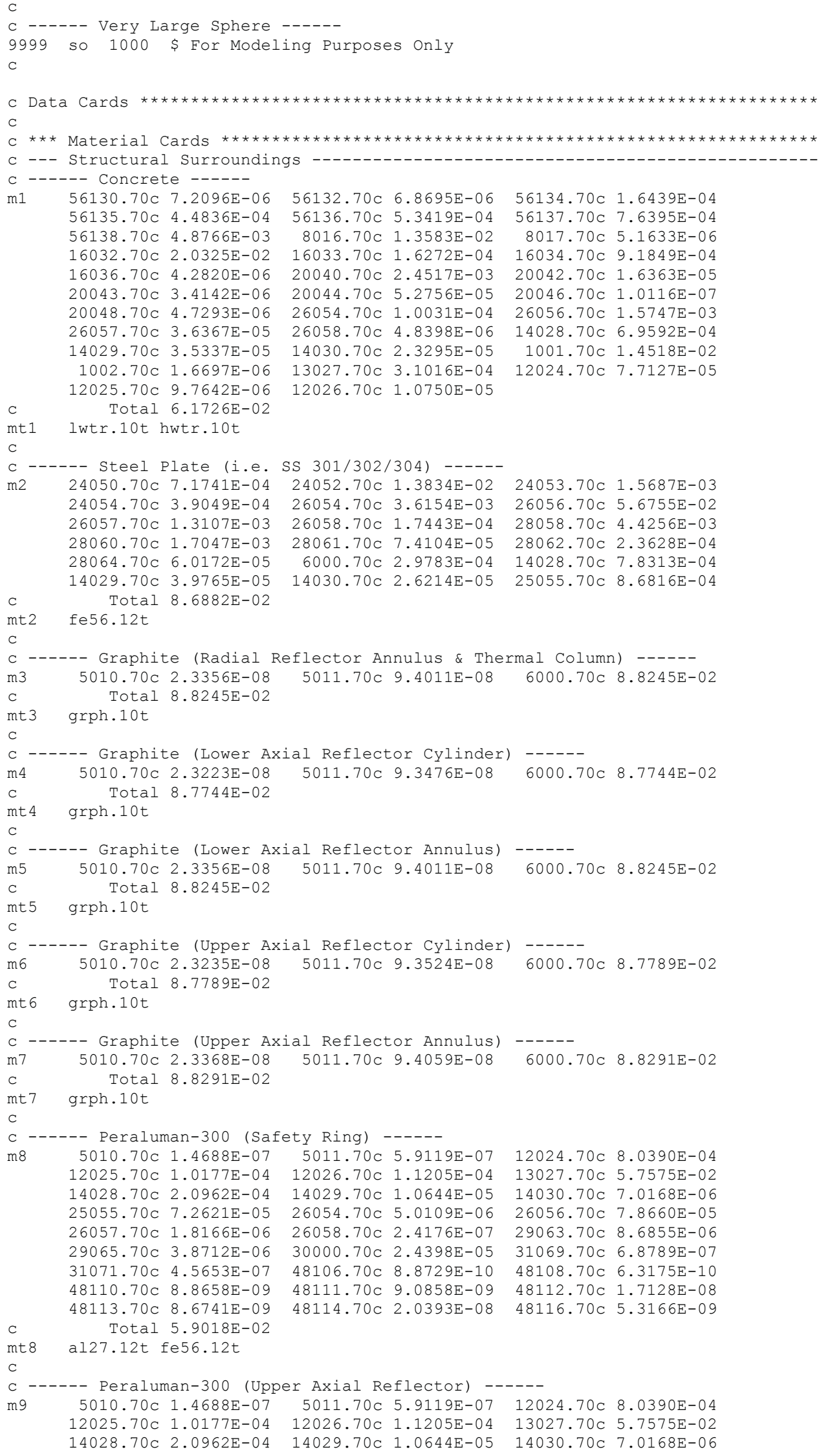

Revision: 1 
NEA/NSC/DOC(2006)1

\section{Gas Cooled (Thermal) Reactor - GCR \\ PROTEUS-GCR-EXP-004 CRIT-REAC}

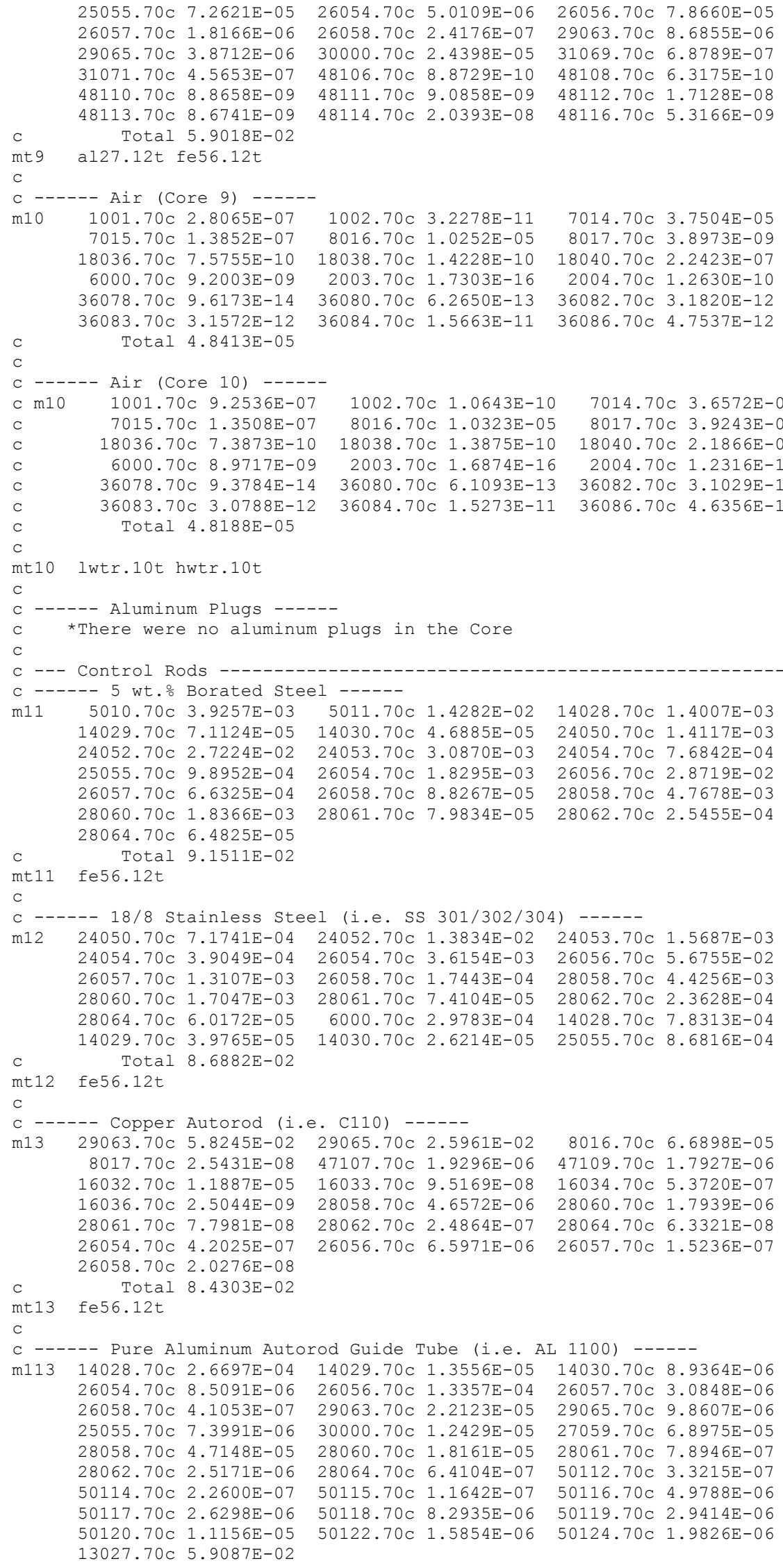

Revision: 1 
NEA/NSC/DOC(2006)1

\section{Gas Cooled (Thermal) Reactor - GCR \\ PROTEUS-GCR-EXP-004 CRIT-REAC}

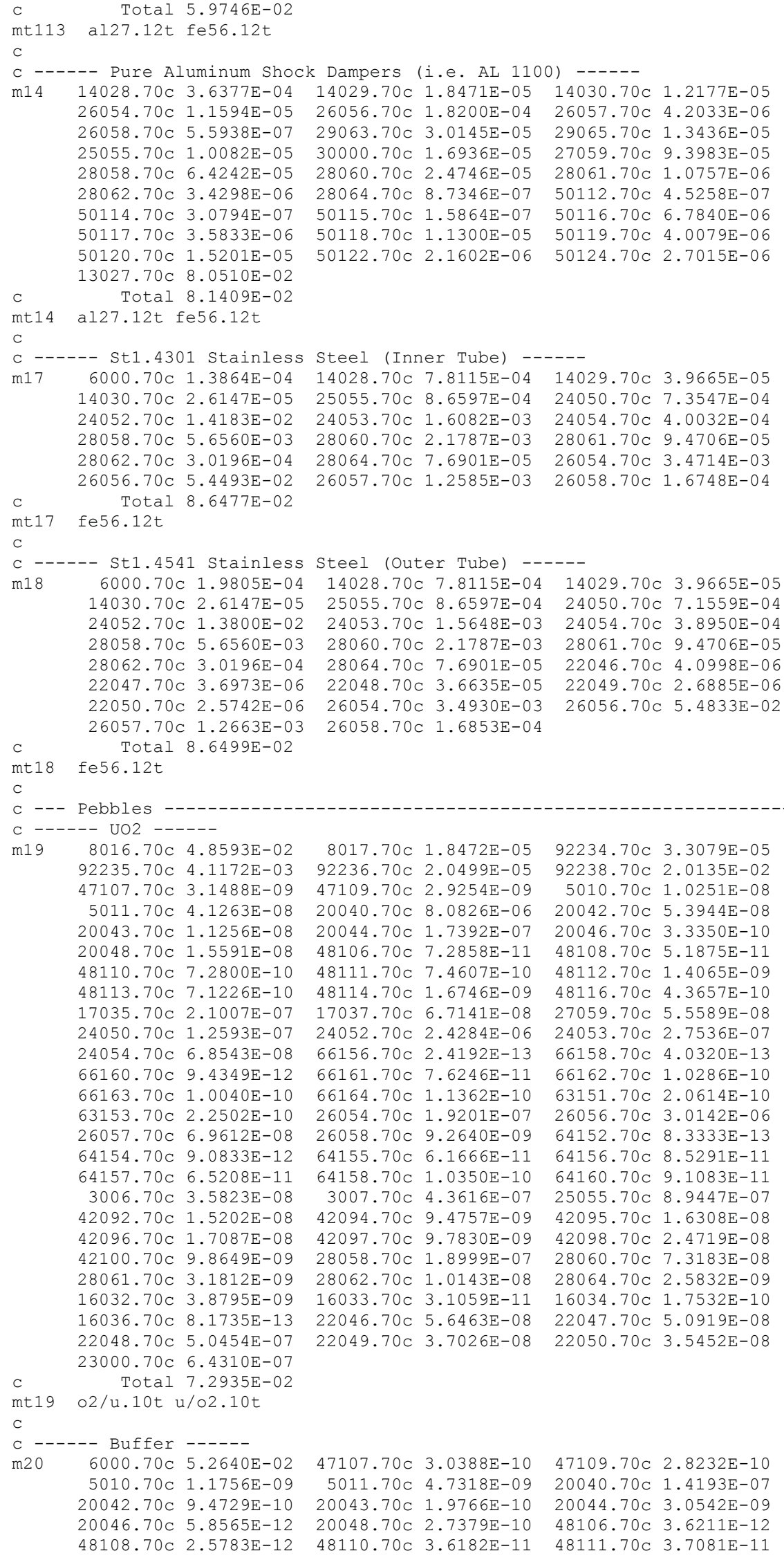
$22049.70 \mathrm{C} \quad 3.7026 \mathrm{E}-08 \quad 22050.70 \mathrm{C} \quad 3.5452 \mathrm{E}-08$

$47107.70 \mathrm{C} 3.0388 \mathrm{E}-10$ $5011.70 \mathrm{C} \quad 4.7318 \mathrm{E}-09$ $20043.70 \mathrm{C} 1.9766 \mathrm{E}-10$ $20048.70 \mathrm{C} 2.7379 \mathrm{E}-10$ $48110.70 \mathrm{C} 3.6182 \mathrm{E}-11$

$92234.70 \mathrm{C} 3.3079 \mathrm{E}-05$ $92238.70 \mathrm{C} 2.0135 \mathrm{E}-02$ $5010.70 \mathrm{C} 1.0251 \mathrm{E}-08$ $20042.70 \mathrm{C} 5.3944 \mathrm{E}-08$ $20046.70 \mathrm{C} 3.3350 \mathrm{E}-10$ $48108.70 \mathrm{C} 5.1875 \mathrm{E}-11$ $48112.70 \mathrm{C} 1.4065 \mathrm{E}-09$ $48116.70 \mathrm{C} \quad 4.3657 \mathrm{E}-10$ $27059.70 \mathrm{C} 5.5589 \mathrm{E}-08$ $24053.70 \mathrm{C} \quad 2.7536 \mathrm{E}-07$ $66158.70 \mathrm{C} 4.0320 \mathrm{E}-13$ $66162.70 \mathrm{C} 1.0286 \mathrm{E}-10$ $63151.70 \mathrm{C} 2.0614 \mathrm{E}-10$ $26056.70 \mathrm{C} 3.0142 \mathrm{E}-06$ $64152.70 \mathrm{C} 8.3333 \mathrm{E}-13$ $64156.70 \mathrm{C} 8.5291 \mathrm{E}-11$ $64160.70 \mathrm{C} 9.1083 \mathrm{E}-11$ $25055.70 \mathrm{C} \quad 8.9447 \mathrm{E}-07$ $42095.70 \mathrm{C} 1.6308 \mathrm{E}-08$ $42098.70 \mathrm{C} 2.4719 \mathrm{E}-08$ $28060.70 \mathrm{C} 7.3183 \mathrm{E}-08$ $28064.70 \mathrm{C} 2.5832 \mathrm{E}-09$ $16034.70 \mathrm{C} 1.7532 \mathrm{E}-10$

$47109.70 \mathrm{C} 2.8232 \mathrm{E}-10$ $20040.70 \mathrm{C} 1.4193 \mathrm{E}-07$ $20044.70 \mathrm{C} 3.0542 \mathrm{E}-09$ $48106.70 \mathrm{C} 3.6211 \mathrm{E}-12$ $48111.70 \mathrm{C} 3.7081 \mathrm{E}-11$

Revision: 1 
NEA/NSC/DOC(2006)1

\section{Gas Cooled (Thermal) Reactor - GCR}

\section{PROTEUS-GCR-EXP-004 CRIT-REAC}

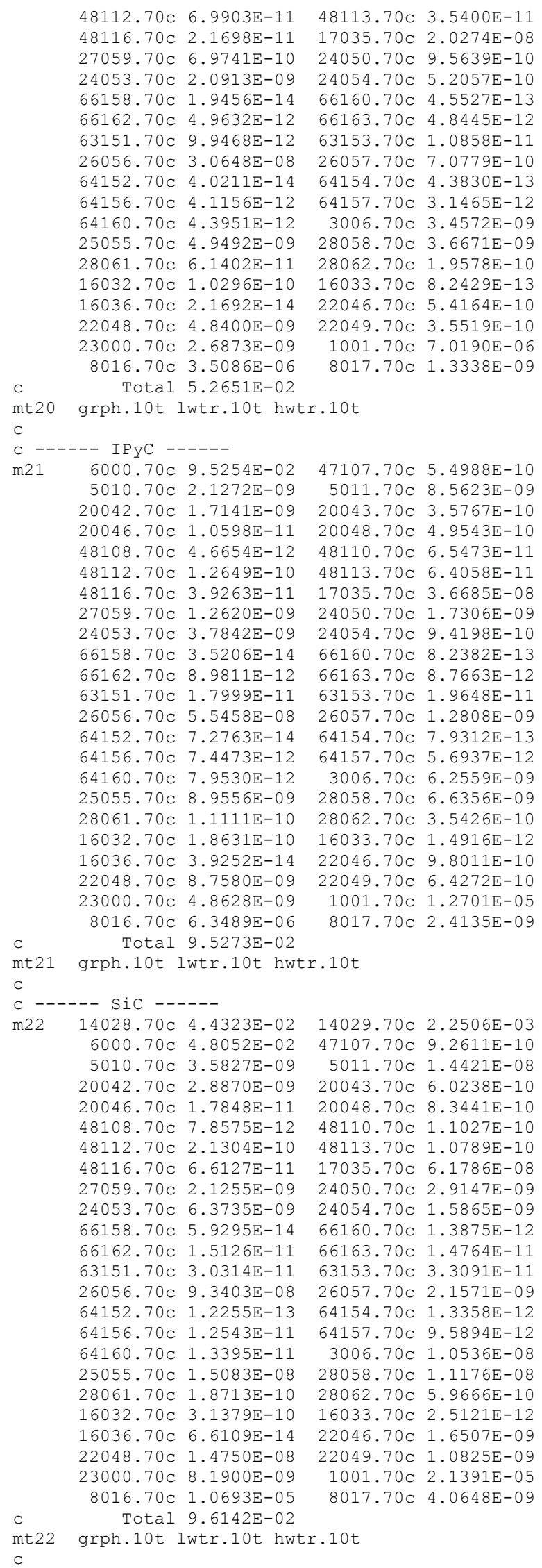

Revision: 1 
NEA/NSC/DOC(2006)1

\section{Gas Cooled (Thermal) Reactor - GCR \\ PROTEUS-GCR-EXP-004 CRIT-REAC}

\begin{tabular}{|c|c|c|c|c|c|c|}
\hline \multirow[t]{23}{*}{$\mathrm{m} 23$} & $70 c$ & $9.4752 \mathrm{E}-02$ & $07.70 \mathrm{c}$ & $8 \mathrm{E}-10$ & $47109.70 \mathrm{c}$ & $5.0817 \mathrm{E}-10$ \\
\hline & $5010.70 \mathrm{c}$ & $2.1160 \mathrm{E}-09$ & $5011.70 \mathrm{c}$ & $8.5172 \mathrm{E}-09$ & $20040.70 \mathrm{c}$ & $2.5548 \mathrm{E}-07$ \\
\hline & $20042.70 c$ & $1.7051 \mathrm{E}-09$ & $20043.70 \mathrm{c}$ & $3.5578 \mathrm{E}-10$ & $20044.70 c$ & $5.4975 \mathrm{E}-09$ \\
\hline & $20046.70 c$ & $1.0542 \mathrm{E}-11$ & $20048.70 \mathrm{c}$ & $4.9283 \mathrm{E}-10$ & $48106.70 \mathrm{C}$ & $6.5181 \mathrm{E}-12$ \\
\hline & $48108.70 c$ & $4.6409 \mathrm{E}-12$ & $48110.70 \mathrm{c}$ & $6.5128 \mathrm{E}-11$ & $48111.70 \mathrm{C}$ & $6.6745 \mathrm{E}-11$ \\
\hline & $48112.70 c$ & $1.2582 \mathrm{E}-10$ & $48113.70 \mathrm{c}$ & $6.3721 \mathrm{E}-11$ & $48114.70 \mathrm{c}$ & $1.4981 \mathrm{E}-10$ \\
\hline & $48116.70 c$ & $3.9056 \mathrm{E}-11$ & $17035.70 \mathrm{C}$ & $3.6492 \mathrm{E}-08$ & $17037.70 \mathrm{c}$ & $1.1663 \mathrm{E}-08$ \\
\hline & $27059.70 c$ & $1.2553 \mathrm{E}-09$ & $24050.70 \mathrm{c}$ & $1.7215 \mathrm{E}-09$ & $24052.70 \mathrm{c}$ & $3.3197 \mathrm{E}-08$ \\
\hline & $24053.70 c$ & $3.7643 \mathrm{E}-09$ & $24054.70 \mathrm{c}$ & $9.3702 \mathrm{E}-10$ & $66156.70 \mathrm{c}$ & $2.1012 \mathrm{E}-14$ \\
\hline & $66158.70 c$ & $3.5021 \mathrm{E}-14$ & $66160.70 \mathrm{c}$ & $8.1949 \mathrm{E}-13$ & $66161.70 \mathrm{c}$ & $6.6224 \mathrm{E}-12$ \\
\hline & $66162.70 c$ & $8.9338 \mathrm{E}-12$ & $66163.70 \mathrm{c}$ & $8.7202 \mathrm{E}-12$ & $66164.70 \mathrm{c}$ & $9.8689 \mathrm{E}-12$ \\
\hline & $63151.70 \mathrm{c}$ & $1.7904 \mathrm{E}-11$ & $63153.70 \mathrm{c}$ & $1.9545 \mathrm{E}-11$ & $26054.70 \mathrm{c}$ & $3.5142 \mathrm{E}-09$ \\
\hline & $26056.70 c$ & $5.5166 \mathrm{E}-08$ & $26057.70 \mathrm{c}$ & $1.2740 \mathrm{E}-09$ & $26058.70 c$ & $1.6955 \mathrm{E}-10$ \\
\hline & $64152.70 c$ & $7.2380 \mathrm{E}-14$ & $64154.70 \mathrm{c}$ & $7.8894 \mathrm{E}-13$ & $64155.70 \mathrm{C}$ & $5.3561 \mathrm{E}-12$ \\
\hline & $64156.70 c$ & $7.4081 \mathrm{E}-12$ & $64157.70 \mathrm{c}$ & $5.6637 \mathrm{E}-12$ & $64158.70 \mathrm{c}$ & $8.9896 \mathrm{E}-12$ \\
\hline & $64160.70 c$ & $7.9111 \mathrm{E}-12$ & $3006.70 c$ & $6.2230 \mathrm{E}-09$ & $3007.70 \mathrm{c}$ & $7.5766 \mathrm{E}-08$ \\
\hline & $25055.70 c$ & $8.9085 E-09$ & $28058.70 \mathrm{c}$ & $6.6007 \mathrm{E}-09$ & $28060.70 \mathrm{C}$ & $2.5426 \mathrm{E}-09$ \\
\hline & $28061.70 c$ & $1.1052 \mathrm{E}-10$ & $28062.70 \mathrm{c}$ & $3.5240 \mathrm{E}-10$ & $28064.70 \mathrm{c}$ & $8.9746 \mathrm{E}-11$ \\
\hline & $16032.70 c$ & $1.8533 \mathrm{E}-10$ & $16033.70 \mathrm{c}$ & $1.4837 \mathrm{E}-12$ & $16034.70 \mathrm{C}$ & $8.3753 \mathrm{E}-12$ \\
\hline & $16036.70 \mathrm{c}$ & $3.9046 \mathrm{E}-14$ & $22046.70 \mathrm{C}$ & $9.7495 \mathrm{E}-10$ & $22047.70 \mathrm{c}$ & $8.7923 \mathrm{E}-10$ \\
\hline & $22048.70 c$ & $8.7119 \mathrm{E}-09$ & $22049.70 \mathrm{c}$ & $6.3933 \mathrm{E}-10$ & $22050.70 \mathrm{c}$ & $6.1215 \mathrm{E}-10$ \\
\hline & $23000.70 c$ & $4.8372 \mathrm{E}-09$ & $1001.70 \mathrm{c}$ & $1.2634 \mathrm{E}-05$ & $1002.70 \mathrm{c}$ & $1.4531 \mathrm{E}-09$ \\
\hline & $8016.70 c$ & $6.3154 \mathrm{E}-06$ & $8017.70 c$ & $2.4008 \mathrm{E}-09$ & & \\
\hline \multirow{4}{*}{$\begin{array}{l}\mathrm{C} \\
\mathrm{mt} 23 \\
\mathrm{C} \\
\mathrm{c}\end{array}$} & Total & $9.4772 \mathrm{E}-02$ & & & & \\
\hline & 30 & 1 unt $r$ 10t but & $10 t$ & & & \\
\hline & & & & & & \\
\hline & - Fueled & Zone ------- & & & & \\
\hline \multirow{23}{*}{$\begin{array}{l}\mathrm{C}- \\
\mathrm{m} 24\end{array}$} & $6000.70 \mathrm{c}$ & $8.6842 \mathrm{E}-02$ & $47107.70 \mathrm{c}$ & $5.0131 \mathrm{E}-10$ & $47109.70 \mathrm{c}$ & $4.6575 \mathrm{E}-10$ \\
\hline & $5010.70 \mathrm{c}$ & $1.9393 \mathrm{E}-09$ & $5011.70 \mathrm{c}$ & $7.8061 \mathrm{E}-09$ & $20040.70 \mathrm{c}$ & $2.3415 \mathrm{E}-07$ \\
\hline & $20042.70 c$ & $1.5628 \mathrm{E}-09$ & $20043.70 \mathrm{c}$ & $3.2608 \mathrm{E}-10$ & $20044.70 \mathrm{C}$ & $5.0385 \mathrm{E}-09$ \\
\hline & $20046.70 c$ & $9.6616 \mathrm{E}-12$ & $20048.70 \mathrm{c}$ & $4.5168 \mathrm{E}-10$ & $48106.70 \mathrm{C}$ & $5.9739 \mathrm{E}-12$ \\
\hline & $48108.70 c$ & $4.2534 \mathrm{E}-12$ & $48110.70 \mathrm{c}$ & $5.9691 \mathrm{E}-11$ & $48111.70 \mathrm{c}$ & $6.1172 \mathrm{E}-11$ \\
\hline & $48112.70 c$ & $1.1532 \mathrm{E}-10$ & $48113.70 \mathrm{c}$ & $5.8401 \mathrm{E}-11$ & $48114.70 \mathrm{c}$ & $1.3730 \mathrm{E}-10$ \\
\hline & $48116.70 c$ & $3.5795 \mathrm{E}-11$ & $17035.70 \mathrm{C}$ & $3.3446 \mathrm{E}-08$ & $17037.70 \mathrm{c}$ & $1.0690 \mathrm{E}-08$ \\
\hline & $27059.70 c$ & $1.1505 \mathrm{E}-09$ & $24050.70 \mathrm{c}$ & $1.5778 \mathrm{E}-09$ & $24052.70 \mathrm{c}$ & $3.0426 \mathrm{E}-08$ \\
\hline & $24053.70 c$ & $3.4500 \mathrm{E}-09$ & $24054.70 \mathrm{c}$ & $8.5879 \mathrm{E}-10$ & $66156.70 \mathrm{c}$ & $1.9258 \mathrm{E}-14$ \\
\hline & $66158.70 c$ & $3.2097 \mathrm{E}-14$ & $66160.70 \mathrm{c}$ & $7.5107 \mathrm{E}-13$ & $66161.70 \mathrm{c}$ & $6.0695 \mathrm{E}-12$ \\
\hline & $66162.70 c$ & $8.1879 \mathrm{E}-12$ & $66163.70 \mathrm{c}$ & $7.9921 \mathrm{E}-12$ & $66164.70 \mathrm{c}$ & $9.0449 \mathrm{E}-12$ \\
\hline & $63151.70 c$ & $1.6409 \mathrm{E}-11$ & $63153.70 \mathrm{c}$ & $1.7913 \mathrm{E}-11$ & $26054.70 c$ & $3.2208 \mathrm{E}-09$ \\
\hline & $26056.70 c$ & $5.0560 \mathrm{E}-08$ & $26057.70 \mathrm{c}$ & $1.1677 \mathrm{E}-09$ & $26058.70 \mathrm{c}$ & $1.5539 \mathrm{E}-10$ \\
\hline & $64152.70 c$ & $6.6337 \mathrm{E}-14$ & $64154.70 \mathrm{C}$ & $7.2307 \mathrm{E}-13$ & $64155.70 \mathrm{c}$ & $4.9089 \mathrm{E}-12$ \\
\hline & $64156.70 c$ & $6.7896 \mathrm{E}-12$ & $64157.70 \mathrm{c}$ & $5.1909 \mathrm{E}-12$ & $64158.70 \mathrm{c}$ & $8.2391 \mathrm{E}-12$ \\
\hline & $64160.70 \mathrm{c}$ & $7.2506 \mathrm{E}-12$ & $3006.70 \mathrm{c}$ & $5.7034 \mathrm{E}-09$ & $3007.70 \mathrm{c}$ & $6.9441 \mathrm{E}-08$ \\
\hline & $25055.70 c$ & $8.1647 \mathrm{E}-09$ & $28058.70 \mathrm{c}$ & $6.0496 \mathrm{E}-09$ & $28060.70 \mathrm{c}$ & $2.3303 \mathrm{E}-09$ \\
\hline & $28061.70 c$ & $1.0130 \mathrm{E}-10$ & $28062.70 \mathrm{c}$ & $3.2298 \mathrm{E}-10$ & $28064.70 \mathrm{C}$ & $8.2253 \mathrm{E}-11$ \\
\hline & $16032.70 c$ & $1.6986 \mathrm{E}-10$ & $16033.70 \mathrm{c}$ & $1.3599 \mathrm{E}-12$ & $16034.70 \mathrm{c}$ & $7.6760 \mathrm{E}-12$ \\
\hline & $16036.70 c$ & $3.5786 \mathrm{E}-14$ & $22046.70 \mathrm{c}$ & $8.9355 \mathrm{E}-10$ & $22047.70 \mathrm{c}$ & $8.0582 \mathrm{E}-10$ \\
\hline & $22048.70 c$ & $7.9846 \mathrm{E}-09$ & $22049.70 \mathrm{c}$ & $5.8596 \mathrm{E}-10$ & $22050.70 c$ & $5.6104 \mathrm{E}-10$ \\
\hline & $23000.70 c$ & $4.4334 \mathrm{E}-09$ & $1001.70 \mathrm{c}$ & $1.1579 \mathrm{E}-05$ & $1002.70 \mathrm{c}$ & $1.3318 \mathrm{E}-09$ \\
\hline & $8016.70 c$ & $5.7882 \mathrm{E}-06$ & $8017.70 \mathrm{C}$ & $2.2003 \mathrm{E}-09$ & & \\
\hline \multirow{3}{*}{$\begin{array}{l}\mathrm{C} \\
\mathrm{mt} 24 \\
\mathrm{C}\end{array}$} & Total & $8.6859 \mathrm{E}-02$ & & & & \\
\hline & $10+t$ & tr.10t hwt & t & & & \\
\hline & & & & & & \\
\hline \multirow{22}{*}{$\begin{array}{l}\mathrm{C}-- \\
\mathrm{m} 25\end{array}$} & -- Un & Zor & & & & \\
\hline & $6000.70 c$ & $8.6842 \mathrm{E}-02$ & $47107.70 c$ & $5.0131 \mathrm{E}-10$ & 47109.70 & $4.6575 \mathrm{E}-10$ \\
\hline & $5010.70 \mathrm{c}$ & $1.9393 \mathrm{E}-09$ & $5011.70 \mathrm{c}$ & $7.8061 \mathrm{E}-09$ & $20040.70 c$ & $2.3415 \mathrm{E}-07$ \\
\hline & $20042.70 c$ & $1.5628 \mathrm{E}-09$ & $20043.70 \mathrm{c}$ & $3.2608 \mathrm{E}-10$ & $20044.70 \mathrm{c}$ & $5.0385 \mathrm{E}-09$ \\
\hline & $20046.70 c$ & $9.6616 \mathrm{E}-12$ & $20048.70 \mathrm{c}$ & $4.5168 \mathrm{E}-10$ & $48106.70 \mathrm{c}$ & $5.9739 \mathrm{E}-12$ \\
\hline & $48108.70 c$ & $4.2534 \mathrm{E}-12$ & $48110.70 \mathrm{c}$ & $5.9691 \mathrm{E}-11$ & $48111.70 \mathrm{c}$ & $6.1172 \mathrm{E}-11$ \\
\hline & $48112.70 c$ & $1.1532 \mathrm{E}-10$ & $48113.70 \mathrm{c}$ & $5.8401 \mathrm{E}-11$ & $48114.70 \mathrm{c}$ & $1.3730 \mathrm{E}-10$ \\
\hline & $48116.70 c$ & $3.5795 E-11$ & $17035.70 \mathrm{c}$ & $3.3446 \mathrm{E}-08$ & $17037.70 \mathrm{C}$ & $1.0690 \mathrm{E}-08$ \\
\hline & $27059.70 c$ & $1.1505 \mathrm{E}-09$ & $24050.70 \mathrm{c}$ & $1.5778 \mathrm{E}-09$ & $24052.70 c$ & $3.0426 \mathrm{E}-08$ \\
\hline & $24053.70 c$ & $3.4500 \mathrm{E}-09$ & $24054.70 \mathrm{c}$ & $8.5879 \mathrm{E}-10$ & $66156.70 \mathrm{c}$ & $1.9258 \mathrm{E}-14$ \\
\hline & 66158.70 & $3.2097 \mathrm{E}-14$ & $66160.70 \mathrm{c}$ & $7.5107 \mathrm{E}-13$ & $66161.70 \mathrm{c}$ & $6.0695 \mathrm{E}-12$ \\
\hline & $66162.70 c$ & $8.1879 \mathrm{E}-12$ & $66163.70 \mathrm{c}$ & $7.9921 \mathrm{E}-12$ & $66164.70 \mathrm{c}$ & $9.0449 \mathrm{E}-12$ \\
\hline & $63151.70 c$ & $1.6409 \mathrm{E}-11$ & $63153.70 \mathrm{c}$ & $1.7913 \mathrm{E}-11$ & $26054.70 \mathrm{c}$ & $3.2208 \mathrm{E}-09$ \\
\hline & $26056.70 c$ & $5.0560 \mathrm{E}-08$ & $26057.70 \mathrm{c}$ & $1.1677 \mathrm{E}-09$ & $26058.70 \mathrm{C}$ & $1.5539 \mathrm{E}-10$ \\
\hline & $64152.70 c$ & $6.6337 \mathrm{E}-14$ & $64154.70 \mathrm{c}$ & $7.2307 \mathrm{E}-13$ & $64155.70 \mathrm{c}$ & $4.9089 \mathrm{E}-12$ \\
\hline & $64156.70 c$ & $6.7896 \mathrm{E}-12$ & $64157.70 \mathrm{c}$ & $5.1909 \mathrm{E}-12$ & $64158.70 \mathrm{c}$ & $8.2391 \mathrm{E}-12$ \\
\hline & $64160.70 c$ & $7.2506 \mathrm{E}-12$ & $3006.70 c$ & $5.7034 \mathrm{E}-09$ & $3007.70 \mathrm{c}$ & $6.9441 \mathrm{E}-08$ \\
\hline & $25055.70 c$ & $8.1647 \mathrm{E}-09$ & $28058.70 \mathrm{C}$ & $6.0496 \mathrm{E}-09$ & $28060.70 \mathrm{c}$ & $2.3303 \mathrm{E}-09$ \\
\hline & $28061.70 c$ & $1.0130 \mathrm{E}-10$ & $28062.70 \mathrm{c}$ & $3.2298 \mathrm{E}-10$ & $28064.70 \mathrm{C}$ & $8.2253 \mathrm{E}-11$ \\
\hline & $16032.70 c$ & $1.6986 \mathrm{E}-10$ & $16033.70 \mathrm{c}$ & $1.3599 \mathrm{E}-12$ & $16034.70 \mathrm{c}$ & $7.6760 \mathrm{E}-12$ \\
\hline & $16036.70 c$ & $3.5786 \mathrm{E}-14$ & $22046.70 c$ & $8.9355 \mathrm{E}-10$ & $22047.70 \mathrm{c}$ & $8.0582 \mathrm{E}-10$ \\
\hline & $22048.70 c$ & $7.9846 \mathrm{E}-09$ & $22049.70 \mathrm{c}$ & $5.8596 \mathrm{E}-10$ & $22050.70 c$ & $5.6104 \mathrm{E}-10$ \\
\hline
\end{tabular}

Revision: 1 
NEA/NSC/DOC(2006)1

\section{Gas Cooled (Thermal) Reactor - GCR \\ PROTEUS-GCR-EXP-004 CRIT-REAC}

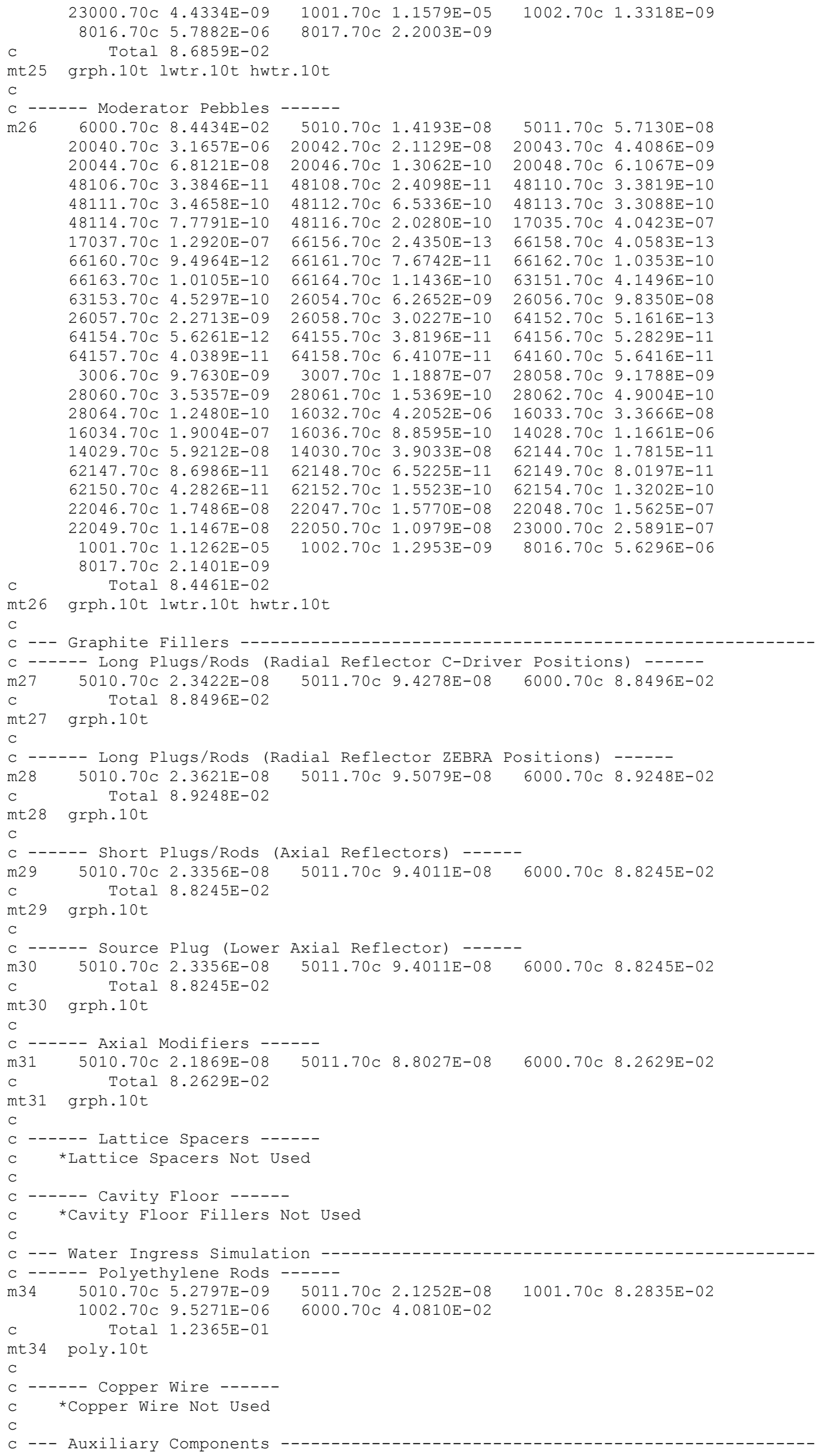

Revision: 1 
NEA/NSC/DOC(2006)1

Gas Cooled (Thermal) Reactor - GCR

PROTEUS-GCR-EXP-004

CRIT-REAC

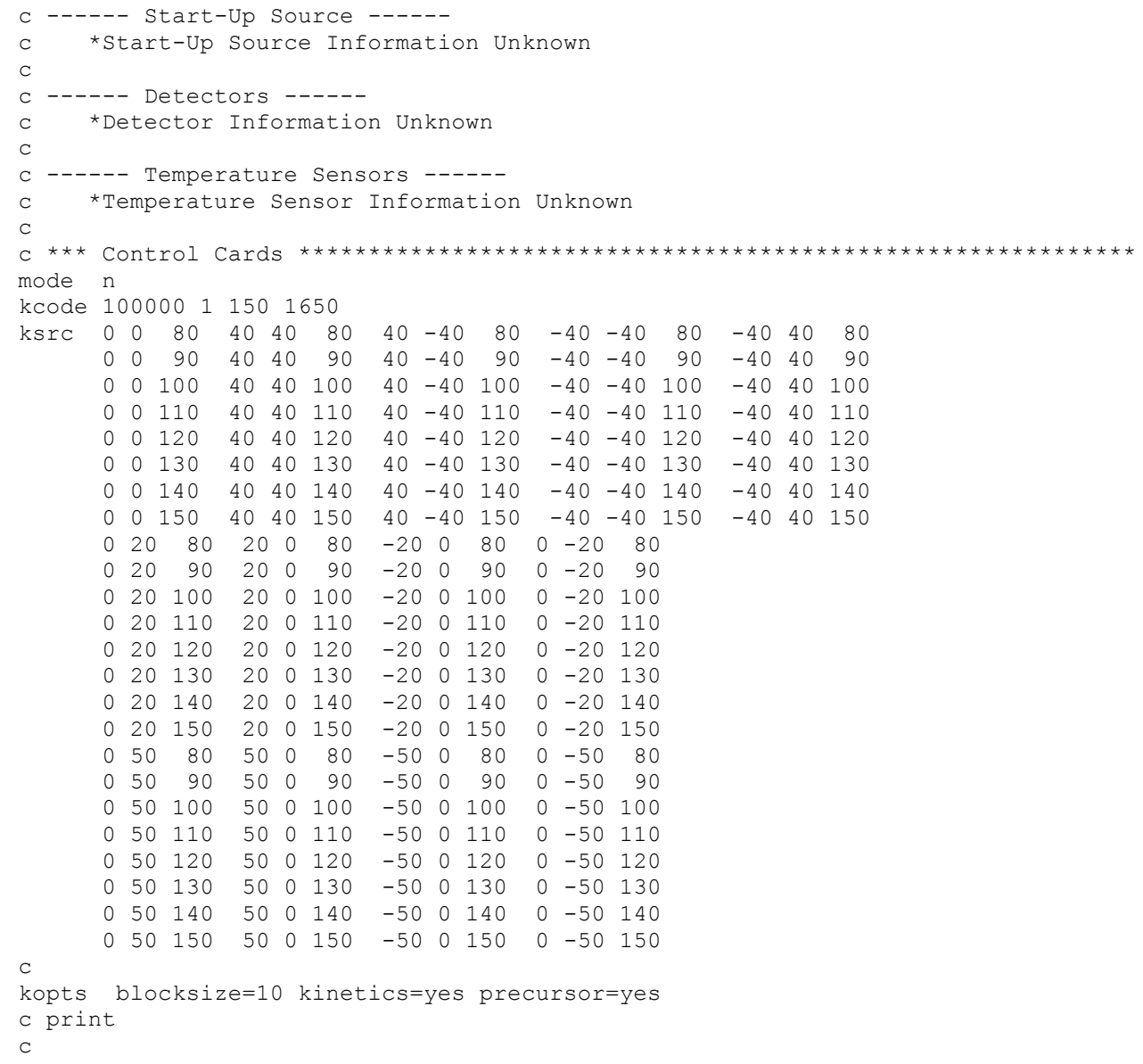

Revision: 1 
NEA/NSC/DOC(2006)1

Gas Cooled (Thermal) Reactor - GCR

PROTEUS-GCR-EXP-004

CRIT-REAC

\section{APPENDIX D: HTR-PROTEUS HISTORICAL DATA}

\section{D.1 Validation of Safety Related Physics Calculations for Low Enriched HTGRs}

The IARA CRP on Validation of Safety Related Physics Calculations for Low Enriched HTGRs (established in 1990) represented a collaboration between China, France, Japan, Switzerland, Germany, the Netherlands, the USA, and the Russian Federation to fill the gaps in validation data for physics methods used in the core design of gas-cooled reactors fueled with low enriched uranium. An international team of researchers assembled at the PROTEUS critical experiment facility of the Paul Scherrer Institute in Villigen, Switzerland to plan, conduct, and analyze a new series of critical experiments focused on the needs of the participating countries.

The following institutes participated in this CRP:

- Paul Scherrer Institute (PSI), Villigen, Switzerland

- Institute for Nuclear Energy Technology (INET), Tsinghua University, Beijing, China

- Forschungzentrum Jülich (FZJ), Jülich, Germany

- Japan Atomic Energy Research Institute (JAERI), Tokai-mura, Japan

- Interfaculty Reactor Institute, Delft University, Delft, the Netherlands

- Centre d'Etudes de Cadarache (CEA), St. Paul les Durance-Cedex, France

- Oak Ridge National Laboratory (ORNL), Oak Ridge, USA

- Russian Research Center Kurchatov Institute (RRC-KI), Moscow, Russia

- Energy Research Center, Petten, the Netherlands

- General Atomics (GA), San Diego, USA

- Experimental Machine Building Design Bureau (OKBM), Nizhny Novgorod, Russia

The PROTEUS graphite moderated LEU critical experiments were planned to fill gaps in the base of validation data. The constraints included room temperature and $5500 \mathrm{LEU}$ fuel pebbles supplied by the KFA Research Center in Jülich, Germany. Specifically, the experiments which could be conducted at the PROTEUS facility with available AVR LEU fuel are summarized in Table D.1-1. The experimental conditions achievable at PROTEUS are summarized in Table D.1-2 (Ref. 3). 
NEA/NSC/DOC(2006)1

\section{Gas Cooled (Thermal) Reactor - GCR \\ PROTEUS-GCR-EXP-004 \\ CRIT-REAC}

Table D.1-1. Summary of PROTEUS Critical Experiments (Ref. 3).

- Clean critical cores.

- LEU pebble-type fuel with $16.76 \%{ }^{235} \mathrm{U}$ enrichment.

- A range of $\mathrm{C} / \mathrm{U}$ atom ratios from 946 to 1890 (achieved by varying the moderator-to-fuel pebble ratio from 0.5 to 2.0 ).

- Core (equivalent) diameter $=1.25 \mathrm{~m}$.

- Core height $=0.843 \mathrm{~m}$ to $1.73 \mathrm{~m}$ (with simulated water ingress smaller core heights possible).

- $\quad$ Core H/D from 0.7 to 1.4 .

- Flux distribution measurements and spectral distribution measurements (including measurements in side reflector).

- Kinetic parameter measurements.

- Worth of reflector control rods (partially and fully inserted).

- Worth of in-core control rod (partially and fully inserted).

- Effects of moisture ingress over range of water density up to $0.25 \mathrm{~g} \mathrm{H}_{2} \mathrm{O} / \mathrm{cm}^{3}$ void (corresponds to $0.065 \mathrm{~g} \mathrm{H}_{2} \mathrm{O} / \mathrm{cm}^{3}$ core for PROTEUS). Water is simulated with polyethylene inserts.

- Effect on core reactivity.

- Effect on worth of reflector control rods.

- Effect on worth of in-core control rod.

- Effect on burnable poison worth.

- Effect on prompt neutron lifetime.

- Effect on flux and power distributions. 
NEA/NSC/DOC(2006)1

\section{Gas Cooled (Thermal) Reactor - GCR \\ PROTEUS-GCR-EXP-004 \\ CRIT-REAC}

Table D.1-2. Experimental Conditions Achievable at PROTEUS (Ref. 3).

- The PROTEUS critical provide validation data for low-enriched uranium fuel with an enrichment near to that planned for advanced GCR designs.

- PROTEUS moisture ingress experiments will investigate the effects which are important for advanced GCR designs (i.e., reactivity worth of moisture, and the effect of moisture on control rod and burnable poison worth and on reaction rate distributions) over the range of moisture densities of interest.

- The achievable range of $\mathrm{C} / \mathrm{U}$ atom ratios at PROTEUS is near to, but higher than, that of advanced GCR designs (this ratio is an important factor in determining the neutron energy spectrum).

- PROTEUS provides the validation data

- For the worth of reflector control rods.

- For the worth of an in-core control rod.

- For the worth of small samples of burnable poison $\left(\mathrm{B}_{4} \mathrm{C}\right)$.

- For fission rate distributions in core and reflector.

\section{D.2 PROTEUS Critical Experiment Facility History and HTR Reconfiguration}

The zero-power reactor facility PROTEUS is a part of the Paul Scherrer Institute (formerly EIR) and is situated near Würenlingen in the canton of Aargau in northern Switzerland. In the past it had been configured as a multi-zone (driven) system for reactor physics investigations of gas-cooled fast breeder and high conversion reactors. Various test configurations were built into a central, subcritical test zone which was driven critical by means of annular, thermal driver zones. PROTEUS was configured, for the first time, as a single zone for the HTR experiments with a pebble bed system surrounded radially and axially by a thick graphite reflector (Ref. 3).

A brief history of the facility is as follows (Ref. 3): ${ }^{a}$

- January 1968 - September 1970

- Operation as a "zero-reactivity experiment" with a thermal, $\mathrm{D}_{2} \mathrm{O}$ moderated test-lattice and a graphite driver.

- $\quad$ September 1970 - April 1972

- Mixed fast-thermal system with a "buffer-zone" and reduced size test-zone.

- April 1972 - April 1979

- Sixteen different configurations of the gas-cooled fast reactor type.

- January 1980 - August 1980

- Preliminary HTR experiments.

- $\quad$ August 1980 - May 1981

- Rebuild of the test-zone to accommodate light-water high conversion reactor experiments.

- May 1981 - October 1982

- Phase I of the advanced light-water reactor experiments. Six configurations were investigated.

- February 1983 - May 1985

- Re-configuration of the test-zone for Phase II of the light-water high conversion reactor experiments.

\footnotetext{
${ }^{a}$ PROTEUS Home Page, http://proteus.web.psi.ch/, Paul Scherrer Institut, Villigen, Switzerland (Accessed January 11, 2011).
}

Revision: 1

Date: March 31, 2014

Page 364 of 375 
NEA/NSC/DOC(2006)1

\section{Gas Cooled (Thermal) Reactor - GCR \\ PROTEUS-GCR-EXP-004 \\ CRIT-REAC}

- June 1985 - December 1990

- Phase II of the advanced light-water experiments. Fourteen different test-zones, containing more representative fuel than in Phase I.

- January 1991 - July 1991

- Rebuild for the LEU-HTR experiments.

- July 1992 - October 1996

- HTR-PROTEUS critical experiments. Ten core configurations, some with multiple reference states.

- $1996-1997$

- Rebuild for LWR-PROTEUS experiments for validation of LWR fuel design and analysis tools.

- $\quad 1997-2001$

- Phase I-SVEA96+ BWR fuel: fission rates and reactivity worths.

- $2001-2003$

- Phase II - PWR fuel: reactivity of burnt fuel segments.

- $2003-2005$

- Phase III - SVEA-96 Optima2 BWR fuel: fission rates and moderator density effects.

- $2005-2011$

- LIFE@PROTEUS experimental program (Large-scale Irradiated Fuel Experiments): power distributions and mismatch, reaction rates, reactivity effects, and characterization of burnt fuel.

A brief summary of the work performed to rebuild the PROTEUS for the HTR-PROTEUS experiments is as follows (Ref. 3):

- All driver and buffer fuel discharged and stored.

- Fuel in test-zone discharged and stored.

- All installations inside graphite reflector removed.

- Construction of upper reflector assembly for HTR, an aluminum tank containing an annular region of old graphite and a central cylinder of new graphite.

- Filling of $\sim 50 \%$ of the $\sim 300$ C-Driver holes with new graphite rods. The other $\sim 50 \%$ were filled with existing graphite rods.

- Renewal of the safety/shutdown rods - increased length to allow for greater core height and better characterization of material properties - for improved benchmark quality of experiments.

- Increased height of radial reflector by $12 \mathrm{~cm}$.

- Reconstruction of lower axial reflector, including central part of new graphite.

- Mounting of graphite panels in core cavity to modify the cavity shape to accommodate deterministic loadings.

- Fuel and moderator pebbles loaded.

- After the rest worths of the original ZEBRA control rods were found to be unacceptably high, these rods were replaced with conventional withdrawable control rods.

\section{D.3 HTR-PROTEUS Timeline and Test Matrix}

The time periods spanned by each configuration is provided in Figure D.3-1. A summary of the test matrix parameters investigated as part of each configuration is presented in Table D.3-3. 
NEA/NSC/DOC(2006)1

Gas Cooled (Thermal) Reactor - GCR

PROTEUS-GCR-EXP-004

CRIT-REAC

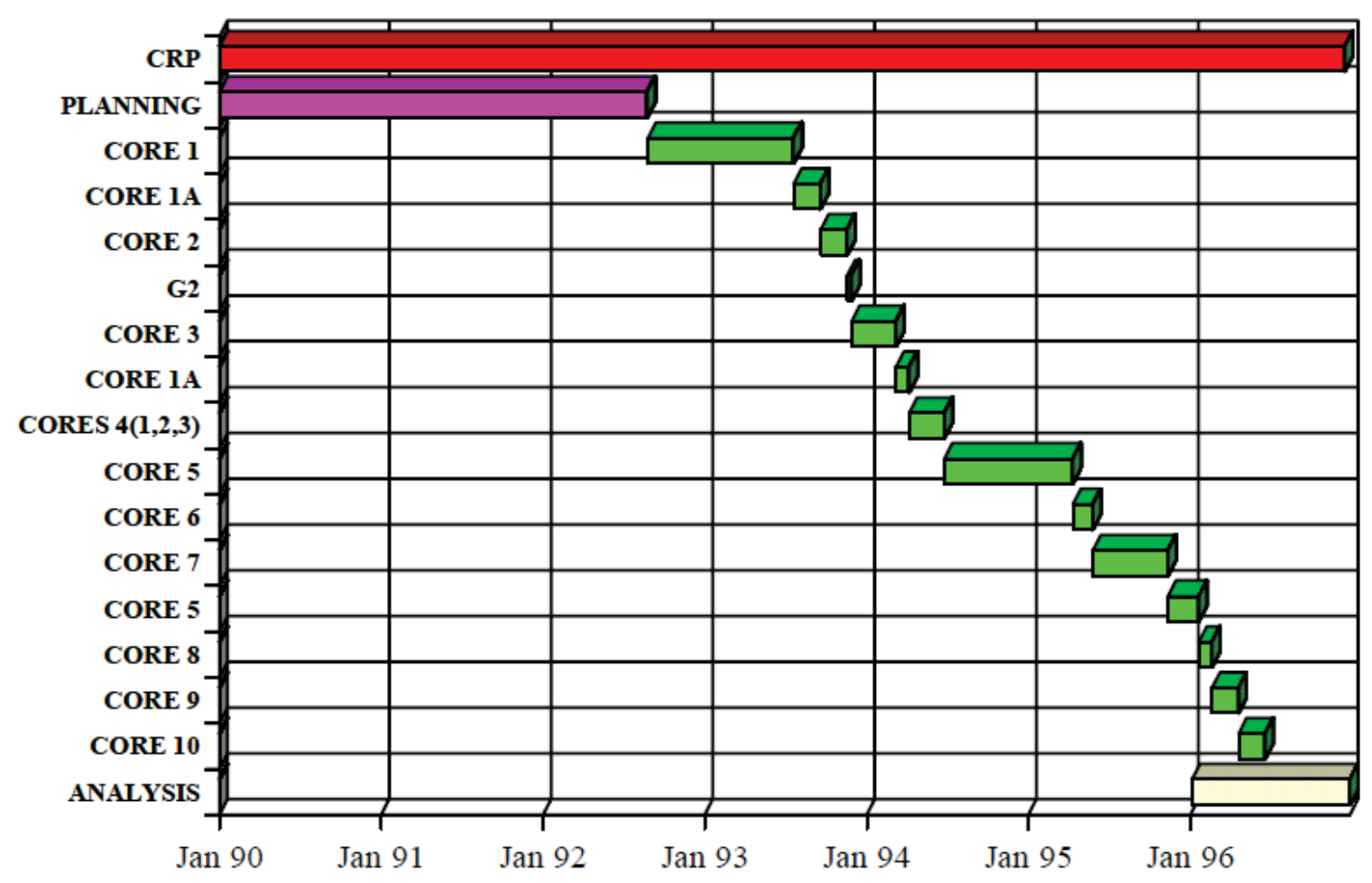

Figure D.3-1. Time Allocation for HTR-PROTEUS Experiments (Ref. 3). 
NEA/NSC/DOC(2006)1

Gas Cooled (Thermal) Reactor - GCR

PROTEUS-GCR-EXP-004

CRIT-REAC

Table D.3-1. Test Matrix for Cores 1 through 10 (Ref. 3).

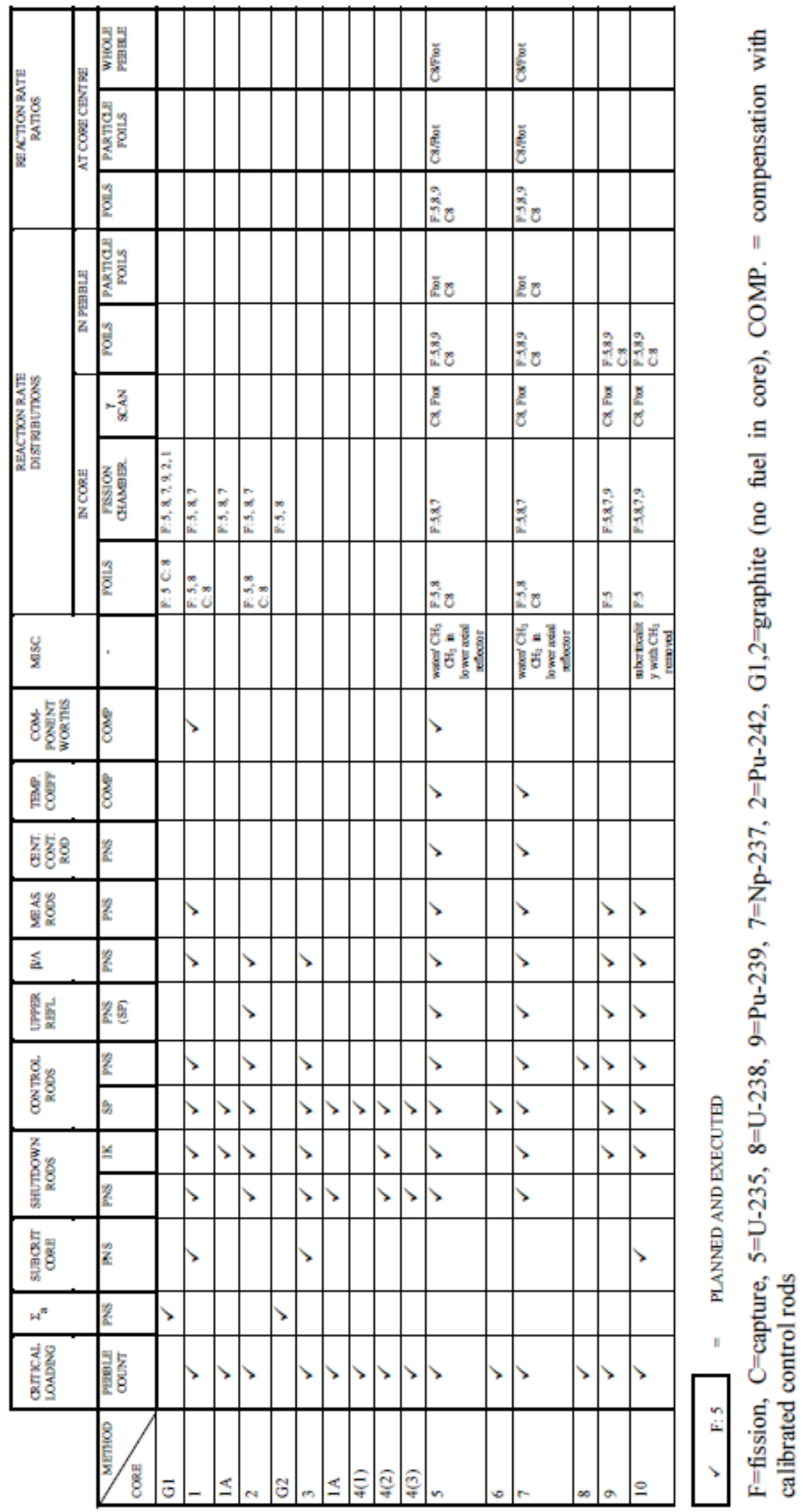

Revision: 1 


\section{Gas Cooled (Thermal) Reactor - GCR \\ PROTEUS-GCR-EXP-004 \\ CRIT-REAC}

\section{APPENDIX E: Data from the 16th edition chart of the Nuclides ${ }^{\text {a }}$}

\section{E.1 Isotopic Abundances and Atomic Weights}

This evaluation incorporated atomic weights and isotopic abundances found in the $16^{\text {th }}$ edition of the Chart of the Nuclides. A list of the values used in the benchmark model or in the generation of the MCNP input deck is compiled in Table E.1-1.

${ }^{a}$ E. M. Baum, H. D. Knox, and T. R. Miller, Nuclides and Isotopes: 16th Edition, Knolls Atomic Power Laboratory (2002).

Revision: 1

Date: March 31, 2014

Page 368 of 375 
NEA/NSC/DOC(2006)1

Gas Cooled (Thermal) Reactor - GCR

PROTEUS-GCR-EXP-004

CRIT-REAC

Table E.1-1. Summary of Data Employed from the $16^{\text {th }}$ Ed. of the Chart of the Nuclides.

\begin{tabular}{|c|c|c|c|c|c|}
\hline $\begin{array}{l}\text { Isotope or } \\
\text { Element }\end{array}$ & $\begin{array}{l}\text { Atomic } \\
\text { Weight } \\
(\mathrm{g} / \mathrm{mol}) \\
\end{array}$ & $\begin{array}{c}\begin{array}{c}\text { Isotopic } \\
\text { Abundance } \\
\text { (at.\%) }\end{array} \\
\end{array}$ & $\begin{array}{l}\text { Isotope or } \\
\text { Element }\end{array}$ & $\begin{array}{l}\text { Atomic } \\
\text { Weight } \\
\text { (g/mol) } \\
\end{array}$ & $\begin{array}{c}\text { Isotopic } \\
\text { Abundance } \\
\text { (at.\%) } \\
\end{array}$ \\
\hline $\mathrm{H}$ & 1.00794 & -- & S & 32.065 & -- \\
\hline${ }^{1} \mathrm{H}$ & -- & 99.9885 & ${ }^{32} \mathrm{~S}$ & -- & 94.93 \\
\hline${ }^{2} \mathrm{H}$ & -- & 0.0115 & ${ }^{33} \mathrm{~S}$ & -- & 0.76 \\
\hline $\mathrm{He}$ & 4.002602 & -- & ${ }^{34} \mathrm{~S}$ & -- & 4.29 \\
\hline${ }^{3} \mathrm{He}$ & -- & 0.000137 & ${ }^{36} \mathrm{~S}$ & -- & 0.02 \\
\hline${ }^{4} \mathrm{He}$ & -- & 99.999863 & $\mathrm{Cl}$ & 35.453 & -- \\
\hline $\mathrm{Li}$ & 6.941 & -- & ${ }^{35} \mathrm{Cl}$ & -- & 75.78 \\
\hline${ }^{6} \mathrm{Li}$ & -- & 7.59 & ${ }^{37} \mathrm{Cl}$ & -- & 24.22 \\
\hline${ }^{7} \mathrm{Li}$ & -- & 92.41 & $\mathrm{Ar}$ & 39.948 & -- \\
\hline B & 10.811 & -- & ${ }^{36} \mathrm{Ar}$ & -- & 0.3365 \\
\hline${ }^{10} \mathrm{~B}$ & 10.012937 & 19.9 & ${ }^{38} \mathrm{Ar}$ & -- & 0.0632 \\
\hline${ }^{11} \mathrm{~B}$ & 11.0093055 & 80.1 & ${ }^{40} \mathrm{Ar}$ & -- & 99.6003 \\
\hline $\mathrm{C}^{(\mathrm{a})}$ & 12.0107 & -- & $\mathrm{Ca}$ & 40.078 & -- \\
\hline $\mathrm{N}$ & 14.0067 & -- & ${ }^{40} \mathrm{Ca}$ & -- & 96.941 \\
\hline${ }^{14} \mathrm{~N}$ & -- & 99.632 & ${ }^{42} \mathrm{Ca}$ & -- & 0.647 \\
\hline${ }^{15} \mathrm{~N}$ & -- & 0.368 & ${ }^{43} \mathrm{Ca}$ & -- & 0.135 \\
\hline $\mathrm{O}$ & 15.9994 & -- & ${ }^{44} \mathrm{Ca}$ & -- & 2.086 \\
\hline${ }^{16} \mathrm{O}$ & -- & 99.757 & ${ }^{46} \mathrm{Ca}$ & -- & 0.004 \\
\hline${ }^{17} \mathrm{O}$ & -- & 0.038 & ${ }^{48} \mathrm{Ca}$ & -- & 0.187 \\
\hline${ }^{18} \mathrm{O}^{(\mathrm{a})}$ & -- & 0.205 & $\mathrm{Ti}$ & 47.867 & -- \\
\hline $\mathrm{Ne}$ & 20.1797 & -- & ${ }^{46} \mathrm{Ti}$ & -- & 8.25 \\
\hline $\mathrm{Mg}$ & 24.3050 & -- & ${ }^{47} \mathrm{Ti}$ & -- & 7.44 \\
\hline${ }^{24} \mathrm{Mg}$ & -- & 78.99 & ${ }^{48} \mathrm{Ti}$ & -- & 73.72 \\
\hline${ }^{25} \mathrm{Mg}$ & -- & 10 & ${ }^{49} \mathrm{Ti}$ & -- & 5.41 \\
\hline${ }^{26} \mathrm{Mg}$ & -- & 11.01 & ${ }^{50} \mathrm{Ti}$ & -- & 5.18 \\
\hline $\mathrm{Al}$ & 26.981538 & -- & $\mathrm{V}^{(\mathrm{a})}$ & 50.9415 & -- \\
\hline $\mathrm{Si}$ & 28.0855 & -- & $\mathrm{Cr}$ & 51.9961 & -- \\
\hline${ }^{28} \mathrm{Si}$ & -- & 92.2297 & ${ }^{50} \mathrm{Cr}$ & -- & 4.345 \\
\hline${ }^{29} \mathrm{Si}$ & -- & 4.6832 & ${ }^{52} \mathrm{Cr}$ & -- & 83.789 \\
\hline${ }^{30} \mathrm{Si}$ & -- & 3.0872 & ${ }^{53} \mathrm{Cr}$ & -- & 9.501 \\
\hline $\mathrm{P}$ & 30.973761 & -- & ${ }^{54} \mathrm{Cr}$ & -- & 2.365 \\
\hline
\end{tabular}


NEA/NSC/DOC(2006)1

Gas Cooled (Thermal) Reactor - GCR

PROTEUS-GCR-EXP-004

CRIT-REAC

Table E.1-1. (cont'd.). Summary of Data Employed from the $16^{\text {th }} \mathrm{Ed}$. of the Chart of the Nuclides.

\begin{tabular}{|c|c|c|c|c|c|}
\hline $\begin{array}{l}\text { Isotope or } \\
\text { Element }\end{array}$ & $\begin{array}{l}\text { Atomic } \\
\text { Weight } \\
\text { (g/mol) } \\
\end{array}$ & $\begin{array}{c}\text { Isotopic } \\
\text { Abundance } \\
\text { (at.\%) } \\
\end{array}$ & $\begin{array}{l}\text { Isotope or } \\
\text { Element }\end{array}$ & $\begin{array}{l}\text { Atomic } \\
\text { Weight } \\
\text { (g/mol) } \\
\end{array}$ & $\begin{array}{c}\text { Isotopic } \\
\text { Abundance } \\
\text { (at.\%) } \\
\end{array}$ \\
\hline $\mathrm{Mn}$ & 54.938049 & -- & Mo & 95.94 & -- \\
\hline $\mathrm{Fe}$ & 55.845 & -- & ${ }^{92} \mathrm{Mo}$ & -- & 14.84 \\
\hline${ }^{54} \mathrm{Fe}$ & -- & 5.845 & ${ }^{94} \mathrm{Mo}$ & -- & 9.25 \\
\hline${ }^{56} \mathrm{Fe}$ & -- & 91.754 & ${ }^{95} \mathrm{Mo}$ & -- & 15.92 \\
\hline${ }^{57} \mathrm{Fe}$ & -- & 2.119 & ${ }^{96} \mathrm{Mo}$ & -- & 16.68 \\
\hline${ }^{58} \mathrm{Fe}$ & -- & 0.282 & ${ }^{97} \mathrm{Mo}$ & -- & 9.55 \\
\hline $\mathrm{Co}$ & 58.933200 & -- & ${ }^{98} \mathrm{Mo}$ & -- & 24.13 \\
\hline $\mathrm{Ni}$ & 58.6934 & -- & ${ }^{100} \mathrm{Mo}$ & -- & 9.63 \\
\hline${ }^{58} \mathrm{Ni}$ & -- & 68.0769 & $\mathrm{Ag}$ & 107.8682 & -- \\
\hline${ }^{60} \mathrm{Ni}$ & -- & 26.2231 & ${ }^{107} \mathrm{Ag}$ & -- & 51.839 \\
\hline${ }^{61} \mathrm{Ni}$ & -- & 1.1399 & ${ }^{109} \mathrm{Ag}$ & -- & 48.161 \\
\hline${ }^{62} \mathrm{Ni}$ & -- & 3.6345 & $\mathrm{Cd}$ & 112.411 & -- \\
\hline${ }^{64} \mathrm{Ni}$ & -- & 0.9256 & ${ }^{106} \mathrm{Cd}$ & -- & 1.25 \\
\hline $\mathrm{Cu}$ & 63.546 & -- & ${ }^{108} \mathrm{Cd}$ & -- & 0.89 \\
\hline${ }^{63} \mathrm{Cu}$ & -- & 69.17 & ${ }^{110} \mathrm{Cd}$ & -- & 12.49 \\
\hline${ }^{65} \mathrm{Cu}$ & -- & 30.83 & ${ }^{111} \mathrm{Cd}$ & -- & 12.8 \\
\hline$Z^{(a)}$ & 65.409 & -- & ${ }^{112} \mathrm{Cd}$ & -- & 24.13 \\
\hline $\mathrm{Ga}$ & 69.723 & -- & ${ }^{113} \mathrm{Cd}$ & -- & 12.22 \\
\hline${ }^{69} \mathrm{Ga}$ & -- & 60.108 & ${ }^{114} \mathrm{Cd}$ & -- & 28.73 \\
\hline${ }^{71} \mathrm{Ga}$ & -- & 39.892 & ${ }^{116} \mathrm{Cd}$ & -- & 7.49 \\
\hline $\mathrm{Kr}$ & 83.798 & -- & $\mathrm{Sn}$ & 118.710 & -- \\
\hline${ }^{78} \mathrm{Kr}$ & -- & 0.35 & ${ }^{112} \mathrm{Sn}$ & -- & 0.97 \\
\hline${ }^{80} \mathrm{Kr}$ & -- & 2.28 & ${ }^{114} \mathrm{Sn}$ & -- & 0.66 \\
\hline${ }^{82} \mathrm{Kr}$ & -- & 11.58 & ${ }^{115} \mathrm{Sn}$ & -- & 0.34 \\
\hline${ }^{83} \mathrm{Kr}$ & -- & 11.49 & ${ }^{116} \mathrm{Sn}$ & -- & 14.54 \\
\hline${ }^{84} \mathrm{Kr}$ & -- & 57 & ${ }^{117} \mathrm{Sn}$ & -- & 7.68 \\
\hline \multirow[t]{5}{*}{${ }^{86} \mathrm{Kr}$} & -- & 17.3 & ${ }^{118} \mathrm{Sn}$ & -- & 24.22 \\
\hline & & & ${ }^{119} \mathrm{Sn}$ & -- & 8.59 \\
\hline & & & ${ }^{120} \mathrm{Sn}$ & -- & 32.58 \\
\hline & & & ${ }^{122} \mathrm{Sn}$ & -- & 4.63 \\
\hline & & & ${ }^{124} \mathrm{Sn}$ & -- & 5.79 \\
\hline
\end{tabular}


NEA/NSC/DOC(2006)1

Gas Cooled (Thermal) Reactor - GCR

PROTEUS-GCR-EXP-004

CRIT-REAC

Table E.1-1. (cont'd.). Summary of Data Employed from the $16^{\text {th }} \mathrm{Ed}$. of the Chart of the Nuclides.

\begin{tabular}{|c|c|c|c|c|c|}
\hline $\begin{array}{l}\text { Isotope or } \\
\text { Element }\end{array}$ & $\begin{array}{l}\text { Atomic } \\
\text { Weight } \\
\text { (g/mol) } \\
\end{array}$ & $\begin{array}{c}\text { Isotopic } \\
\text { Abundance } \\
\text { (at.\%) } \\
\end{array}$ & $\begin{array}{l}\text { Isotope or } \\
\text { Element }\end{array}$ & $\begin{array}{l}\text { Atomic } \\
\text { Weight } \\
\text { (g/mol) } \\
\end{array}$ & $\begin{array}{c}\text { Isotopic } \\
\text { Abundance } \\
\text { (at.\%) } \\
\end{array}$ \\
\hline $\mathrm{Ba}$ & 137.327 & -- & $\mathrm{Gd}$ & 157.25 & -- \\
\hline${ }^{130} \mathrm{Ba}$ & -- & 0.106 & ${ }^{152} \mathrm{Gd}$ & -- & 0.2 \\
\hline${ }^{132} \mathrm{Ba}$ & -- & 0.101 & ${ }^{154} \mathrm{Gd}$ & -- & 2.18 \\
\hline${ }^{134} \mathrm{Ba}$ & -- & 2.417 & ${ }^{155} \mathrm{Gd}$ & -- & 14.8 \\
\hline${ }^{135} \mathrm{Ba}$ & -- & 6.592 & ${ }^{156} \mathrm{Gd}$ & -- & 20.47 \\
\hline${ }^{136} \mathrm{Ba}$ & -- & 7.854 & ${ }^{157} \mathrm{Gd}$ & -- & 15.65 \\
\hline${ }^{137} \mathrm{Ba}$ & -- & 11.232 & ${ }^{158} \mathrm{Gd}$ & -- & 24.84 \\
\hline${ }^{138} \mathrm{Ba}$ & -- & 71.698 & ${ }^{160} \mathrm{Gd}$ & -- & 21.86 \\
\hline $\mathrm{Sm}$ & 150.36 & -- & Dy & 162.500 & -- \\
\hline${ }^{144} \mathrm{Sm}$ & -- & 3.07 & ${ }^{156}$ Dy & -- & 0.06 \\
\hline${ }^{147} \mathrm{Sm}$ & -- & 14.99 & ${ }^{158}$ Dy & -- & 0.1 \\
\hline${ }^{148} \mathrm{Sm}$ & -- & 11.24 & ${ }^{160} \mathrm{Dy}$ & -- & 2.34 \\
\hline${ }^{149} \mathrm{Sm}$ & -- & 13.82 & ${ }^{161}$ Dy & -- & 18.91 \\
\hline${ }^{150} \mathrm{Sm}$ & -- & 7.38 & ${ }^{162} \mathrm{Dy}$ & -- & 25.51 \\
\hline${ }^{152} \mathrm{Sm}$ & -- & 26.75 & ${ }^{163} \mathrm{Dy}$ & -- & 24.9 \\
\hline${ }^{154} \mathrm{Sm}$ & -- & 22.75 & ${ }^{164}$ Dy & -- & 28.18 \\
\hline $\mathrm{Eu}$ & 151.964 & -- & $\mathrm{Pb}$ & 207.2 & -- \\
\hline${ }^{151} \mathrm{Eu}$ & -- & 47.81 & ${ }^{204} \mathrm{~Pb}$ & -- & 1.4 \\
\hline \multirow[t]{7}{*}{${ }^{153} \mathrm{Eu}$} & -- & 52.19 & ${ }^{206} \mathrm{~Pb}$ & -- & 24.1 \\
\hline & & & ${ }^{207} \mathrm{~Pb}$ & -- & 22.1 \\
\hline & & & ${ }^{208} \mathrm{~Pb}$ & -- & 52.4 \\
\hline & & & $\mathrm{Bi}$ & 208.98038 & -- \\
\hline & & & ${ }^{234} \mathrm{U}$ & 234.040946 & $0.0055^{(\mathfrak{b})}$ \\
\hline & & & ${ }^{235} \mathrm{U}$ & 235.043923 & $0.7200^{(\mathrm{b})}$ \\
\hline & & & ${ }^{238} \mathrm{U}$ & 238.050783 & $99.2745^{(\mathrm{b})}$ \\
\hline
\end{tabular}

a. Natural element without isotopic breakdown.

b. Neutronically, ${ }^{18} \mathrm{O}$ is treated as ${ }^{16} \mathrm{O}$.

c. Natural isotopic abundance of $U$. 
NEA/NSC/DOC(2006)1

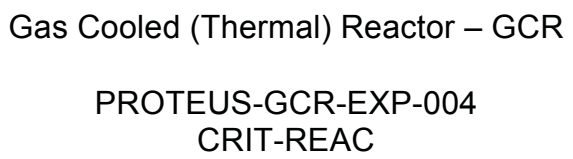

\section{APPENDIX F: MODELS SPECIFICATION FOR SCALED REACTIVITY EFFECTS DATA}

\section{F.1 Evaluated Measurements (NOT BENCHMARK MEASUREMENTS)}

As discussed in Section 2.4, various reported reactivity effects measurements were actually measured on other HTR-PROTEUS core configurations and then scaled via a ratio of control rod bank worths for the core on which the original measurement was performed and the core on which the measurement was applied to allow for model simplifications. Where sufficient information was available, these scaled measurements were evaluated but not considered appropriate to use as benchmark data. A summary of the evaluated scaled measurements in provided in Table F.1-1 for Core 9 and Table F.1-2 for Core 10. Means to model the scaled measurements and sample calculations are provided in subsequent sections of this appendix.

Table F.1-1. Adjusted Experimental Reactivity Effects Scaled Measurements (Core 9).

\begin{tabular}{|c|c|c|ccc|}
\hline \multirow{2}{*}{ Case } & Measured Parameter & \multicolumn{3}{|c|}{ Benchmark } & \multicolumn{3}{|c|}{ Experimental Worth } \\
& & Measurement? & $\mathbf{\rho}(\mathbf{\$})$ & \pm & $\boldsymbol{\sigma}$ \\
\hline \hline 1.F-1 & Autorod Rest Worth & No & -0.13 & \pm & 0.01 \\
\hline 1.F-2 & Graphite in Control Rod Channels & No & -0.026 & \pm & 0.003 \\
\hline 1.F-3 & $\begin{array}{c}\text { Graphite in Empty Channels: } \\
\text { R2-15, -47, \& -63 }\end{array}$ & No & -0.05 & \pm & 0.01 \\
\hline
\end{tabular}

Table F.1-2. Adjusted Experimental Reactivity Effects Scaled Measurements (Core 10).

\begin{tabular}{|c|c|c|ccc|}
\hline \multirow{2}{*}{ Case } & Measured Parameter & \multicolumn{3}{|c|}{ Benchmark } & \multicolumn{3}{|c|}{ Experimental Worth } \\
& & Measurement? & $\mathbf{p ( \$ )}$ & \pm & $\boldsymbol{\sigma}$ \\
\hline \hline 2.F-1 & Autorod Rest Worth & No & -0.081 & \pm & 0.005 \\
\hline 2.F-2 & Graphite in Control Rod Channels & No & -0.021 & \pm & 0.002 \\
\hline 2.F-3 & $\begin{array}{c}\text { Graphite in Empty Channels: } \\
\text { R2-15, -47, \& -63 }\end{array}$ & No & -0.04 & \pm & 0.01 \\
\hline
\end{tabular}

\section{F.2 Modeling Specifications}

Simplifications and discussions provided in Section 3.4 also apply to the models discussed in Appendix F. Only information unique to the simulation of the scaled measurements provided in Table F.1-1 are included herein; all other details for model development are provided as benchmark models in Section 3.1 and 3.4 .

\section{F.2.1 Dimensions Supplemental Data}

\section{F.2.1.1 Autorod Worths}

The rest worth of the autorod is obtained by fully withdrawing the autorod and then comparing this condition with a similar configuration in which the autorod is replaced with void. 
NEA/NSC/DOC(2006)1

\section{Gas Cooled (Thermal) Reactor - GCR \\ PROTEUS-GCR-EXP-004 \\ CRIT-REAC}

\section{F.2.1.2 Graphite Plug Worths}

The locations of the control rod channels and channels R2-15, -47, and -63 are shown in Figure F.1-1. The $x-y$ positions are shown in the figure. The worth of the graphite plugs in the four control rod channels is obtained by taking the benchmark critical configurations (Section 3.1), replacing the control rod components with void, and comparing the condition of voided control rod channels with one in which the channels are filled with graphite. The worth of the graphite plugs in the three R2 positions is obtained by taking the benchmark critical configurations and adding in the three, void-filled, R2 channels that have a diameter of $2.743 \mathrm{~cm}$ and run through the full height of the radial reflector. The modified configurations are then compared with the condition where the three voided channels are filled with graphite.

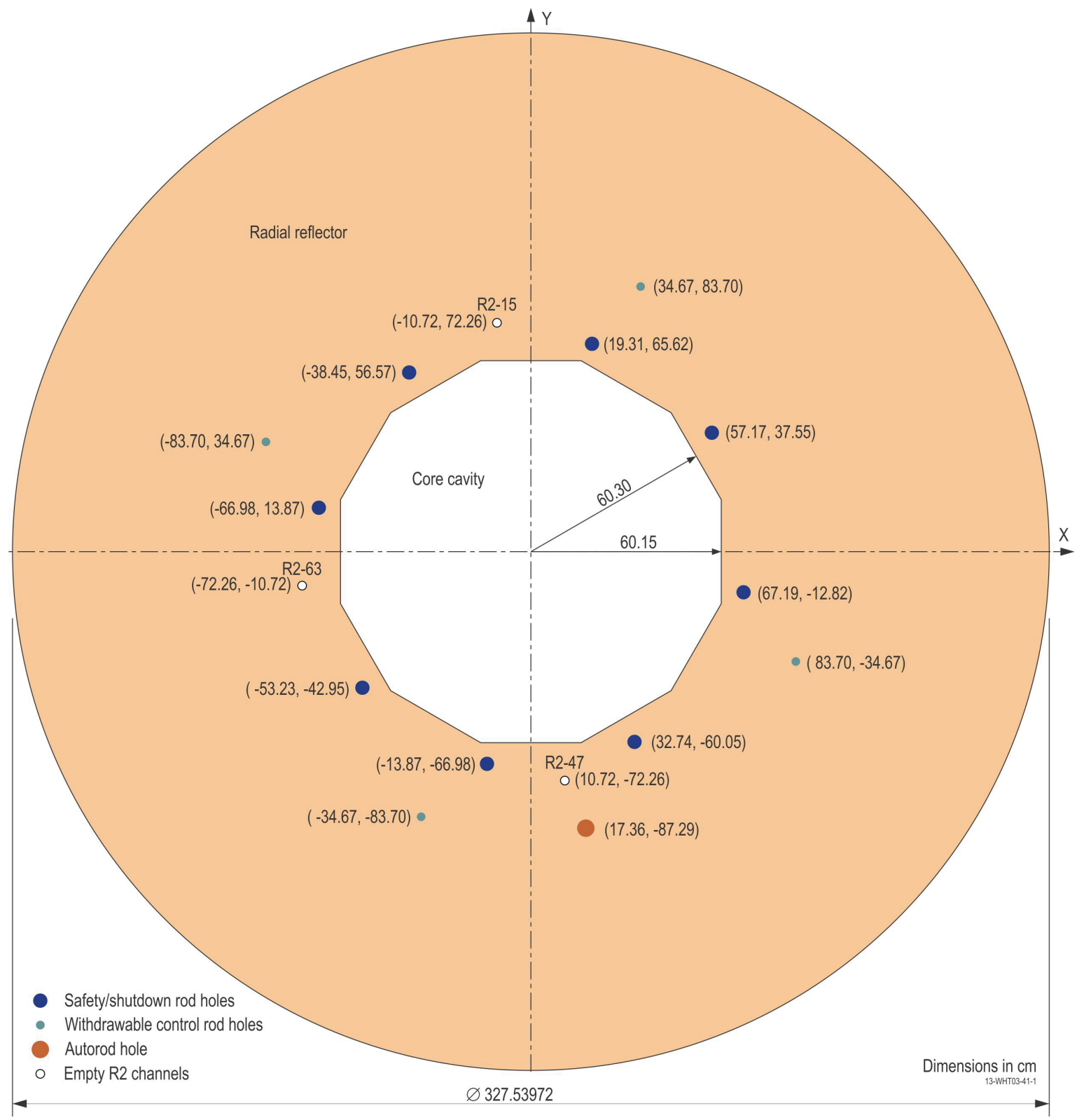

Figure F.1-1. Radial Reflector Surrounding Core Cavity Region Including Empty R2 Channel Positions.

Revision: 1 
NEA/NSC/DOC(2006)1

\section{Gas Cooled (Thermal) Reactor - GCR \\ PROTEUS-GCR-EXP-004 \\ CRIT-REAC}

\section{F.2.2 Material Data Supplemental Data}

\section{F.2.2.1 Graphite Plug Worths}

The graphite plugs have the compositions provided in Table F.1-2, which is the same plug graphite used in the upper axial reflector in Section 3.1.3.2.

Table F.1-2. Graphite Plug Compositions.

\begin{tabular}{|c|c||}
\hline Component & $\begin{array}{c}\text { Plugs in } \\
\text { Radial Reflector }\end{array}$ \\
\hline \hline Isotope/Element & Atoms/barn-cm \\
\hline${ }^{10} \mathrm{~B}$ & $2.3422 \mathrm{E}-08$ \\
${ }^{11} \mathrm{~B}$ & $9.4278 \mathrm{E}-08$ \\
$\mathrm{C}$ & $8.8496 \mathrm{E}-02$ \\
\hline \hline Total & $8.8496 \mathrm{E}-02$ \\
\hline \hline Mass Density $\left(\mathbf{g} / \mathbf{c m}^{\mathbf{3}}\right)$ & 1.765 \\
\hline
\end{tabular}

\section{F.3 Sample Calculations for Scaled Measurements}

The benchmark models described in Section 3.1 were modified as discussed in this appendix and used with MCNP5-1.60 (see Appendix A.1 and A.4 for input deck descriptions) and ENDF/B-VII.0 neutron cross section data. For additional details regarding how the TRISO particles were modeled, see Section 4.1. Monte Carlo calculations were performed with 1,650 generations with 100,000 neutrons per generation. The $\mathrm{k}_{\text {eff }}$ estimates are based on 150 skipped generations and a total of $150,000,000$ neutron histories each.

The difference between various configurations, as described in Section 3.4.2, were simulated to calculate reactivity worths $(\Delta \mathrm{k} / \mathrm{k})$. These worths were then converted into units of $\rho(\$)$ using a $\beta_{\text {eff }}$ value of $0.00693 \pm 0.00035(5 \%, 1 \sigma)$ for Core 9 and $0.00685 \pm 0.00034(5 \%, 1 \sigma)$ for Core 10 . The Monte Carlo statistical uncertainty is approximately $\$ 0.01$. The uncertainty in the calculated values provided in this section also include the uncertainty in $\beta_{\text {eff; }}$, therefore, calculations using additional neutron cross section libraries were not performed.

The worth of a control rod is calculated using the following equation:

$\rho(\$)=\frac{k_{\text {inserted }}-k_{\text {withdrawn }}}{k_{\text {inserted }} \times k_{\text {withdrawn }}} \times \frac{1}{\beta_{e f f}}$.

Worth calculations for other parameters are similarly calculated by comparing the eigenvalues for configurations both with and without a given reactor component.

Results are provided in Table F.1-3 for Core 9 and Table F.1-4 for Core 10. There is generally good agreement between calculated and scaled worths. Most calculations are within $1 \sigma$ to $2 \sigma$ of the scaled experimental values. At the time of this evaluation the statistical uncertainty in MCNP calculations of the HTR-PROTEUS models is $\sim 1 \notin$; it is not practical to further reduce this uncertainty with currently available computing resources. 
NEA/NSC/DOC(2006)1

\section{Gas Cooled (Thermal) Reactor - GCR \\ PROTEUS-GCR-EXP-004 \\ CRIT-REAC}

Table F.1-3. Sample Calculations for Reactivity Effects Scaled Measurements (Core 9).

\begin{tabular}{|c|c|c|c|c|c|c|c|c|c|c|}
\hline \multirow[t]{2}{*}{ Case } & \multirow[t]{2}{*}{ Measured Parameter } & \multicolumn{3}{|c|}{$\begin{array}{l}\text { Scaled } \\
\text { Worth }\end{array}$} & \multicolumn{3}{|c|}{$\begin{array}{l}\text { Calculated } \\
\text { Worth }\end{array}$} & \multirow{2}{*}{$\frac{C-E}{E}(\%)$} & \multirow[t]{2}{*}{ \pm} & \multirow{2}{*}{$\sigma^{(\mathbf{a})}$} \\
\hline & & $\rho(\$)$ & \pm & $\sigma$ & $\rho(\$)$ & \pm & $\sigma$ & & & \\
\hline 1.F-1 & Autorod Rest Worth & -0.13 & \pm & 0.01 & -0.13 & \pm & 0.02 & 0 & \pm & 17 \\
\hline 1.F-2 & $\begin{array}{c}\text { Graphite in Control Rod } \\
\text { Channels }\end{array}$ & -0.026 & \pm & 0.003 & -0.02 & \pm & 0.01 & -23 & \pm & 39 \\
\hline 1.F-3 & $\begin{array}{c}\text { Graphite in Empty Channels: } \\
\text { R2 }-15,-47, \&-63\end{array}$ & -0.05 & \pm & 0.01 & -0.04 & \pm & 0.01 & -20 & \pm & 26 \\
\hline
\end{tabular}

(a) The uncertainty in $\frac{C-E}{E}(\%)$ is calculated by propagating the uncertainties in both the calculated and scaled experiment eigenvalues using the following equation: $\sigma=100 \% \times \sqrt{\left(\frac{\sigma_{C}}{E}\right)^{2}+\left(\frac{\sigma_{E} C}{E^{2}}\right)^{2}}$.

Table F.1-4. Sample Calculations for Reactivity Effects Scaled Measurements (Core 10).

\begin{tabular}{|c|c|c|c|c|c|c|c|c|c|c|}
\hline \multirow[t]{2}{*}{ Case } & \multirow[t]{2}{*}{ Measured Parameter } & \multicolumn{3}{|c|}{$\begin{array}{l}\text { Scaled } \\
\text { Worth }\end{array}$} & \multicolumn{3}{|c|}{$\begin{array}{l}\text { Calculated } \\
\text { Worth }\end{array}$} & \multirow{2}{*}{$\frac{C-E}{E}(\%)$} & \multirow{2}{*}{\multicolumn{2}{|c|}{$\pm \sigma^{(a)}$}} \\
\hline & & $\rho(\$)$ & \pm & $\sigma$ & $\rho(\$)$ & \pm & $\boldsymbol{\sigma}$ & & & \\
\hline 2.F-1 & Autorod Rest Worth & -0.081 & & 0.005 & -0.09 & $\perp$ & 0.01 & 11 & \pm & 14 \\
\hline 2.F-2 & $\begin{array}{c}\text { Graphite in Control Rod } \\
\text { Channels }\end{array}$ & -0.021 & \pm & 0.002 & -0.01 & \pm & 0.01 & -52 & \pm & 48 \\
\hline 2.F-3 & $\begin{array}{c}\text { Graphite in Empty Channels: } \\
\text { R2 }-15,-47, \&-63\end{array}$ & -0.04 & \pm & 0.01 & -0.05 & \pm & 0.01 & 25 & \pm & 40 \\
\hline
\end{tabular}

(a) The uncertainty in $\frac{C-E}{E}(\%)$ is calculated by propagating the uncertainties in both the calculated and scaled experiment eigenvalues using the following equation: $\sigma=100 \% \times \sqrt{\left(\frac{\sigma_{C}}{E}\right)^{2}+\left(\frac{\sigma_{E} C}{E^{2}}\right)^{2}}$. 